\title{
Neural network modeling of a dolphin's sonar discrimination capabilities
}

\author{
Andersen, Lars Nonboe; René Rasmussen, A; Au, WWL; Nachtigall, PE; Roitblat, H.
}

Published in:

Acoustical Society of America. Journal

Link to article, DOI:

10.1121/1.410770

Publication date:

1994

Document Version

Publisher's PDF, also known as Version of record

Link back to DTU Orbit

Citation (APA):

Andersen, L. N., René Rasmussen, A., Au, WWL., Nachtigall, PE., \& Roitblat, H. (1994). Neural network modeling of a dolphin's sonar discrimination capabilities. Acoustical Society of America. Journal, 96(5), 33163316. https://doi.org/10.1121/1.410770

\section{General rights}

Copyright and moral rights for the publications made accessible in the public portal are retained by the authors and/or other copyright owners and it is a condition of accessing publications that users recognise and abide by the legal requirements associated with these rights.

- Users may download and print one copy of any publication from the public portal for the purpose of private study or research.

- You may not further distribute the material or use it for any profit-making activity or commercial gain

- You may freely distribute the URL identifying the publication in the public portal

If you believe that this document breaches copyright please contact us providing details, and we will remove access to the work immediately and investigate your claim. 
The 128th Meeting of the Acoustical Society of America

Stouffer Austin Hotel Austin, Texas 28 November-2 December 1994

NOTE: All Journal articles and Letters to the Editor are peer reviewed before publication. Program abstracts, however, are not reviewed before publication, since we are prohibited by time and schedule.

\title{
Session 1aAO
}

\section{Acoustical Oceanography: Acoustic Inversions for Properties of Gaseous Sediments}

\author{
Michael D. Richardson, Chair \\ Naval Research Laboratory, Stennis Space Center, Mississippi 39529-5004 \\ Chair's Introduction-8:00 \\ Invited Papers
}

8:05

1aA01. Biogeochemical processes controlling gas bubble production and distribution in organic-rich sediments. Christopher S. Martens (Marine Sci., CB-3300, Univ. of North Carolina, Chapel Hill, NC 27599-3300), Daniel B. Albert (Univ. of North Carolina, Chapel Hill, NC), Hannelore Fiedler (Forschungsanstalt der Bundeswehr fur Wasserschall und Geophysik, 2300 Kiel, Germany), and Friedrich Abegg (Geologisch-Palaontologisches Institut und Museum der Universitat Kiel, 2300 Kiel, Germany)

Biogeochemical processes in organic-rich, muddy sediments often result in the net production of biogenic gases including methane. In coastal sediments, methane production ultimately leads to near saturation gas concentrations and bubble formation. Rates of production, oxidation, and transport processes, together with in situ temperature and pressure (depth), combine to determine the actual sediment column depth of methane bubble occurrence. Recent studies along North Carolina's Outer Banks and Eckemfoerde Bay in the Baltic Sea reveal how these processes combine to control saturation gas concentrations and bubble distributions in the upper few meters of coastal sediments. At the North Carolina site, gas production depths vary seasonally, resulting in a bubble layer whose shallowest depth oscillates between 10 - and $30-\mathrm{cm}$ depth from summer to winter, respectively. Large quantities of gas escape the sediments via diffusion and bubble ebullition during the warm months. Similar oscillations in the depth of the bubble (acoustic absorption) layer appear to occur in the sediments of Eckernfoerde Bay; however, competing microbial processes prevent saturation methane concentrations at depths above approximately $50 \mathrm{~cm}$. Stable isotope measurements reveal that microbial methane oxidation consumes methane transported above the bubble layer, resulting in little release of gas into the water column.

$8: 25$

1aAO2. Predictions of the acoustic response of free-methane bubbles in muddy sediments. Anthony $\mathrm{P}$. Lyons, Michael $\mathrm{E}$. Duncan (Dept. of Oceanogr., Texas A\&M Univ., College Station, TX 77843-3146), James A. Hawkins, Jr. (Naval Res. Lab., Stennis Space Center, MS 39529-5004), and Aubrey L. Anderson (Texas A\&M Univ., College Station, TX 77843-3146)

The response of the sediments of Eckernfoerde Bay, Germany to acoustic remote sensing has been attributed to gas features found within the sediment. The existence of features as small as $0.5 \mathrm{~mm}$ equivalent spherical radius has been confirmed by $\mathrm{x}$-ray computed tomography of cores taken and scanned under in situ pressures. The interaction of an acoustic pulse from the Acoustic Sediment Classification System (ASCS) with this type of gassy sediment was modeled. The bubble scattering response included the effects of shear modulus and nonspherical bubbles. Model predictions made using the observed gas feature distribution and normal incidence ASCS data agree and show extended returns (greater than a pulse length) from the seafloor bubble layers as well as high attenuation 
within the bubble layers. These results show that the acoustic returns from the gas bubble layers are probably due to scattering and that differences in level of return can be attributed to random variations in the distributions of scatterers.

\section{8:45}

1aAO3. The effects of free-methane bubbles on the propagation and scattering of compressional and shear wave energy in muddy sediments. Michael D. Richardson, Sean R. Griffin, Kevin B. Briggs (Naval Res. Lab., Stennis Space Center, MS 39529-5004), Aubrey L. Anderson, and Anthony P. Lyons (Texas A\&M Univ., College Station, TX 77843-3146)

Free-methane bubbles cause significant scattering of acoustic energy in the soft sediments of Eckernfoerde Bay, Baltic Sea. In situ and laboratory measurement of sediment geoacoustic and physical properties were made in an attempt to understand the physical mechanisms responsible for this scattering. In situ shear wave velocities (at 100-500 Hz) increased from 5-7 near the surface to 15-20 $\mathrm{m} \mathrm{s}^{-1}$ at $2 \mathrm{~m}$ into the seafloor, whereas in situ compressional wave velocities (at 38 and $58 \mathrm{kHz}$ ) varied little $\left(\sim 1425 \mathrm{~m} \mathrm{~s}^{-1}\right.$ ) with depth. Methane bubbles apparently caused significant attenuation of compressional waves at depths below $1 \mathrm{~m}$, whereas shear wave attenuation was unaffected by gas and decreased with depth. Compressional waves (at $400 \mathrm{kHz}$ ) in cores (1\%-5\% free gas) maintained at in situ pressures were highly attenuated but show little evidence of velocity dispersion. Comparison of geoacoustic data with theory suggest that primary control of the interaction of acoustic profilers with this gassy seafloor is by bubbles larger than about $1 \mathrm{~mm}$ diameter and that the observed high-frequency scattering can be described by models of the seafloor bubbles as individual scatters of acoustic energy. Response of the seafloor as a medium with modified bulk propagation properties would occur at lower frequencies than those used in the Eckernfoerde experiments.

\section{9:05}

1aA04. Modeling of high-frequency acoustic wave scattering by sediment gas voids. Dajun Tang (Dept. of Appl. Ocean Phys. and Eng., Wonds Hole Oceanographic Inst., Woods Hole, MA 02543)

Scattering by volumetric inhomogeneities of marine sediments comes in many forms. It is found in a 1993 CBBL-SRP experiment conducted in Eckernfoerde Bay, Germany, that high-frequency backscattering is caused by gas voids buried at about a meter beneath the seafloor [Tang et al., J. Acoust. Soc. Am. (to be published)]. The backscattering strength at $40 \mathrm{kHz}$ is estimated to be $-10 \mathrm{~dB}$. Assuming the gas voids do not resonate, a simple scattering model is developed based on the Kirchhoff approximation to calculate the backscattering and bistatic scattering strength. Acoustic ray bending due to the sound-speed discontinuity at the water-bottom interface as well as sediment attenuation are taken into account. We find that at $40 \mathrm{kHz}$, only those gas voids whose exposed cross section is larger than the acoustic wavelength contribute to backscattering significantly. The gas void distribution is estimated based on data from the few cores obtained in situ. The model results are compared with backscattering data, and it is intended that this model be used to compare with bistatic scattering data in the future. [Work supported by ONR through NRL.]

\section{9:25-9:40 Break}

\section{Contributed Papers}

$$
\text { 9:40 }
$$

1aA05. Geophysical ground-truthing experiments in Eckernfoerde Bay. Angela Davis, Dei Huws, and Ron Haynes (School of Ocean Sci., Univ. College of North Wales, Menai Bridge, Gwynedd LL59 5EY, U.K.)

During the 1994 Coastal Benthic Boundary Layer Special Research Program's (CBBLSRP) experiment in Eckernfoerde Bay, multichannel digital seismic and electrical resistivity data were collected using surface and bottom towed arrays. Profiling with a bottom towed sledge yielded shear wave velocity and electrical resistivity data indicative of the structural strength of the sediment and of the properties of the sediment's pore space. Shear wave velocities for the gassy mud were, as expected, extremely low, ranging from $<10$ at the surface to around $16 \mathrm{~m} / \mathrm{s}$ at $2 \mathrm{~m}$. Variations in electrical properties were correlatable with lithological change. It is anticipated that analysis of reflection responses will provide significant additional geotechnical ground-truthing.

\section{9:55}

1aAO6. High-frequency bottom hackscattering: Volume scattering from gassy mud. Kevin B. Briggs, Michael D. Richardson (Seafloor Sci. Branch, Naval Res. Lab., Stennis Space Center, MS 39529), and Darrell R. Jackson (Appl. Phys. Lab., Seattle, WA 98195)

Bottom backscattering data at $\mathbf{4 0} \mathrm{kHz}$ were collected from a stationary tripod deployed in a muddy embayment characterized by subsurface methane gas. Near-surface sediment geoacoustic, physical, and roughness properties were measured concurrently with the acoustic data in order to compare model predictions with measured backscatter strength as a function of grazing angle. In situ values for sediment compressional wave velocity and attenuation, sediment density values from diver-collected cores, and values for interface roughness parameters from underwater photogrammetry were used to calculate the parameters for the composite roughness model. The model prediction for scattering strength from the sediment-water interface fell well below the measured value, indicating that volume scattering from within the sediment was the dominant process. Data obtained by other investigators reveal a layer of free methane localed approximately $1 \mathrm{~m}$ below the sediment-water interface. These gas bubbles were evidently responsible for the high level of backscattering observed. A fit of the volume scattering component of the composite-roughness model to the data provides a measure of the contribution of these bubbles to acoustic backscatter.

\section{0:10}

1aAO7. Double monopole resonance of a gas-filled cavity in a sediment. Steven G. Kargl, Kevin L. Williams (Appl. Phys. Lab., Univ. of Washington, 1013 N.E. 40th St., Seattle, WA 98105), and Raymond Lim (Coastal Systems Station, Panama City, FL 32407-7001)

The monopole response of a gas-filled, spherical cavity in a sediment is investigated. The sediment is a saturated, poroelastic medium and we assume that its dynamical behavior satisfies a homogeneous Biot theory. Our method entails the scattering of an incident, (fast) longitudinal field that preferentially excites a monopole response of the cavity. Our main result demonstrates that a gas-filled, spherical cavity in a saturated, poroelastic medium exhibits two distinct monopole resonances. These resonances arise from the two distinct longitudinal modes of propagation in saturated, poroelastic medium as described by Biot's theory. The sensitivity of these resonances to various environmental parameters (such as per- 
meability and porosity) will be presented and discussed. [Work supported by ONR.]

\section{0:25}

1aA08. Acoustic backscatter from bubbles confined in sediment pores. Frank A. Boyle and Nicholas P. Chotiros (Appl. Res. Labs., Univ. of Texas at Austin, P.O. Box 8029, Austin, TX 78713-8029)

A model for acoustic backscatter from trapped gas bubbles in sandy sediments was recently presented [F. A. Boyle and N. P. Chotiros, J. Acoust. Soc. Am. 93, 2397(A) (1993)]. One of the assumptions was that trapped bubbles respond to an ambient acoustic field as if they were free bubbles surrounded by an infinite volume of water. A refinement to this model includes the effects of solid particles surrounding and constraining the fluid around the bubbles. A new expression for the sediment backscattering strength accounts for fluid confinement in pores. This confinement affects sediment acoustic impedances, bubble resonances, and scattering cross sections. Biot fast and slow compressional waves are treated separately. [Work supported by Naval Res. Lab., Stennis Space Center.]

\section{0:40}

1aA09. Bistatic acoustic scattering from trapped gas bubbles in sandy sediments. Frank A. Boyle and Nicholas P. Chotiros (Appl. Res. Labs., Univ. of Texas at Austin, P.O. Box 8029, Austin, TX 78713-8029)

A technique based on acoustic reciprocity has recently been used to calculate backscattering strengths of marine sediments [F. A. Boyle and $N$. P. Chotiros, J. Acoust. Soc. Am. 93, 2397(A) (1993)]. The method begins with computation of the pressure induced at an element of scattering volume in the sediment. The pressure returned to a projector is then obtained via a reciprocal relationship between source and scatterer. A similar technique is developed to permit the calculation of bistatic scattering strengths. This technique is combined with a Biot model for acoustic penetration and a scattering model involving trapped bubble resonance scattering, to arrive at a bistatic sandy sediment acoustic scattering model. [Work supported by Naval Res. Lab. Stennis Space Center under the MCM Tactical Environmental Data System (MTEDS) project.]

\section{0:55}

1aAO10. Experimental determination of broadband propagation under known geophysical conditions. Mohsen Badiey (Office of Naval Res., 800 N. Quincy St., Arlington, VA 22217-5666), Mike Steele (Bolt Beranek and Newman Syst. and Technol., Arlington, VA 22209), William Carey (Adv. Res. Project Agency, Arlington, VA 22203-1714), and Pejhan Peymani (Bolt Beranek and Newman Syst. Technol., Arlington, VA 22209)

Acoustic propagation experiments were conducted with explosives, an air gun, and a continuous source on the New Jersey Continental shelf near the AMCOR borehole 6010. This particular area was extensively surveyed by Davies et al. [Marine Geol. 108, 323-343 (1992)] and is the site of previous acoustic experiments by Carey et al. [Saclantcen, CP 42, a1-a27 (1993)]. Environmental measurements of temperature, conductivity, salinity, sound speed, and bathymetry were made and the signals were received on a vertical array at a sufficient distance from the known source positions. Frequency-time analysis allows for the determination of the dispersive group velocities. The relative position of the sources provides for the assessment of sub-bottom variations on the acoustic propagation. Preliminary results are presented and interpreted in light of normal-mode theory.

\section{1:10}

1aA011. A dynamic penetrometer for rapid assessment of seafloor parameters. T. Akal (SACLANT Undersea Res. Ctr., 19138 San Bartolomeo, La Spezia, Italy) and R. D. Stoll (Lamont-Doherty Earth Observatory of Columbia Univ., Palisades, NY 10964)

Information on geoacoustic and geotechnical parameters of the seafloor is important in sonar performance evaluation as well as in engineering applications. Since measurement of these parameters using traditional methods usually involves some rather elaborate experimental procedures, there is the need for a simple system that can remotely measure some of these parameters from a moving ship or aircraft. To accomplish this a new technique using a probe similar to the expendable bathythermograph (XBT) has been developed wherein the thermistor used to measure water temperature is replaced by an accelerometer. When the probe first impacts the bottom there is rapid deceleration controlled by the shear strength of the sediment, followed by a period of damped oscillation with frequency dependent on the geoacoustic properties of the sediment. Thus when the impact signature is fully analyzed it is possible to obtain information on both the shearing strength of the bottom as well as the geoacoustic properties. At present a series of laboratory and field tests are being carried out in a cooperative program at Lamont-Doherty Earth Observatory and SACLANT Centre. Preliminary results of this work are presented in this paper. [Work supported by ONR.]

\section{1:25}

1aAO12. Shear wave attenuation estimates from inversion of Scholte wave data. Hassan B. Ali and Michael K. Broadhead (Code 7173, Naval Res. Lab., Stennis Space Center, MS 39529-5004)

On the basis of the correlation between sediment stratigraphy and modal dispersion, inversion of Scholte wave data allows one to estimate the shear wave velocities within the sediments. In some cases, this may be a sufficient characterization of the sediments. However, shear velocities contain only part of the wave information, and this will not generally suffice for recovery of the full measured time series. In realistic sediments, a propagating pulse will also be affected by the attenuation profile of the medium. Moreover, the amplitudes of the constituent frequencies will generally be attenuated differently, resulting in distortion of the pulse. Using the results of recent deep-water measurements, examples are presented of the relationship between sediment stratigraphy and Scholte wave modal dispersion. The modeling is then extended to examination of seismogram parameters, using iterative analysis of the shear attenuation profiles and exploitation of the spectral properties of the time series. It is shown that the shear $Q$ profile is crucial in achieving an adequate match to the measured data, but some degree of nonuniqueness is possible. [Work supported by Office of Naval Res., Prog. Element No. 0601153N, with technical management provided by the Naval Res. Lab.]

\section{1:40-11:45 Break}

\section{1:45-12:15}

\section{PANEL DISCUSSION:}

Panel Moderator: Michael D. Richardson

Panel Members: Anthony P. Lyons, Christopher S. Martens, Dajun Tang 


\title{
Session 1aPA
}

\section{Physical Acoustics: Thermoacoustics}

\author{
Robert M. Keolian, Chair \\ Physics Department, Naval Postgraduate School, Monterey, California 93943
}

\section{Contributed Papers}

\section{9:00}

1aPA1. Numerical study of various thermoacoustic refrigerator configurations. Thomas J. Hofler (Phys. Dept., Naval Postgraduate School, Monterey, CA 93943)

The results of numerical models and optimizations for various configurations of thermoacoustic refrigerators will be presented. The refrigeration goals are large-scale refrigeration with low temperature span, high efficiency, and reasonably high power density. The physical equations used are the usual plane wave Rott formulation with improved solutions for the heat exchangers and shaped resonator ducts. The primary emphasis will be on solutions having the highest system efficiency, excluding losses associated with electrical drivers and secondary heat exchange.

\section{9:15}

1aPA2. Simplified model for the study of nonlinear processes in thermoacoustic engines. A. Prosperetti and M. Watanabe (Dept. of Mech. Eng., Johns Hopkins Univ., Baltimore, MD 21218)

Upon integrating the governing equations over the cross section of a thermoacoustic device, a simplified one-dimensional model is obtained. While only approximate, this model renders the study of nonlinear effects very amenable to analysis. In particular, for a thermoacoustic prime mover, the stability limits are calculated and the steady-state amplitude is estimated on the basis of a weakly nonlinear theory. For larger amplitudes, numerical results are presented. The marked propensity of the system to develop shock waves is found to be a very strong factor limiting its efficiency. [Work supported by the Office of Naval Research.]

$$
\text { 9:30 }
$$

1aPA3. A nonlinear analysis of a simple thermoacoustic system. Ronald E. Kumon (Appl. Res. Labs., Univ. Texas at Austin, 10000 Burnet Rd., Austin, TX 78713)

A simple thermoacoustic system was studied to try to better understand the interaction between the temperature, pressure, and velocity modes of the system. The system considered was a one-dimensional "tube," closed and isothermal at both ends and filled with a helium gas. Initially, the gas is static but with a sinusoidal temperature distribution. To obtain a simplified model of the system, a Galerkin-type method was applied to the full hydrodynamic equations in one spatial dimension and the ideal gas law equation of state. By substituting highly truncated sine and cosine series in the spatial variable with time-dependent amplitudes into the aforementioned PDEs, the model was reduced to a set of coupled nonlinear ODEs. First, these equations were linearized and examined for series expansions with different number of terms. Next, the nonlinear ODEs were studied. Finally, these results were compared with direct finite-difference calculations using MacCormack's method to integrate the full hydrodynamic equations.

\section{9:45}

1aPA4. Experimental study of acoustic turbulence and streaming in a thermoacoustic stack. D. Felipe Gaitan, Ashok Gopinath, and
Anthony A. Atchley (Phys. Dept., Naval Postgraduate School, Monterey, CA 93943)

Recent developments in thermoacoustic devices have generated a renewed interest in finite amplitude standing waves and the nonlinear effects associated with them. In a typical thermoacoustic device, a stack and two heat exchangers are placed inside a resonator approximately midway between the velocity and pressure antinodes. These elements, consisting of closely spaced rigid plates, present both a discontinuity in the fluid flow and a rigid boundary with which the fluid can interact. Under these conditions, at least two well-known effects may occur: acoustic streaming and turbulence. These effects are of particular interest since they could significantly affect the thermoacoustic heat transport inside the stack. In this study, the fluid velocity inside and near a stack was measured qualitatively and quantitatively using a stroboscopic lamp and a laser Doppler velocimeter, respectively. Measurements under different conditions will be presented and discussed. [Work supported by ONR.]

\section{0:00}

1aPA5. Numerical modeling of unsteady flow in the neighborhood of a thermoacoustic stack. A. S. Worlikar and O. M. Knio (Dept. of Mech. Eng., Johns Hopkins Univ., Baltimore, MD 21218)

A low-Mach-number compressible flow model for the simulation of acoustically driven flow fields within thermoacoustic couples is constructed. The model is based on the assumption that length of the thermoacoustic stack is much smaller than the wavelength of the driving standing wave. The latter assumption is used to obtain a simplified description of the impact of acoustic waves while retaining all of the essential features of the unsteady flow developing in the neighborhood of solid boundaries. Results of numerical simulations are presented which illustrate the nonlinear response of the flow to different driving amplitudes and frequencies. [Work supported by the Office of Naval Research.]

\section{0:15}

1aPA6. Experimental visualization of heat transfer and fluid flow processes in a thermoacoustic device. C. Herman, C. Bartscher, $M$ Wetzel, and M. Volejnik (Dept. of Mech. Eng., Johns Hopkins Univ., Baltimore, MD 21218-2686)

Different visualization techniques are used to gain insight into the heat transfer and fluid flow processes in a thermoacoustic refrigerator model. For this purpose, an enlarged model of a thermoacoustic device, with transparent viewing windows in regions of interest, was built. The model operates with air at atmospheric pressure as the working fluid. The cross section of the resonant tube is rectangular to obtain essentially twodimensional flow and temperature fields and to allow transillumination of the stack region with parallel laser light. On-line holographic interferometry combined with high-speed cinematography is used to analyze and measure the unsteady oscillating temperature fields in the stack region. The design also allows the visualization of the flow fields by smoke injection and the visualization of the temperature distribution on the stack plates using thermochromatic liquid crystals. The results of the visualization experiments provide new information on the stability and transition of the 
flow in the stack region and on the heat transfer processes. [Work supported by the Office of Naval Research.]

\section{0:30-10:45 Break}

\section{0:45}

1aPA7. A thermoacoustic pin stack F. Scott Nessler and Robert M. Keolian (Dept. of Phys., Code $\mathrm{PH} / \mathrm{Kn}$, Naval Posigraduate School, Monterey, CA 93943)

A comparison of the pin stack geometry with a conventional rolled geometry for thermoacoustic engines is being made in a modular prime mover test rig. By decreasing viscous energy losses, it is hoped that the pin stack will improve the efficiencies of thermoacoustic engines. The stack will consist of over two thousand $75-\mu \mathrm{m}$ wires, separated by $750 \mu \mathrm{m}$, in a hexagonal lattice hand sewn between two finned copper heat exchangers. A temperature gradient will be applied to the stack and the neon working fluid by holding one exchanger at $300 \mathrm{~K}$ and the other at $77 \mathrm{~K}$. A small acoustic driver in the rig will allow us to measure the quality factor $Q$ below onset as a function of the neon pressure. [Work supported by ONR.]

\section{1:00}

1aPA8. Thermoacoustic sound source in the Helmholtz limit. Richard Raspet, Jay A. Lightfoot, James R. Belcher, and Henry E. Bass (Dept. of Phys. and Astron., Univ. of Mississippi, University, MS 38677)

Thermoacoustic engines are placed in resonant cavities for $Q$ amplification. Variations in the cross-sectional area of the resonator serve to reduce device volume and to minimize nonlinear distortion by detuning higher harmonics. In the case of a thermoacoustic sound source, these cross-sectional variations in the resonator area may be carried to an extreme such that the resonator approaches the Helmholtz limit. This limit produces a dimensionally compact, low-frequency thermoacoustic sound source. A thermoacoustic sound source in the Helmholtz limit has been constructed. The measured particle velocity and acoustic pressure in the device will be compared to Helmholtz idealization. [Work supported by ONR.]

\section{$11: 15$}

1aPA9. Radial versus plane wave thermoacoustic engines: Which is best? W. Patrick Arnott (Atmospheric Sci. Ctr., Desert Res. Inst., P.O. Box 60220, Reno, NV 89506), Jay Lightfoot, Richard Raspet, and Henry E. Bass (Univ. of Mississippi, University, MS 38677)

Most previous work in thermoacoustics has considered placing the elements in plane wave resonators. The lowest radial or breathing mode of a cylindrical resonator is also potentially useful for producing thermo- acoustic sound sources or refrigerators. An immediate advantage of the radial geometry is the separation of higher modal frequencies from the higher harmonics of the fundamental when the engine is working at large amplitude. Criteria recently developed for deciding which geometry is best will be presented. This will be followed by a comparison of plane and radial wave refrigerators and sound sources. The short stack approximation for both geometries will be used in the comparison. Our derivation of this approximation is not limited by the additional boundary layer approximation. [Work supported by ONR.]

\section{$11: 30$}

1aPA10. Design of a radial mode thermoacoustic prime mover (sound source) and experimental observations. Jay A. Lightfoot (Dept. of Phys. and Astron., Univ. of Mississippi, University, MS 38677), W. Patrick Arnott (Univ. of Nevada, Reno, NV 89506), Richard Raspet, James R. Belcher, and Henry E. Bass (Univ. of Mississippi, University, MS 38677)

Experimental thermoacoustics has been limited in the past to plane wave systems. Swift briefly discussed the radial mode thermoacoustic engine, but no working models have been built. Radial modes of a cylindrical resonator are naturally anharmonic; thus harmonic generation at high sound-pressure levels by nonlinear processes tend to be less resonance enhanced in the radial mode, potentially leading to higher sound-pressure levels in the fundamental radial mode of oscillation. A radial mode prime mover which is theoretically optimized for a $60 \%$ helium, $40 \%$ argon gas mixture (mole fraction) at atmospheric pressure has been constructed. Details of the design and construction, measurement of the power dissipa ed in the hot heat exchanger (required for onset of self oscillation) as a function of ambient pressure, and harmonic distortion of the fundamental mode of oscillation will be presented. [Work supported by ONR.]

\section{1:45}

1aPA11. Investigation of parametric drive of a longitudinal gas filled resonance tube. Richard Raspet, Bruce Denardo, James Brewster, and John Kordomenos (Dept. of Phys. and Astron., Univ. of Mississippi, University, MS 38677)

High-power drives for thermoacoustic refrigerators are being investigated by several groups. Theoretical calculations have been performed of the feasibility of parametrically driving a longitudinal resonance tube by modulating the temperature with a high-power laser. Parametric drives are promising since the drive mechanism is distributed over the entire volume of gas and because the response may become large before saturation occurs. Although it is demonstrated that laser drive is not attractive as a practical means of high-power drive for longitudinal resonators, much interesting physics has been considered in the analysis of the proposed system and will be discussed. [Work supported by ONR.] 


\title{
Session 1pAO
}

\section{Acoustical Oceanography: Moderate-to-High Frequency Inversions for Sediment Properties}

\author{
Darrell R. Jackson, Chair \\ Applied Physics Laboratory, University of Washington, Seattle, Washington 98105
}

Chair's Introduction-1:30

Invited Papers

1:35

1pAO1. Three-dimensional velocity fluctuation structure of the seabed imaged by high-frequency crosswell tomography. Tokuo Yamamoto (Appl. Marine Phys. Div., RSMAS, Univ. of Miami, Miami, FL 33149)

High-resolution images of the compressional wave velocity fluctuation structure of the seabed are inverted from the travel times measured by high-frequency $(1-50 \mathrm{kHz})$ crosswell acoustic tomography experiments at three different sites in shallow water. The three-dimensional power spectra of the velocity fluctuations are determined from the velocity images. The velocity fluctuation spectra are anisotropic in general, i.e., the fluctuation frequency in the vertical direction is much higher than in the horizontal direction. The aspect ratio of the two ranges from 4 to 10 . In addition, the major and minor axes of anisolropy are often tilted from the vertical and the horizontal direction. The angle of tilt, called dip, is found as large as $30^{\circ}$. The intensity of the fluctuation spectrum depends on the sediment type. These parameters of the three-dimensional power spectrum affect the scatlering of acouslic waves. The strong dependence of acoustic backscattering on the grazing and azimuthal angle observed by Jackson and Briggs (1993) is excellently predicted when the anisotropy and the dip structure of the velocity fluctuations are incorporated in an analytical model of scallering by sediment volume fluctuation (Yamamoto, this meeting). [Work supported by ONR.]

\section{$1: 55$}

1pA02. FM sonar characteristics for normal-incidence sediment classification. Steven G. Schock and Lester R. LeBlanc (Dept. of Ocean Eng., Florida Atlantic Univ., Boca Raton, FL 33431)

The prediction of sediment properties from normal-incidence acoustic measurements made with a broadband sub-bottom profiler requires that several acoustic parameters be estimated from the reflection data using independent numerical techniques to reduce the large potential errors of any one parameter. Examples of acoustic parameters that are combined empirically to estimate the vertical profiles of physical sediment properties are acoustic impedance, inlerlayer volume scattering, and attenuation. From the analysis of normal-incidence data collected in many depositional environments, the characteristics of a quantitative sub-bottom profiler for estimating impedance and volume scattering include: (1) the acoustic bandwidth of the transmitted pulse must be at least 2 oct for the reliable measurement of signal phase from interlayer reflections; (2) the two-way transmission/reception beam should be a cone with a width between $15^{\circ}$ and $20^{\circ}$ at the 3-dB down points to ensure that interlayer reflection amplitude to volume and surface scattering noise ratios are at least $6 \mathrm{~dB}$ (narrower beamwidths result in reflection amplitude measurement errors from sensor motion or seafloor slopes less than $5^{\circ}$ ); (3) the transmitted pulses contain energy from $500 \mathrm{~Hz}$ to $10 \mathrm{kHz}$ to ensure most interlayer impedance gradients can be accurately measured.

$$
\text { 2:15 }
$$

1pA03. Acoustic prediction of sediment impedance. Douglas N. Lambert, Donald J. Walter (Naval Res. Lab., Code 7431, Stennis Space Center, MS 39529), William R. Bryant, Niall C. Slowey (Texas A\&M Univ., College Station, TX 77843), and John C. Cranford (Neptune Sci., Inc., Slidell, LA 70458)

The Naval Research Laboratory has been developing a normal-incidence, high-frequency $(15-30 \mathrm{kHz})$, narrow beamwidth $\left(6^{\circ}-\right.$ $12^{\circ}$ ), high-resolution (>92-dB dynamic range) seismic system with the capability to predict, in near real time, acoustic inpedance of the upper several meters of the seafloor using inversion techniques. Acoustic impedance, predicted in a series of ten selectable time windows, is then used to estimate other sediment properties through empirical relationships. A series of ground truth sediment cores have been collected along a seismic trackline in the southwestern Baltic Sea with sediment types varying from glacial till to soft, methane gas-charged clayey silts. Comparison of the high-resolution seismic data to sediment structure determined from the cores shows excellent correlation for both $15-$ and $30-\mathrm{kHz}$ data. The comparison of laboratory-measured sediment geotechnical properties and acoustically estimated properties shows good correlation in the surficial sediments and somewhat less correlation with depth in the sediments. Since gas bubbles within the sediment are strong acoustic reflectors at these frequencies, the use of standard algorithms for nongassy sediments can lead to overestimation of predicted impedance values.

1pAO4. Measurements of relative bottom backscattering strength by a digital side scan sonar. Gunther Fechner and Ingo $\mathrm{H}$. Stender (Forschungsanstalt der Bundeswehr für Wasserschall-und Geophys., D-24148 Kiel, Germany)

A conventional side scan sonar gives only a qualitative image of seafloor backscatter distribution. It does not allow quantitative measurements of the bottom backscattering strength. Because of the advantages of the side scan sonar (imaging and profiling capacity 
and easy handling), a conventional 100-kHz side scan sonar was redesigned. Signal generation and transducers remained unchanged. The received raw signal is amplified, digitized at $20 \mathrm{kHz}$, and stored on a 486 personal computer. Data are displayed as color coded images on screen and hard copy. Attenuation with distance and angle dependency (using Lambert's law) are compensated. This digital attachment provides relative backscatter data. On the muddy seafloor of the CBBL/JOBEX experimental site in Eckernförde Bay the digital side scan sonar shows a rather uniform bottom with low backscatter values. The spatial variation is less than $8 \mathrm{~dB}$, mainly caused by trawl marks on the bottom. But inside one pockmark the backscatter strength varies as much as $28 \mathrm{~dB}$. This high variation cannot be attributed to sediment changes; all sediment samples inside the pockmark were mud. The strong backscatter is presumably caused by the gassy layer inside the pockmark which nearly coincides with the water-sediment interface.

1pAO5. Acoustic characterization of marine sediments: State of the art. Anatoliy N. Ivakin (N. N. Andreev Acoust. Inst., Shvernika 4, Moscow 117036, Russia)

A review of recent theoretical and experimental investigations of sound scattering from the seafloor and analysis of methods for remote acoustic characterization of marine sediments are presented. For narrow-band signals, echo characteristics have explicit relationships (in some simple cases they are known) to the reflection and scattering coefficients, which can be related in turn to the material parameters of the medium and used for determination of bottom properties. For wideband signals, the relations between the medium and conventional echo characteristics are not so evident. Moreover, one must introduce other characteristics, such as the two-frequency scattering function. Methods for the determination of bottom parameters in this case are not sufficiently studied up to now, but some advantages of wideband signals can be used such as their high spatial resolution and the possibility of their analysis in the time-frequency domain. The second aspect of the problem is connected with modeling the process of sound interaction with different bottom media. Several geoacoustic models for moderate to high frequencies are considered, taking into account regular sediment stratification and different types irregularity: roughness of the interfaces, volume inhomogeneities, and discrete scatterers.

$$
\text { 3:15-3:30 Break }
$$

\section{Contributed Papers}

\section{3:30}

1pAO6. Correlation of acoustic impedance and volume scattering with sediment mean grain size and bulk density. Darryl L. DeBruin, Lester R. LeBlanc, and Steven G. Schock (Ctr. for Acoust. and Vib., Dept. of Ocean Eng., Florida Atlantic Univ., 500 NW 20th St., Boca Raton, FL 33431)

Using high-resolution sonar data, a rule base classifier model is developed to correlate acoustic impedance and volume scattering with sediment mean grain size and bulk density. The acoustic impedance is inverted from the impulse response of the sonar data and the scattering strength is calculated between detected layers using a scattering model. Synthetic data and field data are used to demonstrate the high-resolution layer detection method, based on a local least-squares fit, that extracts the impulse response of the acoustic data. This analysis is heuristically constrained to estimate solutions within the resolution bounds of the sonar data. The classifier uses historic measurements, empirical relations, and fuzzy set theory to build the rule base model that is used to correlate the acoustic properties with sediment mean grain size and bulk density. Comparisons between estimates of mean grain size and bulk density from the rule base classifier model analysis of actual sonar data and geotechnical measurements are made in various depositional environments. The sonar and core data were acquired in Kiel Germany in collaboration with the Coastal Benthic Boundary Layer research team.

\section{3:45}

1pA07. The characterization of near-surface sediments with high-frequency acoustic pulses. James A. Hawkins, Warren T. Wood, Douglas N. Lambert, and Donald J. Walter (Naval Res. Lab., Stennis Space Center, MS 39529-5004)

The degree to which surficial and subsurficial $(5-10 \mathrm{~m})$ sediment properties can be determined from their acoustic response to high-frequency $(15-30 \mathrm{kHz})$ short-duration $(0.1-0.3 \mathrm{~ms})$ acoustic pulses has been investigated. The acoustic response of the sediment (echo) is assumed to be the convolution of the source pulse with the first several meters of the sediment represented in the time domain by a series of impulses for each reflecting surface. The impedance for each is then determined with a standard deconvolution algorithm. The problem is first approached using a synthetic earth model. The algorithm is next applied to field data collected with the Acoustic Sediment Classification System (ASCS). The results indicate the ability to resolve reflecting horizons and determine the impedance of the sediment surface and subsurface.

\section{4:00}

1pA08. Simultaneous reconstruction of velocity structure and boundary geometry in crosshole tomography. Subramaniam D. Rajan, Henry laible, and Cynthia J. Sellers (Woods Hole Oceanogr. Inst., Woods Hole, MA 02543)

In recent years, crosshole tomography has been used to infer the velocity structure in the earth's interior, ocean sediments, and in sea ice. In all cases, velocity estimates were obtained from travel time data for the earliest arrival. In many instances, the field measurements contain not only the information about this direct path but also about paths that have interacted with the boundaries. If one were to use the information contained in such boundary reflected paths, the resolution of the estimates could be considerably improved. This, however, implies that one knows the boundary geometry, which is generally not the case. An approach that one can take is to jointly estimate the boundary geometry and the velocity structure in the material. Different approaches that can be used to implement this idea and evaluate the method using synthetic data and field data are presented. [Work supported by ONR.]

\section{$4: 15$}

1pA09. Determination of physical properties of a porous seabed from reflection amplitude data by using the genetic algorithm. Altan Turgut (Inst. of Marine Sci., METU, P.O. Box 28, Erdemli-Icel, Turkey)

A global optimization technique, the genetic algorithm, is effectively used for the inversion of seabed properties from plane-wave reflection data. The seabed is modeled as a porous viscoelastic medium using Biot's theory and the plane-wave reflection coefficient is calculated using the analytical expressions derived by Stoll and Kan [J. Acoust. Soc. Am. 70, 149-156 (1980)]. A sensitivity analysis indicated that the plane-wave reflection shows strong dependency on the porosity, permeability, and shear modulus of the seabed. Inversion of these parameters is primarily attempted by assigning representative values to other Biot parameters which 
have secondary effects on the plane-wave reflection coefficient. Fast/slow compressional and shear-wave speeds, and density of the seabed, are also calculated using the inverted porosity, permeability, and shear modulus. An experimental technique by using a towed array and chirp signals is also discussed for effective and rapid surveying of the seafloor.

1pA010. A structure function constraint for stable least-squares inversion of reflection data. Kenneth E. Gilbert, Timothy J. Kulbago, and P. Jason White (Appl. Res. Lab. and the Graduate Prog. in Acoust., Penn State Univ., P.O. Box 30, State College, PA 16804)

High-frequency seismic profiles often indicate that the near-surface sediments in shallow water are layered on scales larger than about $0.5 \mathrm{~m}$ but not on smaller scales. Consequently, for a meaningful least-squares inversion of reflection data using a horizontally stratified sediment model, the wavelengths in the insonifying wave should be long enough to "average out" the small-scale sediment structure. With such a finite wavelength inversion, the sediment model must include some resolution constraints in order to yield a stable inversion. A common approach, for example, is to consider a stack of homogeneous layers where each layer is thicker than, say, a quarter of a wavelength. An alternative constraint method based on a structure function or, equivalently, an autocorrelation function, is presented. It is shown that with a structure function constraint, a stable inversion is obtained even if the layered structure approaches a continuous profile. Without the constraint, the inversion becomes meaningless as a continuum is approached. [Work supported by NRL and ONR.]

\section{4:45}

1pA011. Interpreting and extracting sediment attenuation from measured bottom loss data in the frequency range $500-5000 \mathrm{~Hz}$. Charles W. Holland, Greg Muncill, and Peter Neumann (Planning Syst., Inc., 7923 Jones Branch Dr., McLean, VA 22102)

The angle of intromission is the angle at which there is total transmission across an interface. This phenomena occurs between two ideal (lossless) half-spaces when the sound speed in the transmitting medium is lower and the density higher than in the incident medium. In low-energy environments (i.e., where clay and silty-clay sediment types dominate) the angle of intromission is often observed in measured bottom loss data. The loss at and around the angle of intromission is observed to be a strong function of attenuation in the host medium as well as the effects of gradients, random sedimentary layering, discrete sub-bottom reflecting horizons, roughness, and volume inhomogeneities. The physical mechanisms controlling reflection near the angle of intromission are explored in several data sets to demonstrate the potential for inverting the measured data for attenuation. [Work supported by the ONR/AEAS Program.]

\section{$5: 00$}

1pAO12. Acoustical monitoring of sediment transport in the bottom boundary layer at the inner continental shelf LEO-15 site. Peter
Traykovski, James Lynch, James Irish, and Arthur Newhall (Woods Hole Oceanogr. Inst., Woods Hole, MA 02543)

The bottom boundary layer (BBL) is of great importance in oceanography as it determines the amount of frictional stress a flow encounters from the ocean bottom, and it plays a major role in controlling sediment transport processes. The backscatter of acoustical energy from suspended sediments has proven to be a valuable tool in studying the BBL. Preliminary results from acoustical monitoring of sediment transport at the "Long-Term Ecosystem Observatory" (LEO-15) site located in $15 \mathrm{~m}$ of water off of the southern New Jersey coast are presented. The acoustical backscatter system that was deployed at this site during the winter and spring of 1994 is capable of profiling the entire water column by using a downward looking 5-, 2.5-, and 1-mHz sonars and a 1-mHz upward looking sonar. The fine resolution images produced from this system resolved several sediment suspension events and bottom feature movement was observed. [Work supported by NOAA.]

\section{$5: 15$}

1pAO13. The continuous spectrum and ambient noise inversions in shallow water. Nicholas M. Carbone, Grant B. Deane, and Michael J. Buckingham (Marine Phys. Lab.-0238, Scripps Inst. of Oceanogr., 9500 Gilman Dr., La Jolla, CA 92093-0238)

It has been demonstrated that the ambient noise field in the ocean over continental shelf regions contains sufficient environmental information to allow for inversion of the geoacoustic parameters of the seabed. In past studies both compressional and shear wave speeds have been obtained which are in good agreement with measurements made using independent methods. Other factors which affect the coherence and influence the noise inversions for basement parameters include the sea state and sound-speed profile. We have observed that for low sea states, the ambient noise field contains little or no contribution from the continuous spectrum. Theoretical studies, on the other hand, indicate that the effect of the continuous spectrum is significant for soft seabeds. One explanation for its absence is that at low sea states there are few surface acoustic events close to the observation point, implying that the standard statistical model used to calculate the coherence is not valid for overhead sources. The density of surface acoustic sources in relation to the spatial structure of the ambient noise field will be explored. [Work supported by ONR.]

\section{$5: 30-6: 15$}

\section{PANEL DISCUSSION:}

Panel Moderator: Darrell R. Jackson

Panel Members: Anatoliy N. Ivakin, Douglas N. Lambert, Steven G. Schock, Ingo H. Stender, Tokuo Yamamoto 


\title{
Session 1pPA
}

\section{Physical Acoustics: General Topics}

\author{
Christopher L. Morfey, Chair \\ Institute of Sound and Vibration Research, University of Southampton, Southampton S017 1B.J, United Kingdom
}

\section{Invited Paper}

\section{$1: 45$}

\begin{abstract}
1pPA1. New frontiers in molecular acoustics of liquids. Armen Sarvazyan ${ }^{\text {a) }}$ (Dept. of Chem., Rutgers Univ., New Brunswick, NJ 08903)

The major accomplishments in molecular acoustics of liquid systems are summarized. The most promising direction in this field is acoustical thermodynamics. The possibility of acoustical evaluation of thermodynamic parameters of liquids comes from the fact that compressibility of the fluid is the second derivative of Gibbs free energy on pressure. Changes of enthalpy, entropy, free energy, and their pressure and temperature derivatives (heat capacity, volume, expansibility, compressibility, etc.) can be calculated from the pressure and temperature dependencies of sound velocity in the fluid by using additionally the data on the temperature dependencies of density and heat capacity of the fluid at $1 \mathrm{~atm}$. Another new area is related to the studies of the acoustic nonlinearity parameter $B / A$ which is a simple function of the pressure derivative of the bulk modulus and provides unique information on the character of intermolecular forces in the liquid. In aqueous solutions $B / A$ is significantly determined by the structure of water in the hydration shell of the solute. The state of the art in the instrumentation for molecular acoustic studies is presented. New types of acoustical resonators based on the use of cylindrical standing waves enabling one to make measurements in microliter volume samples will be described.

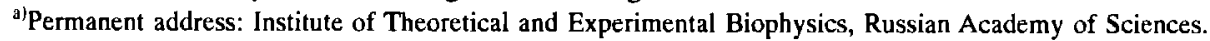

\section{Contributed Papers}

\section{2:15}

1pPA2. Matching pursuits with differential operator dictionaries. Wade Trappe and Joseph D. Lakey (Appl. Res. Labs., Univ. of Texas at Austin, P.O. Box 8029, Austin, TX 78713-8029 and Dept. of Math., Univ. of Texas at Austin, P.O. Box 8029, Austin, TX 78712)

This paper will examine in brevity the matching pursuit algorithm proposed by Mallat and Zhang, which yields an adaptive signal decomposition that may be used to derive a phase plane representation of a signal. Based on these ideas, and motivated by the application of wavelets to linear differential operators, a new numerical method for solving differential equations is proposed which is based on performing a matching pursuit algorithm in an operated dictionary. Using this new algorithm the nonhomogeneous Helmholtz differential operator with zero and nonzero boundary conditions shall be examined. A classical differential equation arising from acoustics shall be presented and solved using this new method. Results are presented for the one-dimensional case, and the extension to higher dimensions is examined using the concept of tensor products to construct a dictionary for higher dimensional Hilbert spaces.

\section{2:30}

1pPA3. Calculation of the transient acoustic wave field emitted by a focused transducer with an arbitrary rim. Adrianus T. de Hoop (Delft Univ. of Technol., Faculty of Elec. Eng., Mekelweg 4, 2628 CD Delft, The Netherlands), Smaine Zeroug, and Sergio Kostek (Schlumberger-Doll Res., Ridgefield, CT 06877-4108)

Closed-form analytic expressions are derived for the transient acoustic wave field emitted by a focused transducer with an arbitrary rim. The radiating part of the transducer is a spherical surface bounded by a simply connected closed curve of arbitrary shape. Starting from the KirchhoffHuygens representation of the emitted acoustic wave field, the expression for the acoustic pressure is transformed into a line integral along the rim of the transducer by employing the Maggi-Rubinowicz transformation in the
Kirchhoff theory of diffraction by a black screen. The resulting line integral for the transient acoustic pressure is evaluated numerically to study the shape of the beam emitted by the transducer in its dependence on the shape of the rim and to analyze the resolving power of the transducer in ultrasonic applications. For a focused transducer with a circular rim, a closedform analytic expression is derived for the transient acoustic pressure on its axis. These results serve as a check on the numerical results obtained for the more general cases. For all cases, the acoustic pressure at the focus admits a closed-form analytic representation. [Adrianus T. de Hoop performed this research as a Visiting Scientist with Schlumberger-Doll Research, Ridgefield, CT.]

\section{$2: 45$}

1pPA4. Transient axial solution for the reflection of a spherical wave from a paraboloidal mirror. Mark F. Hamilton (Dept. of Mech. Eng., Univ. of Texas at Austin, Austin, TX 78712-1063)

A method used previously [J. Acoust. Soc. Am. 93, 1256 (1993)] to derive a transient axial solution for a spherical wave reflected from an ellipsoidal mirror is applied to the case of a paraboloidal mirror. The incident spherical wave is radiated from the focus of the mirror. A solution for the impulse response of the reflected axial pressure is obtained in the form $h(z, t)=\delta\left(t-z / c_{0}\right)-h_{e}(z) \delta\left[t-t_{e}(z)\right]-\left(c_{0} / z_{F}\right) h_{w}(z, t)$, where $\delta$ is the Dirac delta function, $c_{0}$ is sound speed, $z$ is axial distance from the base of the mirror, $z_{F}$ is distance to the focus, $h_{e}$ is the relative amplitude of the edge wave, $t_{e}$ its relative time of arrival, and $h_{w}$ is the wake. Simple expressions are obtained for $h_{e}$ and $h_{w}$. Beyond the focus, the geometrical acoustics result $h_{e} \sim\left(1+d / z_{F}\right)^{-1}$ is recovered for the edge wave, where $d$ is the mirror depth. In the far field, $h_{w}$ becomes a delta function, the impulse response reduces to $h(z, t)-\left(2 z_{F}^{2} / c_{0} z\right) \ln \left(1+d / z_{F}\right) \delta^{\prime}\left(t-z / c_{0}\right)$, and the derivative of the source waveform is thus obtained. Calculations for various source waveforms are presented. Related measurements are discussed in the following presentation by Gelin et al. (Paper 1pPA5). [Work supported by ONR.] 
that occur in these representations satisfy a recurrence scheme, which is well suited for implementation in a symbolic manipulation program. The

1pPA5. Experimental study of reflection of airborne, spark-produced $\boldsymbol{N}$ waves by a paraboloidal dish. Lawrence J. Gelin, Jamie $\mathbf{M}$. Shorey, Daniel M. Hester, and David T. Blackstock (Appl. Res. Labs. and Mech. Eng. Dept., Univ. of Texas at Austin, P.O. Box 8029, Austin, TX 78713-8029)

An experimental investigation of the transient response of a paraboloidal reflector is reported. An inhomogeneous plane $N$ wave was produced by locating an electrical spark at the focus of a machined aluminum paraboloidal reflector (focal length $z_{F}=5.08 \mathrm{~cm}$, radius $a=10.80 \mathrm{~cm}$ ). A second reflector $\left(z_{F}=5.17 \mathrm{~cm}, a=10.05 \mathrm{~cm}\right)$ was constructed by spinning a container of epoxy at constant speed and allowing it to cure. Peak pressure $P$ and arrival time were measured across the beam (fixed axial distance $z$ measured from the reflector surface, variable radial distance $r$ ) and along the axis. The range of measurements was $r \leqslant 80 \mathrm{~mm}$ and 20.74 $\mathrm{mm} \leqslant z \leqslant 95.74 \mathrm{~mm}$. Small-signal $N$ waves $(P=400 \mathrm{~Pa}$, duration $T=9 \mu \mathrm{s})$ were measured as well as stronger ones $(P=1000 \mathrm{~Pa}, T=12 \mu \mathrm{s})$. For small-signal $\boldsymbol{N}$ waves the axial measurements generally confirm Hamilton's theoretical prediction (previous paper, 1pPA4) although the edge waves are weaker than forecast. Transverse measurements agree with raytheory predictions off axis but are up to $10 \%$ low in the axial region. For stronger $N$ waves, transverse measurements of arrival time and peak pressure show evidence of self-refraction (ray bending due solely to finiteamplitude effects). [Work supported by ONR, ARL:UT IR\&D program, and NASA.]

\section{3:15-3:30 Break}

\section{3:30}

1pPA6. Extension of the Huygens-Fresnel principle to a virtual space. Mitsuhiro Ueda (Intl. Cooperation Ctr. for Sci. and Technol., Tokyo Inst. of Technol., O-okayama, Meguro-ku, Tokyo 153, Japan)

The quantitative description of diffraction started with Fresnel, who introduced the interference effect to Huygens' principle where diffracted waves are expressed by an integral of secondary wavelets over an opening in an opaque screen. Although this diffraction formula is an approximate one, it has given precise estimation of waves diffracted by the opening. Most diffraction phenomena arise from 3-D objects. However, attempts to apply the diffraction theory to 3-D objects are unsuccessful since, in these cases, the region for integration of secondary wavelets cannot be determined clearly. In order to explain the diffraction by polyhedrons, a hypothetical observer is assumed at an observation point and the HuygensFresnel principle is extended to a space seen by the observer virtually. In this virtual space the observer can see real images and mirror images through facets of the polyhedron and every point in real and mirror images is considered as a center of the secondary wavelets. The new representation of sound field diffracted by 3-D objects that satisfies both the wave equation and boundary conditions is derived by this extension [J. Acoust. Soc. Am. 95, 2354-2362 (1994)]. The algorithm to obtain solutions from this representation will be discussed in detail.

\section{$3: 45$}

1pPA7. Transient acoustic wave fields in continuously layered fluids with attenuation. Martin D. Verweij (Lab. of Electromagn. Res., Dept. of Elec. Eng., Delft Univ. of Technol., P.O. Box 5031, 2600 GA Delft, The Netherlands)

The space-time domain acoustic wave field in an isotropic, lossy, continuously layered fluid is analyzed using a method that employs a combination of higher-order WKBJ asymptotics and the Cagniard-De Hoop method of inversion. The loss behavior of the fluid is described with the aid of general temporal compliance and inertia memory functions. The continuous layering of the medium manifests itself in the acoustic wave speed, the density of mass, and both memory functions, which are independent, continuous functions of the vertical coordinate. After the application of forward transformations, higher-order WKBJ asymptotic representations of the transform domain solution are derived. The coefficients transform domain WKBJ asymptotic representations are analytically transformed back to the space-time domain with the aid of the Cagniard-De Hoop method. Numerical results for several configurations with an intricate loss behavior are presented.

\section{4:00}

1pPA8. Dependence of axial propagation modes on piezoelectric coupling coefficients in a piezoelectric continuously twisted structurally chiral medium (PCTSCM). Steven F. Nagle and Akhlesh Lakhtakia (227 Hammond Bldg., Dept. of Eng. Sci. and Mechan., Penn State Univ., University Park, PA 16802)

The material properties of a piezoelectric continuously twisted structurally chiral medium (PCTSCM) vary helicoidally along the axial direction. Monochromatic axial wave propagation in a PCTSCM has been solved exactly [A. Lakhtakia, Appl. Acoust. (in press)]. It is shown that specific axial propagation modes are related to specific piezoelectric coupling coefficients. By a judicious choice of these coefficients, one may control certain sets of modes independently of other sets of modes. Since the degree of piezoelectric coupling can be engineered, PCTSCMs are promising candidates for future transduction devices. Emphasis has been placed on PCTSCMs with hexagonal symmetries. Graphical support of the conclusions will be presented. [Work supported by NSF.]

\section{4:15}

1pPA9. Elastic-constant determination of small, near-isotropic crystals using resonant ultrasound spectroscopy: New quasicrystal results. P. S. Spoor, J. D. Maynard (Dept. of Phys., Penn State Univ., University Park, PA 16802), B. Golding (Michigan State Univ., East Lansing, MI 48824), and A. R. Kortan (AT\&T Bell Labs., Murray Hill, NJ 07974)

If a material is very nearly isotropic, quantifying the residual anisotropy may be quite difficult using methods such as pulse superposition or neutron scattering, when only a small sample is available. However, resonant ultrasound spectroscopy using piezoelectric film transducers allows precise $(<0.1 \%)$ measurement of elastic constants even on very small $(<0.25 \mathrm{mg}$ ), fragile samples. This is of interest in the study of icosahedral quasicrystals, which should theoretically be isotropic, but have closely related phases which are crystalline. Results on several samples of quasicrystalline and cubic AlCuLi will be presented, and use of rotations of the elastic tensor and Monte Carlo style error simulations to aid in the data analysis will be discussed. [Work supported by NSF Grant No. DMR9306791 and by ONR.]

\section{4:30}

1pPA10. Measurements of ultrasonic attenuation and sound velocity in a single crystal of $\left(\mathrm{La}_{1-x} \mathrm{Sr}_{x}\right)_{2} \mathrm{CuO}_{4}$. Hong Zhang, $\mathrm{M}$. J. McKenna, Bimal K. Sarma, Moises Levy (Dept. of Phys., Univ. of Wisconsin-Milwaukee, Milwaukee, WI 53201), T. Kimura, K. Kishio, and K. Kitazawa (Univ. of Tokyo, Tokyo, Japan)

Measurements of the ultrasonic attenuation and sound velocity were made at frequencies of 32 and $87 \mathrm{MHz}$ in a large single crystal of $\left(\mathrm{La}_{0.925} \mathrm{Sr}_{0.075}\right)_{2} \mathrm{CuO}_{4}\left(\sim 4 \times 4 \times 4 \mathrm{~mm}^{3}\right)$, which exhibits a sharp $(\Delta T \sim 1 \mathrm{~K})$ superconducting transition at $37 \mathrm{~K}$. For longitudinal waves propagating along the $c$ axis, the sound velocity shows a sharp softening, $\Delta v / v \sim 100$ pprn, concurrent with the sharp superconducting transition in the susceptibility measurements. Following this transition, the susceptibility exhibits a small tail extending to $27 \mathrm{~K}$, where a smaller softening, $\Delta v / v \sim 20 \mathrm{ppm}$, has been observed in the velocity measurements. Accompanying the $\sim 100$-ppm velocity drop, there is a signature in the attenuation; following this feature, the attenuation changes as the temperature is decreased further, with a small kink in the attenuation at $27 \mathrm{~K}$. Additional results of the dependence of the attenuation and sound velocity in external magnetic fields, orientated in the $a-b$ plane, will be presented. [Work supported by ONR.] 
1pPA11. Observation of individual bond breaking events in precursors, cascades, etc. in the onset and progression of fracture. Tianming Zhang, S. R. Savitski, and J. D. Maynard (Dept. of Phys., Penn State Univ., University Park, PA 16802)

Experimental measurements of fracture have been made using a wide bandwidth $(10 \mathrm{MHz})$ transducer and a system with a relatively large bond size, with the result that one is able to observe individual bond breaking events. The data provide a direct test of "fuse" models [L. de Arcangelis, scaling theories of brittle fracture. Measurements have been made with uniform tensile stress and bending stress, with the latter corresponding to a phase transition in the presence of an external field. For samples of the same width, the observations are quite consistent, showing the effects of precursors, onset, progression, stress waves, crack arrest, and other interesting phenomena. A significant difference in precursors for the uniform and bending stress fields is observed, perhaps a result of the dimensionality of the maximum stress field. [Work supported by NSF Grant No. DMR9306791 and by ONR.]

MONDAY AFTERNOON, 28 NOVEMBER 1994

TRINITY A AND B, 1:00 TO 5:00 P.M.

\title{
Session 1pSP
}

\section{Speech Communication: Acoustic Analysis and Perception of Consonants (Poster Session)}

\author{
Harvey Sussman, Chair \\ Department of Linguistics, University of Texas at Austin, Austin, Texas 78712
}

\section{Contributed Papers}

\begin{abstract}
All posters will be on display from 1:00 to 5:00 p.m. To allow contributors an opportunity to see other posters, contributors of odd-numbered papers will be at their posters from 1:00 to 3:00 p.m., and contributors of even-numbered papers will be at their posters from 3:00 to 5:00 p.m. Posters will remain on display until 12:00 noon on Tuesday.
\end{abstract}

1pSP1. Perception of fricatives synthesized by higher-level control of a Klatt synthesizer. David R. Williams (Sensimetrics Corp., 26 Landsdowne St., Cambridge, MA 02139)

Results of perceptual tests of fricatives synthesized using an acousticarticulatory model [K. N. Stevens and C. A. Bickley, J. Phonet. 19, 161174 (1991)] are presented. Parameters of the model permit time-varying control of vocal-tract shape (first four natural frequencies) and of glottal and oral cross-sectional areas. After computing air flow and intraoral pressure values, KLSYN88 synthesizer source parameters are estimated using mapping equations. The current study examines model predictions for intervocalic alveolar and labio-dental fricatives. Stimulus sets were constructed by varying the sizes of peak glottal opening $\left(8-20 \mathrm{~mm}^{2}\right)$ and minimum oral constriction $\left(4-16 \mathrm{~mm}^{2}\right)$, and the rate of oral constriction near closure/release $\left(4,16 \mathrm{~cm}^{2} / \mathrm{s}\right)$. Subjects labeled the synthetic fricatives as voiced or voiceless and rated the "goodness" of the stimuli as exemplars of voiceless fricatives. As expected, stimuli with the smallest and largest glottal openings were judged as yoiced and voiceless, respectively. At intermediate glottal opening values, voicing judgments were influenced by the relative sizes of the glottal and oral openings and, to a lesser extent, by oral constriction rate. Goodness ratings of the stimuli generally correlated with labeling judgments. The results demonstrate the robustness of speech sound categories generated in this manner. [Work supported by NIMH.]

1pSP2. Landmark detection for distinctive feature-based speech recognition. Sharlene A. Liu (Speech Commun. Group, Res. Lab. of Electron., Dept. of EECS, MIT, Rm. 36-511, Cambridge, MA 02139)

This work is a component of a proposed knowledge-based speech recognition system, which uses landmarks to guide the search for distinctive features. In an utterance, landmarks identify localized regions where the acoustic manifestations of the linguistically motivated distinctive features are most salicnt. An algonithm for autommitically detecling landmarks implemented by abrupt articulatory movements is described. The algorithm is a hierarchically structured algorithm rooted in linguistic and production theory. It looks for abrupt energy changes in six frequency bands and at two levels of temporal resolution. Landmarks are found based on information about which bands contain the abrupt change, the steady-state quality in the vicinity of the proposed landmark, segmental duration constraints, and an articulatory continuity requirement. Tested on a database of continuous speech recorded in a silent room by male and female speakers, the landmark detector is shown to perform well, with a $94 \%$ true detection rate and a $10 \%$ false detection rate. Most of the missed landmarks were adjacent to reduced vowels. These promising results indicate that landmarks are robustly identifiable points in the speech waveform and that a landmark detector as a front end in a speech recognition system is feasible. [Work supported by NSF.]

1pSP3. Clear speech does not exaggerate phonemic contrast. John J. Ohala (Dept. of Linguist., Univ. of California, Berkeley, CA 94720)

Structural linguistics teaches that one of the principal properties of speech sounds is being different from each other: The essence of a phoneme is that it is not any other phoneme. In addition, many structuralist theories of sound change are based on notions of preservation of contrast between phonemes. From this one might expect that it should be possible to observe speakers' efforts at maintaining contrasts in speech. One situation where this would be expected is in repeated speech, i.e., where a speaker repeats a word after receiving feedback that it has been misapprehended as another similar word. This paper reports results of an analysis of such repeated speech samples of ten English speakers when they produced 1 of 12 near-minimal syllables (bayed, paid, bed, ped, bet, pet, bid, bit, etc.) under two conditions: control and repetition (in response to feedback that their initial production had been misunderstood as one of the other syllables). Contrary to expectations, there was no significant contrastive exaggerations in VOT or vowel duration as a function of the word presented in feedback. [Work supported by AGT, Ltd.]

1pSP4. The role of segment-internal rate information in the phonetic classification of stop consonants. Thomas Charles Bourgeois and Kerry P. Green (Inst. for Neurogen. Commun. Disord., Univ. of Arizona, Tucson, AZ 85721)

During the course of a normal conversation talkers make frequent and extensive changes in speaking rate which affects the acoustic realization of both consonants and vowels. Previous research has shown that the articu- 
lation rate of a syllable influences both the production and phonetic categorization of its segmental components. However, little certainty exists regarding which portions of the syllable carry information about overall articulation rate. The present study investigated this question by editing the burst and aspiration phases from natural / $\mathrm{bi} /$ and / $\mathrm{pi} /$ syllables produced at a fast rate of speech, and cross-splicing them onto the same tokens produced at slow rates of speech (and vice versa). These tokens were presented to subjects for speeded classification of the initial consonant. The results indicate that subjects were sensitive to the mismatch in articulation rate between the initial burst and aspiration of the stop consonant and the remainder of the syllable created by the cross-splicing technique. Further investigation using / $\mathrm{bi}-\mathrm{pi}$ / continua created in a similar manner indicates that consonant-internal rate information is used during phonetic categorization of the stimuli. These results are discussed in the context of current issues of rate normalization and speech perception. [Work supported in part by Research and Training Center Grant No. P60 DC-01409 from the National Institute on Deafness and other Communication Disorders.]

1pSP5. Cross-language tests of the perceptual magnet effect for /I/ and /1. Paul Iverson (Dept. of Speech and Hear. Sci., WJ-10, Univ. of Washington, Seattle, WA 98195), Eugen Diesch, Claudia Siebert (Tech. Univ. of Berlin, Berlin, Germany), and Patricia K. Kuhl (Univ. of Washington, Seattle, WA)

Recent experiments by Iverson and Kuhl have suggested that the perceptual organization of the American English $/ I /$ and $N /$ categories is strongly influenced by category goodness. American adult listeners exhibit a perceptual magnet effect characterized by low sensitivity and perceptual clustering near the best exemplars of $/ 1 /$ and $/ 1 /$ and high sensitivity and stretched perceptual distances near the worst exemplars. The present study compares the responses of native German and English speakers to evaluate whether the representation of these categories is influenced by language experience. In separate experiments, natural phonemes of adult German $(/ R /$ and $/ / /)$ and American $(/ 1 /$ and $/ / /)$ speakers were recorded, and synthesized American English / $/ /$ and $/ / /$ tokens were identified, discriminated, rated for category goodness, and rated for similarity by both groups of subjects. The results demonstrate that German listeners prefer, produce, and have perceptual clustering for $N /$ phonemes with a higher $F 2$ frequency than do American listeners. German listeners do not perceive American /I/ phonemes to be good examples of the German / $\mathrm{k} /$ category, and German listeners also exhibit somewhat less perceptual clustering for II/ phonemes than do American listeners. The results confirm that the perceptual magnet effect for $/ \mathrm{I} /$ and $/ 1 /$ is determined by language experience. [Work supported by NIH.]

1pSP6. A locus equation study of syllable-final stop place of articulation. Harvey M. Sussman (Dept. of Linguist. and Speech Commun., Univ. of Texas, Austin, TX 78712), Jadine Shore, and David Fruchter (Univ. of Texas, Austin, TX 78712)

Syllable-final stops have different coarticulatory and perceptual properties compared to syllable-initial stops. Locus equations have previously been used to acoustically characterize place of articulation for initial stop consonants [H. Sussman, H. McCaffrey, and S. Matthews, J. Acoust. Soc. Am. 90, 1309-1325 (1991) and H. Sussman, K. Hoemeke, and F. Ahmed, J. Acoust. Soc. Am. 94, 1256-1268 (1993)]. Since locus equations also encode degree of coarticulation, they might also provide an adequate phonetic description of $\mathrm{VC}$ events for final $/ \mathrm{b} /, / \mathrm{d} /$, and $/ \mathrm{g} /$ following varied vowel contexts. Each of ten speakers, five male and five female, produced three repetitions of $90 \mathrm{CVC}$ tokens. For each final stop $(/ \mathrm{b}, \mathrm{d}, \mathrm{g} /)$ there were ten medial vowels and three initial consonants- $b \mathrm{bb}, \mathrm{dVb}, \mathrm{gVb}, \mathrm{bVd}$, $\mathrm{dVd}, \mathrm{gVd}, \mathrm{bVg}, \mathrm{dVg}, \mathrm{gVg}$. Three points along the second formant were measured-F $F$ onset $(\mathrm{Hz}), F 2$ midvowel $(\mathrm{Hz})$, and $F 2$ offset $(\mathrm{Hz})$. Offset locus equations (VC) were generated for each syllable-final stop place, both across initial consonantal contexts and as a function of each initial stop. In addition, 3-D plots were generated to determine how $F 2$ onset $(x)$, midpoint $(y)$, and offset $(z)$ could acoustically capture and differentiate lexical contrasts. [Work supported by NIDCD.]

1pSP7. Speakers nasalize $/ \sigma /$ if it is preceded by $/ n /$, but Msteners don't care-They still hear $/ \mathrm{O} /$. Sharon Y. Manuel (Res. Lab. of Electron., Mass. Inst. of Technol. BIdg. 36-511, Cambridge, MA 02139)
Presumably as a result of coarticulation, $/ \delta /$ often assimilates to a preceding $/ \mathrm{n} /$ in phrases like "win those," but this assimilation is not complete for all features. With respect to the feature [nasal], the assimilation is often radical. The entire consonant region in the middle of the two-word sequence is nasalized. However, acoustic evidence suggests that contextually nasalized $/ \delta /$ retains its dental place of articulation. Specifically, $F 2$ is considerably lower at the release of a contextually nasalized $/ \delta /$ than at the release of a true $/ \mathrm{n} /$, as would be expected for a dental consonant. Perception tests show that listeners can generally tell the difference between natural tokens of pairs like "win nos" and "win those," even when the $/ \delta /$ is completely nasalized. In addition, a synthetic stimulus continuum was constructed in which items differed only with respect to $F 2$ frequency in the vicinity of the nasal consonant regions of phrases like "win nos." Listeners systematically reported hearing "those" more often when $F 2$ was low at the release of the nasal consonant. These results are consistent with the claim of Krakow, Fowler, and others, that listeners can at least sometimes factor out coarticulatory effects. [Work supported by NSF.]

1pSP8. Efrect of fundamental frequency perturbations on medial stop-consonant [voice] judgments. Michelle R. Molis and Randy $L$. Diehl (Dept. of Psychol., Univ. of Texas at Austin, Austin, TX 78712)

Previous research has suggested that the direction of short-duration fundamental frequency $(F 0)$ perturbations following consonants provides a cue to consonant [voice] status. More recently, Silverman [Phonetica 43, 76-91 (1986)] proposed that the [voice] cue is provided by the direction and extent of $F 0$ perturbations relative to the underlying intonational contour. A competing view, the low-frequency hypothesis, suggests that $F 0$ participates in a more general way whereby any low-frequency energy in the region of the consonant will contribute to the perception of a [+ voice] consonant. In this study, 15 speech stimulus series, each ranging perceptually from /aga/ to /aka/, were synthesized by varying VOT from 10 to 45 $\mathrm{ms}$ in $5 \mathrm{~ms}$ steps. Fifteen different pitch contours were generated by designating $F \mathbf{0}$ targets at three points in the stimulus: initial vowel, onset of voicing energy after closure, and $100 \mathrm{~ms}$ after the onset of voicing. Three $F 0$ values were used, arranged into two pairs: 100 and $120 \mathrm{~Hz}$ and 120 and $140 \mathrm{~Hz}$. The results indicated that the value of $F 0$ at vowel onset, rather than the relative movement of $F 0$, is the best predictor of subjects' judgments of consonant [voice] status. [Work supported by NIDCD.]

1pSP9. Devoicing a /z/ does not make an /s/. Caroline L. Smith (Audio/ Speech Path (126), West LA VA Med. Ctr., 11301 Wilshire Blvd., Los Angeles, CA 90073 and Div. of Head and Neck Surgery, UCLA School of Medicine, Los Angeles, CA 90024)

It is well known that voiced stops in English tend not to be fully voiced. For many American speakers, voiced fricatives may also have little or no voicing. In what phonological contexts do speakers devoice underlying /z/? Previous research [T. Veatch, Ling. Soc. Am. mtg., 69 (1989)] emphasized the influence of the following segment. The present study confirms this, and investigates the likelihood of devoicing in different positions in syllable, word, and sentence. Five speakers of American English recorded multiple repetitions of sentences in which $/ \mathrm{s} /$ and $/ \mathrm{z} / \mathrm{oc}$ curred in matched environments. Measures of acoustic durations, airflow, and vocal fold vibration as evidenced by EGG were used to compare the production of $/ z /$ and $/ \mathrm{s} /$. Preliminary data from one speaker show a variety of $/ z /$ 's, with the duration of vocal fold vibrations as a percentage of fricative duration ranging from $0 \%$ to $100 \%$. However, the acoustic vowel length difference preceding $/ \mathrm{s} /$ and $/ z /$ is maintained, and even those tokens of $\mid z /$ in which there was no vocal fold vibration have reduced airflow compared to matched tokens of $/ \mathrm{s} /$, suggesting that devoiced $/ z /$ may differ from underlying $/ \mathrm{s} /$ in glottal constriction or level of pulmonic activity. [Work supported by NIH.]

1pSP10. Spectral discontinuities and the vowel-length effect. Andrew J. Lotto, Keith R. Kluender, and Lori L. Holt (Dept. of Psychol., Univ. of Wisconsin, Madison, WI 53706)

Perception of the voicing contrast in CV syllables can be affected by the duration of the following vowel such that longer vowels lead to more "voiced" responses. On the basis of several experiments, Green, Stevens, and Kuhl [Percept. Psychophys. 55, 249-260 (1994)] concluded that con- 
tinuity of pitch, but not continuity of formant structure, determined the effective length of the following vowel. In attempts to replicate these findings, it was found that both effects were highly dependent upon particular choices of pitch and formant values. First, discontinuity in pitch does not preclude the vowel-length effect when fundamental frequency changes from 200 to $100 \mathrm{~Hz}$ and $200-\mathrm{Hz}$ partials extend continuously through test syllables. Second, spectral discontinuity does preclude the vowel-length effect when formant changes result in a spectral peak moving to another harmonic. Even when perceived identity of the vowel remained constant, formant changes that resulted in a change in peak harmonic precluded the vowel-length effect. Yet, changes that did not change peak harmonic and did change perceived vowel identity did not preclude the vowel length effect. Taken together, the results indicate that neither phonemic continuity nor talker continuity, per se, is critical to the vowellength effect. [Work supported by NSF and NIH.]

1pSP11. Self-organizing maps of stop consonant place from token-level locus equation inputs. Jon Hilbert (Dept. of Comput. Sci., Univ. of Texas, Austin, TX 78712), David E. Fruchter, Mona McWilliams, Joseph Sirosh, and Harvey M. Sussman (Univ. of Texas, Austin, TX 78712)

Previously [D. E. Fruchter, J. Acoust. Soc. Am. 95, 2977 (1994)], identification curves were estimated for English / $b, d, g /$ using synthetic $C V$ stimuli comprehensively sampling the $F 2$-onset $X$ F2-vowel acoustic space in the vicinity of Sussman's $/ b, d, g /$ locus equations. These results were used to delineate "identification surfaces" situated in locus equation space. The current research uses a biologically plausible neural network (the Kohonen algorithm) to model the above perception results. This algorithm is an abstraction of the local, unsupervised map-organizing process thought to occur in the brain. The Kohonen map forms a twodimensional representation of stop consonant place categories from $F 2$ onset and $F 2$-vowel inputs. This emergent representation corresponds well with the experimentally observed identification surfaces and can be used to classify novel inputs and predict phoneme boundaries and confusability regions.

1pSP12. Stimulus intensity and fundamental frequency effects on duplex perception. Houri K. Vorperian (Dept. of Commun. Disord., Univ. of Wisconsin-Madison, Madison, WI 53705), Marleen T. Ochs, and D. Wesley Grantham (Vanderbilt Univ., Nashville, TN 37232-8700)

The simultaneous perception of speech and nonspeech occurs when the intensity of the $F 3$ transition of a three formant synthetic syllable is increased relative to the rest of the syllable (the base). This phenomenon has been interpreted as evidence of a distinct system for speech perception that precedes other specialized systems of general auditory processing [Liberman and Mattingly, Science 243, 489-494 (1990)]. Using F3 transitions with fundamental frequencies different from the base, and referencing their presentation levels to the level at which each $F 3$ transition was barely audible in the context of the base, identification functions were obtained across a wide range of $F 3$ transition intensities. As previously demonstrated, results showed that the $F 3$ transitions contributed to the speech percept over a wide range of intensities and fundamental frequencies. However, at very intense $F 3$ transition levels, /ga/ identification decreased. Also, both / da/ and /ga/ identification progressively decreased as the fundamental frequency of the $F 3$ transition increasingly differed from the base and interacted with intensity. These findings indicate that information from general auditory processing systems is available to the specialized speech perception system. The speech perception system tolerates a range of information from general auditory processing systems before it rejects such information as irrelevant to speech.

1pSP13. Acoustic evaluation of surgical intervention for one speech therapy patient. Robert Hagiwara, Susan Meyers Fosnot (Phonet. Lab., UCLA Dept. of Linguist., 405 Hilgard Ave., Los Angeles, CA 90024), David M. Alessi, and Gerald M. Sloan (Childrens Hospital Los Angeles, Los Angeles, CA 90054)

The development of American / $r$ / (characterized acoustically by a low third formant) in one speech therapy patient (SR, 6;6) after surgical intervention is documented. SR was originally referred to speech therapy for several speech disorders. Most were corrected in less than one year, though he still could not produce American / $/$ /, probably due to restricted movement of his tongue. Fine control of the tongue body and pharyngeal narrowing are necessary for production of $/ r$ /. SR exhibited restricted vertical movement of the tongue body due to a banded tongue base and restricted posterior movement of the tongue body and root duc to enlarged tonsils. Surgery (tonsillectomy, adenoidectomy, and frenuloplasty) was indicated for reduction of SR's occlusive sleep apnea. By hypothesis, these procedures would also alleviate any physiological basis for SR's inability to produce $/ r /$. Recordings were made of multiple repetitions of $/ r /$ in 16 phonological contexts on two occasions before surgery. Two more recordings were made after surgical recovery. (If SR does not develop / $\mathbf{r}$ / after surgical intervention, additional recordings will be made after further speech therapy.) Acoustical analysis of these recordings documents SR's development of $/ r /$, and the efficacy of surgical intervention on his speech.

1pSP14. Postvocalic consonant clusters in Swedish. Peter E. Czigler (Umeaa University, 90187 Umeaa, Sweden) and Dawn M. Behne (Univ. of Trondheim, 7055 Dragvoll, Norway)

In Swedish a postvocalic consonant is phonologically long following a distinctively short vowel and phonologically short following a distinctively long vowel. Previous research has demonstrated that this distinction is reflected in the relative durations of the corresponding segments. In addition, it has been shown that a consonant tends to be shorter in duration when it occurs in a consonant cluster than when it is a single consonant. However, when a consonant occurs in a cluster, it becomes less obvious whether it is a short or long consonant. The goal of this project is to determine whether the short-long dichotomy of consonants is acoustically realized in clusters. Target words were identified in which $/ \mathrm{k} /$ occured occurred in four different codas structures: C, C:, Cs, and sC. Native speakers of Swedish produced the target words in carrier sentences and the duration of the closure of $/ \mathrm{k} /$ and the preceding vowels were measured. The results demonstrate that the structure of a consonant cluster and its neighboring segments can affect the duration of a postvocalic consonant. These findings are discussed in terms of syllable-internal timing and the extent to which consonant duration varies within clusters.

1pSP15. Perceptual evidence of voicing assimilation in Russian. Martha W. Burton (415 Moore Bldg., Dept. of Psychol., Penn State Univ., University Park, PA 16802) and Karen E. Robblee (Penn State Univ., University Park, PA 16802)

Previous research has provided acoustic phonetic evidence of voicing assimilation in obstruent clusters across prepositional boundaries in Russian [Burton et al., J. Acoust. Soc. Am. 95, 2875(A) (1994)]. The current study investigated whether native speakers of Russian perceive the initial consonant in obstruent clusters consistent with the claims of voicing assimilation or whether they show sensitivity to the underlying voicing of the obstruent. Obstruent clusters that were at the boundary between a preposition and a word were excised from sentences produced by three native speakers of Russian. Listeners were presented with four fricative-stop combinations $/ \mathrm{zd}, \mathrm{sd}, \mathrm{zt}, \mathrm{st} /$ and four stop-fricative combinations $/ \mathrm{dz}, \mathrm{tz}, \mathrm{ds}, \mathrm{ts} /$ preceded by $30 \mathrm{~ms}$ of an unstressed vowel and followed by $70 \mathrm{~ms}$ of a stressed vowel. Subjects heard eight repetitions of three tokens of each cluster from each speaker. Results indicated that responses were consistent with the voicing characteristics of the following consonant, not the underlying consonant, supporting voicing assimilation. However, in the /zt/ clusters, there were more voiced responses than in the /st/ clusters, which suggests some evidence of incomplete devoicing of the fricative. Implications of the study for phonetic and phonological theories of speech will be discussed.

1pSP16. Acoustic features differentiating Korean medial tense and lax stops. Ji-Hye Shin (Dept. of Linguist., Univ. of California at Berkeley, Berkeley, CA 94720)

Much research has been done on the cues differentiating the three Korean stops in word initial position. This paper focused on a more neglecied area: the acoustic cues differentiating the medial tense and lax unaspirated stops. Four adult Korean native speakers, two males and two females, pronounced 16 minimal pairs containing these stops. The average duration of vowels before lax stops is $\mathbf{9 3} \mathrm{ms}$ longer than before their tense counterparts (143 ms for lax versus $50 \mathrm{~ms}$ for tense). In addition, the 
average duration of the stop closure of tense stops is $178 \mathrm{~ms}$ longer than that of lax stops ( $74 \mathrm{~ms}$ for lax versus $252 \mathrm{~ms}$ for tense). These durational differences are so large that they may be phonologically determined, not phonetically. Moreover, vowel duration may vary with the speaker's sex. Female speakers have much shorter vowel duration before the lax stops. The quality of voicing, tense or lax, is also a cue to these two stop types, as it is in initial position, but the relative duration of the stops and of the preceding vowel appear to be much more important cues. The consequences of these results for the phonological description of Korean as well as the synthesis and automatic recognition of Korean will be discussed.

1pSP17. Hemispheric differences in the perception of Zulu click consonants. Robert A. Avery and Catherine T. Best (Dept. of Psychol., Wesleyan Univ., Middletown, CT 06459 and Haskins Laboratories, New Haven, CT 06511)

Past research has shown that speech sounds are processed better by the left hemisphere, nonspeech sounds by the right hemisphere, in most righthanded people. To date, however, it is unknown whether phonetic contrasts from an unfamiliar language show this same pattern, and whether they are perceived as speech. It is known that adults' perception of non-native speech contrasts is strongly influenced by their language experience; thus non-native contrasts may be handled by different processes than are native contrasts. Best et al. [JEP:HPP 14, 345-360 (1988)] suggested that English speakers' excellent discrimination of Zulu click consonants occurs because they hear the clicks as nonspeech. Right-handed native speakers of English and of Zulu or Xhosa participated in a dichotic listening study on cerebral dominance for perception of clicks in isolation and in $/ \mathrm{Ca} / \mathrm{syl}-$ lables. Natural tokens of Zulu click consonants were used (apical, lateral, and palatal places of articulation, and voiceless aspirated, voiceless unaspirated, and voiced categories). Native speakers of Zulu and Xhosa showed a left hemisphere dominance for discrimination of both the isolated clicks and $/ \mathrm{Ca}$ / syllables, while native speakers of English demonstrated no such hemispheric dominance. [Work supported by NIH Grant No. HD-01994.]

1pSP18. Combining time averaging and ensemble averaging in analyzing voiceless fricatives in Mandarin. $\mathrm{Yi} \mathrm{Xu}$ and Lorin Wilde (Speech Group at Res. Lab. of Electron., MIT, 36-513 Vassar St., Cambridge, MA 02139)

The random fluctuations and spurious peaks typically seen in fricative spectra can be reduced by time averaging, i.e., averaging spectra obtained with overlapping time windows over an interval of the frication noise. Furthermore, token-to-token as well as individual speaker variations in fricatives can be reduced by ensemble averaging, i.e., averaging over noise spectra of multiple tokens in the same relative time interval. However, for studying coarticulatory variation in the frication noise, neither of these two methods alone is adequate: Time averaging does not handle token-to-token and individual speaker variations; ensemble averaging requires a large number of tokens to produce smooth and consistent spectra. In the present study, time averaging and ensemble averaging were combined in the analysis of coarticulatory variation of fricatives in Mandarin. The size of the time-averaging interval was $20 \mathrm{~ms}$, and the size of the individual FFT windows was $8 \mathrm{~ms}$. The time-averaged spectra were further ensemble- averaged over ten repetitions of the same sentence by the same speaker. Results indicated that the spectra thus obtained were smooth, and they revealed spectral changes over time more clearly than those obtained by either time averaging or ensemble averaging alone. Further ensemble averaging across different speakers was also explored and has produced encouraging data. [Work supported by NIH.]

1pSP19. Ejectives in Babine-Witsuwit'en. Katharine Davis (Dept. of Speech and Hear. Sci., WJ-10 CDMRC, Univ. of Washington, Seattle, WA 98195) and Sharon Hargus (Univ. of Washington, Seattle, WA 98195)

Acoustic properties of ejectives in an Athabaskan language of British Columbia were examined. Data from ten native speakers were recorded in the field. Following Hogan [Phonetica 33, 275-284 (1976)], prerelease closure, burst duration, voice onset time, and postrelease silent interval were measured. Following Ingram and Rigsby [Proceedings of the XIth International Congress of Phonetic Sciences, Talinn, Estonia 2, 134-137 (1987)], burst amplitude and the fundamental frequency of the following vowel were also measured. Values were compared with those of homorganic nonejectives (voiceless unaspirated and voiceless aspirated). Preliminary results show that the ejective stops may be distinguished from plain aspirated stops by VOT alone. Ejectives and plain unaspirated stops have similar VOTs; the principal differences appear to occur in the vowel onset and in the prerelease closure phase. Strong ejective characteristics such as those of Navajo [Lindau, J. Phonet. 12, 147-155 (1984)] do not appear to be present. The present findings, combined with those of Ingram and Rigsby (1987) for the contiguous but unrelated language Gitksan, imply that relatively weak ejectives may be an areal phenomenon. Results will also be contrasted with published data from the related languages Chipewyan and Navajo.

1pSP20. Acoustic correlates of manner of articulation for Urdu stop consonants. Sarmad Hussain (Speech and Lang. Pathol., Dept. of Commun. Sci. and Disord., Northwestern Univ., 2299 Sheridan Rd., Evanston, IL 60208-3570)

This study investigates the acoustic correlates for the four manners of stop articulation ([ \pm voice, \pm aspirated $]$ ) at the four different places of articulation (labial, dental, alveolar, and velar) in Urdu. The study follows up Davis' work [J. Phonet. 22, 177-193 (1994)] on manner in the homologous velar stops in Hindi. Data from four subjects for the 16 stops (ten different tokens of each stop for each subject) support Davis' assertion that $F 2$ "lag" time (between stop-released and onset of $F 2$ for the following vowel) is a more conclusive measure for the feature [aspirated] than the traditional voice onset time (VOT) measure. However in Urdu, unlike Hindi, lag time alone does not exhibit a four-way contrast. A measure of "lead" time (pre-stop-release voicing) is also required. Thresholds for lead time and lag time permit all four manners to be distinguished without reference to the place of articulation for all the subjects. Thus the phonological features [voice] and [aspirated] are more phonetically orthogonal in Urdu than Hindi. The results contribute to the ongoing debate on the invariance versus variability of acoustic cues, and to the discussion concerning the representations relating the phonological and phonetic levels of speech. 


\title{
Session 1pUW
}

\section{Underwater Acoustics: Scattering and Noise}

\author{
Michael F. Werby, Chair \\ Naval Research Laboratory, Code 7181, Stennis Space Center, Mississippi 39529
}

\section{Contributed Papers}

1:00

1pUW1. High-frequency forward scattering from Gaussian spectrum, pressure release, corrugated surfaces: Measurements of twinkling exponents and the dependence of the second moment on distance from surface and pulse length. J. S. Stroud, P. L. Marston (Phys. Dept., Washington State Univ., Pullman, WA 99164-2814), and K. L. Williams (Univ. of Washington, Seattle, WA 98105)

A single realization of a Gaussian spectrum surface (rms roughness 1.5 $\mathrm{cm}$, correlation length $10 \mathrm{~cm}$ ) was manufactured out of Styrofoam. This surface provided a pressure release, corrugated surface for an underwater, forward-scattering investigation. Omnidirectional source and receiver were used in the frequency range of $100-300 \mathrm{kHz}$. Short pulses were used to allow isolation of individual contributions to the scattered field. These individual contributions were then classified using catastrophe theory [K. L. Williams, J. S. Stroud, and P. L. Marston, J. Acoust. Soc. Am. 96, 1687-1702 (1994)]. The frequency dependence of the $m$ th higher-order intensity moments $I_{m}$ was measured and compared to predictions $[\mathrm{M}$. V. Berry, J. Phys. A 10, 2061-2081 (1977)] that $I_{m}$ is proportional to $k^{\nu_{m}}$ (for $m>2$ ), where $\nu_{m}$ is a twinkling exponent. Also, the dependence of $I_{2}$ on distance from the surface was examined at a single frequency utilizing various pulse lengths. It is known that far from a surface the wave field will obey Gaussian statistics $\left(I_{2}=2\right)$. For short pulses, however, the statistics of the wave field are strongly dependent upon individual reflections. For longer pulses this is the case near the surface but as one moves away it is shown that the Gaussian limit is approached. [Work supported by ONR.]

\section{$1: 15$}

1pUW2. Acoustic scattering from a buoyant plume using an antiparallel scattering geometry. John Oeschger (Dept. of Phys., Univ. of Rhode Island, Kingston, RI 02881) and Louis Goodman (Ocean and Atmospheric Phys. Div., Arlington, VA 22217)

An examination is made of the application of the far-field Born approximation to the case of high-frequency acoustic scattering from a thermally produced buoyant plume using a multiple bistatic antiparallel scattering geometry. Initial results indicate the failure of the predictions made by the far-field Born approximation. Further theoretical development includes the higher-order terms (wavefront curvature) in the far-field expansion to fully describe the scattering process. The previously simple relationship, however, between the scattered pressure field and the Fourier transform of the scattering field becomes greatly complicated. The problem is reduced by considering uniform vertical advection of the scattering field and by taking time series measurements of the plume. The resulting prescription relates the complex acoustic data to the two-dimensional Fourier transform of the scattering field through a two-dimensional low-pass filter function which includes the effects of the wavefront curvature terms and the beam pattern. Data are presented for the case of scattering from an unstable plume. Results confirm the predictions made by theory.

\section{$1: 30$}

1pUW3. Sound scattering by a single and by a cloud of air bubbles near the sea surface. G. C. Gaunaurd and H. Huang (Naval Surf. Warfare Ctr., White Oak Detachment, Silver Spring, MD 20903-5640)
Sound scattering by an air bubble in a boundless fluid is an old classical problem [i.e., R. Y. Nishi, Acustica 33, 65-74 (1975)]. If the air bubble is near, and strongly interacting with the surface of a liquid halfspace, then the scattering cross section (SCS) of the bubble is quite different from its value far away from the boundary. The exact solution for this scattering problem is given that is valid for any incidence direction of the (plane) sound waves, and for any bubble depth, obtained by the method of images. This benchmark solution is found by means of the addition theorems for the spherical wave functions. The resulting SCS contains contributions from the interface, the bubble, and from its image, and it is expressible in terms of coupling coefficients containing products of Wigner $3-j$ symbols. The formulation is illustrated with many computed plots and it is finally extended to the case of a round, low-concentration cloud of equal size bubbles, just beneath the sea surface. This generalization is possible by replacing the individual bubble properties by those of an "effeclive medium" describing the bubble cloud just as was found earlier [i.e., J. Acoust. Soc. Am. 85, 541-554 (1989)]. [Work supported by NSWC's IR Program.]

\section{$1: 45$}

1pUW4. Temporal response comparisons between model results and measurements of forward-scattered waves from the sea surface. E. J. Yoerger (Naval Res. Lab., Code 7174, Stennis Space Center, MS 39529) and Suzanne T. McDaniel (Penn State Univ., State College, PA)

The underwater acoustic propagation path for the forward scattering of energy from the sea surface is treated as a linear, time-varying random communications channel in the application of the Helmholtz-Kirchhoff integral formulation of this problem. The model used for data comparisons utilizes the bifrequency system function $\Gamma\left(\omega, \omega^{\prime}\right)$ developed by McDanie] [IEEE J. Ocean. Eng. 17, 216-221 (1992)] for the temporal response from a rough surface. The data analyzed for this work were obtained from a shallow-water high-frequency acoustic experiment conducted on the Baltic Sea during May 1993. Acoustic data included measurements of surface forward-scattering, surface reverberation, and direct-path intensities. These were made utilizing two large stationary towers resting on the seafloor. Each tower was equipped with horizontal and vertical receiving arrays anchored $7.6 \mathrm{~m}$ above the flat bottom depth of $30 \mathrm{~m}$. Concurrent environmental measurements including wave heights, sound velocity profiles, and sample cores were made. The results presented here are for the surface forward-scattered measurements made at 20,40,60, and $90 \mathrm{kHz}$. [Work supported by the Office of Naval Research, Program Element 61153N, with technical management provided by the Naval Research Laboratory, Stennis Space Center, MS.]

\section{2:00}

1pUW5. Modeling and analysis of three-dimensional scattering from Arctic Ice features. Tarun K. Kapoor and Henrik Schmidt (Dept. of Ocean Eng., MIT, Cambridge, MA 02139)

Interpretation of low-frequency acoustic scattering data from Arctic Ice is facilitated with realistic models of the large-scale features observed under the ice sheet. Previous analyses have modeled these, for example, as a hemispherical protuberance on an an infinite plane. Here, the under-ice 
feature is modeled as a solid elastic sphere attached in the center of a thin elastic plate. The sphere is assumed to be completely submerged in water while the plate is assumed to be floating on water. The interaction between the plate and sphere is characterized in terms of equivalent coupling forces and bending moments at the attachment ring which are determined by matching the kinematics of the sphere and the plate at the junction. At our frequencies of interest $[k a \sim O(1)]$, the model demonstrates scattering of the sphere modes into the in-plane and out-of-plane motions of the plate. A comparison will be made between the scattered fields from the free and coupled spheres. The analytical results will also be compared with those obtained from the CEAREX89 scattering experiments conducted in the Central Arctic region with 1.8-lb SUS charges detonated at nominal depths of $800 \mathrm{ft}$. [Work supported by ONR, High Latitude Program.]

\section{2:15}

1pUW6. A study on intensity fluctuations in forward scattering from the sea surface. Peter H. Dahl and Donald B. Percival (Appl. Phys. Lab., College of Ocean and Fishery Sci., Univ. of Washington, Seattle, WA 98105)

Fluctuations in the intensity of sound forward scattered from the sea surface are discussed. The data originate from an experiment off the southern California coastline using the research platform FLIP. Measurements were made using omnidirectional sources suspended from a spar buoy (tethered to FLIP) and receivers mounted on FLIP's hull, with range varying between 500 and $1000 \mathrm{~m}$. The frequency was $20-50 \mathrm{kHz}$ and the roughness parameter $\chi=2 k h \sin \theta$ was $\gg 1$ (where $k$ is acoustic wave number, $h$ rms wave height, and $\theta$ grazing angle); thus the measurements represent high-frequency, incoherent scattering in a single surface bounce channel. An often used statistical model for intensity fluctuations in such conditions is the exponential PDF, or its equivalent Rayleigh PDF for amplitude. Examples are presented wherein the exponential model fails a Lilliefors statistical goodness-of-fit test. The Lilliefors test is used for this purpose because parameters of the distribution are not known a priori. An alternative to the single-parameter exponential PDF is the two-parameter gamma PDF. Candidate variables, based on sea state and acquisition geometry that are covariates to the nature of intensity fluctuations, are discussed. [Work supported by ONR Cade 321.]

1pUW7. Influence of statistical surface models on dynamic scattering of high-frequency signals from the ocean surface. Christian Bjerrum-Niese and Leif Bjorno (Dept. of Indust. Acoust., Tech. Univ. of Denmark, Bldg. 425, DK-2800 Lyngby, Denmark)

Temporal variations of scattering of high-frequency, monochromatic signals from the ocean surface has been studied numerically. In the highfrequency domain the dynamic scattering can be modeled by a coherence function of the scattered pressure field, which is based on the Kirchhoff integral; the surface roughness is described by a spatial surface spectrum and the surface motion is described by the gravity-wave dispersion relation [D. Dowling and D. Jackson, J. Acoust. Soc. Am. 93, 3149-3157 (1993)]. Applying some modifications to this approach, the temporal coherence function is found by numerical evaluation of a double integral. The timevarying scattering can be examined in the frequency domain by the power spectrum (the Fourier transform of the coherence function). It is examined how a monochromatic signal is shifted and smeared in the frequency domain by comparing computations for the Pierson-Moskowitz spectrum (for a fully developed sea) with computations for the JONSWAP spectrum (for fetch-limited seas). The following results, among other issues, have been obtained: As the fetch decreases, the surface waves become shorter, leading to increasing frequency shifting of the scattered signal. [Work sponsored by the Danish Technical Research Council and the EU/MAST programme.]

\section{2:45-3:00 Break}

3:00

1pUW8. Measurements of the scattering of sound from laboratory breaking waves. Pat B. Dandenault, Ali R. Kolaini, and Yi Mao (Natl. Ctr. for Phys. Acoust., Univ. of Mississippi, University, MS 38677)

The results of a laboratory experiment to characterize the backscattered sound field from the bubble cloud generated by spilling breaking waves are described. Gravity waves were generated by a computer controlled plunging-type wavemaker along the length of a $12.7-\mathrm{m}$-long channel where they were made to break in a $3.6 \mathrm{~m} \times 3.6 \mathrm{~m} \times 2.4$ anechoic tank. An underwater F42A transducer with a special parabolic reflector was used to generate incident bursts of sound ranging from 15 to $40 \mathrm{kHz}$. This procedure was conducted in the presence of breaking and nonbreaking gravity waves in order to isolate the acoustic scattering strength of the bubble clouds from surface roughness. Detailed measurements of the average void fraction of the bubble cloud at the instant the sound was incident were made. These observations show that there is a significant increase in backscattering strength from the bubble cloud when compared to surface roughness. The scattering strength of the bubble cloud, with an average void fraction of $0.33 \%$, is also shown to increase as the incident frequency approaches the resonant frequency of the individual bubbles in the cloud. A simple theory will be given to predict some of these observations.

\section{3:15}

1pUW9. Modeling the azimuthal dependence of sea surface backscatter during CST-7. John Dubberley, Richard S. Keiffer (NRL Code 7181, Stennis Space Center, MS 39529-5004), and Jorge Novarini (Planning Syst., Inc., Bay St. Louis, MS)

The operator expansion method is applied to realizations of 2-D roughness measured during CST-7 in order to probe the azimuthal dependence of the backscatter from time-evolving, non-fully developed seas which contain swell from distant surface wave sources. To use wave buoy measured data without extrapolation in frequency beyond the measured surface wave spectra, the measured frequencies examined will be limited to 75 and 150 Hz. The modeling goal will be to determine that if surface scatter was alone responsible for acoustic backscatter from the surface, could there be a measurable azimuthal dependence in the scattering during this experiment? Comparisons to some available measured azimuthal dependence will be shown.

\section{3:30}

1pUW10. Stability of signal plus noise in performance evaluation of processing schemes. Jacob George and Ronald A. Wagstaff (Naval Res. Lab., Code 7176, Stennis Space Center, MS 39529)

The stability of signal plus noise and the mechanisms governing the combination of signal and noise at the receiver are reported. These questions become important in performance evaluation of processing schemes which are designed to enhance the $\mathrm{S} / \mathrm{N}$ ratio. Of special interest is the case of a weak signal combining with a relatively stronger noise. Even though the nonlinear interaction of sound by sound continues to be a hot topic [see, for example, P. J. Westervelt, "Answer to criticism of experiment on scattering of sound by sound," J. Acoust. Soc. Am. 95, 2865 (1994)], the largely linear effects governed by the superposition principle are of primary importance in the present application. Simple rules governing the combination of signal and noise, obtained through analysis of experimental data and model calculations, will be discussed. [Work supported by ONR, with technical management provided by NRL/SSC.]

\section{$3: 45$}

1pUW11. Estimation of Arctic ice thickness from ambient noise. Olga Chernets and J. Robert Fricke (Dept. Ocean Eng., Cambridge, MA 02139)

An estimate of total ice mass in the Arctic is an important parameter for global climate studies, but there are no direct means for providing this 
information. A surrogate measurement, spatial integration of ice thickness, is being pursued by several groups using active acoustic and electromagnetic methods. As a complement to these efforts, the passive estimation of ice thickness using ambient noise caused by thermal and mechanical stress cracks in the ice sheet is focused on. The acoustic signal received from propagating stress cracks is the convolution of an elemental fracture source function and the spatial distribution function (array) of the propagating crack. This analytic result predicts a tonal spectral component of $O(1 \mathrm{kHz})$ that relates directly to ice thickness. Experimental data collected in the Beaufort Sea during the spring of 1994 demonstrates the presence of this spectral peak, which correlates well with the measured ice thickness at the site.

\section{4:00}

1pUW12. Shallow-water ambient noise due to surf. Marc Stewart (Combat Syst. Sci. and Technol. Curriculum, Naval Postgraduate School, Monterey, CA 93943), O. B. Wilson, J. H. Wilson, and R. H. Bourke (Naval Postgraduate School, Monterey, CA 93943)

Forecasting levels and directionality of ambient noise in the littoral environment is important for predicting performance of acoustic sensor systems and developing naval warfare tactics for that environment. Noise due to waves breaking at the beach contributes significantly to the lowfrequency ambient noise in shallow water, as shown by the observations of O. B. Wilson, S. N. Wolf, and F. Ingenito [J. Acoust. Soc. Am. 78, 190195 (1985)]. At some geographic points worldwide, unique geoacoustic features contribute to the detectability of "surf point noise" (a term coined by submariners) dozens of kilometers out to sea. The objective of this work is to apply more recently developed and powerful shallow-water propagation loss models and the increased knowledge of the geoacoustic properties of the seabed to obtain a quantitative understanding of observations reported by $\mathrm{O}$. B. Wilson et al., and to make possible the prediction of the noise fields from surf in other locations. The results of the use of the SNAP programs applied to the Monterey Bay environment will be presented.

\section{$4: 15$}

1pUW13. A semiempirical model for breaking wave noise fields. Steven Finette and Richard Heitmeyer (Acoust. Div., Naval Res. Lab., Washington, DC 20375)
Mean noise properties, traditionally used for underwater acoustics systems analysis, are not suitable for evaluating the performance of full spectrum processors because they don't have sufficient space/time/frequency resolution. A computer model of the noise field associated with breaking surface waves has been developed in an attempt to address this need. Due to the localized structure of the breaking wave sources, the true ambient noise field is inherently discrete and an attempt has been made to include some properties of the discrete nature of the space-time noise field in the model. The model computes a realization of a time-delay beamformed representation of the acoustic field at a vertical array in a shallow-water environment, given some statistical information about the noise sources. The approach taken is stochastic and semiempirical, incorporating recently obtained experimental data on the acoustic coverage (acoustic analog of whitecap coverage), duration, and source level distributions associated with individual breaking waves (Farmer 1994). Sources are considered to radiate as dipoles, and the radiated fields from the individual discrete sources (breaking waves) are propagated to the array using a wave-number integration technique. [Work supported by ONR.]

\section{$4: 30$}

1pUW14. Effect of forward-scattered signal on beam noise estimation in shallow water. Yung P. Lee and John Hanna (Sci. Appl. Intl. Corp., 1710 Goodridge Dr., MS T1-3-5, McLean, VA 22102)

In deterministic situations, adaptive processors resolve strong coherent interferers and eliminate their contribution at locations away from them. But, in operation, it is found that the beam noise away from strong interferers is affected by their presence. Stochastic mechanisms involved with the interferer's signal are assumed to be responsible for this behavior. A comparison of ocean data with a simulation has been made to illustrate the effect of the forward-scattered signal from the ocean surface on adaptive beam noise estimation in shallow water. The stochastic forward-scattering mechanism is dependent on the environment and its effect is apparently influenced by undersampling in the spatial aperture of the measurement array. The dependency of beam noise on waveheight and bathymetry have been observed.

\title{
Session 1eID
}

\section{Interdisciplinary: Tutorial on Acoustic Cavitation: Sonic Effervescence}

\author{
Anthony A. Atchley, Chair \\ Department of Physics, Naval Postgraduate School, Monterey, California 93943
}

Chair's Introduction-7:00

\section{Invited Paper}

\section{7:05}

\begin{abstract}
1eID1. Acoustic cavitation: Sonic effervescence. Robert E. Apfel (Ctr. for Ultrasonics and Sonics, Yale Univ., New Haven, CT
\end{abstract} 06520-8286)

Bubbles intrigue and amuse young and old, scientist and nonscientist, alike. When acoustical waves either cause or enhance the activity of bubbles, a variety of phenomena can be observed, ranging from sonoluminescence to ultrasonic cleaning, and from acoustical attenuation in the ocean to microbubble formation in blood. This tutorial will review these and other phenomena associated with acoustic cavitation, focusing on the interesting basic questions underlying the observations and summarizing the nature of the beneficial applications that cavitation permits and the strategies for avoiding undesirable cavitation. In addition, points of contact will be made with other bubble phenomena, such as boiling, hydrodynamic cavitation, decompression sickness, and radiation-induced bubble formation in superheated liquids (the bubble chamber). Participants in this tutorial should be able to tell if the glass of beer they are drinking from is clean. (If possible, samples will be available...for testing purposes.) 
Session 2aAO

\title{
Acoustical Oceanography: Acoustical Determination of Ocean Processes
}

\author{
Michael D. Collins, Chair \\ Naval Research Laboratory, Code 7140, Washington, DC 20375
}

Chair's Introduction-8:00

\section{Contributed Papers}

\section{8:05}

2aAO1. Eficient navigation of parameter landscapes. Michael D. Collins (Naval Res. Lab., Washington, DC 20375)

The covariance matrix of the gradient of the cost function contains a great deal of information about a parameter space. The eigenvectors of the covariance matrix form an optimal basis (in the sense of data compression) for the gradient. Since search algorithms base their decisions on the gradient (often in an indirect fashion), the eigenvectors in some sense form an optimal set of generators for navigating parameter landscapes. For problems involving a long valley, there is usually an eigenvector oriented parallel to the valley. Search algorithms based on the optimal generators may find the deepest point in the valley several times faster than algorithms based on other generators. The covariance matrix also contains information about the key underlying parameters. The most important parameters correspond to the eigenvectors associated with the largest eigenvalues. This information can be exploited to reparametrize with a smaller number of parameters. The covariance matrix is the integral of the outer product of the gradient over the parameter space. Obtaining a good estimate of this integral with the Monte Carlo method usually requires relatively little effort, even for high-dimensional parameter spaces. Examples are presented for geoacoustic inverse problems involving acoustic sources and receivers located in the ocean.

$$
\text { 8:20 }
$$

2aAO2. Matched-field inversion of multifrequency data. Peter Gerstoft, Dave Hannay, and Don Gingras (SACLANT Undersea Res. Ctr., I-19138 La Spezia, Italy)

Geoacoustic inversion using single-frequency data measured on a vertical array has become a popular method for estimating sub-bottom parameters. Inclusion of multiple frequencies in the inversions allows exploitation of both the waveguide dispersion and the different penetration into the sub-bottom at different frequencies. This is achieved, however, at the expense of greater required computing time, and more complicated processing schemes. Several approaches exist for defining a broadband object function, i.e., coherent or incoherent addition across frequencies and hydrophones. Each approach has specific requirements with regard to the recording geometry and the source spectrum. For coherent addition across frequencies not only the source spectrum but also the absolute propagation time must be estimated. When a useful object function has been defined it is optimized using genetic algorithms [P. Gerstoft, J. Acoust. Soc. Am. 95, 770-782 (1994)] for both the sediment and the geometric parameters. Different approaches are illustrated on broadband data from the Mediterranean North Elba site with center frequencies of 170 and $330 \mathrm{~Hz}$. The water depth was $130 \mathrm{~m}$ and the source range was $5.5 \mathrm{~km}$. The normalmode code SNAP was used as the forward model.

\section{8:35}

2aAO3. Inversion of acoustic field data using genetic algorithms: Shallow-water results. D. F. Gingras and P. Gerstoft (SACLANT Undersea Res. Crr., Viale San Bartolomeo, 400, 19038 La Spezia, Italy)
Precise knowledge about environmental parameters is highly desirable for precise acoustic modeling of the underwater channel. Simulations have shown that global inversion methods such as simulated annealing and genetic algorithms are effective for the estimation of both geoacoustic and geometric (source location, receiver locations, and water depth) parameters. In this paper genetic algorithms are applied to acoustic field observations. The field observations were collected at a shallow-water site in the Mediterranean Sea near the Italian coast, where the depth was approximately $130 \mathrm{~m}$. The source was stationary and produced a broadband signal at $170 \mathrm{~Hz}$; a vertical array of receivers which spanned most of the water column was employed. The inversion process used the Bartlett processor for the object function. A range-independent version of the SACLANTCEN normal-mode acoustic propagation model SNAP was used for forward modeling. Inversion was carried out simultaneously over source location parameters, range and depth, and environmental parameters. A 20-min sample of observations was considered; at 1-min intervals estimates of the parameters were computed. As a function of both time and frequency, over a limited frequency band, it was shown that the genetic algorithms produced stable estimates for both the environmental and source location parameters.

\section{8:50}

2aAO4. Adaptation of genetic-algorithm search for matched-field inversion of ocean bottom compressional wave speed profiles. Thomas J. Hayward and T. C. Yang (Naval Res. Lab., Washington, DC 20375-5350)

Matched-field inversion for ocean bottom compressional wave speeds using a synthetic horizontal aperture has been demonstrated previously in simulations and data analyses employing optimization by simulated annealing [M. Collins, W. Kuperman, and H. Schmidt, J. Acoust. Soc. Am. 92, 2770 (1992)] and genetic-algorithm search [P. Gerstoft, J. Acoust. Soc. Am. 95, 770 (1994)]. The first reference noted a lack of sensitivity of the matched-field ambiguity function to zero-mean functional variations of the sound-speed profile. This lack of sensitivity sometimes results in slow convergence of the search algorithm when applied to profiles with several unknown parameters. This problem is observed in simulations using genetic algorithm search. Improved convergence of the genetic algorithm is then achieved by using some nonstandard procedures, including autologous gene exchanges, to generate new solutions. The simulations indicate the practical feasibility of inversion for deep-bottom (up to $500 \mathrm{~m}$ ) compressional wave speeds at a shallow-water site using a single receiver and coherent synthetic horizontal aperture processing of a towed-source signal.

\section{9:05}

2aA05. Optimizing shallow-water sound-speed estimation using parameter resolution bounds. Nicholas C. Makris (Naval Res. Lab., Washington D.C. 20375)

A technique is under development to optimize experimental design for estimation of 3-D sound-speed structure by inversion of acoustic data. The motivation is to take advantage of $a$ priori knowledge of invariant envi- 
ronmental parameters to estimate the minimum number of sensors necessary and their optimal deployment geometry for a well constrained inversion. First, static environmental information, such as bathymetry, geoacoustic parameters of the sediment, and mean sound-speed structure of the water column, is input to an appropriate range-dependent acoustic model for a given sensor deployment geometry. Next, a theoretical lower bound on estimation error for the water-column sound-speed structure is obtained via the Cramer-Rao bound. The deployment geometry is then perturbed until the estimation error is within acceptable bounds for oceanographic and acoustic modeling. However, the choice of sound-speed parametrization can also severely affect the accuracy of an inversion. For example, an empirical orthogonal function (EOF) representation typically has higher resolution for fewer parameters than a discrete cell representation. But this is at the cost of more limiting assumptions. These issues are addressed by computing the theoretical lower bound on estimation error for discrete cell, EOF, and Fourier internal wave representations of 3-D sound-speed structure.

\section{9:20}

2aA 06. Coherent acoustic bubble sizing in the ocean. B. K. Choi, H. R. Lee, and S. W. Yoon (Dept. of Phys., Sung Kyun Kwan Univ., Suwon 440-746, Republic of Korea)

Bubbles are well known to induce variations of acoustic characteristics in water. The bubble size distribution gives significant information for understanding underwater sound propagation and acoustic roles of bubbles in the ocean. Since the conventional acoustic bubble sizing method only considered sound attenuation due to bubbles around resonance frequencies in a bubbly mixture, a bubble size distribution was incoherently estimated. In the present coherent acoustic bubble sizing method, sound speed as well as sound attenuation are considered to have a coherent estimation of bubble size distribution in the bubbly mixture. The present coherent method is proved to describe a bubble size distribution more accurately than the conventional incoherent one. [Work supported by the Korea Science and Engineering Foundation.]

2aA07. Multiple bubbles: Collective modes and the origin of superresonances. C. Feuillade (Naval Research Laboratory, Stennis Space Center, MS 39529-5004)

Acoustic resonances and scattering from systems of multiple air bubbles in water are described using a coupled differential equation method. By recombination, the problem may be analyzed by scattering from the individual normal modes of vibration of the ensemble. The modes are of two types: "Symmetric" modes, where the bubbles vibrate in phase with each other, typically show downward shifts in frequency and increased damping; "antisymmetric" modes, where some or all of the bubbles vibrate in antiphase, generally show upward frequency shifts and reduced damping and may become super-resonant. For two bubbles the method reproduces frequency shifts measured experimentally. Examination of the modal response functions shows that super-resonances may occur for specific bubble spacings if the individual bubbles are primarily radiation damped. For two and three bubble systems super-resonant scattering is strongly dipolar and propagates little energy in the far field, making the phenomenon difficult to observe experimentally. Scattering from a bubble reflected in a pressure release surface should show the phenomenon strongly. By extending multiple bubbles in a line the damping rates predicted by Weston for an infinite line array may be approached. The method outlined can easily be applied, using matrix methods, to describe acoustic scattering from large ensembles. [Work supported by ONR (Element $602435 \mathrm{~N}$ ). Technical management provided by NRL-SSC.]

\section{$9: 50$}

2aA08. Acoustic characterization of laboratory breaking waves in fresh and salt water. Ali R. Kolaini (Natl. Ctr. for Phys. Acoust., Univ. of Mississippi, University, MS 38677)

Results of a laboratory experiment to characterize the underwater acoustic noise radiated from breaking waves in fresh and salt water are described. The underwater sound field radiated by various breaking waves intensities in fresh water in the range from 0.1 to $20 \mathrm{kHz}$ were reported earlier [A. R. Kolaini and L. A. Crum, J. Acoust. Soc. Am. (in press); 92, 2349(A) (1992)]. These waves were generated by a computer-controlled plunging-type wavemaker and propagated along a $12.7-\mathrm{m}$-long channel where they were made to break at the midsurface of a $3.6-\times 3.6-\times 2.4-\mathrm{m}$ anechoic tank. Specific attention is given to a comparison of the results obtained in salt and fresh water. The sources of acoustic radiation from bubble clouds, the average sound-pressure level, bubble clouds shape, bubble concentration, and size distribution in these medium are discussed. These results may provide considerable insight into the likely source mechanisms for ocean ambient noise.

\section{0:05}

2aA09. Acoustic daylight: Preliminary results from an ambient noise imaging system. John R. Potter, Michael J. Buckingham, Grant B. Deane, Chad L. Epifanio, and Nicholas M. Carbone (MPL 0238, Scripps Inst. of Oceanogr., Univ. Calif. at San Diego, 9500 Gilman Dr., La Jolla, CA 92093-0238)

A broadband $(8-80 \mathrm{kHz})$ multibeam (126) acoustic daylight system has been developed which forms real-time images at a $27-\mathrm{Hz}$ frame rate using only natural ambient noise in the ocean. The color and intensity of each pixel of the final image is determined by the spectral shading and intensity of the received signal in each beam normalized to a runningaverage ambient noise spectrum. The first deployment of this system will be made from a floating research platform (ORB) during August in San Diego Bay. The high-frequency ambient noise at this site is dominated not by surface waves, but by colonies of snapping shrimp (Alpheus and Synalpheus) associated with nearby structures and other habitats. The preliminary results from this deployment will be presented, including a description of the data processing and color mapping algorithm, the ambient noise environment, and some preliminary image results from simple targets at close range. [Work supported by ONR.]

\section{0:20-10:35 Break}

\section{$10: 35$}

2aA010. Forward-scattering simulations through ocean-ridge hydrothermal-vent diffusive layer turbulence: Feasibility of vent monitoring. T. F. Duda (Woods Hole Oceanogr. Inst., Bigelow 2, Woods Hole, MA 02543) and D. A. Trivett (Dalhousie Univ., Halifax, NS B3H 4J1, Canada)

Temperature and velocity fluctuations in a bottom boundary layer down-current of Juan de Fuca Ridge hydrothermal sites were monitored in 1990 from tripods. The diffuse flow from relatively cool vents remains near the bottom, and acoustic refractive index fluctuations decay away slowly. Rytov theory-based forward-scattering simulations through structures consistent with the observations are used to verify the validity of Rytov theory, compare the usefulness of various propagation path lengths and frequencies on monitoring, and evaluate the ability of acoustic phase and amplitude recordings to monitor desired vent parameters. The Rytov theory is valid for some ranges and frequencies, but issues such as turbulence homogeneity, energy dissipation rate, boundary-layer depth, beam patterns, and sensor motion would have strong effects on the measurements.

\section{0:50}

2aA011. Continuous wave and $M$-sequence transmissions across the Arctic. Peter N. Mikhalevsky (Sci. Appl. Intl. Corp., McLean, VA 22102), Arthur B. Baggeroer (MIT, Cambridge, MA 02139), Alexander Gavrilov (Andreev Inst. of Acoust., Moscow, Russia), and Mark Slavinsky (Inst. of Appl. Phys., Nizhny Novgorod, Russia)

In the spring of 1994 a series of $\mathrm{cw}$ and maximal length sequences (M sequences) were transmitted $2600 \mathrm{~km}$ across the Arctic Ocean. The transmissions were centered at $19.6 \mathrm{~Hz}$ from a Russian source located $300 \mathrm{~km}$ north of the Svalbard Archipelago. The transmissions were received in the 
Lincoln Sea (900-km range) on a 32-element vertical line array and in the Beaufort Sea $(2600-\mathrm{km}$ range) on a 32-element vertical line array and a 32-element horizontal planar array. Analysis of the transmission loss, phase stability, array gain, and pulse compression gain are being used to develop an acoustic monitoring strategy in the Aretic for climate studies. The exceptional stability of the Arctic sound channel first noted in the early 1980s [P. N. Mikhalevsky, J. Acoust. Soc. Am. 70, 1717 (1981)] make acoustic thermometry potentially a very sensitive indicator of Arctic Ocean temperature and ice changes.

\section{1:05}

2aA012. Performance limits on acoustic thermometry of ocean climate in the presence of mesoscale sound-speed variability. Jeffrey L. Krolik and Sunil Narasimhan (Dept. of Elec. Eng., Duke Univ., Box 90291, Durham, NC 27708)

The ability to measure climatic changes in ocean temperature is fundamentally limited by the presence of mesoscale variability. Because ocean acoustic propagation depends on the range-averaged sound-speed (and hence temperature) profile, long-range acoustic transmissions have been proposed as a means of filtering out mesoscale variability in order to measure a global warming related trend in mean temperature. The Cramer-Rao lower bound (CRLB) on the estimation of a change in the mean depth-dependent temperature profile is presented to determine the highest accuracy which could be achieved by acoustic thermometry. This work extends [A. B. Baggeroer, J. Acoust. Soc. Am. 95, 2850 (A) (1994)] by evaluating the CRLB for different representations of the mean depthdependent temperature profile perturbation with different levels of a priori knowledge about the mesoscale sound-speed variability. With prior statistical knowledge of the mesoscale variability, the CRLB indicates that accurate measurement of the climate signal may be possible using a general Chebyshev polynomial representation of the mean depth-dependent temperature pertubation. [Work supported by ONR.]

\section{1:20}

2aAO13. High-frequency ( $>1$ cpd) travel time variability of long-range reciprocal acoustic transmissions in the western North Atlantic. Brian D. Dushaw (A.P.L., Univ. of Washington, 1013 NE 40th St., Seattle, WA 98105-6698), Peter F. Worcester, Bruce D. Cornuclle (Univ. of California, La Jolla, CA 92093-0213), and Bruce M. Howe (Univ. of Washington, Seattle, WA 98105-6698)

Time series of ray travel times were obtained at 350-, 410-, and $670-\mathrm{km}$ ranges in the western North Atlantic during the 1991-2 Acoustic Mid-Ocean Dynamics Experiment (AMODE). Transmissions were recorded for approximately $\mathbf{3 0 0}$ days between six transceivers in a pentagonal array. Sound-speed (current) variability is observed by calculating the sum (difference) of reciprocal travel times. The sum and difference of reciprocal travel times are high-pass filtered by removing a daily average. The barotropic-tide current is measured by the differential travel times. Both phase-locked and narrow-band internal-tide sound-speed variability, caused by the internal-tide isotach displacement, are observed by the sum travel times. The acoustic array acts as a high-directivity antenna for the incident internal tide. The observed internal tide is likely generated at the continental shelf surrounding the North American Basin, or perhaps the Mid-Atlantic Ridge. The nontidal, high-frequency variability (>1 cpd) is due to internal-wave sound-speed and current variability.

\section{1:35}

2aA014. The Kaneohe acoustic thermometry experiment and rays. John L. Spiesberger (Dept. of Meteorol. and the Appl. Res. Lab., 512 Walker Bldg., Penn State Univ., University Park, PA 16802)

In the 1980s, the Kaneohe source, on the north shore of Oahu, transmitted sound (133 Hz; $60-\mathrm{ms}$ resolution) from 183-m depth to a U.S. Navy receiver at $3709-\mathrm{km}$ distance near northern California. Despite the fact that sound reflects from the Oahu slope before being trapped in the sound channel, ray theory is shown capable of determining the spatial coordinates of the stable acoustic pulses. Rays that bounce from the bottom are prob- ably chaotic, but the coordinates and travel times of eigenrays are insensitive to initial conditions and ocean fluctuations. The coda of the reception can probably not be explained with a propagation model in which the sound-speed field is smoothed to suppress scales smaller than the mesoscale. Instead, it appears that scattering of sound from smaller scales distributes otherwise axially trapped sound over $1000 \mathrm{~m}$ in the vertical, thus limiting the vertical resolution achievable with tomography. [Work supported by Advanced Research Projects Agency and Office of Naval Research.]

\section{1:50}

2aAO15. The Kaneohe acoustic thermometry experiment and waves. Fred D. Tappert (Appl. Marine Phys., RSMAS, Univ. of Miami, 4600 Rickenbacker Cswy., Miami, FL 33149) and John L. Spiesberger (Penn State Univ., University Park, PA 16802)

A new parabolic approximation is derived and implemented that efficiently gives travel time predictions that are accurate to within a few milliseconds at a range of $1000 \mathrm{~km}$ and center frequency $75 \mathrm{~Hz}$. Sensitivity of the full-wave travel time predictions to oceanographic and bathymetric inputs along a $3700-\mathrm{km}$ track is studied by comparisons to acoustic data between a source at Kaneohe having center frequency $133 \mathrm{~Hz}$ and a bottom mounted receiver near the northem California coast. Path stability for a time interval of several years is also examined with the new broadband PE model, and full-wave generalizations of eigenrays, called eigentubes, are computed. [Work supported by SERDP, ARPA, and ONR.]

\section{2:05}

2aA016. Change of sound ray by various equations of the sound velocity in the 621-km propagation experiment. Toshio Tsuchiya, Toshiyuki Nakanishi, Yasutaka Amitani, Hiroshi Ochi, Hidetoshi Fujimori (Deep-Sea Technol. Dept., Japan Marine Sci. and Technol. Ctr., 2-15 Natsushima-cho, Yokosuka 237, Japan), and Akio Hasegawa (Natl. Defense Acad. of Japan, 1-10 Hashirimizu, Yokosuka 239, Japan)

Generally, sound velocity used to be calculated according to equations by Wilson (1960), DelGrosso (1974), Chen and Millero (1977), and others, which were obtained by direct measurement in laboratories based on water depth, temperature, and salinity data collected by the XBT (expendable Bathey-tharmograph) or CTD (conductivity temperature depth) system. However, in observations such as ocean acoustic tomography (OAT) where it is necessary to make accurate measurement of the sound wave propagation time over the distance of hundreds to thousands of kilometers, even the slightest difference of the sound velocity can produce significant errors in the observatory accuracy. Thus the effect of various equations of the sound velocity on the formation of the specific sound ray in the SOFAR channel was examined by using the simulation method by the sound ray theory. The simulation results were compared with the specific sound ray data obtained in the 621-km propagation experiment in the northeast $\mathrm{Pa}$ cific Ocean, and as a result, the equation by Chen and Millero is found to be the most consistent among the three equations.

\section{2:20}

2aAO17. Long-range propagation tests of a $200-\mathrm{Hz}$ sound source for ocean acoustic tomography. Toshiaki Nakamura, Toshio Tsuchiya, Iwao Nakano, Toshiyuki Nakanishi (Japan Marine Sci. and Technol. Ctr., 2-15 Natsushima-cho, Yokosuka 237, Japan), Ieharu Kaihou, Akio Kaya (Oki Electric Industry Co., Ltd., 688 Ozuwa, Numazu 410, Japan), and Toshiaki Kikuchi (National Defence Academy of Japan, 1-10 Hashirimizu, Yokosuka 239, Japan)

A 200-Hz low-frequency sound source for ocean acoustic tomography was developed by using a supermagnetostrictive material made of the rare-earth metal $(\mathrm{Tb}, \mathrm{Dy}) \mathrm{Fe}_{2}$ aimed at increasing the transmission level and the reduction of the sound source size. A sound-pressure level above 190 $\mathrm{dB}(r e: 1 \mu \mathrm{Pa}$ at $1 \mathrm{~m}$ ) with a frequency bandwidth of over $50 \mathrm{~Hz}$ were obtained in the depth range between 800 and $1200 \mathrm{~m}$. Field tests for the long-range propagation of several hundreds of kilometers were carried out 
near the Japan Trench in November 1993. The sound source was moored at a depth of $1242 \mathrm{~m}$ and the hydrophone array was moored at a depth of $1385 \mathrm{~m}$. The distance between the sound source and the hydrophone array was $621 \mathrm{~km}$. Received data were recorded with a sufficient signal-to-noise ratio after achieving beamforming to discriminate the sound waves inci- dent upon the hydrophone array from the upper and lower direction. The signals were also received by the acoustic monitoring system suspended from the ship at the distance of $772 \mathrm{~km}$ from the sound source. From these results, it is confirmed that this sound source has the capability of $1000-\mathrm{km}$ propagation necessary for the tomography transceiver systems.

\title{
Session 2aNS
}

\author{
Noise: Fan Noise and General Topics in Noise \\ Gerald C. Lauchle, Chair \\ Applied Research Laboratory, Pennsylvania State University, P. O. Box 30, State College, Pennsylvania 16804
}

Chair's Introduction-8:30

\section{Invited Papers}

\section{$8: 35$}

2aNS1. Turbofan noise research at NASA. John F. Groeneweg (NASA Lewis Res. Ctr., M.S. 77-6, 21000 Brookpark Rd., Cleveland, $\mathrm{OH} 44135$ )

Results of recent NASA research to reduce aircraft turbofan noise are described. For very high bypass ratio turbofan engines, the dominant source of engine noise is the fan. A primary mechanism of tone noise generation is the rotor blade wakes interacting with downstream stator vanes. Methods of analyzing rotor-stator tone noise generation are described and sample results are given. The analysis includes the unsteady aerodynamic response of the stators to gusts, coupling to duct modes, and finite element calculation of the far-field radiation accounting of for flow in the fan ducts. Wind tunnel tests of model fans and nacelles are described including comparisons between measured and predicted tone directivities. A novel rotating microphone technique is used to measure the acoustic modes in the fan inlet, and the results indicate that a mechanism associated with unsteady fan tip loading can be important in addition to rotor-stator interaction. Finally, concepts for active fan noise control which emphasize control at the source are addressed by an unsteady aerodynamic analysis of compliant stator vanes.

\section{9:00}

2aNS2. Historical developments in the control of noise generated by small air-moving devices. George C. Maling, Jr. (Empire State Software Syst., Ltd., P. O. Box 2880, Poughkeepsie, NY 12603)

The scaling laws for air-moving device noise developed by R. D. Madison form a useful starting point in the development of techniques for the control of air-moving device noise. The commercial building boom after World War Il provided the impetus for research into air-moving device noise, and the increase in the use of home air-conditioning systems provided the motivation for further research. Beginning in the $1960 \mathrm{~s}$, noise generated by computing machinery became important, and the development of air-cooled computing equipment provided a further stimulus to noise control research. Developments in the control of air-moving device noise are reviewed with emphasis on scaling laws, dimensional analysis techniques, mechanisms of noise generation, noise emission measurements, noise reduction techniques, design guidelines, and system effects. Some suggestions for future research on air-moving device noise are given.

\section{9:25}

2aNS3. Efficient sound generation in a ducted axial fan due to a rotating wavelike flow pattern. M. H. Krane, P. H. Bent, ${ }^{\text {a) }}$ and D. A. Quinlan (AT\&T Bell Labs., 600 Mountain Ave., Murray Hill, NJ 07974)

Previous investigations have shown the presence of narrow-band peaks in the acoustic spectra of ducted axial fans at frequencies other than harmonics of the blade passage frequency. This phenomenon is of practical importance because these peaks are strongest when the fan is operating near its best efficiency point. Measurements of the fan flowfield were conducted in the rotating frame using hot-wires and surface-mounted hot-film sensors in order to identify the source of the narrow-band tones. These measurements have shown a rotating wavelike motion composed of several discrete modes. The wave pattern was strongest on the pressure side of the blade in the region between the tip and the midspan. One of the modes of the rotating pattern was found to be an integer multiple of the number of fan blades, resulting in efficient sound generation at that frequency. ${ }^{\text {a) }}$ Present address: Acoustics Technology, McDonnell Douglas Aerospace, Mail Code 71-35, 1510 Hughes Way, Long Beach, CA 90810-1864.

\section{9:50}

2aNS4. A modified transfer function method for source impedance in ducts. M. G. Prasad and W. S. Kim (Dept. of Mech. Eng., Stevens Inst. of Technol., Castle Point on the Hudson, Hoboken, NJ 07030)

Experimental characterization studies of source impedance have resulted in several direct and indirect methods. The transfer function (TF) method, which is a well-known direct method, has been quite successful in measuring source impedance. However, one 
important drawback of the TF method is that it fails for the case of low signal-to-noise ratio in the system. The noise in the system could be due either to the operating source (to be characterized) or to the flow in the system. A novel method to overcome the problem due to low signal-to-noise ratio is presented. A modified transfer function (MTF) is determined by using an additional measurement of transfer function of the system with a very low level of signal of the secondary source used in the method. Then the two transfer functions are used to estimate the MTF. The source impedance is then calculated using the reflection coefficient which is obtained using the MTF. This new MTF method has given very good results on a model electroacoustic speaker-duct system. A modified coherency function is used for error analysis. This MTF method can be used to measure the impedance of sources such as fans and blowers used in cooling systems.

10:15-10:30 Break

\section{Contributed Papers}

10:30

2aNS5. Applications of the proposed new method for computing attenuation of fractional octave bands of a wideband noise by atmospheric absorption. Paul D. Joppa (Boeing Commercial Airplane Group, P.O. Box 3707, MS 67-ML, Seattle, WA 98124-2207), Louis C. Sutherland (Rancho Palos Verdes, CA 90274), and Allan J. Zuckerwar (NASA Langley Res. Ctr., Hampton, VA 23681-0001)

A simple method for computing the attenuation due to atmospheric absorption for fractional octave bands of noise, outlined in a previous paper [P. D. Joppa, L. C. Sutherland, and A. J. Zuckerwar, J. Acoust. Soc. Am. Suppl. $188, S 73$ (1990)], is briefly reviewed and example applications to aircraft noise and room acoustics presented. The method uses an analytical approximation of a "representative" frequency for which the pure-tone attenuation loss due to atmospheric absorption is equal to the actual loss for the band of noise. The resulting total band attenuation, $\delta \mathrm{Lb}$ for propagation over a distance $s$, for a band with an exact midband frequency $f_{m}$, is equal to a nonlinear function of the total pure-tone attenuation $a\left(f_{m}\right) \cdot s$ over this path where $a\left(f_{m}\right)$ is the pure-tone attenuation coefficient at the exact midband frequency $f_{m}$. The method provides better estimates than in SAE ARP 866A for the true band attenuation when the total true band attenuation is less than about $50 \mathrm{~dB}$. For application to room acoustics, it is shown that the "distance" $s$ involved is simply equal to the reverberation time, $T$ times the speed of sound in the room.

\section{0:45}

2aNS6. Noise assessment and prediction system (NAPS) evaluation. John M. Noble, Robert O. Olsen (U.S. Army Res. Lab., Battlefield Environment Directorate, ATTN: AMSRI-BE-S, White Sands Missile Range, NM 88002), and Richard Okrasinski (New Mexico State Univ., Las Cruces, NM 88001)

A system has been designed to provide an assessment of noise levels that result from testing activities at Aberdeen Proving Ground, MD. The system receives meteorological data from surface stations and an upper air sounding system. The meteorological data are used as input into an acoustic ray trace model which projects sound level contours onto a twodimensional display of the surrounding area. This information is also provided to the range control office where a decision can be made to proceed or delay the test activity depending upon acoustic propagation conditions. To evaluate the noise level predictions, a series of microphones is located off the reservation to monitor sound-pressure levels. Any events of significant level are transmitted back to the central display unit, allowing for comparison between prediction and data. The computer models are modular, allowing for a variety of models to be utilized and tested to achieve the best agreement with data. This technique of prediction and model validation will be used to improve the noise assessment system.

11:00

2aNS7. Abstract withdrawn.
11:15

2aNS8. Legal frame of environmental noise in México. Rafael Trovamala Landa (Ctr. de Investigación y de Estudios Avanzados del I.P.N., Av. Inst. Politéc. Ncl. 2508, San Pedro Zacatenco, México, D.F. 07000, México)

The regulations, accepted methods of measurement, and standards used in México related to the determination of noise in environmental and labor environments, as well as the maximum allowable values in contrast with their equivalents used in the United States and Canada, are reviewed.

11:30

2aNS9. Noise regulations in Mexico. Fernando J. Elizondo (Lab. de Acúst., Facultad de Ingeniería Mecánica y Eléctrica, Univ. Autónoma de Nuevo León, P.O. Box 28 sucursal F, Cd. Universitaria, San Nicolás, N.L. 66450, México)

An overview of the regulations on noise in Mexico is presented. Included are historic development, classification, categorization, contents, and sources. Information on how to update and obtain the laws and regulations is also mentioned.

\section{1:45}

2aNS10. Noise levels in Mexican industry. Juan Antonio Ortiz Garcia (Ingenieria Acúst. Spectrum, S. A. de C. V. Apaseo el Allto No. 21-2, Colonia San Bartolo Atepehuacán, Delegación Gustavo A. Madero, México 07730 D.F., Mexico)

Since 1985, labor regulations on noise exposure have existed, and as in other countries, the quantity employed is the A-weighted sound-pressure level. Maximum allowable exposure levels were taken from ISO-19991975. According to local statistics of professional illness, hearing loss occupies second place in importance. The measurement results of A-weighted sound-pressure levels in different types of Mexican industries, from 1990 to 1993, are described. These measurements were made in concordance with official methodology. The results showed that less than $50 \%$ of a sampled population stayed in a noisy ambient (continuous or fluctuating) with levels higher than $90 \mathrm{dBA}$. It is considered that A weighted sound-pressure level is not enough to properly describe noise at the workplace; due to high level values usually encountered at low frequencies, which, even though they are not normally audible, may cause workers' low performance, beside other biological and psychological effects. Frequency analysis results of the same industries and how they are employed for the calculation of other quantities, like loudness and noisiness, are also described. 


\title{
Session 2aPA
}

\section{Physical Acoustics: Sonoluminescence and Sonochemistry I}

\author{
Ronald A. Roy, Chair \\ Applied Physics Laboratory, University of Washington, Seattle, Washington 98105 \\ Chair's Introduction-8:25 \\ Invited Papers
}

8:30

2aPA1. Sonoluminescence. Lawrence A. Crum and Sean Cordry (Appl. Phys. Lab., Univ. of Washington, Seattle, WA 98105)

In 1896, Henri Becquerel discovered that a uranium salt could darken a photographic plate, and from this effect went on to discover radioactivity. In 1934, Frenzel and Schultes discovered that acoustic waves, generated in a water bath, could also darken a photographic plate. They attributed this effect to luminescence from the sound field, a phenomenon that would later be known as sonoluminescence. A sound field by itself does not cause luminescence; it is through the mechanism of acoustic cavitation, whereby microscopic gas bubbles are caused to oscillate so violently that a remarkable energy concentration occurs. Under certain conditions, the cavitation event is transitory, and self-destructs within a few acoustic cycles; under other conditions, a stable bubble will luminesce for extended periods of time. This initial presentation will serve as a general review of the phenomenon of acoustic cavitation and demonstrate how bubble dynamics leads to many of the observed effects. Later papers in this session will provide more detail of various aspects of the general phenomenon. [Work supported in part by the Office of Naval Research.]

\section{8:55}

2aPA2. Probing the unknowns of sonoluminescence. Bradley P. Barber, Robert Hiller, Ritva Löfstedt, Keith Weninger, and Seth Putterman (Phys. Dept., UCLA, Los Angeles, CA 90024)

The mechanism whereby a bubble transduces sound into a clocklike stream of picosecond flashes of ultraviolet light is robust, complex, and unknown. A theoretical understanding of the key bubble parameter, its ambient radius, is lacking. An explanation as to why this phenomenon has so far only been seen in water is elusive. In addition, we do not understand why cooling the water dramatically increases the light output or why sonoluminescence is so sensitive to doping with a noble gas. Experimentally, the spectrum has been unable to be followed pasi 7 electron volts and so the limits of energy concentration which can be achieved with sonoluminescence from a single bubble are not yet measured. In addition to yielding clues experiments in progress will most likely serve to deepen the mystery! [Work supported by the US DOE Division of Advanced Energy Projects; RL is an AT\&T Fellow.]

\section{9:20}

2aPA3. Experimental determinations of transient conditions during cavitation. Kenneth S. Suslick and Kathleen A. Kemper (Dept. of Chemistry, Univ. of Illinois, 505 S. Mathews Ave., Urbana, IL 61801)

The collapse of cavitation bubbles generates intense local energy release, resulting in extreme local heating, high local pressures, and high energy chemistry. Determining the local conditions formed during cavitation, however, has proved to be a difficult problem. In our work, sonochemical reactions and the sonoluminescence that results have been used as quantitative probes of both temperature and pressure during cavitation. The effective temperatures reached during cavitation can be probed by measurement of the relative rates of a series of chemical reactions whose temperature dependence is already known. Comparative rate thermometry of multibubble cavitation (immersion horn at $20 \mathrm{kHz}$ and $\approx 30 \mathrm{~W} / \mathrm{cm}^{2}$ ) yields effective temperatures of $\approx 5200 \mathrm{~K}$ in hydrocarbons [ $\mathrm{K}$. S. Suslick, Science 247, 1439 (1990)]. Consistent with this, sonoluminescence in these liquids closely resembles flame emission from excited states of $C_{2}$; rotational and vibrational fine struclure permits a spectroscopic determination of the emission temperature of $C_{2}$ excited states at $5080 \pm 160 \mathrm{~K}$ [E. B. Flint and K. S. Suslick, Science 253, 1397 (1991)]. Sonoluminescence from excited state metal atoms is produced from sonolysis of organometallic compounds, for which the linewidth determines the collisional lifetimes of emitting atoms and the effective local pressures. For excited state $\mathrm{Cr}$ atoms produced from $\mathrm{Cr}(\mathrm{CO})_{6}$, emission lifetime is only $0.20 \mathrm{ps}$, corresponding to local densities of $--0.15 \mathrm{~g} / \mathrm{cm}^{3}$ or pressures of $=1.5 \mathrm{kBar}$ at $5000 \mathrm{~K}$. [Supported by NSF.]

\section{9:45}

2aPA4. Picosecond sonoluminescence from macroscopic to microscopic models. T. Lepoint, F. Mullie, Y. Lambillotte (Lab. de Sonochimie et d'Elude de la Cavitation, Inst. Meurice, 1, Av. E. Gryzon, 1070 Brussels, Belgium), M. Goldman, A. Goldman, and P. Tardiveau (Ecole Supérieure d'Electricité, 1192 Gif-sur-Yvette Cedex, France)

The discovery of stable "picosecond" sonoluminescence (SL) has stimulated a great deal of theoretical effort aimed at explaining this striking result. Instead of assuming a perfectly smooth spherical bubble (as in the hot-spot theory, the converging shock-wave model or the Casimir effect model), our model is based on bubble deformation and the subsequent disruption of the interface toward 
the end of the collapse. The formation of an intracavity spray via the Taylor (hydrodynamic) or the Rayleigh (electrohydrodynamic) instabilities and the electrification of the tiny droplets due to the disturbances of the interfacial electrical double layer during the spraying process are described. The high electrical field $\left(E \subseteq 10^{9} \mathrm{Vm}^{-1}\right)$ characterizing a small area of a droplet surface is considered to be responsible for the field emission process. The formation and expansion of a dense microplasma inside the cavity as a consequence of the emission of electrons is proposed to be the origin of picosecond sonoluminescence. By means of this model, which is based both on hydrodynamics and on the microstructure of the bubble interface, the main experimental observations (SL flash temporal localization and duration, shape of the SL spectrum) can be explained.

\section{0:10-10:25 Break}

\section{0:25}

2aPA5. Spherical shocks in a van der Waals gas and their stability C. C. Wu (Dept. of Phys., Univ. of California, Los Angeles, CA 90024) and P. H. Roberts (Univ. of Califomia, Los Angeles, CA 90024)

A theory of sonoluminescence has been developed based on the compression and eventual ionization of air in a bubble by the converging shock waves generated by the applied acoustic radiation. The air is compressed to such high densities that departures from the ideal gas law become very significant. An extension to the van der Waals gas has been made to the similarity theory of Guderley for the structure of spherical shocks in an ideal gas. The linear stability of these shocks has also been examined.

\section{0:50}

2aPa6. Hydrodynamic simulations of bubble collapse and picosecond sonoluminescence. William C. Moss (Lawrence Livermore Natl. Lab., L-200, P. O. Box 808, Livermore, CA 94550), Douglas B. Clarke, John W. White, and David A. Young (Lawrence Livermore Natl. Lab., Livermore, CA 94550)

Numerical hydrodynamic simulations of the growth and collapse of a 10- $\mu \mathrm{m}$ air bubble in water were performed. Both the air and the water are treated as compressible fluids. The calculations show that the collapse is nearly isentropic until the final $10 \mathrm{~ns}$, after which a strong spherically converging shock wave evolves and creates enormous temperatures and pressures in the inner $0.02 \mu \mathrm{m}$ of the bubble. The reflection of the shock from the center of the bubble produces a diverging shock wave that quenches the high temperatures $(>30 \mathrm{eV})$ and pressures in less than $10 \mathrm{ps}$ (FWHM). The picosecond pulse widths are due primarily to spherical convergence/ divergence and nonlinear stiffening of the air equation of state that occurs at high pressures. The peak temperature at the center of the bubble is affected strongly by the ionization model used for the air. The results are consistent with recent measurements of sonoluminescence that had optical pulse widths less than $50 \mathrm{ps}$ and $30-\mathrm{mW}$ peak radiated power in the visible. [This work was performed under the auspices of the U.S. Department of Energy by Lawrence Livermore National Laboratory under Contract No. W-7405-Eng48.]

\section{1:15}

2aPA7. Is sonoluminescence collision-induced emission? Lothar Frommhold (Phys. Dept., Univ. of Texas at Austin, Austin, TX 78712-1081) and Anthony A. Atchley (Naval Postgraduate School, Monterey, CA 93943)

An estimate is attempted of the collision-induced emission (CIE) intensity and spectral profile in the visible and near UV region of the spectrum of $\mathrm{N}_{2}-\chi$ pairs, where $\chi$ represents another $\mathrm{N}_{2}$ molecule or an argon atom, etc., for conditions that correspond to shock waves believed to exist in sonoluminescence experiments. Calculated profiles consist of superimposed high overtone bands and resemble measured profiles of sonoluminescence spectra. The intensities calculated on the basis of a few, simple assumptions concerning the induced dipole surface compare favorably with measurements. The agreement obtained suggests that collision-induced emission is a viable or even an attractive alternative to bremsstrahlung to explain sonoluminescence. According to the theory presented, the CIE source is optically thin so that the spectral emission profile is not related to Planck's radiation law. 


\title{
Session 2aSA
}

\section{Structural Acoustics and Vibration: Scattering from Elastic Structures}

\author{
Linda P. Franzoni, Cochair \\ Department of Mechanical Engineering and Aerospace Engineering, North Carolina State University, Raleigh, \\ North Carolina 27695-7910 \\ Geoffrey L. Main, Cochair \\ Office of Naval Research, 800 North Quincy Street, Arlington, Virginia 22217
}

\section{Contributed Papers}

\section{8:15}

2aSA1. Energy flux vector field in an ensonified elastodynamic sphere. Cleon E. Dean (Dept. of Phys., Georgia Southern Univ., Landrum Box 8031, Statesboro, GA 30460) and Michael F. Werby (NRL, Stennis Space Center, MS 39529-5004)

Recent interest has been shown in the energy flux density inside an ensonified elastodynamic scatterer. A spherical model was chosen as the simplest and most general geometry to analyze. To properly model the energy flow in an elastodynamic medium, it is necessary to employ the elastodynamic equivalent of the Poynting vector. Plots of the vector field of the elastodynamic intensity are presented with an eye toward the classification of the type of resonance behavior displayed. Analogies with other resonance phenomena in related geometries suggest the possibility of fruitful comparison with resonance modes in shells and plates. [Work in this area supported by ONR/NRL and by ASEE/ONT Summer Fellowship Program.]

\section{8:30}

2aSA2. On the scattering cross section of bodies in inhomogeneous medium. Yueping Guo (Dept. of Ocean Engineering, MIT, Cambridge, MA 02139)

A theorem is proven that relates the scattering cross section of arbitrarily shaped scatterers to the scattered far field in one or two particular directions. This can be regarded as a generalization of the classical optical theorem (also known as the extinction theorem) for homogeneous medium of infinite extent. In that case, the scattering cross section is given by the far field in the forward scattering direction. For a half-space with a bounding surface of arbitrary mechanical properties, it is shown that the scattering cross section is given by the scattered far field in the specular reflection direction. For an inhomogeneous medium with abrupt density and soundspeed change at an interface, the scattering cross section is given by the far field in both the specular reflection direction and the transmission direction that obeys the Snell's law. [Work supported by ONR.]

\section{$8: 45$}

2aSA3. Forward scattering from hemispherically end-capped cylindrical shell in shallow water. Angie Sarkissian (Naval Res. Lab., Washington, DC 20375-5350)

When measuring the scattered field from a target in the exact forward direction, the presence of a significantly stronger indicident field makes the extraction of the scattered field difficult because the two signals arrive almost simultaneously. As the position of the target changes, the two signals arrive at distinct times to make the scattered field easier to extract. In a bounded medium, multipath arrivals produce additional complications by increasing the duration of the incident field. Frequency and time domain computations are made for the scattered field from a hemispherically endcapped cylindrical shell placed in a bounded medium where the geometry consists of a fixed source, a fixed receiver, and a target placed in between. The location of the target is varied, to compare the incident field to the scattered field in both intensity and arrival times.

9:00

2aSA4. Acoustic scattering from a fluid-loaded elastic plate with a distributed mass inhomogeneity. J. M. Cuschieri (Ctr. for Acoust. and Vib., Dept. of Ocean Eng., Florida Atlantic Univ., Boca Raton, FL 33431) and D. Feit (David Taylor Research Center, Bethesda, MD 20084)

The scattering of a plane acoustic wave by a fluid-loaded, thin, elastic plate, of infinite extent, with a distributed inhomogeneity is investigated by solving the equation of motion in the wave-number domain using the hybrid numerical analytical approach [J. Acoust. Soc. Am. 95, 1998-2005 (1994)]. The presence of the distributed inhomogeneity in the equation of motion, when expressed in the wave-number domain, results in a Fredholm integral equation of the third kind. By substituting for the product of the response and the plate characteristic equation, the Fredholm integral of the third kind is reduced to a Fredholm integral of the second kind. The plate response in the wave-number domain is obtained from the solution of the Fredholm integral. Inverse Fourier transforming the wave-number domain response function gives the spatial domain solution for the response. The hybrid approach is used to perform the inverse Fourier transform. The scattered pressure is obtained in a similar manner. Response and scattered pressure results for distributed mass inhomogeneities, with different distribution functions, are presented and compared to the results for a line inhomogeneity. [Work sponsored by ONR.]

\section{9:15}

2aSA5. Influence of rib resonances on the acoustic scattering from rib reinforced elastic plates. Angela K. Karali and Sabih I. Hayek (Dept. of Eng. Sci. and Mech., Penn State Univ., University Park, PA 16802)

In a previous paper [A. K. Karali and S. I. Hayek, J. Acoust. Soc. Am. 95, 2805 (1994)] the analytic model for the prediction of the acoustic scattered field by infinite elastic plates reinforced with periodic arrays of line discontinuities was presented. In this paper the analysis is extended to infinite elastic plates reinforced with periodic arrays of ribs. If actual ribs are attached to a plate, instead of line discontinuities, their impedances are frequency dependent. The ribs are modeled as Timoshenko beams attached to an elastic plate, whose flexural vibrations are modeled using Mindlin's plate theory. Analytic expressions for the rib impedances are obtained and plotted against the normalized frequency of an incoming plane acoustic waye. The rib impedances show resonances and antiresonances in the frequency range of 1 to 15 times the classical coincidence frequency of the plate. The influence of the rib impedance resonances and antiresonances on the nonspecularly reflected field is demonstrated by direct calculation of the acoustic scattered field at several critical frequencies of the ribs. 
as predicted by SARA 2D finite-element methods. [Work supported by ONR.]

2aSA6. Analytical numerical matching for efficient calculation of acoustic scattering from cylindrical shells. Richard $C$. Loftman and Donald B. Bliss (Mech. Eng. and Mater. Sci., Duke Univ., Box 90300, Durham, NC 27708)

Structural discontinuities in highly coupled fluid/structure systems are modeled by a novel approach called analytical numerical matching (ANM). ANM separates the low-resolution global influence of a discontinuity from the relatively high-resolution local effects. A continuous, smoothed replacement for a fundamental structural discontinuity is constructed so that the system is identically unchanged beyond a small smoothing region. Simultaneously, the precise local effect of smoothing the discontinuity is retained in analytical form. The smoothed problem is solved by numerical techniques, with rapid convergence and reduced computational cost. The original discontinuous character is restored, using the analytical expression for the local difference between the smoothed and the original problems. ANM has been successfully applied to acoustic scattering from a thin, infinitely long cylindrical shell, with multiple structural discontinuities. Local solutions for line discontinuities with radial, tangential, and rotational constraints have been formulated using ANM. Line constrained scattering problems, as well as line driven problems, are investigated. The ability of analytic numerical matching to replace a discontinuous physical problem by a well-behaved continuous one for numerical evaluation, while ultimately retaining the original geometry and physical behavior, is illustrated. [Work supported by ONR.]

\section{9:45}

2aSA7. Approximate closed-form analysis of modal coupling for radiation and scattering from fluid loaded structures. Linda $P$. Franzoni (Mech. and Aerospace Eng., North Carolina State Univ., Raleigh, NC) and Donald B. Bliss (Duke Univ., Durham, NC 27708)

Modal analysis is often used in problems involving radiation and scattering from fluid loaded structures. The modal formulation leads to a coupled system of equations for the modal amplitudes. Typically, structural modes are coupled through the effects of the fluid. In practice, modal coupling is sometimes assumed unimportant, enabling modal coefficients to be solved independently in closed form. This approach is particularly appealing when subsequent analytical treatment of expressions is desirable. The validity of this approach, including the order of the error involved in neglecting coupling is discussed. It is shown that the principal effects of weak modal coupling can be incorporated in a simple way without solving the fully coupled system. An approximate closed-form solution for weakly coupled systems of equations is developed. The approximation is found to work well, even in systems where coupling is fairly strong. The approximate form gives physical insight into the dominant effects of modal coupling, and shows that neglecting coupling may lead to significant errors. The effects of coupling are assessed by solution of model problem involving acoustic scattering from a fluid loaded plate. Solutions are compared for three methods: fully coupled, uncoupled, and the approximate analysis. [Work supported by ONR.]

\section{0:00-10:15 Break}

\section{0:15}

2aSA8. Reciprocity relations for endcap scattering of natural waves on a fluid-loaded cylindrical shell. P. W. Smith, Jr., Kevin D. LePage, and J. Gregory McDaniel (Bolt Beranek and Newman, Inc., 70 Fawcett St., Cambridge, MA 02138)

The response field on a finite axisymmetric elastic shell is approximated by a limited set of lightly damped natural waves which are coupled to each other and to far-field sound by the scattering process at the endcaps. In this talk the constraints upon the scattering functions that are a consequence of the principle of reciprocity are obtained through analysis. Results show that the reciprocity constraints predicted for a thin shell example agree well with the observed behavior of natural wave scattering

\section{0:30}

2aSA9. Experimental investigation of backscatter from an internally loaded cylindrical shell with a constrained layer damping treatment. Michael Klausbruckner, Matthew Conti, Ira Dyer, and J. Robert Fricke (Dept. of Ocean Eng., MIT, Cambridge, MA 02139)

Previous investigations have examined the backscatter from empty and internally loaded shells with no damping treatment, demonstrating the primary importance of helical shear waves. The helical waves contribute to large backscatter for aspect angles within $30 \mathrm{deg}$ of normal for the empty shell. The region increases to $\mathbf{4 5} \mathrm{deg}$ of normal for the internally loaded shells. A constrained layer was applied to the cylindrical section of an internally loaded shell to damp the helical waves while decoupling them from the intemal structures. The intended effect was to lower the backscatter levels and reduce the angular width of strong backscatter to within $30 \mathrm{deg}$ of normal. This preliminary investigation demonstrates the achievement of each of these goals. In addition, the importance of direct acoustic backscatter from the attachment locations of the internal rings was highlighted. The bistatic measurements were conducted over a frequency range of $2.5<k a<10$, corresponding to $3 / 4$ to 3 times the ring frequency of the shells. [The authors acknowledge the assistance of NRI for acquisition of data. Work supported by ONR.]

\section{0:45}

2aSA10. Point-matching technique for scattering of Stokesian flows around axisymmetric bodies. Scott $A$. Wymer, Renata S. Engel, and Akhlesh Lakhtakia (Dept. Eng. Sci. and Mech., The Pennsylvania State Univ., 227 Hammond Bldg., University Park, PA 16802-1401)

In solving for velocity and pressure fields in a slow, viscous, incompressible fluid, frequency-domain scattering techniques can be brought into action by taking the Fourier transform of the equations describing Stokesian flows. This is exemplified by considering the scattering of a transverse plane wave by an impenetrable axisymmetric body immersed in a fluid modeled by the unsteady Stoke's equation, the wave number in the ambient Stoke's fluid being necessarily complex. The pressure and velocity phasors satisfy the Laplace and the Helmholtz equations, respectively, and are written as sums of incident and scattered components. These components are then expanded in terms of spherical harmonic functions. The point-matching technique is used to satisfy boundary conditions on a discrete set of points on the surface of the scattering body. Convergence of the resulting series solutions is studied for spheroids of different aspect ratios and for varying wave numbers.

\section{1:00}

2aSA11. Acoustics wave diffraction on a cylindrical shell with two local masses. Rostislav A. Dudnik and Andrei B. Kolpakov (Dept. of Phys., Inst. Arch. \& Civil Eng., 65 Il'inskaya St., 603600 N.-Novgorod, Russia)

The diffraction of a plane sound wave on a thin cylindrical shell having inhomogeneity presented by two identical inertia masses fastened symmetrically on its surface is considered. It is shown that the field scattered by such a shell presents a superposition of the fields radiated by both the symmetrical and antisymmetrical low-frequency azimuth modes excited depending on the direction of initial wave propagation toward the vertical plane of the shell symmetry $(\Phi=0 ; \Phi=180)$. In support of the existence of corresponding nonsingular normal velocity distributions, a specialized experimental investigation was carried out for the force-excited shell models. The experimental investigation of near as well as far acoustical fields of these shell was executed also. The calculation results of frequency responses and directionality diagrams of the field scattered by the shell with two masses fastened diametrically opposed at the initial only symmetrical oscillations are presented. These results are compared with analogous results for the shell having one inertia mass (at $\Phi=180$ ). It was found that 
the radiation level control is possible for the investigated oscillation structure by such division of a unit inhomogeneity in certain frequency ranges.

\section{1:15}

2aSA12. Scattering of sound by a free-flooded cylindrical shell. $\mathrm{K}$. Steven Kim (Signatures Directorate, Carderock Div., Naval Surface Warfare Ctr., Bethesda, MD 20084-5000)

Scattered acoustic pressure fields are obtained when a free-flooded cylindrical shell of finite length is subjected to plane waves incident obliquely to the axis of the cylindrical shell. Integral equations are formulated for the cases of a rigid thin shell and a elastic thin shell with simply supported boundary condition at both ends. Equations are solved by a Rayleigh-Ritz type approach. Near-field and far-field acoustic pressures of the scattered sound are calculated. Scattered far-field acoustic pressures for a short shell and a long shell are discussed.

\section{1:30}

2aSA13. Enhanced backscatter in finite reverberant systems. Richard L. Weaver (Dept. of Theor. and Appl. Mech., Univ. of Illinois, 104 South Wright St., Urbana, IL 61801)

Enhanced backscatter, in which reverberant signal levels at the position of a transient source are greater than mean reverberant signal levels elsewhere, is studied analytically and numerically. Popular weak localization arguments, in which descriptions of responses in terms of incoherent rays are modified to include some effects of coherent interference, indicate that the mean-square response at the source should be twice as large as it is at other points. A modal analysis, however, shows that the actual ratio should be three. A more detailed theory shows that the enhanced return factor is 2 at moderate times, but 3 at late times comparable to the modal density. The theory indicates that the factor of 2 is achieved only if the eigenfrequency statistics have the spectral rigidity predicted by random matrix theory. Thus a connection between spectral rigidity and weak localization is hinted at. Numerical solutions in model undamped two-dimensional reverberation rooms are found to agree with the theory. The effect of damping on these results is also investigated.

\section{$11: 45$}

2aSA14. Scholte interface wave on a cylindrical shell. G. Maze, F. Léon, F. Lecroq, D. Décultot (LAUE, URA 1373 of the CNRS, Univ. of Le Havre, 76610 Le Havre, France), and $H$. Überall (Univ. of Le Havre, France and Catholic Univ., Washington, DC 20064)

The fluid-borne interface wave on an evacuated cylindrical shell immersed in water is known as Scholte-Stoneley wave, or also as $A$ wave [Talmant $e t$ al., J. Acoust. Soc. Am. 86, 278 (1989)]. The resonances of this wave, caused by phase matching upon its multiple circumnavigations of the scattering object, can be observed in the backscattering spectra of incident sound waves within a frequency window that depends on the shell thickness, for thicknesses less than $40 \%$ of the cylinder radius. The interaction between the flexural wave $A_{0}$ in the shell, and the Scholte wave is studied in detail as a function of shell thickness. Dispersion curves for the $A$ and the $A_{0}$ waves are presented, indicating a region of repulsion between these two curves corresponding to a change of the physical nature of the two waves.

\title{
Session 2aSP
}

\section{Speech Communication and Engineering Acoustics: Microphone Arrays: Design and Applications I}

\author{
James L. Flanagan, Cochair \\ CAIP Center, Busch Campus, Rutgers University, Core Building 706, Piscataway, New Jersey 08855-1390 \\ Harvey F. Silverman, Cochair \\ Laboratory for Engineering Man/Machine Systems, School of Engineering, Brown University, Providence, \\ Rhode Island 02912 \\ Qiguang Lin, Cochair \\ CAIP Center, Rutgers University, Core Building, Frelinghuysen Road, Piscataway, New Jersey 08855-1390
}

Chair's Introduction-8:30

Invited Papers

8:35

2aSP1. Microphone systems and signal processing: New opportunities in sound capture. J. L. Flanagan (CAIP Ctr., Rutgers Univ., Piscataway, NJ 08855-1390) and H. F. Silverman (Brown Univ., Providence, RI 02912)

Over the recent past, advances in two technical areas have opened new opportunities for sophisticated sound capture at distances. High-quality low-cost acoustic sensors, primarily in the form of electret microphones, are plentiful and can be used in large numbers. Continued progress in microelectronics now provides enormous amounts of economical computation. These incentives have stimulated research in new techniques for sound capture in practical acoustic environments-such as conference halls, noisy computer rooms, teleconferencing facilities, and mobile communicalion. Recent research has cmbraced the design of large-scale two- and threedimensional arrays of microphones, and the implementation of algorithms for automatic sound source location. This report reviews the status of research in these areas. It also highlights the emerging applications of distant-talking sound capture for hands-free operation of communications equipment such as automatic speech recognizers. [This research is supported by the National Science Foundation under Contract \#MIP 9314625.] 
2aSP2. Practical issues in the use of a frequency-domain delay estimator for microphone-array applications. John Adcock, Joe DiBiase, Michael Brandstein (Box D, Brown Univ., Providence, RI 02912), and Harvey F. Silverman (Brown University, Providence, RI 02912)

A frequency-domain delay estimator has been used as the basis of a microphone-array talker location and beamforming system [M. S. Brandstein and H. F. Silverman, Techn. Rep. LEMS-116 (1993)]. While the estimator has advantages over previously employed correlation-based delay estimation methods [H. F. Silverman and S. E. Kirtman, Comput. Speech Lang. 6, 129-152 (1990)], including a shorter analysis window and greater accuracy at lower computational cost, it has the disadvantage that since delays between microphone pairs are estimated independently of one another, there is nothing to ensure that a set of estimated delays corresponds to a single location. This not only introduces errors in talker location but degrades the performance of the beamformer. A method for delay estimation and talker location with a microphone array is described that preserves the low computational complexity and rapid tracking ability of the frequency-domain delay estimator, while improving the coherence and stability of the estimated delays and derived source locations. Experimental results using data from a real 16-element array are presented to demonstrate the performance of the algorithms. [Early work principally funded by DARPA/NSF Grant IRI-8901882, and current work by NSF Grant No. 9314625.]

\section{9:15}

2aSP3. A constant-directivity beamforming microphone array. Gary W. Elko, Thomas C. Chou, Robert J. Lustberg, and Michael M. Goodwin (Acoust. Res. Dept., AT\&T Bell Labs., 600 Mountain Ave., Murray Hill, NJ 07974)

The quality of audio teleconferencing in large rooms and noisy environments can be increased with the use of steerable directional microphone arays. A minimum bandwidth of $4 \mathrm{oct}$ is required to faithfully transmit the speech signal. In a typical teleconferencing arrangement, only discrete angular directions are of interest and therefore the microphone steering directions are quantized. A standard delay-sum beamformer can result in noticeable frequency response changes as the talker moves between these steening locations. In an effort 10 mitigate this problem, a broadband constant-directivity beamformer bas been designed and constructed. A few of the algorithms developed in this work will be discussed and compared to existing techniques. Basically, the solution revolves around the design of FIR filters that are inserted in the delay-sum beamformer after each element. A constant-beamwidth 4 oct steerable linear array microphone using directional elements will be described. A real-time implementation utilizing multiple AT\&T DSP3210 digital signal processors is also described.

2aSP4. Hands-free mobile telephony by means of an adaptive microphone array. Sven Nordholm, Ingvar Claesson, Sven Nordebo, and Mattias Dahl (Dept. of Signal Process., Univ. of Karlskrona/Ronneby S-372 25 Ronneby, Sweden)

By employing speech generation models and new algorithms more and more a priori information about speech signals is utilized in speech recognition and speech coding. A fair signal-to-noise ratio is therefore required to ensure that the $a$ priori information is correct. This implies a need for noise reduction under adverse conditions, such as hands-free operation of telephones in the car compartment or speech recognition in cars [S. Nordholm et al., "Adaptive Array Noise Suppression of Handsfree Speaker Input in Cars," IEEE Trans. Veh. Tech. 42, 514-518 (1993)]. The paper presents two adaptive microphone array schemes, aimed for this situation. The first, denoted spatial filtering generalized sidelobe canceller (SFGSC), gives good noise suppression with little distortion of the speech but requires careful calibration. The second, denoted adaptive microphone array employing calibration signals recorded on-site (AMAEC), facilitates a simple built-in calibration. It is beneficial from a user point of view to use a calibration signal recorded on site eliminating amplifier tuning and microphone selection. The calibration can be done within $60 \mathrm{~s}$. The AMAEC calibrates the array to the speakers' location, microphone positions and lobe gains, amplifiers, and to the acoustic environment in the car. No $a$ priori information about signal statistics or array geometry is utilized. [Work supported by Nutek.]

\section{9:55-10:10 Break}

10:10

2aSP5. Robust hands-free speech recognition. Qiguang Lin, Chi Wei Che, and James Flanagan (CAIP Ctr., Rutgers Univ., Piscataway, NJ 08855-1390)

When speech recognition technology moves from the laboratory to real-world applications, there is increasing need for robustness. This paper describes a system of microphone arrays and neural networks (MANN) for robust hands-free speech recognition. MANN has the advantage that existing speech recognition systems can directly be deployed in practical adverse environments where distanttalking sound pickup is required. No retraining nor modification of the recognizers is necessary. MANN consists of two synergistic components: (1) signal enhancement by microphone arrays and (2) feature adaptation by neural network computing. High-quality sound capture by the microphone array enables successful feature adaptation by the neural network to mitigate environmental interference. Through neural network computation, a matched training and testing condition is approximated which typically elevares performance of speech recognition. Both computer-simulated and real-room speech input are used to evaluate the capability of MANN. Measurements of isolated-word recognition in noisy, reverberant, and distant-talking conditions show that MANN leads to a word recognition accuracy which is within $4 \%-6 \%$ of that obtained under a close-talking condition in quiet.

10:30

2aSP6. Wideband microphone array for hearing aid preprocessing. Kung Yao (Elect. Eng. Dept., Eng. IV, 68-113, UCLA, Los Angeles, CA 91403-1594), Sigfrid D. Soli (House Ear Inst., Los Angeles, CA 90057), and Dan Korompis (UCLA, Los Angeles, CA 91403-1594)

Speech communication in environments with low signal/noise ratios (SNRs) is a primary complaint of the hearing impaired. Microphone beam formation techniques provide an effective approach to improving SNR in these environments. A novel, fixed 
microphone array is being developed with user-controlled mainlobe spatial look direction and attenuation band(s), and with a flat frequency response over the speech bandwidth. The array of $R$ microphones and $L$ taps per microphone maximizes energy concentration over a spatial look region and frequency band, subject to spatial and frequency constraints. Constrained maximization of $\mathrm{w}^{*} \mathrm{Aw} / \mathrm{w}^{*} \mathrm{Bw}$ is required, where $\mathrm{A}$ and $\mathrm{B}$ are matrices specifying spatial and frequency factors, and $\mathbf{w}$ is the RL dimensional weight vector. The constraining subspace is specified by the array values, derivative values, and spatial directional constraints; $w$ is obtained as the solution of a tractable unconstrained full-rank lower dimensional generalized eigenvalue problem. Numerical and simulation results for different values of $\mathrm{R}$ and $\mathrm{L}$ and for different bandwidths will be reported, as well as results of preliminary listening tests with normally hearing and hearing impaired individuals. The feasibility of real-time acoustic beamformers with arrays for hearing aids, and the advantages of this scheme over conventional adaptive schemes will also be discussed.

10:50

2aSP7. A large microphone array for outdoor sound propagation studies. David I. Havelock (Inst. for Microstructural Sci., National Research Council, Ottawa, ON K1A 0R6, Canada)

A sound field propagating outdoors is perturbed by the turbulence in the atmosphere. To study the fluctuations due to turbulence, the sound field is measured simultaneously at a large number of points using a microphone array. The array consists of 64 microphones which can be configured in a variety of geometries ranging from small patches of only a few square meters to an elongated array spanning $700 \mathrm{~m}$. Remote "satellite" arrays are also possible. The data are collected and processed in a mobile equipment trailer. The microphones, electronics, data collection, and processing are described and practical aspects of deploying the array are discussed. The design criteria and example applications of the array are also discussed.

11:10-11:15 Break

11:15-11:45 PANEL DISCUSSION

TUESDAY MORNING, 29 NOVEMBER 1994

BALLROOM B, 8:00 A.M. TO 12:00 NOON

Session 2aUW

Underwater Acoustics: Moderate-to-High Frequency Bottom Interacting Acoustics I

Mohsen Badiey, Chair

Code 3240A, Office of Naval Research, 800 North Quincy Street, Arlington, Virginia 22217-5660

Chair's Introduction $-8: 00$

Invited Papers

8:05

2aUW1. The investigation of millimeter scale heterogeneity in Coastal Benthic Boundary Layer sediments using microresistivity and x-ray imaging of "diver" cores. Peter D. Jackson (British Geological Survey, Keyworth, Notts NG12 5GG, UK), Kevin B. Briggs (Naval Res. Lab., Stennis Space Center, MS 39529-5004), Robert Flint (British Geological Survey, Keyworth, Notts NG12 5GG, UK), Michael A. Lovell, and Peter K. Harvey (Univ. of Leicester, Leicester LE1 7RH, UK)

A micro-resistivity imaging technique, developed for use on 70-mm "half-round" slabbed core has been adapted for use with larger "diver" cores, $33 \mathrm{~mm}$ thick, $350 \mathrm{~mm}$ wide, and $\mathbf{4 4 0} \mathrm{mm}$ long. This resistivity technique is shown to have a resolution of $5 \mathrm{~mm}$ and to operate in a manner which is complimentary to x-ray photography. Theoretical and practical examples are presented showing responses to individual heterogeneity such as shells. The benefits of 3-D investigations are explored via numerical models are shown to be beneficial even in cores such as those above having a "flat" aspect ratio. The microresistivity method is shown to be particularly sensitive to layered structures and the presence of shells within the very high porosity surface layers in Eckernforde Bay (89\% porosity $0-10 \mathrm{~cm}$ below sea floor). [Work supported by U.S. Navy.]

$8: 25$

2aUW2. Correlation functions for sediment acoustic properties. Kevin B. Briggs (Seafloor Sciences Branch, NRL, Stennis Space Center, MS 39529)

Models for sediment volume scattering require knowledge of the correlation functions for sediment sound velocity and density (or porosity). High-resolution vertical correlation functions of these properties have been estimated using sediment porosity and compressional wave velocity data measured vertically at regular, closely spaced intervals from diver-collected core samples. Because the data series are truncated owing to a limited core length, the correlation functions were estimated using a first-order autoregressive 
model and autocorrelations were calculated with Burg's algorithm. Correlation length estimates were from a variety of shallow-water sites. Relationships between grain size and conelation length are discussed.

\section{$8: 45$}

2aUW3. Modeling the near-bottom seafloor. Robert D. Stoll (Lamont-Doherty Earth Observatory of Columbia Univ., Palisades, NY 10964)

In mine counter measures work and other seafloor engineering applications, there are two distinctly different classes of sediment properties that play important roles in object detection and sonar performance-those that determine if an object will sink into the bottom or stay fully or partially exposed and those that control the penetration of high-frequency sonar signals into the bottom. The ability of the seafloor to support an object depends largely on shearing strength which is a nonlinear, large-strain property associated with plastic deformation and complete disruption of the in-situ structure. In contrast, the complex velocity (speed and attenuation) of low-amplitude geoacoustic signals is a small-strain property that can be treated to a good approximation using linear theories. Both classes of response and their interaction are discussed using insights gained from the Biot-Gassmann theory. This theory allows extrapolation from low- to high-frequency response and examination of the effects of various parameters such as gas content and low overburden pressure. In particular the effect of recently measured high attenuation and velocity gradients is discussed and a correlation between shear strength and shear-wave velocity is shown. [Work supported by NRL, CBBLSRP.]

\section{9:05}

2aUW4. Microstructural modeling of clay sediments in Eckernfoerde Bay. Jack J. Kolle, Alan C. Mueller (QUEST Integrated, Inc., 21414 68th Ave. S., Kent, WA 98032), and Jack Dvorkin (Stanford Univ., Stanford, CA 94305)

A finite-element microstructure analysis code was used to model the geoacoustic properties of shallow marine clay sediments in Eckernfoerde Bay which has been the subject of an intensive geoacoustic and geotechnical study (Coastal Benthic Boundary LayerSpecial Research Program). Uniaxial strain and pure shear were applied to two-phase microstructure models with generalized, periodic plane-strain boundary conditions to generate anisotropic bulk and shear moduli. Analyses were carried out on two length scales: clay microfabric $(\mu \mathrm{m})$ and gas-filled inclusions $(\mathrm{mm})$. The clay microstructure was modeled as a random lattice of Illite platelets with a density and porosity equivalent to average values observed in shallow Eckernfoerde Bay sediments. Saturated and dry framework moduli were calculated and the effective medium velocities were compared with observed velocities. The analysis suggests that shear wave propagation in these sediments is not supported by the framework structure but by differential compression of fluid-filled pores. Radiographic images of pressurized cores containing gas-filled inclusions with dimensions of a few millimeters were also analyzed. The analysis indicates a shear wave velocity anomaly related to the inclusion structure. The calculated effective medium velocities are compared with analytical models of spheroidal inclusions. [Work supported by ONR.]

\section{Contributed Papers}

9:25

2aUW5. A propagation model for wave motion in saturated granular sediments. M. H. Sadd, A. Gautam (Mech. Eng. and Appl. Mech. Dept., Univ. of Rhode Island, Kingston, RI 02881), A. J. Silva (Univ. of Rhode Island, Narragansett, RI 02882), and G. E. Veyera (Univ. of Rhode Island, Kingston, RI 02881)

Wave propagation in saturated granular sediments is modeled through discrete element simulation. The sediments under study are assumed to be primarily sandy, and are thus modeled as cohesionless granular material saturated with pore fluid. Numerical simulation is based on the discrete element method, a numerical scheme employing a modeling strategy to determine the translational and rotational motion of all particles in model material assemblies. For application to wave propagation, the movements of individual particles are a result of the propagation of disturbances through the media. Contact laws between adjacent particles are constructed using elastohydrodynamic lubrication theory, and these relations determine the contact force as a function of the relative displacement and relative velocity between neighboring particles. Using these new contact laws, wave motion simulations of one- and two-dimensional computer-generated model assemblies have been conducted. Results indicate that the wave speed and amplitude attenuation are functions of the physical microstructure or fabric of the discrete medium. Wave speed is inversely related to the porosity of the solid phase, while attenuation studies indicate that a branch vector distribution correlates with interparticle force transmission. Model development and simulation results will be presented.

\section{9:40}

2aUW6. Acoustical properties of undisturbed sands from the West Florida sand sheet. Horst G. Brandes and Armand J. Silva (Dept. of Ocean Eng., Univ. of Rhode Island, Narragansett, R1 02882)
Eight undisturbed large-diameter $(10.1-\mathrm{cm})$ gravity cores, ranging in length from 13.5 to $50.5 \mathrm{~cm}$, were collected at the CBBL-SRP study site southeast of Panama City, FL. The recovered sediments varied from coarse sand and shell fragments near the surface to fine/medium sand at depth. The Multi Sensor Core Logger was used to measure compressional wave velocity, density, and attenuation as a function of depth in each of the unsplit cores in two mutually perpendicular directions. Two spring-loaded compressional wave Iransducers operating at $500 \mathrm{kHz}$, mounted on the outside of the liner, were used to measure velocity and attenuation, and a Cs-137 gamma ray source with scintillation counter were used to measure bulk density. In the longest of the cores, PC-GC-623, compressional wave velocity varied between 1700 and $1730 \mathrm{~m} / \mathrm{s}$ in the upper $10 \mathrm{~cm}$ and between 1650 and $1700 \mathrm{~m} / \mathrm{s}$ below that depth. Attenuation decreased slightly with depth, with most values falling between 0.3 and $0.5 \mathrm{~dB} / \mathrm{kHz} / \mathrm{m}$ (with reference to core liner filled with water). Density was relatively depthindependent and ranged between 1.8 and $1.9 \mathrm{~g} / \mathrm{cm}^{3}$.

\section{9:55}

2aUW7. Optimizing finite difference schemes for wave propagation in high-loss viscoelastic materials. Mark A. Hayner and J. Robert Fricke (Dept. Ocean Eng., MIT, Cambridge, MA 02139)

An optimization technique is discussed which improves the numerical efficiency of the finite difference method with memory variables used to analyze wave propagation in high-loss materials $[\eta=O(1)]$ [Blanch et al., "Viscoelastic finite difference modeling," Rice University Tech. Rep. TR93-04 (1993)]. With the conventional use of memory variables, the relaxation function of a viscoelastic material is modeled with a series of decaying exponential functions. The amplitudes and relaxation times of these exponentials are then matched, as closely as possible, to the behavior of the viscoelastic material. Using the new optimization technique, the error of the finite difference method, which is completely predictable using 
the von Neuman method, is accounted for during the matching process reducing the total error. For narrow-band models, the reoptimization process can reduce run times and memory requirements for 2-D models by about $8 X$ and $4 X$, respectively. The usefulness and accuracy of this technique versus analytic methods are demonstrated.

\section{0:10-10:30 Break}

\section{0:30}

2aUW8. The effects of a thin slow sediment layer on shallow water acoustic field. E. C. Shang and Y. Y. Wang (CIRES, Univ. of Colorado/ NOAA/Environmental Tech. Lab., Boulder, CO 80303)

The seabottom often has a thin layer on its top and the sound speed in this thin layer is less than in water. It has been found that this thin layer can significantly affect the acoustic field in water column. First, it changes the bottom reflection character, and, on the other hand, it changes the modal wave-number difference: $D_{m n}=2 \pi\left(k_{m}-k_{n}\right)^{-1}$. Those two factors determine the basic features of the acoustic field-the modal interference pattern and the modal attenuation rate. Based upon the sensitivity analysis, bottom parameter estimation scheme is proposed.

\section{0:45}

2aUW9. Consistent geoacoustic models in the frequency range 50-5000 Hz. Charles W. Holland, Peter Neumann, and Greg Muncill (Planning Systems Inc., 7923 Jones Branch Dr., McLean, VA 22102)

The process of creating a geoacoustic model begins with the process of defining the dominant mechanisms that control bottom interaction for the frequency range and application of interest. For example, a geoacoustic model that treats the seafloor reflection process might be much simpler than a geoacoustic model which treats bottom interaction (i.e., general bi-static scattering including reflection and backscattering as special cases). The following step is to determine values for the parameters that describe those physical mechanisms of importance. This is typically approached by a combination of direct measurements (e.g., core data), empirical modeling \{e.g., Hamilton relations [E. L. Hamilton, J. Acoust. Soc. Am. 68, 1313-1340 (1980)] , or by inversion the acoustic data. The result can be tested by comparing the geoacoustic model predictions (used as primary inputs to an acoustic model) against measured acoustic data. Geoacoustic model predictions and comparisons with acoustic data and are given for a variety of marine environments. A geoacoustic model that is successfully employed for both seafloor reflection and scattering is demonstrated. [Work supported by the ONR/AEAS Program.]

\section{1:00}

2aUW10. Boundary conditions and the theory of acoustic attenuation in lossy fluid seabeds. Grant B. Deane (Marine Phys. Lab.-0238, Scripps Inst. of Oceanogr., 9500 Gilman Dr., La Jolla, CA 92093-0238)

During the last decade the traditional view that acoustic attenuation in marine sediments is linear in frequency from seismic to ultrasonic frequencies has been re-examined [A. C. Kibblewhite, J. Acoust. Soc. Am. 86, 716-738 (1989)]. In particular, there is a growing body of evidence from the ocean acoustic and marine seismology communities that the characteristics of attenuation in marine sediments are compatible with the BiotStoll theory for porous media. The Biot-Stoll formulation predicts a range of frequency dependencies, according to which of a number of attenuation mechanisms, including viscous dissipation, is dominant. The question of how different attenuation mechanisms can be accounted for in a fluid description of sediments will be examined. The convention of complex sound speed does not account for all the effects of absorption due to viscous dissipation when quantities involving both the pressure and velocity field are considered. An example of such a problem is the Pekeris waveguide with a lossy basement. The effect of viscous dissipation can be accounted for by adopting a convention that amounts to introducing a complex quiescent density.

\section{1:15}

2aUW11. Acoustic backscattering by the anisotropic and dipped structure of velocity fluctuations within the bottom sediments. Tokuo Yamamoto (Appl. Marine Phys. Div., RSMAS, Univ. of Miami, Miami, FL 33149)

The three-dimensional power spectra of the velocity fluctuations within the seabed measured by high-frequency $(1-50 \mathrm{kHz})$ crosswell acoustic tomography experiments indicate that the velocity fluctuation spectra are anisotropic in general and are often dipped; i.e., the major and minor axes of anisotropy are tilted from the vertical and the horizontal direction (Yamamoto, this meeting). The aspect ratio of the horizontal scale to the vertical scale ranges from 4 to 10 in the shallow-water sediments. The angle of tilt, called dip, is found as large as $30 \mathrm{deg}$. The intensity of the fluctuation spectrum depends on the sediment type. These parameters of the three-dimensional power spectrum affect the scattering of acoustic waves. An analytical solution to acoustic wave scattering by a dipped anisotropic 3-D velocity fluctuations in the sediments has been obtained. The strong dependence of acoustic backscattering on the grazing and azimuthal angle observed by Jackson and Briggs (1993) is excellently predicted when the realistic anisotropy and dip structures of the velocity fluctuations are incorporated in this analytical model of scattering by sediment volume fluctuation. [Work supported by ONR.]

\section{1:30}

2aUW12. Influence of sediment transport events upon bottom backscattering, Eckernfoerde Bay. Darrell Jackson (Appl. Phys. Lab., Univ. of Washington, 1013 NE 40th St., Seattle, WA 98195) and L. D. Wright (Virginia Inst. of Marine Sci., College of William and Mary, Gloucester Point, VA 23062-1346)

In Spring 1993, oceanographic and acoustic apparatus were deployed in Eckernfoerde Bay, Baltic Sea. An instrumented tetrapod recorded physical data, revealing turbidity events associated with resonant internal waves. Acoustic bottom backscattering data at $40 \mathrm{kHz}$ were acquired simultaneously and show features that correlate with the turbidity events. Such a correlation is surprising as estimates of shear velocity suggest that bottom stress never reached the critical magnitude necessary to resuspend sediment; rather the turbidity events appear to involve advection from a distant location. Correlations are evident in comparisons of acoustically derived time series for change in temperature and change in echo character with time series from current meters, optical backscattering sensors, and thermistors. [Work supported by the NRL CBBL Special Research Program.]

\section{1:45}

2aUW13. Normal-mode description of reverberation with a finite area bottom patch average. J. LeMond (Appl. Res. Labs., Univ. of Texas at Austin, Austin, TX 78713)

A normal-mode reverberation model is presented that includes the effects of modal interference among spatially correlated fields scattered within a patch of (nearby) bottom locations. At each frequency the integrated scattered field from a bottom patch as a function of patch size is calculated from an explicit normal-mode expression. The time integrated reverberation level at each frequency is computed by squaring this contribution from each patch and summing over bottom patches. As a function of source/receiver separation, the time integrated reverberation levels computed with the finite patch description oscillate about a mean that is consistently lower than the levels obtained in the zero patch area limit. Results from a study of the dependence of the level differences and oscillation magnitudes on environment, frequency and patch area are presented. [Work supported by the Advanced Surveillance and Prediction System (ASAPS) Program of the Space and Naval Warfare Systems Command (SPAWAR, PMW 183-32).] 


\title{
Session 2pAA
}

\section{Architectural Acoustics: Performance and Recording Spaces for Popular Music and Topics in Isolation and Reverberation}

\author{
Richard E. Boner, Chair \\ Boner Associates, Inc., 200 East 30th Street, Austin, Texas 78705
}

Chair's Introduction-1:00

\section{Invited Papers}

1:05

\begin{abstract}
2pAA1. "...through the glass darkly..." or designing for the needs of traveling groups in a multipurpose performing arts facility. Dana S. Hougland and Edward L. Logsdon (David L. Adams Associates, Inc., 1701 Boulder St., Denver, CO 80211)

Predicting and designing for the everchanging acoustic and electroacoustic needs of a traveling show utilizing multipurpose performing arts facilities is an ongoing challenge. Most communities cannot afford the luxury of building and maintaining singlepurpose performing arts facilities. There is a need to accommodate a variety of local or resident performing arts groups, as well as traveling shows. Shows may range from simple lectures to Broadway show productions, as well as the everincreasing number of multimedia performances. Measures to facilitate the traveling show performances must be simultaneously unobtrusive when not in use, and readily accessible to the crew of each traveling show. The successes and failures of various techniques are presented and critiqued.
\end{abstract}

1:35

2pAA2. Popular music performance and acoustics in spaces designed primarily as sports halls. Jack Wrightson (WJHW, Inc., 13714 Gamma Rd., Ste. 110, Dallas, TX 75244)

Large indoor sports arenas have always served a secondary function as venues for other types of entertainment and as gathering spaces. The reasons include available capacity to serve large crowds, proximity to dense population centers, and the economic needs of the facilities themselves, which cannot afford to operate as a single-season sport franchise. The rise in popularity of the touring "rock acts" is examined, as well as other types of musical presentations, and some of the acoustical planning considerations needed to reasonably successfully accommodate the diverse acoustical needs of various types of entertainment in these halls are discussed. Examples and lessons from recent work will be presented.

2:05

2pAA3. Current trends in pop music recording facilities. Russ Berger (Russ Berger Design Group, Inc., 4004 Beltline, \#110, Dallas, TX 75244)

The design of current recording facilities is being influenced by several factors. The proliferation of digital signal processing equipment, signal acquisition, and storage media have sparked changes in the way recording studio facilities are used. The mixture of programmed synthesis with acoustic studio performance and the resulting changes in recording technique also provide a significant influence on the design of pop music recording facilities. The impact these changes are having on facility layout, configuration, and function; noise and vibration control; the architectural acoustic environment of studios and control rooms; and the electroacoustic interface will be discussed.

\section{Contributed Papers}

\section{2:35}

2pAA4. Auditorium characteristics and a cappella music. William W. Ryan, J. (Harding Univ., Box 2239, Searcy, AR 72149-0001)

Many authors have presented the typical curves of reverberation time versus volume for various auditorium functions, such as religious, concert hall, opera, etc. Most of the religious curves suggest that the auditorium will be used for instrumental (piano or organ) accompaniment of the singing, whether congregational or choir. This paper presents the preliminary results of a congregational survey to discover what the participants of a cappella music desire. A plot of the reverberation times and volumes for the auditoriums of the participating congregations is also presented. From these preliminary results, it appears that the desire is for a more lively environment, and that the reverberation time should be somewhat higher than that of the "protestant church" curve as presented in Acoustics by Beranek. [Work supported by Ryan \& Associates.]

\section{2:50}

2pAA5. Music critic listening experiment at Dallas' Meyerson Symphony Center-A progress report. David Lubman (D. Lubman \& Associates, 14301 Middletown Lane, Westminster, CA 92683) and Gary W. Siebein (Univ. of Florida, Gainsville, FL 32611)

Professional music critics often pass judgment on concert hall acoustics in their reviews. Because of the wide circulation given to their reviews, music critics may strongly influence public perceptions of the acoustical 
merits of concert halls. Surprisingly, no systematic study has been reported comparing critics' acoustical judgments with those of musicians and of ordinary listeners in the same hall, despite its potential value to both acoustical and critic communities. For this reason, the ASA launched a cooperative effort with the Music Critics Association of North America (MCANA). Its first step was an informal experiment at Dallas' Meyerson Symphony Center during MCANA's June 1994 meeting. The critics' meeting provided a potential opportunity to obtain acoustical assessments from a significant number of critics attending symphony concerts in the same hall. Standard survey forms and instructions were placed in the registration packets of about 75 music critics at check-in, and were distributed randomly to about 1000 volunteers attending concerts on two consecutive nights. Disappointingly, of 138 survey forms returned so far, only about 10 are from critics. This could improve by presentation time. Survey results and lessons learned will be reported. [Work supported with private donor and TCAA Technical Initiative Funds.]

\section{3:05-3:15 Break}

\section{$3: 15$}

2pAA6. Acoustics of large homes. James E. Bruce, Charles T. Moritz, and Robert D. Bruce (Collaboration in Sci. and Technol., Inc., 15835 Park Ten Place, Ste. 105, Houston, TX 77084-5131)

Many large houses (in excess of 5000 square feet) have recently been built in the Houston, TX area. Some of these houses are constructed on speculation by builders and some are designed and built for specific individuals. This paper summarizes the results of a survey of the builders of both types of homes. Since many individuals who build their own homes design into their spaces features that reflect their personality and needs, it was thought that it would be interesting to investigate if "good acoustics" were one of these features. In addition, measurements of background noise, reverberation time, noise reduction between critical spaces, and the noise reduction from outside sources will be discussed. Finally, suggestions for improving the acoustics of large homes will be presented.

\section{3:30}

2pAA7. Computer-based system for reverberation room design. Eduardo Méndez Castañeda (Ingeniería Acústica Spectrum, S.A. de C.V., Apaseo el Alto 21-2, San Bartolo Atepehuacán, Del Gustavo A. Madero, México D.F., CP 07730, Mexico)

A simple computer system named SIDIART developed in QBASIC language is proposed, which permits the manipulation of a database of materials and their respective absorption coefficients, making their election easy. Knowing the volume and the areas, if the user wishes, the system suggests the optimal $500-\mathrm{Hz}$ reverberation time (RT) from one of ten optional types of rooms. The Sabine or Eyring equations, and relative humidity between $10 \%$ to $90 \%$ for the calculus of air absorption, can be chosen. Given a number of surfaces, its areas and a group of candidate materials for each surface, the system reports the ratio between the optimal and calculated RT for each possibility and for each frequency. The system has the option of searching and reporting only the combination that offers deviations from the optimal RT defined by the user as acceptable, if that combination exists. The algorithm of search is based on the traditional way to solve the problem without a computer. The advantage of the system takes root in the velocity of calculus provided by the computer; however, the final result of the project depends upon the designer.

\section{$3: 45$}

2pAA8. Sound isolation at low frequencies, only an extension of the traditional frequency range? Tor Kihlman (Dept. of Appl. Acoust., Chalmers Univ. of Tech., S-412 96 Gothenburg, Sweden)

The expression "low frequencies" implies a frequency range where the rooms' dimensions are comparable with the wavelength of sound in the rooms. In this range (below $150 \mathrm{~Hz}$ for normal room sizes) the sound field in the rooms consists of a few modes only, and changing of parameters that are not connected to the partition influences the sound insulation. This is not a problem of a correct measurement procedure. It is far more the description of the sound insulation itself in which problems arise. The measured or calculated sound insulation is only valid for the specific case under consideration. Numerical studies of the sound insulation of a flexible partition (characterized by a limp mass) between two rooms show that the dimensions of the sending and receiving rooms have the most important influence. This has to be taken into account when extending the frequency range in the standards concerning sound insulation which is necessary since neighbors low frequency stereo equipment, as well as the all present traffic noise from outdoors, demands a sufficient sound insulation even below $100 \mathrm{~Hz}$.

\section{4:00}

2pAA9. Sound isolation-A review. Ilhuicamina Servin Rivas (Academia de Acústica, Escuela Superior de Ingeniería Mecánica y Eléctrica, Instituto Politécnico Nacional, Laboratoristas No. 8, Colonia Sifón, Delegación Iztapalapa, Mexico 09400 D.F., Mexico)

When treating the property of sound attenuation provided by partitions, different ways are found for its specification. In this paper most common descriptors employed to specify airborne and structureborne sound insulation are summarized and reviewed. Advantages and disadvantages of single-number specifications for sound insulation are commented upon. On the other hand, emphasis is made as to the importance and convenience of field measurements of sound insulation, even though they are timeconsuming. Also included is a brief description of the situation prevailing in some countries, Mexico among them, regarding minimum sound insulation requirements between dwellings. Finally, reference is made to the use of acoustic intensity techniques for the measurement of sound insulation and the need of standardization on this type of evaluation.

\section{$4: 15$}

2pAA10. Initial tests in AT\&T Bell Labs' Varechoic Chamber. William C. Ward (WARD Lab., 2441 Camino Capitan, Santa Fe, NM 87505), Gary W. Elko, Robert A. Kubli (AT\&T Bell Labs., Murray Hill, NJ 07974), and W. Craig McDougald (Acoustic Systems, Austin, TX 78764)

Results are available for the first measurements in the recently completed Varechoic chamber, a digitally controllable variable acoustics facility at AT\&T Bell Laboratories in Murray Hill, NJ. The $118 \mathrm{~m}^{3}(6.7 \times 6.1$ $\times 2.9 \mathrm{~m}$ ) room has 368 independently actuated surfaces in the walls, ceiling, and floor. The room boundary conditions can be completely changed in a fraction of a second. This chamber is a unique facility for acoustical measurements, testing of electroacoustic and signal processing systems, perception tests, or for recording. The panels from which it is constructed could be applied in multipurpose rooms, recording studios, or performance spaces. Reverberation time in the $750-\mathrm{Hz}$ octave band is variable from $0.1-1.6 \mathrm{~s}$; this talk will present full $1 / 3$-oct decay rates of the room. Some interesting impulse responses, and comparisons with image model calculations, will also be given.

\section{$4: 30$}

2pAA11. Developments in the "chopper" theory of rotating diffuser sideband production. David Lubman (D. Lubman \& Associates, 14301 Middletown Lane, Westminster, CA 92683)

Periodic motion of a sound reflector in a tonally excited reverberant sound field results in a multitonal reverberant spectrum consisting of symmetrical "sidebands" centered around the forcing frequency. This surprising and useful phenomenon does not violate linear system theory! It was first reported in 1968 , late in the history of what some consider to be the "mature" field of architectural acoustics. It was discovered in connection with the invention of rotating diffusers (RDs), which were themselves a surprise. (Moving diffusers were shown to be the only class of diffuser that can improve spatial uniformity of reverberant sound.) Interest in RDs was spurred by their ability to improve the precision of tonal sound power determination in reverberation rooms. But neither the reason for multitonal "sidebands" nor their role in improving spatial uniformity was understood. 
Some workers pronounced sidebands to be trivial doppler shifts. Others denied their very existence, dismissing data as mere illusions of reactive sound. At the recent Sabine Centennial, this writer proposed a new "chopper" hypothesis to account for RD sidebands. More physically compelling than the doppler hypothesis, it shows strong potential for analytical development, and may facilitate RD design optimization. Chopper theory is reviewed and recent developments are reported.
4:45-5:00

PANEL DISCUSSION: Compatibility of Performing Spaces for Popular Versus Classical Music

Panel Moderator: Richard Boner

Panel Members: Russ Berger, Dana Hougland, Ed Logsdon, Jack Wrightson

\title{
Acoustical Oceanography and Animal Bioacoustics: Effects of Sounds on Marine Mammals: Update and Discussion on the Need for Standards I
}

\author{
James H. Miller, Chair \\ Department of Electrical and Computer Engineering, Naval Postgraduate School, Monterey, California 93943
}

Chair's Introduction-1:40

Invited Papers

1:45

2pAO1. A review of the effects of sound on cetaceans. Adam S. Frankel (Dept. of Oceanogr., Univ. of Hawaii, 1000 Pope Rd., Honolulu, HI 96822) and Christopher W. Clark (Cornell Bioacoust. Res. Prog., Ithaca, NY 14850)

Studies to determine the effects of various acoustic stimuli on whales are reviewed, with an emphasis on those studies that quantified sound level. Most of these studies have tested the effects of anthropogenic sound on bowhead and gray whales, with some work on other species. The variables used to measure the whales' response to sound typically include course deviations and changes in rates of respiration and other behaviors. Examples include the relationship of received level with the probability of avoidance of the sound source [Malme et al., MMS Rep. $(1983,1984)$ ] and changes in respiration rate after the beginning of a sound playback [Richardson et al., Mar. Environ. Res. 29, 135-160 (1990)]. Response thresholds for continuous sounds have typically been measured at 110-124 dB, with responses to orca "screams" near $0 \mathrm{~dB} \mathrm{~S} / \mathrm{N}$ ratio [Malme et al., MMS Rep. (1983)]. These studies have led to the use of the 120-dB level as a regulatory criterion for cetacean disturbance. The results of these studies will be presented considering which variables may be important for determining or predicting a whale's response to sound.

2pAO2. MMATS: Acoustic localization of whales in real time over large areas. David $S$. Clark (NRaD, San Diego, CA 92152-5000), John Flattery (ORINCON Corp., San Diego, CA 92121), R. Gisiner (NRaD), L. Griffith (NRaD), J. Schilling (ORINCON), T. Sledzinksi (ORINCON), and R. Trueblood (ORINCON)

The marine mammal acoustic tracking system (MMATS) provides real-time display and signal processing of ten channels of acoustic data. In this study analog signal data from ten sonobuoys were radioed to a circling aircraft carrying the MMATS hardware; the data were transformed into an intensity/time/frequency display scrolling in real time. Whale species were determined from the signal characteristics; the system includes a neural network for automated detection. Arrival time delays of a signal at three or more siles were used to localize the whale. Acoustic identification and localization of the whales were visually confirmed by observers making an independent visual survey of the area at the same time. Acoustic monitoring capabilities of the type provided by MMATS can significantly reduce the number of whales missed by traditional visual-only monitoring and provides a means of calibrating both methods, reducing the statistical uncertainty of population estimates made using either technique alone. Because MMATS can monitor large areas for long periods of time, it is well suited to monitoring the effects of manmade noise on the activities of whales.

\section{2:25}

2pAO3. Acoustic detection and location of hlue whales (Balaenoptera musculus) from SOSUS data by matehed filtering. Kathleen M. Stafford (Hatfield Marine Sci. Ctr., Oregon State Univ., Newport, OR 97365), Christopher G. Fox (Natl. Ocean. and Atmos. Admin., Newport, OR 97365), and Bruce R. Mate (Oregon State Univ., Newport, OR 97365)

Blue whale calls were recorded off central California in the Fall of 1993. These calls were characterized as to duration, frequency downsweep, intercall interval, and sound-pressure level. Average values were determined from 303 calls, including up to three harmonics (fundamental downsweep from 18.9 to $17.2 \mathrm{~Hz}$ over $16 \mathrm{~s}$ ). These frequency-domain characterizations were then used to develop numerical time series (kernels) that, when convolved with the original time series, produce correlation peaks indicating the presence of blue whale calls (matched filter). When harmonics were present in the data, a combined kernel, including the fundamental frequency and first harmonic, improved the signal to noise ratio over use of the fundamental kernel alone. These matched filters were able to detect blue whale calls even in very "noisy" time series. When applied to hydrophone recordings from three U.S. Navy SOSUS (SOund SUrveillance System) arrays, it is possible to produce locations for blue whale calls by timing the arrival of individual calls 
and applying least-squares techniques. This information can be used to increase our knowledge of blue whale distribution in the northeast Pacific. The methods described here may also be extended to other species that employ low-frequency vocalizations or to other ocean areas.

2pA04. Effects of boat noise on the acoustic behavior of humpback whales. Thomas F. Norris (Dept. of Vertebrate Zoology, Moss Landing Marine Labs., P.O. Box 450, Moss Landing, CA 95039)

The effects of boat noise on cetacean acoustic behavior are not well understood. To examine these, real sources of boat noise were experimentally introduced to singing humpback whales (Megaptera novaeangliae). Humpback whales were chosen as subjects because they sing long songs that are easy to record. Also, they are often distributed in nearshore environments with heavy boat traffic. Songs from nine animals were analyzed $(n=9)$. Ten variables describing time and frequency characteristics of humpback song signals and the structure of song patterns were compared before and during exposure to boat noise. Means of two variables (unit duration and phrase duration) were significantly less during boat passes than during control periods. Means of eight other variables were not significantly different. The statistical power of detecting a difference between the means was $>90 \%$ for all variables describing frequency characteristics of songs. Because the durations of some variables were shortened, these results indicate that boat noise might affect humpback whale singing behavior. However, power analyses indicate that frequency structure is probably not affected. The significance of these effects concerning the behavioral biology of humpback whales is uncertain at this time.

3:05

2pA05. Temporal and spatial distribution of whale calls off Monterey, California. Khosrow Lashkari (Monterey Bay Aquarium Res. Inst., Pacific Grove, CA 93950)

Twenty-seven hours of acoustic data were recorded from a horizontal array in deep waters off the central coast of California. These data were analyzed to determine the characteristics of diverse underwater acoustic sources. Some of the identified sources were: moored RAFOS sources at ranges of $150-1000 \mathrm{~km}$, low-frequency ship and machinery noise, and sounds of biological origin. Over 400 whale calls were identified and analyzed to determine the distribution of these calls in both time and azimuth. Spectral analysis of the vocalizations indicate that most of the calls were from humpback whales. [Work supported by the United States Navy, Naval Postgraduate School, and Monterey Bay Aquarium Research Institute.]

\section{Contributed Papers}

\section{3:25}

2pAO6. The influence of acoustic signals on a juvenile gray whale. Peter J. Rovero, Robert M. Keolian, and James H. Miller (Code PH/Kn, Naval Postgraduate School, Monterey, CA 93943)

In May 1994, a juvenile gray whale, Eschrichtius glaucus, entered the Petaluma River, which empties into the north end of San Francisco Bay, CA. The Marine Mammal Center of Sausalito, CA, coordinated a rescue and asked us to lure the whale to deeper water with sound. The Petaluma River is muddy and brackish, $20 \mathrm{~km}$ long, and generally $75 \mathrm{~m}$ wide and 3 to $4 \mathrm{~m}$ deep. Recorded gray whale calls and synthetic signals in the range of $100-900 \mathrm{~Hz}$ were broadcast with a $\mathrm{J}-9$ acoustic transducer providing a source level of $153 \mathrm{~dB}$ re: $1 \mu \mathrm{Pa}$ at $1 \mathrm{~m}$. Over several hours, the whale, who surfaced for air every $140 \mathrm{~s}$, seemed to be attracted to these sounds as we traveled at a few knots down river, our sound boat typically $50 \mathrm{~m}$ ahead of the whale. The whale appeared to lose interest in the sound boat much beyond $100 \mathrm{~m}$. A split step parabolic equation model of acoustic propagation in the river suggests that the sound level was $123 \mathrm{~dB}$ near the river bottom at a range of $50 \mathrm{~m}$ and $120 \mathrm{~dB}$ at $200 \mathrm{~m}$. On one occasion the whale approached to within $3 \mathrm{~m}$ of the active source. The sound level at this distance would be about $144 \mathrm{~dB}$.

\section{3:40-3:55 Break}

\section{3:55}

2pA07. A procedure for the calculation of the noise impacts from a spatially stochastic source. Rodolfo T. Arrieta (Spectrum Sciences and Software, Ft. Walton Beach, FL 32547)

There is growing concern over the impact of human intrusion into the habitat of certain wild animal species. A major part of this intrusion is in the form of noise from moving vehicles. The level on the ground or underwater caused by moving noise sources has been dealt with as singleevent intrusions that may cause startle and associated physiological responses, and as cumulative noise exposures. The later approach allows correlation between the cumulative noise exposure of the whole animal population, and the change in population numbers and overall health. Currently, the most difficult part of this analysis lies in determining the sound exposure of the population since both the animals and the noise sources are spatially and temporally varying. There is a certain amount of knowledge about the movement of both the noise sources and the population; this knowledge can be used to create a kinematic simulation of the motions of both entities. Such a simulation has been used to yield long-term spatial probability distributions of noise sources that can then be superimposed over similarly obtained distributions of the population. This superimposition yields the required estimates of the total noise exposure of the population.

\section{4:10}

2pA08. Low-frequency hearing in California sea lions and harbor seals. David Kastak (Long Marine Lab., 100 Shaffer Rd., Santa Cruz, CA 95060) and Ronald J. Schusterman (California State Univ., Hayward, CA 94542)

Studies on pure-tone detection thresholds were conducted on two female California sea lions and on a harbor seal. The older sea lion and the harbor seal were trained to wear custom-fitted headphones in order to determine minimum audible pressures in a binaural listening task. All three animals were trained to respond to underwater signals at frequencies ranging from $100-1600 \mathrm{~Hz}$ at a depth of about $1.5 \mathrm{~m}$. Results were very reliable, owing to a combination of psychophysical threshold determining measures. Sensitivity to low-frequency sounds by both species were 25-30 $\mathrm{dB}$ better underwater than in air. The low-frequency hearing of the harbor seal was $2-25 \mathrm{~dB}$ better than the older seal lion. At $100 \mathrm{~Hz}$, the sensitivity of the harbor seal was $17 \mathrm{~dB}$ superior to that of the younger seal ion, and $23 \mathrm{~dB}$ superior to the older seal lion. Results at low frequencies support the notion that the harbor seal (phocid) ear is more water adapted than the sea lion (otariid) ear. 
2pA09. Behavioral response of migrating gray whales (Eschrichtius robustus) to acoustic stimuli along the central California coast. Nicole M. Angiel (School of Fisheries, Univ. of Washington, WH-10, Seattle, WA 98195)

The gray whale (Eschrichtius robustus) migrates close to shore along the central California coast, and is easily observable from land. The objective of this study is to determine the effects of low-frequency sounds on gray whale behavior. A J-15 transducer will be used to project tones of varying low frequencies and intensities from a vessel platform located off the coast of central California. Gray whales will be observed from shore during the south and northbound migrations past this region, in the presence and absence of sound production. Observed behaviors will then be compared and any disturbance due to sound will be determined. Results of this experiment will be important in regulating future sound-producing activities along the gray whale migration route. This study will also help resolve some of the controversy over another experiment known as the acoustic thermometry of ocean climate (ATOC). The ATOC program involves the projection of low-frequency sounds at higher intensities to measure changes in the ocean temperature over extended time periods. One of the sound projectors for ATOC is proposed to be placed off the central California coast and within the auditory range of migrating gray whales.
2pAO10. Acoustically enhanced bubble growth at low frequencies and its implications for human diver and marine mammal safety. Lawrence A. Crum and Yi Mao (Appl. Phys. Lab., Univ. of Washington, Seattle, WA 98105)

Computations are made of the conditions necessary to obtain bubble growth by rectified diffusion under a variety of conditions associated with low-frequency sound propagation in the ocean. The complex issue of microbubble nuclei stabilization is treated by assuming either a sufficient level of supersaturation to stabilize the initial bubble size, or by examining a microbubble nucleus with zero surface tension. The bubble growth rates and thresholds are obtained for a range of SPLs (re: $1 \mu \mathrm{Pa}$ ) from 150-220 $\mathrm{dB}$, for initial bubble radii from 1-10 $\mu \mathrm{m}$, and for levels of the dissolved gas concentration from $100 \%$ to $223 \%$ of saturation. It was determined that for the range of conditions examined, it was necessary to utilize three different formulations of the equations for bubble growth. The results of these calculations and assumptions indicate that for SPLs in excess of 210 $\mathrm{dB}$, significant bubble growth can be expected to occur, and divers and marine mammals exposed to these conditions could be at risk. For SPLs below about $190 \mathrm{~dB}$, however, significant bubble growth is unexpected. [Work supported in part by NSMRL.]

\title{
Session 2pPAa
}

\section{Physical Acoustics: Sonoluminescence and Sonochemistry II}

\author{
Anthony A. Atchley, Chair \\ Physics Department, Naval Postgraduate School, Monterey, California 93943
}

\section{Contributed Papers}

1:00

2pPAal. Transient, high-pressure solidification associated with cavitation in water. Robert Hickling (Natl. Ctr. for Phys. Acoust., Univ. of Mississippi, MS 38677)

The very high pressures $(>1 \mathrm{GPa})$ that occur during the final stages of collapse of a cavitation bubble force the water in the vicinity of the bubble wall briefly $(\sim 1 \mathrm{~ns})$ into a metastable state of subcooling, relative to the equilibrium phase diagram. Estimates show that the subcooling can fall below the critical temperature for homogeneous nucleation of freezing and that high-pressure ice particles form at a sufficient rate to affect the collapse. Because of the greater density of high-pressure ice, a sudden drop in pressure occurs that triggers a shock wave that converges at the center of the compressed gas in the bubble. Such microshocks are believed to be the cause of the extremely short duration of the flashes of sonoluminescence ( $<50 \mathrm{ps}$ ) that have been observed from single cavitation bubbles. The occurrence of transient, high-pressure solidification can explain different phenomena associated with cavitation, specifically the decrease in cavitation erosion and the increase in sonoluminescence as the overall water temperature approaches $0^{\circ} \mathrm{C}$, together with the nucleation of freezing by cavitation in subcooled liquids. A single explanation for such diverse effects provides strong support for the solidification hypothesis.

\section{1:15}

2pPAa2. Comparisons between multibubble and single-bubble sonoluminescence. Thomas J. Matula, William B. McNamara, III, Pierre D. Mourad, Ron A. Roy, and Kenneth S. Suslick (Appl. Phys. Lab., Univ. of Washington, Seattle, WA 98195 and Univ. of Illinois, Urbana, IL 61801)
Recent advances in the use of single bubble cavitation [D. F. Gaitan et al., J. Acoust. Soc. Am. 91, 3166 (1992)] have made it possible to examine both the temporal and spectral characteristics of sonoluminescence [B. P. Barber et al., J. Acoust. Soc. Am. 91, 3061 (1992)]. Direct comparison of the spectra of single-bubble sonoluminescence to multibubble sonoluminescence has proved difficult, however, due to differences in experimental conditions. Examination has begun of both single-bubble and multibubble sonoluminescence spectra of various aqueous solutions under closely similar conditions, where, in a systematic way, a varicty of dissolved gases, volatile organic liquids, and involatile inorganic salts have been introduced. Qualitative comparisons of these spectra will be discussed. [Work supported by ONR and NSF.]

\section{$1: 30$}

2pPAa3. Effect of ambient pressure on sonoluminescence from a single bubble. Bradley P. Barber (Phys. Dept., UCLA, Los Angeles, CA 90024)

The dynamic sound field pressure $\boldsymbol{P}_{a}$ required to generate sonoluminescence (SL) from a single trapped bubble is a little higher than the ambient pressure $P_{0}$ (e.g., $P_{0} \approx 1.2 P_{0}$ ). Since the acoustic energy density is proportional to the square of $P_{a}$ observation of SL at lower drive levels would imply that even greater degrees of energy concentration accompany the transduction of sound into light. Motivated by this perspective, the dependence of SL on ambient pressure is being measured. Light emission at $P_{0}=0.3 \mathrm{Atm}$ has already been achieved. Pressures higher than an atmosphere are also being investigated, especially with attempts to find single bubble SL in liquids other than water. [Work supported by the U.S. DOE Division of Advanced Energy Projects.] 
2pPAa4. Time scales for sonoluminescence. Keith Weninger, Robert Hiller, Seth Putterman, and Bradley P. Barber (Phys. Dept., UCLA, Los Angeles, CA 90024)

The establishment of stable sonoluminescence from a single trapped bubble of air in water requires more than $5 \mathrm{~s}$. During this time the bubble goes through a transition period (about $1 \mathrm{~s}$ long) that is characterized by an emitted intensity which is over ten times smaller than the steady state. Pure noble gas bubbles turn on to their steady state values on a much shorter time scale (say less than $0.2 \mathrm{~s}$ ). During the transient period light from an air bubble is weaker than light from an Argon bubble but in the steady state the air bubble is brighter. In view of the long time scale required for the establishment of sonoluminescence from a single bubble of air it is concluded that this is a fundamentally different phenomenon from the transient multibubble sonoluminescence that has been studied since its discovery in 1934. [Work supported by the U.S. DOE Division of Advanced Energy Projects.]

\section{2:00}

2pPAa5. Searching for an isotope effect in sonoluminescence. Robert A. Hiller and Seth Putterman (Phys. Dept., UCLA, Los Angeles, CA 90024)

The only pure liquids in which sonoluminescence from a single stable bubble has been observed are water and heavy water. With regard to the content of the trapped bubble there are a number of gases which yield light. Helium is particularly interesting because its spectrum is strongly peaked in the far ultraviolet. In order to learn about the mechanism responsible for sonoluminescence, the search is on for differences berween the spectra of $\mathrm{He}^{4}$ and $\mathrm{He}^{3}$ bubbles in water and heavy water. Other isotope pairs to be compared include hydrogen and deuterium. [Research supported by the U.S. DOE Division of Advanced Energy Projects.]

\section{2:15}

2pPAa6. Sonoluminescence from large plasma-induced vapor bubbles. Gary R. Hess, Ellis L. Loree, James D. Wieting, Forest E. White, and Chris M. Young (Tetra Corp., Albuquerque, NM 87109-4512)

The light emitted during the collapse of large vapor bubbles has been measured. The emission lasts for tens of microseconds and is so intense it must be filtered, under our experimental conditions, to record the complete time-dependent behavior. The majority of the energy appears to be emitted well below $630 \mathrm{~nm}$. Such a long duration emission appears to be more consistent with adiabatic heating than shock phenomena. The temporal shape of the emission compares well with the temporal shape of the pressure produced prior to and after collapse, as well. Such bubbles promote study of the collapse processes in regions of parameter space which are highly accessible. [Work supported by the U.S. Navy.]

\section{2:30-2:45 Break}

\section{2:45}

2pPAa7. Numerical simulations of single bubble sonoluminescence. Pierre D. Mourad (Appl. Phys. Lab., Univ. of Washington, Seattle, WA 98195), Daniel L. Marcus (Lawrence Livermore Natl. Lab., Livermore, CA 94551), Ron A. Roy, and Thomas J. Matula (Univ. of Washington, Seattle, WA 98195)

A higher-order Godunov method is used to solve the spherically symmetric, compressible Euler equations with an ideal gas equation of state as a model for single bubble sonoluminescence. Basic shock physics is discussed in this context, exploring how modeled variations of the bubble interior support or suppress the generation and propagation of shock waves within the bubble as well as the interaction of a shock with the bubble interface. [Work supported by ONR through the ONR/ARL program.]
2pPAa8. Compressibility in bubble dynamics and scattering. Paul E. Barbone, Ali Nadim, and Daniel Goldman (Dept. of Aerospace and Mech. Eng., 110 Cummington St., Boston Univ., Boston, MA 02215)

The Rayleigh-Plesset equation describes the oscillations of a spherical bubble wall under the assumption that the fluid surrounding the bubble is incompressible. Many modifications of this equation have been proposed to incorporate slight fluid compressibility, and though different in form, they are asymptotically equivalent. The different forms of the equations, however, reveal remarkably different properties. In some forms of the modified Rayleigh-Plesset equation, for example, spurious unstable solutions are present while in other cases they are not. Here, physically motivated restrictions on the form of the modified Rayleigh-Plesset equation are discussed. These restrictions are discussed in the context of causality requirements and higher-order corrections to the modified RayleighPlesset equation.

\section{3:15}

2pPAa9. On asymmetrical motions of cavitation bubbles. Tao Shi and Robert E. Apfel (Yale Univ., New Haven, CT 06520-8286)

The phenomenon of sonoluminesence has been of considerable recent interest due to a better understanding of two types of mechanisms: asymmetrical collapse of transient bubbles and single gas bubble oscillation. Asymmetrical collapse of transient bubbles result in lower sonoluminescence temperature; asymmetrical shape modes may also disturb the periodic stability of single gas bubble oscillation. The boundary integral method has been used to study the asymmetrical motions of bubbles of initial diameters of 1-100 $\mu \mathrm{m}$. The role of initial bubble shape perturbation, dissolved gas saturation, surface tension, and maximum bubble size are observed up to a point where assumptions conceming the maximum bubble wall velocity and the internal bubble dynamics are expected to break down. It is observed that the initial shape perturbation grows when the bubble collapses and decays when it expands. Therefore, it appears that there are circumstances when cyclic single bubble oscillations can occur even with significant shape distortion. [Work supported by Jet Propulsion Lab through Contract No. 958722.]

\section{3:30}

2pPAa10. An aspect of sonoluminescence from hydrodynamic theory. H. Kwak, H. Yang (Mech. Eng. Dept., Chung-Ang Univ., Seoul, 156-756, Korea), and J. Hong (Ohsan Tech. College, Ohsan, 447-749, Korea)

Sonoluminescence (SL), the phenomenon of light emission associated with the collapse of bubbles oscillating under an ultrasonic pressure field has been studied by solving the continuity, momentum (Euler), and energy equations for the gas inside the bubble analytically. Heat transfer in the liquid layer adjacent to the bubble wall has also been considered in this analysis. It has been found that the gas behavior is neither adiabatic nor isothermal for a bubble under ultrasound conditions. In this analysis, the launch condition and the Hugoniot curve for the shock propagation has been identified, and the shock duration of 2.7 to $17 \mathrm{ps}$, which is comparable to experimental results, has been obtained with the help of a similarity solution (Guderley) for converging spherical shock. For SI, the gas temperalure after the shock focusing has been found to be $7000-44000 \mathrm{~K}$, depending on the equilibrium bubble radius and the driving amplitude of ultrasound. It has also been found that the heat flux at bubble collapse is as large as $47 \mathrm{GW} / \mathrm{m}^{2}$, which could be more than enough to cause an explosion of an explosive crystal.

\section{$3: 45$}

2pPAall. Theoretical prediction of luminescence from acoustically driven cracks. Ritva Löfstedt and Seth Putterman (Phys. Dept., UCLA. Los Angeles, CA 90024)

Under the effect of an intense long wavelength sound field the length of a crack in a solid medium should oscillatc. When the crack length is increased the imposed acoustic energy is focused down to regions of 


\title{
Session 2pPAb
}

\author{
Physical Acoustics: Porous Media and Ducts \\ Carl K. Frederickson, Chair \\ National Center for Physical Acoustics, University of Mississippi, University, Mississippi 38677
}

\section{Contributed Papers}

2:00

2pPAb1. On the use of probe microphone and level difference measurements to characterize air-filled porous media. Carl $\mathrm{K}$. Frederickson and James M. Sabatier (Natl. Ctr. for Phys. Acoust., Univ. of Mississippi, Coliseum Dr., University, MS 38677)

Air-filled porous media have been characterized acoustically using level difference measurements and an impedance model that depends on a pore-shape factor, porosity, tortuosity, and bulk flow resistance. Leastsquares fitting of level difference spectra only allows two of the above parameters to be independently determined. Probe microphone measurements have been used to determine the propagation constant in both washed sand and glass beads. Values of tortuosity calculated from probe microphone measurements were used in the analysis of level difference data to determine porosity and bulk flow resistance. An average pore-shape factor is used in the analysis. For the unconsolidated porous media used, the effect of the pore-shape factor variation was within the error range of the measurement. The availability of both probe microphone and level difference data has also allowed for the comparison of porosity and flow resistance values calculated from each set of data. There are some discrepancies between the frequency dependence of the model and the probe microphone data. [Work supported by USDA.]

\section{2:15}

2pPAb2. Acoustic probe microphone measurements of Biot type I and II waves in air-filled sands. Craig Hickey, Wayne Prather, and James M. Sabatier (Natl. Ctr. for Phys. Acoust., Univ. of Mississippi, University, MS 38677)

Probe microphone measurements of air-borne sound penetrating into air-filled sands and soils indicate two absorption coefficients for the frequency range $40-4000 \mathrm{~Hz}$. The probe microphone signal attenuates rapidly with depth in a region near the surface. Below that region the microphone signal attenuates significantly slower with depth. Rigid-capillarytube porous models, which allow for pore-fluid motion only, correctly describe the rapid attenuation of probe pressure. Using these models, pore properties (tortuosity and air permeability) are typically deduced from the measured complex absorption coefficient. The Biot poro-elastic-capillary tube model describes both attenuation regimes. The two absorption coefficients are associated with the Biot type I and II waves. The large attenuation in the region near the surface is associated with the Biot type II wave. The much smaller attenuation coefficient is a consequence of the elasticity of the matrix and is associated with the Biot type I wave. Biot's model is used to calculate the microphone pressure from both Biot type waves as a function of depth. [Work supported by USDA.]
2:30

2pPAb3. The influence of pore-size distributions on complex wave number in air-filled porous materials. David W. Craig, Carl K. Frederickson, and James M. Sabatier (Natl. Ctr. for Phys. Acoust., Univ. of Mississippi, University, MS 38677)

Probe microphones have been developed to determine tortuosity and effective flow resistivity for air-filled porous materials, such as agricultural soils. They are used to measure complex wave number as a function of frequency. These data are then inverted using a single-pore-size capillary tube model for propagation in the material. However, the frequency dependence of the measured wave number in sand differs from the predictions of such single-scale models. It is shown that distributions of pore sizes can produce a similar dependence. By summing over pore sizes, wave number as a function of frequency is calculated for lognormal, fractal (power-law), and empirical distributions derived from porosimetry data. Results are also compared with Wilson's relaxation-matched fractal model [D. K. Wilson, J. Acoust. Soc. Am. 94, 1136-1135 (1993)]. [Work supported by USDA.]

$$
\text { 2:45 }
$$

2pPAb4. Sound propagation in capillary-tube-type porous media: Effects due to the presence of absorbed water in the capillary walls. Miguel Bernard and James M. Sabatier (Natl. Ctr. for Phys. Acoust., Univ. of Mississippi, University, MS 38677)

The effects on sound propagation is investigated in air-filled capillarytube-type ceramic porous media that result from the presence of small quantities of absorbed water in the tube walls. Specific acoustic impedance measurements are performed for a rigid-backed sample for the cases of both porous and nonporous tube walls. For the nonporous tube walls measurements are performed on dry and water wetted walls. Preliminary results suggest a contribution to the increased attenuation of sound in the air-filled porous samples due to the presence of a thin water film. [Work supported in part by ONR.]

3:00

2pPAb5. Sound attenuation in a cylindrical tube due to evaporation-condensation. Yi Mao and James M. Sabatier (Natl. Ctr. for Phys. Acoust., Univ. of Mississippi, University, MS 38677)

The influence of evaporation-condensation processes on the sound propagation in a cylindrical tube were studied in an attempt to understand the sound attenuation in porous materials. In the theoretical model, the tube wall was rigid and kept a constant temperature. A very thin layer of water 
on the wall was allowed to evaporate into or condense from the sound field propagating in the tube. In addition to the acoustical, thermal, and vorticity modes in Kirchhoff's theory, there exists a mass-diffusion mode. The sound attenuation was obtained by applying the boundary conditions on the tube wall to these four modes. Analytic expressions for the asymptotic behaviors of both high and low-frequency limits were derived. While the sound attenuation due to viscosity could be identified, those due to thermal conduction and evaporation-condensation were coupled. The sound attenuation due to the evaporation-condensation process would increase to a substantially large level when the percentage of vapor in the tube was high, but it still underestimated the experimental results in porous materials. [Work supported by ONR.]

\section{3:15}

2pPAb6. Wave dynamics and flow in multiple-porosity media. Timothy S. Margulies (908 Marine Dr., Annapolis, MD 21401)

The purpose of this paper is to describe the fluid flow and dynamics of wave propagation in multiple-porosity media (such as applied to porous rock with fissures of different sizes). Coupled partial differential equations for matrix deformation and Darcy flow with compressible mass conservation equations obtained from continuum mixture theory are developed. For the limiting case of a single porosity medium the transient response (pressure decay) solution for drilling into a gas saturated rock layer, for example, that is homogeneous and isotropic, will be derived exactly by transformation of the nonlinear diffusion equation describing the motions. Furthermore, a Burgers equation which admits solitons will be presented. Finally a generalization to the case of dual/triple continua will be treated.

\section{$3: 30$}

2pPAb7. Modeling sound propagation in a high porosity fibrous material. Christopher L. Morfey, Bradley P. Semeniuk, and Maurice Petyt (Inst. of Sound and Vib. Res., Univ. of Southampton, Southampton SO17 1BJ, UK)

Sound propagation in an air-filled, high porosity fibrous material involves the elastic response of the fiber skeleton as well as thermal and viscous effects at the fiber-fluid boundaries. A theoretical model of wave propagation in such a medium has been constructed based on the idea of an equivalent fluid, which occupies the entire space and whose properties approximate the locally averaged properties of the actual fluid. Once the elastic properties of the skeleton are known-e.g., from in vacuo experiments - the model yields phase speeds, attenuation rates, and complex characteristic impedances for both fast and slow waves in the composite medium. Typical results are presented for fiberglass blankets of the type used as thermal insulation in aircraft fuselages. [BRAIN project supporied by the European Commission.]
2pPAb8. Sound transmission in a pipe with developing laminar flow: Upstream/downstream phase speed differences. Christopher L. Morfey (Inst. of Sound and Vib. Res., Univ. of Southampton, Southampton SO17 1BJ, UK) and Malcolm G. Smith (Univ. of Southampton, Southampton SO17 1BJ, UK)

In a uniform rigid-walled duct, plane waves propagate with axial phase speed $(1 \pm M)$ times the sound speed, according to the inviscid plug-flow model. The difference between the downstream and upstream phase speeds provides a measure of the flow rate; the same concept can be extended to realistic profiles of $M$ (Mach number) across the duct section, although the lowest-order mode is not a plane wave any more. Calculations were carried out to provide a means of converting phase speed differences to flow rates, assuming that propagation is confined to a single low-order mode (i.e., near-axial propagation). The results were then compared with phase speed measurements made in a cylindrical steel tube, through which air was pumped at a controlled steady flow rate. Close but not perfect agreement was found, which raises the question of whether our neglect of thermoviscous phenomena is justified, particularly near the duct walls. [Work supported by British Gas.]

\section{4:00}

2pPAb9. Propagation of sound in a lined circular duct with sheared mean flow. Jinlong Wu and Mark F. Hamilton (Dept. of Mech. Eng., Univ. of Texas at Austin, Austin, TX 78712-1063)

This presentation describes an analytical investigation of the propagation of sound in a lined circular duct with sheared mean ambient flow. The main assumptions are that the ambient flow is turbulent and unaffected by the sound, the Mach number for the flow is small compared to unity, the thickness of the viscous boundary layer is small in comparison with the radius of the duct, and the acoustical lining is locally reactive. The mean flow profile away from the wall is assumed to be uniform, but the profile within the boundary layer can take one of several analytic forms. Outside the boundary layer, the acoustic mode structure is described by Bessell functions. The solution within the boundary layer is expressed in terms of Kummer functions, and the dispersion relation is obtained by matching the inner and outer solutions, taking the wall impedance into account. The dispersion relation is solved numerically for the attenuation and phase speed of the sound as a function of mode number, complex wall impedance, boundary layer thickness, and flow profile within the boundary layer. Both upstream and downstream propagation are considered. Comparisons are made with published numerical results. 


\title{
Session 2pPP
}

\section{Psychological and Physiological Acoustics: Emissions, Localization, Rhythm, Masking, and More}

\author{
Ted L. Langford, Chair \\ U.S. Army Aeromedical Research Laboratory, Fort Rucker, Alabama 36362
}

Chair's Introduction-1:00

\section{Contributed Papers}

\section{1:05}

2pPP1. Studies of the relationships between ear canal and cochlear signals for external tones and spontaneous and distortion product otoacoustic emissions, and their connection with middle ear transmission. Carrick L. Talmadge and Arnold Tubis (Dept. of Phys., Purdue Univ., West Lafayette, IN 47907)

Some knowledge about the transmission characteristics of the human middle ear may be obtained by comparing ear canal levels of external tones and spontaneous emissions of the same frequency, which when used with another tone give similar ear canal levels of distortion product otoacoustic emissions. It is assumed that the cochlear activity patterns of an external tone of frequency $f 2$ and a spontaneous emission of frequency $f 2$, which produce the same levels of ear canal cubic distortion products when used in conjunction with an external tone of frequency $f 1$ and fixed level, are very similar. The degree of similarity is studied using nonlinear active cochlear models that give spontaneous emissions [Talmadge and Tubis (1993)]. The relationship of the cochlear activities at the place of peak excitation and at the base of the cochlea is also studied in detail. The model results indicate that the comparison of ear canal levels of external tones and spontaneous emissions, which are equivalent with respect to the production of distortion product emissions, may be used to give estimates of the reflection and transmission of cochlear waves at the stapes. [Work supported by the Deafness Research Foundation.]

\section{$1: 20$}

2pPP2. Wavelet analysis of transient-evoked otoacoustic emissions. Jeffrey D. Travis and R. Joe Thornhill (Appl. Res. Labs., Univ. of Texas at Austin, P.O. Box 8029, Austin, TX 78713-8029)

A novel approach to finding information in transient-evoked otoacoustic emissions (TEOAEs) was used: the discrete wavelet transform. It is argued that wavelet transforms are more appropriate than fast Fourier transforms (FFTs) for signal decomposition because of the transient, nonstationary nature of TEOAEs. Several wavelets were used to obtain timefrequency maps of TEOAEs collected in past experiments. The latency of several frequency bands can be clearly determined for any given subject with this map. Another set of data collected from an experiment in which subjects were administered quinine sulfate was also studied. Quinine sulfate suppresses TEOAEs and induces some hearing loss [D. McFadden and E. G. Pasanen, J. Acoust. Soc. Am. 95, 3460-3474 (1994)]. Wavelet analysis on this data revealed any temporal shifts of frequency bands during the effect of the drug, and correlated the TEOAE waveform with the amount of temporary hearing loss at each frequency band.

\section{$1: 35$}

2pPP3. Effect of anticholinergic drugs on human spontaneous and click-evoked otoacoustic emissions, frequency discrimination, and lateralization using interaural intensity differences. Narriman Lee Callaway and Dennis McFadden (Dept. of Psychol., Mezes Hall 330, Univ. of Texas, Austin, TX 78712)
The primary neurotransmitter for the cochlear efferent system is widely believed to be acetylcholine. If anticholinergic drugs reduce neurotransmitter activity in the efferent pathway, they should produce effects in accord with those seen in animal studies where the olivocochlear bundle (OCB) was severed. Experiments were conducted to measure effects of three common anticholinergic drugs, diphenhydramine (Benadryl ${ }^{\boxplus}$ ), hyoscyamine (Levsin ${ }^{\text {}}$ ), and scopolamine (Transderm-Scop ${ }^{\text {(O) }}$ ) on two psychophysical tasks and two physiological measures. There was no evidence of impaired performance in a complex frequency-discrimination task or in a lateralization task using interaural intensity differences. Also, no compelling evidence was found to indicate that either spontaneous or click-evoked otoacoustic emissions were enhanced by these drugs. After the beginning of these experiments, a report appeared [S. G. Kujawa et al., Hear. Res. 74, 122-134 (1994)] indicating that, in guinea pigs, suppression of distortionproduct otoacoustic emissions was reversed by anticholinergics having antinicotinic action, but less so by those having antimuscarinic action. The three anticholinergic drugs used here are primarily antimuscarinic in their action, which may explain the failure to observe the predicted effects. [Work supported by NIDCD.]

\section{1:50}

2pPP4. Evidence for heritability and prenatal masculinization of cochlear mechanisms from measures of otoacoustic emissions. Dennis McFadden and John C. Loehlin (Dept. of Psychol. and Inst. for Neurosci., Mezes Hall 330, Univ. of Texas, Austin, TX 78712)

Spontaneous and click-evoked otoacoustic emissions (SOAEs and CEOAEs) were measured in twin pairs of various sorts-monozygotic (MZ), same-sex dizygotic (SSDZ), and opposite-sex dizygotic (OSDZ)-as well as in nontwins. Comparison of the number of SOAEs exhibited by $\mathrm{MZ}$ and SSDZ twin pairs previously led to the conclusion that about $75 \%$ of the individual variation in SOAE expression can be attributed to genes. Parallel calculations will be shown for the CEOAE data. In accord with past surveys, females generally exhibited more SOAEs than males; however, OSDZ females exhibited, on average, less than half as many SOAEs as other females, and thus were comparable to males on this measure. Similarly, the strength of the CEOAEs was generally greater in females than in males, but not in OSDZ females. The interpretation is that the cochleas of OSDZ females have been masculinized by exposure to the high levels of androgens produced prenatally by their male co-twins. (Prenatal masculinizing effects are well known in other mammals.) If correct, the genes apparently lay a groundwork for the expression of emissionsand, correspondingly, good hearing sensitivity-and prenatal exposure to androgens operates to reduce both emission strength and sensitivity. [Work supported by NIDCD.]

\section{2:05}

2pPP5. Individual differences in sensitivity to interaural disparities of time and of level. Ted L. Langford (U.S. Army Aeromed. Res. Lab., Fort Rucker, AL 36362)

The sensitivities of 22 women and 23 men to interaural temporal differences (ITDs) in low-frequency noise bursts and interaural level differ- 
ences (ILDs) in high-frequency noise bursts were measured in discrimination and absolute judgment tasks. The discrimination abilities of both groups of observers were symmetrical in that there were no differences in performance between interaural disparities favoring either the right or the left ear. The women were somewhat less sensitive and more variable in their performances than were the men in both the temporal and level difference discrimination tasks. However, the mean differences between the sexes, $28 \mu \mathrm{s}$ and $0.9 \mathrm{~dB}$, were not large compared with the variability across individuals. Individuals who performed well-discriminating ILDs also performed well with ITDs. Many observers exhibited ILD subjective midlines which were offset to the left of objective center in the absolute judgement task. This effect was unrelated to ILD discrimination ability, to monaural thresholds, or to absolute judgements based on ITDs.

\section{$2: 20$}

2pPP6. Localizing broadband noise in a reverberation room. Timothy J. VanderVelde, Millicent M. Ow, Wendy R. Thorpe, William Morris Hartmann, and Brad Rakerd (Michigan State Univ., East Lansing, MI 48824)

Sound localization experiments were performed to determine the relative importance of steady-state information and onset-transient information to the localization of broadband noise in a reverberant environment. Experiments used a source identification method with an array of 24 speakers, separated by $2 \mathrm{deg}$ of azimuth, in a teverberation room (RT $60 \approx 4 \mathrm{~s})$. Noise signal onsets were either abrupt, or slowly ramped, or entirely masked by other noise. The ratio of direct to reverberant sound was controlled by positioning the listener with respect to the speakers. Data showed that (1) localization of sound with abrupt onsets is particularly insensitive to direct/ reverberant ratio. (2) Slowly ramped onsets allow listeners to use localization information in the weak direct sound before the reverberant field has fully formed. (3) Some listeners (all young) have remarkable ability to extract localization cues from apparently overwhelming reverberated noise when onsets are masked. Further experiments suggested that these listeners make use of cues at high frequencies where reverberated sound is minimized by wall absorption; performance for these listeners decreased dramatically when the noise was lowpassed at $5 \mathrm{kHz}$. [Work supported by the NIDCD.]

\section{$2: 35$}

2pPP7. Differential sensitivity to increases and decreases in tempo: Evidence for an entrainment model. J. Devin McAuley (Dept. of Comput. Sci., Cognitive Sci. Program, Indiana Univ., Bloomington, IN 47405) and Gary R. Kidd (Indiana Univ., Bloomington, IN 47505)

Listeners' abilitics to detect changes in tempo were investigated with two- and four-tone isochronous sequences with interonset intervals (IOI) of $100,400,700$, and $1000 \mathrm{~ms}$. Separate thresholds were measured for increases and decreases in tempo using an adaptive-tracking procedure. On each trial a standard pattern was followed by two comparison patterns, one of which was faster or slower than the standard. Listeners judged which comparison pattern was different from the standard. Consistent with previous studies of tempo discrimination, thresholds were found to be lower with four-tone sequences than with two-tone sequences, especially at the faster tempos. However, at the fastest tempos, listeners showed greater sensitivity to increases than to decreases in tempo, while the reverse was true at the slower tempos. The crossover point occurred at an IOI between 400 and $700 \mathrm{~ms}$. The findings are consistent with the predictions of an entrainment model [J. D. McAuley, J. Acoust. Soc. Am. 95, 2966 (A) (1994)] in which tempo sensitivity is reflected by the degree to which a system of adaptive oscillators is entrained by the rhythm of a stimulus pattern. [Work supported by NIMH and NIDCD.]

\section{$2: 50$}

2pPP8. A novel architecture for rhythmic pattern recognition, Fred Cummins (Depts. of Linguistics and Cognitive Sci., Indiana Univ., Bloomington, IN 47405)

The problem of pattern recognition in time is usually addressed by buffering, which converts time into a spatial dimension, and allows the application of standard pattern recognition methods. But buffering of high bandwidth sensory input is implausible and problematical. An architecture based on the adaptive oscillator model [J. D. McAuley, J. Acoust. Soc. Am. 95, 2966 (A) (1994)] is presented which generates a spatial pattern from the rhythmic content of the acoustic input. The acoustic signal is passed through a bank of gammatone filters, each channel is half-wave rectified and down sampled, allowing a simple differencing procedure to identify onsets, which serve as inputs to a 2-D array of oscillators organized by frequency channel and by intrinsic period. Each oscillator adjusts its intrinsic period to match periodic onsets present in the signal. Only those oscillators that succeed in synchronizing their activity to a period in the input signal produce persistant output. After synchronization, the output distribution produces a stable spatial pattern over the 2-D array. The procedure allows treatment of pattern distributed in time without recourse to sensory buffering. Output patterns for a variety of stimuli, including animal gaits, musical rhythms, and prosodic structure will be presented. [This project was supported by ONR.]

\section{3:05-3:20 Break}

\section{$3: 20$}

2pPP9. Contralateral stimulation and the masking overshoot effect. Christopher W. Turner, Rebecca Waite, Karolyn Cummings, and Melanie Rosen (Syracuse Univ., Program in Commun. Sci. and Disord. and Inst. for Sensory Res., 805 South Crouse Ave., Syracuse, NY 13244)

Overshoot is the increase in masked threshold for a short signal presented at the onset of a masker compared to the threshold for a signal presented in the temporal center of the masker. One hypothesis to explain this effect is the slow onset time for masker-stimulated ipsilateral efferent activity to influence the response to the masked signal. Recent physiological experiments have shown that noise presented to the contralateral ear, which provides an increment in efferent activity, can increase the neural detectability of short tones masked by noise [Kawase et al., J. Neurophys. 70, 2533-2549 (1993)]. The present behavioral experiment took advantage of these findings and measured the amount of overshoot under conditions where a brief contralateral noise was presented prior to the masker onset, in an attempt to "prime" the efferent system prior to the masker onset. All measurements were conducted using insert earphones, which provide approximately $80 \mathrm{~dB}$ of interaural attenuation. The preceding contralateral noise reduced or eliminated the overshoot across a wide range of masker levels. Possible mechanisms for the overshoot effect will be discussed. [Work supported by NIDCD.]

\section{3:35}

2pPP10. Effects of rise/fall time on masked detection thresholds and temporal integration for noise band signals. M. G. Heinz (Dept. of Elec. and Comput. Eng., Johns Hopkins Univ., Baltimore, MD 21218), C. Formby (Univ. of Maryland School of Medicine, Baltimore, MD 21201), and K. L. Mortimer (Georgetown Univ., Washington, DC 20057)

Formby et al. [J. Acoust. Soc. Am. 96, 102-114 (1994)] noted that masked detection thresholds (MDT) and temporal integration (TI) for brief noise band signals may be influenced by the rise/fall time (RFT) used to gate the signals. Also, the definition of signal duration $(T)$, defined (1) exclusive of RFT or (2) inclusive of RFT, affected time constant $(\tau)$ estimates for TI. In this study, the role of RFT was evaluated systematically for signal bandwidth $(W=62-6000 \mathrm{~Hz})$ and masker conditions reported by Formby et al. The MDTs were measured for a range of rise/fall time $(\mathrm{RFT}=1-40 \mathrm{~ms})$ and plateau $(P=1-20 \mathrm{~ms})$ values to extend Formby et al.'s original durations ( $T=P=10-480 \mathrm{~ms})$. In general, for a given $W$ and $P$ condition, MDT decreased and became asymptotic with increasing RFT. For a given $W$, the effect of RFT increased as $P$ decreased, and the effect was greatest for small $W$. For $P \geqslant 10 \mathrm{~ms}$ and RFT $\geqslant 1 \mathrm{~ms}$, MDTs were independent of RFT for all $W$. For $T$ defined exclusive of RFT (i.e., $T=P$ ), $\tau$ was inversely proportional to RFT for $W \leqslant 1000 \mathrm{~Hz}$ and invariant with RFT for $W>1000 \mathrm{~Hz}$. For $T$ defined inclusive of RFT (i.e., 
$\left.T=2^{*} R F T+P\right), \tau$ increased monotonically with RFT for all $W$. [Research supported by NIH.]

2pPP11. A computational model of temporal integration. Neil $P$. McAngus Todd (Dept. of Psychol., Univ. of Manchester, Manchester M13 9PL, UK)

In this paper a computational model of temporal integration is demonstrated. The model has the following architecture. The first stage computes a representation of the auditory nerve response based on the Sheffield ear. The nerve response is summed and low-pass filtered with a temporal window of about $10 \mathrm{~ms}$ [Plack and Moore, J. Acoust. Soc. Am. 87, 2178-2187 (1988)]. In the second stage the summed and smoothed nerve response is coupled to a transmission line model of auditory sensory memory. Close to the periphery the impulse response of the transmission line is sharp. As a pulse transmits into the system though, it becomes progressively more attenuated and spread out in time. In the third stage the line is tapped at different points (approximately 10-ms spacing) and peak responses detected, which then become input samples for higher order leaky integrator with a time constant of about $200 \mathrm{~ms}$. The model thus resembles the "multiple looks" model as proposed by Viemeister [Viemeister and Wakefield, J. Acoust. Soc. Am. 90, 858-865 (1991)] and is able to account for the "resolution-integration" paradox. Quantitative predictions of the model are shown for the temporal integration example from the Houtsma, Rossing, and Wagenaars compact disk.

\section{4:05}

2pPP12. Signal processing to improve speech quality for hearing-impaired people. Zlatan Ribic and Jun Yang (Viennatone Ges. M.B.H., Fröbelgasse 26-32, A-1164 Vienna, Austria)

A signal processing scheme is proposed to improve speech quality for the hearing-impaired people (HI). In this scheme, Hilbert transform and vector sum generator are used to obtain the Hilbert envelope AM, and it follows a voltage-controlled amplifier (VCA) with the gain inversely proportional to AM, the output signal of VCA is sent to a modulator. On the other hand, AM is dynamically processed by a lowpass filter and nonlinear amplitude processor, then sent to the said modulator to restore the dynamic range of the speech. In comparison with the original speech, the resulting one is of a little spectral enhancement, the intermodulation-distortion (ID), and some compression effect because of the nonlinear amplitude processing. Towards the end of our research, the informal listening experiment was performed, 25 people with sensorineural hearing impairment who wear their own hearing aids routinely participated in the experiment. The experiment results have shown that $80 \%$ listeners preferred the processed speech to the original one. This result is quite consistent with one of our expectations: HIs lose the nonlinear active characteristics to some extent, therefore, introducing appropriately ID can help HIs to perceive speech.

\section{4:20}

2pPP13. Acoustic characteristics of voiceless plosives: For the improvement of consonant recognition ability in cochlear implant. Koichi Omori, Stanley M. Blaugrund (Ames Vocal Dyn. Lab., Lenox Hill Hospital, 100 East 77th Street, New York, NY 10021-1883), Hisayoshi Kojima, and Kazuhiko Shoji (Kyoto Univ., Sakyo-ku, Kyoto 606, Japan)

Recognition of consonants, especially voiceless plosives, is inadequate in patients who undergo multichannel cochlear implants. This study was undertaken to ascertain acoustic characteristics of voiceless plosives $/ \mathrm{p} /$, $/ \mathrm{k} /$, and $/ \mathrm{k} /$. Consonant-vowel syllables $/ \mathrm{pa} /, / \mathrm{ta} /$, and $/ \mathrm{ka} /$ of a normal human voice were digitized, and processed by computer in three different ways. First, a portion of consonant signals was deleted. Second, a preceding consonant in one syllable and a following vowel in the other syllable were combined. Third, vowel /a/ was synthesized by repetition of the first cycle of following vowels, and was examined by spectral analysis. All processed sounds were perceived by five experienced listeners. Each voiceless plosive was recognized correctly by its processed syllables which had at least a 1-ms signal of consonant from the onset. Following vowels served as a cue for recognizing voiceless plosives. In following vowels, frequency information, especially in the high-frequency range, was an important factor. From these results, recognition of a short signal at the onset of preceding consonants and emphasis of high-frequency power in following vowels are essential for the improvement of speech perception of voiceless plosives in cochlear implantation.

\title{
Session 2pSA
}

\section{Structural Acoustics and Vibration: Energy Methods in Active Control}

\author{
Scott D. Sommerfeldt, Chair \\ Applied Research Laboratory, Pennsylvania State University, P.O. Box 30, State College, Pennsylvania 16804
}

Chair's Introduction-1:00

Invited Papers

1:05

2pSA1. Active control of flexural and extensional power flow in beams using time domain wave vector sensors. Gary $P$. Gibbs and Chris R. Fuller (Vib. and Acoust. Labs., Dept. of Mech. Eng., Virginia Polytech. Inst. and State Univ., Blacksburg, VA 24061-0238)

Active control of beam vibration using power flow considerations has been a topic of interest in recent years. Traveling waves in structures can be considered "carriers" of energy. For thin beams the magnitude of the energy carried by the wave is proportional to the square of the amplitude of the traveling wave. Thus if traveling waves in structures can be sensed in the time domain then the corresponding power flow will be estimated in the time domain. In this paper, a time domain method for the estimation of flexural and extensional waves in beams will be presented. The method uses a spatial array of sensors in conjunction with digital filters to estimate the separate power flow associated with positive and negative traveling flexural and extensional waves. In an example, surface- 
-mounted PVDF sensors were used in conjunction with a digital filter network to estimate the traveling waves for bandlimited spectra. Experiments where conducted in which the simultaneous flexural and extensional power flow in semi-infinite and finite beams was controlled using the wave vector sensors and surface mounted piezoceramic actuators. These experiments demonstrate the control of beam vibration using power flow-based methods requires fewer actuators and sensors then corresponding modal control techniques. [Work supported by NASA Langley Research Center.]

\section{1:30}

2pSA2. Active structural vibration control via sliding modes: Links to Lyapunov design. Shawn E. Burke (The Charles Stark Draper Lab., 555 Technology Square, Mail Stop 53, Cambridge, MA 02139) and John E. Meyer (Failure Analysis Associates, Menlo Park, CA 94025)

A nonlinear active vibration control design method is developed based upon an extension of variable structure control (VSC) techniques, in particular sliding mode control, to distributed parameter systems. The temporal compensator design utilizes a generalized wave equation representation of the plant. The control is implemented via a series of decentralized single-input/single-output (SISO) local loops around collocated transducers. No a priori knowledge of the temporal plant model is assumed, hence the resulting designs are insensitive to variations in the plant modal frequencies. The equivalent control reduces to output velocity feedback, a known stabilizing control. Active damping performance is enhanced through the introduction of an additive nonlinear term which selectively increases the velocity feedback control with a constrained nonlinear gain profile away from the zero-velocity phase plane origin. Stability constraints are discussed. For simple structural components such as beams and plates, the design method yields controllers identical to those derived using Lyapunov's direct method, which extremize total system energy. Example controllers for beams and plates are presented. In order to demonstrate the application of the nonlinear control, closed-loop vibration control experiments on a $56-\times 59$-in. nine-bay aluminum grillage are summarized.

\section{1:55}

2pSA3. Sensor location considerations for active noise control in enclosures. John W. Parkins and Scott D. Sommerfeldt (Appl. Res. Lab. and Graduate Program in Acoustics, Penn State Univ., P.O. Box 30, State College, PA 16804)

Minimizing the squared pressure at a discrete point(s) is one method of achieving global control in an enclosure, but this strategy will fail when the error sensor(s) lie close to nodal planes of the pressure field. In this case, the secondary modes dominate the pressure measurement, and the active control will create a minimum with little consideration given to the dominant mode. Subsequently, primary mode amplification may result, and the total potential energy in the enclosure will increase. A control based on energy density, on the other hand, can generally sense the dominant mode when the error sensor is close to a pressure field nodal plane, due to its dependence on velocity as well as pressure. Nodal patterns of the energy density field consist of nodal lines and nodal points that lie on the pressure field nodal planes. At these locations, energy density measurements will also be dominated by the secondary modes, and may cause primary mode amplification. Computational results of pressure and energy density fields will be presented which provide insight to optimal error sensor placement for the two aforementioned control methods.

2pSA4. Active control of structural volume velocity using shaped PVDF sensors. Alain Berry (G.A.U.S., Dépt. de génie mécanique, Univ. de Sherbrooke, Sherbrooke, PQ J1K 2R1, Canada)

In low frequency, the sound power radiated from planar structures is simply related to the net structural volume velocity. A cost function based on the volume velocity in active control of structural radiation has the advantage of keeping the control simple (one error sensor). The implementation of volume velocity error sensors in feedforward control of flexural beams and plates using shaped PVDF films is presented. For a beam, a single extended strip of prescribed shape is needed, while in the case of a panel, a number of shaped strips related to the number of flexural modes contributing to the volume velocity is required. The sensor obtained is independent of the type and frequency of excitation. A procedure for deriving the appropriate sensor shape, based on analytical or experimental modes, is discussed. The experimental implementation of volume velocity sensors is addressed and results of active control using piezoceramic (PZT) actuators are presented in the case of a simply supported beam, and simply supported or clamped panels. The strategy of minimizing the volume velocity is shown to provide significant acoustic attenuation for structural free-field radiation or transmission problems.

\section{2:45-3:00 Break}




\section{3:00}

2pSA5. Active control in three-dimensional enclosures using multiple secondary sources and error sensors. Scott $D$. Sommerfeldt and John W. Parkins (Appl. Res. Lab. and Graduate Program in Acoustics, Penn State Univ., P.O. Box 30, State College, PA 16804)

The use of multiple secondary sources and multiple error sensors can significantly improve global attenuation whether one employs a control method based on the squared pressure or energy density. A single source positioned close to a pressure node will be inefficient at exciting the corresponding mode, therefore the secondary modes will dominate the pressure field, and attenuation is unlikely at the related frequency. Increasing the number of secondary sources improves the probability that at least one source will not lie close to a pressure node, thereby mitigating this problem. Problems also arise when error sensors are close to nodes. Adding multiple error sensors increases the probability that the sensors will be able to observe the dominant modes, which will yield improved attenuation. Using a greater number of error sensors than secondary sources will yield a determined control system, with a unique optimal solution. If more sources are used than sensors, an underdetermined control system will result which can be uniquely solved by adding more constraints to the system, such as minimum effort. The performance of the energy density versus squared pressure control methods are compared as they relate to the use of multiple secondary sources and multiple error sensors.

\section{3:15}

2pSA6. A structural-based acoustic intensity sensor. James $P$. Carneal and Gary P. Gibbs (Vib. and Acoust. Labs., Dept. of Mech. Eng., Virginia Polytech. Inst. and State Univ., Blacksburg, VA 24061)

A structural-based acoustic intensity (SBAI) sensor has been developed for low-frequency applications. The sensor is comprised of a structural mounted accelerometer and pressure sensor. Local acoustic intensity is calculated by the time average of the product of the velocity and pressure measurements. The ability to use a structural-mounted pressure sensor was confirmed when the phase between a near field and structural-based pressure sensor was determined to be less than $5 \mathrm{deg}$ up to approximately 1500 Hz. Verification of the SBAI sensor proceeded as follows. The structuralbased acoustic intensity sensor output was shown to be proportional to the output of a calibrated two microphone intensity sensor from a piston source. Active control of the piston source using the SBAI as an error sensor showed global reduction of radiated acoustic power of approximately $15 \mathrm{~dB}$ for several barmonic excitation frequencies. Active control of a complex structure (plate) at various frequencies displayed mixed results. For the $(3,2)$ resonance and a position of $(x / L x=0.85, y / L y=0.78)$, global reduction of radiated acoustic power of approximately $9 \mathrm{~dB}$ was achieved. However, other tests did not show this type of reduction primarily due to the SBAI sensor acting as a local intensity estimator. It is evident that when applied to a complex structure, the point (or points) at which the intensity measurement is taken must be chosen carefully to obtain a global estimate of the far field radiation.

\section{3:30}

2pSA7. Active sound extraction for noise control. Sameer I. Madanshetty (Aerosp. and Mech. Eng., Boston Univ., 110 Cummington St., Boston, MA 02215) and Boa-Teh Chu (Yale University, New Haven CT 06520)

A method of controlling the noise level in duct flows is described. The method is based on the principle of energy extraction by active source(s), rather than by wave cancellation as in "antisound." As such the method of energy extraction is robust; it does not need the delicate signal processing, perfect phase, and amplitude matching, crucial to sound cancellation. Two useful modes of control are discussed, one for quieting low frequencies and the other effective for higher frequencies. The possibility of perfect "noise trapping" in a finite region is also considered, even though it is difficult to implement such complete control in practice. A preliminary experiment that substantiates the concept is described. Extension of this method of noise control to three-dimensional cases is also briefly discussed.

\section{$3: 45$}

2pSA8. One-dimensional sound field control by sound pressure and energy-density sensor. J. Warner Soditus and Jiri Tichy (Graduate Program in Acoustics, Penn State Univ., P.O. Box 30, State College, PA 16804)

The use of an energy density error quantity for adaptive filtered-x active noise control has been demonstrated to provide advantages over pressure squared control in a one-dimensional experiment [S. D. Sommerfeldt and P. J. Nashif, "An Adaptive Filtered-X Algorithm for EnergyBased Active Control," J. Acoust. Soc. Am. 96, 300-306 (1994)]. The performance of energy-density-based control was further explored in comparison to pressure-squared control. By using two small microphones, the pressure and energy-density sensor was moved through regions of the upstream and downstream of the secondary source in a one-dimensional low-frequency harmonic sound field. Extensive data were collected for various sensor locations to demonstrate that the pressure-based control strongly depends on sensor location, while the energy-density-based control is more sensor location-independent.

\section{4:00}

2pSA9. Optimization of multiple piezoelectric actuator locations for active control of sound radiation from plates with arbitrary boundary conditions. Tao Song and Chris R. Fuller (Vib. and Acoust. Labs., Dept. of Mech. Eng., Virginia Polytech. Inst. and State Univ., Blacksburg, VA 24061)

Active control of sound radiation from plates with arbitrary boundary conditions is studied in this paper. An optimization procedure for the locations of piezoelectric actuators is developed. The objective function in the optimization is chosen as the sound power radiated from the plates. The influence of the changes in the support conditions on the eigenproperties of the plate is evaluated. The numerical results show that the control performance with respect to a boundary condition change is dependent upon the excitation frequency for the case where actuators are optimized for a certain boundary condition. However for the case of simply supported boundary condition, it is observed that a change in the translational stiffness will result in degraded control performance only when the translational stiffness is significantly less than infinite (i.e., simply supported). [Work supported by ONR.]

\section{$4: 15$}

2pSA10. Experiments on active control of wave propagation in fluid-filled elastic cylindrical shells. Bertrand J. Brévart and Chris R. Fuller (Vib. and Acoust. Labs., Dept. of Mech. Eng., Virginia Polytech. Inst. and State Univ., Blacksburg, VA 24061-0238)

Experimental results of studies on active vibration control in fluidfilled piping systems are presented. Reductions of the total power now, i.e., the power flow in the shell wall and the power flow in the fluid field, have been achieved by means of extemal radial forces applied to the structure. Axisymmetric wave propagation was first considered, using PVDF cables both as actuators and sensors. The shell radial displacement beyond the control discontinuity was reduced by 7 to $20 \mathrm{~dB}$ in a large frequency range. In addition, the internal pressure field was globally attenuated by the external forces. Considering a point force disturbance at low frequencies, attenuations of the shell motion associated with higher-order modes ( $n$ $=1,2$ ) have been achieved, by means of shaped PVDF films as error sensors and point forces as structural control inputs. [Work supported by ONR.] 
2pSA11. The application of a biologically inspired controller to control sound transmission. James P. Carneal and Chris R. Fuller (Vib. and Acoust. Labs., Dept. of Mech. Eng., Virginia Polytech. Inst. and State Univ., Blacksburg, VA 24061)

A biologically inspired control approach for reducing sound transmission through a distributed elastic system has been theoretically and experimentally verified for narrow-band excitation. The control paradigm approximates natural biological systems for initiating movement, in that a low number of signals are sent from an advanced, centralized controller (analogous to the brain) and are then distributed by local rules and actions to multiple actuators (analogous to muscle fiber). A local learning rule that was developed from linear quadratic optimal control theory and solved $a$ priori was implemented. The investigation considered a plate excited by normal plane wave, oblique plane wave, and reverberant acoustic fields. Radiated sound power was the defined cost function and therefore used as the controller error signal. Four control inputs in the form of piezoelectric actuators were mounted on the plate in a two-by-two anay. Results indicate that increases in transmission loss of approximately $18 \mathrm{~dB}$ are attainable for off-resonance excitation. In general, comparisons of theoretical and experimental data show good agreement. This investigation has demonstrated that the biological control approach has the potential to control multimodal response in distributed elastic systems using an array of many actuators with a reduced order main controller. Thus significant reductions in control system computational complexity have been realized by this approach. [Work supported by NASA Langley.]

\section{4:45}

2pSA12. Experimental results from hybrid control with a sensoriactuator. Robert L. Clark and Jeffrey S. Vipperman (Dept. of Mech. Eng. and Mater. Sci., Duke Univ., Durham, NC 27708-0300)

Both transient and persistent disturbance rejection were demonstrated experimentally on a cantilevered beam configured with a piezoelectric sensoriactuator. The transient response of the system was suppressed through direct-rate feedback control, and adaptive feedforward control was utilized to minimize the response to a harmonic input disturbance. A timeaveraged gradient descent algorithm was implemented to adapt a finite impulse response filter in the feedforward control approach. Experimental results demonstrate that rate-feedback control can be utilized to enhance the transient adaptation of the feedforward control algorithm. Furthermore, the sensoriactuator provides a convenient method of performing both sensing and actuation simultaneously in feedback and feedforward control of adaptive structures.

\section{5:00}

2pSA13. Determination of effective secondary source locations for active noise control. Jihe Yang (Automated Analysis Corp., $2805 \mathrm{~S}$. Industrial, Ste. 100, Ann Arbor, MI 48104) and David K. Holger (Iowa State University, Ames, IA 50011)

A numerical method for determining effective secondary source locations for active control of interior sound fields has been investigated. The method uses intermediate results from an indirect boundary element simulation of a sound field to determine effective boundary locations for secondary sources. In the indirect boundary element method (IBEM), an interior sound field is simulated by replacing the physical boundaries with a fictitious source distribution that is determined from the geometry, the properties of the physical boundaries, and the primary source location(s). Locations of high fictitious source strength, as determined by the IBEM, are found to be particularly effective locations for secondary sources that are components in three dimensional active noise control systems. Numerical results for simple geometries are in agreement with previous experimental results [Elliott et al., J. Sound Vib. 117, 35-58 (1987)], and numerical predictions of active noise control using the proposed method for locating secondary sources resulted in sound pressure level reductions of more than $20 \mathrm{~dB}$ in reverberant and semi-reverberant spaces. The results obtained suggest that the method has significant potential for efficiently locating effective secondary sources for a variety of active noise control applications.

\title{
Session 2pSP
}

\section{Speech Communication and Engineering Acoustics: Microphone Arrays: Design and Analysis II}

\author{
James L. Flanagan, Cochair \\ CAIP Center, Busch Campus, Rutgers University, Core Building 706, Piscataway, New Jersey 08855-1390 \\ Harvey F. Silverman, Cochair \\ Laboratory for Engineering Man/Machine Systems, School of Engineering, Brown University, Providence, \\ Rhode Island 02912 \\ Qiguang Lin, Cochair \\ CAIP Center, Rutgers University, Core Building, Frelinghuysen Road, Piscataway, New Jersey 08855-1390
}

\section{Contributed Papers}

\section{1:30}

2pSP1. Microphone arrays for reducing reverberation and noise in speech communication. James G. Ryan (Inst. for Microstructural Sci., Natl. Res. Council, Ottawa, ON K1A 0R6, Canada)

Microphone pickup of sound in typical rooms is impaired by the combined effects of reverberation and noise. This degrades speech intelligibility and quality particularly in applications where the microphone is located far away from the talker. Recent advances in microphone array technology suggest a potential solution to such problems. This paper gives an overview of current microphone array techniques and discusses the potential benefits for speech communication. Various criteria for measuring the performance of a microphone array are described. A fexible, experimental microphone array intended for research in speech communications is under construction and will be described.

1:45

2pSP2. Stable dereverberation using microphone arrays for speaker verification. A. C. Surendran and J. L. Flanagan (Ctr. for Comput. Aids for Indust. Productivity, P.O. Box 1390, Piscataway, NJ 08855-1390)

The impulse response of a reverberant environment, in general, is a nonminimum phase and cannot be inverted. But an exact inverse of the 
environment can be obtained by modeling the room as a multiple inputoutput (MINT) system [M. Miyoshi and Y. Kaneda ICASSP (1986)]. In this report, this model is applied to a microphone array and is used as a front-end processor for a speaker verification system. The $G$ matrix is inverted using row action projection (RAP), an iterative approach to solving a system of linear equations. Starting from an initial guess, the solution is repeatedly projected onto each hyperplane of the equation system until it converges. The method is stable, robust to noise, and converges to the pseudo-inverse solution. In computer-simulated experiments, the signal-toreverberant-noise ratio is found to improve with the number of microphones in the array. A speaker verification system using the array is evaluated at various signal-to-competing-noise ratios (SCNR). Results suggest that verification performance can be substantially elevated in adverse acoustic environments.

\section{2:00}

2pSP3. Binaural arrays for hearing enhancement. Michael V. Scanlon and Stephen M. Tenney (Army Res. Lab., AMSRL-SS-SH, 2800 Powder Mill Rd., Adelphi, MD 20783-1197)

Two hearing augmentation devices developed at the Army Research Laboratory can enhance normal listening abilities and restore hearing degraded by encapsulating headgear. Surrounding sounds are localized with a head-mounted binaural pinna attachment that recreates the head-related transfer function associated with the normal listening. The user's brain interprets the recreated stereo signals that enter the ear canals through intra-aural speakers, giving excellent restoration of omnidirectional hearing. A hand-held, ultra-directional array extends the user's listening range. The use of delay and sum beamforming in the array assures maximum directivity in the pointing direction. The binaural long-range hearing device has two linear endfire arrays of eight cardioid microphones each. The slightly offset directivity patterns of the two arrays create stereo outputs, so that the user can interpret differences in amplitude, phase, time-of-arrival, and frequency content of sounds in the forward area. These devices provide aural protection and an intra-aural input for communications, without removing the user from his acoustic environment. Both devices can be monitored remotely, and are ideally suited for detecting speech, personnel, equipment, or vehicles during military or law enforcement missions. Performance measurements of various array configurations will be shown.

\section{2:15}

2pSP4. A multisensor connectivist model for the preprocessing of the speech signals. Turker Kuyel and Elmer L. Hixson (Dept. of Elec. Eng., Univ. of Texas at Austin, Austin, TX 78712)

Due to the inherent redundancy of the speech data, the design of a redundancy reducing speech preprocessor is very important. Preprocessor design is also very important because it can greatly reduce the computational load on the later stages of speech processing. A special laboratory oriented method in speech data acquisition, which is called near-field spectral wave number estimation is implemented. In this method multiple microphones are used. The goal is to incorporate air flow velocity into speech feature vector. This extra feature is used in addition to the short time cepstrum of the sound data to make the final speech vectors. The speech vectors are then quantized into a determined number of categories using a self-organizing neural network. These quantized and extended vectors are then used for the modeling of higher speech constructs such as phonemes and words. The preprocessing scheme reduced the computational complexity considerably at the expense of slight reduction of the recognition accuracy.

\section{2:30}

2pSP5. Position-tolerant differential microphones for noisy environments. James E. West, G. W. Elko, D. R. Morgan, and R. A. Kubli (Acoust. Res. Dept., AT\&T Bell Labs., 600 Mountain Ave., Murray Hill, NJ 07974)

Directional microphones are best noted for their noise reduction properties in communication systems. Close-talking differential microphones are particularly useful when the noise environment disturbs the ability to communicate without error, such as in public and cellular telephony, aircraft communications, etc. These differential microphones work best when they are placed within $1 \mathrm{~cm}$ from the lips of the talker where the sound field has a large gradient. For a plane-wave sound field the sensitivity rises proportional to $\omega^{n}$, where $n$ is the order of the difference. Users of differential microphones do not always correctly position the sensor at the proper distance from the mouth and therefore the sensitivity of the microphone may also rise proportional to $\omega^{n}$ especially at high frequencies. A method is described of correcting for this high-frequency gain without significantly degrading the noise canceling properties of first- and secondorder differential microphones.

\section{2:45}

2pSP6. A new adaptive differential microphone array. Gary W. Elko and Anh-Tho Nguyen Pong (Acoust. Res. Dept., AT\&T Bell Labs., 600 Mountain Ave., Murray Hill, NJ 07974)

An adaptive differential microphone has been implemented by combining two omnidirectional elements to form back-to-back cardioid directional microphones. By combining the weighted subtraction of these two outputs, any first-order array can be realized. If certain simple constraints are placed on the combination weighting, the null location can be constrained to defined angular regions. Three algorithms that control the constrained adaptation are presented and discussed for the array: the LMS algorithm, Newton's algorithm, and a time-varying least-squares Wiener filter. A real-time implementation utilizing an AT\&T DSP32C digital signal processor is also described.

\section{3:00-3:15 Break}

\section{3:15}

2pSP7. An adaptive subband differential microphone. Juergen Cezanne and Anh-Tho Nguyen Pong (Acoust. Res. Dept., AT\&T Bell Labs., 600 Mountain Ave., Murray Hill, NJ 07974)

In a previous talk, "A new adaptive differential microphone array" by Elko and Pong, a differential microphone has been introduced that adapts its directivity pattern to the particular acoustic environment to provide for a good signal-to-noise ratio. There, the selected pattern remains more or less constant with respect to frequency. In this talk an approach is described that contains one more degree of freedom. The spectrum of the signals is partitioned in uniform subbands and different directivity patterns are adaptively chosen in each subband. This allows to cancel multiple noise sources with nonoverlapping spectra. An LMS-based algorithm will be derived with focus on a low computational load and a short delay for the desired signal. Consequences on the speed of adaptation are discussed. Further, experimental results of a first implementation with 33 subbands on a PC-based DSP32C board will be presented. The measurements verify the ability of the algorithm to cancel multiple noise sources with disjoint spectra without distorting the desired signal.

\section{3:30}

2pSP8. Adaptive enhancement of microphone array signals. Carsten Sydow (Inst. for Electroacoust., Tech. Univ. of Darmstadt, Merckstr. 25, D-64283 Darmstadt, Germany)

The signal-to-noise ratio of a speech signal picked up by a microphone array can be improved by adaptive post processing. Enhancement techniques known from single microphone or dual microphone signal processing, like noise canceling and spectral subtraction can be extended to a multimicrophone array system. The noise canceling technique and derived structures try to model the room impulse response by an adaptive transversal filter. Thus the performance of these algorithms is limited by the ratio of filter length to reverberation time and by the capability to track the nonstationary impulse response. Reduction of the noise of approximately 8 $\mathrm{dB}$ can be achieved with acceptable filter length in a stationary environment, but precautions must be taken to avoid canceling of the desired speech signal. The spectral subtraction met' sd yields higher improvements 
in signal-to-noise ratio up to $12 \mathrm{~dB}$, but due to false estimation of magnitude short time spectra the processed speech signal contains distortion known as "musical tones." Different structures for adaptive post processing of microphone array signals based on both enhancement techniques are presented and discussed. Their performance in stationary and nonstationary environments is characterized by means of improvement of the signalto-noise ratio and subjective speech quality.

\section{$3: 45$}

2pSP9. Crosscoupling effects on large wideband arrays. Richard $\mathrm{K}$. Johnson (Siemens Med. Systems, 22010 SE 51 St., Issaquah, WA 98027)

The effects of crosscoupling on array performance can be well described by spatiotemporal or spatiofrequency correlation matrices. The data acquisition and analysis required for this approach, however, are very demanding tasks for large wideband arrays. The most important effects of crosscoupling within an array are latent in single element beam patterns which are relatively simple to measure. This paper describes the use of these single element beam patterns to predict array performance and to establish crosscoupling requirements.

\section{4:00}

2pSP10. Minimum error sound source localization. D. Rainton (ATR Human Information Processing Res. Labs., 2.2 Hikaridai, Seika-cho, Soraku-gun, Kyoto 619-02, Japan)

A novel approach to the problem of computing the direction of arrival (DOA) of a sound source using a two-element microphone array is presented. Typically, the DOA is computed by peak picking from the resulting cross-correlation function. In order to improve such estimates it is usually desirable to pre-filter the signals prior to cross correlation. However, proper selection of these pre-filters is often problematic. The proposed algorithm adapts the filters during an initial training session to directly minimize the number of location estimation errors. The only information provided is the signal DOA, no explicit knowledge of the signal or noise spectra are required. It is assumed however that the overall signal/noise statistics are long term stationary over the training and subsequent testing. Examples of both linear and nonlinear filter design are presented for a talker location identification task.

\section{$4: 15$}

2pSP11. A model for the holographic reconstruction of sound fields disturbed with temperature gradients. Martín Gutiérrez V. (Inst. of Acoust., Univ. Austral de Chile, Casilla 1130, Valdivia, Chile) and Jorge P. Arenas B. (Inst. of Acoust., Univ. Austral de Chile, Valdivia, Chile)

Holographic techniques are used to detect noise sources in a wide range of devices. Some of these techniques are commonly employed in the reconstruction of sound fields produced by automobile or motorcycle engines. These sound fields are characterized by having several, rather than single, temperalures; in fact, temperature gradients are frequently found in regions near the engine. These gradients produce changes in every point of the sound wave velocity. The mathematical model presented in this paper was designed to minimize the effect of assuming a constant sound velocity in the entire space in reconstruction of the sound field. It is based on the space transformation of sound field (STSF) technique [Hald, Jorgen, B\& $\mathbf{K}$ Tech. Rev. 1, 1-50 (1989)], which uses a microphone array to measure pressure or determine the principal component of cross spectra over a hologram plane. The model scans the sound field in two planes. The first plane is used as an STSF initial condition (hologram plane), and the second plane is considered the boundary condition to Rayleigh integral's propagation.

\title{
Session 2pUW
}

\section{Underwater Acoustics: Moderate-to-High Frequency Bottom Interacting Acoustics II}

\author{
Kevin L. Williams, Chair \\ Applied Physics Laboratory, University of Washington, 1013 NE 40th Street, Seattle, Washington 98105-6698
}

Chair's Introduction-1:00

Invited Papers

1:05

2pUW1. Propagation in range-dependent poro-elastic media. Michael D. Collins (Naval Res. Lab., Washington, DC 20375), W. A. Kuperman (Scripps Inst. of Oceanogr., La Jolla, CA 92093), and William L. Siegmann (Rensselaer Polytech. Inst., Troy, NY 12180)

Biot's theory of poro-elasticity is derived for heterogeneous media and reduced to a system of three coupled equations. Previous formulations of this problem include a redundant fourth equation. The reduced system factors into incoming and outgoing wave equations and may therefore be solved with the parabolic equation (PE) method, which is useful for range-dependent problems. The operator square root is approximated using rational-linear functions that were originally designed for the elastic PE and provide accuracy and stability. An initial condition for the poro-elastic $\mathrm{PE}$ is obtained with the self-starter, which has been generalized to handle compressional and shear sources in poro-elastic media. Qualitative tests involving the propagation and reflection of slow and fast compressional wave beams and shear wave beams demonstrate that the poro-elastic PE handles all wave types. A solution based on the wave-number spectrum has been developed to test the poro-elastic PE quantitatively. The PE and spectral solutions are nearly identical for problems involving a water column overlying a poro-elastic sediment. A nonlinear relationship involving the coefficients of the wave equation and the Biot moduli has been worked out so that the natural parameters (i.e., porosity, density, wave speeds, and attenuations) may be used as inputs to propagation models. 
2pUW2. A new high-frequency ocean bottom backscattering model. Nicholas P. Chotiros and Frank A. Boyle (Appl. Res. Labs., Univ. of Texas at Austin, P.O. Box 8029, Austin, TX 78713-8029)

A new model of acoustic backscatter from the ocean bottom is presented. Based on the analysis of existing data, three main physical mechanisms have been identified. These are gas bubbles, sediment grains, and interface roughness. Gas bubbles are particularly prevalent in shallow water sediments. A single-scatter approximation is used to model the bubble scattering. Scattering from sediment grains is a multiple-scatter problem which is still under investigation, but an empirical relationship is available. Scattering from interface roughness is modeled in terms of a Kirchhoff-Helmholtz scattering integral. The total backscattering strength is an incoherent sum of the three contributions. It is found that the interface roughness is dominant near normal incidence. The other two mechanisms tend to dominant at shallow grazing angles. [Work supported by Naval Research Laboratory, Stennis Space Center under the MCM Tactical Environmental Data System (MTEDS) project.]

\section{1:45}

2pUW3. High-frequency acoustic bottom reverberation. S. Stanic, E. Kennedy (Naval Res. Lab. Code 7174, Stennis Space Center, MS 39529), and R. Goodman (Appl. Res. Lab., Penn State Univ., State College, PA 16804)

A series of high-frequency bottom backscattering experiments were conducted off the coast of Panama City, Florida. Reverberation results will be presented as a function of frequency $(20$ to $180 \mathrm{kHz})$ and grazing angle $\left(5^{\circ}-30^{\circ}\right)$. Geoacoustic parameters taken during the experiments will be used as inputs to an existing seafloor backscattering model initially developed by D. R. Jackson et al. [J. Acoust. Soc. Am. 79, 1410-1422 (1986)], and modified by A. Lyons et al. [J. Acoust. Soc. Am. 95, 2441-2451 (1994)]. The experimental data will then be compared to these model simulations.

2:05

2pUW4. Modeling the sonar backscatter by objects buried in very shallow water. Raymond Lim (Code 130B, Coastal Systems Station, Panama City, FL 32407-7001)

Conventional sonar systems for bottom searching underwater coastal environments have limited long-range classification capabilities. This is especially true when objects searched for are significantly buried so that imaging is difficult. To investigate the feasibility of nonimaging methods of classification (e.g., based on isolating resonances in the echo from the desired object), models that faithfully predict the acoustic response of known objects in various states of burial are required. Because the response of the object can be strongly modified by the structure of its local environment, an appropriate model must account for effects due to propagating the acoustic field to and from the object, the scattering itself, and the interaction of the object with its local environment. The present talk will discuss how these issues can be handled in an exact fashion via a transition-matrix formulation both for fully buried and partially buried objects. In either case, the solution involves identifying a suitable set of global basis functions. [Work supported by ONR and the CSS IR program.]

\section{Contributed Papers}

2:25

2pUW5. Acoustic form function for porous solid spheres: Comparison between theory and experiments. Kerry $W$. Commander, Raymond Lim (Coastal Systems Station, Panama City, FL 32407-7001), Theodore W. L. Huskey, Steven R. Baker (Naval Postgraduate School, Monterey, CA 93943-5000), and Steven G. Kargl (Univ. of Washington, Seattle, WA 98105)

An underwater acoustic scattering experiment was performed on three porous solid spheres of varying grain size to determine their acoustic form functions. The spheres were constructed from bonded glass beads, sized to yield permeabilities in the range of fine to medium grained sand. Measurements of required material and lattice parameters were determined on analogous cylindrical samples, including a measurement of the dry lattice moduli per the method described by Garrett [S. L. Garrett, J. Acoust. Soc. Am. 88, 210-221 (1990)]. Quantitative agreement between the acoustic measurements and predictions from a theoretical Biot model [S. G Kargl and R. Lim, J. Acoust. Soc. Am. 94, 1527-1550 (1993)] was found but only at the low-frequency end $(<20 \mathrm{kHz})$ of the measurements and for the smaller grained spheres. Although the measured and predicted form functions were still qualitatively similar at higher frequencies, deviations due to inhomogeneities in the porous spheres became evident. For each discrete frequency measurement, the spheres were rotated $360 \mathrm{deg}$ and variations in the backscattering strength noted. For values of $k a$ where little variation with sphere orientation was obtained, there was good agreement with the theoretical model.
2:40

2pUW6. Moderate frequency scattering from objects in an elastic bottom with rough interfaces. Jaiyong Lee and Henrik Schmidt (Dept. of Ocean Eng., MIT, Cambridge, MA 02139)

The diffuse reverberation from small scale bottom roughness severely affects the spatial coherence of the scattered field from near-bottom buried objects. To investigate this phenomenon theoretically, a numerical model is being developed for moderate frequency scattering from an elastic inclusion in a stratified, rough bottom. The model combines theories for object scattering and small-scale rough interface scattering. For the numerical implementation, a global approach is introduced, which combines three different methods into a consistent hybrid scheme. Boundary elements are used to compute the effects due to an elastic inclusion [P. Gerstoft and $\mathrm{H}$. Schmidt, J. Acoust. Soc. Am. 89, 1629-1642 (1991)], wave-number integration provides numerical Green's function for the horizontally stratified medium, and finally the rough interface scattering is handled using a selfconsistent perturbational method [W. A. Kuperman and H. Schmidt, J. Acoust. Soc. Am. 86, 1511-1522 (1989)]. Compared to earlier implementations, the efficiency of the boundary element component of the model has been significantly improved by introducing a new analytical integration approach to the computation of the influence matrices. The modeling approach will be described and its application to realistic sonar scenarios will be demonstrated. [Work supported by ONR.] 
2pUW7. Moderate frequency acoustic penetration of a sandy shallow water sediment. Nicholas P. Chotiros (Appl. Res. Labs., University of Texas at Austin, P.O. Box 8029, Austin, TX 78713-8029), Äge Kristensen, and Enzo Michelozzi (SACLANT Undersea Res. Ctr., 19026 LaSpezia, ltaly)

An experiment was conducted to investigate acoustic penetration of a sandy shallow water sediment, in the band $500 \mathrm{~Hz}$ to $2 \mathrm{kHz}$, in a site off LaSpezia, Italy. Acoustic sensors were buried in the sediment, forming a sparse three-dimensional array, to measure the sediment penetrating signals. The sound source was a sparker. The collected signals are processed coherently to give direction and speed of the sediment acoustic waves. From a theoretical point of view, the medium is treated as a poro-elastic solid governed by Biot's theory of acoustic propagation. It is predicted to support two acoustic waves. Comparisons are made between theory and experiment. [Work supported by Office of Naval Research, Ocean Acoustics Program, Code 11250A, under the initiative for basic research in the physics of moderate to high frequency acoustics.]

\section{3:10-3:30 Break}

\section{3:30}

2pUW8. High-frequency acoustic penetration of ocean sediments. Nicholas P. Chotiros, Robert A. Altenburg (Appl. Res. Labs., University of Texas at Austin, P.O. Box 8029, Austin, TX 78713-8029), and Stephen J. Stanic (Naval Res. Lab., Stennis Space Center, MS 39529-5004)

Two experiments were conducted to investigate acoustic penetration of ocean sediments: one in a muddy site in the Baltic Sea and the other in a sandy site in the Gulf of Mexico. In both experiments, acoustic sensors were placed in the sediment to measure the acoustic signals. The signals are processed coherently to give direction and speed of the acoustic waves. The data from the two sites give very different results. In the muddy site, the sediment sound speed is very close to that of the water, the situation in the sandy site is more complicated. The medium is treated as a poro-elastic solid governed by Biot's theory of acoustic propagation. For the sandy site, the theory predicts two acoustic waves, one faster than the sound speed in water, and the other slower. Comparisons are made between theory and experiment. [Work supported by Office of Naval Research, under the Coastal Benthic Boundary Layer (CBBL) Special Research Program.]

\section{3:45}

2pUW9. The effect of roughness on acoustic penetration of the ocean bottom. John E. Moe and Darrell R. Jackson (Appl. Phys. Lab., Univ. of Washington, 1013 NE 40th St., Seattle, WA 98195)

The acoustic intensity penetrating a rough surface is analyzed using Rayleigh-Rice perturbation theory. When the grazing angle of the incident field is below the critical angle in relation to the mean surface, only the zero-order component of the transmitted field is evanescent; the higherorder components contain downward traveling waves. For an incident field below the critical angle, first-order computations using parameters appropriate to a sandy bottom show that the field below the rough surface can be much greater than the corresponding field below a flat surface. These computations are carried out using a low-frequency cutoff for the bottom relief spectrum. With regard to the accuracy of the calculation, both the short-wavelength portion of the relief profile that is retained as well as the long-wavelength portion that is discarded are considered. It is shown that the rms height of the portion retained and the rms slope of the portion discarded are sufficiently small to lend confidence in the perturbation approach. Further work is required, however, to unequivocally establish the accuracy of the method. [Work supported by ONR.]
2pUW10. On measuring sediment/Biot properties in shallow water at moderate to high frequencies. Ahmed Abawi, W. S. Hodgkiss, W. A. Kuperman (Scripps Inst. of Oceanogr., Univ. of Califomia San Diego, La Jolla, CA 92093-0701), and Michael D. Collins (Naval Res. Lab., Washington, DC 20375)

A broadband line array is being designed and constructed to study the acoustics of shallow water in the moderate to high-frequency regime. The 64-element array will consist of nested apertures half-wavelength spaced at 4,8 , and $16 \mathrm{kHz}$ with a total bandwidth of $2-20 \mathrm{kHz}$. The intention is to study both the forward and inverse problem, the latter with respect to sediment properties. The poroelastic parabolic equation [Collins et al., this meeting] is being used for simulations to (1) aid in the design of the array, (2) study the forward problem, and (3) investigate the potential observables with respect to the inverse problem. In particular, it is of great interest as to whether effects related to the Biot description are observable by measuring waveguide propagation. The use of the parabolic equation formulation will allow for a consistent extension to range-dependent environments in a future study. [Work supported by ONR Code 321OA.]

\section{4:15}

2pUW11. Hybrid melhods for incorporating density variations into the split-step PE. David Yevick (Dept. of Elec. Eng., Queen's Univ., Kingston, Ontario K7L 3N6, Canada) and David J. Thomson (Defence Res. Estab. Pacific, FMO Victoria, BC V0S 1B0, Canada)

Density variations are easily analyzed in the context of finite difference parabolic equation (PE) solvers by discretization of an appropriate differential operator. In split-step Fourier solution algorithms, however, variations in the density $\rho$ are instead modeled by adding terms to the refractive index. Since these extra terms depend on derivatives of $\rho$, geoacoustic density profiles must be smoothed appropriately to remove any step discontinuities. In this paper, a new hybrid method is proposed for treating density inhornogeneities in the split-step PE. This approach involves splitting the differential operator into density-independent and densitydependent components. While the former component is propagated using the split-step Fourier technique, the influence of density changes is computed through a finite difference procedure. Such an algorithm is especially attractive as it may be transparently incorporated into the recently proposed hybrid split-step/finite-difference and split-step/Lanczos solvers [J. Acoust. Soc. Am. 96, 396-405 (1994)]. Both the hybrid and the standard finite-difference procedures are applied to a shallow-water test case involving jump discontinuilies in both the sound speed and the density and are found to be in excellent agreement with reference solutions.

\section{4:30}

2pUW12. Finite difference simulations of propagation in a shallow water environment. John B. Schneider, Shira Lynn Broschat, and Patrick J. Flynn (School of Elec. Eng. and Comput. Sci., Washington State Univ., Pullman, WA 99164-2752)

The propagation of acoustic energy in a shallow water environment is studied using a two-dimensional, staggered-grid finite-difference model. Such a model allows one to view the propagation of energy in the time domain throughout the region of interest. Since fields are available "everywhere," the model can be used to guide the development of robust schemes for the detection of scatterers in the water column or buried in the sediment. In addition, it can be used to benchmark approximate models for acoustic propagation and scattering in shallow water. This paper considers the effect of different physical features on the possible identification of scatterers. Such scattering mechanisms as top and bottom roughness and inhomogeneities in the water column are considered. Monte Carlo simulations are used to gauge the relative effects of these features. Frequencies on the order of $7.5 \mathrm{kHz}$ are considered for depths up to $30 \mathrm{~m}$. The implementation used here includes several enhancements over traditional finitedifference models. For example, typical finite difference models approximate continuously varying material interfaces by an interface that appears like a staircase - the material properties change only at discrete location. For this research a conformal technique is used to model more accurately the continuous interface. [Work supported by ONR.] 
2pUW13. Optimum beam widths for seafloor scattering problems. R. A. Stephen (Woods Hole Oceanogr. Inst., Woods Hole, MA 02543)

Gaussian beams are often used for the insonifying field in scattering problems from interfaces. They have the advantage of restricting the grazing angle content of the incident field while localizing the scattering region on the interface. However a disadvantage is that the beams spread significantly. For oblique incidence, the width of the beam can vary significantly over the region of interaction on the interface and the scattering problem work has been carried out for the propagation of Gaussian beams in homogeneous and heterogeneous media [for example, Cerveny et al., Geophys. J. R. Astron. Soc. 70, 109-128 (1982)]. For a given propagation distance there is an optimal initial beamwidth that will minimize the width of the beam along the whole path. Consequently for a given angle of incidence and a given incident amplitude threshold, there will be a minimum surface scattering area. Optimum beamwidths can be defined and these should be used by all investigators in order to standardize results. [Work supported by Office of Naval Research.]

\title{
Session 3aAA
}

\section{Architectural Acoustics: Case Studies in Architectural Acoustics}

\author{
J. Christopher Jaffe, Chair \\ Jaffe Acoustics, Inc., 114A Washington Street, Norwalk, Connecticut 06854
}

\author{
Chair's Introduction $\longrightarrow \mathbf{8 : 0 0}$
}

Invited Papers

\section{$8: 05$}

3aAA1. Report on the Cerritos Centre for the Performing Arts. Jerry Godden (Theatre Projects Consultants, 3 Apollo Studios, Charlton Kings Rd., London NW5 2W, U.K.)

Small and medium sized communities wanting a high quality performing arts space are faced with reconciling the conflicting demands that different arts forms make on the performance space. The recently completed Cerritos Centre for the Performing Arts in California is unusual in its design in that it utilizes modern technology to provide a space for the performing arts that can become a 1896-seat concert hall, a 1450-seat lyric theatre, a 900-seat drama theatre, a 1934-arena theatre, or a cabaret space seating 1472. The auditorium is transformed including its seating, its sightlines, and its acoustics by the use of movable seating towers. The design demonstrates how a small town can afford to build and operate a facility in scale with its capabilities and aspirations and may well prove to be a key point in the concept of multi purpose spaces of the future.

\section{8:35}

3aAA2. Problems associated with the renovation of existing music practice facilities. David P. Walsh (Walsh, Norris $\&$ Associates, Inc., 25 Drumm St., San Francisco, CA 94111)

The acoustical design of music practice and rehearsal facilities is always an interesting and challenging task. However, the challenges increase significantly when the project is a renovation of existing facilities rather than a new design. In renovation projects, extensive investigation and evaluation of the building structure and construction assemblies are required since, in a great number of cases, the original drawings are no longer available. It is also not unusual for undocumented modifications to have been completed to the structure over the years. A working knowledge of outdated construction techniques and materials is typically required. This paper presents a case study of the renovation of the practice and rehearsal facilities for a university music department originally constructed in 1956. The original wall and ceiling systems did not provide satisfactory airborne sound isolation between practice rooms and major modifications were begun less than a year after opening. These modifications improved the overall degree of sound isolation between practice rooms somewhat, however, they were not completely satisfactory. This paper discusses the current modifications, together with the complications inherent in working on a building over 30 -years-old for which minimal information on the original construction assemblies was available.

$$
\text { 9:05 }
$$

3aAA3. Sound isolation criteria for Cornell University Music Department Timothy J. Foulkes and William J. Cavanaugh (Cavanaugh Tocci Associates, Inc., 327F Boston Post Rd., Sudbury, MA 01776)

A detailed case study in sound isolation criteria for a new music education facility at a major university is presented. Part of the department is to be housed in an existing, historic building with wood floor structures. Part of the new facility will be an addition (new construction).

9:35-9:50 Break 
3aAA4. Acoustic project of "Mexico Radio ABC" broadcasting rooms. Ana Maria Valdés (Av. Popocatepetl 295-7, México 03340 D.F., Mexico) and Mario Vázquez-Raña (Organización Editorial Mexicana, Guillermo Prieto 7, Col. San Rafael, México 06470 D.F., Mexico)

Design for room acoustic insulation, reverberation time, and homogeneous diffusion of acoustic field, results in high building budgets for broadcasting and TV stations, theaters, etc., projected in Mexico. For this reason, they are often constructed without an adequate acoustic design. This paper presents the acoustic design applied to the construction of three broadcasting studios for "Mexico Radio ABC," considering both a low cost project and long life materials fitted for the Mexico City's environment, and taking into account a new national draft standard. The project was planned for noisy surroundings (sound pressure levels averaged of 78 $\mathrm{dB}$, A-weighted). External noise comes mainly from traffic, nearby schools and helicopters; indoor noise comes from air conditioned and background office noise. The original volume shape was a parallelepiped, which had to be transformed to avoid undesirable reflections. The reverberation time chosen for control rooms was $0.35 \mathrm{~s}$ at $1000 \mathrm{~Hz}$, and $0.22,0.25$, and 0.35 $\mathrm{s}$ at $1000 \mathrm{~Hz}$ for broadcasting studios; these values were achieved in practice. Final costs were low, in comparison with a construction without previous acoustic design.

\section{0:05}

3aAA5. Sound isolation of an audiometric test room. Arno $S$. Bommer and Charles T. Moritz (Collaboration in Sci. and Technol., Inc., 15835 Park Ten Place, Ste. 105, Houston, TX 77084-5131)

Modifications were required to reduce sound levels in a former office so that the room would meet the ambient noise requirements for audiometric testing. Sound sources in the area included people in neighboring offices, transformers in a neighboring mechanical equipment room, ventilation ducts in the plenum above the room, and air-conditioning vents. The transformers created harmonically related vibration tones in the concrete floor slab and walls that radiated sound into the proposed audiometric test room. Acoustical recommendations consisted primarily of building a vibration-isolated room within the existing room (including a floating floor) and acoustically treating the ventilation system. Initial measurements revealed unexpectedly high sound levels that were the result of unintended (erroneous) structural connections between the inner and outer room. When these connections were cut, the room met the noise requirements.

\section{0:20}

3aAA6. Seat-dip phenomenon. David Greenberg (Jaffe Holden Scarbrough Acoustics, Inc., 114A Washington St., Norwalk, CT 06854) and Courtney Burroughs (Appl. Res. Lab., State College, PA 16804)

The "seat-dip phenomenon" is so called because an anomalous attenuation (dip) in the frequency response of auditoriums is found to be caused by the presence of rows of seats. As sound from the stage grazes over seats, it is subject to broad attenuation of the low frequencies from 75 to $300 \mathrm{~Hz}$. This frequency dip can be as much as $20 \mathrm{~dB}$ below the sound level without the presence of seats. Experiments were performed with a simple 1:10 scale model of an auditorium floor. It is found that the dip is caused by a coincidence of effects rather than the single effect dominant in the literature. The effects are considered. (1) A standing wave between the seat and source with nulls relating to the quarter-wavelength distance, (2) half-wave cancellations where reflected and/or diffracted waves interfere with the on-coming wave, and (3) the propagation of the sound of interest at nearly zero degrees over the seats. It is shown that the standing waves and sound pressure cancellations associated with each successive row of seats occur at frequencies that are very close to each other. The effect of frequency spreading of (1) and (2) are presented.
3aAA7. BACH, a new binaural room acoustical measure. António Pedro O. Carvalho (Architecture Technol. Res. Ctr., $331 \mathrm{ARCH}$, Univ. of Florida, Gainesville, FL 32611-2004)

This study reports on acoustical field measurements made in a major survey of 41 Catholic churches in Portugal built in the last fourteen centuries. Binaural measurements were taken in each church using a dualchannel real-time frequency analyzer to calculate the coherence values between the signals at both ears in 1/3-octave frequency bands. From the coherence values a new binaural acoustical measure was developed and called BACH (Binaural Acoustical CoHerence). Monaural acoustical measurements were also taken at several source/receiver locations using the impulse response method. $B A C H$ was shown to be an orthogonal parameter in statistical tests with nine other monaural acoustical measures (RT, EDT, C80, D, TS, L, BR_RT, BR_L, and RASTI) and fifteen architectural parameters. Information was collected regarding the quality of music in each church. ANOVA tests were performed to examine the significance of the differences among the groups of equal subjective quality ratings. The author wanted to test the hypothesis that this new binaural acoustical measure can be useful in estimating the general subjective quality of churches regarding music. A linear correlation coefficient near 0.7 was found between the $B A C H$ and the subjective quality ratings that supported the stated hypothesis. This is exploratory research and developments are being tested to improve the model. [Work supported by JNICT/Ministry of Planning and Univ. of Porto, Portugal.]

\section{0:50}

3aAA8. Field impact insulation class (FIIC)-A case study. John J. LoVerde and Gary Mange (Western Electro-Acoust. Lab., 1711 Sixteenth St., Santa Monica, CA 90404)

Impact noise in buildings constitutes a potentially serious problem because of the short duration, high intensity sounds involved [U.S. Department of Housing and Urban Development, Airborne, Impact, and Structure Borne Noise, Chapter 7 (1967)]. Since 1974, the State of California has tried to manage this problem by instituting the California Noise Insulation Standards, which require any multi-family dwelling to provide an impact insulation class (IIC) rating of 50 based on laboratory tests, or a field impact insulation class (FIIC) rating of 45 based on field tests [Office of Noise Control, California Noise Insulation Standards, 1-7 (1988)]. Concem about the acceptability of floor ceiling assemblies is increasing due to increased awareness of the problem and larger numbers of people moving into apartments, condominiums and townhomes throughout California. Westem Electro-Acoustic Laboratory (WEAL) had the opportunity to witness the installation of floor ceiling assemblies in an apartment complex in Bakersfield, California. Six different assemblies were tested to determine how the FIIC value changed when minor modifications were made to the standard floor ceiling assembly. WEAL will show the results of the field-tested assemblies and compare the data with typical laboratory results for similar constructions.

\section{1:05}

3aAA9. Effect of concave sound reflecting surfaces on speech intelligibility and the articulation index. Sami A. Khaiyat (Dept. of Architecture, Texas A\&M Univ., P.O. Box 2844, Bryan, TX 77805-2844) and Lester L. Boyer (Texas A\&M Univ., College Station, TX 77843)

Two different methods, the calculation of the articulation index (AI) and the rapid speech transmission index (RASTI) measurement using the speech transmission meter, were utilized to obtain the speech intelligibility in spaces with concave sound reflecting surfaces. Many factors were considered such as room size, size of curvature, position of the sound source, and background noise level. The base cases, spaces without curvatures, showed highly correlated results indicating no significant differences between calculated AI and measured RASTI. With curvatures and the sound 
source at $4 \mathrm{ft}$. away from the center position of the front wall it was found that the effect of room size is significantly different between the two methods under the 2- and 16-ft. radius curvatures only; also, the background noise level showed almost the same effect on both calculated AI and RASTI. Under low background noise levels, spaces with curvatures showed that the effect of change in room size for both methods is identical. The center location for the sound source showed better speech intelligibility under all different testing conditions. Finally, a modification factor is developed and applied to the calculated AI so that reliable estimates of speech intelligibility in spaces with curvatures may be obtained.

\section{1:20}

3aAA10. Relations between the apparent source width (ASW) of the sound field in a concert hall and its sound pressure level at low frequencies (GL), and its inter-aural cross correlation coefficient (IACC). Toshiyuki Okano, Takayuki Hidaka (Takenaka Res. and Develop. Inst., 1-5, Ohtsuka, Inzai-machi, Inba-gun, Chiba, Japan, 270-13), and Leo L. Beranek (975 Memorial Dr., Ste. 804, Cambridge, MA 02138)

The influence of GL (amplifier gain the low-frequency range below $355 \mathrm{~Hz}$ ) and IACC on ASW was determined by psychoacoustic experi- ments with simulated concert-hall sound fields using anechoic symphonic music presented to subjects by multiple loudspeakers. "Equal ASW curves" were determined for $1 / 1$ octave band filtered source signals with mid-frequencies from 125 to $4000 \mathrm{~Hz}$. The ASW's for the upper four bands are found to be equal for the same IACC and SPL band values, indicating equal importance of those bands in determining overall ASW's. Combinations of GL's and LACCE3's (average of LACC's in the 500, 1 and $2 \mathrm{kHz}$ bands) for wide-band musical source signals were determined that produced the same ASW's. The early sound was comprised of 2 to 11 early "reflections" and judgments were made with and without later reverberation. It was found that both larger values of GL and smaller values of IACCE3 result in larger values of the subjectively determined ASW's. It is shown that GL and LACCE3 jointly are physical measures of spatial impression in a concert hall and that, combined, they cover the frequency range from low to high frequencies.

$$
\text { 11:35-12:05 }
$$

PANEL DISCUSSION: Weighing Cost Versus Benefit in Music Education and Performance Facilities

Panel Moderator: J. Christopher Jaffe

Panel Members: Timothy J. Foulkes, J. Godden, David P. Walsh

\title{
Session 3aAO
}

\section{Acoustical Oceanography and Animal Bioacoustics: Effects of Sounds on Marine Mammals: Update and Discussion on the Need for Standards II}

\author{
Charles R. Greene, Jr., Chair \\ Greeneridge Sciences, Inc., 4512 Via Huerto, Santa Barbara, California 93110
}

Chair's Introduction- 8:00

Invited Papers

8:05

3aA01. Modulation detection interference on presentation of two amplitude-modulated tones. William F. Dolphin (Dept. of Biomed. Eng., Boston Univ., Boston, MA 02215)

Psychophysical studies have shown that the processing of modulation in one frequency channel is interfered with when a similar modulation pattern is present in another frequency channel. To test for physiological correlates to this phenomena a sinusoidally amplitude-modulated (SAM) probe tone was presented in the presence of a simultaneously gated, modulated, or unmodulated interfering tone while recording from the scalp of anesthetized gerbils. Responses were recorded from the scalp, amplified, digitized, and averaged. The amplitude of the response was determined as the magnitude of the Fourier transform measured at the frequency corresponding to the probe $f_{\text {mod }}$ and $f_{c}$. Responses in the presence of the interfering tone were compared to responses obtained to the SAM probe tone alone. The shape of the interference pattern was highly dependent on the modulation frequency of the interfering tone. With some modulation frequencies an increase in interference relative to control responses was observed at the probe $f_{\text {mod }}$, while at other modulation frequencies either no interference, or even enhancement of the response was apparent. In contrast, at the probe $f_{c}$ interference patterns were very similar for all interference tone modulation frequencies.

\section{8:25}

3aA02. A change in sperm whale (Physeter macroephalus) distribution correlated to seismic surveys in the Gulf of Mexico. Bruce R. Mate (Hatfield Marine Sci. Ctr., Oregon State Univ., Newport, OR 97365), Kathleen M. Stafford (Oregon State University, Newport, OR 97365), and Donald K. Ljungblad (Elk Mountain, WY 82324)

From 7 to 29 June 1993, vessel surveys for sperm whales were conducted in the Gulf of Mexico off the Louisiana coast. Ninety sperm whales were seen in water 600 to $1400 \mathrm{~m}$ deep. On four of the first five survey days, whales were found routinely in an area $100 \mathrm{~km}$ S.E. of the Mississippi River before a seismic survey operation began ( 0.092 whales $/ \mathrm{km})$. Within the seismic operations area, whale abundance changed significantly to 0.038 whales $/ \mathrm{km}$ during the first two days and then to 0.0 whales $/ \mathrm{km}$ for the following five days $(p$ value $<0.001$ ). During the first two days of seismic activity, whales were only seen around the periphery of the seismic area. Survey effort for the last 5 days $(920 \mathrm{~km})$ and revealed only one group of four animals $61 \mathrm{~km} \mathrm{S.W.} \mathrm{of} \mathrm{the} \mathrm{seismic} \mathrm{survey} \mathrm{area} \mathrm{and} \mathrm{also}$ $56 \mathrm{~km} \mathrm{N.E.} \mathrm{from} \mathrm{another} \mathrm{active} \mathrm{seismic} \mathrm{survey.} \mathrm{Although} \mathrm{the} \mathrm{observation} \mathrm{of} \mathrm{seismic} \mathrm{survey} \mathrm{activity} \mathrm{was} \mathrm{serendipitous,} \mathrm{it} \mathrm{was} \mathrm{highly}$ 
correlated to numbers of sperm whales. This relationship deserves further investigation. If validated, additional efforts will be needed to identify areas used by sperm whales and assure that the effects of simultaneous seismic surveys do not overlap and prevent sperm whales from using important habitat.

3aA03. Developing standards for protecting marine mammals from noise: Lessons from the development of standards for humans. Ann E. Bowles (Hubbs-Sea World Res. Inst., 2595 Ingraham St., San Diego, CA 92109)

Models for protecting marine mammals from noise have been suggested that are analogous to human noise criteria, specifically (1) weighting functions that model species-specific auditory threshold functions (analogous to A-weighting); (2) threshold models for predicting the proportion of individuals that avoid a noise (analogous to the Schulz model of annoyance); and (3) the equal-energy hypothesis for predicting hearing loss. Models for reducing sleep interference, speech interference, and attention deficits might also be applicable. All these models will be reviewed. Unfortunately, human noise criteria do not apply to a number of effects that could occur in free-ranging marine mammals. Noise could affect nonauditory physiology. Noise could also mimic natural sounds (e.g., seismic impulses that are similar to tail beats), or attract marine mammals into dangerous areas (e.g., attracting killer whales to fishing gear). Given the paucity of research available on noise effects in marine mammals, standards may be difficult to establish, although they are badly needed. At present, management agencies have adopted extremely conservative noise criteria. The experience of regulating noise in human communities suggests that such stringent criteria cannot be enforced consistently. Solutions that have proved practical for human communities will be reviewed.

\section{9:05}

3aAO4. Basic understandings of whale bioacoustics: Potential impacts of man-made sounds from oceanographic research. Christopher W. Clark (Cornell Lab. of Ornithology, Bioacoust. Res. Program, 159 Sapsucker Woods Rd., Ithaca, NY 14850)

The recent increased public awareness and concern over the potential impact of acoustic sources for oceanographic research, particularly the source for the Acoustic Thermometry of Ocean Climate (ATOC) study, has raised the difficult issue of assessing both short-term and long-term effects. A baseline study in Kauai, HI has been underway for two seasons as part of the Marine Mammal Research Program associated with ATOC. This research specifically addresses the questions related to short-term $(<4$ months), small scale $(<30-\mathrm{km}$ radius zone of influence) issues using traditional visual and acoustic field methods. This includes shore-based and aerial observations, passive hydrophone array tracking, and aerial survey methods. These efforts, conducted prior to any operation of an ATOC source, provide a baseline measure of the level of short-term impact under "normal" conditions off Kauai, where normal includes regular exposure to noise from small craft, ships, helicopters, and airplanes. Potential long-term impact is addressed through statewide aerial surveys and through integration of the inter- and intra-seasonal variability of whale behaviors and distributions. Results of the Kauai research will be presented and discussed in terms of baseline impact and the need for standards.

\section{9:25-9:40 Break}

9:40

3aAO5. Marine mammals and ocean-acoustic experiments: A personal view from Monterey Bay. Stanley M. Flatté (Dept. of Phys., Univ. of California, Santa Cruz, CA 95064)

Experience in presenting technical descriptions of the relationship between marine mammals and the Acoustic Thermometry of Ocean Climate project to environmental groups, business groups, students, and researchers is described. It is pointed out that the greatest gap in the knowledge base of the general public is the lack of realization that low-frequency acoustic noise in the ocean is at present dominated by anthropogenic sources. [Work supported by ONR Ocean Acoustics.]

10:00

3aA06. Auditory-evoked potentials for assessment of hearing in marine animals. S. H. Ridgway and D. A. Carder (Biosci. Div., Naval Command, Control and Ocean Surveillance Ctr., RDTE Div., Code 5107B, 49620 Beluga Rd., Rm. 200, San Diego, CA 92152)

Brain-wave activity (EEG) even at minute levels recorded from the dolphin head surface may be processed in synchrony with sound to reveal an auditory-evoked potential (AEP). AEPs can provide objective information about the auditory system and many features are consistent across species so that experience with common laboratory animals and humans may be of help in evalualing responses. Although AEPs do not require a behavioral response, they may be compared with behavioral responses as sound is attenuated toward threshold. Some components of the AEP are unaffected by level of consciousness, allowing their use to evaluate hearing in sleeping infants and to determine brain damage or brain death. Auditory thresholds, and related information such as temporary threshold shifts, are critical for evaluating the potential impacts of ocean noise pollution on marine animals. Most species that are of concern, such as the great whales will not likely be brought into the laboratory so that their auditory system can be studied; however, many opportunities exist for brief studies when such animals become stranded or entrapped. Physiological studies, including AEPs, could go a long way toward providing critical information needed to define some limits for safe noise exposure for marine animals.

10:20

3aA07. Whale ears: Structural analyses and implications for acoustic trauma. D. R. Ketten (Dept. of Otolaryngol., Harvard Medical School, MEEl, 243 Charles St., Boston, MA 02114)

Over 75 species of dolphins and whales are spread throughout every aquatic habitat. Although echolocation abilities of some dolphins are well documented, little is known about hearing in most whales. Dolphin signals range as high as $200 \mathrm{kHz}$, while baleen whales routinely produce $10-$ to $20-\mathrm{Hz}$ signals. Whales have, therefore, two important auditory considerations: (1) the broadest signal 
range of any mammal group; and (2) the only mammalian ears adapted to underwater hearing. In this study, three-djmensional morphometric models of middle and inner ears from noncaptive toothed and baleen whales were used to estimate their hearing ranges. The analyses show echolocating species have basilar membrane stiffness coefficients $3 \times$ that of bats. Baleen whales have stiffness coefficients lower than elephants that hear infrasonics. Ganglion cell densities in whales are $2 \times$ bat and $3 \times$ human values. These data suggest echolocating dolphins may use multiple, parallel processed signal deteclion mechanisms. Cellular hypertrophy of inner ear support structures (stria vascularis, spiral ligament, basement membrane, ete.) in all whales may mean whales have inner ear mechanisms that decrease the potential for acoustic trauma. [Work supported by ONR Grant No. N00014-92-J-4000.]

\title{
Contributed Papers
}

10:40

3aA08. Late night fish choruses in the shallow waters off San Diego. G. L. D'Spain, L. Berger, A. M. Richardson, G. Clapp, and J. Rice (Marine Phys. Lab., Scripps Inst. of Oceanogr., Mail Code 0704, La Jolla, CA 92093-0701 and NCCOSC.RTDE DIV, San Diego, CA 92152-5001)

Two major experiments, SWellEx-1 and SWellEx-3, and one engineering sea test recently have been conducted in 200-m-deep water 8 miles west of the mouth of San Diego harbor. SWellEx-1 was conducted in August of 1993, the engineering sea test in May 1994, and SWellEx-3 in July 1994. For both of the summertime SWellEx experiments, an unusual oscillation in the background levels between $275-550 \mathrm{~Hz}$ and $575-700 \mathrm{~Hz}$ was observed, typically starting around midnight local time and lasting until sunrise. The repeating pattern to the oscillations is that of a 3-5 dB increase in spectral levels, which lasts on the order of $20 \mathrm{~s}$, and then followed by $25 \mathrm{~s}$ or so of lower levels. Azimuthal beamforming has indicated that the predominant direction of this "cycling sound" is from near coastal waters. In the data from the May 1994 engineering sea test, no such cycling sound was present. The character of these signals, i.e., their spectral content, their diumal variation, their seasonal variation, and their directionality, correspond to those described in year-long, single-hydrophone measurements made from a shallow water tower off San Diego in the early 1960s and ascribed to sounds made by fish of the croaker family [G. A. Clapp, NEL Tech Mem 1027 (1966)]. Additional properties of these signals, including a recording of the sounds made by a single individual, will be presented. [Work supported by ONR Code 321.]

\section{0:55}

3aA09. Using singing whales to track nonsingers. Nicholas $\mathrm{C}$. Makris (Naval Research Laboratory, Washington, DC 20375) and Douglas H. Cato (Defence Sci. and Technol. Organization, Pyrmont, NSW 2009, Australia)
The ability to track vocalizing whales over large distances $(>100 \mathrm{~km})$ using horizontal arrays has recently received much attention in the underwater acoustics community ["Use of Naval Facilities for Ocean Acoustic Research," J. Acoust. Soc. Am. 95, 2851-2854 (1994)]. The high source levels of the whales, up to $190 \mathrm{~dB}$ re: $1 \mu \mathrm{Pa}$ at $1 \mathrm{~m}$, make this possible. However, tracking vocal whales may provide an incomplete behavioral picture because a significant proportion of whales may be nonvocal at any given instance. (Visual observations are limited to the small proportion of time that whales surface.) Alternatively, the high source levels invite the possibility of treating the vocalizing whale as an active source. Sound from this source of opportunity that is scattered by other members of the herd may be sufficient to locate nonvocal whales with a towed array. Historical data are used to model the spatial distribution of vocal and nonvocal humpback whales migrating off the Eastern Continental Shelf of Australia. A spectral model for scattering from an object in a waveguide based on Ingenito's method is used to determine the scattered field from the whales. Waveguide noise is modeled via the Kuperman and Ingenito approach. Simulations indicate that localization of nonvocal whales is plausible for towed arrays within roughly $10 \mathrm{~km}$ of the herd in typical ambient noise conditions. This range may be extended significantly with time-domain matched filtering.

\section{1:10-11:15 Break}

11:15-12:15

\section{PANEL DISCUSSION:}

Panel Moderator: Charles R. Greene, Jr.

Panel Members: Ann E. Bowles, Christopher W. Clark, William F. Dolphin, Jeannie Drevenak, Carol Fairfield, Stanley M. Flatté, Darleen R. Ketten, Bruce R. Mate, Sam H. Ridgway, Ann Terbush

\section{Session 3aEA}

\section{Engineering Acoustics: Acoustical Measurement and Instrumentation I}

\author{
George S. K. Wong, Cochair \\ Institute for National Measurement Standards, National Research Council, Montreal Road, Ottawa, \\ Ontario K1A OR6, Canada \\ J. S. Echeverria-Villagomez, Cochair \\ Centro Nacional de Metrologia, km 4.5 Carr, Los Cues, Mplo. El Marqués, Querétaro, CP 76900, Mexico
}

Chair's Introduction-8:30

Invited Papers

8:35

3aEA1. Progress in acoustic transducer design and calibration. Gunnar Rasmussen (G.R.A.S. Sound \& Vibration aps, Vedbaek, Denmark)

The accuracy of acoustic measurements will play an increasing role, if legislation, prediction, and noise control are to be taken seriously. If the traffic intensity is doubled by year 2000 , one should expect a $3 \mathrm{~dB}$ higher noise level, unless the emitted sound power 
from each vehicle is reduced by $3 \mathrm{~dB}$. It is unlikely that emitted power will be cut in half, unless a number of sources the sum of which is half are attacked. This calls for better and more accurate measurements. Better monitoring equipment and better source location and source ranking equipment is needed. Outdoor monitoring has undergone a development to more rugged, stable, and accurately calibrated systems. Intensity measurements are getting out of the crib. Standards have evolved and calibration procedures are starting to be evolved. Instrumentation has been improved. It is important that intensity probes can now be produced to narrow specifications to fulfill the demand for precise and reproducible measurements; they are no longer selected from large populations, but manufactured to exact specifications. It is important to make proper calibrations, not just assume that certain parts of a probe are working properly. If intensity measurements shall gain in popularity it is important to develop the instrumentation from magic machines into practical tools for the practitioner. Practical systems, calibration, and applications will be discussed.

\section{9:05}

3aEA2. Free-field calibration and characterization of microphone systems. Victor Nedzelnitsky (Natl. Inst. of Stds. and Technol., Sound Building 233, Rm. A147, Gaithersburg, MD 20899-0001)

Significant discrepancies occurred in results from the recent European intercomparison of free-field calibration of IEC type LS2aP (13.2 mm nominal diameter) laboratory standard microphones [K. Rasmussen and E. Sandermann Olsen, The Acoustics Laboratory, Technical University of Denmark (DTH) Report PL-07 (1993)]. Consequently, Dr. Richard Barham of the National Physical Laboratory, U.K. (NPL) visited NIST to resolve one such discrepancy between NPL and DTH by obtaining a NIST calibration of a microphone that also had been calibrated by NPL and DTH in the European comparison. All laboratories used the reciprocity method, with independently implemented apparatus at each laboratory. The NIST calibration agreed relatively well with that of DTH, but not NPL, if all laboratories used the same values for the frequency-dependent acoustic center positions of the microphones. The problematical DTH and NPL determinations of acoustic center positions for the type LS2aP microphone, which disagree with the values obtained by scaling the positions standardized for another microphone type, are considered. Various implications for the current draft IEC standard on primary free-field calibration are discussed. Selected NIST free-field comparison calibration methods and transducer characterization procedures, recently applied to relatively novel devices such as micro-machined silicon microphones, are described.

\section{9:35}

3aEA3. Microphone monitoring by charge injection calibration. Erling Frederiksen (B\&K Res. and Develop. Dept., 2850 Naerum, Denmark)

Charge injection calibration is a new method for monitoring operation conditions of measurement chains consisting of a microphone with preamplifier, cables, and conditioning amplifier. The method replaces the insert-voltage calibration technique which is frequently used with large or unmanned systems. A great advantage of the new method is its ability to check the transducer which is practically ignored by the insert-voltage calibration. From a remote voltage source, a constant charge is "injected" into the microphone capacitance and preamplifier input circuit using a small capacitance integrated with the preamplifier. This leads to an output signal that is inversely proportional to the combined impedance of the transducer and input circuit. An accidental change in the microphone impedance-which is a good transducer condition indicator-would appear as a change in the preamplifier output. High stability and extremely high leakage resistance (typically $10^{14} \Omega$ ) are required for the charge injection capacitor (typically $0.2 \mathrm{pF}$ ). This problem was solved and the (patented) method was found to work reliably over a wide frequency range ( $2 \mathrm{~Hz}-200 \mathrm{kHz})$. The new method is less costly and simpler to implement. It also preserves the systems resistance to extraneous electrical fields. The method and its ability to indicate malfunctioning are discussed.

\section{0:05}

3aEA4. Traceability of acoustics and vibrations standards at the National Center of Metrology in Mexico. J. S. Echeverría-Villagomez (Centro Nacional de Metrologia, km 4.5 Carr, Los Cues, Mplo. El Marqués, Querétaro, CP 76900, Mexico)

After the creation of the Mexican Primary Laboratory of Metrology, primary standards on acoustics and vibrations are being established for the first time in the country. For this task, CENAM staff has relied on the technical assistance of the corresponding groups at NIST and NRC. The process has involved several specific features due mainly to the particular needs and conditions of the Mexican laboratory. On the acoustics side, for the instrumentation of the reciprocity method for microphone calibration, the altitude of the site at which CENAM is located and the consequent low pressure lead to the proposal for a pressure-controlled chamber. Both the proposal and its completion has been carried out at NIST, with direction of its staff and participation of CENAM staff. The stage in which the project is at the moment will be shown. Also the instrumentation and methods used for giving traceability from CENAM to transfer and working standards of external customers. On the vibrations side, instrumentation of the reciprocity method for accelerometer calibration has been achieved with the Bouche Labs 5000CR system. Besides an interferometric system is being obtained as a complementary aid and for cross checking purposes. Traceability to transfer and working standards at CENAM is already being accomplished through the comparison method. An evaluation of the systems performance will be presented and a first estimation of their uncertainties will be discussed.

\section{0:35}

3aEA5. Velocity of sound measurement in liquids. Zhu Shi-ming (Inst. of Acoust., Tongii Univ., Shanghai, People's Republic of China) and George S. K. Wong (Inst. for Natl. Measurement Stds., Natl. Res. Council, Ottawa, ON K1A 0R6, Canada)

Sound velocities in pure water at various temperatures were measured. A PZT transducer sends a 2-MHz pulse towards a target stationed at an accurately known distance of $120 \mathrm{~mm}$ away. The transit time between the forward pulse and the reflected pulse was measured precisely with statistical averaging and a reference timebase derived from the NRC cesium frequency standard. The 
transducer-target probe and liquid chamber was immersed in a temperature controlled bath that was under the control of a computer. Measurements were repeated with known concentrations of alcohol and distilled water. Uncertainties of the measurements will be discussed. The authors would like to thank Lu-Jie of the Tongji University for his contribution to the construction of the measurement circuits.

11:05

3aEA6. Considerations of errors in computer-based measurements. Alan D. Wallis (Cirrus Research plc, Acoustic House, Hunmanby YO14 OPH, UK)

The use of computers has come to dominate acoustical measurements as was predicted at ICA 1983 in Paris. However, what was not predicted was that a generation of acousticians would arrive with no previous experience of older analogue systems. To some of these new generation workers, "the computer is always right" and little or no thought is given to the limitations and accuracy restrictions of a totally digital system. Automatic checks routinely done before and after measurements on previous generations of instruments are sometimes ignored, while instrument tolerances are assumed to be zero and calibrations are assumed to be perfect even though logic suggests otherwise. Consideration is given to making basic checks on computer based instruments, so that reasonable reliance can be placed on the results, often using old and traditional methods brought up to data and translated from the analogue to the digital world. Sample case histories of particular "real world" errors are given, with some idea of the practical limitations of new technology instrumentation and where they sometimes fall short of older units. These are compared with some requirements of ANSI and international standards.

\title{
Session 3aNS
}

\section{Noise: Nonoccupational Noise Exposures and Implications of Recent TLV Changes}

\author{
Larry H. Royster, Cochair \\ MAE Department, North Carolina State University, Raleigh, North Carolina 27695-7910 \\ Julia D. Royster, Cochair \\ Environmental Noise Consultants, Inc., P.O. Box 30698, Raleigh, North Carolina 27622-0698
}

Chair's Introduction-8:20

\section{Invited Papers}

8:30

3aNS1. On comparing noise metrics applied to hearing conservation. John J. Earshen (Angevine Acoust. Consultants, Inc., 1021 Maple St., P.O. Box 725, East Aurora, NY 14052-0725)

A number of different metrics are in use for measuring and reporting exposure to noise for hearing conservation including noise dose, time-weighted average level, sound exposure, and sound exposure level. Functions for computation of individual metrics from measurement of acoustic pressure vary widely. Functions defining particular metrics in some instances contain choices of parameters (e.g., exchange rates and threshold levels). In most instances, once a metric has been evaluated for a particular time profile of acoustic pressure, it is not possible to convert it to another metric if the original time function is not retained. In addition to the defining functions, dynamic and frequency response characteristics of measuring instruments have influences on results obtained and can present obstacles to conversion among metrics. Large bodies of data exist which only contain the finally processed metric. The degree to which comparisons can be made because of recent changes in TLV requires inferential interpretation of the values of specific metrics which are produced by time functions that are identified only in a statistical sense. This paper examines the inferential relationships among prominent metrics for various classes of sound-pressure profiles as obtained with measuring instruments meeting ANSI and IEC performance standards.

8:55

3aNS2. Noise exposures in U.S. coal mines. John P. Seiler, M. P. Valoski, and M. A. Crivaro (U.S. Dept. of Labor, MSHA, P.O. Box 18233, Pittsburgh, PA 15236)

Mine Safety and Health Administration (MSHA) inspectors conduct full-shift environmental noise surveys to determine the occupational noise levels to which coal miners are exposed. These noise surveys are performed to determine compliance with the noise standard promulgated under the Federal Mine Safety and Health Act of 1977. Data-from over 60000 full-shift noise surveys conducted 
from Fiscal Year 1986 through 1992 were entered into a computer database to facilitate analysis. This paper presents the mean and standard deviation of over 60000 full-shift noise dose measurements for various underground and surface coal mining occupations. Additionally, it compares and contrasts the levels with historical noise exposure measurements for selected coal mining occupations that were published in the 1970 s. The findings were that the percentage of miners surveyed that were subjected to noise exposures above $100 \%$, neglecting personal hearing protectors, were $26.5 \%$ and $21.6 \%$ for surface and underground mining, respectively. Generally, the trend is that the noise exposures for selected occupations have decreased since the 1970 s.

9:20

3aNS3. Comparison of daily noise exposures in one workplace based on 3-dB vs 5-dB exchange rates and criteria recommended by the ACGIH versus OSHA. Michelle Petrick (Occupational Health and Safety, IBM, Dept. 692, Bldg. 002, P.O. Box 12195, Research Triangle Park, NC 27709), Larry Royster (NC State University, Raleigh, NC), Julia D. Royster (Environmental Noise Consultants, Inc., Raleigh, NC), and Parker Reist (UNC, Chapel Hill, NC)

In May 1994, the American Conference of Governmental Industrial Hygienists (ACGIH) recommended as a threshold limit value (TLV) for noise exposure an 8-h equivalent level of $85 \mathrm{dBA}$, based on a 3-dB exchange rate. The U.S. Department of Labor's Occupational Safety and Health Administration (OSHA) requires that employees be included in a hearing conservation program (HCP) if their 8-h time-weighted average (TWA) noise exposure equals or exceeds $85 \mathrm{dBA}$ based on a 5-dB exchange rate. To investigate the number of additional employees who would be included in the HCP at one industrial facility if the ACGIH TLV were used, new personal dosimetry samples were collected for all noise-exposed employees. Employees at this facility have low noise exposures characterized by time-varying or intermittent noise and impact components. To allow analysis of each sample using both exchange rates, each monitored worker wore either two single-setting dosimeters or one double-setting dosimeter. Based on this data the potential impact if OSHA changed its present procedures for determining employee noise exposure will be presented.

3aNS4. The impact of using a 3-dB vs 5-dB exchange rate on the predicted employee's 8-h equivalent A-weighted sound-pressure level in three different types of industrial work environments. Larry H. Royster (MAE Dept., NC State Univ., Raleigh, NC 27695-7910) and Julia Doswell Royster (Environmental Noise Consultants, Inc., Raleigh, NC 27622-0698)

A noise exposure database was previously collected using a 5-dB exchange rate for three different types of industrial work environments: paper manufacturing, aluminum casting and rolling, and meat packaging. This database will be reanalyzed to estimate the impact on the percentage of additional employees that would have to be included in each company's hearing conservation program if a 3-dB exchange rate replaces the present OSHA 5-dB exchange rate. In addition, the effect of using different combinations of criterion and action levels in conjunction with the use of a $3-\mathrm{dB}$ exchange rate will be discussed.

10:00

3aNS5. Contribution of off-the-job noise to hearing thresholds of employees with occupational noise exposure. Julia D. Royster (Environmental Noise Consultants, Inc., P.O. Box 30698, Raleigh, NC 27622), Larry H. Royster (NC State University, Raleigh, NC 27695), Pamela S. Calliari (Associated Hear. Services, Raleigh, NC), and Kelly L. Tennyson (Developmental Evaluation Ctr., Raleigh, NC)

Employers often assert that it is fruitless to protect employees from noise at work because workers' off-the-job noise exposures are sufficient to cause significant hearing loss. Certainly, the lower the action level for including employees in a hearing conservation program $(\mathrm{HCP})$ is set, the greater the potential contribution of nonoccupational exposures becomes. This paper will describe the types of nonoccupational noise exposures reported by employees in HCPs at several industries on annual auditory history questionnaires and in interviews. In order to evaluate the contribution of nonoccupational noise exposure to workers' hearing thresholds, employees were grouped according to their self-reported amount of off-the-job noise. Results of analyses on age-corrected hearing levels will be presented.

\section{0:15}

3aNS6. Nonoccupational noise exposures and estimated daily $L_{\text {eq }}$ values for attendance at college basketball games and shopping centers. Larry H. Royster (MAE Dept., NC State Univ., Raleigh, NC 27695-7910) and Julia Doswell Royster (Environmental Noise Consultants, Inc., Raleigh, NC 27622-0698)

Noise exposure data were collected during 1988-1989 home basketball games at North Carolina State University. Thirteen exposure samples averaging $2 \mathrm{~h}$ in duration were collected utilizing Larson-Davis 700 noise dosimeters. Measured noise level results were as follows: average unweighted peak pressure was $133.1 \mathrm{~dB}$, average maximum sound level $\left(L_{\mathrm{A}}\right)$ was $115.2 \mathrm{~dB}$, and average $L_{\text {Aeq2 } \mathrm{h}}$ was $97.1 \mathrm{dBA}$. The equivalent 40 -h exposure level $L_{\text {Aeq40 } \mathrm{h}}$ was $84 \mathrm{dBA}$. As another example of nonoccupational noise exposure, between 1982 and 1994 seven shopping malls in four different cities were visited to establish the sound level distributions. Over 800 noise level samples were obtained. When the data were restricted to malls with at least $500000 \mathrm{sq}$ ft, it was determined that measured noise levels $L_{\mathrm{A}}$ could be approximated utilizing the following equations (where $N$ is the number of shoppers within a radius of $7.6 \mathrm{~m}$ of the measurement location): $L_{\mathrm{A}}=61+10 \log \left(N^{0.55}\right)$, for $N \leqslant 10$, and $L_{\mathrm{A}}=54+10 \log \left(N^{1.20}\right)$, for $N>10$.

$$
\text { 10:35-10:50 Break }
$$


10:50

3aNS7. Noise exposure measurements of the Tokyo String Quartet. Larry H. Royster (MAE Dept., NC State Univ., Raleigh, NC 27695-7910) and Julia Doswell Royster (Environmental Noise Consultants, Inc., Raleigh, NC 27622-0698)

During the Spring 1994 Meeting of ASA a special concert was given by the Tokyo String Quartet. During the first part of the program the Tokyo String Quartet played two music selections using each of four different sets of instruments: Old Italian instruments and modern instruments crafted by Carleen Hutchins, Curtin and Alf, and Robert and Deena Spear. The Tokyo String Quartet had previously agreed to allow noise dosimeter monitoring of their exposures during the first half of the program. In addition, one member of the audience also wore a noise dosimeter during the performance. The measured $L\left({ }_{6}\right)$ values across instrument groups for the audience member exhibited a range of $0.7 \mathrm{dBA}$ for the first musical selection and 0.6 dBA for the second selection. The remaining recorded database findings and its analysis will be presented. The authors are grateful to the members of the Tokyo String Quartet for allowing them the opportunity to obtain additional musician noise exposure information.

\section{1:05}

3aNS8. Investigation of lawn-maintenance equipment noise. Gordon T. Lepley, IV and L. Marcelyn Bruce (Collaboration in Sci. and Technol., Inc., 15835 Park Ten Place, Ste. 105, Houston, TX 77084-5131)

As the lawn-maintenance industry grows in suburban residential areas, it has become a new and significant source of environmental noise and occupational noise exposure. Due to the short duration at any one location and public acceptance of this activity, there are few community complaints about the noise (except at unreasonable hours). This paper will concentrate on recreational and occupational exposure, where individuals are exposed to high noise levels for long periods of time. Most lawn-maintenance workers spend from 8-10 h per day exposed to A-weighted sound levels greater than $85 \mathrm{~dB}$, and it appears that few employees wear hearing protection. Sound levels were measured and monitored at the operator's ear and measured at a distance of $\mathbf{1 0} \mathrm{ft}$ for the following equipment: lawn mowers, gas and electric edgers, gas and electric trimmers, electric blowers, and an electric hedge trimmer. A-weighted sound levels at the operator's ear ranged from 82 to $102 \mathrm{~dB}$. A comparison of equipment sound levels will be presented as well as a comparison of noise levels for gas versus electric lawn equipment. Finally, daily noise exposure for lawnmaintenance employees will be developed using the data.

\section{1:20}

3aNS9. Sampling strategy for noise dosimetry measurements. Felix Z. Sachs (U.S. Army Ctr. for Health Promotion and Preventive Medicine (Provisional), Aberdeen Proving Ground, MD 21010-5422)

The U.S. Army has adopted most of the new ACGIH TLVs as criteria in its hearing conservation program. Most significant to the Army program was the change from a non-time-weighted inclusion criterion to the 8-h time-weighted average noise exposure level (TWA). The TWA must now be identified for all Army civilian personnel subjected to hazardous noise exposure and for soldiers working in noise-hazardous industrial operations. This can be accomplished without making noise dosimetry measurements on every single individual suspected of being exposed to hazardous noise.
The U.S. Army Environmental Hygiene Agency Technical Guide 181 on noise dosimetry and risk assessment was developed as a guide for evaluating types of noise exposure patterns and includes sampling strategies for dosimetry measurement of TWAs. The statistical basis of the sampling approach will be discussed in some detail. Other changes in the Army hearing conservation program resulting from the adoption of the TLVs will be outlined.

\section{1:35}

3aNS10. Active noise reduction for circumaural hearing protectors by digital feedforward control. G. J. Pan, A. J. Brammer, J. Ryan, J. Zera (Inst. for Microstruct. Sci., Natl. Res. Council, Montreal Rd., Ottawa, ON K1A 0R6, Canada), and R. Goubran (Carleton Univ., Ottawa, ON K1S 5B6, Canada)

A commercial analog active noise reduction (ANR) communication headset, constructed using a circumaural hearing protector, has been modified to explore the performance of a digital ANR system for this application. The apparatus retains one ear cup and cushion, together with the earphone and attached microphone of the commercial device. The sound field outside the ear cup is sensed by a miniature microphone attached to the exterior of the ear cup, and forms the "reference" signal. This signal is fed forward to an adaptive controller, which drives the earphone within the ear cup. Control of the sound pressure within the volume enclosed by the hearing protector is maintained by an "error" microphone attached to the earphone, using the filtered-X LMS algorithm. The error-path impulse response is identified off-line. A Texas Instruments TMS320c31 floating point digital signal processor has been used to implement the control system in real time. The noise reduction of the adaptive digital system will be discussed when the hearing protector is subjected to bandlimited noise in the frequency range from 50 to $1000 \mathrm{~Hz}$. [Work done in collaboration with the Defence and Civil Institute of Environmental Medicine, Toronto, Ont.]

\section{1:50}

3aNS11. New method dose for a 24-h estimation of occupational and nonoccupational noise. Alexander A. Menshov (Dept. of Phys. Factors, Inst. for Occupational Diseases, $252033 \mathrm{Kiev}$, Ukraine)

Over $24 \mathrm{~h}$ the influence of occupational and nonoccupational noise may be distinguished by three periods: job, rest, and sleep. If the level of noise for each period is described in dBA there is no possibility of summing up these levels and receiving mean arithmetical values (average daily) because $\mathrm{ABA}$ is a logarithmic value. But if one describes levels of noise in doses there is a possibility of receiving a "medium-weighted" dose of noise load for 24-h because the doses are arithmetical values and it is possible to sum up and divide the total. If the dose level during the work is 1 dose $-80 \mathrm{dBA}$, at rest 1 dose $-40 \mathrm{dBA}$ (one dose), and during sleep 1 dose $-30 \mathrm{dBA}$ (Ukraine and Russian sanitary standards), then the medium-weighted sanitary standard for the 24-h level of noise is one dose. The increase of the level for each a third 24-h period at $3 \mathrm{dBA}$ above the norm will increase the dose level twice (two doses); at $6 \mathrm{dBA}$ it will increase to four doses; at $9 \mathrm{dBA}$ it will increase to eight doses; at $12 \mathrm{dBA}$, to sixteen doses, etc. If such high doses for each period are summed up and are then devised in three, a real medium-weighted dose of noise load for 24 $h$ above the standard, which is 1 dose, will be found. This method is simpler than estimating the noise load above the standard for each third period of a 24-h influence of noise. 


\title{
Session 3aPAa
}

\section{Physical Acoustics: Outdoor Sound Propagation}

\author{
Michael J. White, Chair \\ U.S. Army Construction Engineering Research Laboratory, P.O. Box 9005, Champaign, Illinois 61826-9005
}

\section{Contributed Papers}

\section{8:00}

3aPAa1. Detection of direct and indirect sonic booms from space shuttle landings. Joseph Cates, B. Sturtevant (Graduate Aeronaut. Labs., California Inst. of Technol., Caltech 301-46, Pasadena, CA 91125), Hiroo Kanamori (California Inst. of Technol., Pasadena, CA 91125), and J. Mori (U.S. Geological Survey, Pasadena, CA 91106)

Two hundred seismic stations covering over 50000 square kilometers in Southern California were used to analyze the sonic boom patterns from the landing of STS-42 in November 1993 and STS-58 in January 1994 at Edwards Air Force Base (EAFB). The instrument ground motion traces show separate arrivals which can be identified as the primary boom, creeping waves generated at the edge of the primary boom carpet, and multiple indirect sonic booms refracted from high altitude. The measured arrival times are compared with the results of linear ray tracing calculations through reference temperature and wind profiles for EAFB. The ray calculations accurately predict the measured arrival times and wavefront angles for the primary sonic boom and the creeping waves in shadow regions, and accurately predict the wavefront angles for the indirect booms. Ray theory using the reference atmosphere fails to predict indirect boom arrival time, observed multiple booms within the first shadow region, and extensive overlap of the multiply refracted sonic booms. These results suggest that boom exposure under the real atmosphere may be larger than previously expected.

\section{8:15}

3aPAa2. Sonic boom rise time. Robin $O$. Cleveland and David $T$. Blackstock (Appl. Res. Labs. and Mech. Eng. Dept., Univ. of Texas at Austin, P.O. Box 8029, Austin, TX 78713-8029)

The rise time of a sonic boom shock in air depends strongly on the relaxation processes of nitrogen and oxygen. Stratification of the atmosphere leads to significant variation of the relaxation processes with altitude. J. Kang [Ph.D. thesis, Penn State (1991)] argues that the shock profile can adjust quickly enough to changes in attenuation that it always appears to be in steady state. If correct, then rise time at the ground can be calculated from local conditions only. A time domain computer algorithm, based on work by Lee and Hamilton ("Time domain modeling of pulsed finiteamplitude sound beams," submitted to J. Acoust. Soc. Am. in March 1994), is presented for a Burgers-type equation. The algorithm includes the effects of nonlinear distortion, thermoviscous absorption, molecular relaxation, and ray tube spreading. A parametric study of the effect of change in relative humidity shows that the steady-state assumption is not justified for sonic boom shocks in the atmosphere. A sonic boom propagated through a real atmosphere is shown to have a rise time that, in all cases run so far, is shorter than that of a steady-state shock calculated using atmospheric properties at the ground only. [Work supported by NASA.]

\section{$8: 30$}

3aPAa3. Weakly nonlinear propagation of $N$ waves through turbulence. Bart Lipkens (Macro-Sonix, 1054 Technol. Park Dr., Glen Allen, VA 23060) and Philippe Blanc-Benon (Ecole Centrale de Lyon, 69131 Ecully Cedex, France)
Reported here is a numerical investigation to explain experimental observations of the effect of turbulence on $N$ wave propagation [B. Lipkens, Ph.D. thesis, Mech. Eng. Dept., Univ. of Texas at Austin (1993)]. An adaptation of Von Kármán's spectral model for incompressible, isotropic turbulence is used to generate a statistical realization of a turbulent field. The 2-D, random, isotropic velocity or temperature fields consist of a collection of discrete Fourier velocity modes [Ph. Blanc-Benon et al., Theoret. Comput. Fluid Dynamics 2, 271-278 (1991)]. The nonlinear propagation model consists of two parts: (1) Linear geometric acoustics is used to trace rays through each realization of the turbulent field, and (2) a nonlinear transport equation is derived for the propagation along the eigenrays and solved by a Pestorius-type algorithm. The input waveform to the algorithm is a plane $N$ wave similar to that used in the model experiment to simulate sonic boom propagation through a turbulent atmosphere. Statistics of peak pressure and rise time, parameters that determine the loudness of sonic booms, are calculated over 100 realizations. The effect of turbulence is to reduce the nonlinear distortion of the $N$ wave. On average, turbulence reduces peak pressure and increases rise time. Qualitatively, the same conclusions are observed as in the model experiment.

\section{$8: 45$}

3aPAa4. A numerical study of random focusing for plane waves propagating in scalar or vectorial turbulent media. Philippe Blanc-Benon and Daniel Juvé (Lab. de Mécan. des Fluides et d'Acoust., URA-CNRS 263, BP 163, Ecole Centrale de Lyon, 69131 Ecully Cedex, France)

A numerical simulation has been developed to investigate the random focusing for an acoustic plane wave propagating through scalar or vectorial turbulent fields. The turbulence is represented as a set of realizations of random fields (temperature or incompressible velocity) generated by a limited number of random Fourier modes [M. J. Karweit et al., J. Acoust. Soc. Am. 89, 52-62 (1991)]. Through each realization the propagation of a plane wave is considered in either the geometric or the parabolic approximation. Using the ray equations the ray trajectories and the location of the instantaneous caustics are computed. The acoustic pressure field and the intensity fluctuations are obtained by solving a wide-angle parabolic equation. Ensemble averaging is then performed to evaluate the probability distribution of the occurrence of caustics and the variance of acoustic intensity fluctuations. For 2-D and 3-D scalar simulations the results demonstrate that the position of caustics as well as the maximum peak in the scintillation index are governed by universal parameters related to the transverse spatial derivatives of the correlation function of the fluctuating components of the turbulent medium. Results will be given to generalize the definition of these universal parameters for sound propagation in random velocity fields.

\section{9:00}

3aPAa5. Short term fluctuations in the sound field within a shadow region near the ground. David I. Havelock (Inst. for Microstruct. Sci., Natl. Res. Council, Ottawa ON K1A 0R6, Canada)

An acoustic shadow region can be created near the ground by an upwardly refracting atmosphere or masking by prominent terrain features. 
Acoustic energy arrives deep within the shadow region principally by scattering from turbulence in the insonified region above the shadow. It is expected that the structure and dynamics of the turbulence ultimately determine the characteristics of the sound field observed in the shadow region. By identifying observable characteristics of this sound field, it is hoped that useful relationships between meteorological dynamics and sound field fluctuations within the shadow region can be established. A large array of microphones has been deployed on an asphalt runway to investigate the sound field fluctuations in a refractive shadow. The frequency range being investigated is $40-1000 \mathrm{~Hz}$, at ranges up to $700 \mathrm{~m}$. The evolution of the sound field over the period of a few seconds is discussed. Examples of the observed sound field are presented.

\section{9:15}

3aPAa6. Atmospheric sound propagation in a turbulent atmosphere over irregular terrain. Xiao Di and Kenneth E. Gilbert (Appl. Res. Lab. and the Graduate Program in Acoust., Penn State Univ., P.O. Box 30, State College, PA 16804)

A phase screen method for atmospheric turbulence and a cascaded conformal mapping technique for inregular terrain are used together to investigate scattering of sound by turbulence into a terrain-generated shadow zone. Comparisons with and without atmospheric turbulence are made for frequencies from 50 to $500 \mathrm{~Hz}$. It is shown that with the nearground turbulence model of Daigle and a hill similar to that in the Joint Acoustic Propagation Experiment (JAPE), turbulence effects are negligible below approximately $125 \mathrm{~Hz}$. Above $125 \mathrm{~Hz}$, there is considerable scattering into the shadow zone behind the hill. For the data currently available from the JAPE measurements, the theoretical predictions are in good agreement with experiment. [Work supported by the Army Research Laboratory.]

\section{9:30}

3aPAa7. Scattering of sound by turbulence into a shadow region. Michael R. Stinson (Inst. for Microstruct. Sci., Natl. Res. Council, Ottawa, ON K1A 0R6, Canada)

The sound field above ground due to a point source in an upwardly refracting atmosphere has been computed to determine the nature of turbulent scattering into an acoustic shadow region. A Green's function parabolic equation (GFPE) method that includes turbulence via a phase screen approach [X. Di and K. E. Gilbert, J. Acoust. Soc. Am. 92, 2405(A) (1992)] is used for the simulations. Flat ground with finite surface impedance and a logarithmic sound-speed profile are assumed, and propagation ranges of $1 \mathrm{~km}$ are considered. Different realizations of a turbulent atmosphere were generated employing a Gaussian spectrum to describe the turbulence. For $500-\mathrm{Hz}$ source tones, it was found that the components of the turbulence spectrum that contributed most to the scattered field in the acoustic shadow region corresponded to dimensions of the order of 2-5 m; this scale of structure can be understood in terms of a Bragg reflection condition. The sound pressure at a receiver position in the acoustic shadow appears to be dominated by contributions from a small number of localized scattering regions.

\section{9:45}

3aPAa8. Scale model experiments on the diffraction and scattering of sound by a partially rough curved surface. James $P$. Chambers, Yves H. Berthelot, and T. Shane Stone (School of Mech. Eng., Georgia Inst. of Technol., Atlanta, GA 30332-0405)

Scale model experiments have been conducted to study the propagation of sound over a curved surface partially covered with rigid random roughness elements in the high-frequency diffraction/low-frequency scattering regime. The effects of roughness are found by comparing the sound field with that obtained with a fully smooth curved surface. Experiments indicate that roughness in the shadow zone acts to tunnel more sound into the shadow zone of the partially rough surface, relative to the curved smooth surface, via a "creeping boundary wave." This wave qualitatively follows the trends of both creeping waves due to diffraction and Tolstoy-type "boundary waves" due to coherent scattering. However, roughness in the bright zone causes less sound to propagate into the shadow zone of the partially rough surface, relative to the curved smooth surface, because of incoherent scattering. [This work is supported by a Fannie and John Hertz Fellowship.] ")Current address: National Center for Physical Acoustics, University, MS 38677.

\section{0:00-10:15 Break}

\section{0:15}

3aPAa9. Estimation of time-varying phase for multiple tones in atmospheric sound propagation. John C. Burgess (Dept. of Mech. Eng., Univ. of Hawaii, 2540 Dole St., Honolulu, HI 96822) and David I. Havelock (Natl. Res. Council, Ottawa, ON K1A 0S1, Canada)

Atmospheric turbulence affects both the amplitude and phase of transmitted sound. Tones at different frequencies will be affected differently. In an earlier presentation [J. C. Burgess and D. I. Havelock, "Estimation of time-varying phase (instantaneous frequency) in atmospheric sound propagation," J. Acoust. Soc. Am. 95. 2838(A) (1994)], the time-varying phase of a single tone was estimated by two methods, one based on an FFT with optimum data windows, the other based on the discrete Hilbert transform. In this presentation, the optimum data window method is extended to multiple tones. Accuracy of the method is examined using a synthesized signal with and without additive noise.

\section{0:30}

3aPAa10. Helicopter field data compared with fast field program. Michael T. Barnes (U.S. Army Res. Lab., Battlefield Environment Directorate, White Sands Missile Range, NM 88002)

A study was conducted to compare the fast field program (FFP) to data obtained from helicopters. The purpose of the study was to see how well FFP predictions would compare to helicopter data over ranges out to $20 \mathrm{~km}$ and determine if the FFP could be used to reliably predict the propagation conditions for acoustic arrays listening for helicopters. Over a period of 2 weeks acoustic data from several helicopters with various flight paths out to a range of $20 \mathrm{~km}$ was recorded. At the same time meteorological conditions were also noted. These included temperatures up to $400 \mathrm{~m}$, relative humidity, barometric pressure, wind speed, and wind direction up to 2000 $\mathrm{m}$. The results were very promising and in most cases provided a good comparison until the helicopter signal was buried in background noise.

\section{0:45}

3aPAa11. Sensitivity analysis of the Green's function parabolic equation model for atmospheric sound propagation. Michael $R$. Dobry (Dept. of Mech. Eng., New Mexico State Univ., Box 30001-3501, Las Cruces, NM 88004), Robert P. Hansen (U.S. Military Academy, West Point, NY), and David H. Marlin (U.S. Army Res. Lab., White Sands Missile Range, NM)

A sensitivity analysis was performed on the Green's function parabolic equation (GFPE) model to determine parameter bounds which maintain model validity. To determine these bounds, inverse theory was used to determine the "best" combination of input parameters over a twodimensional domain using a normalized sum of squared residuals. A twodimensional version of the fast field program (FFP), a widely accepted atmospheric propagation model, was used as comparison. Plots of the error surface were then made to show sensitivity bounds. Upward and downward refracting atmospheres were considered for a range of frequencies. The GFPE model was found to require a beight increment parameter of about 0.05 wavelengths while the range increment parameter extended from 10 to 120 wavelengths depending on atmospheric profile and frequency. The thickness of the attenuation layer was found to be frequency dependent and ranged from 50 to over 200 wavelengths. The surface wave integral height was found to be a minimum of 30 and 13 wavelengths for the upward and downward atmospheres, respectively. The CPU time for the GFPE model ranged from 10 to $60 \mathrm{~s}$, depending on frequency, and was approximately $60-1000 \times$ faster than the two-dimensional FFP. 
3aPAa12. When is downwind propagation really upwind propagation? John M. Noble (U.S. Army Res. Lab., Battlefield Environment Directorate, White Sands Missile Range, NM 88002)

For a long time, atmospheric acoustic propagation has used phases like "upwind propagation," "downwind propagation," and "crosswind propagation" to describe the type of propagation conditions present in the atmosphere. For short-range propagation $(<3 \mathrm{~km})$, the portion of the atmosphere between 50 and $100 \mathrm{~m}$ will primarily influence the propagation of sound. This region of the atmosphere is called the surface layer. Typically, through this region there is not much change in the mean wind direction. Therefore the terms mentioned earlier have some meaning for short-range propagation. This is due to the effective speed of sound for a given direction not varying much due to the small changes in the mean wind direction with height. For longer range propagation, this terminology could have little to do with the actual propagation conditions due to the effect of wind speed and wind direction variations with height above the surface layer. The purpose of this presentation is to discuss why this terminology is not appropriate and for what conditions it is appropriate for use in atmospheric acoustics.

\section{1:15}

3aPAa13. Complex image theory for sound propagation above ground. Michael J. White (U.S. Army Construction Eng. Res. Lab., P.O. Box 9005, Champaign, IL 61826-9005) and Y. L. Li (Univ. of Illinois, Urbana, IL 61801)

A very simple and accurate method is introduced for the computation of the sound field above an impedance ground or a porous half-space. The procedure is based on complex image theory. Using Prony's method, the slowly varying part of the integrand in the Sommerfeld integral is approximated by a short series in terms of complex exponential functions. Combining the series of exponential functions and the remaining integrand, the resulting integral can be exactly evaluated by the Sommerfeld identity. A new closed-form expression of the sound field is represented as the original source, its quasistatic image, and three "images" with complex amplitudes and positions. Avoiding numerical evaluation of the Sommerfeld integral makes these closed-form expressions computationally very efficient. $\mathrm{Nu}$ merical results show that these closed-form Green's functions are very accurate for the computation of the near and intermediate fields.
3aPAa 14. Determining the acoustic parameters of snow by impulsive waveform inversion. Donald G. Albert (U.S. Army Cold Regions Res. and Eng. Lab., 72 Lyme Rd., Hanover, NH 03755-1290)

The amplitude and waveform shape of atmospheric acoustic pulses propagating horizontally over a seasonal snow cover are profoundly changed by the air forced into the snow pores as the pulses move over the surface. Attenborough's four-parameter, rigid-ice-frame porous treatment of snow is applied to the inverse problem, where the observed waveform is used to determine the acoustic parameters of the snow. Horizontally propagating acoustic pulses were generated using blank pistol shots fired 1 $\mathrm{m}$ above various seasonal snow covers and measured by surface microphones $60 \mathrm{~m}$ away. Various inversion strategies, both frequency and time domain, were investigated to determine automatically the acoustic parameters that accurately predict the observed waveforms. Preliminary results show that the effective flow resistivity and depth of the snow are the controlling parameters. [Work supported by the Directorate of Research and Development, U.S. Army Corps of Engineers.]

\section{1:45}

3aPAa15. New fast field programs for a multiple-source system. Y. L. Li (Dept. of Elect. and Comput. Eng., Univ. of Illinois, Rm. 332, 3rd fl. North CSRL, 1308 W. Main St., Urbana, IL 61801) and Michael J. White (U.S. Army Construction Eng. Res. Lab., Champaign, IL 61826-9005)

A new fast field program (FFP) has been developed for numerical computation of sound pressure from a phased multiple-source or multiplereceiver system in a layered medium. The new FFP is based on a far-field approximation. Using the stationary phase method, the twofold integral for sound pressure in the far field is reduced to a onefold integral. The onefold integral makes the new FFP very efficient and more practical than computation performed individually for each source. A criterion for validity of the far-field approximation is also given. The sound pressure of several phased-array systems in a layered medium has been computed. Numerical results from the new FFP are compared with some well-known results. The agreement is excellent.

\title{
Session 3aPAb
}

\section{Physical Acoustics: Bubble Dynamics and Cavitation}

\author{
Lev A. Ostrovsky, Cochair \\ Institute of Applied Physics, Nizhini Novgorod 603600, Russia \\ Michalakis A. Averkiou, Cochair \\ Applied Physics Laboratory, University of Washington, Seattle, Washington 98105
}

\section{Contributed Papers}

\section{8:30}

3aPAb1. A nanosecond acoustic source: Experimental investigation of pulsed dielectric breakdown of water and its dependence on pressure and conductivity. Hugh M. Jones (Dept. of Phys. and Eng. Phys., Stevens Inst, of Technol., Hoboken, NJ 07030) and Erich E. Kunhardt (Stevens Inst. of Technol., Hoboken, NJ 07030)

Spark gaps are finding use as underwater acoustic sources in sonar and prospecting applications. It is thus important to determine the influence of pressure and conductivity on the dielectric breakdown of water. Well defined rectangular pulses $(80-\mathrm{kV}, 3-\mathrm{ns}$ rise time, 100-ns duration) have been applied to a gap $(0.04-0.21 \mathrm{~cm})$, between Rogowski profile electrodes, containing de-ionized, nondistilled water, de-ionized, distilled water, or sodium chloride solutions $(0.001-1.0 \mathrm{M})$. Breakdown in these liquids has been studied at pressures up to $400 \mathrm{~atm}$. Calibrated voltage dividers situated on the source and load sides of the test gap permitted measurement of the interelectrode potential and the current response. From these measurements, the time lag to breakdown, breakdown voltage, power input to the 
liquid, and temporal characteristics of the breakdown process have been determined. The breakdown time lag increases with increasing pressure and gap width, and decreases with increasing field. Moreover, it is weakly dependent on the conductivity of the liquid. An Ohmic current-voltage relation has been observed during the prebreakdown stage. A dynamical model has been developed to explain these results and is also presented at this meeting. [Work supported by ONR.]

\section{8:45}

3aPAb2. Processes leading to cavity formation and dielectric breakdown in liquids subjected to high electric fields. Hugh $M$. Jones (Dept. of Phys. and Eng. Phys., Stevens Inst. of Technol., Hobaken, NJ 07030) and Erich E. Kunhardt (Slevens Inst. of Technol., Hoboken, NJ 07030)

The use of liquid-filled gaps as acoustic sources requires an understanding of the contributions from various microscopic processes to the dynamics of current growth, cavity formation, and dielectric breakdown. A model is presented for the development of cavities and dielectric breakdown in liquids subjected to uniform high amplitude electric fields with durations of microseconds or less. In this model, breakdown proceeds in four stages: formation of a nucleation site in the liquid near an electrode, growth and expansion of this site until the local density is reduced sufficiently for electron-impact ionization to occur, growth of an electron avalanche and its transformation into an ionizing front, and propagation of the front via a sequence of processes occurring in the region ahead of the front, namely, heating by electron injection, lowering of the liquid density, and avalanche growth and retardation. Expressions for the duration of each of these stages are derived and their behavior with pressure and field strength analyzed. From this, a critical energy criterion for breakdown is obtained. Comparison is made with experimental results for water, salt solutions, and the liquid noble gases, and for pulse durations in the microsecond and submicrosecond time scales. [Work supported by ONR.]

\section{9:00}

3aPAb3. An experimental investigation of bubble shapes from a nozzle in water. B. K. Choi and S. W. Yoon (Dept. of Phys., Sung Kyun Kwan Univ., Suwon 440-746, Republic of Korea)

The shape oscillations of bubbles from a nozzle in water have been well investigated by Longuet-Higgins [M. S. Longuet-Higgins, J. Fluid Mech. 201, 543-565 (1989)]. In the present paper the elliptical deformation due to a bubble rising in water is introduced in the Longuet-Higgin's theory. The bubble shape oscillations in water are also experimentally observed using a stroboscope photographic technique. The theory with an elliptical deformation condition well describes the experimental observation of bubble shape oscillations. The dependence of antipole time on bubble radius shows good agreement between the theoretical estimation and the experimental observation. [Work supported by the Korea Science and Engineering Foundation.]

\section{9:15}

3aPAb4. Radiation force, equilibrium position, and shape for acoustically levitated bubbles driven above resonance. Thomas $\mathbf{J}$. Asaki and Philip L. Marston (Phys. Depl., Washington State Univ., Pullman, WA 99164-2814)

The equilibrium positions and shapes of stably levitated bubbles on the Earth in an ultrasonic standing wave have been measured and calculated for bubbles larger than resonance size (radius $\approx 140 \mu \mathrm{m}$ ). The method of levitating bubbles typically having radii $>400 \mu \mathrm{m}$ was previously described [T. J. Asaki, P. L. Marston, and E. H. Trinh, J. Acoust. Soc. Am. 93, 706-713 (1993)]. The equilibrium position gives a measure of the radiation force on the bubble. Trapped bubbles exhibit an oblate shape of primarily quadrupole distortion. They are positioned near to and above the axial pressure minimum in the levitation chamber. These results are in good quantitative agreement with predictions based upon the multipole projections of the acoustic radiation pressure on the bubble, taking into account the monopole acoustic response of the bubble. The monopole response is limited by inertia and radiation damping for the size range of bubbles considered. A simplified theory for the shape (based on the deformation resulting from the Bernoulli pressure of incompressible potential flow around a sphere) is generally consistent with the measurements for the smaller bubbles observed. [Research supported by ONR.]

\section{9:30}

3aPAb5. Evolution of surface properties of acoustically levitated bubbles in aqueous solutions and seawater and sources of anomalous damping. Thomas $\dot{J}$. Asaki and Philip L. Marston (Washington State Univ., Pullman, WA 99164-2814)

The complex free-decay frequency was measured for quadrupole oscillations of acoustically levitated bubbles. Single bubbles were stably levitated for periods of up to $3 \mathrm{~h}$. During this time the natural frequency and damping were measured at approximately 2 -min intervals. Bubble oscillations were detected by means of an optical extinction technique. Each free-decay record was taken by abruptly terminating the amplitude modulation used to induce shape oscillations. The method is remarkably sensitive to the presence of impurities which adsorb onto the surface from solution. Damping associated with "clean" small bubbles agrees with predictions associated with viscous and boundary layer considerations for oscillations about a spherical shape. The complex frequency obtained for controlled bulk concentrations of surfactant (Triton X-100) agrees qualitatively with planar surface capillary-wave studies. Salt water solutions and Puget Sound seawater are also examined. Anomalous additional damping was found in association with larger bubbles (radius $\geqq 750 \mu \mathrm{m}$ ) and possible sources will be considered. [Research supported by ONR.]

\section{9:45}

3aPAb6. Phase locking of nonlinear monopoles with reactive coupling. Svetlana Felman, Yuri Kobelev, Lev Ostrovsky, ${ }^{\text {a) }}$ and Irina Soustova (Inst. of Appl. Phys., Nizhni Novgorod, Russia)

Recently it was shown that a system of coupled nonlinear acoustic oscillators such as bubbles in a liquid may generate a coherent radiation starting from the initially incoherent state. However, such an active (radiative) coupling is typically weak, especially if the size of the system is smaller than the acoustic wavelength. Here a stronger, reactive coupling, such as an interaction of bubbles via the potential fluid motion around them, is considered. It is shown that an initial incoherent phase distribution is unstable only for the excitation level exceeding a definite threshold. For a nonlinear stage of generation a three-mode analytical model is used, permitting one to perform a phase-plane investigation of a system and to estimate the amplitude and duration of the generated coherent pulse. From the viewpoint of nonlinear dynamics, such an effect is an example of a "chaos-order" transition in a Hamiltonian system (although chaos may be just a lack of coherence). The role of small dissipation is also considered. Some estimates are made for the ensemble of bubbles in a liquid. ${ }^{\text {a) Now at }}$ Univ. of Colorado at Boulder, CIRES, ETL/ERL/NOAA, 325 Broadway, R/E/ET, Boulder, CO 80303.

$$
\text { 10:00-10:15 Break }
$$

\section{0:15}

3aPAb7. Mass diffusion in spark-Induced vapor bubbles. J. S. Allen, M. A. Averkiou, L. A. Crum (Appl. Phys. Lab., Univ. of Washinglon, Seattle, WA 98105), C. M. Young, and G. R. Hess (Tetra Corp., Albuquerque, NM 87109-4512)

The diffusion of gas into a spark-generated vapor bubble is shown to be an important factor when attempting to generate repeated oscillations of the bubble. Predictions for the radius-time curve based on the Trilling 
equation are matched with experimental measurements and the equilibrium radius is deduced. The effect of the ambient, hydrostatic, and vapor pressures of the liquid on the bubble collapse time is demonstrated. The highfrequency approximation of the first-order solution of the Eller-Flynn formulation for mass diffusion is utilized to estimate the amount of gas diffused into the bubble at the end of a single cycle. An increase in the bubble radius and collapse time is noted and this process is repeated for an extended number of cycles. The frequency signature of the resulting bubble oscillation is analyzed. The increase in the bubble radius and the period of oscillation due to diffusion, and the gas-microbubble residues remaining after collapse, have potentially important effects when generating a repeated sequence of vapor bubbles, as in extracorporeal shock wave lithotripsy. [Work supported in part by the U.S. Navy and the National Institute of Health.]

\section{0:30}

3aPAb8. Acoustic modeling of plasma-induced vapor bubbles. Gary R. Hess, Arturo E. Rodriguez, Niels K. Winsor, Chris M. Young (Tetra Corp., Albuquerque, NM 87109-4512), Lawrence A. Crum (Univ. of Washington, Seattle, WA 98105), and Robert F. Stellingwerf (Los Alamos Natl. Lab., Los Alamos, NM 87544)

Plasma-induced bubbles respond to time-dependent injection of energy which modifies the acoustic output relative to "classical" gas bubbles. Additionally, they consist primarily of hot vapor which complicates the thermodynamics, elevates the importance of some traditionally untreated variables, and requires additional physical process treatments beyond the basic hydrodynamics. A code that is based upon fundamental physical principles was developed to study the importance of many of these variables. The code is capable of treating the actual driving circuit, resultant plasma behavior, transition from cylindrical to spherical geometry early in the discharge, radiation production, plasma chemistry, thermal transport, and hydrodynamics in a modified Flynn formulation. Primary output consists of bubble wall acceleration, radius, etc., energy balance, and far field pressure. The code has been validated with experimental data and will be compared with ongoing hydrocode model development as well. Experimental and theoretical results are presented for large vapor bubbles. [Work supported by the U.S. Navy.]

\section{0:45}

3aPAb9. Acoustic microstreaming within a gas-filled bubble. Wesley L. Nyborg (Dept. of Phys., Univ. of Vermont, Burlington, VT 05405)

When surface waves occur on the boundary of a gas-filled space surrounded by liquid, the resulting motions of the gas include velocity components parallel to the gas-liquid interface. Because these satisfy a nearly nonslip condition, boundary layers are established and small-scale acoustic streaming is generated in the gas phase. Approximate theory suggests that significant convection can occur, and may play a role in sonic enhancement of rate processes which occur at gas-liquid interfaces. For example, intrabubble streaming may contribute to the enhancement of rectified diffusion observed by Gould [J. Acoust. Soc. Am. 56, 1740-1746 (1974)].

\section{1:00}

3aPAb10. A model for cavitation damage during ultrasonic cleaning. Quan Qi, John G. Harris, and Robert E. Johnson (Dept. of Theor. and Appl. Mech., Univ. of Illinois at Urbana-Champaign, 216 Talbot Lab., 104 S. Wright St., Urbana, IL 61801)

A model is proposed in this report to describe the potential growth of an existing crack in the structural component caused by collapsing bubbles during ultrasonic cleaning. A local pressure rise is generated by the collapse of a single bubble and the momentum of the collapse is described by the Kelvin impulse $I(t)$. By assuming a uniform pressure distribution and using dynamic fracture mechanics, we obtain a simple relation that expresses the dynamic stress intensity factor $K(t)$ as a function of the Kelvin impulse. Further simplification is achieved by assuming spherical bubble deformation in the description of the Kelvin impulse, as suggested by Blake and Gibson [Ann. Rev. Fluid Mech. 19, 99-123 (1987)], and this leads to a relation between the dynamics of a single bubble and the stress intensity factor $K(t)$. The time history of $K(t)$ is discussed for a completely collapsed bubble obeying the Rayleigh equation. Once the dynamical stress intensity factor exceeds the fracture toughness $K_{I C}$ crack will start to grow. Repeated action of collapsing bubbles eventually leads to the failure of the component. [Work supported by the Manufacturing Research Center of the University of Illinois.] 


\title{
Session 3aPP
}

\author{
Psychological and Physiological Acoustics: Posters on Hearing \\ (Poster Session) \\ Craig A. Champlin, Chair \\ Communication Disorders, University of Texas, Austin, Texas 78712
}

\section{Contributed Papers}

\begin{abstract}
All posters will be on display from 8:00 a.m. 1o 12:00 noon. To allow contributors an opportunity to see other posters, contributors of odd-numbered papers will be at their posters from 8:00 to 10:00 a.m., and contributors of even-numbered papers will be at their posters from 10:00 a.m. to 12:00 noon. Posters will remain on display until 5:00 p.m.
\end{abstract}

\begin{abstract}
3aPP1. Forward-masked intensity increment thresholds at two recovery times. Lance Nizami and Bruce A. Schneider (Dept. of Psychol., Erindale College, Univ. of Toronto, 3359 Mississauga Rd., Mississauga, ON LSL 1C6, Canada)
\end{abstract}

Forward-masked just-noticeable differences were obtained from two listeners using a two-interval forced-choice procedure. In one of the intervals, a 2-kHz, 200-ms masker preceded a 2-kHz Gaussian-shaped tone pip ( $\sigma=0.5 \mathrm{~ms}$ ). The other interval contained the same masker followed by a comparison tone pip. The listener's task was to identify the interval containing the louder tone pip. Within a block of trails both masker intensity (range $=10-80 \mathrm{~dB}$ SPL) and masker-target time gap ( 3 or $80 \mathrm{~ms}$ ) were kept constant. The intensity of the standard tone pip (before multiplication by the Gaussian envelope) was always set to $20 \mathrm{~dB}$ above the level of the masker. When intensity-increment thresholds are plotted as a function of masker level at both time gaps, thresholds rise to a maximum at a masker level of $50 \mathrm{~dB}$ SPL before declining again. This pattern may be consistent with the idea of two intensity-processing channels, one for louder sounds and one for softer ones [see F.-G. Zeng, C. W. Turner, and E. M. Relkin, Hear. Res. 55, 223-230 (1991)]. [Work supported by NSERC.]

3aPP2. Abstract withdrawn.

3aPP3. Effect of the level difference between leading and trailing stimuli on pitch, loudness, and binaural perception. Shigeaki Aoki (NTT Human Interface Labs.; 1-2356, Take Yokosuka-shi, Kanagawa 238-03, Japan) and Tammo Houtgast (TNO Inst. for Human Factors, Soesterberg, The Netherlands)
The total perception for pitch and loudness as a function of level difference between the leading and trailing stimuli is investigated using the same stimulus configuration and measuring the paradigm which clearly showed that the dominance of the leading stimulus depends on the level difference [S. Aoki and T. Houtgast, Hear. Res. (1992)]. A brief stimulus of $20 \mathrm{~ms}$ is subdivided into two parts with durations $T_{1}$ and $T_{2}$. Each part consists of a sinusoidal signal of either 1 or $2 \mathrm{kHz}$ for pitch perception, or each part consists of a $2-\mathrm{kHz}$ sinusoidal signal with either + or $-3 \mathrm{~dB}$ for loudness perception. The measuring paradigm aims at assessing the critical ratio $T_{1} / T_{2}$ for which both parts contribute equally to the overall sensation of pitch or loudness for these brief stimuli. In contrast to the earlier study on dichotic cues, the effect of the level difference between the leading and trailing stimuli on pitch perception is found to be smaller and discontinuous. The effect on loudness perception is found to be greater and symmetrical. It is also found that the characteristic curves of these perceptions form a meaningful triangular area.

3aPP4. A computational model of auditory localization. Keith D. Martin (The Media Lab., MIT, 20 Ames St., Rm. E15-401, Cambridge, MA 02139)

A novel computational model of localization has been implemented. Using a simplified model of the cochlea filter bank, maximum-likelihood estimates of azimuth and elevation are formed from interaural intensity and phase differences at energy onsets in multiple critical bands. Preliminary results show that the model is capable of resolving front/back confusions and other elevation ambiguities resulting from the "cones of confusion." The onset sampling strategy is intended to model psychoacoustic phenom ena such as the precedence effect and allows the model to deal with multiple sound sources and reverberant environments. Examples of the model's ability to resolve ambiguous signals will be presented and compared with human performance. [Work supported by NSF.]

3aPP5. Efferent adaptation and fatigue: The effect of contralateral noise on spontaneous otoacoustic emissions. Gregory L. Dykstra (Dept. of Phychol., Mezes Hall 330, Univ. of Texas, Austin, TX 78712)

Mott et al. [Hear. Res. 38, 229-242 (1989)] have shown that both frequency and amplitude of spontaneous otoacoustic emissions (SOAEs) can be modulated by contralateral acoustic stimulation. They also examined the effects of stimulus durations up to $4 \mathrm{~min}$ in search of long-term adaptation, but none was found. Because the driven rate of efferent fibers declines by only about one spike per second per minute [M. C. Liberman and M. C. Brown, Hear. Res. 24, 17-36 (1986)], longer durations might be necessary to observe efferent adaptation/fatigue. In the present study, SOAEs of three subjects were measured in the presence of contralateral narrow-band noises with various frequency characteristics and with durations of up to $60 \mathrm{~min}$. The hypothesized effect-an initial decline in SOAEs amplitude under contralateral stimulation followed by a return to preexposure levels with the onset of efferent adaptation-was not consis- 
tently observed. While seen in some sessions for some subjects, it could not be brought under experimental control. Thus these results were in approximate agreement with the findings of Mott et al. Some methodological and functional implications of these findings will be discussed. [Work supported by a NIDCD grant to D. McFadden.]

3aPP6. Processing of nonlinguistic auditory stimuli in Landau-Kleffner Syndrome: A case study. Clifton Kussmaul, Kathleen Baynes (Ctr. for Neurosci., Univ. of California, Davis, CA 95616), and Judy Kegl (Rutgers Univ., Newark, NJ 07102)

"Acquired aphasia with convulsive disorder" or Landau-Kleffner Syndrome (LKS) was first described in 1957 [W. M. Landau and F. R. Kleffner, Neurology 7, 523-530 (1957)]. This language disorder typically manifests as a decline in verbal expression and comprehension in children who had been developing normally. EEG is always abnormal, although clinical seizures are not always observed. This report presents a 26-yr-old right-handed female who was diagnosed with LKS at age 4 with an abnormal EEG and seizure activity. Her EEG, MRI, and audiogram are now normal, but PET demonstrates bilateral temporal lobe hypometabolism. A detailed linguistic comparison of her limited spoken English and relatively good signed English is reported elsewhere [K. Baynes et al., Int. Neuropsychol. Soc. (submitted)]. Interestingly, she listens to and enjoys several types of music. This discrepancy motivates our initial investigation of her ability to perform nonlinguistic auditory tasks. Despite easy recognition of environmental sounds, discrimination thresholds of pitch and rhythm are greatly exaggerated. Such data can clarify which brain processes are specific to language, and which correspond to more general auditory or hierarchical processing. [Work supported by NIH.]

3aPP7. Spontaneous remediation of sensorineural hearing loss in an infant girl. Richard P. Meier (Depts. of Linguist. and Psychol., Univ. of Texas, Austin, TX 78712), Renée A. E. Zakia, and Craig A. Champlin (Univ. of Texas, Austin, TX 78712)

An infant girl (JD) was assessed as having a mild-to-moderate sensorineural hearing loss in her left ear and a moderate-to-severe sensorineural loss in her right ear, based on results of auditory brain-stem response $(A B R$ ) at age 3 months and soundfield testing at 4,5 , and 9 months (Better ear PTA $=55 \mathrm{~dB}$ HL). JD was fitted with hearing aids binaurally. The one risk factor in her birth history is prenatal polydrug exposure, including cocaine. She had recurrent otitis media, but tympanometry and other evidence weigh against middle ear problems as the cause of her hearing loss. A follow-up ABR at 13 months revealed JD to have normal hearing, a result confirmed by audiometric testing of her unaided hearing thresholds (PTA $=10 \mathrm{~dB}$ HL). The implication is that, between ages 9 and 13 months, JD's hearing improved to normal levels, possibly as a result of delayed maturation of the peripheral auditory tracts. Early detection of hearing loss may reveal more children like JD who have sensorineural losses that spontaneously remediate with development. Such cases would highlight the need for repeated testing of unaided thresholds in hearing-impaired infants, especially if aggressive intervention is contemplated. [Work supported by NIH and the Texas Advanced Research Program.]

3aPP8. Acoustic evaluation of pharmacodynamics. D. B. Daly and D. M. Daly (Box 210855, Dallas, TX 75211)

Ability to classify sets of certain synthetic sounds fluctuates with vigilance. In patients with genetic hypersomnia, performance can fluctuate from well-defined classes with narrow transitions to intervals with wide transitions where only stimuli at extremes are identified consistently. LRCs were used to measure whether performance differed from chance and if so, whether it differed from a composite of normals [Daly et al., J. Neurophysiol. 44, 200-222 (1980)]. In a group of subjects independently treated for genetic hypersomnia (11-13 yr old: IQ $>120$; meds: $15-20$ mg methylphenidate, $\tau=2 \mathrm{~h}$ ), rested untreated performance over 4 -min periods in 24 -min intervals changed $\sim 100$-fold (from normal to periods with wide transitions). At 10-12 min following dissolution of single, sublingual 10 -mg doses, transitions narrowed and performance began to improve (re: normal composite). Performance continued to improve for 10-15 min, then remained relatively consistent (in some cases within normal limits for 40-50 min). Performance returned to untreated levels in an orderly fashion: Well-defined transition areas gradually widened, then spread toward extremes. Under similar conditions subsequent dose-response functions for an individual differed little. Name brand and generic preparations apparently dissolve at different rates; some differences among individuals may reflect variations in excipients or compounding.

3aPP9. The effect of a diver's glove on vibrotactile thresholds. Jeffrey D. Travis and Joan Schoppe (Appl. Res. Labs., The Univ. of Texas at Austin, P.O. Box 8029, Austin, TX 78713-8029)

An experiment measuring vibrotactile thresholds in human subjects was performed. Vibration thresholds at frequencies between 100 and 400 Hz under normal conditions were compared with other researchers' published results. To examine how diving gear would affect a sea diver's vibratory sensitivity, vibrotactile thresholds were determined on the middle phalanx of subjects with and without diver's gloves. Two kinds of diver's gloves were tested, one designed for cold water and the other for warm water. It was established that diver's gloves do have an effect on vibrotactile thresholds, and that the specific type of glove (cold water or warm water) alters the normal shape of the threshold curves in different ways.

3aPP10. Personal sound. W. F. Druyvesteyn and R. M. Aarts (Philips Res. Labs., Bldg. WAK, P.O. Box 80000,5600 JA Eindhoven, The Netherlands)

Introducing more audio/video equipment (CD, DCC, TV, CD-I, etc.) into a room creates a new audio problem, one of multiple sound sources with multiple listeners. When different persons, denoted as A, B, C, etc., listen to different programs, denoted as "a," "b," "c," person A experiences program " $a$ " as sound and programs " $b$ " and " $c$ " as noise. Each person would prefer a high sound/noise $(\mathrm{S} / \mathrm{N})$ ratio at his listening position. In this contribution the situation of two persons working with their (multimedia) PCs in an office, or watching in a living room two TVs with accompanying sound, is considered. In order to obtain a specification, listening tests were done, which gave a desired $S / N$ ratio of about 20 $\mathrm{dB}(\mathrm{B})$. In an anechoic environment such a specification is easily realized using a loudspeaker array to control the directivity. In a real (living) room this is not sufficient and active noise control (to decrease $\mathrm{N}$ ) and active sound control (to increase S) are used, particularly in the low-frequency region. During the presentation at the conference, recordings will be reproduced of realized $\mathrm{S} / \mathrm{N}$ ratios of $34 \mathrm{~dB}(\mathrm{~B})$ in the anechoic room and of more than $20 \mathrm{~dB}(\mathrm{~B})$ in a living room.

3aPP11. Multisensory interaction: Influence of spatial visual cues on auditory detection. Dietrich Parlitz (Graduate College Psychoacoust., FB 8, Oldenburg Univ., D-26111 Oldenburg, Germany)

Recent physiological and psychological studies suggest spatiotopic maps of auditory space often build up by groups of multisensory neurons. At this neuronal level stimulus representations of different modalities seem to be transformed into a common coordinate system. A multimodal stimulus may produce enhancement, depression, or no interaction effect on the cell activity, depending on its certain spatiotemporal combination. In several experiments the influence of visual cues on auditory perception was examined. The results indicate a dependence on spatial and temporal parameters. [Work supported by DFG.] 


\title{
Session 3aSP
}

\section{Speech Communication: Vowel Production and Perception (Poster Session)}

\author{
Linda Thibodeau, Chair \\ Department of Speech Communication, Jones Communication Complex, University of Texas, Austin, Texas 78712
}

\section{Contributed Papers}

\begin{abstract}
All posters will be on display from 8:00 a.m. to 12:00 noon. To allow contributors an opportunity to see other posters, contributors of odd-numbered papers will be at their posters from 8:00 to 10:00 a.m., and contributors of even-numbered papers will be at their posters from 10:00 a.m. to 12:00 noon. Posters will remain on display until 10:00 p.m.
\end{abstract}

\begin{abstract}
3aSP1. Extending the perceptual magnet effect to a CVC word context. Richard Eyraud and Patricia K. Kuhl (Dept. of Speech and Hear. Sci., WJ-10, Univ. of Washington, Seattle, WA 98195)
\end{abstract}

Recent research by Kuhl and her colleagues has demonstrated a perceptual magnet effect in vowels and consonants suggesting that perceptual organization is strongly influenced by category goodness. Equidistant stimuli cluster around the best exemplars of a category, demonstrating poorer discriminability and decreased perceptual distances. Conversely, regions surrounding the worst exemplars of a category are "stretched," demonstrating relatively better discriminability and increased perceptual distances. The present experiments extend this finding to a word context by investigating the perceptual organization of the American English vowel /i/ in isolation and in a CVC word context, specifically /bid/. In the vowel experiment, eight variants of $/ \mathrm{i}$ / were synthesized by independently changing $F 1$ and $F 2$. Similarly, in the CVC word experiment, eight variants of the word / bid/ were synthesized by independently changing the vowel steady states of $F 1$ and $F 2$. Adult monolingual speakers were asked to judge the goodness of each variant and the similarity of paired tokens on a seven-point scale. Multidimensional scaling analyses demonstrated a similar perceptual magnet effect in both the vowel and CVC word experiments. These results suggest that the perceptual magnet effect observed with phonetic units extends to the lexical level, possibly contributing to lexical access. [Work supported by NIH.]

3aSP2. Effect of voice quality on the tense/lax distinction for English vowels. Lori L. Holt, Andrew J. Lotto, and Keith R. Kluender (Dept. of Psychol., Univ. of Wisconsin, Madison, WI 53706)

For a variety of East and West African languages, voice quality covaries with tongue root advancement. In these vowel systems, an advanced tongue root vowel is produced with breathy phonation, whereas, a modal voice quality is used with nonadvanced tongue root. A potential explanation for this regularity, based on the interaction between acoustic effects of vocal-tract shape and of voice quality, was evaluated. Because it has been suggested that the advanced tongue root contrast is similar to the tense/lax contrast in English, male and female series varying perceptually from tense to lax were synthesized for several English vowels. Breathy versions of each series were created by increasing spectral tilt. Results from identification tasks indicated that, in general, breathy phonation led to more high vowels being identified as tense. In addition, the effect of breathiness was greater for vowels modeled after female productions. These results suggest that the covariation in African languages may be consistent with the general principle of auditory enhancement and adaptive dispersion. The findings may also be relevant to gender differences in voice quality. [Work supported by NIDCD Grant No. DC-00719 and NSF Grant No. DBS9258482.]

3aSP3. Limitation on labial anticipation in [i] in a language with an [i] $-[y]$ contrast. Cécile Fougeron (Phon. Lab., Dept. of Linguist.,
UCLA, 405 Hilgard Ave., Los Angeles, CA 90024-1543 and Inst. de Phonét. CNRS URA1027, Paris, France)

In French, the acoustic effects of anticipation of a final high front rounded vowel, $[y]$, can be observed in a preceding [i]. This labial anticipation induces a lowering of the formants being measured $(F 2, F 3, F 4)$ both in the middle and, to a greater extent, at the end of the $[\mathrm{i}]$ in $\left[\mathrm{iC}_{\mathrm{n}} \mathrm{y}\right]$ sequences. Although significant, this contextual influence does not jeopardize the acoustic contrast between [i] and [y]. To understand the articulatory basis of these results, acoustic consequences of the gestures involved in the so-called rounding contrast between [i] and [y] are investigated through articulatory modeling. An [i]-like area function is simulated with different degrees and combinations of anticipatory lip protrusion, reduction of the area at the lips, and larynx lowering. [i] allows little variation in lip gestures; beyond a limited range of coarticulation [i] shifts acoustically to the region occupied by [y]. While [i] appears to be quantal when we consider variation in tongue constriction, it shows a sensitive response to lip perturbation. In the [iCy] sequences observed, it seems that constraints on distinctiveness prevent a greater labial anticipation.

3aSP4. Effects of lexical status on native and non-native English-speaking adults' vowel perception. Victoria L. Michela, Lauren A. Randazza, Amanda C. Walley (Dept. of Psychol., Univ. of Alabama at Birmingham, Birmingham, AL 35294), and James E. Flege (UAB)

Monolingual, English-speaking adults and native Chinese (Mandarin) speakers who learned English as a second language heard stimuli from two "native," synthetic continua, in which the vowels ranged from English /I/ to $/ i /$ in the context $/ b_{-} b /$ or $/ b_{-} p /$. Thus the end points of the first continuum constituted an English word and a nonword ("bib" vs * "beeb"); the reverse held for the second continuum (" "bip" vs "beep"). These same subjects also heard stimuli from two "foreign" continua, where the vowels ranged from English / / to a foreign (non-English) vowel $/ Y /$ in the contexts described above. Thus the end points of the first continuum corresponded to a word and a nonword ("bib" vs *"bYb"); both end points of the second continuum corresponded to nonwords ("bip" vs " "bYp"). After training on end points, subjects' identifications of the nine stimuli of a given continuum were examined to assess whether: the Chinese speakers, like native English speakers, exhibit a "lexical bias" effect for English vowels (from the native continua); vowel categories not bounded by another native/English vowel (as in the foreign continua) expand outward or become better defined with increased exposure to English and/or lexical status. [Work supported by NIH.]

3aSP5. Learning to perceive and produce American English vowels: A study of speakers of Brazilian Portuguese. Marialice Szpigel (Dept. Speech, Commun. Sci., and Theatre, St. John's Univ., 8000 Utopia Parkway, Jamaica, NY 11439) and Fredericka Bell-Berti (St. John's Univ., Jamaica, NY 11439 and Haskins Labs., New Haven, CT 06511) 
Brazilians who are fluent in English have more difficulty perceiving and producing American English vowels than consonants. This study examines vowel perception and production by ten native speakers of Brazilian Portuguese who had begun the sixth year of study of American English (at the Instituto Brasil-Estados Unidos, Rio de Janeiro, Brazil). At this level, all students are able to speak English fluently, but with varying degrees of proficiency; within the program constraints, the subjects were chosen to represent different levels of English proficiency. These bilinguals' perception of American English vowels (produced by native speakers of American English) will be compared to the reported perception of American English vowels [G. Peterson and H. Barney, J. Acoust. Soc. Am. 39, 151-184 (1952)]. The bilinguals' productions of 15 American English vowels were identified by six teachers of English (three native Americans and three native Brazilians), with extensive experience teaching English. Ability to perceive the American English vowels will be related to production ability, for the group and for the two best and two poorest students. [Work supported by St. John's University and by NIH Grant No. DC00121 to the Haskins Laboratories.]

3aSP6. Baseline acoustic parameters for nasalized vowels in English and French. Marilyn Y. Chen (Res. Lab. of Electron. and Dept. of Elec. Eng. and Comput. Sci., MIT, Rm. 36-511, Cambridge, MA 02139)

Acoustic analysis of nasalized vowels in the frequency domain indicates the presence of extra peaks, one between the first two formants with amplitude $P 1$ and one below the first formant with amplitude $P 0$; the first-formant amplitude $A 1$ is also reduced relative to its amplitude for a nonnasal vowel. These acoustic characteristics may be explained by speech production theory. The objective of this study is to determine the base line values for the acoustic parameters in quantifying nasality. A1-P1 and $A 1-P 0$ were tested as measures of nasality by comparing vowels adjacent to nasal consonants and those adjacent to stops for English speakers. For high vowels, $A 1-P 1$ is a better parameter to distinguish nasal vowels from non-nasal vowels, with an average difference of $10 \mathrm{~dB}$; however, $A 1-P 0$ is better for low vowels, with an average difference of $7 \mathrm{~dB}$. French nasal vowels adjacent to stops were also examined. To further test these parameters as measures of nasality, they were systematically manipulated in synthetic speech and presented in perceptual experiments. High correlation was obtained between nasality perception and normalized acoustic parameters. [Work supported by NSF, NIH, and LeBel fellowship.]

3aSP7. Dialect differences in vowel production and perception. Alice Faber (Haskins Labs., 270 Crown St., New Haven, CT 06511) Catherine T. Best (Wesleyan Univ., Middletown, CT 06459 and Haskins Labs., New Haven, CT 06511), and Marianna Di Paolo (Univ. of Utah, Salt Lake City, UT 84112)

Earlier work [Faber et al., J. Acoust. Soc. Am. 94, 1865 (1994)] reported differences among American-English-speaking listeners from Utah and Connecticut/NY in perception of the HEEL-HILL and POOL-PULL contrasts (pairs that are nearly merged in Utah but distinct in the northeast US), measured by three tasks, labeling, AXB discrimination, and keyword identification. On these tasks, a few CT/NY listeners (those with parents from the southern US) performed differently from the other subjects. Their vowel spaces also were qualitatively different from those of the other listeners, based on acoustic analysis of three readings of the keywords. The CT/southern listeners had more high back crowding and did better on Utah POOL/PULL than the other listeners, while the Utah listeners had more high front crowding and did better on Utah HEEL/HILL. These results accord with the literature reviewed by Bradlow [Cross-Linguistic Study of Vowel Inventories, Cornell (1993)] relating listeners' ability to discern small vowel differences to the number of vowels in their language, but were tentative because of the small number of $\mathrm{CT} / \mathrm{southern}$ subjects. The current study presents perception and production data from additional Connecticut/NY subjects confirming the original finding. [Work supported by NIH.]

3aSP8. Formant discrimination in femalelike vowels as a function of $F_{1}-F_{0}$ Bark distance. Dennis L. Hughes and Randy L. Diehl (Dept. of Psychol., Univ. of Texas, Austin, TX 78712)

Hughes and Dieh] [J. Acoust. Soc. Am. 95, 2978(A) (1994)] reported that discrimination of first formant frequency for both single and multiple formant stimuli was strongly influenced by both a formant/harmonic interaction and $F_{1}-F_{0}$ Bark distance. No evidence for a peak in discriminability near $F_{1}-F_{0}=3.0-3.5$ Bark was observed, however. The current study extended these findings to stimuli more characteristic of female talkers. Listeners performed a formant discrimination task on one of five series of four-formant stimuli varying in $F_{1}-F_{0}$ distance. $F_{1}$ ranged from 3.2 to 7.7 Bark within a series, and $F_{0}$ from 1.75 to 2.75 Bark between series. Preliminary results confirm both the strength of the formant/ harmonic interaction and the lack of a peak in discriminability at $F_{1}-F_{0}=3.0-3.5$ Bark. Results also point up the need for a more adequate characterization of how formant frequency is represented in the auditory system. [Work supported by NIDCD.]

3aSP9. The perception of vowel height in Castilian Spanish: Effects of varying $F_{1}-F_{0}$ Bark distance. Richard P. Fahey (Dept. of Psychol., Univ. of Texas, Austin, TX 78712) and Luis E. Lopez-Bascuas (Universidad Complutense de Madrid, 8223, Madrid, Spain)

Analyzing American English vowels, Syrdal and Gopal [J. Acoust. Soc. Am. 79, 1086-1100] found that $F_{1}-F_{0}$ Bark distance was highly correlated with vowel height. The $[ \pm$ high $]$ distinction could be based on a boundary at 3-3.5 Bark $F_{1}-F_{0}$ distance. This corresponds to the distance under which the center of gravity effect is thought to occur [Chistovich and Lublinskaya, Hear. Res. 1, 185-195]. Since a feature distinction with an auditory basis is a good candidate for a phonetic universal, one would expect the [ \pm high] boundary to be at 3-3.5 Bark $F_{1}-F_{0}$ distance in many languages. This is true of the [ \pm high] distinction in American English, for both front vowels [Hoemeke and Diehl, J. Acoust. Soc. Am. 93, 2422(A) (1993)] and back vowels [Fahey, J. Acoust. Soc. Am. 95, 2978(A) (1994)]. In the current study, native Castilian Spanish speakers identified synthetic vowel height series ranging between $/ \mathrm{i} /$ and $/ \mathrm{e} /$ or $/ \mathrm{u} /$ and $/ \mathrm{o} /$. Within each set, $F_{0}$ and formant pattern varied orthogonally. Data were analyzed to determine (1) whether $F_{1}-F_{0}$ distance is a correlate of perceived vowel height, and (2) whether the [ \pm high] boundary is at 3-3.5 Bark $F_{1}-F_{0}$ distance.

3aSP10. The effect of removing the fundamental component on the perception of vowel height in stimuli with varying $F_{0}$. Randy $L$. Diehl and Richard P. Fahey (Dept. of Psychol., Univ. of Texas, Austin, TX 78712)

Using front vowels, Hoemeke and Diehl [J. Acoust. Soc. Am. 93, 2422(A) (1993)] found that $F_{1}-F_{0}$ Bark distance is a correlate of perceived vowel height. This finding is consistent with a suggestion that perceived vowel quality depends on the pattern of the neural "place" representation, rather than the absolute position of spectral peaks [Potter and Steinberg, J. Acoust. Soc. Am. 22, 807-820 (1950)]. On this view, vowel perception should be disrupted if a critical component of the place pattern is removed. In the current study, listeners identified synthetic vowels varying between $/ \mathrm{L} /$ and $/ \varepsilon /$, with $F_{0}$ varied orthogonally, in three conditions: in quiet, in quiet with the fundamental component removed, and in low-pass noise with the fundamental component removed. The results reported by Hoemeke and Diehl were replicated in all three conditions: Increasing $F_{0}$ shifted the phoneme boundary towards the $/ \varepsilon /$ end point. There was no apparent decrease in the size of this effect in the conditions where the fundamental component was absent. Thus the role of $F_{0}$ in vowel height perception is not as a formantlike peak in the place representation of the spectrum. [Work supported by NIDCD.]

3aSP11. Priming and the enhancement of concurrent vowels. D. D. Paschall (The Univ. of Texas at Dallas, P.O. Box 830688, GR4.1, Richardson, TX 75083-0688)

Experiments were conducted to determine whether listeners with normal hearing can exploit information in a prior sample of speech to segregate a target voice from a second, competing voice. There are at least two ways that exposure to a prior sample of speech might aid the perceptual segregation of voices. First, peripheral adaptation processes can reduce the auditory response to preexisting sounds in order to enhance newly arriving energy associated with the onset of a second voice. Second, perceptual grouping based on harmonicity or spectrum envelope cues can reduce the interfering effects of preexisting sounds. To separate the contribution of these factors, concurrent vowel pairs were preceded by a precursor which 
had either the same $F_{0}$ and spectrum envelope as one of the vowels ("voiced vowel" precursor), the same $F_{0}$ but a flat spectrum envelope ("buzz" precursor), or the same spectrum envelope as one of the vowels, but with noise excitation ("whispered vowel" precursor). All three types of precursors resulted in increased accuracy when the precursor was brief $(200 \mathrm{~ms})$. When the precursor was $1 \mathrm{~s}$, only the voiced vowel precursor led to an increase in identification accuracy. These results suggest that both adaptation and perceptual grouping may play a role.

3aSP12. Modeling formant frequency discrimination for isolated English vowels using excitation patterns. Yijian Zheng and Diane Kewley-Port (Dept. of Speech and Hear. Sci., Indiana Univ., Bloomington, IN 47405)

Thresholds for formant discrimination across three sets of female and male vowels with different $F 0$ were significantly different in a recent report [Kewley-Port et al., J. Acoust. Soc. Am. 95, 2978(A) (1994)]. This analysis examined whether excitation patterns could model these and other effects of stimulus parameters on formant thresholds. The goal was to determine if an "auditory metric" would be constant across the three stimulus sets when $\Delta F$ thresholds varied by $25 \mathrm{~Hz}$. A separate discrimination study showed that listeners only attend to harmonic components within a restricted region near the formant [Sommers and Kewley-Port, J. Acoust. Soc. Am. 93, 2422(A) (1993)]. Based on those results, four critical bands around the altered formant were selected, and the area between the critical-band spectra for the standard and just discriminable vowel was calculated. This spectral distance across formant frequency and gender was shown to be constant in three analyses: (1) $\Delta F$ threshold differences across the three sets of vowels were no longer significant; (2) slopes for $\Delta F$ thresholds (approximately 1.0 ) were flat for spectral distance; (3) variability of spectral distance across $F 1$ and $F 2$ is significantly smaller than that of $\Delta F$ thresholds. Results suggest that the auditory system has an inherent nonlinear transformation which changes threshold differences to be almost constant in the internal representation.

3aSP13. Modeling listeners' categorization of a large $F 1-F 2-F 3$ continuum. Terrance M. Nearey and Michael Kiefte (Dept. of Linguist., Univ. of Alberta, Edmonton, AB T6G 2E7, Canada)

A number of alternate spectral representations have been suggested for vowel spectra [see H. Hermansky, J. Acoust. Soc. Am. 87, 1738-1752 (1990)]. To better evaluate the perceptual relevance of some of these, 972 vowels were synthesized. The stimuli were each $115 \mathrm{~ms}$ in duration with a falling $F 0$ contour $(125-100 \mathrm{~Hz}$ ). $F 1$ ranged (in 0.5 Bark steps) from 250 to $760, F 2$ from 750 to 2260 , and $F 3$ from 1360 to $3080 \mathrm{~Hz}$. F 4 and $F 5$ were fixed at 3500 and $4500 \mathrm{~Hz}$, respectively. (Constraints were placed on formant separations to ensure relatively natural stimuli.) Fifteen speakers of Western Canadian English categorized the stimuli as the vowels $/ i, 1, e$, $\varepsilon, æ, \Lambda, \mathrm{D}, \mathrm{o}, \mathrm{U}, \mathrm{u}, \Im \%$. Preliminary results indicate that while nominal synthesis formant frequencies can provide a relatively good fit to the data, alternate representations such as cepstral coefficients based on Hermansky's PLP analysis may provide moderate improvements of fit. However, linear transformations of the PLP cepstra show strong correlations with formant frequencies [similar to those noted by D. Broad and F. Clermont, J. Acoust. Soc. Am. 86, 2013-2017 (1985)]. [Work supported by SSHRC.]

3aSP14. Formant movement and duration cues in the identification of vowels. Amy T. Neel and Diane Kewley-Port (Dept. of Speech and Hear. Sci., Indiana Univ., Bloomington, IN 47405)

Traditionally, target values of $F 1$ and $F 2$ are viewed as primary determinants of vowel identity. Several recent studies, however, have demonstrated the importance of dynamic formant information to vowel identification. The present study examines the contribution of both dynamic and durational information to vowel identification using sine-wave vowel analogs. Sine-wave stimuli consisting of two tones representing $F 1$ and $F 2$ were constructed using careful LPC measurements of ten vowels produced in $/ \mathrm{dVd} /$ context by male and female speakers. Four types of stimuli were constructed by varying two factors: (1) appropriate versus fixed vowel duration and (2) variation in tones representing formant movement throughout the token versus static target formant values. Listeners identified the sine-wave vowel analogs using a key-word response form. Results demonstrated that stimuli with appropriate vowel duration were identified with significantly greater accuracy than those with fixed length. For appropriate duration stimuli, there was no significant difference for dynamic versus static tokens. However, for fixed length stimuli listeners identified dynamic tokens with significantly greater accuracy than static stimuli. This suggests that intrinsic vowel duration, as expected, is an important cue to vowel identity and that dynamic formant information is used more by listeners when duration cues are unavailable.

3aSP15. A linear model of boundary shifts in $/ U-L /$ and $/ 0-\varepsilon /$ continua. Anna K. Nabelek and Alexandra Ovchinnikov (Dept. of Audiol. and Speech Pathol., The Univ. of Tennessee, Knoxville, TN 3796-0740)

Boundary locations were tested for $/ U-1 /$ and $/ J-\varepsilon /$ continua with steady-state or linearly changing formants in which $F 2$ was varied. $F 1$ and $F 2$ trajectories had upward and downward directions. Boundary shifts were calculated for changing formant stimuli relative to the boundary for steady-state stimuli. The directions of boundary shifts indicated perceptual emphasis of the final segments of $F 2$ trajectory which might be a consequence of low-frequency spread of masking from $F 1$ to $F 2$. A linear model was developed in which boundary shifts were related to spectral distance between $F 1$ and $F 2$ trajectories. Parameters were initial and final frequencies of $F 1$ and boundary $F 2$ established for each continuum with steady-state stimuli. When the distance was constant in time shifts depended on directions of $F 1$ and $F 2$ trajectories, described by two model terms containing differences of initial and final frequencies of $F 1$ and $F 2$. When the distance was changing in time additional shift was toward the greater spectral distance, described by two model terms containing ratios of initial and final frequencies. [Work supported by NIH.]

3aSP16. The spectral center of gravity effect and auditory filter bandwidth. Marc Fagelson (Dept. of Speech Commun., Prog. in Commun. Sci. and Disord., Univ. of Texas-Austin, CMA 2.200, Austin, TX 78712) and Linda M. Thibodeau (Univ. of Texas, Austin, TX 78712)

The spectral center of gravity refers to a listener's averaging of frequency and intensity components when formant peaks in a speechlike signal are separated by 3.5 Bark units or less. In this paper a total of 18 synthetic vowels whose spectra approximated $/ \mathrm{ae} /$ or $/ \mathrm{N} /$ were generated digitally; each stimulus contained the first 40 harmonics of a $100-\mathrm{Hz}$ fundamental. Nine spectra contained three formants, while the balance contained only two. Subjects with normal hearing and mild high-frequency hearing loss above $3000 \mathrm{~Hz}$ were instructed to identify synthetic vowels as either $/ \mathrm{ae} /$ or $/ \mathrm{N} /$ as $F 2$ frequency was varied between nine different values in $100-\mathrm{Hz}$ steps for both the two-formant and three-formant stimuli. Probit analysis indicated that the normal-hearing subjects identified stimuli more consistently than the mildly hearing-impaired listeners across $F 2$ frequencies for three-formant than for two-formant spectra. The $F 2$ frequency corresponding to the perceived increase in vowel frontness occurred at a lower frequency for normal-hearing listeners. Auditory filter bandwidth was negatively correlated with the $F 3-F 2$ Bark difference. Results suggest that spectral averaging may help listeners disambiguate confusing speech signals.

3aSP17. The effects of pitch changes on the perception of vowel sequences. Benita K. Nordenstrom, Magdalene $H$. Chalikia, and Elizabeth M. Ebsen (Dept. of Psychol., Moorhead State Univ., Moorhead, MN 56563)

When listeners hear a repeated sequence of steady-state vowels (of the same duration and pitch) phonemic transformations occur, and they report hearing words and phrases absent in the original stimulus. A previous study [M. H. Chalikia, R. Meyer, and R. Lindemann, 34th Psychon. Society Meet. (1993)] investigated the possible effects of variations in vowel duration, pitch, or both. Listeners successfully matched these vowel sequences to the verbal forms heard with vowels of equal duration and pitch (base-line stimuli). In this study a broader variation in pitch was employed, and the vowel sounds were computer-generated rather than naturally produced. Six base-line sequences of six 60 -ms vowels (at $100 \mathrm{~Hz}$ ), followed by a $300-\mathrm{ms}$ silent gap, were used. Four variations of these were created by randomly changing the pitch of individual vowels. Most listeners were able to match the modified vowel sequences to the verbal forms heard with the base-line stimuli, thus confirming the robust, stable nature of the verbal 
organizations. It is suggested that these organizations are based upon objective acoustic characteristics, and the present stimulus manipulations were probably perceived as prosodic variations.

3aSP18. The effects of duration changes on the perception of vowel sequences. Magdalene $\mathrm{H}$. Chalikia and Tammy Dresser (Dept. of Psychol., Moorhead State Univ., Moorhead, MN 56563)

Previous studies have shown that listeners exposed to a repeated sequence of steady-state vowels (of same duration and pitch) experience phonemic transformations, and report hearing words and phrases absent in the original stimulus. A previous study [M. H. Chalikia, R. Meyer, and R. Lindemann, 34th Psychon. Society Meet. (1993)] investigated the possible effects of variations in vowel duration. Duration variations ranged from $\mathbf{3 0}$ to $120 \mathrm{~ms}$ per vowel. Listeners successfully matched these stimuli to the verbal forms heard with vowels of equal duration and pitch, confirming the robust, stable nature of the verbal organizations. It was suggested that these organizations are based upon objective acoustic characteristics, and the stimulus manipulations were probably perceived as prosodic variations. In this study a broader variation in duration was employed $(10-300 \mathrm{~ms})$, in an attempt to examine listeners' limitations in performing the matching task. Six base-line sequences of six 80 -ms vowels (at $100 \mathrm{~Hz}$ ), followed by a 300 -ms silent gap, were used. Four variations of these were created by randomly changing the duration of individual vowels within a sequence. Most listeners were not able to perform the task. Implications concerning the perceptual organization of speech will be discussed.

3aSP19. Perceptual implications of selected aspects of vocal behavior. Shari L. Campbell (Dept. of Commun. Sci. and Disord., 576 Aderhold Hall, Univ. of Georgia, Athens, GA 30602)

The perceptual dimensions derived from acoustical changes associated with vowel quality, voice quality, voice classification, and gender are not clearly defined. This project uses the trained singing voice as a source of controlled variation in parameters important to the timbre of both speech and voice. A database of vowels was collected from 18 singers representing six major voice classifications. Repetitions were made of three vowels at three or four fundamental frequencies and at three dynamic levels in normal register. Six subjects also attempted to match loudness (as indicated by a target of equal phons) for this isoparametric condition. Spectral measures (e.g., FFTs, STFTs, 1/3-octave band levels) were transformed in a manner intended to approximate perceptually relevant aspects of auditory processing (e.g., loudness and sharpness densities and loudness and sharpness over time). By comparing the spectral measures with their transformations, it may be possible to make inferences concerning which acoustical changes are perceptually relevant, and about the nature of their perceptual representation. [Work supported by the Institute for Perception Research, Eindhoven, The Netherlands.]

3aSP20. Evaluating speech quality with adaptive psychophysical methods. Muralidhar Kudumala (Elec. Eng., Univ. of Oklahoma, Norman, OK) and Blas Espinoza-Varas (Univ. Oklahoma Health Sciences Ctr., Oklahoma Cily, OK 73190)

This study examines some advantages of adaptive psychophysical tests for obtaining reliable and efficient estimates of speech quality. Specifically, in vowels corrupted by additive noise, noise-detection thresholds were measured adaptively. The 350-ms vowels were obtained by addition of the first 32-35 harmonics of 100-200 fundamental frequencies, with spectral envelopes appropriate for $/ \mathrm{i} /$ and $/ \mathrm{a} /$. Noise was synthesized by randomphase addition of harmonics of a $10-\mathrm{Hz}$ fundamental; the noise bandwidth and the spectral envelope were equal to those of the vowels. In a 2IFC task, subjects had to determine the observation interval that contained a "noisy vowel." During a block of trials, the noise level was varied using a "twodown, one-up" rule (Levitt, 1971); the test terminated when the subject completed 6-8 reversals, and thresholds were defined as the average noise level obtained in the last three reversals. The adaptive method yielded threshold estimates in much fewer trials than a constant stimuli procedure used in previous studies. The adaptive method was implemented in LABVIEw software, National Instruments DSP boards, and a Macintosh computer. [Work supported by OCAST.]
3aSP21. Event-related vertex potentials preceding vowel onset. Nancy Holland and A. Yonovitz (Conley Speech and Hear. Ctr., Dept. of Commun. Disord., Univ. of Maine, Orono, ME 04469)

Neural events prior to speech were determined by averaging vertexrecorded potentials $512 \mathrm{~ms}$ before vocalization. In a manner analogous to auditory-evoked potentials, it would seem plausible to identify specific sites along motor pathways that yield cortically recordable electrical potentials that are precursory to vocalization. In this study, subjects repeatedly phonated a schwa 500 times. The repetition rate was approximately $8 / \mathrm{min}$ with the subject initiating each trial. The onset of vocalization was detected by a throat microphone that provided the trigger. The sample rate (25 000 samples/s) was sufficient to observe brief time periods $(10 \mathrm{~ms}$ ) along the averaged prespeech time epoch. The results indicated that repeatable, biphasic potentials were obtained during the time period between 45 and $250 \mathrm{~ms}$ before the onset of the vowel trigger. Interwave latencies between wavelets were highly related within and between subjects. The technique of event-related potentials may be a useful procedure for studying the electrophysiology of speech production in normal and disordered speakers.

3aSP22. Development of a microcomputer based system for evaluating hypernasality in vowels of cleft palate patients. Kyuta Kataoka (UNC Craniofacial Ctr., Univ. of North Carolina, Chapel Hill, NC 27514), Ken-ichi Michi (Showa Univ., Tokyo, Japan), and Kaoru Okabe (Coretex Corp., Tokyo, Japan)

To evaluate hypernasality quantitatively, frequency analyses were performed on the Japanese isolated vowel /i/ produced by sixteen cleft palate patients with or without speech appliances and four normal subjects. These 26 speech samples were rated by 20 listeners. Analysis of the one-thirdoctave power spectra revealed an increase in power level between the first and second formant and a reduction in second and third formant regions among hypernasal speech samples. Factor analysis of the five-point listener's ratings revealed that the consensus perception of hypernasality accounted for $75 \%$ of the total variance. Multiple regression analysis revealed a high correlation $(0.82)$ between the perception of hypernasality and the peak levels of two clusters of 1/3-octave bands: the sixth, seventh, and eighth 1/3-octave multiples, and the tenth, eleventh, and twelfth multiples from the band containing the fundamental frequency. Based on these findings, a system for evaluating hypernasality was developed using a computer and 1/3-octave bandpass filter. This approach is presently being used to assess the relationship between velopharyngeal port area obtained from pressure-flow study and hypernasality evaluated by this system.

3aSP23. A noninvasive EM based speech feature estimation method for analysis and recognition of vocal fold cancer. Liliana Gavidia-Ceballos (Robust Speech Processing Lab., Dept. of Biomedical Engineering, Duke Univ., Box 90291, Durham, NC 27708-0291) and John H. L. Hansen (Duke Univ., Durham, NC 27708-0291)

The focus of this study is to formulate a speech parameter estimation algorithm for analysis/detection of vocal fold cancer, which does not require direct estimation of the glottal flow waveform. The proposed method separates speech components under healthy and assumed pathology conditions using a mixed excitation speech model. This problem is addressed using an iterative maximum-likelihood (ML) estimation procedure, based on the estimation-maximization (EM) algorithm. Two new features, termed enhanced spectral pathology component (ESPC) and mean area peak value (MAPV) index are estimated and shown to vary consistently between healthy and pathology conditions. For classification, a hidden Markov model recognizer is formulated using MAPV and/or ESPC spectral features. Classifier evaluations using speech recordings from healthy and vocal fold cancer patients for sustained vowels, showed that while MAPV is a useful feature for vocal fold cancer detection (88.7\%), superior performance was achieved using a finer spectral representation of ESPC (92.8\%). Since direct glottal flow estimation is not necessary, the inability to accurately characterize vocal fold pathology due to incomplete glottal closure is no longer an issue. The results suggest that the ESPC feature can provide a noninvasive approach for analysis, detection, and characterization of speech production under vocal fold pathology. 


\title{
Session 3aUW
}

\section{Underwater Acoustics: Moderate-to-High Frequency Bottom Interacting Acoustics III}

\author{
Nicholas P. Chotiros, Chair \\ Applied Research Laboratories, University of Texas at Austin, P.O. Box 8029, Austin, Texas 78713-8029
}

\section{Contributed Papers}

\section{8:00}

3aUW1. Acoustic phase stability in a shallow-water environment. Christine T. Mire and Stephen Stanic (Naval Res. Lab., Code 7174, Stennis Space Center, MS 39529)

The formation of a synthetic aperture is dependent on the phase stability of the medium. Temporal and spatial phase stability of the medium were estimated from measurements taken during a high-frequency experiment conducted in August 1993 in the shallow-water coastal environment off Panama City, Florida. Phase stability was estimated from sequences of 1500.5 -ms and $1.0-\mathrm{ms}$ pulses separated by $1-\mathrm{s}$ intervals at experimental frequencies of $20,40,60$, and $90 \mathrm{kHz}$. Phase fluctuations were found to contain both deterministic and random components which changed with time, indicating a changing propagation environment. Phase fluctuations were spatially correlated between both vertical and horizontal components of the receiving array. The relationship between acoustic phase stability and environmental factors is examined. Results of this analysis are compared with previous phase stability measurements [J. T. Christoff, C. D. Loggins, and E. L. Pipkin, J. Acoust. Soc. Am. 71, 1606-1607 (1982); P. T. Gough and M. P. Hayes, ibid. 86, 837-839 (1989)]. [Work sponsored by ONR.]

\section{8:15}

3aUW2. Acoustic pulse to pulse variability due to seawater temporal temperature fluctuations. $\mathrm{Li}$ Zhang (Ctr. for Marine Sci., Univ. of Southern Mississippi, Stennis Space Center, MS 39529), Ralph Goodman (Penn State Univ., State College, PA 16804), and Steve Stanic (Naval Res. Lab., Stennis Space Center, MS 39529)

Mire and Stanic (preceding abstract) have observed significant pulse to pulse fluctuations in high-frequency seafloor backscattering measurements in which the sound sources and receivers were mounted on a tower that stood on the seafloor in a shallow-water environment (about $30 \mathrm{~m}$ ). This paper presents the hypothesis that temperature fluctuations that are associated with turbulence can account for the acoustic variability. The model is based on a random distribution of fixed discrete scatters on the surface of the seafloor. Computations that sum the arrivals of scattered $\mathrm{CW}$ pulses for a range of changes in travel times that are due to varieties in the average temperature along the paths reveal fluctuations similar to Mire and Stanic's observations for a range of tens of meters, temperatures changes as small as a tenth of a degree Celsius, and frequencies of about $40 \mathrm{kHz}$ and higher. Comparisons between computations and measurement are presented in this paper. [Work supported by NRL/Stennis.]

\section{8:30}

3aUW3. Laboratory measurements of variability in high-frequency bottom backscatter due to thermal fluctuations. P. Jason White, Kenneth E. Gilbert, and Ralph R. Goodman (Appl. Res. Lab. and the Graduate Prog. in Acoust., Penn State Univ., P.O. Box 30, State College, PA 16804)

From the World War II era to the present, researchers in ocean acoustics have observed fluctuations (scintillation) in high-frequency backscatter from the seafloor. In general, the fluctuations have been regarded as a nuisance to be gotten rid of if possible. Here the possibility that the fluctuations may actually provide a means to leam more about the scattering process than would be possible with an unchanging signal is considered. Laboratory experiments are described in which known targets are insonified in the presence of artificially induced thermal fluctuations. The resulting backscattered signals are discussed and analyzed within the framework of a weak scatter theory. Possible applications to ocean data are pointed out. [Work supported by ONR and NRL.]

\section{$8: 45$}

3aUW4. Bistatic bottom scattering at high frequencies: Experiment and model comparison. Kevin Williams and Darrell Jackson (Appl. Phys. Lab., Univ. of Washington, 1013 NE 40th St., Seattle, WA 98105)

In 1993 a special research program on Coastal Benthic Boundary Layers (CBBL) was begun. As part of that program an autonomous, spatially scanning, sonar system was deployed in a well characterized regions in the Baltic Sea and off the coast Florida. The goal was to examine highfrequency acoustic scattering from the sediment. This sonar operated at 40 $\mathrm{kHz}$, had a $5^{\circ}$ horizontal beamwidth, and acquired backscattering data within a $50-\mathrm{m}$ radius area at least once an hour for several weeks at each site. During part of each deployment it operated in conjunction with a mobile receiving array so as to acquire bistatic data. The bistatic experimental apparatus, procedures, and results are presented. Finally, a recently developed bistatic model (driven, in part, by the environmental parameters measured at the experimental site) is tested against the experimental results. [Work supported by NRL CBBLSRP program.]

\section{9:00}

3aUW5. Acoustic backscatter analysis from a smooth sand surface. Julia Laughlin and Nicholas P. Chotiros (Appl. Res. Labs., P.O. Box 8029, Austin, TX 78713-8029)

A multiscattering theory to describe backscatter behavior in waterfilled sands has been developed for smooth sand surfaces. The theory is based on a two-dimensional solution of the diffusion equation and a Biot propagation theory for porous materials. Experiments were conducted at high frequencies $(80-120 \mathrm{kHz})$ to measure backscatter at shallow grazing angles from smooth sand surfaces at varied sand depths. In addition, reflection measurements were made at normal incidence to verify the smoothness of the sand surface. Comparisons of theoretical model backscatter results to experimental measurements were then made. [Work supported by ONR, Ocean Acoustics Program, Code 11250A.]

\section{9:15}

3aUW6. The effect of gradients on high-frequency bottom scattering. John E. Moe and Darrell R. Jackson (Appl. Phys. Lab., Univ. of Washington, 1013 NE 40th St., Seattle, WA 98195)

The first-order perturbation expression for the bistatic scattering cross section of a rough surface [Moe and Jackson, J. Acoust. Soc. Am. (to be published)] is used to study the effect of gradients on high-frequency bistatic bottom scattering. Strong gradients on scales of several centimeters are often observed in shallow-water environments. It is shown that upward 
refraction or reflection by strong gradients can lead to significant enhancement of high-frequency scattering. This enhancement occurs over a wide range of bistatic angles, but is strongest when the incident and scattered grazing angles are equal. These effects can be explained in terms of the reflection coefficient of the corresponding mean (flat) surface. [Work supported by ONR.]

\section{9:30}

3aUW7. Temporal coherence and stability of bottom interacting sound pulses. Xin Tang and Fred D. Tappert (Appl. Marine Phys., RSMAS, Univ. of Miami, 4600 Rickenbacker Cswy., Miami, FL 33149)

Sound propagation at moderate frequencies (depth to wavelength ratio between 20 and 100 ) in shallow-water environments is extremely complicated, variable, and poorly understood due to multiple processes that cover a wide range of temporal and spatial scales. Broadband full-wave numerical simulations are performed with the UMPE model at a large number of geophysical times to display the temporal variability, stability, and coherence of acoustic pulse arrivals in a multipath environment that includes multiple rough bottom forward scattering and multiple forward volume scattering by internal waves that are described by either a Garrett-Munk type of spectral model or a soliton type of model. It is found that the effects of rough bottom scattering change the phase structure while the effects of internal waves account for the observed temporal fluctuations, leading to coherence times of minutes for individual multipaths at $20-\mathrm{km}$ ranges, and a stable envelope having a coherence time of hours. [Work supported by ONR.]

\section{9:45-10:00 Break}

\section{0:00}

3aUW8. Temporal model of high-frequency seafloor acoustic backscatter. Christian de Moustier and Daniel Sternlicht (Marine Phys. Lab., Scripps Inst. of Oceanogr., 9500 Gilman Dr., La Jolla, CA 92093-0205)

A monostatic temporal model of seafloor acoustic backscatter has been developed to investigate the relative contributions of interface roughness and inhomogeneities in the sediment volume for measurements made at various angles of incidence. The model takes into account sonar parameters such as acoustic wavelength, beam pattern, pulse length, and angle of incidence, and it includes a theoretical angular dependence of seafloor scattering strength that is controlled by the roughness statistics of the surface insonified, by its refraction index, and by a volume reverberation term. Simulations have been carried over angles of incidence from $0^{\circ}$ to $60^{\circ}$, and at acoustic frequencies ranging from 10 to $100 \mathrm{kHz}$ for yarious types of substrates. The theoretical angular dependence predicts that the contribution from the sediment volume becomes noticeable for angles of incidence greater than about $6^{\circ}$; however, in the temporal model it is shown that this contribution can be detected closer to normal incidence for small beamwidths (e.g., $2^{\circ}$ ). When applied to a multi-narrow-beam sonar geometry this temporal model shows greater potential for bottom classification than the angular dependence function of acoustic backscatter obtained by integrating the returns received in each beam. [Work supported by ONR.]

\section{0:15}

3aUw9. Spatial coherence of high-frequency $(67-\mathrm{kHz})$ acoustic fluctuations in fully developed turbulence. Michael J. Buckingham, John R. Potter, and Grant B. Deane (Marine Phys. Lab., Scripps Inst. of Oceanogr., La Jolla, CA 92093-0213)

As part of ONR's high-frequency initiative, a field experiment to determine the temporal and spatial coherence of acoustic fluctuations induced by propagation through a fully developed turbulent flow has been planned in collaboration with several other research groups for late September 1994. Narrow-band measurements $(67-\mathrm{kHz}$ coded signals) will be taken in Cordova Channel, British Columbia, where strong, semidiurnal tidal flows (up to $1.0 \mathrm{~m} / \mathrm{s}$ ) create fully developed turbulence (Reynolds number $\approx 10^{7}$ ), and strong mixing prevents stratification (bulk Richardson number $\approx 0.02$ ). Prior experiments have established the turbulent nature of the flow and examined the horizontal arrival angle and intensity fluctuations with time. In the present experiment, a new compact acoustic array, consisting of 128 hydrophones in an elliptical configuration, is being adapted to monitor the acoustic arrivals in the vertical and horizontal, with finer spatial resolution than has previously been obtained. Amplitude and phase data will be recorded from 15 channels. Environmental data from ADCPs, turbulence meters, side-scan sonars, microstructure instruments, CTDs, etc., will be available to help interpret the acoustic results. Preliminary results of the acoustic fluctuation experiment will be presented, with an analysis of the fine-scale phase and intensity coherences, which are intimately related to the microstructure of the transmitting medium.

\section{0:30}

3aUW10. High-frequency sound variation in a coastal environment. Jules S. Jaffe (Marine Phys. Lab., Scripps Inst. of Oceanogr., La Jolla, CA 92093-0238)

Despite the proliferation of high-frequency sonar imaging systerns, the variability of high-frequency sound propagation in shallow water has only recently been measured. In a series of experiments adjacent to the Scripps Institution of Oceanography pier, the variability of sound at a frequency of $450 \mathrm{kHz}$ has been measured. The experimental measurement consisted of deploying a multibeam imaging system on a tripod in approximately $20 \mathrm{ft}$ of water. Repeated insonification and subsequent measurement of the backscattered waveforms indicate that a large degree of variability can exist in the backscattered data. In order to define whether the source of the variability in the backscattered data was originating from the bottom or the volume, a corner reflector was deployed. Assuming that the variability in the sound reflected from the bottom consists of the superposition of both volume and the bottom variability, and that the sound from the corner reflector consists of variability only from the volume, assignment of the relative importance between the two forms of variability can be made. The importance of these two sources of variability as a function of environmental conditions such as tide and surface conditions will be presented. [Work supported by ONR.]

\section{0:45}

3aUW11. Dereverberation of shallow-water, high-frequency signals. Jean O. Nam and J. Robert Fricke (Dept. Ocean Eng., Cambridge, MA 02139)

Propagation of high-frequency signals, $O(10 \mathrm{kHz})$, in a shallow-water channel, $O(10 \mathrm{~m})$, is complicated by multipath effects associated with the surface and bottom. The goal of this research is to dereverberate the time spread and dispersed received signal into a processed signal with a temporal duration comparable to that of the outgoing pulse. Given the impulse response of the channel and the received signal, an inverse filtering method utilizing minimum-phase components can be effective. However, because of channel dynamics caused by turbulence, internal waves, and thermal stratification, calculation of the exact impulse response is impossible. A more robust signal recovery method, known as cepstral smoothing, has been developed to separate the signal from the channel impulse response in the cepstral domain. Using this method, the recovery is independent of the channel impulse response and is robust in the presence of additive noise. The cepstral smoothing method dereverberates the original signal, but absolute time delay information is lost. A moving rms window applied to the original received signal recovers the lost time delay.

\section{1:00}

3aUW12. Shallow-water reverberation measurement and prediction. James H. Miller, Charles E. Muggleworth, Ching-Sang Chiu (Code EC/ Mr, Naval Postgraduate School, Monterey, CA 93943), and James F. Lynch (Woods Hole Oceanogr. Inst., Woods Hole, MA 02543)

In August 1992, a shallow-water, low-frequency reverberation measurement was made in the Barents Sea utilizing explosive SUS charges as sound sources and a middepth hydrophone as the receiver. The objectives 
of this work were to analyze the reverberation data from this experiment, compare several theories which have been proposed to model reverberation, develop a technique for estimating the bottom backscattering coefficient in shallow water from single hydrophone acoustic data, and determine the reverberent characteristics of the experimental region. The threedimensional Hamiltonian Acoustic Ray-tracing Program for the Ocean (HARPO) was used as the primary propagation modeling tool. The temporal signal processing consisted of a short-time Fourier-transform spectral estimation method applied to data from the single hydrophone. Chapman's source spectrum model was used. A bistatic Lambert's Law with a frequency-dependent constant was found to fit the data from the experiment. A statistical analysis showed that the reverberation from the Barents Sea experiment was Gaussian to a high degree of confidence.

\section{1:15}

3aUW13. Shallow-water backscatter reduction through selective mode excitation. D. F. Gingras (SACLANT Undersea Res. C1r., Viale San Bartolomeo, 400, 19038 La Spezia, Italy)

In shallow-water areas the utilization of active sonar is complicated by the fact that in these environments the performance of active sonar systems is degraded by backscatter generated by source signal interaction with channel boundaries. In this paper knowledge about the shallow-water acoustic channel, namely the normal-mode structure, is used to design vertical source arrays which minimize source signal boundary interaction. The boundary signal interaction is minimized for many environments by exciting only the first mode. Mode one excitation is accomplished by weighting the array sources by the amplitude and polarity of the mode one eigenfunction. Simulations across the frequency range from 200 to $700 \mathrm{~Hz}$ using a two-way PE backscatter model were conducted. It was shown that a vertical array of sources, containing only 15 elements, can reduce bottom generated backscatter by as much as $15-20 \mathrm{~dB}$.

\section{1:30}

3aUW14. Source localization via received arrival time structure analysis. S. A. Stotts and N. R. Bedford (Appl. Res. Labs., The Univ. of Texas at Austin, P.O. Box 8029, Austin, TX 787123)

An efficient and robust method has been developed to locate impulsive sources in an ocean environment. A sonobuoy field was first deployed in a ring-type pattern. GPS receivers were installed on the buoys to obtain their locations within a few meters of accuracy. Charges were then set off at arbitrary locations within the ring. High-resolution plots were used to obtain direct path and first bottom bounce anivals on each buoy. A model grid of arrival times was constructed, corresponding to the dimensions of the buoy field. A ray model previously developed here at ARL:UT [E. K. Westwood and P. J. Vidmar, J. Acoust. Soc. Am. 81, 912-924 (1987)] was used to obtain model travel times. The minimum value of the least-square error between the real arrival times and the modeled travel times resulted in an unambiguous location of the source, within the limits of the grid spacing chosen. This value was calculated by picking one phone as the reference and then summing the errors of each phone relative to the reference. Successive iterations with finer grid spacings results in source location within the accuracy of the buoy locations. Results obtained at Lake Travis and the Gulf of Mexico will be presented. [This work is supported by Navy Strategic Systems Programs.]

\section{$11: 45$}

3aUW15. Broadband source Iocalization in a shallow-water environment with a small bottom slope. David $P$. Knobles and Robert A. Koch (Appl. Res. Labs., The Univ. of Texas at Austin, Austin, TX 78712)

Results from an analysis of matched-field source localization in range and depth for a range-dependent shallow-water ocean environment are presented. The matched-field localization is based on a broadband crosscorrelation technique. The measured data are the time series of acoustic arrivals that propagated to a bottom mounted array from small omnisources at a location $0.5-30 \mathrm{~km}$ downslope. Over these ranges the water depth was $125-180 \mathrm{~m}$. The measured data contain many strong multipaths because the acoustic environment was a downward refracting water column and a seafloor with a thin sand-silt sediment over a thick limestone layer. Matching fields for the localization of the sources in the measured data were computed with a broadband adiabatic normal-mode model in the $100-500-\mathrm{Hz}$ band. The presentation also includes a comparison of the measured and modeled time series as a validation of the environmental and propagation descriptions. [Work supported by the Office of Naval Research (Code 322). The data measurements were sponsored by ARPA.]

WEDNESDAY MORNING, 30 NOVEMBER 1994

NUECES ROOM, 9:00 A.M.

\title{
Meeting of Accredited Standards Committee S2 on Mechanical Vibration and Shock
}

to be held jointly with the

\section{U.S. Technical Advisory Group (TAG) Meeting for ISO/TC 108 Mechanical Vibration and Shock}

\author{
D. J. Evans, Chair S2 \\ National Institute of Standards and Technology (NIST), Building 233, Room A 147, Gaithersburg, Maryland 20899
}

D. F. Muster, Chair, U.S. Technical Advisory Group (TAG) for ISO/TC 108, Mechanical Vibration and Shock 4615 O'Meara Drive, Houston, Texas 77035

\begin{abstract}
Standards Committee S2 on Mechanical Vibration and Shock. Working group chairs will present reports of their recent progress on writing and processing various shock and vibration standards. There will be a report on the interface of $\mathbf{S} 2$ activities with those of ISO/TC 108 (the Technical Advisory Group for ISO/TC 108 consists of members of S2, S3, and other persons not necessarily members of those committees) including a report on the activities of ISO/TC 108, including its September 1994 meeting in Berlin, Germany.
\end{abstract}

Scope of S2: Standards, specifications, methods of measurement and test, and terminology in the fields of mechanical vibration and shock, and condition monitoring and diagnostics of machines, but excluding those aspects which pertain to biological safety, tolerance and comfort. 


\title{
Session 3pEA
}

\section{Engineering Acoustics: Acoustical Measurement and Instrumentation II}

\author{
Elmer Hixson, Cochair \\ Department of Electrical and Computer Engineering, University of Texas, Austin, Texas 78712 \\ Kevin Baugh, Cochair \\ Applied Research Laboratories, University of Texas, P. O. Box 8029, Austin, Texas 78713-8029
}

Contributed Papers

\section{1:00}

3pEA1. Target response measurement system for the NUWC Lake Seneca Test Facility. David M. Deveau, Lynn F. Carlton (Naval Undersea Warfare Ctr. Range Development Div., B104/Code 3822, Newport, RI 02841), and Peter J. Stein (Scientific Solutions, Inc., Nashua, NH 03062)

Here is presented an overview of the target response measurement system (TRMS) deployed at the NUWC Seneca Lake test facility. This system is capable of measuring the target strength of objects at ranges from 10 to $300 \mathrm{~m}$. The frequency range for monostatic measurements is from $300 \mathrm{~Hz}$ to $400 \mathrm{kHz}$. The current bistatic capability is measurements every $5^{\circ}$ over a $90^{\circ}$ window from 2 to $15 \mathrm{kHz}$. A multichannel PC-based digital data-acquisition system has been developed which includes high-speed A/D boards, baseband translation, and real-time monitoring of TS as a function of target aspect angle. Target aspect angle and acoustic source signatures are measured at the target and telemetered to the acquisition for digital storage along with the acoustic data. Accuracies of $1 \mathrm{~dB}$ in TS and $1^{\circ}$ resolution in target aspect angle are possible. Measurements are generally clutter limited to a target strength less than $-30 \mathrm{~dB} r e: 1 \mathrm{~m}$. Clutter of the target harness is usually a major issue and is discussed. The target strength of various targets and support structures is presented.

\section{1:15}

3pEA2. Five axis programmable positioning system for underwater acoustic measurements at Penn State. Kyle M. Becker (Graduate Prog. in Acoust., Penn State Univ., Appl. Res. Lab., Student Area, P. O. Box 30, State College, PA 16804)

To facilitate underwater acoustic measurements at Penn State, a five axis computer controlled positioning system has been developed by the author and installed in the new water tank facilities at the Applied Research Laboratory. Providing linear motion in three directions and rotations about two axes, the system allows an acoustic transducer to be positioned and oriented at virtually any position and direction within the water tank. Motion is controlled along each axis via programmable controllers (indexers) which are in turned accessed by a computer via RS 232. Resolution in the linear directions is $0.005 \mathrm{in}$. Angular resolution is $0.18^{\circ}$. Working in conjunction with the appropriate data-acquisition hardware and software, the positioning system allows the entire measurement procedure to be automated. Although designed initially for use in monostatic and bistatic surface scattering experiments, the system is also being used for other interesting underwater experiments, including bottom sediment studies, and the effects of thermal gradients on acoustic signals.

\section{$1: 30$}

3pEA3. Wideband reverberation reduction in long-time acoustic signals produced by transducers in small test chambers. Niels $\mathrm{K}$. Winsor, Gary R. Hess, Forest E. White, Chris M. Young (Tetra Corp.,
3701 Hawkins St. NE, Albuquerque, NM 87109-4512), and Russ Roberts (Enigmatics, Inc., New Harmony, UT 84757-0537)

For times long compared to the acoustic wave transit time in a test tank, the signal from point acoustic sources may be dominated by internal reflections and the modal response of the experimental tank in which experiments are performed. If the free-field source behavior of the acoustic source is desired, it can be extracted by taking advantage of special properties of the source/tank system. The transducer can be operated in a manner which permits characterization of the tank response; then the processed results can be used to reduce the effects of the tank response in the signals of interest. Spectral methods for this signal processing will be described and illustrated. The result is that signal characteristics in spectral regions of interest can be more clearly resolved and enhanced. [Work sponsored by U.S. Navy.]

\section{$1: 45$}

3pEA4. The design, construction, and performance testing of an acoustical agglomeration chamber. Laura A. Dudes, M. G. Prasad, and R. F. McAlevy, III (Dept. of Mech. Eng., Stevens Inst. of Technol., Castle Point on the Hudson, Hoboken, NJ 07030)

An acoustical agglomeration system is an apparatus which can be used to enhance the efficiency of current particulate removal devices. This study presents the design and construction of an acoustical agglomeration chamber and associated measurement instrumentation. The system consists of a main chamber $3.05 \mathrm{~m}$ long with an inner diameter of $0.075 \mathrm{~m}$. The dimensions are sufficient to develop the particulate gas stream as well as acoustical plane-wave fields up to $2500 \mathrm{~Hz}$. The inlet flow velocities of the gas stream range from 0.5 to $5 \mathrm{~m} / \mathrm{s}$ with operating pressures ranging from 101 to $1010 \mathrm{KPa}$. A midrange (e.g., $200-4000 \mathrm{~Hz}$ ) heavy duty electroacoustic driver is used as the sound source. The particulate size distribution is optically measured with a Malvem 2600 laser particle sizer. A silencer was used to reduce the noise level coming out of the agglomeration chamber. The performance testing of the agglomeration apparatus was carried out using aluminum oxide particulate with frequencies of 1 and $2 \mathrm{kHz}$ in the range of sound-pressure levels between 140 and $160 \mathrm{~dB}$. An increase in particulate size from 16 to $26 \mu \mathrm{m}$ was achieved in the preliminary measurements. This study will have applications in reducing emission of hazardous, fine particulate into the atmosphere. [Work supported by New Jersey Hazardous Substance Management Research Center.]

\section{2:00}

3pEA5. Characterizing the performance of piezoelectric ceramic as a function of stress, electric field, and thermal history. R. Lowell Smith and Alan V. Bray (Texas Res. Inst., 9063 Bee Caves Rd., Austin, TX 78733)

The relatively recent development of large flextensional and other lowfrequency, high-power transducers has stimulated renewed interest in the performance of piezoelectric ceramic under conditions of high stress and 
electric field. This paper addresses the development of special fixturing for the assembly and testing of structures emulating the use of PZT-8 material in high-power projectors. The test fixtures themselves are Tonpilz oscillators. Using a system of four tie rods, axial loads as high as $2.2 \times 10^{5} \mathrm{~N}$ ( $50000 \mathrm{lbf}$ ) can be applied. The tie rods are fitted with strain gauges so that load can be monitored as often as desired during the course of aging and stress and electrical drive sensitivity studies. Initially data were acquired for combined static and dynamic stress in a controlled thermal environment. The performance characteristics of the piezoelectric ceramic are inferred from electrical admittance measurements. Providing for the incorporation of extensometers and closed-loop control of axial stress allows the fixtures to be used for a number of broader applications such as measurement of the viscoelastic properties of ceramic. [Work supported by SPAWAR.]

\section{2:15}

3pEA6. Determination of effective piston area by three methods. $S$. L. Garrett and D. F. Gaitan (Phys. Dept. Code PH/Gx, Naval Postgraduate School, Monterey, CA 93943)

The volumetric velocity of an acoustic source is sufficient to characterize its source strength if the acoustic impedance of the load presented to the source is known. Although a calibrated accelerometer can accurately determine the velocity of a piston, the volumetric velocity requires a determination of the effective area of the "piston." For a thermoacoustic refrigerator operating at resonance, this load is purely resistive. Three methods for determining the effective area of an electrodynamic thermoacoustic driver will be presented. For in situ measurements, good agreement ( $\pm 2 \%-4 \%$ ) was obtained between direct measurement of the force, using the known $B l$ product and the resonant reciprocity method using the transfer impedance calculated by Rudnick [G. W. Swift, A. Migliori, S. L. Garrett, and J. C. Wheatley, Rev. Sci. Instrum. 53, 1906-1910 (1982)]. The result for effective area obtained by measurement of pressure under the reactive load presented by a small coupler [T. Hofler, J. Acoust. Soc. Am. 83, 777-786 (1988)] was smaller and more pressure dependent. A possible explanation for this discrepancy, based on bellows deformation under different loading, will be presented. [Work supported by ONR, the Naval Postgraduate School Direct Funded Research Program, and the Navy Science Assistance Program.]
3pEA7. Measurement of throat impedance and far-field directivity for acoustic horns. John T. Post (Klipsch Professional, P. O. Box 1320, Hope, AR 71801) and Elmer L. Hixson (Univ. of Texas, Austin, TX 78712)

Experiments suitable for the measurement of throat impedance and directivity of acoustic horns were designed and implemented using an axisymmetric exponential and tractrix horn. The horns have equal length $(55.9 \mathrm{~cm})$, throat radius $(2.54 \mathrm{~cm})$, and mouth radius $(27.1 \mathrm{~cm})$. A modern implementation of the "reaction on the source" method was compared with a two-microphone method for the measurement of acoustic impedance. The indirect reaction on the source method was found to be less accurate but has the capability of in situ-type impedance measurements. The far-field pressure was derived by measuring the near-field pressure and mathematically extrapolating to the far field, a technique which has been used in the area of antennas since the early 1970s.

\section{2:45}

3pEA8. Measurement of three-dimensional transient motion of surfaces by optical vibrometry. Joseph Vignola, Harry Simpson (SFA, Landover, MD 20785), and Brian H. Houston (Naval Res. Lab., Washington DC 20375-5000)

A three-dimensional laser vibrometer for measuring simultaneously the three components of vibration induced displacement on a surface has been constructed and tested [Vignola and Houston, J. Acoust. Soc. Am. 94, 1853(A) (1993) and ASME Winter Meeting, 93-WA/NCA-10 (11/93)]. The device, which is fully submersible, was developed for structural acoustic studies where measurements of full three-dimensional motion can provide insight into the analysis of the sound radiated by a structure. This presentation is principally concerned with the methodology and design of the signal processing schemes for the detection of transient events. Some preliminary data are shown together with an analysis of the sensitivity, noise sources, and other performance characteristics of the system. The data presented has been collected from both a test structure (a piezoelectric cylinder with three accelerometers for comparison) and in a water tank half-filled with filtered sand (mean diameter of $140 \mu \mathrm{m}$ ). In the sand experiment the motion of the water-saturated sand interface was monitored.

\title{
Interdisciplinary: Hot Topics in Acoustics
}

\author{
Uwe J. Hansen, Chair \\ Physics Department, Indiana State University, Terre Haute, Indiana 47809
}

A special session on "Hot Topics on Acoustics" is presented at each meeting of the Society. A member is chosen from each of three or four of the Society's technical committees or specialty groups to present a tutorial talk on topics of current special interest. The talks are intended to help acousticians become familiar with issues and achievements that are not within their own primary fields of interest.

\author{
Chair's Introduction-1:30
}

\section{Invited Papers}

\section{1:35}

3pID1. Hot topics in architectural acoustics. Gary W. Siebein (Arehitecture Technol. Res. Ctr., Univ. of Florida, 231 ARCH, Gainesville, FL 32611-2004)

This lecture will review some of the current topics of interest in the architectural acoustics community. There has been much recent work in trying to determine what physical factors contribute to subtle perceptions of spaciousness and source width in rooms. Efforts 
to standardize measurement techniques for recently proposed acoustical measures derived from the impulse response of rooms is underway as well as experiments to determine the utility of these measures in applied situations. These measures are also predicted in rooms by tests in physical scale models and computer models. Aural simulation of the effects of the architectural features of rooms on the qualities of music and speech is also being pursued. Measurements of the effects of flanking transmission, plumbing noise, and impact noise are also being refined as are recommended methods for assessing background noise levels in rooms. A significant area of activity in the architectural community that differentiates it from other technical committees occurs through the design and construction of real buildings to meet design goals based on current understandings of sound perception and propagation. Several case studies of actual buildings that have been designed to test some of the emerging knowledge gained in the laboratory will be presented.

1:55

3pID2. Hot topics in acoustical oceanography. Lawrence A. Crum (Appl. Phys. Lab., Univ. of Washington, 1013 N.E. 40 th St., Seattle, WA 98105-6698) and Michael J. Buckingham (Scripps Inst. of Oceanogr., La Jolla, CA 92093-0213)

Since acoustical oceanography was given full Technical Committee status within the ASA in November 1991, there has been a rapid development of novel techniques for interrogating the ocean using sound. For example, the total global precipitation amount is poorly known; however, by examining the underwater noise produced by rainfall, it may be possible to use underwater acoustic monitoring devices to obtain an estimate of this precipitation and from that better estimates of the global heat flux. Recently, it has been demonstrated that the sound pulses can be propagated over extremely large distances in the ocean; consequently, a precise timing of the arrival of these sound pulses over extended periods would enable changes in the average ocean temperature to be monitored, and thus provide information on global warming. Similarly, ambient noise provides the basis of a new acoustic imaging technique, designated acoustic daylight, that is analogous to conventional photography but based on sound rather than light. These and a variety of other topics will be presented.

\title{
2:15
}

3pID3. Increasing the Acoustical Society's role in noise control and noise effects. B. M. Brooks (P.O. Box 322, Vernon, CT 06066), T. J. Dubois (Tujunga, CA 91042), R. M. Hoover (Houston, TX 77082), G. C. Maling (Poughkeepsie, NY 12603), and L. C. Sutherland (Rancho Palos Verdes, CA 90274)

At the meeting of the Technical Committee on Noise at the fall 1991 meeting of ASA in Houston, a discussion was held on how, or if, the Acoustical Society should develop more concrete policies or action concerning noise control and noise effects. As a result of that discussion, a Noise Task Group was formed by the authors at the direction of the Chair of the Technical Committee to explore the issues involved. Since that time, several special sessions have been held to help establish a direction for this activity. This talk will briefly review some of the more important elements of that activity which, properly, are beginning to involve members of other technical committees within the Society in such areas as hearing screening tests at ASA meetings, development of lecture materials for use in presenting talks to youth on acoustics and noise, and potential development of room noise criteria and/or rating schemes for meeting spaces. Other such action areas that have evolved from these special sessions will be mentioned. These offer other opportunities for ASA members to help achieve a goal worthy of the Society-to preserve quiet and tranquility in concert with advances in technology and population growth.

WEDNESDAY AFTERNOON, 30 NOVEMBER 1994

PECOS ROOM, 1:00 TO 3:15 P.M.

\section{Session 3pSA}

\section{Structural Acoustics and Vibration: Numerical and Physical Experimental Methods}

\author{
Takeru Igusa, Chair \\ Civil Engineering Department, Northwestern University, 2145 Sheridan Road, Evanston, Illinois 60208
}

\section{Contributed Papers}

1:00

3pSA1. Wave propagation in truss structures. Yueping Guo (Dept. of Ocean Eng., MIT, Cambridge, MA 02139)

A wave-based approach is used to study truss vibrations. Each truss member is treated as a wave-bearing system so that the number of unknowns is the same as the number of wave types, independent of the physical dimension and frequency. A global matrix system is then formed to solve the overall problem, which, because of the wave approach used for the truss members, is very sparse and numerically absolutely stable. Thus even large-scale trusses can be studied with computation that is almost trivial. An example is given for a truss with 35 joints, connecting 109 beams, which is also used in our experimental studies. Results are obtained to discuss features such as pass/stop bands, modal density, and energy sharing between different wave types. [Work supported by ONR.]

\section{1:15}

3pSA2. Effect of mass loading on the dynamic behavior of a three-dimensional truss. Joseph E. Bondaryk and Ira Dyer (Dept. of Ocean Eng., MIT, 77 Massachusetts Ave., Rm. 5-435, Cambridge, MA 02139)

Recent trends in submarine design suggest the use of trusslike structures, connected to the hull by a limited number of attachment points, to 
support rotating machinery and decks with passive loads. The ratio of the supported mass to the truss mass is high, typically $5-10$. This loading must affect the dynamic response of the truss. It is believed that resiliently mounted mass will have a "fuzzy" effect on the truss and result in damping of structural waves. The MIT Structural Acoustics Group is making experimental measurements on a 1:15 scale model of a nonpractical, 3D, submarine truss, constructed of 0.5 -in. aluminum tubing, up to frequencies of $5 \mathrm{kHz}$. The dynamic response of two identical trusses, one unloaded and one mass-loaded at a ratio of 5:1, to octave-band noise are measured and compared. The results show the effects of passive and resiliently mounted mass loading on a truss structure. [Research supported by ONR.]

\section{$1: 30$}

3pSA3. Influence of dynamic absorbers on a three-dimensional truss. Denis Branthonne and Ira Dyer (Dept. of Ocean Eng., MIT, Cambridge, MA 02139)

The objective of this research is to explore experimentally the effects of adding dynamic absorbers to a three-dimensional truss and to design analytical models which predict the phenomena of attenuation. The truss is built with cells made of aluminum rods and connected in series. $110 \mathrm{dy}-$ namic absorbers are mounted on the rods in the center cell to achieve a local mass ratio of 3 . The truss is excited at one end with white noise to measure the spatial attenuation averaged on octave bands as a function of axial distance. The data are compared to the spatial attenuation for the undamped structure. The differences determine the effect of the dynamic absorbers. The overall shape of the differential curves of attenuation versus axial distance is a step function, with the step located in the dynamic absorbers attachment area. Assuming the equipartition of energy between the different wave types and using the classical theory of attenuation of waves propagating on a semi-infinite rod loaded with a continuous layer or dynamic absorbers, one predicts stronger attenuation than experimental data at low frequencies. The assumption of equipartition of energy proves to be incorrect and the applicability of the semi-infinite model is questioned. Subsequently, a new theory is derived and validated by experiment to describe the attenuation of waves on finite rods loaded with a layer of dynamic absorbers. At low frequencies, this model achieves a better estimation of the axial attenuation along the truss. [Work supported by ONR.]

\section{$1: 45$}

3pSA4. The effect of surface characterization and laser beams polarization on laser Doppler vibrometry. Ming Yang, Jacek Jarzynski, and Yves H. Berthelot (School of Mech. Eng., Georgia Inst. of Technol., Atlanta, GA 30332)

In a laser Doppler vibrometry, the characterization of the measured surface plays an important role in both in-plane and out-of-plane surface motion measurements. Experimental data is presented for the in-plane optical probe configuration, where the vibrating surface is illuminated symmetrically by two laser beams. However, some of the conclusions of the present study apply also to out-of-plane vibration measurements. It is found that certain surfaces perform better than others. How the surface character affects the in-plane surface motion measurement (in particular, the signal-to-noise ratio and the probe alignment) is studied. The surfaces studied are lathe-finished, polished aluminum and steel surfaces and two types of retroreflecting tapes from 3M company. Also, the profiles of the metallic surfaces are obtained with a profilometer. A simple model is used to relate the surface characteristics to the performance of in-plane motion measurement. The effect of polarization of the two laser beams is also studied, including the depolarization of the light by the surface. [Work supported by ONR.]

\section{2:00}

3pSA5. Dispersion of longitudinal waves in periodic septate liquid-elastic waveguides. L. Sheiba (EG\&G WASC, Inc., 1396 Piccard Dr., Rockville, MD 20850)

The propagation of longitudinal waves in a composite liquid-elastic waveguide is analyzed. The waveguide is a periodic structure consisting of alternating liquid-elastic cylinders joined by rigid septa. The elastic ma- terial (Lamé constants: $\mu \ll \lambda$, Poisson relation $\sigma \Rightarrow 0.5$ ) and the liquid material $(\mu=0, \sigma=0.5)$ are assumed to have low compressibility, and the septa are rigid and weightless. Consequently, the boundary between the cylinder and the septum's radial displacement is absent, and the axial displacements are planar. The admittance matrix $\mathbf{Y}$ of the unit cylinder is initially constructed within a framework of the hypothesis of plane cross sections, neglecting strains induced by hydrostatic stress. Hydrostatic stress waves are approximately included by adding to the unit cylinder admittance matrix correction $\Delta \mathbf{Y}$ associated with the strains of hydrostatic stress in the cylinder. Transfer matrix of the liquid-elastic waveguide and its elements, expressed through the waveguide sections elastic parameters, have been obtained. It has been shown that damping in a waveguide with a periodic structure is much greater in comparison with a regular waveguide, even when the latter is made with a high-loss material.

\section{2:15}

3pSA6. On crack identification and characterization in a beam by nonlinear vibration analysis. Jeffry $N$. Sundermeyer and Richard $L$. Weaver (Dept. of Theor. and Appl. Mech., Univ. of Illinois, $104 \mathrm{~S}$. Wright St., Urbana, IL 61801)

The weakly nonlinear character of a cracked vibrating beam is exploited for the purpose of determining crack location, depth, and opening load. The approach is motivated by examining the response of a bilinear spring-mass system to excitation at two frequencies, such that the difference between the two frequencies is the resonant frequency of the system. The numerically generated steady-state response of the system clearly betrays the presence of the bilinear spring, even if the difference between the compressive and tensile stiffness is very small. The same idea is applied to a cracked beam forced at two frequencies, with the crack providing a local bilinear stiffness in the beam. The numerically generated steady-state response shows the effect of the opening and closing of the crack. The prominence of this nonlinear effect is then correlated with crack position and depth. It is shown that the nonlinear effect is maximized if a static load is also placed on the beam that would cause the crack to be on the verge of opening, thus determining the opening load. A perturbation analysis is applied to the problem, and some preliminary experimental results are discussed. [Work supported by the Army Research Office.]

3pSA7. A new method for damage detection in structures using transient bending wave propagation. K.-L. Ting and Zongabo $\mathrm{Li}$ (Dept. of Mech. Eng., Tennessee Technol. Univ., Cookeville, TN 38505)

With the increasing demand for safety and reliability on structures and mechanical systems, damage detection by nondestructive evaluation (NDE) methods has attracted considerable attention from many researchers. This paper presents a new method for damage detection based on time-frequency analysis of transient bending wave propagation. The wavelet transform and short-time Fourier transform are applied to the analysis of impulse-induced bending wave propagation in a cracked finite beam. The time-frequency representation resulting from the transformation of measured acceleration response is utilized to identify the reflection part of the transient bending wave by a crack and to determine its reflection coefficient and the arrival time (from the location of the crack to the transducer position) for different frequency components contained in the transient bending wave. The quantity of the crack is evaluated from the reflection coefficient, and its location is estimated from the arrival time. To demonstrate the effectiveness of the presented method in quantifying and locating cracks, a cantilever beam and a free-free beam with the same crack are investigated.

\section{$2: 45$}

3pSA8. Changes in sound phenomena of a beam due to rib and weld properties. Matthew S. Carney, J. Adin Mann, III, and Patricia L. Driesch (Dept. of Aerosp. Eng. and Eng. Mech., Iowa State Univ., 2019 Black Engineering, Ames, LA 50011)

Experiments will show how rib and weld discontinuities on a beam effect wave propagation and far-field sound radiation. Numerous alumi- 
num and steel beams were constructed using a different rib and weld configuration. The beam dimensions were $5 \mathrm{~cm} \times 0.635 \mathrm{~cm} \times 2.44 \mathrm{~m}$ and the ribs had T-shaped cross sections of slightly varying dimensions. The beams were then suspended and sound pressure $2.5 \mathrm{~cm}$ from the beam surface was measured with a microphone. By changing the physical construction of the attachment area of the ribs it was possible to isolate and analyze the effects of the different construction parameters. Also, the existence and effect of residual stresses in the beams due to the construction process was investigated. Analysis of the data showed that both the ribs and the welds produced changes in the far-field sound radiation and the phase speed of the propagating waves. Also, the geometry and the means of attachment of the rib affected the sound field. Transmission and reflection coefficients were calculated, along with travel times of the waves, to verify the changes in phase speeds that were found. [Work supported by ONR, Contract No. N00014-93-1-0493.]

3:00

3pSA9. Wave propagation in thermoporoelastic plate. H. S. Paul and V. M. Murali (Dept. of Math., Indian Inst. of Technol., Madras 600 036, India)
Governing equations for wave propagation of a thermoporoelastic plate are derived. The material obeys the theory of Pecker and Dersiewicz [C. Pecker and H. Dersiewicz, Acta Mech. 16, 45-64 (1973)]. The temperatures of solid and liquid phases are assumed to be different. Due to temperature difference in both phases at every point in the medium, there is a coupling parameter in heat conduction equations. The frequency equation is obtained for stress-free and thermally insulated boundary conditions. Numerical results are calculated for isothermal and adiabatic wave propagation corresponding to kerosene filled sandstone. Phase velocities and the attenuation factor are plotted against frequencies for symmetric and antisymmetric mode. In an isothermal case, the phase velocity oscillates sharply for the symmetric mode but it is not so sharp for the antisymmetric mode. But the behavior of the attenuation factor is reverse. In the adiabatic case, the phase velocity oscillates very less, up to frequency of $2 \mathrm{~Hz}$; afterwards, it oscillates for both modes. In the attenuation factor, there is rapid oscillation throughout for the antisymmetric mode but it is less in the symmetric mode. Oscillation is almost absent between 2.5 and $4.5 \mathrm{~Hz}$ in the symmetric mode. The phase velocity is higher for the isothermal case than the adiabatic case whereas it is opposite for the attenuation factor.

WEDNESDAY AFTERNOON, 30 NOVEMBER 1994

SAN MARCOS ROOM, 1:15 TO 3:00 P.M.

\title{
Session 3pSP
}

\section{Speech Communication: Auditory Front Ends for Speech Recognition}

\author{
Terrance M. Nearey, Chair \\ Department of Linguistics, 42-32 Assiniboia Hall, University of Alberta, Edmonton, Alberta T6G 2E7, Canada
}

Chair's Introduction-1:15

\section{Contributed Papers}

$1: 20$

3pSP1. Recognition of speech separated from acoustic mixtures. Martin Conke and Phil Green (Dept. of Comput. Sci., Univ. of Sheffield, 211 Portobello St., Sheffield S1 4DU, U.K.)

A perceptually plausible solution to the problem of automatic recognition of speech in arbitrary noise backgrounds involves computational auditory scene analysis (ASA) followed by recognition of the separated patterns. However, it is not generally possible to recover a complete representation of individual acoustic sources, so a new approach is required to recognize partial descriptions. Suitable modifications to the powerful stochastic framework of hidden Markov models (HMM) have recently been described [M. P. Cooke, P. D. Green, and M. D. Crawford, Proc. Int. Conf. Spoken Language Processing (1994)]. The studies reported here demonstrate HMM-based digit recognition in noise. An auditory-nerve firing rate representation undergoes auditory scene analysis, producing a mask of time-frequency locations where the speech is dominant. Each mask frame defines a marginal distribution for the HMM probability calculation. Results show robust performance even when the mask has most of its elements removed. Further, these studies suggest a solution to the problem of sensitivity to $F 0$ in matching auditory representations of speech in which $F 1$ is represented by a set of resolved harmonics. The new approach ensures that the matching process operates on a partial description consisting largely of harmonic peaks.

\section{1:40}

3pSP2. A computational model of speech segmentation. Neil $P$. McAngus Todd (Dept. of Psychol., Univ. of Manchester, Manchester
M13 9PL, U.K.) and Guy Brown (Univ. of Sheffield, Sheffield S10 2TN, U.K.)

Recently a computational model of prosody perception based on a multi-time-scale decomposition of the output from a cochlear model has been demonstrated [Todd and Brown, "A multi-scale auditory model of prosodic perception," Proceedings of the International Conference on Spoken Language Processing (1994)]. This model determines the temporal grouping and prominence of syllables from a speech signal. In this paper we present evidence to show that the model is able to carry out a complete segmentation of a speech signal, from the level of individual phonemes and phoneme clusters up to the phrase and utterance level. Implications for speech recognition are discussed.

\section{2:00}

3pSP3. Composite wavelet transform as an auditory model. Wade Trappe and Joseph D. Lakey (Appl. Res. Labs., Univ. of Texas at Austin, P.O. Box 8029, Austin, TX 78713-8029 and Dept. of Math., Univ. of Texas at Austin, Austin, TX 78712)

Motivated by the human auditory system, a new signal transform is presented which models the way humans hear. Cochlear processing acts like a constant bandwidth bank of filters in the low-frequency range but is of proportional bandwidth at higher frequencies. This new transform, which we call the composite wavelet transform, is better able to model this process than standard signal processing techniques such as the short-time fourier transform (STFT) and the continuous wavelet transform (CWT). The composite wavelet transform in fact provides a signal analysis tool that is able to examine signals with competing signal structures whereas 
the STFT and the CWT do not. Numerical results for this transform are presented along with a comparison to the STFT and the CWT.

3pSP4. Modeling the separation of acoustical signals based on the simulation of spike firing patterns. Kyrill A. Fischer (Drittes Physikalisches Inst., Univ. of Göttingen, Bürgerstrasse 42-44, D-37073 Göttingen, Germany)

It is investigated to what extent the separation of overlapping acoustical sounds may be achieved by detecting and grouping comodulated frequency bands. Comodulation is defined by coherent envelope fluctuations which are detected by means of physiological motivated algorithms involving spike representation and coincidence detection. Groups of comodulated frequency bands are defined by cluster analysis and their temporal variations are traced. Every group detected can then be separated from its "acoustical background" by suppressing all channels not belonging to the given group. The paper addresses problems concerning the definition of suppression gain values and the problem of overlapping groups.

\section{2:40}

3pSP5. Processing of continuous speech by a hierarchical neural network. Wolf Dieter Brandt and Holger Behme (Drittes Physikalisches
Inst. der Univ. Goettingen, Buergerstr. 42-44, D-37073 Goettingen, Germany)

A multilevel neural network has been developed for the tasks of psychoacoustic preprocessing of speech, segmentation, segment classification, and recognition. Various neurophysiological and psychoacoustical results have been taken into account. The network relies heavily on unsupervised learning. On increasing and nonlinear time scales, each level of the network extracts segments from the stream of input data and classifies them using topology-conserving vector quantizers (self-organizing feature maps SOFM, "neural gas" algorithm (NGA) and passes the results to the next level. Modified learning algorithms for feature maps have been developed to achieve better representation of low-energetic consonants and transient parts in the first level. On higher levels variants of the NGA are used. The segmentation algorithm uses, depending on the level, the topological relationships within the SOFM or statistical information extracted from the training data. Within each segment, dynamic time normalization is achieved by appropriate temporal integration. The output of the topmost level is passed to a recognition network containing a linguistic model to perform continuous speech recognition. Results using this purely neural and in many parts self-organizing method will be presented and compared to classical methods. [Work supported by BMFT.]

WEDNESDAY AFTERNOON, 30 NOVEMBER 1994

BALLROOM B, 1:30 TO 3:00 P.M.

\title{
Session 3pUW
}

\section{Underwater Acoustics: Signal Processing I: Matched Field}

\author{
Homer P. Bucker, Chair \\ Naval Command and Control Ocean Surveillance Center, San Diego, California 92152-5001
}

\section{Contributed Papers}

\section{$1: 30$}

3pUW1. Calculation of a source spectrum using matched-field tracking. Homer Bucker (Code 541, NRaD, NCCOSC, San Diego, CA 92152)

An underwater acoustic source can be detected by finding the best match between a measured set of covariance matrix elements and those calculated for possible tracks [H. Bucker, "Matched-field tracking in shallow water," J. Acoust. Soc. Am. (to be published)]. Let $\left(g_{l f}\right)$ be the measured elements and $\left(\hat{g}_{1 f t}\right)$ be the elements calculated for the best track of a unit source. Here, index $l$ refers to a pair of sensors, $f$ to a frequency bin, and $t$ is for different times. Then a plot of $S(f, t)=\Sigma_{l g} g_{l f t} / \Sigma_{l} \dot{g}_{l f t}$ is a Lofargram in which the effects of the multipath propagation have, at least in part, been removed. Of course, the success of the deconvolution depends upon the accuracy of the propagation model and contamination of other sound sources. Several examples will be presented to illustrate the method.

\section{$1: 45$}

3pUW2. Exploiting source dynamics in matched-field processing. Zoi-Heleni Michalopoulou and Michael B. Porter (Dept. of Math. and Ctr. for Appl. Math. and Statistics, New Jersey Inst. of Technol., Newark, NJ 07102)

Matched-field processing is typically considered as a static problem of locating a fixed, narrow-band source. However, the source does not randomly jump from point to point during the course of time and much can be gained by exploiting this extra information. Various schemes bave been proposed in the past for treating source motion. A key problem is that even when the source motion is uniform the source may appear to have a nonuniform speed due to environmental mismatch. We show that by allowing for these random perturbations in source motion, an improvement in the localization results is obtained.

\section{2:00}

3pUW3. Environmental source tracking using measured and modeled replicas. John S. Perkins, Laurie T. Fialkowski, Michael D. Collins, W. A. Kuperman, ${ }^{\text {a) }}$ Timothy L. Krout, Jon Jannucci (Naval Res. Lab., Washington, DC 20375), Ross F. Barrett, L. J. Kelly, Ashley Larsson (Defence Sci. and Technol. Organization, Salisbury, SA, Australia), Lindsay Hall (Defence Sci. Establishment, Auckland, New Zealand), John A. Fawcett, and Anthony Hoffmann (Defence Res. Establishment Pacific, Victoria, BC, Canada)

Preliminary results will be presented for the second TTCP Environmental Signal Processing Exercise (TESPEX 2), which was conducted in June 1994 in shallow waters north of Darwin, Australia. There were two objectives: (1) demonstrate improvements in environmental source tracking [Collins et al., J. Acoust. Soc. Am. 94, 3335 (1993)] (a singlefrequency, single-hydrophone synthetic-aperture tracking technique) by relying on a second, horizontally separated hydrophone, and (2) determine the horizontal resolution the environment provides to a vertical array through matched-field processing [Perkins and Kuperman, J. Acoust. Soc. Am. 87, 1553 (1990)]. The approach was to first characterize the region by towing a high-SNR source throughout the region and measure the replica fields using two vertical line arrays separated by several kilometers (one with 32 elements, the other 4 elements), and then to use this data to form the replicas needed to track the source as it traversed the region alone a variety of tracks. The measured replicas are supplemented by modeled 
replicas; the environment used in the modeling is determined by inverting the measured data. We present preliminary processing results relevant to both objectives, including environmental inversions and tracking with measured and modeled replicas. ${ }^{\text {a)} P r e s e n t ~ a d d r e s s: ~ S c r i p p s ~ I n s t i t u t i o n ~ o f ~}$ Oceanography, La Jolla, CA 92093.

\section{2:15}

3pUW4. Performance stability of broadband matched-field processors subjected to sound speed and array-element location error in a shallow-water environment. Howard A. Chandler, Murty Akundi, and G. B. Smith (Naval Res. Lab., Stennis Space Center, MS 39529-5004)

The effects of array-element location errors on the performance of the three broadband matched-field processing algorithms (Barlett, maximum likelihood, and sector focusing) have been investigated. The KRAKEN propagation model was used to generate the replica acoustic pressure field at multiple frequencies for a shallow-water channel with a depth variable sound-speed profile typical of a midlatitude summer environment. It was also used to simulate a "detecled" field due to an acoustic source in the presence of both uncorrelated and correlated (modal) noise. These fields were then correlated using the three algorithms for selected degrees of sound speed and array deformation error. Results will be presented. [Work sponsored by the Office of Naval Research, Program Element 61153N, with technical management provided by NRL-SSC.]

\section{$2: 30$}

3pUW5. Acoustic array navigation in shallow water. D. F. Gingras, L. Troiano (SACLANT Undersea Res. Ctr., Viale San Bartolomeo, 400, 19038 La Spezia, Italy), and R. B. Williarns (Naval Ctr. for Command, Control, and Ocean Surveillance, San Diego, CA)

The inversion of acoustic field data for estimation of unknown environmental or geometric parameters is receiving considerable attention. The environmental parameters usually consist of bathymetry, sound speed in the water, and bottom properties such as sound speed, attenuation, and density. The geometric parameters consist of source and array sensor positions. In many situations it is assumed that the array sensor positions are known and in these cases it is important to the inversion process that the array sensor positions are known precisely. In this paper an acoustic navigation method for precisely determining sensor position is presented. Acoustic travel time measurements were used to navigate the sensors of a 94-m vertical array in shallow water $(130 \mathrm{~m})$. Array navigation performance was evaluated during a vertical array deployment in the Mediterranean from the R/V Alliance. A network of four bottom-moored acoustic transponders were interrogated from the Alliance and their replies were received by the vertical array sensors and telemetered to the Alliance and their replies were received by the vertical array sensors and telemetered to the Alliance for navigation processing. In October, over a 2-day period, current versus depth and array shape was monitored. During this period the local currents were small; the array shape was estimated to be almost straight and nearly vertical.

\section{$2: 45$}

3pUW6. Matched beam processing for geoacoustic inversion of bottom sonnd-speed profiles. T. C. Yang (Naval Res. Lab., Washington, DC 20375) and T. Yates (Vector Research Co., Rockville, MD 20852)

A broadband signal (shot) received on a vertical array was processed for geoacoustic inversion of bottom sound-speed profile. The data were beamformed to show the distribution of waterborne and bottom interacting signals. By correlating the (complex) beams containing the bottom interacting signals with the equivalently formed beams for the replica field (matched beam processing), one evaluates the ambiguity function maximum at the source location and searches for the bottom sound-speed profile which yields the highest correlation for the matched beam processing. Different frequency bands of the signal are investigated to evaluate the sensitivity to boltom sound-speed variations. The inverted sound-speed profiles are found consistent (within the resolution of $50-100 \mathrm{~m} / \mathrm{s}$ ) with the sound-speed profiles estimated from (1) core samples and other geoacoustic dala at the site, and (2) the arrival time of the Head waves [J. Wolf, J. Acoust. Soc. Am. 94, 1769 (1993)].

\title{
Plenary Session, Business Meeting, and Awards Ceremony
}

\author{
Jiri Tichy, Chair \\ President, Acoustical Society of America
}

Business Meeting

Presentation of certificates to New Fellows and Science Writing Award recipients

Presentation of Awards

Distinguished Service Citation to William J. Cavanaugh

Silver Medal in Noise to Kenneth M. Eldred

Silver Medal in Physical Acoustics to Julian D. Maynard

Silver Medal in Speech Communication to Peter Ladefoged

Electronic music performance presented by Haeyon Kim Lent and Keith Lent 


\title{
Session $\mathbf{4 a A B}$
}

\section{Animal Bioacoustics: Animal Bioacoustics Research Methodology I}

\author{
Whitlow W. L. Au, Chair \\ Hawaii Institute of Marine Biology, P.O. Box 1106, Kailua, Hawaii 96734
}

Chair's Introduction-8:00

Invited Papers

8:05

4aAB1. A novel mechanism for directional hearing in a parasitoid fly. D. Robert, R. R. Hoy (Sec. of Neurobiol. and Behavior, Cornell Univ., Ithaca, NY 14853-2702), and R. N. Miles (State Univ. of New York, Bingbamton, NY 13902-6000)

Sound localization is a basic bebavioral task of the auditory system. Incident sound waves arrive at the ears and generate interaural differences in time of arrival and in amplitude that are key cues for the computation of sound direction. In small animals, both cues can become vanishingly small, posing a challenge for directional hearing. Yet nearly all animals that hear can localize sound. In the fly Ormia ochracea, the two acoustic sensors are separated by only about $520 \mu \mathrm{m}$, and are contained within an undivided air-filled chamber, an arrangement that results in minimal differences in interaural time $(<2 \mu s)$ and no intensity cues from an incident sound wave. Using laser vibrometry, it is shown that the mechanical response of the tympanal membranes has a pronounced directional sensitivity. Using probe microphones and neurophysiological recording techniques, it is demonstrated that this fly utilizes a novel mechanism for the detection of an incident sound wave. This mechanism relies on the mechanical coupling between the two tympanal membranes. In effect, the fly's ears operate by mechanical preprocessing, converting interaural acoustic time differences of $2 \mathrm{~ms}$ or less into neural time differences large enough to encode in the central nervous system.

8:30

4aAB2. Seismic communication in amphibians. Peter M. Narins (Dept. of Biol., UCLA, 405 Hilgard Ave., Los Angeles, CA 90024)

The white-lipped frog, Leptodactylus albilabris, exhibits the greatest sensitivity to substrate-borne vibrations (seismic stimuli) reported to date for any terrestrial animal. Nerve fibers from the sacculus, the source of this extraordinary sensitivity, show clear responses to sinusoidal seismic stimuli with peak accelerations less than $0.001 \mathrm{~cm} / \mathrm{s}^{2}$. In addition, this animal generates substrate-bome vibrational signals during calling. As the male's vocal sac expands, it strikes the substrate impulsively, generating a vertically polarized surface (Rayleigh) wave that is detected by neighboring males. These Rayleigh waves are used as intraspecific communication signals to coordinate chorus behavior in this species. Recently, a particularly unusual behavior has been described for one species of Malaysian treefrog (Polypedates). During noctumal courtship, females living in dense mats of floating vegetation perch conspicuously on a reed or blade of grass and tap their rear toes rbythmically. Males on neighboring reeds were observed to quickly locate and mate with the tapping female. Thus it is likely that toe tapping functions as a vibrational signal indicating the female's presence to neighboring males. It is becoming clear that seismic communication and sensitivity to whole-body vibrations are more ubiquitous among the vertebrates than had been previously imagined. [Work supported by NIH.]

\section{8:55}

4aAB3. Studying the evolutionary history of communication: The ghost of signals past. Michael J. Ryan (Dept. of Zool., Univ. of Texas, Austin, TX 78712)

The efficiency of communication can be maximized by a tight match between signal and receiver. This view underlies the field of animal communication and has led biologists to propose functional and evolutionary models that are firmly grounded in such linkage. Recent empirical studies have shown, however, that tight linkage between sender and receiver need not be the case. Using the phonotactic response of female frogs to variation in mating calls, it has been shown that some aspects of the receiver are quite broad relative to the conspecific signal. For example, females show preferences for signals of their own species to which are added components from the signals of other species. Also, using algorithms for reconstruction of ancestral traits, it is shown that key stimuli needed for species-specific pattern recognition have not been tightly linked with receiver evolution. These studies suggest a more liberal interpretation for the evolution of animal communication systems.

4aAB4. Effects of aircraft noise on wildlife: Techniques used in Air Force research. Robert C. Kull (AL/OEBN, 2610 Seventh St., Wright-Patterson AFB, OH 45433-7901)

Many researchers bave set out to study the effects of noise on animals, especially aircraft noise, for the past 20 years yet have failed in properly describing the noise their subject animals were exposed to. In 1989 the Air Force began a series of wildlife studies to determine the effects of aircraft noise. Research included effects on Desert Bighom Sheep, caribou, kit fox, domestic turkeys, dairy cows, horses, and raptors. One major objective in these studies was to accurately determine the noise exposure levels from aircraft. 
Conventional methods were used for research on domestic animals, but studies on wild animals posed special problems. Various techniques and procedures of how noise exposures were determined will be described.

9:45

4aAB5. Physiological monitoring of noise effects in wildlife. D. W. DeYoung (Univ. Animal Care, Univ. of Arizona, Tucson, AZ 85724) and R. C. Kull, Jr. (AL/OEBN, Wright-Patterson Air Force Base, OH 45433-7901)

Noise as a stress to animals will be discussed with respect to the physiological parameters that characterize it. Known currently available radiotelemetry systems that can monitor some of these parameters will be presented. Data that can be reliably derived from parameters obtained by available radiotelemetry systems will be presented. Possible pathology due to long-term stress as well as information for animal studies, telemetry implant surgery and anesthesia, and the potential complications of implant surgery will be discussed. Finally, recommendations for future study of noise as a stressor to animals will be made. [Work supported by USAF.]

\section{0:05-10:15 Break}

10:15

4aAB6. Passive acoustic localization of calling animals in 3-D space. John L. Spiesberger (Dept. of Meteorol. and the Appl. Res. Lab., 512 Walker Bldg., Penn State Univ., University Park, PA 16802)

Fluctuations and uncertainties in winds (currents) and sound speed place lower limits on the accuracy with which calling animals can be passively localized from their calls in terrestrial and marine environments. The most accurate acoustic passive localizations of animals require simultaneous mapping of these environmental fluctuations using tomographic techniques [J. Spiesberger and $\mathrm{K}$. Fristrup, Am. Nat. 135, 107-153 (1990)]. One localization experiment conducted in a wood found tomographic localization of a cap gun possible but tomographic localization of birds difficult because of echoes. Marine environments may be conducive to tomographic localizations because of a paucity of reflecting surfaces at low frequencies. A new oceanographic instrument called a surface suspended acoustic receiver (SSAR) has the potential for passively localizing and censusing calling animals throughout the world's oceans in real time. [Work partially supported by Advanced Research Projects Agency.]

10:35

4aAB7. Using FFT spectrum analyzers to measure animal sounds. Frank T. Awbrey (Biol. Dept., San Diego State Univ., San Diego, CA 92182-0057)

Sound-pressure levels of animal sounds have been measured in so many different ways that comparison between studies is very nearly impossible. The transient, repetitive nature of most vocalizations means that standard sound level meters may yield very different numbers, depending on meter time constant and frequency weighting. Integrating sound level meters, which average sound over various periods and measure sound exposure level directly, are an improvement, but are hard to use for measuring single, transient sounds such as a frog call or an echolocation pulse. A better way, with brief signals, is to record them through calibrated transducers, along with calibration tones of known amplitude, onto tape recorders with linear response to varying levels. These recordings can then be measured with digital spectrum analyzers in a way that is reliable, accurate, and repeatable. Possible measures include peak, octave, one-third octave, maximum, time-average, sound exposure, and spectrum levels. Comparability requires attention to and reporting of important details, such as averaging times, filter bandwidth and weighting functions, and adherence to standards for measuring and reporting sound.

\section{0:55}

4aAB8. Determining the effects of low-frequency sound on the fish auditory system. Mardi C. Hastings, James J. Finneran (Dept. of Mech. Eng., Ohio State Univ., 206 W. 18th Ave., Columbus, OH 43210), Arthur N. Popper, and Pamela J. Lanford (Univ. of Maryland, College Park, MD)

A 15 -m-long flexible waveguide was successfully uscd to create a traveling wave for frequencies below $300 \mathrm{~Hz}$ in water. A cylindrical waveguide that allows only plane-wave propagation was designed and fabricated from Plexiglas. This flexible material reduced the effective stiffness and thus the sonic speed and wavelength of the disturbance generated by a J9 underwater sound projector flanged to one end. In addition, the energy of the wave was dissipated as it traveled along the waveguide; consequently, little if any reflection occurred at its end. The fish were placed inside a PVC mesh cage and positioned in the waveguide approximately $3 \mathrm{~m}$ down from the J9. Then they were exposed to either a continuous wave or a pulsed wave similar to manmade sources. Afterwards the fish were held for a specified time period and then sacrificed. The auditory organs were treated with fixative and removed. Then they were shipped ovemight to the University of Maryland where the tissue was immediately prepared for scanning electron microscopy to determine if sensory hair cells bad been destroyed. This method has yielded dependable data to help assess the effects of low-frequency sound on hearing in fish. [Work supported by ONR.]

\section{Contributed Papers}

\section{1:15}

4aAB9. Abiotic controls on elephant communication. David Larom, Michael Garstang (Dept. of Environmental Sci., Univ. of Virginia, Charlottesville, VA 22903), Richard Raspet (Univ. of Mississippi, University, MS 38677), and Malan Lindeque (Etosha Ecological Institute, Namibia, Africa)

Atmospheric conditions conducive to long-range transmission of lowfrequency sound as used by elephants are found to exist in the Etosha
National Park in Namibia during the late dry season. Meteorological measurements show that strong temperature inversions form at the surface before sunset and decay with sunrise, often accompanied by calm wind conditions during the early evening. These observations are used in an acoustic model to determine the sensitivity of infrasound to the effects of (a) the strength, thickness, and elevation of temperature inversions, and (b) the growth and decay of an inversion typical of dry, elevated African savannas. The results suggest that elephant communication range more than doubles at night. Optimum conditions occur 1-2 h after sunset on 
clear, relatively cold, calm nights. At these times ranges of over $10 \mathrm{~km}$ are likely, with greatest amplification occurring at the lowest frequency tested. This strong diurnal cycle in communication range may be reflected in longer period changes in weather and may exert a significant influence on elephant behavior on time scales from days to many years.

\section{1:30}

4aAB10. Applications of multifrequency acoustics: Spatial patterns in a freshwater lake. C. F. Greenlaw, R. W. Player, and U. S. Samilo (Tracor Appl. Sci., Inc., 9150 Chesapeake Dr., San Diego, CA 92123)

Vertical profiles of acoustical scattering at four frequencies were obtained at a mesh of points covering the major regions of a small, manmade freshwater lake. Inversion methods were used to estimate size-abundance profiles of zooplankters using a scattering model validated for oceanic zooplankton such as copepods. These profiles were then used to form estimates of lake-wide 3-D spatial patterns sorted by size classes of the dominant zooplankters. Results illustrate the value of multifrequency acoustical surveys as an adjunct to conventional sampling for rapid assessment of spatial distributional features of zooplankton populations in lakes. Problems with applications of ocean-based assessment techniques to lacustrine environments, such as the lack of validated scattering models for freshwater zooplankton, are discussed.

\section{1:45}

4aAB11. A publicly accessible database for marine mammal call classification research. David K. Mellinger and Christopher W. Clark (Bioacoust. Res. Prog., Cornell Univ., 159 Sapsucker Woods Rd., Ithaca, NY 14850)

A central problem in bioacoustic transient (animal sound) detection and classification has been the lack of standards against which to compare various methods. A similar problem existed in speech recognition research; it was solved by the creation of databases (e.g., TIMIT) used as common data sources for testing and comparing different methods. A similar database has been initiated for use in marine mammal sound detection and classification. Initially, mysticete sounds have been placed in the database; since their low frequency makes possible a low sampling rate, a large number of sounds may be stored in the space available. The database currently holds sounds of four species of mysticete-1765 blue, 3909 finback, 356 minke, and 589 bowhead whale vocalizations-at sampling rates from $100 \mathrm{~Hz}$ to $2 \mathrm{kHz}$. It was desired to categorize sounds by signalto-noise ratio, since performance of detection methods will depend on this; since no measure of SNR for transient signals is well known, a simple measure of energy ratio within the sounds' frequency band was used. The database includes performance data of some detection methods, and it is hoped that other researchers will contribute both sounds and performance data. The database is accessible at ftp.ornith.comell.edu.

THURSDAY MORNING, 1 DECEMBER 1994

SAN ANTONIO ROOM, 8:30 TO 11:45 A.M.

\title{
Session 4aEA
}

\section{Engineering Acoustics: Piezocomposite Transducers}

\author{
Thomas R. Howarth, Chair \\ Naval Research Laboratory, Underwater Sound Reference Detachment, P.O. Box 568337, Orlando, Florida $32856-8337$
}

\section{Invited Papers}

8:30

4aEA1. Development of 0-3 piezocomposite transducers for underwater applications. Fred G. Geil and Linda L. Matteson (Westinghouse Electric Corp., Oceanic Div., Annapolis, MD 21404)

Piezorubber is a composite material consisting of lead titanate powder dispersed in a neoprene rubber matrix. Piezorubber has certain acoustic properties that sets this transduction material apart from the traditional ceramics. Among these are some good qualities such as a high hydrostatic sensitivity, a relatively low lateral sensitivity, high resistance to shock, and conformability. Some less attractive qualities are the low dielectric constant and high density. Nevertheless, piezorubber has been the material of choice for several US Navy projects. These include a hydrophone flank array for ASW, a high-frequency array of small elements, a 700-element wide bandwidth listening array, a conformal shell-mounted low-frequency hydrophone, a continuous element for towed arrays, and high-frequency elements for side-looking sonars. These six projects will be described briefly, concentrating on the achieved benefits and what has been learned about applying piezorubber to hydrophone applications, also indicating the areas where the application was less than completely successful.

\section{9:00}

4aEA2. U.S. Navy 0-3 piezocomposite transducers. Mark L. Pecoraro (Naval Res. Lab., Underwater Sound Reference Detachment, P.O. Box 568337, Orlando, FL 32856-8337)

Interest in 0-3 piezoelectric composite materials for use in hydrophone applications has grown considerably in recent years. Designs which require operation in the hydrostatic mode with a large hydrostatic voltage coefficient $g_{h}$, are desired to increase free-field voltage sensitivity and meet the ever increasing demands placed on hydrophone performance. U.S. Navy scientists at the Naval Research Laboratory, Underwater Sound Reference Detachment, have investigated the utilization of 0-3 piezocomposite for sonar applications and have developed various hydrophone configurations based on specific program requirements. The requirements of the programs, such as a lightweight, low-profile, and high receive sensitivity, demanded unique designs and the need for utilizing the unique characteristics of 0-3 composite materials. The hydrophones presented in this discussion used a $0-3$ composite known at NRL simply as piezorubber or PZR. This material consists of lead titanate particles embedded in a neoprene elastomeric matrix. This discussion will cover the development, including problems and solutions, fabrication, and testing of two unique 0-3 composite hydrophones. 
4aEA3. Development of 1-3 and 2-2 piezocomposite transducers. Leslie Bowen, Richard Gentilman, Hong Pham, William Serwalka, and Daniel Fiore (Mater. Syst., Inc., 521 Great Rd., Littleton, MA 01460)

1-3 and 2-2 piezoelectric ceramic/polymer composiles offer design versatility and performance advantages over monolithic piezoelectric ceramic and monolithic piezoelectric polymer devices in both resonant and nonresonant transducer applications. Recently, a new process, viz., ceramic injection molding, has been applied to solve the difficulties of cost-effectively manufacturing the arrays of fine PZT ceramic elements required for composite transducer assembly. As a result, these materials are now available in commercial quantities for the first time. In this paper, Materials Systems, Inc. briefly introduces its PZT ceramic injeclion molding Iransducer manufacturing process, and then reviews the performance of various 1-3 composite transducer designs as a function of composite layoul, materials, and transducer performance enhancement features, such as stiff cover plates and Poisson's ratio decoupling. The performance of several transducer designs in both nonresonant sensing and actuating undersea applications, and in high-frequency resonant applications, e.g., acoustic imaging, is discussed. [Work supported by ONR.]

10:00

4aEA4. 1-3 Piezocomposite transducer materials for commercial underwater applications. Victor Murray (Fugro-UDI Ltd., Bridge of Don, Aberdeen, Scotland AB23 8JW, United Kingdom)

Fugro-UDI Limited has gained several years experience in developing piezo-composite materials for use in underwater imaging sonars operating in the frequency range $100-500 \mathrm{kHz}$. A substantial amount of this work has concentrated on multielement arrays for military applications such as mine counter measures. The low mechanical quality factor $\left(Q_{m}\right)$ and high electro-mechanical coupling coefficient $\left(K_{t}\right)$ of the material has provided wideband and efficient transducer materials. These parameters and others such as dielectric constant and electrical impedance can be tailored by varying the type and quality of both the ceramic material and polymer phase within the piezocomposite device. This has contributed to reducing the development and manufacluring costs of piezocomposite transducers. This presentation will discuss the various Iransducers developed by Fugro-UDI for commercial applications ranging from simple echo-sounder transducers, to sidescan transducers, through to multielement arrays for electronically scanned sonars. Sensitivities and beam patterns for piezocomposite devices will be compared wilh their ceramic counterparts. Other important transducer parameters such as pressure and temperature dependence of the piezocomposite materials will be presented.

10:30

4aEA5. Evaluations of 1-3 piezocomposite transducers for underwater applications. Robert $Y$. Ting and Thomas R. Howarth (Naval Res. Lab., Underwater Sound Reference Detachment, P.O. Box 568337, Orlando, FL 32856-8337)

This presentation begins with discussions on 1-3 piezocomposite transducer designs and how the designs may be tailored for specific underwater acouslic applications. For instance, 1-3 piezocomposite materials with cover plates for actuator applications shall be shown and compared with other 1-3 piezocomposite transducers that feature finer scale piezoceramic rods for wideband underwater acoustic imaging applications. Other 1-3 piezocomposile transducers to be shown include multilayer plates with elements stacked in mechanical series and wired electrically in parallel as well as monolithic structure designs with elements elched into the piezocomposite structure. Underwater electroacoustic evaluations of each of the 1-3 piezocomposite transducers will be presented. The in-water measurements include the transmitting and receiving performances as well as directivity patterns over exlended frequency bands. Discussions on how the 1-3 piezocomposite transducers may be applied towards future underwater acoustic systems shall conclude the presentation. [Work sponsored by ONR.]

\section{Contributed Papers}

\section{1:00}

4aEA6. Surface-mounted sensors on a large-area 1-3 composite actuator. Robert D. Corsaro and Brian Houston (Naval Res. Lab., Code 7130, Washington, DC 20375-5350)

Surface-mounted sensors can be used to monitor the performance of an underwater acoustic projector, or to control its impedance in a complex dynamic environment. However, there are few examples in the literature of studies of the issues involved in marrying sensors and actuators. These issues include spatial sampling, near field sensing, internal resonances, and both direct and extraneous coupling mechanisms, all of which can contribute to complicate the system transfer functions and limit the applicability of this approach. The work presented here includes measurements made on a 1-3 composite actuator with integrated pressure and velocity sensor layers. Both free-field sensitivities and actuator-sensor coupling transfer functions are evaluated in the NRL Large Pool Facility. These are compared with predictions from simple acoustic models. Implications for local control of the acluator's surface are also discussed.

\section{1:15}

4aEA7. A dynamic model for piezoelectric composite transducers and coupling coefficient $\boldsymbol{k}_{\boldsymbol{t}}$. Yongan Shui (Inst. of Acoust., Nanjing Univ., Nanjing 210008, People's Republic of China) and Qiang Xue (Analogic Corp., Peabody, MA 01960)
Piezoelectric composite materials are increasingly used in medical and other ultrasonic transducers. In order to design composite transducers with desirable performance, especially in high-frequency range where polymer and ceramic structures are not much smaller than wavelength, a good understanding of dynamic behavior of the material is necessary. This work developed a dynamic model to investigate wave propagation along thickness direction and resonant behavior of the transducer. Relationships of thickness resonant frequencies as well as lateral periodic resonant frequencies with composite configuration and material (polymer and ceramic) properties were emphasized. Discussions on coupling coefficient $k_{t}$ of transducers as a function of volume fraction and aspect ratio of composite are presented. A series of composite transducers with varied parameters were fabricated and measured. Experimental results of $k_{t}$ and resonant frequencies are compared with the theory.

\section{1:30}

4aEA8. 1-3 composite transducer for partial discharge detection in high-voltage transformers. Valsala Kurusingal (Clr. for Mater. Technol., Univ. of Technol., P.O. Box 123, Broadway, NSW 2007, Australia)

Ultrasonic techniques alone or in combination with electrical methods are increasingly being used for the detection and/or location of partial discharge (PD) in high-voltage transformers and other electrical plants. 
Ultrasonic transducers used for fault location are traditionally coupled externally on to the transformer wall. Mounting transducers inside the transformer steel tank has certain definite advantages like better noise immunity, increased sensitivity due to better acoustic matching to oil, and less complicated signals (only longitudinal waves). Transformers are filled with mineral oil whose temperature could rise up $10110^{\circ} \mathrm{C}$ during normal operation. Also, there are large electric and magnetic fields and strong mechanical vibrations as well inside the transformer. The authors has developed 1-3 piezopolymer composite transducers which are capable of operating in this adverse environment. These transducers were life tested in mineral oil at $130{ }^{\circ} \mathrm{C}$ for over 500 days and their $k_{t}$ values monitored as a function of time. Life test and vibration test data for several transducers as well as sensitivity, frequency response, pulse widih, and constructional details are presented.

\title{
Session 4aMUa
}

\author{
Musical Acoustics: General Topics \\ Uwe J. Hansen, Chair \\ Physics Department, Indiana State University, Terre Haute, Indiana 47809
}

Chair's Introduction-8:15

\section{Contributed Papers}

8:20

4aMUa1. A study of lip vibrations in a trombone. David C. Copley and William J. Strong (Dept. of Phys. and Astron., Brigham Young Univ., Provo, UT 84602)

More than 50 years ago, Martin investigated lip vibrations in a coronet mouthpiece using stroboscopic photography [D. Martin, J. Acoust. Soc. Am. 13, 305-307 (1942)]. Since then, several researchers have based lip models on Martin's data. Unfortunately, due to the quality of the photographs, it is difficult to obtain anything more than a limited quantitative description of the lip motion. The purpose of this study is to obtain more detailed photographic sequences and lip motion data on which new models may be based. The trombone was selected as representative of the lip reed family. A computer-controlled fiber optic stroboscope was used to capture the motion of a player's lips on video. By inserting the optic bundle through small holes drilled in the mouthpiece, lip motion was observed from the front and side for six notes (Bb2, F3, B b3, D4, F4, G4) played at loud and soft dynamic levels. Video sequences and resulting lip motion data will be presented and discussed.

\section{8:35}

4aMUa2. Brass sound simulation with a lip vibration model having two degrees of freedom. Sciji Adachi and Masa-aki Sato (ATR Human Information Processing Res. Labs., 2-2 Hikaridai, Seika-cho, Soraku-gun, Kyoto 619-02, Japan)

Brass sound simulation is carried out with the use of a two-dimensional lip vibration model, where lips execute both rolling and reciprocating motions. This model allows lips to operate on both the lower and higher frequency sides of the air-column resonance frequencies. Oscillations generated by the total sound production system are on both the lower and higher frequency sides in the first and second resonance modes, while in the higher modes they are realized only on the lower side. The harmonic series selected from these oscillations in the second and higher modes to achieve the optimum pitch tums out to comprise an oscillation on the higher side of the second resonance frequency and oscillations on the lower sides of the higher resonance frequencies. This result closely matches the transition of lip vibration states from the outward-striking vibration at the second mode (i.e., the lowest mode among the ones used musically) to the vocal-cord-like vibration at the higher modes, which is observed in the simultaneous measurement [S. Yoshikawa, submitted to $\mathrm{J}$. Acoust. Soc. Am.] of mouthpiece pressure and lip vibration.
8:50

4aMUa3. On the use of Schrödinger's equation in the analytic determination of horn reflectance. David Berners and Julius $O$. Smith, III (Ctr. for Comput. Res. in Music and Acoust., Dept. of Music, Stanford Univ., Stanford, CA 94305-8180)

The flaring horn has traditionally been modeled in one dimension using piecewise conical or cylindrical elements. Acoustic properties within each element are known, and scattering between the elements is computed. Under the piecewise model, a shape for the wavefront of the acoustic disturbance within the horn is implicitly assumed (planar for cylindrical elements, spherical for conical elements). For horns of significant flare, the true wavefront shape will be neither planar nor spherical. A more general model is thus desirable. Here an alternate model is presented: The flaring hom is modeled according to Webster's equation. A change of variables transforms the equation into the form of the Schrodinger wave equation using in one-dimensional particle scattering. Boundary conditions can be derived directly from the physical dimensions of the horn, and the solution of the equation gives estimates of acoustic properties in terms of frequency dependent reflection and transmission coefficients. Here, Webster's equation is solved along the entire length of the horn, with no lumped scattering. Advantages over piecewise modeling techniques include the ability to specify arbitrary axisymmetric wavefront shapes for the acoustic disturbance within the hora. Under appropriate assumptions for wavefront shapes, results converge to those obtained with traditional piecewise models.

\section{9:05}

4aMUa4. The wave digital hammer: A computationally efficient traveling wave model of the piano hammer and the felt mallet. Scott A. Van Duyne and Julius O. Smith, III (CCRMA, Stanford Univ., 660 Lomita Dr., Stanford, CA 94305)

Recent work has led to traveling wave string and membrane models using the digital waveguide and the 2-D digital waveguide mesh. This paper introduces a new development for these musical instrument models which extends their usefulness: a traveling wave model for the piano hammer, or felt mallet. When a mallet strikes an ideal membrane or string, it sinks down into it, feeling a pure resistive impedance. In the membrane case, the depression induces a circular traveling wave outward. If the membrane is bounded, reflected waves return to the strike point to throw the mallet away from the membrane. This complex mallet-membrane interaction can have very different and difficult to predict acoustical effects, particularly when a second or third strike occurs while the membrane 
is still in motion, as in a drum roll. The piano hammer, or felt mallet, is viewed as a nonlinear mass/spring (inductor/capacitor) system, the nonlinear spring representing the felt portion. By decomposing the system into appropriate traveling waves, a unit of delay is extracted in the discrete time version, greatly simplifying the implementation. This wave digital hammer can be attached to any waveguide string or membrane model at a timevarying lossless scattering junction.

4aMUa5. Rhythmogram analysis of human and synthetic performance. Neil P. McAngus Todd (Dept. of Psychol., Univ. of Manchester, Manchester M13 9PL, United Kingdom), Eric Clarke, and Luke Windsor (Univ. of Sheffield, Sheffield S10 2TN, UK)

Previously, a method of performance analysis has been described which is based on a multitimescale decomposition of the acoustic signal [Todd, "Wavelet analysis of rhythm," J. Acoust. Soc. Am. 93, 2290(A) (1993)]. This analysis is sensitive to a variety of expressive devices employed by musical performers including tempo, dynamics, and articulation. The output of the analysis is a "rhythmogram" which resembles the timespan reduction of the theory of Lerdahl and Jackendoff. In this paper, the results of some analyses of real and synthetic performances are presented [Todd, "The dynamics of dynamics: a model of musical expression," J. Acoust. Soc. Am. 91, 3540-3550 (1992)].

\section{9:35}

4aMUa6. The pitch of elements of a harmonic complex. Blane Anderson and W. Dixon Ward (Dept. of Commun. Disord., Univ. of Minnesota, Minneapolis, MN 55455)

It is well established that the pitch of a sinusoid is shifted away from the frequency of a simultaneous noise band or sinusoid. Attempts to determine whether or not such pitch shifts occur among elements of a harmonic complex tone have given results that are apparently conflicting. In an endeavor to shed light, or at least a few shadows, on the question, seven listeners adjusted a sinusoid to match the pitch of either the 3rd or 7th harmonic of a preceding $220-\mathrm{Hz} 8$-harmonic complex in which the harmonic in question was periodically intertupted. Both monotic and dichotic procedures, with corrections for diplacusis, were employed in order to control for possible adaptation effects. On avcrage, the pitch of the 7th harmonic was shifted upward by about 25 cents due to the presence of its neighbors, but the pitch of the 3rd harmonic was unaffected. In both cases, however, consistent individual differences were found, so that no unequivocal conclusions can be reached.

\section{9:50}

4aMUa7. Historical temperaments analysis system. Alejandro $S$. Esbri (Dept. of Electron. Music, Superior School of Music, Concepción
Beistegui 2103-C-203, Colonia Vertiz Narvarte, Delegación Benito Juárez, México 03020 D.F., Mexico)

This paper describes in a succinct form an historical temperaments analysis system designed for musicians with advanced knowledge of intervalic theory. By introducing the concept of positive trajectory reduction in the fifth circle diagram of the temperament to be analyzed, it is possible to do a very fast calculation of the size of any interval inside that temperament, within an error of \pm 1 cent. The corresponding exact frequency ratio becomes obvious in many cases and it is easy to summarize all the intervalic information in one analysis sheet. In addition to the fifth circle diagram, this sheet includes frequency ratios for the chromatic, diatonic, and hexatonic scales (especially their size in cents), intervals with respect to the tonic, and the deviation in cents of each note from equal temperament. With this system, piano tuning students can make comparisons of different temperaments, classify them, and understand their practicability for different types of keyboard music. Besides, the practical tuning instructions for each temperament can be comprehended much better. Also, with this analysis system, composers and keyboardists are able to program any temperament in an electronic synthesizer with user-tuning option, and the system is even helpful for creating new temperaments.

\section{0:05}

4aMUa8. Musicians' tendency to stretch larger-than-octave melodic intervals. Andrzej Rakowski (Music Acoust. Lab., Chopin Academy of Music, Okolnik 2, Warsaw 00-368, Poland)

Four musicians experienced in identifying musical intervals, but not possessing absolute pitch, tuned a pure-tone oscillator in individual sessions to obtain various melodic intervals with a standard pure tone $500 \mathrm{~Hz}$. Within a single task the standard and variable tones were interchanging until the intended value of a melodic musical interval was obtained. Stimuli were presented via earphones at a loudness level 50 phons. An isosonic filter was used to maintain equal loudness of tones. The variable oscillator was set at either very low or very high frequency at the beginning of each task. The range of 3 octaves above and 2 octaves below standard was investigated and each subject tuned each of the 60 musical intervals within this range 10 times. The sequence of tunings within each octave was quasirandom. The results are presented as mean deviations of each interval from its equally tempered value. The dispersion of results as well as intersubject and intrasubject variability is shown. A tendency appears toward stretching large melodic intervals; they are stretched the more the larger they are. Maximum stretch across 5 octaves investigated is about 1 semitone. [Work supported by the Polish National Committee for Scientific Research.] 


\title{
Session 4aMUb
}

\author{
Musical Acoustics: Computer Music \\ Uwe J. Hansen, Chair \\ Physics Department, Indiana State University, Terre Haute, Indiana 47809 \\ Chair's Introduction-10:30 \\ Inviled Papers
}

10:35

4aMUb1. Technical and aesthetic considerations in interactive computer music systems. Todd Winkler (Dept. of Music, Brown Univ., Box 1924, Providence, RI 02912)

The proliferation of reliable interactive computer music systems has created opportunities for performers to directly influence computer music processes. Performing musicians are highly valued for their unique sense of musical expression and taste: the ever-changing subtleties of tempo, dynamics, timbre, and articulation encompassed in the larger musical framework of musical gesture and phrasing. How can musicians communicate their highly refined skills to a computer, and elicit similarly musical results? This paper describes techniques whereby performers influence highly flexible compositional algorithms that are subtly responsive to musical nuances. These algorithms create MIDI data used to control synthesizers and signal processors. Musical examples will be demonstrated using FollowPlay, a computer program for interactive music and a real-time environment for music composition. The program consists of a large collection of software modules organized into three functional types: Listener Objects analyze and record aspects of a musician's performance, Composition Objects respond by generating MIDI data, and Interpreter Objects unify the entire collection with a graphical user interface that handles timing and intermodular communications.

\section{1:05}

4aMUb2. A touch sensitive dance Hoor/MIDI controller. Russell F. Pinkston (Dept. of Music, Univ. of Texas at Austin, Austin, TX 78712)

A prototype MIDI Dance Surface has been developed which is capable of transmitting precise position coordinates, velocity, and pressure information in the form of standard MIDI messages. The surface consists of a large number of force sensing resistors (FSRs) which are attached to heavy duty plastic sheeting and covered with polyethylene foam. The sheets may either be placed on top of or beneath a standard Marley Dance floor. The FSRs are typically arranged in a grid with 16 columns (left to right) and 4 rows (front to back), which results in a $16 \mathrm{ft}$. square dance surface with $641 \times 4 \mathrm{ft}$. velocity and pressure sensitive regions, each of which is assigned a separate input channel of a Voltage to MIDI Interface Box which has 64 analog inputs, plus MIDI Out. The MIDI Box incorporates a Motorola MC68HC11 microprocessor and can be programmed to convert input/output analog signals to/from any desired MIDI messages, on multiple MIDI channels. Hence, used in conjunction with an "intelligent" external MIDI processing system, it is ideal for use in interactive dance compositions in which one or more dancers can affect both the music and lighting by the nature of their movements and by their precise position(s) on the surface.

\section{Contributed Papers}

\section{1:35}

4aMUb3. Real-time computer simulation of concert hall acoustics: Arbitrary geometries. Turker Kuyel, Elmer L. Hixson (Dept. of Elec. Eng., Univ. of Texas at Austin, Austin, TX 78712), and Russell Pinkston (Univ. of Texas at Austin, Austin, TX 78712)

Artificial reverberation is a challenging application of the computer technology in the fields of musical acoustics, architecture, multimedia, or even in home audio. Real-time operation and high perceptional quality are the two challenges for artificial reverberator design. In this paper, the theory and the implementation of "real-time computer simulation of concert hall acoustics" is discussed. Efficient real-time algorithms based on theoretical models have been built. The memory and computational requirements of these algorithms have been determined. Successful implementation of these theoretical models and software algorithms have been implemented in UT Computer Music Studios using "Accelerando" audio processor. "Accelerando" implementation assumes arbitrary room geometries for the early reverberation response, and rectangular room geometries for the late reverberation response. Extensions of the model and algorithms are made to enable the effective simulation of the late reverberation responses of concert halls with arbitrary geometries. For real-time implementation of the extended algorithms, MasPar MP1 massively parallel computer is used.

\section{1:50}

4aMUb4. Effects of processing audio signals through equivalent color matching filters. Andrew Blackford (Elec. Eng., Univ. of Oklahoma, Norman, OK 73019) and B. Espinoza-Varas (Univ. of Oklahoma Health Sci. Ctr., Oklahoma City, OK 73190)

In applications that combine musical sounds with visual colors, it is relevant to define rules that may relate color dimensions to the pitch and timbre of sounds. In a first attempt toward defining such rules, this investigation examined effects of processing audio signals through equivalent color matching filters. Using Butterworth bandpass filters, a three filter bank $(f 1, f 2, f 3)$ was designed to approximate the response of filters used in the CIE 1931 Standard Observer [G. Wyszecki and W. S. Stile, Color Science (1982)]. The bandwidths of the color-matching filters were trans- 
posed to the audible frequency range $(0.02-20.0 \mathrm{kHz})$; the filter passbands were $0.05-0.6 \mathrm{kHz}$ and $9.0-11.0 \mathrm{kHz}$ for $f 1,1.0-4.0 \mathrm{kHz}$ for $f 2$, and $9.0-11.0 \mathrm{kHz}$ for $f 3$; the passband gains were $1.15,0.35,1.0$, and 1.75 , respectively. Synthesized audio signal were passed through the filter bank and the power, $P$, at the filter outputs was computed. The $P$ values were used to specify the $X$ and $Y$ coordinates of CIE chromaticity diagrams as follows: $\quad X=P f 1 /(P f 1+P f 2+P f 3) ; \quad Y=P f 2 /(P f 1+P f 2+P f 3)$. Chromaticity diagrams were obtained for: (a) $0.07-8.0 \mathrm{kHz}$ sinusoids; (b) vowels with different fundamentals; and (c) complex sounds consisting of 2-21 equal-energy sinusoids. [Work supported by OCAST.]

\title{
Session 4aNS
}

\section{Noise: Progress Report and Discussion on the Continuing Activity on ASA's Role in Noise and its Control}

\author{
Robert M. Hoover, Chair \\ Hoover and Keith, Inc., 11381 Meadowglen, Suite I, Houston, Texas 77082
}

\section{Invited Paper}

\section{0:30}

4aNS1. Noise: Progress report and discussion on the continuing activity on ASA's role in noise and its control. Robert M. Hoover, Chair (Hoover and Keith, Inc., 11381 Meadowglen, Houston, TX 77082)

A discussion meeting is being sponsored by the Technical Committee on Noise to review progress made to date on the actions initiated by the Technical Committee on Noise at the Denver 1993 meeting to increase the role of the ASA in noise and its control. Members of the steering committee will each review the specific activities undertaken in the areas of education, collaboration with other societies, increasing public awareness of noise and the establishment of a task force to determine feasibility of establishing an ASA clearinghouse on noise.

\section{Session 4aPAa}

\section{Physical Acoustics and Structural Acoustics and Vibration: Ray Methods in Radiation and Scattering from Elastic Objects I}

\author{
Philip L. Marston, Cochair
}

Department of Physics, Washington State University, Pullman, Washington 99164-2814

Allan D. Pierce, Cochair

Department of Aerospace and Mechanical Engineering, Boston University, 110 Cummington Street, Boston, Massachusetts 02215

Chair's Introduction-8:25

\section{Invited Papers}

\section{8:30}

4aPAal. Waves and rays on fluid-immersed shells: Beyond spheres, cylinders, Donnell, and Watson. Allan D. Pierce (Boston Univ., Dept. of Aerospace and Mech. Eng., 110 Cummington St., Boston, MA 02215)

Waves propagating along ray paths on shells have various descriptors, such as the dispersion relation that connects frequency and the two principal wave-number components for each point on the surface. Other descriptors inelude polarization relations: complex ratios of amplitudes of quantities that oscillate under the influence of a propagating wave. Such oscillating quantities include the components of the displacement vector for points on the middle surface, the acoustic pressure at the external surface, and the locally-spatially-averaged passive forces exerted on the shell by the internal structure. Energy balance relations are also wave descriptors. Such descriptors are derivable directly from the equations of elasticity and fluid mechanics. In any frequency and 
wave-number regime (associated with the ranges of scales for the intended application) there are a limited number of possible waves types, but analogies with waves on flat plates are often not appropriate. The use of wave-based theory depends on whether the wavelength along the direction of propagation is somewhat smaller than the effective shell radius associated with that direction. It is not necessary for the frequency to be high and/or for the wavelength to be smaller than both of the principal radii of curvature. Radiation of sound into the fluid from waves traveling slower than the speed of sound is possible as long as there is some point above the surface at which the extrapolated phase velocity is supersonic; the explanation is analogous to why propellers with subsonic tip speeds radiate sound. [Work supported by ONR.]

\section{9:00}

4aPAa2. Backscattering enhancements and the impulse response of shells: Observations and ray theory. Philip L. Marston, Greg Kaduchak (Dept. of Phys., Washington State Univ., Pullman, WA 99164-2814), and David H. Hughes (Naval Res. Lab., Washington, DC 20375-5350)

Observations of backscattering by thin spherical shells in water were carried out using tone bursts and a novel broadband transient source [G. Kaduchak, Ph.D. dissertation, WSU (1994)]. Ray theory pertaining to some of the underlying features observed will be discussed including: (a) a bipolar specular feature of the impulse response sensitive to the shell's mass-per-area; (b) a high-frequency enhancement of the tone burst response due to a backwards wave [G. Kaduchak et al., J. Acoust. Soc. Am. (in press)]; (c) the coincidence frequency enhancement of the tone burst and impulse responses associated with the $a_{0}$ - wave; (d) low-frequency features evident in the impulse response associated with the effect of curvature on the $a_{0-}$ wave (or what some authors describe as the "Junger wave"); and (e) periodic $s_{0}$ wave packets giving resonance dips up to moderate frequencies. Feature (b) is sometimes called the thickness quasiresonance and for the stainless steel shell studied the ray theory uses the $s_{2}$ leaky Lamb wave properties in the negative group-velocity region. The development of ray theory for situations where conventional thin shell theory is not applicable will be reviewed. [Work supported by ONR.]

\section{9:30}

4aPAa3. Selected topics in computational ray methods. Roger H. Hackman (Lockheed Palo Alto Res. Lab., Palo Alto, CA 94304-1191) and Gary S. Sammelmann (Coastal Systems Station, Panama City, FL 32407-5000)

Several selected topics intimately involved with the application of ray theory to acoustic scattering are discussed. The first topic is the application of quantitative ray theory to the low-frequency acoustic scattering from large aspect ratio solids. In this approach, the scattering amplitude is developed as a time-ordered perturbation series. The series for the elastic response is explicitly summed to obtain a closed form expression that is analogous to results obtained for spherical and infinite cylindrical geometries through application of the Sommerfeld-Watson transformation. Emphasis is placed on novel features that have no counterpart on these simpler geometries (e.g., "bipolar" coupling phenomena) and their implications for resonance excitation. A second topic deals with a near-field/far-field target strength model for more complicated geometrical shapes that is under development at CSS. But theoretical and experimental results are presented. A third topic is the high-frequency "quasi-resonance" phenomenon of thin shelled structures. The structure of the "quasiresonance" mode is obtained for both complex wave number (real frequency) and complex frequency (real wave number) extensions of the reflection coefficient for a fluid loaded flat plate. The results are used to synthesize the scattering amplitude for spherical shells using forms previously derived for the Sommerfeld-Watson transform.

\section{0:00-10:15 Break}

\section{0:15}

4aPAa4. Implementation of a ray tracing algorithm to calculate the acoustic scattering from fluid loaded, doubly curved shells. Douglas A. Rebinsky, Andrew N. Norris (Dept. of Mech. and Aerosp. Eng., Rutgers Univ., P.O. Box 909, Piscataway, NJ 08855-0909), and Yang Yang (SFA, Inc., Landover, MD 20785)

Ray tracing is used to calculate the acoustical and structural response of smooth, elastic shells of nonseparable shape. The frequency range of interest is below flexural coincidence but still high enough that asymptotic methods are applicable. The structure and development of ray-like solutions on arbitrarily doubly curved shells is reviewed with a discussion of two mechanisms: (1) a "background" response determined by the local inertial impedance, and (2) phase matching to longitudinal and shear waves. The background response can be approximated by specular reflection, but the membrane waves require global treatment over the whole structure. After first calculating the coupling curves, which are the closed loci defined by phase matching with the incident wavefield, "pressure" rays are then sent out over the shell with each ray and its amplitude evolving according to a ray equation and a transport equation. Illustrative examples of ray paths and ray-tube areas will be presented for ellipsoidal and quasicylindrical shells. The use of the Gaussian beam summation method to describe the wavefields will be discussed. Numerical comparisons are made with the exact results for the canonical geometries, and extensions to nonseparable shapes and discontinuous shells will be shown and discussed. [Work supported by ONR.]

\section{0:45}

4aPAa5. Rays, modes, and spectra: Footprints in phase space. Leopold B. Felsen (Dept. of Aerosp. and Mech. Eng., Boston Univ., 110 Cummington St., Boston, MA 02215)

Ray methods have been used extensively to describe mid- and high-frequency, as well as pulsed, phenomena associated with acoustic propagation in the presence of submerged solid or layered elastic structures. The relevant wavefields include externally reflected and diffracted, as well as externally-internally coupled, progressing constituents, repetitive multiples of which combine into oscillatory (modal) forms. Full exploitation of ray methods for radiation and scattering scenarios, either forward (for classification) or inverse (for identification), is aided by systematic footprinting of the (space-time)-(wave number-frequency) characteristics of the 
various wave objects in a (configuration)-(spectrum) phase space catalog. "Clean" footprints obtained from forward asymptotics for certain test problems are shown to be diffused (a) by windowed processings that are applied to extract these footprints from data (imaging), and (b) by space-time limits imposed on the data set. Examples include submerged elastic cylindrical, and finite flat plate, geometries. Also included are simple models of truncated strict or perturbed periodicity, with illustration of superresolution and backpropagation [L. Carin et al., J. Acoust. Soc. Am. (submitted)]. [Work supported by ONR and AFOSR.]

\title{
11:15
}

4aPAa6. Postmodern quantum mechanics: Chaos and the Schrödinger wave equation. Steven Tomsovic (Dept. of Phys., Washington State Univ., Pullman, WA 99164-2814)

Recent theoretical and experimental developments are driving a growing interest in the asymptotic wave behavior of quantum mechanics. The underlying ray theory is based on the trajectories found in the corresponding classical mechanical system. Many systems exhibit some chaotic motion and present a number of challenges for the theory. Recent advances will be illustrated with the stadium billiard which has become one of the premiere paradigms of highly chaotic dynamics [E. J. Heller and S. Tomsovic, Phys. Today 46(7), 38 (1993)]. Both the time-dependent and stationary solutions will be discussed. [Work supported by NSF Grant No. PHY-9305582.]

Session 4aPAb

\section{Physical Acoustics and Bioresponse to Vibration and to Ultrasound: Bioeffects of Ultrasound and Cavitation}

\author{
Wesley L. Nyborg, Chair \\ Physics Department, University of Vermont, Burlington, Vermont 05405
}

\section{Contributed Papers}

\section{9:00}

4aPAb1. Bioeffects of positive and negative pressure pulses in vivo. Michael R. Bailey, David T. Blackstock (Appl. Res. Labs. and Mech. Eng. Dept., Univ. of Texas at Austin, Austin, TX 78713-8029), Diane Dalecki, Carol H. Raeman, Sally Z. Child, and Edwin L. Carstensen (Univ. of Rochester, Rochester, NY 14627)

Murine lung and Drosophila larvae were used to compare effects of isolated positive and negative pulses on tissue containing gas bodies. The basic pulse, produced by an underwater spark, was positive. It could be isolated by floating absorptive, nonreflecting rubber on the water surface to prevent reflection. When an isolated negative pulse was desired, the nubber was removed and the direct, positive pulse was blocked by inserting a jagged-edge barrier between the spark and the exposure area. The jagged edge rendered the diffracted wave (from the barrier edge) incoherent and therefore negligible in the exposure area. Exposure levels were varied by altering the proximity of the exposure area to the source. For each level, lung damage and larva mortality due to 20-pulse exposures were measured. Positive pulses were found to be at least as damaging, to both tissues, as negative pulses having the same amplitude. If the damage was due to cavitation, this result is contrary to conventional wisdom, which holds negative pressure largely responsible for the violence of inertial cavitation. It is speculated that the expansion and ensuing catastrophic collapse of a bubble are hindered in the presence of tissue. [Work supported by ONR, NIH, and ARL: UT IR\&D program.]

\section{9:15}

4aPAb2. Sound transmission to the human fetus. Christopher $\mathrm{L}$. Morfey and Roger J. Pinnington (Inst. of Sound and Vib. Res., Univ. of Southampton, Southampton SO17 1BJ, United Kingdom)
A common noninvasive test for fetal well-being involves transmitting an audio-frequency acoustic signal into the amniotic fluid by means of a mechanical vibrator, which is applied to the mother's abdomen close to the fetal head. This test has been simulated in the laboratory, using a silicone rubber model uterus of spherical shape filled with water. The outside of the model was excited by an impedance head and a load-distributing contact disk. Mechanical impedance measurements were compared with similar measurements on volunteer subjects at about 30 weeks' pregnancy, and showed similar trends as a function of frequency. The model was then used to establish a transfer function between the exciting force and the acoustic pressure in the water-filled cavity. A simple theoretical model is presented which accounts for the main features observed experimentally. Results indicate that commercial fetal stimulators can produce intrauterine sound pressures as high as $30 \mathrm{~Pa}$ rms (180 Pa peak pressure) close to the point of excitation. The actual level depends on the thickness of the subcutaneous fat layer which couples the vibrator to the uterus.

\section{9:30}

4aPAb3. Propagation of elastic waves inside concretions to simulate dynamic failure of kidney stones and gallstones during lithotripsy. Girish Dahake and S. M. Gracewski (Dept. of Mech. Eng., Univ. of Rochester, Rochester, NY 14627)

Reflection and superposition of stress waves is analyzed using finite difference techniques to better understand the effect of stone parameters and geometry on the distribution of strains within kidney stones and gallstones during lithotripsy. Concretions of irregular geometries are subjected to ultrasonic wave sources that simulate lithotripter pulses. The time evolution of strain is calculated inside cylinders of rectangular and circular cross sections, due to an incident radially diverging source in the liquid surrounding the solid. Two schemes are considered to explicitly account 
for the liquid-solid interface conditions. Both schemes account for varying grid sizes and give identical results for straight interfaces, but the second scheme also handles irregular interfaces. The time sequence obtained numerically for strain at the center of a rectangular cylinder also matches well with the experimental results [S. M. Gracewski et al., J. Acoust. Soc. Am. 94, 652-661 (1993)]. In addition, strain contours are plotted for the propagation of $P$ (longitudinal) and $S V$ (shcar vertical) waves inside a circular cylinder. It is shown that the reflection from the concave back surface of the circular cylinder has a focusing effect with the subsequent formation of focal zones (caustics).

\section{9:45}

4aPAb4. The propagation of ultrasonic waves through a bubbly liquid into tissue: A linear analysis. Quan Qi, John G. Harris (Theor. Appl. Mech. UIUC, 216 Talbot Lab., 104 S. Wright St., Urbana, IL 61801), and William D. O'Brien (Elec. Comput. Eng., UIUC, Urbana, IL 61801)

The steady-state response induced by an ultrasonic wave in a structure comprised of two layers, a bubbly liquid, and a viscoelastic solid with a rigid boundary, is studied in the linear approximation. This structure models a steadily cavitating liquid in contact with tissue. The upper surface of the liquid is driven harmonically and models the source. The lower surface of the solid is rigid and models bone. Though the cavitation processes are nonlinear, the propagation is approximated as linear. The model of the bubbly liquid is a simple continuum one, supplemented by allowing for a distribution of different bubble radii and for damping of the oscillations of each bubble. The model contains three functions, the probability distribution describing the distribution of bubble radii, and two functions modeling the mechanical response of, respectively, the individual bubble and the tissue. Numerical examples are worked out by adapting data taken from various published sources to deduce the parameters of these functions. These examples permit an assessment of the overall attenuation of the structure, and of the magnitude of the pressure and particle velocity in the bubbly liquid, and of the traction and the particle displacement in the tissue. [Partial support from Arjo, Inc., Morton Grove, IL.]

\section{0:00}

4aPAb5. Cavitation in water generated by pulse Doppler ultrasound. Ronald A. Roy (Appl. Phys. Lab., Univ. of Washington, Seattle, WA 98105) and J. Brian Fowlkes (Univ. of Michigan Medical Cir., Ann Arbor, MI 48109)

A recently published study reports the direct observation of transient microcavitation in water produced by clinical diagnostic ultrasound [Holland et al, IEEE UFFC 39, 95-101 (1992)]. Results are presented from a similar study in which an aqueous suspension of $0.2-\mu \mathrm{m}$-diam polystyrene spheres was insonated by an ATL UM9 scanner operating in the pulse Doppler mode at 3.5 and $5.0 \mathrm{MHz}$. The suspension was degassed to approximately $90 \%$ of saturation. Using a computer automated active cavitation detector [Roy et al., J. Acoust. Soc. Am. 87, 2451-2458 (1990)], cavitation production rates were obtained for a variety of pulse parameters such as intensity, peak negative pressure, pulse length, and pulse repetition frequency. Results suggest a cavitation threshold which is fairly well predicted by the mechanical index calculated from the various pulse waveforms obtained in situ. [Work supported by NIH through Grant No. RO1 CA39374.]

\section{0:15}

4aPAb6. Cavitation from short pulses high-frequency ultrasound: $A$ study of cavitation production rates and their dependence on acoustical parameters. Adam Calabrese (Dept. of Phys., Univ. of Mississippi, Oxford, MS 38677) and Ronald A. Roy (Univ. of Washington, Seattle, WA 98105)
Previously, cavitation due to pulsed megahertz-frequency ultrasound was detected using a technique that only looked for the onset of detectable cavitation [Calabrese et al., Advances in Nonlinear Acoustics, edited by $\mathrm{H}$. Hobæk, pp. 394-399 (1993)]. This method provides little information about cavitation production rates, which may be better correlated to a mechanical bioeffect than the threshold alone. A modified approach using a passive cavitation detector [Roy et al., J. Acoust. Soc. Am. 87, 2451$2458(1990)]$ is used to determine the rate of cavitation as a function of acoustic pressure amplitude. Measurements are repeated for a variety of pulse lengths (from 3 to $100 \mu \mathrm{s}$ ) and duty cycles (from $0.1 \%$ to $20 \%$ ) and at frequencies of $1,2.25$, and $5 \mathrm{MHz}$. Initial results suggest that the threshold pressure for cavitation is weakly dependent on pulse length and decreases with increasing duty cycle. [Work supported by NIH through Grant No. RO1 CA39374.1

\section{0:30}

4aPAb7. Focused acoustic pulses of finite amplitude and their influence on cavitation bubble dynamics. M. A. Averkiou, L. A. Crum (Appl. Phys. Lab., Univ. of Washington, Seattle, WA 98105), and M. F. Hamilton (Univ. of Texas at Austin, Austin, TX 78712-1063)

A computer code that solves the KZK equation in the time domain [Lee and Hamilton, Ultrasonics Intl. 91 Conference Proceedings (ButterworthHeinemann, Oxford, 1991), pp. 177-180] is modified to include the effects of focusing on pulsed sound beams of finite amplitude. Single cycle pulses, similar to those used in lithotripsy and high intensity focused ultrasound (HIFU) surgery, are considered. The numerical results demonstrate the combined effects of nonlinearity, absorption, diffraction, and focusing on the waveform distortion and shock formation in short acoustic pulses. Self-defocusing associated with shock formation is also taken into account. Propagation curves are shown for the positive peak pressure amplitude $\left(P_{+}\right)$and the negative peak pressure amplitude $\left(\boldsymbol{P}_{-}\right)$along the axis of the source. The calculated pressure waveforms are used as input to computer codes that model bubble dynamics. Predicted radius-time curves are presented for the response of a bubble due to a focused acoustic pulse of finite amplitude. Gas diffusion from the liquid into the vapor bubble, and the roles of $\boldsymbol{P}_{+}$and $\boldsymbol{P}_{-}$in the diffusion process, are also considered. [Work supported by the National Institute of Health.]

\section{0:45}

4aPAb8. The biological relevance of transient cavitation measurements performed in water: The effect of shear viscosity. John Allen (Dept. of Mech. Eng., Univ. of Washington, Seattle, WA 98105) and Ronald A. Roy (Univ. of Washington, Seattle, WA 98105)

Recent years have seen a host of direct experimental evidence of transient microcavitation in water produced by short pulses of megahertzfrequency ultrasound [Roy et al., J. Acoust. Soc. Am. 87, 2451-2458 (1990); and others]. However, to our knowledge there have been no published accounts of direct observations of such microcavitation activity in biological media. It is useful to relate the results of the aqueous experiments to the likelihood of producing cavitation in tissue. To do this, one must assess the relevant differences between the two media. As a first step in this process, a numerical implementation of the Gilmore equation for adiabatic bubble pulsations is employed [Church, J. Acoust. Soc. Am. 83, 2210-2217 (1988)] and compare predicted thresholds in water and in biological media modeled as a viscous fluid. Comparisons are made with the analytical theory of Holland and Apfel [IEEE UFFC 36, 204-208 (1989)]. The requirement that, at the threshold pressure, the bubble collapse temperature exceed $5000 \mathrm{~K}$ provides the rigorous comparison criterion. Results are viewed in light of recent experimental results obtained in water [Calabrese et al., J. Acoust. Soc. Am. 95, 2856 (1994)] and in tissuemimicking phantom materials [Zheng et al., J. Acoust. Soc. Am. 95, 2855 (1994)]. [Work supported by NIH through Grant No. RO1 CA39374.] 


\title{
Session 4aSPa
}

\section{Speech Communication: Production and Perception of Speech by Children I}

\author{
Peter Assmann, Cochair \\ School of Human Development, University of Texas at Dallas, Box 830688, Richardson, Texas 75083 \\ William Katz, Cochair \\ Callier Center for Communication Disorders, 1966 Inwood Road, Dallas, Texas 75235-7298
}

\author{
Chair's Introduction-8:00 \\ Invited Papers
}

8:05

\begin{abstract}
4aSPa1. Anatomic development of the vocal tract: Implications for speech motor control and acoustic properties of speech. Ray D. Kent (Dept. of Commun. Disord., Univ. of Wisconsin, 1975 Willow Dr., Madison, WI 53706)

It has been proposed that models of speech production should be developed with consideration of age and gender differences. Although some important age differences in anatomy and acoustic patterns of vocalization have been described between infants and adults, much less attention has been given to the anatomic development of the speech production system between infancy and adulthood. This talk reviews the anatomic development of the craniofacial, oral, and laryngeal systems of speech production between birth and young adulthood. Implications of the developmental patterns are considered for the ontogeny of speech production, especially speech motor control and acoustic patterns of speech. Consideration also is given to gender and race as they relate to vocal tract anatomy and its development in children. Topies to be reviewed include: craniofacial skeleton, nasopharynx, tongue, lips, and larynx. The overall pattern of development will be summarized for each system and intervals of especially rapid growth will be identified. A major hypothesis to be evaluated is whether growth is harmonious across the major anatomic systems.
\end{abstract}

\section{8:30}

4aSPa2. Development of skilled speech production in children. Shari R. Baum (School of Commun. Sci. \& Disord., McGill Univ., 1266 Pine Ave. W., Montreal, PQ H3G 1A8, Canada) and William F. Katz (Univ. of Texas at Dallas, Dallas, TX)

There has been much recent interest in the manner in which children develop mature speech production capabilities. Investigations of both normal speech acquisition and developmental speech production impairments have contributed to our current knowledge base. A consistent finding is that young children's speech is highly variable, both in its temporal and spectral attributes, and that this variability diminishes gradually with age. However, the exact manner in which articulatory precision emerges (or fails to emerge in speech disorders) continues to be the subject of debate. For example, some research on anticipatory coarticulation suggests a developmental progression from syllable- to segment-based timing strategies while other research suggests the opposite. Similarly, some studies of compensatory articulation show comparable degrees of motor equivalence in young children and adults, while others show that this aspect of motor control emerges gradually with maturation. This presentation will review key experiments (primarily based on acoustic analyses), consider potential explanations for the controversial data, and discuss methodological alternatives that may assist in the resolution of outstanding questions. Recent evidence indicates that specific motor patterns of different articulators will play a major role.

\section{$8: 55$}

4aSPa3. The developmental role of acoustic boundaries in speech perception. Ralph N. Ohde (Div. of Hear. and Speech Sci., Box 552, Station 17, Vanderbilt Univ. School of Medicine, Nashville, TN 37232-8700)

During production, there are frequent abrupt changes in the amplitude or in the spectrum of the sound, and these variations are regarded as boundaries between speech sounds. The information appears rich in cues for phonetic features of the segments occurring within 10 to $30 \mathrm{~ms}$ of the acoustic boundaries. Recent research examining children's speech shows that the information contained within the acoustic boundaries of their productions provides important cues for consonant place of articulation. In addition, interspeaker variability for these segments is low, as compared to properties such as formant transitions. Current studies also reveal that for both adult and child speech a principal mechanism involved in processing acoustic boundary cues involves short-term memory, and that the elimination of boundary information negatively affects perception of place of articulation, particularly for children's speech. Furthermore, the importance of acoustic boundaries in children's speech extends to their perception of place of articulation of both consonants and vowels. Young children accurately process place of articulation from very short duration stimuli, which includes the period of rapid spectrum change. In the current paper, this evidence supporting the salience of acoustic boundary information in children's speech and children's perception of speech will be presented. [Work supported by NIH, DC00464.] 
4aSPa4. The development of speech perception: Beyond infancy. Amanda C. Walley, Victoria L. Michela (Dept. of Psychol., Univ. of Alabama at Birmingham, Birmingham, AL 35294), and James E. Flege (Univ. of Alabama at Birmingham, Birmingham, AL 35294)

In comparison to what is known about speech perception in infancy and adulthood, much less is known about perception in childhood. Nevertheless, there is a growing consensus that phonetic/phonemic segments are not present in the "initial state," but only emerge gradually over the course of childhood as units of speech representation and/or processing. How perception may become more segmentally based by virtue of vocabulary growth will be considered-as the mental lexicon grows in overall size, as individual lexical items become more familiar, and as particular regions of the lexicon become more "crowded" (i.e., as the acoustic-phonetic similarity among different words increases). The research to be presented includes recent data bearing on children's perception of native and non-native vowels in word and nonword contexts. Age-related changes in spoken word representation and processing may have important consequences for other developmental achievements, such as the growth of metaphonological knowledge/skills and early reading ability, as well as for success in learning a second language. More generally, the purpose is to help set an agenda for future research that will provide a more complete picture of the developmental trend for speech perception. [Work supported by NIH.]

\title{
Session 4aSPb
}

\section{Speech Communication: Production and Perception of Speech by Children II (Poster Session)}

\author{
Peter Assmann, Cochair \\ School of Human Development, University of Texas at Dallas, Box 830688, Richardson, Texas 75083 \\ William Katz, Cochair \\ Callier Center for Communication Disorders, 1966 Inwood Road, Dallas, Texas 75235-7298
}

\section{Contributed Papers}

\begin{abstract}
All posters will be on display from 8:00 a.m. to 12:15 p.m. To allow contributors an opportunity to see other posters, contributors of odd-numbered papers will be at their posters from 10:15 a.m. to 11:15 a.m., and contributors of even-numbered papers will be at their posters from 11:15 a.m. to 12:15 p.m. Posters will remain on display until 10:00 p.m. on Friday.
\end{abstract}

\begin{abstract}
4aSPb1. Prosodic features of foreigner talk register in the speech of 10- to 11- and 6- to 7-year-old American children. Jennifer Sullivan and Andrea G. Levitt (Wellesley College, 106 Central Street, Wellesley, MA 02181-8289)

At what age and in what ways do children begin to modify their speech in the presence of a non-native speaker? Two groups of six American children each, three girls and three boys, were asked to teach two children, one a native speaker of English and another with limited English proficiency, to recite the pledge of allegiance and two children's poems. The children in the older group were between 10 and 11 years old, and those in the younger group were between 6 and 7. When speaking to the non-native child, the older group of children produced shorter utterances, repeated often, and spoke significantly more slowly than when speaking to the native speaker. Repetitions were generally spoken with a lower $F 0$, and the older children also used a significantly reduced $F 0$ range when addressing the non-native speaker. There were no differences in the prosodic adjustments made by the boys and the girls. The children in the younger group generally made few modifications in their speech to the non-native child, although some of the older subjects in this group occasionally modified their speech to the non-native speaker in ways similar to those of the older children. [Work supported by NIH Grant No. 1 R15 HD28173-01.]
\end{abstract}

4aSPb2. Prosodic cues for phrasal boundaries in productions by children and adults. Sushama Verma, Anne M. Mannering, Branden
M. Komell, William F. Katz (Dept. of Commun. Disord., Univ. of Texas at Dallas, Callier Center, 1966 Inwood Rd., Dallas, TX 75235), and Cheryl Beach (3408 Custer St., Cincinnati, OH 45208)

Although recent evidence suggests that young children use prosodic information to "bootstrap" themselves into syntactic comprehension, little is known about how children use prosody to signal syntactic boundaries in their spoken utterances. A speech elicitation task examined the extent to which children and adults control duration and intonation to mark phrase boundaries in spontaneous speech. Adults and young children (ages 5 and 7) were asked to describe groupings of colored blocks in a manner such that a blindfolded listener could tell "which blocks go together." This procedure elicited utterances corresponding to three syntactic bracketings of the phrase "pink and green and white." Acoustic analysis of subjects' productions indicated that adults reliably controlled duration (i.e., syllable lengthening, pauses) and intonation fall-rise patterns to signal phrase boundaries. In contrast, children's productions were highly variable and showed no evidence of intonation cues being involved. Individual subject analyses suggested that some of the 7-year-old children used duration cue to signal phrase boundaries, although the contrasts were not as marked as in the adult data. The results were interpreted as showing that by age 7 , most children have not yet mastered prosodic cues for phrasal boundaries in their speech production. 
4aSPb3. A preliminary investigation of how prosodic cues may help young infants' to extract words from natural sentences. Francisco Lacerda, Ulla Sundberg, and Christin Andersson (Inst. of Linguist., Stockholm University, S-10691 Stockholm, Sweden)

This paper presents preliminary results of a study assessing 6-monthold infants' capacity to pick up linguistically relevant information presented in complex naturalistic linguistic contexts. The head-turn procedure was used to investigate the infants' ability to discriminate target words that are embedded in natural carrier sentences. The sentences were produced as infant-directed or adult-directed speech ("direction") and for each direction of the target word occurred either in emphatic or nonemphatic position ("focus"). A large number of sentences, including all the possible combinations of direction and focus, was first submitted to adult scrutiny in order to select unambiguous carrier sentences in which adequate target words were subsequently edited. Both adults and infants were tested by the headturn procedure, to assess the differences between the infants' and adults' use of the prosodic information. [Research supported by The Bank of Sweden Tercentenary Foundation, Grant No. 94-0435.]

4aSPb4. The nature of top-down representations that affect bottom-up phonemic processing in children and adults. Angela $\mathbf{L}$. Bauman (Dept. of Psychol., SUNY-Buffalo, Park Hall, Box 604110, Buffalo, NY 14260-4110) and Judith C. Goodman (Univ. of California at San Diego, La Jolla, CA 92093)

Adults and children use lexical knowledge to interpret phonemes in speech [W. F. Ganong, JEP: HPP 6, 110-125 (1980); Hurlburt and Goodman, in preparation; S. Nittrouer and A. Boothroyd, J. Acoust. Soc. Am. 87, 2705-2715 (1990)]. This study examines the nature of lexical representations necessary for top-down effects. In a baseline assessment, 5-yearolds and adults heard stimuli from seven-step voice onset time (VOT) continua. The continua endpoints formed nonwords (e.g., beb $\rightarrow$ peb). Subjects were then trained on one endpoint from each continuum in one of two learning conditions. Subjects in the exposure condition heard items in a carrier phase five times (e.g., "The sound you will hear is peb."). Subjects in the concept condition heard five instances of each item used as a picture label, in definitional sentences, and in a sentence identifying its superordinate category. After training, subjects again heard the baseline VOT continua. Children's and adults' phoneme boundaries shifted such that a greater proportion of tokens were perceived as compatible with the trained end of the continuum regardless of learning condition. This suggests that minimal exposure to a sound pattern, rather than fully-specified lexical knowledge, can result in top-down effects in speech perception.

4aSPb5. Voice pitch as an aid to speechreading in young children. Joanna D. Fagg (School of Commun. Sci. and Disord., McGill Univ., 1266 Pine Ave. West, Montreal, PQ H3G 1A8, Canada)

The speechreading ability of a group of young children with normal hearing was assessed using a simple, closed-set, single word identification task. The speechreading test was devised such that the accurate identification of half of the test items would be expected to be facilitated by the ability to perceive voicing contrasts. All of the subjects performed the task twice, once with visual speech information only and once with visual information supplemented by a voice pitch signal, derived using an electrolaryngograph. Subjects were found to be able to identify familiar words through speechreading, both in the silent condition and with the addition voice pitch signal. No significant overall improvement was found to result from the provision of voice pitch information. However, subjects were found to significantly improve in their ability to identify those test items which required the discrimination of voicing contrasts. Conclusions were drawn about the normal speech perceptual processes of young children and about the passible benefits of providing simple speech pattern information, such as the voice pitch signal, for children with profound hearing losses.

4aSPb6. A developmental study of audiovisual speech perception using the McGurk paradigm. Neil S. Hockley and Linda Polka (School of Commun. Sci. and Disord., McGill Univ., 1266 Pine Ave. West, Montréal, PQ H3G 1A8, Canada)

The development of audiovisual speech perception was examined in this experiment using the McGurk paradigm (McGurk and MacDonald, 1976), in which a visual recording of a person saying a particular syllable is synchronized with the auditory presentation of another syllable. Previous audiovisual speech studies have shown that adult perception is strongly influenced by the visual speech information whereas the perception of young children (5-8 years) shows a very weak influence of visual speech patterns and a strong bias favoring the auditory speech information. In this investigation 46 children in four age groups $(5,7,9$, and 11 year olds) and 15 adults were presented with conflicting audiovisual syllables in which an auditory / $\mathrm{ba} /$ sequence was combined with visual $/ \mathrm{va} / / / \mathrm{Oa} /, / \mathrm{da} /$, and $/ \mathrm{ga} /$ sequences, respectively. The results indicated that the influence of auditory information decreased with increasing age, while the influence of visual information and the integration of auditory and visual information increased across the age groups. In addition, an adult-like response pattern was observed in only half of the children in the oldest child subject group (10-12 years old) suggesting that the ability to utilize visual speech information continues to develop beyond the age of 12 . [Work supported by NSERC.]

4aSPb7. Infant dependence on acoustic cue redundancy: Discrimination of the word-final voicing contrast. Margret A. Orme and Linda Polka (School of Commun. Sci. and Disord., McGill Univ., 1266 Pine Ave. West, Montréal, PQ H3G 1A8, Canada)

Discrimination of a word-final stop consonant voicing contrast, /bid//bit/, by adults and infants at two ages (6-8 and 10-12 months) was investigated in a category-change conditioned headtum procedure across three stimulus conditions: full cue (FC), burst and closure cues neutralized (BCCN), and vowel duration neutralized (VDN). Adults performed at ceiling levels for all three conditions. No infant age differences were observed. However, there was some evidence that infants benefitted from the presence of redundant acoustic cues (FC $>\mathrm{BCCN}$, but $\mathrm{FC} \ngtr \mathrm{VDN}$ ). Infants performed significantly better with the VDN stimuli indicating that final release burst information is more salient to infants than vowel duration differences for this $/ \mathrm{bid} /-/ \mathrm{bit} /$ contrast. This result differs from findings of prior research on adult and infant perception of such contrasts which showed a prominent use of the preceding vowel duration cue. This finding suggests that vowel duration becomes useful as a cue to final stop voicing with linguistic sophistication. [Work supported by NSERC.]

4aSPb8. Sensitivity to the distinctive features of vowels in newborn speech segmentation. Kelley L. Kaye (Univ. of Texas at Dallas, School of Human Develop. GR41, P.O. Box 830688, Richardson, TX 75083-0688)

A previous study investigating the unit of speech perception in newborns [K. L. Kaye, J. Acoust. Soc. Am. 92, 2299(A) (1992)] yielded results suggesting that speech processing in newborns requires both the definition of the multidimensional perceptual space of the utterances in terms of small perceptual units, and the chunking of phenoemes into syllables for cognitive processing in memory. The present study focused on the perceptual process, investigating whether newborns are sensitive to the distinctive features of vowels. A vowel can be characterized by three opposed distinctive features. Using an operant $(|\mathbf{S}|)$ sucking procedure, one group of newborns received, over earphones, a presentation set with 0 distinctive feature variation, a second group with greater than 0 but less than 1 distinctive feature variation, and a third group with 2 distinctive features variation between the computer synthesized vowels in the set. Results indicated that responding is affected by the number of distinctive features required to define the vowel set, and together these studies indicate that newborns can segment speech in terms of syllables, phonemes, and distinctive features. Distinctive features are normally defined in articulatory terms, yet newborns do not have the appropriate articulatory equipment. The theoretical implications of this will be discussed.

4aSPb9. Prototype preference and magnet effects in newborns: Evidence for an innate component. Michelle Aldridge (Callier Ctr. for Commun. Disord.-UTD, 1966 Inwood Rd., Dallas, TX 75235)

Within the vowel space there are subareas which correspond to specific vowel caregories. Within each subarea there is a point which defines the best exemplar or prototype of that vowel. Kuhl [J. Phon. 21, 125-139 (1993)] has argued that the area immediately surrounding a prototype is perceptually shrunken, with reduced variance in responding to points in that immediately surrounding area, a magnet effect [Kuhl (1993)]. Empiri- 
cally, prototype points correspond to the averages of large numbers of utterances. This leads to the hypothesis that prototypes are learned. It is equally possible that prototypes are innate [Kuhl (1993)]. To decide between these two hypotheses an experiment was run with newborns. The vowel $/ y /$ was used in the experiment; $/ y /$ does not occur in Texas. The newborns' only experience was provided in the experiment. "Best exemplar" was equated with "most attractive" measured by listening time in an operant choice task. Results indicated that experience did not determine preference. The prototype / $y$ / was preferred, regardless of the experience provided. No significant magnet effect occurred. A second, slightly different, experiment did find a magnet effect. The difference between the two experiments may explain why the magnet effect occurs.

4aSPb10. Deficits in linguistic experience delay the development of mature speech perception abilities and of phonemic awareness. Susan Nittrouer (Boys Town Natl. Res. Hospital, 555 North 30th St., Omaha, NE 68131)

One model of how mature patterns of speech perception develop suggests that the weighting of various kinds of acoustic information changes as a result of experience with a native language. It has further been suggested that this developmental shift in perceptual weighting is related to the development of mature sensitivity to the phonemic structure of one's native language (i.e., phonemic awareness). Thus it could be predicted that deficits in linguistic experience would delay both the development of mature patterns of speech perception and of phonemic awareness. This preliminary study tested this prediction by administering labeling and phonemic-awareness tasks to two groups of children: those of mid socioeconomic status (SES) and those of low SES. Several studies have shown that parental language directed to low-SES children differs from that directed to mid-SES children in kind and amount, such that low-SES children may be thought of as enduring deficits in linguistic experience. Results showed that low-SES children performed differently from mid-SES children on both experimental tasks, displaying results similar to those of younger, mid-SES children in other studies. These results suggest that linguistic experience plays a role in the development both of mature perceptual weighting schemes for speech signals and of phonemic awareness.

4aSPb11. Maturational limitations to pronunciation accuracy in a second language. Grace $\mathrm{H}$. Yeni-Komshian (Dept. of Hear. and Speech Sci., Univ. of Maryland, College Park, MD 20742) and James E. Flege (Univ. of Alabama, Birmingham, AL 35233)

This study examines pronunciation proficiency in a second language (L2) as a function of the age of (L2) acquisition. Accuracy of pronunciation of words and sentences produced by Korean-English bilinguals who began learning their L2 (English) between 2 and 24 years of age is reported. All subjects were born in Korea and had resided in the U.S. for at least 8 years. Their average age at the time of testing was about 24 years. The results are based on the subjects' accuracy of pronunciation of vowels and consonants in English words and sentences. Preliminary findings, regarding segmental phonemes, indicate no major difficulties up to about 11 years for $\mathbf{L} 2$ acquisition. Those who learned English (L2) at or later than 12 had significantly lower pronunciation accuracy scores. These difficulties were more evident in vowels than in consonants. The results support the critical period for language leaming hypothesis. The discussion will address the problem of specifying the optimum conditions for second language acquisition and first language retention.

THURSDAY MORNING, 1 DECEMBER 1994

BALLROOM B, 8:00 A.M. TO 12:15 P.M.

Session 4aUW

\title{
Underwater Acoustics: Signal Processing II
}

\author{
Gary R. Wilson, Chair \\ Applied Research Laboratories, University of Texas, P.O. Box 8029, Austin, Texas 78713-8029
}

\section{Contributed Papers}

8:00

4aUW1. Towed vertically directive source for shallow-water scattering measurements. Tommy G. Goldsberry (Office of Naval Res., 800 N. Quincy St., Arlington, VA 22217-5660), Joseph M. Monti, and Michael Werbicki (Naval Undersea Warfare Ctr., Newport Div./ Detachment New London, New London, CT 06320-5594)

A unique tool for low/mid-frequency (LF/MF) shallow-water reverberation and scattering measurements has been developed. The towed vertically directive source (TVDS) is designed to overcome the two critical deficiencies in most attempts to measure LF scattering characteristics of the ocean bottom in shallow water-insufficient source level and inadequate directivity of the sound source. Although TVDS provides high source level and directivity at LF and MF (220 to $230 \mathrm{~dB}$ over the frequency band from 400 to $4000 \mathrm{~Hz}$ ) it is designed for use on 3000 ton research vessels at tow depths to $180 \mathrm{~m}$. An articulated strut allows aperture for a $20 \mathrm{deg}$ vertical beamwidth at $600 \mathrm{~Hz}$ but permits TVDS to be deployed and retrieved with the low-frequency array retracted. An MF array with center frequency of $3000 \mathrm{~Hz}$ is mounted in the vertical stabilizer of TVDS and also provides a $20 \mathrm{deg}$ vertical beamwidth. Provision for simultaneous transmission at LF and MF will permit frequency diversity in measurements over a decade of frequency with scattered signal reception on the TVDS transducer arrays or an adjunct towed array. TVDS design and test results will be discussed. [Work sponsored by ONR.]
8:15

4aUW2. Performance of adaptive beamforming (ABF) for fixed receiver bistatic active systems. Yung $P$. Lee and John Hanna (Sci. Applications Int. Corp., 1710 Goodridge Dr., MS T1-3-5, McLean, VA 22102)

Data collected from a sea test have been processed and analyzed to examine the performance of adaptive spatial beamforming techniques in fixed receiver bistatic active systems. Adaptive beamforming using both the least-mean-squared (LMS) method and block sample-matrix-inversion (SMI) method have been studied. The LMS method uses the current input signal to recursively update the adaptive weight; it converges slowly and provides only a little adaptive gain (over the conventional beamforming) for reverberation suppression. On the other hand, the SMI method uses an estimate of the current covariance matrix to recursively update the adaptive weight; it converges rapidly and provides more than $10 \mathrm{~dB}$ adaptive gain. It is well known that adaptive processing is very sensitive to mismatch due to system errors or environmental fluctuations. To recover the signal lost in mismatch, two robust SMI adaptive beamformers, Feedback-Loop WhiteNoise-Constrained (FLWNC) method and Signal-Coherence-Constrained Reduced-Rank (SCCRR) method, were applied. They successfully recover signals and maintain excellent clutter suppression. 
4aUW3. An assessment of the variability of low-frequency active sonar performance in shallow water. Jeroen Franken, Ching-Sang Chiu, James H. Miller (Code EC/Mr, Naval Postgraduate School, Monterey, CA 93943), and Warren W. Denner (EOS Res. Assoc., Monterey, CA 93940)

The performance of a generic low-frequency, active sonar was simulated using a broadband, coupled mode, propagation model. The lowfrequency active system consisted of a $1000-\mathrm{Hz}$ source with source level of $220 \mathrm{~dB}$ and a towed horizontal array of hydrophones. The ocean environment used in the simulation was 159 tomographic snapshots of the Barents Sea Polar Front, taken every 5 min. These tomographic images over a range of $35 \mathrm{~km}$ provide a virtual ocean in which system performance and environmental data requirements can be assessed. The probability of detection calculated as a function of time for $13 \mathrm{~h}$ is compared with that estimated using a range- and time-independent assumption. The importance of coastal acoustic tomography for tactical applications will be discussed.

\section{$8: 45$}

4aUW4. Reverberation characterization and suppression in the presence of channel spreading. Geoffrey S. Edelson (Lockheed Sanders, Inc., MAN6-2000, P.O. Box 868, Nashua, NH 03061-0868) and Ivars P. Kirsteins (SACLANT Undersea Research Centre, La Spezia, Italy)

In active sonar, the reverberation plus target time series can be modeled as the joint convolution of the signal with the channel and scatterer impulse responses. Especially in shallow water, the channel response cannot be adequately modeled as propagation over discrete paths. A maximum likelihood type approach is proposed for estimating the arrival times of signals which have propagated via a continuum of paths, i.e., temporally spread channels. The channel spreading is included in the model by using a discrete prolate spheroidal sequence (DPSS) to represent the channel impulse response of given duration, but unknown shape. Thus the unknown parameters are the arrival times and the scale factors of the DPSS expansion. The parameters are estimated using an iterative methodology which decomposes the original data into its constituent components and then estimates the parameters of the individual components through a sequence of one-dimensional searches. Experimental data and computer simulation examples indicate that the method performs well.

\section{9:00}

4aUW5. Dynamic stereo imaging of hard-skinned sonar targets. Terry L. Henderson (Appl. Res. Lab., Univ. of Texas at Austin, Austin, TX 78713-8029)

The visible portion of a hard-skinned sonar target can be scanned in $x y z$ coordinates by beamforming a planar array, to give a 3-D representation. However, a simple line array running along the $x$ axis can provide a projection view of a target that lies along the $z$ axis, with its upper and lower surfaces being projected semitransparently onto the $x z$ plane, giving a picture analogous to that of a biological specimen viewed with a slide microscope. If the line array is tumbled about the $z$ axis, it will appear that the target is revolving acound the $z$ axis. When this tumbling view is presented $14^{\circ}$ out of phase to the left and right eyes, a stereoscopic view of the tumbling target is obtained, with its 3-D form revealed by the brain's innate tomographic signal processing capability. In-water experiments have been conducted for several target shapes. Other means of generating dynamic displays have been developed that are suitable for scanning objects on the seafloor. A videotape of results for several targets has been prepared. Further simplifications of the array structure and processing are possible, with some limitations and weaknesses. [Work supported by ARL:UT IR\&D.]
4aUW6. Improvement of ultrasonic synthetic aperture imaging via deconvolution preprocessing. Nobuyuki Ishikawa, Yoshio Fujii (Control \& AI Lab., Japan Atomic Energy Res. Insl., Tokai-mura, Naka-gun, Ibaraki, 319-11 Japan), and Yoshikuni Shinohara (Nuclear Power Eng. Cooperation, Toranomon, Minato-ku, Tokyo, 105 Japan)

Ultrasonic synthetic aperture imaging is a useful technique for detecting cracks in the material and viewing the underwater environment. Since high-frequency ultrasonics are utilized for such purposes, transducers with sufficient transient response have been made available. On the other hand, ordinary transducers for measurements in air are of the resonant type because of comparatively low practical frequency used. However, the utilization of resonant type transducers for synthetic aperture imaging in air makes the measurement resolution poor. This degradation comes from the narrow-band characteristics of transducers, which cause signal distortion in transduction. In this study, to compensate for the narrow-band characteristics of transducers, a deconvolution technique is applied for preprocessing of synthetic aperture imaging and improvement is attained thereby in the case of using the resonant type transducer. The filter used for deconvolution is of the FIR type which is designed in the time domain. The desired filter output has bandpass characteristics with a center frequency of $40 \mathrm{kHz}$ corresponding to the resonant frequency of the transducer and the passband is $6 \mathrm{kHz}$. Experimental results show that resolution of synthetic aperture imaging is improved by a factor of 3.0 in the range direction and 3.5 in the lateral direction by using deconvolution processing.

4aUW7. Optimum detection of solid spherical elastic objects of uncertain composition in an uncertain reverberation-limited environment. M. Wazenski, V. Premus, D. Alexandrou, and L. W. Nolte (Dept. of Elec. Eng., Duke Univ., Box 90291, Durham, NC 27708-0291)

Detection of solid spherical elastic objects is approached via optimal detection and estimation theory in conjunction with acoustic scattering models for both the object and the environment. The objective is the development of full field (amplitude and phase) optimal decision methods for high-frequency active detection of a target in the vicinity of the seafloor. This parametric approach incorporates the inherent uncertainty of both object composition and seafloor geomorphology. The acoustic model used for the spherical object is the well-known modal series solution and is parametrized by object size, density (object and medium), compressional wave speed (object and medium), and shear wave speed. This deterministic model is used to predict the target signature measured at an array of sensors. Acoustic modeling of the seafloor is performed by applying the 3-D Helmholtz-Kirchoff formulation to the anisotropic, power-law description of seafloor relief due to Goff and Jordan and is parametrized by correlation length (two directions), lineation direction, rms height, and fractal dimension. This model is used to predict the spatial and temporal coherence of the scattered sound field. Illustrative detection examples are presented along with receiver operator characteristic (ROC) performance. [Work supported by ONR: Ocean Acoustics.]

\section{$9: 45$}

4aUW8. A generalized framework for high-frequency optimum target detection in a shallow water, random wave scattering environment. V. Premus, D. Alexandrou, and L. W. Nolte (Dept. of Elec. Eng., Duke Univ., Durham, NC 27708-0291)

It has been shown that for the case of Gaussian reverberation, the likelihood ratio detector can be made robust with respect to inexact knowledge of the acoustic scattering medium by incorporating a probabilistic description of environmental uncertainty and a model for the wave-number spectrum of the bottom-interacting acoustic field [Premus et al., J. Acoust. Soc. Am. (submitted)]. In this work a generalized form of the optimum detector is presented which requires full-field (amplitude and phase) modeling capability for the scattered field and relies on Monte Carlo integration to treat environmental uncertainty. The performance of the generalized 
detector formulation is compared to that of the original detector formulation, which is a special case based on explicit modeling of the reverberation second-order statistics that is exact for the case of Gaussian reverberation. Tradeoffs involving the prior knowledge, modeling assumptions and computational requirements are discussed. [Work supported by ONR, Ocean Acoustics.]

\section{0:00}

4aUW9. Equal-likelihood probability modeling in active and passive sonar. Richard Pitre (Naval Res. Lab., Washington, DC 20375-5350)

The equal-likelihood rationale [R. Pitre and N. R. Davis, J. Acoust. Soc. Am. (to be published)] for the selection of probability models has been extended and strengthened. The method is shown to be optimally robust, testable, and correctable. Nonlinear parameter estimation and filtering equations are developed based on this formalism and its application to modeling active sonar in a random waveguide is formulated. [Work sponsored by ONR.]

\section{0:15-10:30 Break}

\section{0:30}

4aUW10. Reconstruction of a periodic time-domain signal "buried" in Gaussian noise. Stefan T. Sidahmed and Antal A. Sarkady (Dept. of Elec. Eng., U.S. Naval Acad., Annapolis, MD 21402)

Power spectral analysis does a reasonably good job of signal detection but fares poorly in characterization because the phase information is ignored in the spectral computations. From spectral analysis, an initial or seed value for the period of the target signal is obtained. The original digitally sampled noisy time signal is resampled (up/down sample rate conversion) at such a rate that precisely an integer number of time samples are obtained on each cycle of the quasiperiodic target signal. These noisy cycles are synchronously averaged in the time domain and an estimate for the shape of the target signal is computed. The sample rate conversion, and the synchronous averaging is repeated until the rms value of the result is maximized (i.e., phase cancellation is minimized). The voltage signalnoise ratio of the reconstructed target signal is proportional to the square root of the number of target cycles averaged. This procedure was tested with laboratory type signals and found to have an excellent performance at signal-to-noise voltage ratios as low as $-40 \mathrm{~dB}$.

\section{0:45}

4aUW11. Asymptotic behavior of the matched-phase noise filter in the limit of low signal-to-noise ratio. B. Edward McDonald and Gregory J. Orris (Naval Res. Lab., Washington, DC 20375-5000)

It has been shown elsewhere that information about the shape of the noise spectrum with no phase knowledge can be used to construct a noise rejection algorithm which we call the matched-phase filter. Here, it is demonstrated that to first order in the signal-to-noise ratio (SNR), the matched-phase filter is capable of improving an arbitrarily low SNR ( $\ll 1)$ to order unity when the spectrum of the noise is known exactly. This analysis accounts for properties of numerical results with computergenerated signal and noise, with SNR values below $-100 \mathrm{~dB}$. Since computers have dynamic ranges far in excess of audio equipment, these results in an actual experiment would be limited by the dynamic ranges of the equipment used. Also shown are examples illustrating the performance of the algorithm when the noise spectrum is known only approximately.

\section{1:00}

4aUW12. A multiresolution, likelihood-based approach to pattern classification with application to characterization and automated recognition of marine mammal sounds. Thomas J. Hayward (Naval Res. Lab., Washington, DC 20375-5350)

A multiresolution, likelihood-based statistical approach is presented for characterizing labeled classes of samples (e.g., measured time series or time-frequency distributions of acoustic transients) and for classifying new samples based on this statistical characterization. The labeled classes are characterized by a histogram associated with a multiresolution decomposition of the data in each class. Classification of a new sample is then performed by calculating, for each labeled class, the conditional probability of the sample given the statistics of that class. These conditional probabilities, which are interpreted as relative likelihoods that the sample belongs to each of the classes, are calculated in a recursive computation that proceeds from coarse to fine resolution. A simple, efficient computer implementation using associative arrays is presented. Successful classification of both time series and time-frequency distributions of marinemammal vocalizations is demonstrated using relatively small numbers of labeled samples ( 10 per class). [Work supported by ONR.]

\section{1:15}

4aUW13. Remote identification of moving acoustic objects. Roger F. Dwyer (Naval Undersea Warfare Ctr., New London, CT 06320)

Often in applications it is desired to remotely identify an object from its acoustic signature. In active sonar the object is insonified by a transmitted waveform and the received pressure is processed to localize and identify the object. Here, the received pressure time series from spherical acoustic objects in motion that have been insonified by linear frequency modulated (LFM) waveforms are used to identify the objects. The unknown object's unknown constant velocity using a unique property of the fourth-order cumulant spectrum [J. Acoust. Soc. Am. 93, 1460-1465 (1993)] is extracted and then its transfer function is extracted. The results for five spherical acoustic objects in motion are discussed. Three methods, deconvolution, matched filtering, and fourth-order cumulant spectrum deconvolution to extract the object's transfer function are compared.

\section{1:30}

4aUW14. Towed array geometry estimation during ship's maneuvering. S. M. Jesus, P. Felisberto (UCEH-Univ. of Algarve, PT-8000 Faro, Portugal), and F. Coelho (Hidrographic Inst.-PT-1000 Lisbon, Portugal)

Towed arrays of hydrophones are commonly used as receiving apparatus for determining the directionality of the underwater acoustic field. It is well known that a line array beam response has an inherent left-right ambiguity and that any deformation of the array will produce a distortion on the estimated acoustic field directionality. In particular, the array cannot be operated during ship's maneuvering which is a potential drawback to its operational usage. In theory, if the array is not straight but the hydrophone's position are known at each time instant, the beamformer could be compensated in order to obtain a corrected beam response. More, depending on the array shape, the left-right ambiguity could also be resolved. In practice, it is extremely difficult to obtain sufficiently accurate measurements of the hydrophone positions. This paper presents the measurements obtained at sea, with a $156 \mathrm{~m}$ aperture array, instrumented with several high precision tiltmeters, compasses, depth sensors, and accelerometers. After filtering and preliminary analysis of the sensor position data it is concluded that the array is never a straight horizontal line. The array has approximately a catenary shape with vertical deviations at the tail up to 15 $\mathrm{m}$ at constant tow speed. Under maneuvering, the array is largely deformated and a consistent shape could be estimated on real time from the nonposition sensors. The use of the estimated geometry for acoustic data processing, shows that consistent beam responses (close to theoretical) could be obtained even under strong array distortion. It is also shown, with real data, that the knowledge of array geometry significantly improves full-field matching for source localization and/or bottom characterization.

\section{$11: 45$}

4aUW15. A fundamental study of multipath localization using bottomed receivers in shallow water. Randall $W$. Smith, Jaime $F$. Nualart, and David E. Grant (Appl. Res. Lab., Univ. of Texas at Austin, P.O. Box 8029, Austin, TX 78713-8029)

The basic characteristics of multipath propagation from a shallow source to a bottomed receiver in shallow water are investigated, with an 
emphasis on application to the problem of source localization. For this fundamental study, the environment is assumed to be ideal (perfectly reflecting bottom and surface, range invariant depth, isovelocity). The delays between travel times along different propagation paths depend on the location (range, depth, and bearing) of the source relative to the receiver. Mcasurements of several such time delays can be used to determine the location of the source. It was found that the travel time difference between paths having differing numbers of traversals of the water column depends strongly on range but weakly on depth. In contrast, the travel time difference between paths having an equal number of traversals but which have different source angles (i.e., up or down) depends strongly on depth but weakly on range. As an example, these observations are applied to localize a towed sound source in a real environment. The example provides a background for an assessment of how accurately a real environment must be modeled in order to obtain reasonable results.

\section{2:00}

4aUW16. Time domain based optimal a posteriori source localization in a shallow-water environment. J. A. Shorey and L. W. Nolte (Dept. of Elec. Eng., Box 90291, Duke Univ., Durham, NC 27708-0291)

An optimal a posteriori probability approach to matched field processing using time domain data in the form of arrival times is implemented. The inherent robustness of this technique is demonstrated with respect to conventional narrow-band frequency domain based matched field processing techniques. A ray-tracing method and a wideband normal mode method are compared as modeling mechanisms for this application. Localization performance for a shallow-water source localization problem is presented using probabilities of correct localization. Specific results for this shallowwater environment are presented as a function of signal-to-noise ratio. [Research sponsored by ONR Ocean Acoustics and NUWC.]

\title{
Meeting of Accredited Standards Committee S12 on Noise
}

to be held jointly with the

\section{U.S. Technical Advisory Group for ISO/TC 43/SC1 Noise and ISO/TC 94/SC12 Hearing Protection}

\author{
D. L. Johnson, Chair S12 \\ EG\&G Special Projects, Albuquerque Operations, Albuquerque, New Mexico 87119-9024
}

P. D. Schomer, Chair, U.S. Technical Advisory Group (TAG) for ISO/TC 43/SC1, Noise (and Vice Chair S12) U.S. CERL, P.O. Box 4005, Champaign, Illinois 61820

\author{
H. E. von Gierke, Vice Chair, U.S. Technical Advisory Group (TAG) for ISO/TC 43/SC1, Noise \\ 1325 Meadow Lane, Yellow Springs, Ohio 45387
}
E. H. Berger, Chair, U.S. Technical Advisory Group (TAG) for ISO/TC 94/SC12, Hearing Protection Cabot Safety Corporation, 7911 Zionsville Road, Indianapolis, Indiana 46268-1657

\begin{abstract}
Standards Committee S12 on Noise. Working group chairs will report on their progress for the production of noise standards. The interaction with ISO/TC 43/SCl and ISO/TC $94 / \mathrm{SC} 12$ activities will also be discussed, with reference to the international standards under preparation. The Chairs of the respective U.S. Technical Advisory Groups (H. E. von Gierke and E. H. Berger) will report on current activities of these International Technical Subcommittees under ISO and the meeting of ISO/TC 43/SC1, held in London, U.K. in November 1994.

Scope of S12: Standards, specifications and terminology in the field of acoustical noise pertaining to methods of measurement, evaluation and control; including biological safety, tolerance and comfort and physical acoustics as related to environmental and occupational noise.
\end{abstract}




\title{
Meeting of Accredited Standards Committee S1 on Acoustics
}

to be held jointly with the

\section{U.S. Technical Advisory Group for ISO/TC 43 Acoustics and IEC/TC 29 Electroacoustics}

\author{
G. S. K. Wong, Chair S1 \\ Institute for National Measurement Standards (INMS), \\ National Research Council, Ottawa, Ontario KIA OR6, Canada \\ R. L. McKinley, Vice Chair S1
}

U.S. Air Force AAMRL/BBA Aerospace Medical Research Laboratory, Wright-Patterson AFB, Dayton, Ohio 45433

P. D. Schomer, Chair, U.S. Technical Advisor (TA) for ISO/TC 43, Acoustics

U.S. CERL, P.O. Box 4005, Champaign, Illinois 61820

H. E. von Gierke, Vice Chair, U.S. Technical Advisory Group (TAG) for ISO/TC 43, Acoustics

1325 Meadow Lane, Yellow Springs, Ohio 45387

V. Nedzelnitsky, U.S. Technical Advisor (TA) for IEC/TC 29, Electroacoustics

National Institute of Standards and Technology (NIST), Building 233, Room A149, Gaithersburg, Maryland 20899

\begin{abstract}
Standards Committee S1 on Acoustics. Working group chairs will report on their preparation of standards on methods of measurement and lesting, and terminology, in physical acoustics, electroacoustics, sonics, ultrasonics, and underwater sound. Work in progress includes measurement of noise sources, noise dosimeters, inlegrating sound-level meters, and revision and extension of sound level meter specifications. Open discussion of committee reports is encouraged.
\end{abstract}

The intemational activities in ISO/TC 43 Acoustics, and IEC/TC 29 Electroacoustics, will also be discussed. The chairs of the respective U.S. Technical Advisory Groups for ISO/TC 43 (H. E. von Gierke), and IEC/TC 29 (V. Nedzelnilsky), will report on current activities of these Technical Committees, including their most recent meetings. (ISO/TC 43 and IEC/TC 29 both met in London, U.K. during November 1994).

Scope of S1: Standards, specifications, methods of measurement and test and terminology in the field of physical acoustics including architectural acoustics, electroacoustics, sonics and ultrasonics, and underwater sound, but excluding those aspects which pertain to biological safety, tolerance and comfort.

\section{Session 4pAB}

\section{Animal Bioacoustics: Animal Bioacoustics Research Methodology II}

Mardi C. Hastings, Chair

Department of Mechanical Engineering, The Ohio State University, 206 West 18th Avenue, Columbus, Ohio 43210-1107

\author{
Chair's Introduction-1:30 \\ Invited Papers
}

1:35

4pAB1. Otoacoustic emissions in animals: An intricate view of the peripheral stage of acoustic processing. Brenda $L$ Lonsbury-Martin, Glen K. Martin, and Martin L. Whitehead (Univ. of Miami Ear Inst. (M805), P.O. Box 016960, Miami, FL 33101)

Otoacoustic emissions (OAEs) represent sounds generated by the ear that can be simply measured from the ear canal. In particular, OAEs provide a means to examine in detail the encoding properties of the outer hair-cell system, which presumably generates emitted responses. Animal research using emissions as a response measure consists of four general classes of utilization including the use of 
OAEs: (1) to describe the basic limits of auditory function for a particular species, (2) to monitor or index cochlear function during experimental manipulations of the auditory system, (3) to enhance understanding of the fundamental nature of the various emissions, and (4) to further knowledge about the physical basis of certain afflictions that affect human hearing using animal models of specific pathologies. The purpose of this presentation will be to provide an overview of OAEs including their essential basis, to review the equipment and procedures used to adequately detect this response measure, and to present examples of the unique applications of emission measurements in animals. By reviewing these many aspects of OAEs, the great benefits along with the limitations inherent to this attractive research technique can be appreciated. [Work supported by PHS (DC01668, ES03500).]

\section{2:00}

4pAB2. Application of Navy IUSS for whale research. Christopher W. Clark and David K. Mellinger (Cornell Lab. of Ornithol., Bioacoust. Res. Prog., 159 Sapsucker Woods Rd., Ithaca, NY 14850)

The recent application of Navy Integrated Undersea Surveillance System (IUSS) data (Whales '93) for detecting and tracking low-frequency sounds from whales has led to a renewed interest in the use of these arrays for marine mammal research. Previous work with sparse arrays has limited the detection and tracking to ranges of tens of kilometers, while the IUSS allows for orders of magnitude greater coverage. SOSUS data are processed via computer systems specifically developed for detecting and tracking whales, as well as through annotations, to determine individual, geographic, and seasonal variability in vocalizations for at least four species (blue, finback, humpback, and minke), and there are also numerous sounds from unidentified whales. Given that these animals use these deliberate patterns of intense low-frequency sounds for either communication, navigation, or food finding, verification of sound function will be deduced through careful examination of behavioral context and other environmental conditions such as local productivity, bathymetry, and oceanography. Results from the Whales ' 93 and continuing Dual Use projects will be presented. This will include descriptions of signal variability for the different species of marine mammals and preliminary interpretation of the results in terms of sound function. [Work supported by ONR and NRL.]

2:25

4pAB3. The role of passive sonar technology in marine mammal population assessment. William E. Evans (Texas Inst. of Oceanogr., Texas A\&M Univ., P.O. Box 1675, Galveston, TX 77553) and Robert Benson (Texas A\&M Univ., College Station, TX 77843-3367)

When sonar detection of marine organisms is mentioned most every one visualizes active sonars. This is because development of passive sonar systems has been essentially limited to military use until recently. With the development of new designs, submarines could run deeper and more silent than in the past. The development of more sensitive listening systems was essential. With the development of improved passive sonars, a vast variety of biological noise mostly generated by cetaceans was documented. Most dolphins and whales are either direct or incidental noise makers. In many cases sound generating cetaceans can be identified as to family and in some cases to species (e.g., Physeter macrocephalus, Tursiops truncatus). In 1992 The Texas Institute of Oceanography, Center for Bioacoustics, Texas A\&M University, in partnership with the National Marine Fisheries Service, Southeast Research Center, conducted a three-year acoustic/visual seasonal census of the cetaceans of the Gulf of Mexico. A linear towed array and sonobouy arrays were used. Thirteen of the 21 species of cetaceans known to occur in the Gulf of Mexico were detected acoustically using the towed array. Acoustic contacts were compared with the distribution and density of these species determined by the visual survey. The use of a mobile directional acoustic array had the advantage of detecting the presence of cetaceans $24 \mathrm{~h}$ a day. Because of this the number of acoustic contacts was significantly greater than those for the visual survey. In the case of the sperm whale it was possible to assess the distribution and also estimate the population density.

\section{2:50}

4pAB4. Vocalization tracking of blue and fin whales in the North Pacific. Mark A. McDonald, John A. Hildebrand, and Spahr C. Webb (Scripps Inst. of Oceanogr., Univ. of California, La Jolla, CA 92093-0205)

Arrays of seafloor seismometers provide the ability to track vocalizing marine mammals in the deep ocean over a radius of $20 \mathrm{~km}$ or more with position accuracy as good as several hundred meters. These tracking data can be used to infer swimming speed and apparent respiration rate, as measured by position change and by repeated pauses in the vocalization sequence. Speed and respiration rate can be compared during times of relative quiet, during the passage of large merchant vessels, and during times of man-made acoustic transmissions to evaluate possible effects of low-frequency noise. Seafloor seismometer arrays also allow study of fin whale call sequences, which often are interactions between multiple whales located several kilometers apart. That these fin whales are interacting, rather than calling independently, is suggested by: (1) consistent spacing between calls with rarely overlapping calls, (2) a distinctive spectral signature for each whale, and (3) synchronization of respiration among vocalizing whales.

\section{3:10}

ApABS. Zooplankton population density assessment with acoustics. D. V. Holliday (Tracor Appl. Sci., Anal. and Appl. Res. Div., 9150 Chesapeake Dr., San Diego, CA 92123)

There are a variety of acoustical methods which can be used as tools in basic and applied research in biological oceanography, estuarine ecology, and in limnology. The mode of application of these tools is often as important and interesting as are the sensor characteristics. Two such sensor systems, the TAPS and the BITS, are being used to sense and describe temporal and spatial distributions of small zooplankton in situ. These sensors operate as active sonars at discrete, multiple frequencies from hundreds of kilohertz to several megahertz. Acoustical design parameters and the mode of deployment for several of these systems will be presented. TAPS operates in either a profiling mode or in a cast mode. BITS estimates zooplankton abundance at discrete depths from a mooring and telemeters the data to shore. Data from both will be used to illustrate their application in both marine and estuarine ecosystems science. Illustrations of the acoustical data each of these systems produces will be illustrated, along with several ancillary 
abiotic measurements and derived estimates of the size structure and biomass of the underlying population of zooplankton that causes the scattering. [Work supported by ONR Oceanic Biology, NSF Biological Oceanography, and Tracor.]

\section{3:30-3:40 Break}

$3: 40$

4pAB6. Auditory-evoked potentials obtained using continuous amplitude-modulated tones. William F. Dolphin (Dept. of Biomed. Eng., Boston Univ., Boston, MA 02215)

Auditory-evoked potentials (AEPs) recorded from the scalp surface are neurogenic potentials arising from the massed discharge of neural populations in response to acoustic stimuli and provide a "window" on the physiological basis of auditory processing. AEPs, in particular the transient-evoked auditory brain-stem response (ABR) have been widely used in human clinical settings for the evaluation of auditory functioning. Recently, several new techniques using continuous amplitude-modulated tonal stimuli have been investigated. Many of these techniques take advantage of auditory nonlinearities which result in the auditory system acting as an envelope extractor. This envelope following response (EFR), phase locking of neural discharge to waveform periodicities, has been measuring using two-tone, sinusoidal amplitude-modulated, and multitone, multiple envelope components signals. The EFR offers great promise for frequency specific threshold measurements, especially for low-frequency audiometry and for the examination of auditory processing of complex sounds.

\section{4:00}

4pAB7. A portable system for physiological assessment of hearing in marine animals. D. A. Carder and S. H. Ridgway (Biosci. Div., Naval Command, Control and Ocean Surveillance Ctr., RDTE Div., Code 511, 49620 Beluga Rd., Rm. 200, San Diego, CA 92152)

A compact field portable system has been developed and tested for presenting sound stimuli $(20 \mathrm{~Hz}-200 \mathrm{kHz})$ and acquiring and analyzing sound and microvolt-level electrophysiological responses in marine animals. Stimulus sound is controlled for frequency, amplitude, duration, rise/fall times, interstimulus interval, phase, and masking noise. Analysis is by synchronous averaging, with artifact rejection, and is displayed, stored, and may be printed, if desired. The weather resistant system includes hardware based on a 486 with fast bus and software for presenting shaped tones and pulses, acquiring up to eight channels of data for display, storage, and analysis. The system includes all necessary functional units (suction cup electrodes, high gain (X500K) amplifiers, analog-to-digital and digital-to-analog converters, fiber-optic intermodule connection, computer with LCD screen that can be seen in bright sunlight, an internal 1.2-gigabyte hard disk, power amplifier, transducers, power source) and is constructed in a set of small cases that can be hand carried to remote sites. To date, the system has been used for hearing tests in dolphins, Tursiops, white whales, Delphinapterus, and a beached pygmy Sperm whale, Kogia. [Work supported by ONR.]

4pAB8. Dynamics of dolphin biosonar search and detection. J. E. Sigurdson (Code 511, NCCOSC, RDT\&E Div., 53405 Front St., San Diego, CA 92152-6530)

The biosonar emissions of a free-swimming dolphin were measured with respect to direction and timing while the animal searched for and reported objects at various ranges and bearings from a test enclosure. The technology allows a quantitative description of the pulse-by-pulse scan pattern of the freely moving animal and subsequent inferences about search strategies, echo-integration time, functional beam coverage, and learning effects on biosonar performance. Presently, the methods are being extended to test an animal from a boat in the open ocean with measurement of the animal's attitude and movement in three dimensions as well as the spectral content of emitted pulses during search, detection, and discrimination. The technique combines standardized test paradigms, and instrumentation pack carried by the animal, and a positioning system. The animal's pack is a self-contained, data-acquisition system with sensors for magnetic azimuth, acoustic emissions, attitude, and motion. It is based on a high-speed, real-time microprocessor with open software architecture for maximum flexibility. The positioning system employs a high-accuracy real-time differential GPS for prepositioning targets and monitoring the position of the boat and animal during a search.

\section{Contributed Papers}

\section{$4: 40$}

4pAB9. Neural network modeling of a dolphin's sonar discrimination capabilities. Lars N. Andersen, A. René Rasmussen (Tech. Univ. of Denmark, Lyngby, Denmark), Whitlow W. L. Au, Paul E. Nachtigall (Hawaii Inst. of Marine Biol., Kailua, HI 96734), and Herbert Roitblat (Univ. of Hawaii, Honolulu, HI)

The capability of an echo-locating dolphin to discriminate differences in the wall thickness of cylinders was previously modeled by a counterpropagation neural network using only spectral information of the echoes [W. W. L. Au, J. Acoust. Soc. Am. 95, 2728-2735 (1994)]. In this study, both time and frequency information were used to model the dolphin discrimination capabilities. Echoes from the same cylinders were digitized using a broadband simulated dolphin sonar signal with the transducer mounted on the dolphin's pen. The echoes were filtered by a bank of continuous constant- $Q$ digital filters and the energy from each filter was computed in time increments of $1 /$ bandwidth. Echo features of the standard and each comparison target were analyzed in pairs by both a counterpropagation and a backpropagation neural network. The backpropagation network performed better than the counterpropagation network, and the use of both time and frequency domain features resulted in better performance than if only time or frequency domain features were used. [Work supported by ONR, Grant No. ONR N00014-93-1378.]

\section{4:55}

4pAB10. Considerations for designing underwater acoustical playback experiments. William $T$. Ellison and Karen $S$. Weixel (Marine Acoust., Inc., 14 Pelham St., Newport, RI 02840)

The continued high interest in the effects of man-made sounds on marine wildlife has resulted in increased use of playback experiments. In such experiments, the intent is often to simulate a larger more powerful 
source of sound, e.g., a drill ship, with a playback of a recording, but using a small portable source system such as a J-11 to play the sound back. Simulation accuracy is assumed to be met in these playbacks if the receive level of the sound at the animals being observed is the same as they would be exposed to by the full scale source. To achieve this same receive level the playback source must often be moved to within short range of the animals. The purpose of this paper is to identify and discuss those components of the actual full scale exposure that may not be accurately simulated in an experiment where sound level at the animal is the only control variable. Components examined include the temporal, spectral, and spatial properties of the noise field, as well as the relative motion of animal and source with emphasis on the highly variable nature of the noise field at short playback ranges. Recommendations are made to assist experimental designers in developing more accurate simulations.

\section{5:10}

4pAB11. Methods for automatic detection of mysticete calls. David K. Mellinger and Christopher W. Clark (Bioacoust. Res. Prog., Cornell Univ., 159 Sapsucker Woods Rd., Ithaca, NY 14850)
The automatic detection of animal cells has several potential applications: for range, distribution, and, census efforts; for acoustic behavior studies, both local and wide area; for screening of large volumes of data for sounds of interest. Methods were developed for detecting the vocalizations of three species of mysticete: blue, finback, and minke whales. Each call is modeled as a sequence of either bandlimited pulses or frequency sweeps. Sweeps and pulses are detected by cross-correlating a specially designed kernel with a spectrogram of the target sound signal; the kernel is built from the call model, and includes excitatory regions corresponding to the sweeps or pulses in the call, and flanking inhibitory regions to inhibit response to noise and interfering sounds. This method produces as output a time series with values corresponding to the likelihood that the modeled call is present. A time-windowed autocorrelation is then performed on this output, and the result of that is percentile-normalized and thresholded. The final output is a yes/no decision about the presence or absence of a periodic sequence of the desired calls. This process is currently being used for scanning large amounts of sound data from Navy SOSUS arrays; results of this scanning are presented.

THURSDAY AFTERNOON, 1 DECEMBER 1994

SAN ANTONIO ROOM, 1:00 TO 5:00 P.M.

\title{
Session 4pEA
}

\section{Engineering Acoustics: Transducers and Transducer Modeling}

\author{
R. Lowell Smith, Cochair \\ Texas Research Institute, 9063 Bee Caves Road, Austin, Texas 78733
}

\author{
Elizabeth A. McLaughlin, Cochair \\ Naval Undersea Warfare Center, New London Detachment, New London, Connecticut 06320
}

\section{Contributed Papers}

\section{$1: 00$}

4pEA1. Theoretical model for a $(3,1)$ drive Iow-frequency piezofilm hydrophone. P. Philip Thomson (Dept. of Appl. Phys., School of Phys., Univ. of New South Wales, Sydney 2052, Australia)

An innovative $(3,1)$ drive transducer design has been discussed in the literature [J. J. Bhatt, P. P. Thomson, and P. R. S Pillai, J. Acoust. Soc. Am. 94, 3053-56 (1993)] using a poled piezofilm adhered to a driver pin which is fixed to the center of a rigidly clamped circular plate. This paper attempts to analyze the above transducer for a particular frequency range in terms of the theory of transverse vibrations of a clamped circular plate carrying a concentrated mass at its center [R. E. Roberson, J. Appl. Mech. 18, 349-352 (1951)]. The system satisfies the requirements for vibrational analysis and the natural frequencies can be estimated from above theory. $A$ statistical approach is made to calculate the theoretical sensitivity below resonance. The prestretched piezofilm acts as a longitudinal vibrator with the electric field perpendicular to its length and the transducer system can be represented by a six-terminal network with an electrical input voltage driving the mechanical load acting only on one edge of the piezofilm. The surface strain developed in the diaphragm in response to the acoustic pressure and electroacoustic sensitivity are evaluated by thin plate theory and piezoelectric theory. The calculated values are in agreement with experimental results.

\section{1:15}

4pEA2. State switched acoustic source. Gregg D. Larson and Peter H. Rogers (School of Mech. Eng., Georgia Inst. of Technol., Atlanta, GA 30332-0405)
To produce high amplitude low-frequency signals, an underwater transducer musı generate a relatively large volume displacement. Since water exerts a large reaction force back on the transducer, "conventional wisdom" dictates that such a transducer would have to be a high $Q$ resonant device and thus not be broadband as seemingly required for many applications. However, a transducer does not have to be broadband in the conventional sense to work in communication and SONAR systems. A transducer capable of switching between two discrete frequencies is adequate for communication and one capable of switching among several frequencies could produce chirp signals for active sonars. Ordinarily, a broadband transducer is needed to switch frequencies rapidly. It is theoretically possible, however, to instantaneously switch frequencies with a high $Q$ resonant system provided that the system's resonant and drive frequencies are altered simultaneously. Such a "state-switched" transducer [Munk, Webb, Birdsall, unpublished (1980), (1981)] would retain the advantages (high power, high efficiency, and large displacements) of a high $Q$ resonant transducer without the accompanying disadvantage of slow response time. A state-switched acoustic source with an active spring of PZT has been built to demonstrate the state switching concept. [Research supported by ONR 334.]

\section{$1: 30$}

4pEA3. A planar-omnidirectional sensor for broadband and high-frequency underwater acoustic applications. Thomas $R$. Howarth, Sam Petrie, and Larry E. Ivey (Naval Res. Lab., Underwater Sound Reference Detachment, P.O. Box 568337, Orlando, FL 32856-8337)

A prototype hydrophone with planar-omnidirectional radiation characteristics has been developed for broadband, high-frequency, underwater 
acoustic applications. A 9- $\mu \mathrm{m}$ sheet of KYNAR-type polyvinylidene fluoride (PVDF) was wrapped about a $2.5-\mathrm{mm}$ solid Rho-c rod with a height of $86 \mathrm{~mm}$ such that the resulting cylindrical shape had an omnidirectional radiation behavior in the $X-Y$ plane. The PVDF was oriented with the 3-1 direction radially wrapped and the 3-2 axis positioned along the mounting rod for improved vibration strum rejection from the mounting rod with improved radial sensitivity because of the orthotropic nature of the KYNAR material. Further discussions will detail the selection of an impedance matching mounting rod and waterproofing elastomer and a cable with the low capacitance of $0.018 \mathrm{pF} / \mathrm{m}(8.5 \mathrm{pF} / \mathrm{ft})$. Measured data to be shown includes the free-field voltage sensitivity (FFVS) and $X$-Y plane receiving radiation patterns from $100 \mathrm{kHz}$ to over $1.5 \mathrm{MHz}$. The presentation will conclude with a conceptual discussion on an advanced version of this same sensor design for a broadband and high-frequency, three-dimensional omnidirectional sensor.

\section{$1: 45$}

4pEA4. A miniature, high-sensitivity, electron-tunneling accelerometer. Thomas B. Gabrielson (NAWC Aircraft Div., Code 5044, Warminster, PA 18974-0591), Howard K. Rockstad, and Tony K. Tang (Ctr. for Space Microelectron. Technol., Pasadena, CA 91109-8099)

A prototype low-noise accelerometer has been fabricated with an electron-tunneling transducer. By measuring the tunneling current between an electrode on the proof mass and a feedback-controlled monitor electrode, very small accelerations can be detected with high responsivity. This particular prototype $(10 \times 10 \times 1.5 \mathrm{~mm})$ was designed for underwater acoustic measurement from a few hertz to $1 \mathrm{kHz}$. The measured responsivity below the fundamental device resonance at $100 \mathrm{~Hz}$ is roughly 1500 $\checkmark$ per $\mathrm{m} / \mathrm{s}^{2}$ with a measured noise spectral density of $10^{-6} \mathrm{~m} / \mathrm{s}^{2}$ per root hertz or less between 30 and $300 \mathrm{~Hz}$. The noise floor is controlled primarily by $1 / f$ noise in the tunneling current although the noise floor reaches the theoretical molecular-agitation limit at $100 \mathrm{~Hz}$. The responsivity and directivity of the device were measured in a standard gradient-hydrophone calibrator; the noise floor was determined in a vacuum-isolation chamber assembled from commercial off-the-shelf components; and the detailed dynamics of the proof-mass motion were examined using a heterodyne laser interferometer that was scanned across the surface and synchronously detected with respect to the excitation. [Work supported by ONR.]

\section{2:00}

4pEA5. The improvement of low-frequency driver performance using an amplifier with negative output resistance. Thomas D. Kite and Elmer L. Hixson (Dept. of Elec. and Comput. Eng., Univ. of Texas at Austin, Austin, TX 78712)

A low-frequency dynamic driver (woofer) was modeled on PSPICE, an electric circuit simulator. The far-field pressure response of the driver was computed as the dc resistance of the voice coil, $R_{e}$, was varied. In the limit of zero $R_{e}$, the on-axis response for a constant voltage input was shown to rise at $6 \mathrm{~dB} /$ octave. The input to the driver was therefore equalized with an integrator to produce a flat pressure response with frequency. A beneficial side effect of reducing $R_{e}$ was the improvement of the transient response of the driver, since it became possible to critically damp the system. The system was found to be stable for values of $R_{e}$ greater than zero. The simulated results were tested in an anechoic chamber using a power amplifier with variable negative output resistance. The far-field pressure response of the driver was found to be substantially flat over a broad range of frequencies around the natural resonance of the driver, and transient response was improved, as predicted by the circuit simulation.

\section{2:15}

4pEA6. A low-frequency projector using giant magnetostrictive rods. Akiyoshi Kawamori, Takashi Yoshikawa, Tomohiro Tuboi (OKJ Electric Industry Co., Ltd., Electron. Syst. Lab., 688 Ozuwa, Numazu-shi, Shizuoka, 410 Japan), Iwao Nakano, Toshio Tsuchiya, and Tosiaki Nakamura (Japan Marine Sci. and Technol. Ctr., Yokosuka-shi, Kanagawa, 237 Japan)
A superior underwater projector having low frequency, high power, high efficiency, and broad bandwidth for the Ocean Acoustic Tomography (OAT) system has been developed. The OAT system is proposed to measure temperature and current distributions of a wide range of sea area with a diameter of about $1000 \mathrm{~km}$ on real time by using sound waves. The projector vibrates in the radial mode of octagonal radiating plates connected with eight driving units having giant magnetostrictive rods. The projector is able to emit sound of more than $190 \mathrm{~dB}[0 \mathrm{~dB}=1 \mu \mathrm{Pa}-\mathrm{m}]$ at a low frequency of about $200 \mathrm{~Hz}$ under the operation depth of about $1000 \mathrm{~m}$ when it is excited by electric input power of about $900 \mathrm{~W}$. The performance is enough to cover the range of $1000 \mathrm{~km}$ for the OAT system. Also, the derived values from measurements are in good agreement with the calculations based on the simple equivariant circuit model.

\section{2:30}

4pEA7. A reexamination of mode coupling and loss phenomena for simple piezoelectric oscillators. R. Lowell Smith (Texas Res. Inst., 9063 Bee Caves Rd., Austin, TX 78733)

Applications of both linear and nonlinear theories of piezoelectricity to transduction problems commonly ignore internal material dissipation effects. Such losses can be represented phenomenologically via the introduction of complex dielectric, elastic, and piezoelectric coefficients. However, there is some confusion in the literature with respect to the proper partitioning of electrical and mechanical attributes in the presence of piezoelectric coupling. This paper is a review of this situation wherein dissipation coefficients are motivated from more fundamental physical insights. Equivalent circuit models are useful both for developing this perspective and summarizing the results. Suggestions are also presented for generalizing the celebrated Mason model to include the coupling of orthogonal modes via Poisson dilation. This formalism is intended to provide straightforward procedures for computing piezoelectric oscillator performance characteristics using a personal computer. The approach is evaluated via regression analysis in terms of its self-consistency for interpreting Tonpilz oscillator admittance data. The procedures developed are expected to be applicable for the interpretation of data associated with a variety of other electroacoustic structures.

\section{$2: 45$}

4pEA8. Some second thoughts on electromechanical coupling. Li-Feng Ge (Anhui Bureau of Tech. Supervision, 30 Ma An Shan Rd., Hefei, Anhui 230001, People's Republic of China)

An electromechanical transducer can be regarded as an energy converter, and also as an impedance inverter. It could be significant for understanding the transduction process to introduce a new generalized concept, inversive impedance, which is defined as a ratio of the open-circuited voltage caused by a motion at the mechanical end of a transducer to the exciting current required to produce the same motion, i.e., a ratio of the receiving (voltage) sensitivity of a transducer to its sending (current) sensitivity. Thus the electrical driving-point impedance is composed of an impedance without coupling and a inversive impedance, which is induced by electromechanical coupling, and is constant at a given frequency for a particular transducer. The transduction process or the impedance inversion process can be described by only three independent parameters [L.-F. Ge, J. Acoust. Soc. Am. 91, 2326 (A) (1992)], and determined uniquely by a mapping between the electrical driving-point impedance and its mechanical load impedance. It is an inherent physical property independent of analogy-type chosen and mathematical representation that a piezoelectrictype transducer is reciprocal, and an electrodynamic-type transducer is antireciprocal. Thus there exists, respectively, an optimum way to perform reciprocity calibration for the two types of electromechanical transducers.

\section{3:00-3:15 Break}

\section{$3: 15$}

4pEA9. Variational model for the radiation impedance of class IV transducers. Harold C. Robinson and Elizabeth A. McLaughlin (Naval 
Undersea Warfare Ctr., New London Detachment, New London, CT 06320)

In a previous paper [H. C. Robinson, J. Acoust. Soc. Am. 95, 2833 (A) (1994)], a variational method for determining the modal radiation impedance of baffled transducer arrays, based on the radiated power, was presented. In this paper, these variational methods will be extended to include unbaffled arrays of radiators with arbitrary spatial orientation. The surface velocity of each of the radiators is represented by in vacuo eigenmode expansions, while the surface pressure is given by expansions in suitable trial functions. The arbitrary orientation of the array elements will be represented by transforming the surface velocities and pressures using Euler angles. As a specific example, the case of two coplanar class IV flextensional transducers will be examined in detail. The in vacuo eigenmodes of a single element will be determined using finite element methods and parametrized in elliptical cylindrical coordinates $(\xi, \eta, z)$. The surface pressure trial functions will be judiciously chosen functions of $(\xi, \eta, z)$ as well. The behavior of the modal radiation impedances as a function of dimensionless separation distance $k d$, as well as of relative orientation, will be investigated. Comparisons of the results to equivalent circuit models and experimental data will be presented. [Work sponsored by ONR.]

\section{$3: 30$}

4pEA10. The combined finite element and hybrid-type infinite element analysis for complex structure underwater transducers. Jong-Rak Yoon and Chun-Duck Kim (Dept. of Telematics Eng. and Dept. of Elec. Eng., Natl. Fisheries Univ. of Pusan, Pusan, Korea 608-737)

A numerical method for evaluating the electroacoustical characteristics of a complex structure of transducer immersed in an infinite acoustic medium is adopted. The mechanical, electrical, and acoustical response of the transducer in the finite region is modeled by the finite element method; the Sommerfeld radiation condition to the infinite region is modeled by the hybrid-type infinite element method, and the boundary between both regions is formulated with compatibility of the acoustical admittance in both regions. The numerical results, which include the elastic response, the radiation impedance, and the input electrical admittance, are given for a single piezoelectric cylinder and a coaxial two piezoelectric cylinder array with various polyurethane windows. The technique allows one to interpret and to optimize design parameters such as geometrical shape and mechanical properties of structure components, electrical characteristics of piezoelectric elements, etc.

\section{$3: 45$}

4pEA11. Wideband array transducer. Chun-Duck Kim and Jong-Rak Yoon (Dept. of Elec. Eng. and Dept. of Telematics Eng., Natl. Fisheries Univ. of Pusan, Pusan, Korea 608-737)

There is a need to develop a wideband underwater sound source. This need can be accomplished by an array of different resonance frequencies of transducers. An axisymmetric array transducer, which consists of three different size piezoelectric cylinder elements, is designed and its coupled electrical, mechanical, and acoustical characteristics are evaluated using the combined single code finite element method and hybrid-type infinite element method. The numerical result of electrical input impedance is consistent with the measurement result using an impedance analyzer and is shown to be better than that by Warren P. Mason's equivalent circuit modeling method. Its bandwidth and directivity pattern are also obtained and its limits as underwater sound projector are discussed.

\section{4:00}

4pEA12. Coupling finite element and impedance element to model the radiation of piezoelectric transducers in boreholes. Didace Ekeom and Bertrand Dubus (Inst. d'Electron. et de Microélectron. du Nord, UMR CNRS 9929, Dépt. ISEN, 41 boulevard Vauban, 59046 Lille Cedex, France)

In the context of petroleum acoustics, it is of great interest to modelize the radiation of the piezoelectric transducer in a borehole surrounded by an homogeneous isotropic elastic formation of infinite extent without restrictive assumptions on the geometry, radiation pattern, or types of waves. The finite element method is well suited to solve such problems if the troncature of the infinite formation is correctly treated. This troncature generates ingoing waves which normally do not exist in infinite domain. In this paper, classical finite elements (ATILA code) are used to model in steady state the transducer, the fluid-filled borehole, and part of the formation inside a spherical boundary. On this exterior boundary, impedance elements are used to take into account the infinite character of the formation. These elements are obtained by discretizing the mechanical impedance of outgoing spherical $P$ and $S$ waves. The method is validated by studying two configurations having analytical solutions: the pulsating sphere and the oscillating point. Results include displacement fields and radiation patterns for $P$ or/and $S$ waves. Finally, the radiation of a cylindrical piezoelectric transducer in an oil-filled borehole [S. Kostek et al., J. Acoust. Soc. Am. 95, 109 (1994)] is analyzed. $P$ and $S$ components of the displacement field, electrical admittance of the transducer, directivity patterns, and distribution of radiated energy are displayed.

\section{$4: 15$}

4pEA13. Thermodynamic property considerations for modeling piezoelectric ceramic vibrators including thermal effects. Won-kyu Moon and Ilene J. Busch-Vishniac (Mech. Eng. Dept., Univ. of Texas at Austin, Austin, TX 78712)

The thermodynamic characteristics of piezoelectric ceramics are investigated by considering two thermodynamic material property models: isentropically linear and isothermally linear models. Those two models are two possible interpretations of the data on temperature-dependent linear coefficients of piezoelectric ceramics. Thermodynamic differences of the two models are examined and effects of the thermo-electro-mechanical couplings are evaluated in examples. Application of the thermo-electromechanical model to PZT4 shows that the difference observed in piezoelectric constants obtained by static and dynamic measurements cannot be explained by the difference between isothermal and isentropic processes.

\section{4:30}

4pEA14. Bond graph model for one-dimensional heat conduction for modeling of piezoelectric ceramic vibrators including thermal effects. Won-kyu Moon and Ilene J. Busch-Vishniac (Mech. Eng. Dept., Univ. of Texas at Austin, Austin, TX 78712)

A new bond graph model for conduction heat transfer is developed and applied to consideration of thermal energy balance in the piezoelectric thickness vibrator. In formulation of the model, the mechanical and electrical effects are included, so it can be applied directly to the temperaturedependent thickness vibrator. Although the analysis is applied to the onedimensional case only, the method can be used for general heat conduction problems. For the purpose of evaluation of the new method, the model predictions for one-dimensional heat conduction excluding other variable effects are compared with the results of the analytic solutions in simple cases. The simulation illustrates the validity and the accuracy of the model.

\section{$4: 45$}

4pEA15. Cooling low-frequency sonar projectors by heat transfer optimization. Bertrand Dubus, Patrice Bigotte (Inst. d'Electron. et de Microélectron. du Nord, UMR-CNRS 9929, Dépt. ISEN, 41 boulevard Vauban, 59046 Lille Cedex, France), Gilles Grosso (Eramer, $83500 \mathrm{La}$ Seyne sur Mer, France), and Didier Boucher (DCN-Ingénierie Sud, 83140 Six Fours les Plages, France)

Heating has become a critical issue for low-frequency projectors due to their high dissipated power density and long excitation time. In this paper, the cooling of sonar projectors in terms of heat transfer optimization is presented. An analysis of the projector heating requires the description of the heat conduction in the structure and the heat exchanges with the surrounding medium. This analysis is performed in steady state using both 
analytical (thermoelectrical analogies) and numerical (finile element) models. For the reference transducer (double-ended longitudinal piezoelectric vibrator), the temperature distribution and heat fluxes are computed. The temperature decrease and the importance of various heat paths are evaluated for various techniques of cooling. Practical considerations (simplicity, fiability, cost, size) and cooling efficiency are both taken into account to determine the optimal solution. At constant dissipated power density, the maximum temperature is theoretically divided by 3 . Temperature measurements on reference and optimized projectors are provided and compared to predictions. Extension of the technique to other geometries of projectors and other types of active materials (magnetostrictive, electrostrictive) is discussed.

\title{
Session 4pPAa
}

\section{Physical Acoustics: Nonlinear Acoustics}

\author{
Oleg V. Rudenko, Cochair \\ Department of Acoustics, Physics Faculty, Moscow State University, Moscow 119899, Russia \\ E. A. Zabolotskaya, Cochair \\ Department of Mechanical Engineering, The University of Texas at Austin, Austin, Texas 78712-1063
}

\section{Contributed Papers}

\section{1:00}

4pPAa1. Nonscattering of sound by sound resulting from a head-on collision of two plane-wave pulses. Peter J. Westervelt (Dept. of Phys., Brown Univ., Box 1843, Providence, RI 02906)

Two arbitrary pulses $p_{2}\left(t-c_{0}^{-1} x\right)$ and $p_{1}\left(t+c_{0}^{-1} x\right)$ each of width $c_{0} \tau$ pass through one another at $x=0$, generating a differential surface mass rate source density $d \sigma_{s}(t, x)=q_{s} d x$ where $q_{s}=A d\left(p_{1} p_{2}\right) / d t$ and $A$ $=\left(\rho_{0} c_{0}^{4}\right)^{-1}\left[2+\rho_{0} c_{0}^{-2}\left(d^{2} p / d \rho^{2}\right)_{p_{0}}\right]$. The equation for the scattered pressure $p_{s}$ is $d^{2} p_{s} / d x^{2}-c_{0}^{-2} d^{2} p_{s} / d t^{2}=-\dot{q}_{s}$ and its solution is $p_{s}\left(x^{\prime}, t\right)=\int d p_{s}=\left(c_{0} / 2\right) \int d \sigma_{s}=\left(c_{0} / 2\right) \int q_{s}\left(x, t^{\prime}\right) d x, t^{\prime}$ being the retarded time; thus, $\left(p_{s}\right)_{ \pm}=\left(c_{0} / 2\right) A \int_{ \pm c_{0} x^{\prime} / 2}^{x^{\prime}}\left[d\left(p_{1} p_{2}\right)_{1-t^{\prime} \pm} / d t\right] d x$, where + is chosen when $x^{\prime}<x$, - when $x^{\prime}>x$, and $t_{ \pm}^{\prime}=t \pm c_{0}^{-1}\left(x^{\prime}-x\right)$. Suppose $x^{\prime}>c_{0} \tau / 2$ then $x^{\prime}$ lies outside the interaction zone, it is found that $\left(p_{s}\right)_{-}=\left(c_{0}^{2} A / 4\right)\left(p_{2} p_{1}+\dot{p}_{2} \int^{\prime}{ }_{\infty} p_{1} d t\right)=0$ unless $p_{1}$ has a nonzero average which is possible for plane waves but it is assumed that this is not the case [L. D. Landau and E. M. Lifshitz, Fluid Mechanics (Addison-Wesley, Reading, MA, 1959), p. 267]. In the event $x^{\prime}<-c_{0} \tau / 2 x^{\prime}$ again lies outside the interaction zone and $\left(p_{s}\right)_{+}=\left(c_{0}^{2} A / 4\right)\left(p_{1} p_{2}+\dot{p}_{1} f_{-\infty}^{t} p_{2} d t\right)=0$. Within the interaction region $-c_{0}^{2} / 2<x^{\prime}<c_{0} \tau / 2$ so that $p_{s}=\left(p_{s}\right)_{+}$ $+\left(p_{s}\right)_{-}=\left(c_{0}^{2} A / 4\right)\left(2 p_{1} p_{2}+\dot{p}_{2} \int_{-\infty}^{t} p_{1} d t+\dot{p}_{1} \int_{-\infty}^{t} p_{2} d t\right)$, which is precisely the result obtained previously [P. J. Westervelt, J. Acoust. Soc- Am. 94, 1774 (A) (1993)], recalling that $p_{1}$ is here a plane wave; thus in the earlier result $p_{m}=p_{1} / 2$. The zero scattering results obtained here conflict with nonzero results obtained by Trivett and Rogers [J. Acoust. Soc. Am. 71, 1114 (1982)] in a related problem, which most likely stems from spurious sources arising from discontinuities similar to those that plagued Ingard and Pridmore-Brown.

\section{1:15}

4pPAaz. Scattering of sound by sound within the interaction zone. Peter J. Westervelt (Dept. of Phys., Brown Univ., Box 1843, Providence, RI 02912)

Starting with Eckart's equation for $\rho_{s}$ the scattered density [P. J. Westervelt, J. Acoust. Soc. Am. 29, 934 (1957)], $\square^{2} \rho_{s} c_{0}^{2}=\square^{2} E_{12}$ $-\nabla^{2}\left(2 T_{12}+\Lambda V_{12}\right)$, the variables $x^{0}=c_{0} t$ and $\psi_{0}=-\left(4 \rho_{0} c_{0}^{2}\right)^{-1 / 2} p$ are introduced for which $\square^{2} \psi=0, \quad \square^{2} \psi^{2}=T_{12}-V_{12}$, and $\nabla^{2} V_{12}=\square^{2} V_{12}+\left(V_{12}\right)_{00}$ to obtain $\square^{2}\left[\rho_{s} c_{0}^{2}+T_{12}+(\Lambda-1) V_{12}\right.$ $\left.+2\left(\psi^{2}\right), 00\right]=-2(2+\Lambda)\left[\left(\psi_{0}\right)^{2}\right]_{, 00}$. Next it is assumed that $\psi=\phi+\chi$, where $\phi\left(x^{0}-\mathbf{n} \cdot \mathbf{r}\right)$ is a plane wave and $\chi_{, 0}=\sigma_{, 0}+n \cdot \nabla \sigma$, where $\sigma\left(x^{0}, \mathbf{r}\right)$ is an arbitrary wave. Terms bilinear in $\phi$ and $\chi$ are retained; thus $\left(\psi_{0}\right)^{2}=2 \chi_{, 0} \phi_{0,0}$, and since $\nabla \phi=-\mathbf{n} \phi_{, 0}$ it is found $\square^{2}(\sigma \phi)=2 \nabla \sigma \cdot \nabla \phi-2 \sigma_{0,0} \phi_{0}=-2 \phi_{.0} X_{0}$, leading to the solution of Eckart's equation, $\rho_{s} c_{0}^{2}=(2-\Lambda) V_{12}-E_{12}$ $+2\left[(2+\Lambda) \sigma \phi-\psi^{2}\right]_{00}$, valid within the interaction zone, but vanishing outside where $V_{12}=E_{12}=\sigma=\phi=\psi=0$. The feasibility of making optical measurements of $\rho_{s}$ is being investigated.

\section{1:30}

4pPAa3. Criticism of experiments on the scattering of sound by sound. D. H. Trivett (Naval Res. Lab., Underwaler Sound Ref. Det., P.O. Box 568337, Orlando, FL 32856-8337) and Peter H. Rogers (Georgia Inst. of Technol., Atlanta, GA 30332-0405)

At the 127th meeting of the Acoustical Society of America, Westervelt [Peter J. Westervelt "Answer to criticism of experiments on the scattering of sound by sound," J. Acoust. Soc. Am. 95, 2865 (A)] stated that a recent experiment by Roy and Wu [R. Roy and J. Wu, in Proceedings of the 13th ISNA, edited by H. Hobæk (Elsevier Science, London, 1993)] supported the position that there was no signal scattered out of the interaction region of two perpendicular, collimated sound beams. In this paper, an analysis of the Roy and Wu experiment is presented, specifically, and of previous experiments, in general, which have failed to find any scattering of sound by sound. The analysis indicates that the null results of the experiments do not necessarily support theoretical null predictions.

\section{$1: 45$}

4pPAa4. Experimental difficulties in measuring the scattering of sound by sound. James A. TenCate) (Appl. Res. Labs. and Mech. Eng. Dept., Univ. of Texas at Austin, P.O. Box 8029, Austin, TX 78713-8029)

The question of whether one sound beam can interact with another at nonzero angle and scatter nonlinearly generated sound outside the mutual interaction region has been debated since the 1950s. Experimental work on this problem has left the question unresolved. This presentation describes experimental difficulties associated with measuring scattered sound produced by real diffracting primary beams. Optimal conditions for observing scattered sound, as outlined by Berntsen et al. [J. Acoust. Soc. Am. 86, 1968 (1989)] and by Darvennes and Hamilton [J. Acoust. Soc. Am. 87, 1955 (1990)], are reviewed in relation to the design of our own experiments. Our experiments were performed with either two uniform circular sources in water (megahertz frequencies), or with one circular source and the other a shaded source with lower sidelobes. A variety of primary frequency ratios, interaction angles, and other parameters were considered. 
Comparison of the primary beam patterns with measured sum and difference frequency field patterns reveals the difficulty in identifying which components of the latter correspond to "scattered" sound. It is concluded that two Gaussian-type sources with exceedingly good sidelobe suppression are needed to perform a reasonable experiment. [Work supported by

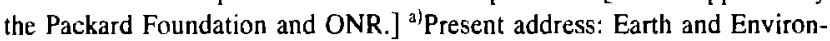
mental Sciences Division, Mail Stop D443, Los Alamos National Laboratory, Los Alamos, NM 87545.

\section{2:00}

4pPAa5. Analytical method for describing the paraxial region of finite amplitude sound beams. Mark F. Hamilton (Dept. of Mech. Eng., Univ. of Texas at Austin, Austin, TX 78712-1063), Vera A. Khokhlova, and Oleg V. Rudenko (Moscow State Univ., Moscow 119899, Russia)

The paraxial region of finite amplitude sound beams in lossless fluids is studied theoretically. Both focused and unfocused beams are considered. A special analytical method which combines the parabolic approximation ( $\mathrm{KZ}$ equation) with nonlinear geometrical acoustics (NGA) is developed to model nonlinear and diffraction effects near the axis of the beam. The corresponding system of nonlinear equations describing waveform evolution is derived. For the case of an initially sinusoidal wave radiated by a Gaussian source, an analytical solution of the coupled equations is obtained along the axis of the beam. The solution is expressed in both the time and frequency domains. In the high-frequency limit, classical simple wave solutions are recovered (plane-wave solution for unfocused beams and spherical wave solution for focused beams). In the limit of weak nonlinearity, the quasilinear axial solutions of the $\mathrm{KZ}$ equation for the fundamental and second-harmonic components are recovered. The analytical solution is in good agreement with numerical solutions of the $\mathrm{KZ}$ equation for a wide range of ratios between the focal length, Rayleigh distance, and shock formation distance. Harmonic propagation curves, waveform distortion, focusing amplification factors, and other characteristics are calculated. [Work supported in part by NATO and ISF.]

\section{2:15}

4pPAa6. A study of the role of diffraction in the behavior of focusing step shocks using NPE-type equations and a finite difference program. Andrew A. Piacsek (Graduate Prog. in Acoust., Penn State Univ., P.O. Box 30, State College, PA 16804)

A finite difference solution to an NPE-like equation is used to study the linear and nonlinear behavior of focusing step shocks in regions where caustics are formed. The initial condition is a nominally plane step pulse with a hyperbolic tangent rise profile of thickness $l_{s h}$; the wavefront has a single ripple of length scale $L_{w f}$, concave towards the direction of propagation. When $\epsilon=l_{s h} / L_{\mathrm{wf}}$ is much less than unity, the linear numerical simulation showed that the shock behaves nearly according to geometric theory. Several different values of $\epsilon$ were investigated, verifying the linear theory predictions of normalized amplitude $\sim \epsilon^{-1 / 4}$ at the cusp [J. Hunter and J. Keller, Wave Motion 9, 429-443 (1987)]. Numerical solutions are also obtained for three cases of nonlinear propagation with dissipation. In addition to $\epsilon$, the distinguishing parameters are shock amplitude and dissipation. It is observed that for cases in which nonlinearity is stronger relative to diffraction and focusing, the wavefront forms a cusp at a later time, caustics are less pronounced, and the (normalized) rise time is shorter. Implications of these results on the effect of caustics on sonic boom rise times is discussed.

\section{2:30}

4pPAa7. Modification of the spectral method for deseribing nonlinear acoustic waves containing shocks. Vera A. Khokhlova and Oleg A. Sapozhnikov (Dept. of Acoust., Phys. Faculty, Moscow State Univ., Moscow 119899, Russia)

Numerical simulations of finite amplitude sound waves become particularly time consuming in the case of strongly distorted waveforms that contain shocks. To improve the efficiency of time domain numerical codes, weak shock theory can be used to separate the description of the shock fronts from the evolution of the continuous segments of the wave. The idea of the present work is to modify the spectral method by taking into account the known asymptotic behavior of high-frequency components in shock waves. Use of asymptotic results permits the number of spectral components retained in the numerical computations to be reduced. A finite set of coupled equations for the spectral amplitudes, which approximates the infinite set of spectral equations corresponding to an exact formulation, is obtained for the simple wave equation. This reduction is achieved by introducing asymptotic expressions for the high-frequency components in sawtoothlike waves. Several model problems are studied. It is shown that less than 15 harmonics require numerical calculation in order to obtain an adequate description of the evolution of an initially sinusoidal wave to a sawtoothlike waveform. [Work supported by ISF and the Russian Fund for Fundamental Investigations.]

\section{2:45-3:00 Break}

\section{3:00}

4pPAa8. Generation of streaming and rarefaction of the gas in the far field of the weakly nonlinear plane waves. Takeru Yano and Yoshinori Inoue (Dept. of Eng. Sci., Hokkaido Univ., Sapporo 060, Japan)

The propagation of weakly nonlinear plane waves emitted from a harmonically oscillating plate into an ideal gas of semi-infinite extent is considered under the condition that the energy dissipation is negligibly small everywhere except for discontinuous shock fronts. Recently, the authors have numerically shown that, in the case of strongly nonlinear waves, contrary to the result of the conventional weakly nonlinear theory, streaming due to shocks occurs in the direction of wave propagation and thereby the gas near the source is rarefied as time proceeds [Y. Inoue and T. Yano, J. Acoust. Soc. Am. 94, 1632-1642 (1993)]. In the present paper, the evolution of the weakly nonlinear waves including shocks is determined up to $O\left(M^{2}\right)$, where $M$ is the acoustic Mach number $(M \ll 1)$. In this order, the wave profile develops to an asymmetrical sawtoothlike one in the far field and weak streaming is excited in the region beyond the shock formation distance. For $M \leqslant 0.2$, the results quantitatively agree with those in the previous work. Furthermore, by taking into account both the production of entropy and the generation of reflected wave at each shock front, the physical mechanism is clarified for the rarefaction of the gas in $O\left(M^{3}\right)$.

\section{$3: 15$}

4pPAa9. Prediction of the time-averaged pressure distribution for finite amplitude standing waves. Reh-lin Chen and Victor W. Sparrow (Graduate Prog. in Acoust., Penn State Univ., 157 Hammond Bldg., University Park, PA 16802)

A finite difference numerical approach to studying two-dimensional nonlinear wave propagation was recently developed [V. W. Sparrow and R. Raspet, J. Acoust. Soc. Am. 90, 2683-2691 (1991)]. In this talk the approach is modified and applied to a one-dimensional finite amplitude standing wave in a rigid tube. The numerical method can solve for lossless, weakly nonlinear waves in the tube including all second-order nonlinearities from the continuity equation, momentum equation, and the equation of state. The acoustic pressure in the time, space, and frequency domains and the time-averaged acoustic pressure distribution are all determined. Generation of harmonics of the fundamental frequency along with the development of a nonzero, time-averaged pressure are seen from discrete Fourier transforms of the numerical results. The results for the time-averaged pressure distribution are compared to recent analytical predictions [C. P. Lee and T. G. Wang, J. Acoust. Soc. Am. 94, 1099-1109 (1993)]. The results show agreement between the present work and that of Lee and Wang when the nonlinearities from both the momentum equation and equation of state are included. A slightly different result is obtained when one also includes nonlinearity from the continuity equation. 
4pPAa10. Extensions of the theory for nonlinear Rayleigh waves. $E$ Yu. Knight, M. F. Hamilton, Yu. A. II'insky, and E. A. Zabolotskaya (Dept. of Mech. Eng., Univ. of Texas at Austin, Austin, TX 78712-1063)

The derivation of Zabolotskaya's coupled spectral equations for nonlinear Rayleigh waves in isotropic solids [J. Acoust. Soc. Am. 91, 2569 (1992)] applies to plane, progressive, periodic waves. These assumptions are removed here in a more general derivation based on the Hamiltonian formalism employed in the original work. Via methods described in Paper 4pPAa12 of this scssion, the resulting spectral equations are transformed into time domain evolution equations. Model equations for cylindrical waves and diffracting beams, obtained previously via ad hoc modification of the plane-wave model, can be derived rigorously from the new generalized equations. The nonlinear terms are shown to be equivalent to those obtained previously by Parker [Int. J. Eng. Sci. 26, 113 (1985)] by entirely different methods. Numerical results for the shock formation distance are used to define an effective coefficient of nonlinearity that is consistent with the corresponding parameter for sound waves in fluids. The coefficient is of order one for common isotropic solids, as for the case of fluids, but it is several orders of magnitude larger in rock with microcracks and other inhomogeneous features. [Work supporled by DOE, ONR, and NSF.]

\section{3:45}

4pPAal1. Pulsed nonlinear Rayleigh waves. E. Yu. Kright, M. F. Hamilton, and E. A. Zabolotskaya (Dept. of Mech. Eng., Univ. of Texas at Austin, Austin, TX 78712-1063)

Pulsed, plane, nonlinear Rayleigh waves in isotropic solids are investigated with numerical solutions of the coupled spectral equations derived by Zabolotskaya [J. Acousi. Soc. Am. 91, 2569 (1992)]. Calculations of waveform evolution are presented as functions of distance from the source and depth within the solid. For the case of weak nonlinearity (characteristic absorption length on the order of the shock formation distance), selfdemodulation of tone bursts is investigated. Self-demodulation refers to the nonlinear generation of a low-frequency waveform by a highfrequency pulse. Comparisons are made with the analogous process in fluids. Whereas demodulated Rayleigh and acoustic waveforms have similar shapes, the demodulated Rayleigh waveforms have substantially smaller relative amplitudes. The difference in amplitude is due to the frequency dependence of the nonlinearity coefficient matrix for Rayleigh waves. For the case of strong nonlinearity, shock formation is investigated in a variety of transient waveforms. Via comparison with acoustic waveform evolution in fluids, precursors generated by certain Rayleigh waveforms are identified as consequences of nonlocal nonlinearity. [Work supported by DOE, ONR, and NSF.]

\section{4:00}

4pPAa12. Evolution equations lor nonlinear Rayleigh wave propagation. M. F. Hamilton, Yu. A. Il'insky, and E. A. Zabolotskaya (Dept. of Mech. Eng., Univ. of Texas at Austin, Austin, TX 78712-1063)

Time domain evolution equations are derived for nonlinear Rayleigh wave propagation along the free surface of an isotropic solid. The evolution equations are expressed in different forms: one for the horizontal displacement component, and one for a complex displacement variable. Both equations are derived from spectral equations published earlier [Zabolotskaya, J. Acoust. Soc. Am. 91, 2569 (1992)]. To simplify the derivation of equations for the displacement variables, not all components of the nonlinearity coefficient matrix are retained. However, the terms which are retained possess the same mathematical properties (symmetries in the frequency domain, singularities in the time domain, and others) as the terms that are not taken into account. Moreover, with appropriate definition of the shock formation distance, it is shown that numerical solutions based on the simplified equations yield close agreement with numerical solutions based on the complete nonlinearity matrix. The nonlinear terms in the new evolution equations are expressed as time derivatives and Hilbert transforms of the displacement variables. Waveforms calculated with the time domain evolution equations are shown to agree with results based on the original frequency domain equations. [Work supported by the Packard Foundation, ONR, and NSF.]

\section{4:15}

4pPAa13. Theoretical model for nonlinear Stoneley and Scholte waves. G. Douglas Meegan, Mark F. Hamilton, and E. A. Zabolotskaya (Dept. of Mech. Eng., Univ. of Texas at Austin, Austin, TX 78712-1063)

The Hamiltonian formalism used previously to derive model equations for nonlinear Rayleigh waves [Zabolotskaya, J. Acoust. Soc. Am. 91,2569 (1992)] is used here to obtain a mathematical model for nonlinear Stoneley and Scholte waves. Planar interfaces formed by contact between two isotropic materials are assumed. The resulting coupled spectral equations are expressed in the same form as those for Rayleigh waves. In particular, the nonlinearity coefficient matrix can be written as $R_{m l}^{S}=R_{m}^{(1)}+K R_{m}^{(2)}$, where $K$ is a constant and $R_{m l}^{(i)}$ (conesponding to medium $i=1,2$ ) are matrices having the same mathematical form as the matrix obtained for Rayleigh waves. Since $R_{m l}^{S}$ exhibits the same symmetries as the nonlinearity matrix for Rayleigh waves, the model equations for Stoneley and Scholte waves can be analyzed with the same mathematical techniques that have been employed in recent investigations of nonlinear Rayleigh waves (see, e.g., other papers in this session). The coupled spectral equations were solved numerically for Stoneley and Scholte waves at interfaces formed by a variety of media pairs, including some geological materials. Comparisons of harmonic generation and shock formation are made with the corresponding processes in Rayleigh waves. [Work supported by AASERT and ONR.]

\section{4:30}

4pPAa14. Observations of nonlinear effects associated wh ice cracks. Lev A. Ostrovsky, Alexander Ekimov, Alexey Korotkov, Andrey Lebedev, and Alexander M. Sutin (Inst. of Appl. Phys., Nizhni Novgorod, Russia)

The hypothesis that cracks in ice may provide anomalously strong acoustic nonlinearity is experimentally verified. Two series of experiments were performed. One of them was done in situ, on a freshwater lake covered by a 40 -cm-thick ice. Flexural waves were excited in ice by a vibrator in frequency range $0.2-2 \mathrm{kHz}$ and received by accelerometers. Air temperature was -5 and $+3{ }^{\circ} \mathrm{C}$ for two different series of measurements. A high level of nonlinearity was registered. In particular, a pronounced subharmonic signal was generated which testifies for parametric instability. The second experiment was performed in laboratory conditions, when a "crack" was created artificially, as a contact between two pieces of ice. We studied modulation of ultrasound (about $26 \mathrm{kHz}$ ) by low-frequency ( $30-90$ $\mathrm{Hz}$ ) vibrations. For vibration acceleration amplitudes up to 0.1 and $0.3 \mathrm{~g} \mathrm{a}$ pronounced modulation spectrum appeared; moreover, modulation frequency subharmonics were observed in this case. Some theoretical considerations of the effects observed are also given. It is believed that nonlinear effect can be effectively used for Arctic ice characterization. ${ }^{\text {a) }}$ Now at Univ. of Colorado, CIRES, ETL/ERL/NOAA, 325 Broadway R/E/ET, Boulder, CO 80303.

\section{4:45}

4pPAa15. Diffusing light photography of solitons and capillary-wave turbulence. W. Wright, R. Budak, and S. Putterman (Phys. Dept., UCLA, Los Angeles, CA 90024)

The attenuation of light propagating through a slab of water (containing a dilute concentration of polyballs) is approximately proportional to its thickness. Application of this insight to the local elevation of a fluid surface has enabled us to use photography to determine the instantaneous global topography of the surface of a fluid in motion. Use of diffusing light enables us to obtain images that are free of the caustics which plague shadowgraphs. Applications include breather solitons and wave turbulence which results from the nonlinear interaction of a broadband spectrum of high amplitude surface ripples. Measurements indicate that as the amplitude of excitation of the surface of water is increased the wave number of the capillary motion displays a transition to a broadband spectrum. The 
temporal response of a single pixel yields the power spectrum of the surface height as a function of frequency " $f$." The numerous harmonics which can be seen at low amplitude merge at high amplitude into a broadband spectrum which goes as $1 / f^{3}$. This technique should permit the measurement of turbulent parameters which go beyond the purported range of current theories. [Work supported by US DOE Division of Engineering and Geophysics and NASA Microgravity.]

$5: 00$

4pPAa16. Experimental study of streaming in acoustic resonators. Joseph C. Jankovsky, R. Glynn Holt, and Robert E. Apfel (Dept. of Mech. Eng., Yale Univ., New Haven, CT 06520-8286)

Anomalous rotations of solid and liquid drops have been observed in resonant chambers in both $1 \mathrm{~g}$ and in the microgravity environment of space in the Drop Physics Module (DPM) aboard the United States Microgravity Laboratory (USML-1) mission in 1992. The observed torques are produced by acoustic streaming, typically in the nondegenerate plane where rotations are undesired, and have been attributed to an imbalance in the paired driver amplitudes. To quantify the torque exerted on the inclusion, measurements were made using suspended and levitated spherical samples with single and paired driver configurations. Experiments were conducted in both degenerate and nondegenerate planes to study the effects of orthogonal coupling. Absorption effects of active and passive drivers are presented, along with asymmetrical driver configurations. The results will be used to control and eliminate unwanted tumbling rotations in the USML-2 DPM experiments to be flown in 1995. [Work supported by NASA through JPL Contract No. 958722.]

\section{$5: 15$}

4pPAa17. Generation and propagation of high intensity short acoustic pulses in dispersive media. I. B. Esipov, K. A. Naugol'nykh, O. B. Ovchinnikov, A. E. Pashin, and O. M. Zozuly (N. Andreev Acoust. Inst., 117036, Schvernik st. 4, Moscow, Russia)

Experiments on laser generation of acoustical pulses, amplitude 10 MPa and duration $1 \mu s$, and their propagation in different liquids have been carried out. Propagation was measured in both pure liquids and structured media. Nonlinearity and dispersion caused evolution of the propagating signals. Some theoretical treatment of the results indicate the possibility of obtaining data on spectral features of the nonlinear response of the medium.

THURSDAY AFTERNOON, 1 DECEMBER 1994

WEDGWOOD ROOM, 1:15 TO 4:45 P.M.

\title{
Session 4pPAb
}

\section{Physical Acoustics and Structural Acoustics and Vibration: Ray Methods in Radiation and Scattering from Elastic Objects II}

\author{
Douglas A. Rebinsky, Chair \\ Department of Mechanical and Aerospace Engineering, Rutgers University, Piscataway, New Jersey 08855-0909
}

\section{Contributed Papers}

\section{$1: 15$}

4pPAb1. Geometrical theory of acoustic scattering by thin elastic shells. Jin-Meng Ho (SFA, Inc., 1401 McCormick Dr., Landover, MD 20785 and Naval Res. Lab., Washington, DC 20375)

The sound field scattered by a smooth thin elastic shell immersed in fluid arises largely from specular reflection and acoustic-membrane coupling, unless both source and observer are located near or on the shell. These two contributions have been found to be well described by ray fields for canonical shells in the mid-frequency regime, and hence may be generalized by the principle of localization to accommodate more general geometries. By examining the excitation, propagation, and radiation processes of the supersonic membrane waves on the shell, this paper defines the excitation and radiation coefficients associated with these shell-guided leaky waves as well as the divergence coefficients of the ray tubes characterizing the variation of the wave amplitudes. It extends the concepts of Keller and Karal's geometrical theory for surface waves [J. B. Keller and F. C. Karal, Jr., J. Appl. Phys. 31, 1039-1046 (1960)]. The explicit evaluation of these coefficients, and therefore the leaky fields, is demonstrated for two classes of shells-cylindrical shells of arbitrary cross section and shells of revolution-based on the knowledge of cylindrical and spherical shell prototypes and differential geometry. The reflected waves are more straightforward and the related coefficients are readily determined. [Work supported by ONR.]

4pPAb2. Radiation from fluid-loaded smooth elastic shells of arbitrary shape. Yang Yang (SFA, ${ }^{\text {a) }}$ Inc., 1401 McCormick Dr., Landover, MD 20785)
A ray method is systematically used to derive a relation between radiated acoustic waves and elastic waves traveling over a smooth elastic shell of arbitrary shape. The radiated acoustic field is found to be intimately connected with the geometry of the shell's surface and the elastic wavefronts. This connection leads to an asymptotic expression for the local radiation impedance associated with each surface ray under the condition $k_{f} R \gg 1$, where $k_{f}$ is the wave number in fluid and $R$ the smallest radius of curvature of the shell. The first term in this formula is actually the result for an infinite flat plat, which is homogeneous and isotropic, while the second term introduces inhomogeneity and anisotropy into the radiation impedance because it explicitly depends on the local curvatures of the shell's surface and the elastic wavefronts. The general result is further simplified for a cylindrical shell with cross sections of arbitrary shape. Comparisons are made between the present asymptotic solution and the exact solution in the two special cases of vibrating circular cylindrical shells and spherical shells. It turns out that the first two terms in the asymptotic expansions of these solutions have exactly the same expression. [Work supported by ONR and NRL.] 'ander contract to Naval Res. Lab., Washington, DC 20375-5000.

\section{$1: 45$}

4pPAb3. Acoustic scattering from fluid-loaded elastic shells: A Gaussian beam approach. Yang Yang (SFA, Inc., 1401 McCormick Dr., Landover, MD 20785), Andrew N. Norris (Rutgers Univ., Piscataway, NJ 08855-0909), and Luise S. Couchman (Naval Res. Lab., Washington, DC 20375-5000)

Acoustic scattering problems from fluid-loaded elastic shells are usually formulated in terms of surface Helmholtz integrals. The numerical evaluation of these integrals is very time consuming, especially at high 
frequencies. A new method is proposed here for the computation of the scattered fields. The basic idea is to use ray methods to first represent the supersonic surface fields. The surface integral is then represented as a summation of individual integrals along Gaussian beams. Each integral is approximated as a line integral along the center ray of the beam, and finally, the acoustic far ficld is obtained by asymptotically evaluating each line integral at its stationary points. The asymptotic expression for the membrane wave part is uniform and valid at all the observation angles. The difficulties of dealing with caustics and infinite sets of rays do not arise in the present formalism. This approach allows the acoustic response to be easily calculated without resorting to two-point ray tracing or surface Helmholtz integrals. It is therefore numerically fast. The numerical results agree well with the available exact solution and the pure ray results on a spherical shell for $k_{f} a>10$, where $k_{f}$ is the fluid wave number. [Work supported by ONR and NRL.]

\section{2:00}

4pPAb4. Impulse response of thin shells: Source development, analysis of the bipolar specular contribution, and computations showing the effect of water on the inside of the shell. Gregory Kaduchak and Philip L. Marston (Dept. of Phys., Washington State Univ., Pullman, WA 99164-2814)

The response of shells to a delta function pressure impulse is well suited to interpretation with ray methods. We examine the theory for PVDF sheet sources [C. S. Kwiatkowski, G. Kaduchak, and P. L. Marston, J. Acoust. Soc. Am. 94, 1831 (A) (1993)] which facilitated wideband impulse scattering measurements in modest-sized water tanks. Response features for spherical and nonspherical shells will be discussed. An approximation is developed for the bipolar specular feature which for an empty sphere of radius $a$ becomes $\delta(T)-2 x_{N} \exp \left(-x_{N} T\right) \theta(T)$, where $T=t c / a$, the delta function is the initial specular echo, $\theta$ is a unit step function, and $x_{N}=\rho a / \rho_{E} h$ is the dimensionless null frequency. The densities of the shell and water are $\rho_{E}$ and $\rho$. Thus the mass-per-area $\rho_{E} h$ for the shell of thickness $h$ affects the magnitude and decay time of the negative feature. Observations for an empty spherical shell and an end cone piece of an MIT/ NRL model shell will be examined. Computations show that both the bipolar feature and the coincidence frequency wave packet for spherical shells are not quenched when water is inside the shell. [Work supported by ONR.]

\section{2:15}

4pPAb5. Asymptotic determination of the eigenfrequencies of a sphere in a fluid. G. C. Gaunaurd (Naval Surface Warfare Center, White Oak Detachment, R-34, Silver Spring, MD 20903-5640)

Starting from a phase-matching principle that is the acoustical analog of the Bohr-Somerfeld-Wilson quantization rule of the old "quantum theory," it is analytically shown how to asymptotically obtain the eigenfrequencies of an insonified sphere immersed in a fluid. This technique was first illustrated by J. B. Keller [cf. Ann. Phys. 4, 180-188 (1958)] and it has been extended by many authors, notably L. B. Felsen and J. M. Ho, who have renamed it the "ray-acoustic algorithm." It is shown here how the acoustical counterpart of this quantum principle leads to a resonance condition for the (external) eigenfrequencies of a sphere (rigid, soft, or to some extent, elastic) that exactly coincides with F. W. J. Olver's (1954) classical asymptotic formula for the (complex) zeros of the spherical Hankel functions. The poles of the scattering amplitude of an elastic sphere fall into two great families. one depending on shape, and the other on elastic composition. The asymptotic spacings in between the shape-dependent zeros in the (complex) $k a$ plane are shown to reduce to a uniform value, obtained earlier by numerical means, which manifests itself in all the (RST) "background" curves of the sphere. [Work supported by NSWC-DD IR Program.]

\section{2:30}

4pPAb6. Concerning the extinction cross section of an arbitrary body in Stokesian fluid flow. Scott A. Wymer (Dept. Eng. Sci. and Mech., Penn State Univ., 227 Hammond Bldg., University Park, PA
16802-1401), Akhlesh Lakhtakia, and Renata S. Engel (Penn State Univ., University Park, PA 16802-1401)

Time-dependent Stokesian fluid flow around an arbitrary body can be analyzed in terms of time-harmonic phasors. Analytical techniques commonly used for frequency-domain scattering can therefore be brought to use. The scattering response of a body is often quantitated by the extinetion cross section. However, the wave number for Stokesian flows is necessarily complex, so the usual interpretation of the extinction cross section is untenable in the present instance. It is shown, however, that a detectorbased interpretation of the extinction cross section is unambiguous and experimentally relevant. An almost exact formula is derived for the extinction coefficient for the far-field, forward scattering case. Computed values of extinction cross sections are presented for spheres and spheroids.

\section{2:45-3:00 Break}

\section{3:00}

4pPAb7. Approximate diffraction coefficients. Paul E. Barbone (Dept. of Aerospace and Mech. Eng., 110 Cummington St., Boston Univ., Boston, MA 02215)

Expressions for diffraction coefficients for canonical shapes, joints, and discontinuities are necessary in applications of the geometrical theory of diffraction to scattering from submerged structures. In many cases of practical interest, however, the diffraction coefficients are either not available or are very difficult to evaluate. The use of perturbation theory and matched asymptotic expansions in obtaining suitable approximations of diffraction coefficients is described. These two methods can yield approximations that are simple to compute, easy to apply, and are valid in complementary parametric ranges. The perturbation method assumes that the properties of the solid or its geometry are nearly homogeneous. Matched asymptoties, on the other hand, is a useful tool when the solid is nearly hard, or the fluid is light. The accuracy of these methods is demonstrated by comparing them to the exact solution for diffraction by an impedance discontinuity. When the effort is made to obtain uniformly valid asymptotic expressions, the results prove to be remarkably accurate even at values of $\epsilon=1$ (here, $\epsilon$ is a "small" parameter).

\section{3:15}

4pPAb8. Acoustic and flexural wave interaction at the junction of two fluid-loaded plates. Andrew N. Norris, Douglas A. Rebinsky (Dept. of Mech. and Aerosp. Eng., Rutgers Univ., Piscataway, NJ 08855-0909), and G. Wickham (Univ. of Manchester, Manchester M13 9PL, UK)

A general solution is developed for the acoustic and structural scattered response from the junction of two flat plates under unilateral fluid loading. The plates are modeled by the classical theory of flexure, and the solution is found using the Wiener-Hopf technique for the dual integral equations for the unknown pressure on the plates. Explicit formulas are obtained for the pressure transform when the plates are in welded or clamped contact, and corresponding explicit and relatively simple expressions are given for the various diffraction coefficients associated with the fluid/struclure interaction. Numerical results for the redistribution of energy from flexural to flexural plus acoustic at a thickness discontinuity will be discussed in detail. [Work supported by ONR.]

\section{3:30}

4pPAb9. Longitudinal wave diffraction generated at curved plate junctions. Douglas A. Rebinsky (Dept. of Mech. and Aerosp. Eng., Rutgers Univ., P.O. Box 909, Piscataway, NJ 08855-0909)

A perturbation solution is developed to study diffracted longitudinal membrane waves originating from the junction of two curved plates which are joined so that their tangent is continuous. It is shown that the diffracted longitudinal wave field in the plates is dependent upon or forced by a background solution which characterizes the acoustic interaction between two joined, flat plates and the surrounding fluid. Two forms of background 
wave fields are used: (1) a simple solution based on the acoustic diffraction from two massive plates, and (2) the solution of acoustic diffraction from two flat plates governed by the classical theory of flexure. Explicit expressions are shown for the longitudinal diffraction coefficients, associated with this fluid/structure interaction. The reciprocal problem of an incoming longitudinal wave to the joint is discussed. Numerical results of the diffraction coefficient will be shown. [Work supported by ONR.]

\section{$3: 45$}

4pPAb10. Retroreflective backscattering of ultrasound due to Rayleigh waves on an elastic solid rectangular parallelepiped. Karen Gipson and Philip L. Marston (Dept. of Phys., Washington State Univ., Pullman, WA 99164-2814)

High-frequency (1.5-MHz) sonar experiments were performed on a solid stainless-steel rectangular parallelepiped block in water in order to determine the most likely mechanism for producing a strongly backscattered signal. When the angle of incidence is near the Rayleigh angle of the steel, a leaky Rayleigh wave is launched on the flat surface of the block. When this wave reaches the edge of the block, part of it is reflected and propagates to the adjacent edge forming the corner, where it is reflected again. The net result is that the wave vector of the Rayleigh wave is reversed, and the resulting leaky radiation gives a pronounced enhancement of the backscattering. For a randomly oriented block, this effect is more likely to be observed than specular reflection, since the latter requires that two of the Euler angles of the surface normal lie in a small range, while for the Rayleigh mechanism only one angle is narrowly constrained. The observed peak amplitude of the retroreflected backscattered signal agrees well with an approximate theoretical model [Marston et al., J. Acoust. Soc. Am. 94, 1861 (A) (1993)] modified for the present case of Rayleigh waves. [Work supported by ONR.]

\section{4:00}

4pPAb11. Structural and acoustic responses of mass-spring loaded elastic cylindrical shells: Spectral formulation and ray synthesis. Jin-Meng Ho (SFA, Inc., 1401 McCormick Dr., Landover, MD 20785 and Naval Res. Lab., Washington, DC 20375)

This paper applies the spectral theory to the formulation of both the pressure field and the displacements of a source-excited cylindrical shell with the internal attachment of a mass-spring substructure at two arbitrary locations. Respective alternative representations in the series and integral forms, with a distinct correspondence between each other, may be readily written down. The series form generalizes Guo's solution which is limited to the diametrical attachment of the spring to the shell [Y. P. Guo, J. Acoust. Soc. Am. 91, 926-938 (1992)], while the integral form is suitable for further ray acoustic reductions which are also affected here. Furthermore, the internal loading is equivalent to the spring-shell interaction forces acting at the attachment locations. The radiation from the induced forces superimposed with the contribution due to the empty shell then gives rise to the total structural and acoustic responses of the internal-shell-fluid system. The equivalent forces, which in general have both normal and tangential components, are functions of the shell displacements at the attachments; the latter may be in turn determined from their continuity conditions and evaluated either by normal-mode series or the geometrical acoustics fields and shell guided modes. [Work supported by ONR.]

\section{$4: 15$}

4pPAb12. Structural and acoustic responses of mass-spring loaded elastic cylindrical shells: Numerical implementation. Jin-Meng Ho (SFA, Inc., 1401 McCormick Dr., Landover, MD 20785 and Naval Res. Lab., Washington, DC 20375)

An important step in dealing with the mass-spring loaded cylindrical shell is to evaluate the total shell displacements at the attachment points, which determine the strength of the equivalent forces. Through dynamical balance at such points, these displacements satisfy a system of linear equations whose coefficients involve the shell displacements generated by onsurface forces of unit strength, a Green's-function problem with both source and observer located on the shell surface. It is thus evident that flexural waves, though subsonic, must be accounted for in the ray acoustic solution to the latter problem. Numerical examples show that they produce high $Q$ intense resonances because of their extremely small radiation damping, which would be dominated by structural damping for dissipative shell material, imprinting their signature in the far field through radiation of the induced forces and carrying much of the information on the internal load. By including the flexural as well as membrane waves, the ray model accurately predicts the induced forces, the shell displacements, and the surface and far-zone pressures in the frequency range of interest; $1 \leqslant k a \leqslant 25$ (where $k$ is the fluid acoustic wave number and $\alpha$ is the shell mean radius). [Work supported by ONR.]

\section{4:30}

4pPAb13. The impact of a rigid body of revolution with an elastic layer of finite thickness. Yu. A. Rossikhin and M. V. Shitikova (Dept. of Theor. Mech., Voronezh State Academy of Construction and Architecture, ul.Kirova 3-75, Voronezh 394018, Russia)

The problem of the impact of a rigid body of revolution with an elastic isotropic layer is considered in the initial stage of dynamic interaction. The initial stage is characterized by the fact that the velocity of the displacement of the intersection points of the sphere with the upper boundary of the layer is larger than the velocity of longitudinal waves; in so doing the free surface external to the contact domain with the body is undisturbed. The method of successive approximations as well as the ray method, according to which the solution behind the fronts of incident and reflected waves is constructed in terms of power series (ray expansions), are used as methods of solution. In the problem under consideration, use has been made of one-term ray expansions whereby the main characteristics of the shock interaction have been obtained, and the possibility of localized damage of the material of the layer at the points lying along the central ray has been examined. 


\title{
Session 4pSP
}

\section{Speech Communication: Speech Gestures}

\author{
Joseph S. Perkell, Chair \\ Massachusetts Institute of Technology, Room 36-591, 50 Vassar Street, Cambridge, Massachusetts 02139-9992
}

Chair's Introduction-1:30

\section{Contributed Papers}

1:35

4pSP1. Evidence of gestural overlap across speaking rate: Acoustic data. Kris Tjaden and Gary Weismer (Waisman Ctr. and Dept. of Commun. Disorders, 1500 Highland Ave., Univ. of Wisconsin-Madison, Madison, WI 53705)

The form of phonetic gestures has been assumed to remain stable across variations in speaking rate. Segmentalization or amount of gestural overlap has been shown to covary with speaking rate. However, the precise relationship between speaking rate and acoustic measures of segmentalization has not been delineated. In the present investigation, speakers produced multiple repetitions of the words "build," "dill," and "gill" in carrier phrases at graded, self-selected speaking rates ranging from slow to fast. $F 2$ onset frequency of the vocalic nucleus served as an index of segmentalization and was used to predict the time delay to the onset of the major $F 2$ transition associated with /11/. Previous work suggests $F 2$ onset frequency is sensitive to degree of gestural overlap in vocalic nuclei preceded by alveolar consonants. Preliminary results suggest (1) longer vocalic nuclei tend to be associated with $F 2$ onset frequencies closer to the preceding consonantal articulatory locus, and (2) the linear relationship between $F 2$ onset and time to target deteriorates above certain speaking durations, suggesting that the basic form of the gesture is altered at slower rates. [Work supported by DC 00319 .]

\section{1:50}

4pSP2. Articulatory evidence for acoustic goals for consonants. J. S. Perkell, M. L. Matthies, and J. A. Svirsky (Speech Commun. Group, Res. Lab. of Electron., Rm. 36-511, MIT, Cambridge, MA 02139)

Evidence has been found for trading relations between tongue-body raising and upper lip protrusion (measured with an EMMA system) for the vowel $/ \mathrm{u} /$, reflecting a "motor equivalence" strategy that should help to constrain acoustic variation [Perkell et al., J. AcousI. Soc. Am. 93, 29482961 (1993)]. Theoretically, analogous relations in the transformation between the area function and the acoustic transfer function are possible for the consonants $/ \mathrm{r} /$ and $/ \mathrm{J} /$, which are also produced with two independently controllable constrictions, formed by the tongue and by the lips. Such relations might occur more among tokens that are least protolypical, i.e., closest to perceptual boundaries. In a preliminary test of these hypotheses, a single speaker has produced the sounds $/ \mathrm{r} /, \mathrm{K} /$, and $/ \mathrm{L} /$, embedded in contexts that might induce differences in prototypicality. Motor equivalence was observed for the $/ \mathrm{u} / \mathrm{in} / \mathrm{tut} /$ (least prototypical, with the highest $F 2$ values) but not in /pub/ or /hud/. For $/ \mathrm{r} /$ and $/ \mathrm{S} /$, anterior displacernent of the tongue constriction was positively correlated with upper lip protrusion, providing initial support for the hypothesis that movement goals for the consonants also include acoustic components, which are manifested in a tendency to maintain sufficient front cavity length to help assure acoustic-phonetic distinctiveness. [Work supported by NIDCD.]

\section{2:05}

4pSP3. On explaining intrinsic vowel duration differences: An electromagnetic midsagittal articulometer (EMMA) study. Alice
Turk, Melanie Matthies, Joseph Perkell, and Mario Svirsky (Res. Lab. of Electron., Rm. 36-531, MIT, Cambridge, MA 02139)

The purpose of this study is to determine whether intrinsic vowel duration differences arise from an explicit timing control strategy or solely from the distance an articulator travels. Presumably, a difference in control strategy would be reflected in a change in the relation of peak velocity to distance travcled, or in the amount of time position is maintained. An EMMA system was used to track movements of points on the tongue and jaw during the production of $/ \mathrm{dV} \mathbf{s} /(V=/ \mathrm{e}, \mathrm{E}, \mathfrak{x}, \mathrm{a}, \mathrm{N} /)$ tokens in a constant frame at two different speaking rates. Measurements were made of target position, distance traveled, and peak velocity for each gesture toward and away from the vowel target. Preliminary results from one speaker suggest that the duration of articulatory movement can be well predicted by articulatory distance and peak velocity alone. Furthermore, all of the unreduced vowels measured in the study showed a similar relationship between peak velocity and displacement, suggesting that the same control strategy was used for all of them. [Work supported by NIH.]

4pSP4. On the perceptual characteristics of "speech gestures." René Carré, Samir Chennoukh (ENST, 46 rue Barrault, Paris, France), Pierre Divenyi (Veterans Affairs Med. Ctr., Martinez, CA), and Björn Lindblom (Univ. of Stockholm)

The notion of "speech gesture" or "phonetic gesture" has been developed as a theoretical construct during the past few years. For present purposes, the term is specified as a "phonologically significant task defined on the vocal tract area function." A given task generally involves the coordination of the movements of several articulators. It appears reasonable to expect speech gestures to have perceptual characteristics like the following: (a) Within certain limits, the time course of speech gestures is not important; (b) within certain limits, the precise synchronization of two, or more, gestures (e.g., constriction displacement and lip opening) is also unimportant; (c) vowel reduction is a nalural consequence of speech gesture characteristics. The commands of the distinctive region model were studied as possible speech gestures. Preliminary tests show, as hypothesized, that there are no significant perceptual differences (a) when the shape of a gestural transition is changed to be linear, quadratic, logarithmic, or cosine; (b) when the time separation between two gestures varies from -30 to $+30 \mathrm{~ms}$; (c) when gestural dynamics (transitional rate of change) is kept constant but the target is not reached (because of movement undershoot).

\section{2:35}

4pSP5. The articulatory kinematics of two levels of stress contrast. K. Bretonnel Cohen, Mary E. Beckman (Linguist. Dept., Ohio State Univ., Columbus, $\mathrm{OH}$ 43210-1298), Jan Edwards, and Marios Fourakis (Ohio State Univ.)

Intonation pattern and syllable duration are thought to be the most salient perceptual cues to phrase stress. However, intonation is an inherently ambiguous cue, since not all English pitch accents involve higher 
pitch on the accented syllable, and because there are stress contrasts at a lower level, where stress cannot be equated with accentuation. Duration, too, is ambiguous, since it can cue other prosodic contrasts, such as phrasal position. This study examines finer-grained timing cues to stress. A straingauge system was used to examine jaw opening and closing movements in /pap/ sequences in intonationally accented full-voweled syllables, unaccented full-voweled syllables, and completely stressless (reduced-vowel) syllables produced by fou speakers. Measured values for movement duration, displacement, and velocity were consistently larger in accented than in unaccented full-voweled syllables. However, these differences were nowhere near as large as the differences between full- and reduced-vowel syllables. Reduced syllables also had steeper velocity-displacement relationships, suggesting a durational difference at the level of gestural dynamics as well. However, no such consistent difference was observed between accented and unaccented full-voweled syllables. These results support the notion that stress contrasts are not uniformly interpreted in the phonetics at different levels.

\section{2:50-3:05 Break}

\section{3:05}

4pSP6. The IPA problem. Peter Ladefoged (Phonet. Lab., Linguist. Dept., UCLA, Los Angeles, CA 90024-1543)

Every few years it is appropriate to review our knowledge of the phonetic structures of the world's languages and try to assess how many different speech sounds there are. Problems arise because it is not easy to say whether two sounds occurring in different languages are the same or not. Two sounds are definitely different if they distinguish words within a language. But if they occur in different languages this test cannot be used. This is the basis of the IPA problem. The International Phonetic Association tries to provide a way of symbolizing every distinct speech sound, but it cannot tell whether two sounds are potentially distinct when they have been observed only in different languages. The data from recent phonetic studies indicate that there are limitless ways in which the sounds of one language can differ from another, but that the number of parameters along which sounds can vary is fairly small. In most cases the variation in the sounds that occur in different languages is the result of the use of a different value of one of the parameters, rather than the use of some novel parameter that has not been observed in other languages.

\section{3:20}

4pSP7. High vowel devoicing in Turkish. Stefanie Jannedy (Dept. of Linguist., Ohio State Univ., 222 Oxley Hall, 1712 Neil Ave., Columbus, $\mathrm{OH} 43210$ )

Jun and Beckman (ICSLP94) explain vowel devoicing in Japanese and Korean in terms of gestural overlap [Browman and Goldstein, LabPhon1 (1990)]; the glottal gestures for preceding and following voiceless consonants overlap to a greater or lesser extent with the glottal gesture for high vowels. The devoicing of the four short high Turkish [i i y u $]$ can be explained by the same model which predicts that vowels are more likely to undergo devoicing if they are short and the adjacent voiceless consonants have strong and large glottal opening gestures. This study evaluates influencing factors such as preceding and following environment, rate, stress, and syllable type. Subjects read 210 words positioned utterance initially in a carrier phrase at three rates. Faster rates and lack of stress facilitated devoicing most. As in Japanese and Korean, there were more devoiced vowels before following stops than before following fricatives, since vocal fold vibration ceases gradually before fricatives, but abruptly before stops. Devoicing also occurred in utterance-initial position. This result is less easy 10 interpret in terms of the model, since only the following laryngeal gesture can overlap with the vocalic gesture. More devoicing occurred in open syllables, where vowels are significantly shorter in Turkish.

\section{3:35}

4pSP8. Control of lip closure in stop consonant production. Anders Löfquist and Vincent L. Gracco (Haskins Labs., 270 Crown St., New Haven, CT 06511-6695)

Studies applying mechanical perturbations to the lower lip and the jaw have shown a trade off between movements of perturbed and unperturbed articulators in making the lip closure for a bilabial stop. While this concept is attractive, evidence for such trading relationships in normal speech has remained elusive, partly due to methodological problems. This study examines the vertical positions of the upper and lower lip at closure for bilabial stops. An electromagnetic transduction system was used to track receivers on the upper and lower lips, and the lower incisors for transducing jaw movements. The speech material consisted of VCV sequences where the first vowel was $/ \mathrm{a} /$, the middle consonant $/ \mathrm{p}, \mathrm{b} /$, and the second vowel $/ \mathbf{i}, \mathbf{a} /$; stress occurred on the second vowel. Preliminary results from three subjects showed upper lip positions at closure to occur over a range of $0.3 \mathrm{~cm}$; the range for lower lip positions was $0.5 \mathrm{~cm}$. Examination of 40 tokens for each subject revealed a positive relationship between upper and lower lip positions at closure, with correlation coefficients ranging from 0.5 to 0.7 . The results will be discussed in relation to theories of speech motor control. [Work supported by NIH.]

\section{3:50}

4pSP9. Exploring the relationship of breath intake to utterance duration. D. H. Whalen and J. M. Kinsella-Shaw (Haskins Labs., 270 Crown St., New Haven, CT 06511)

Previous work has indicated that there may be a positive relationship between the duration and extent of breath intake and the length of an upcoming utterance. However, none of that work has been free of alternative interpretations. An attempt was made to avoid some of those by forcing subjects to utter single sentences ranging in length from 5 to 82 syllables (mean of 27), after inspiring fully and then expiring down to a set level before uttering the sentence. For three subjects, there was a significant positive relationship between breath intake and utterance length $(0.65$, 0.50 , and 0.29 individually). This was still true without those utterances containing a secondary breath $(0.53,0.54$, and 0.29$)$. The two subjects with the higher correlations also exhaled more quickly for the shorter sentences than longer ones. The other subject had no correlation with exhalation rate: He might have been selecting a point within a rather large range of starting lung volumes, with only the longest sentences requiring that the range's lower part be avoided. The individual differences need further investigation, but there is a correlation between the duration of the sentence to be said and the breath before it. [Work supported by NIH Grant No. HD01994.]

\section{4:05}

4pSP10. An analysis of the variation of vocal tract configurations in continuous speech utterances. Tokihiko Kaburagi and Masaaki Honda (Information Sci. Res. Lab., NTT Basic Research Laboratories, 3-1, Morinosato-Wakamiya, Atsugi-shi, Kanagawa, 243-01 Japan)

This paper studies the variation of vowel and consonant articulation to examine the invariant features of phoneme-specific vocal tract configurations. The establishment of these features may be one of the goals of organizing articulatory movements, and they can be useful in constructing models of those movements. Articulatory data were gathered with a magnetic position-sensing device that traced the position of receiver coils placed on the lower jaw, upper lip, lower lip, and tongue in the midsagittal plane. Experiments were performed using (a) $V_{1} V_{2} V_{3}$, (b) $V_{1} V_{2} V_{3} V_{4} V_{5}$, (c) $\mathrm{V}_{1} \mathrm{CV}_{2}$, and (d) $\mathrm{CV}_{1} \mathrm{CV}_{2} \mathrm{CV}_{3} \mathrm{CV}_{4} \mathrm{CV}_{5}$ contexts, where $\mathrm{V}_{i}$ was a combination of vowels $/ \mathrm{a}, \mathrm{i}, \mathrm{u}, \mathrm{e}, \mathrm{o} /$ and $\mathrm{C}$ was one of consonants $/ \mathrm{p}, \mathrm{t}, \mathrm{k}, \mathrm{b}, \mathrm{d}, \mathrm{g} /$. A Japanese male subject uttered these sequences at several speeds with different stress. Articulatory data were sampled when the vocal tract constricted for vowels or when the vocal tract closed for plosive consonants: For each vowel and consonant, 200 data frames were sampled on average from the whole data set of about 150000 frames $(10 \mathrm{~min})$. The variability of the vocal tract configuration was then quantitatively analyzed for each 
phoneme by calculating the variation of each measurement point from its average position. The variation was very small when the vocal tract closed or constricted, especially in the traversal direction. How phoneme context, utterance speed, and stress affect the vocal tract configuration was also investigated.

4pSP11. Perceptual evaluation of articulatory movement recovered from acoustic data. Richard S. McGowan and Philip E. Rubin (Haskins Labs., 270 Crown St., New Haven, CT 06511-6695)
A method involving task dynamics and a genetic algorithm [see McGowan, Speech Commun. 14, 19-48 (1994)] was used to recover articulation from the speech acoustics of a human subject. Six utterances from a single subject were chosen to test the applicability of the proposed method to human beings: $/ \mathrm{ga} /, / \mathrm{gi} /, / \mathrm{da} /, / \mathrm{di} /, / \mathrm{ba} /$, and $/ \mathrm{bi} /$. The first three formant trajectories of the natural utterances were extracted and used to represent the acoustic data. A genetic algorithm was used in constrained optimization of task-dynamic parameters applied to the Haskins Laboratories articulatory synthesizer, ASY. The articulatory movements recovered in this manner were assessed using perceptual tests of the resulting speech generated by ASY and by a visualization of the resulting ASY articulation.

THURSDAY AFTERNOON, 1 DECEMBER 1994

BALLROOM B, 1:00 TO 5:00 P.M.

\title{
Session 4pUW
}

\section{Underwater Acoustics: Bottom Scattering}

\author{
Charles Loeffler, Chair \\ Applied Research Laboratories, University of Texas, P.O. Box 8029, Austin, Texas 78713-8029
}

\section{Contributed Papers}

\section{1:00}

4pUW1. Bistatic bottom scattering observed by seafloor vertical arrays during the July 1993 ARSRP bottom reverberation experiment. K. B. Smith and W. S. Hodgkiss (Marine Phys. Lab., Scripps Inst. of Oceanogr., La Jolla, CA 92093-0704)

During the July 1993 ARSRP bottom reverberation experiment, the R/V KNORR deployed bottom-moored, 64-element, vertical line arrays (VLAs) with the data being recorded by autonomous digital recording packages (GARRPs). Bistatic scattering data were recorded by these VLAs from R/V CoRY Chouest and R/V AluiAnCE transmissions. In this paper. bottom scattering observed at Site $B^{\prime}$ in the SRP Natural Laboratory with these VLAs will be shown and the received scatter related to the sourcearray positions and bathymetry. Modeling using UMPE/PEREV [K. B. Smith, F. D. Tappert, and W. S. Hodgkiss, J. Acoust. Soc. Am. 94, 1766 (A) (1993)] and BB-UMPE [K. B. Smith and W. S. Hodgkiss, J. Acoust. Soc. Am. 95, 2826 (A) (1994)] will be used to provide insight into the spatial and temporal structure of the observed scatter. [Work supported by ONR Code 3210A.]

\section{1:15}

4pUW2. Computer model simulations of reverberation from the ARSRP acoustics experiment. Stanley A. Chin-Bing (Naval Res. Lab., Stennis Space Center, MS 39529-5004) and Joseph E. Murphy (Univ. of New Orleans, New Orleans, LA 70148)

Detailed reverberation measurements were made from the mid-Atlantic ridge region during the 1993 Acoustics Experiment, Acoustic Reverberation Special Research Program (ARSRP). The reverberation scenario (designated as "Seg 076") that ensonified a segment of sedimented seafloor overlying an anelastic subbottom has been examined. Computer simulations of the reverberant field were generated and compared with the ARSRP measured reverberation. Time domain and $\mathrm{cw}$ computer simulations were made using high-resolution ocean acoustic-seismoacoustic computer models. Several realizations of the rough range-dependent sediment and sub-bottom were used (from the Webb-Jordan sediment distribution model and the Goff-Jordan fractal seafloor/basement model). Computer simulations allowed "numerical experiments" to be performed whereby parts of the environment were changed or eliminated. Simulation results that help identify those seafloor regions responsible for the reverberant returns are presented. [Work supported by ONR, Acoustic Reverberation SRP, and the High Performance Computing DoD Shared Resource Center: U.S. Army
Corps of Engineers Waterways Experiment Station (CEWES) HPC Center, Vicksburg, MS.]

4pUW3. Simulations of the 1993 ARSRP deep ocean seafloor scattering experiments using HARVEST-An adaptive wave propagation regime simulation technique. Johan $O$. A. Robertsson, Alan Levander (Dept. of Geol. and Geophys., Rice Univ., P.O. Box 1892, Houston, TX 77251-1892), and Klaus. Holliger (Inst. of Geophys. CH-8093 Zürich, Switzerland)

A new general hybrid technique, HARVEST (hybrid adaptive regime viscoelastic simulation technique), to solve the 2-D acoustic/viscoelastic equations for problems which encompass different wave propagation regimes, such as the relatively smooth water column and the highly heterogeneous seafloor in deep ocean seafloor scattering experiments, is presented. A Gaussian beam method is used to propagate the source field through a vertically heterogeneous water column to the scattering region near the seafloor. This extrapolated source field is inserted into a viscoelastic finite-difference method used to compute the complex acoustic/ anelastic interaction of the wave field with the rough seafloor. The backscattered field is extrapolated to a receiver array distant from the scattering locale by means of the Kirchhoff integral. HARVEST is employed to simulate ocean acoustic reverberation data collected during the 1993 ARSRP (Acoustic Reverberation Special Research Program) Acoustics Cruise. HARVEST is found to successfully reproduce ocean acoustic data within the confidence limits imposed by knowledge of seafloor morphology and material properties. The nature of scattering targets is interpreted from acoustics data in terms of synthetic HARVEST simulated data. In particular, rough basement that barely protrudes the sediment cover may cause strong returns in the backscattered field.

\section{1:45}

4pUW4. Shallow-water acoustic experiment in the Strait of Sicily Area: Preliminary results. J. M. Berkson (Naval Res. Lab., Washington, DC 20375), A. Kristensen, M. Max (SACLANT Undersea Research Ctr., 19138 LaSpezia, Italy), T. W. Yates, and G. M. Vermillion (Vector Res., Rockville, MD 20852)

Shallow-water acoustic and geoacoustic measurements were made in the southwest margin of the Adventure Bank area of the Strait of Sicily in a region having about $120-\mathrm{m}$ water depth and a generally smooth calcar- 
eous sand seafloor. Concurrent propagation and reverberation experiments were conducted using horizontal and vertical array receivers with both broadband explosive and controlled narrow-band sources. Propagation is frequency dependent with minimum transmission loss at long range occurring in the frequency band $200-800 \mathrm{~Hz}$. Overall reverberation envelope of duration was about $60 \mathrm{~s}$ and includes both diffuse returns and arrivals from major bathymetric features. [Work supported by ONR.]

\section{2:00}

4pUW5. Acoustic wave scattering by 3D variability of deep ocean sediments. D. Tang, D. Li, G. V. Frisk, and C. J. Sellers (Dept. of Appl. Ocean Phys. and Eng., Woods Hole Oceanogr. Inst., Woods Hole, MA 02543)

Volumetric inhomogeneities of marine sediments, especially their horizontal changes, are of great importance in understanding bottom scattering processes, but difficult to assess. Though certain theoretical models are available, so far little is known about sediment variability experimentally. During the 1993 ARSRP experiment, bottom scattering data over a sediment pond were obtained using the Deep Towed Acoustics/Geophysics System (DTAGS) near the bottom with its receiving acoustic array configured vertically. By analyzing this data set, the following results have been found: (1) The sediment is layered, but with gentle changes horizontally. (2) Beamforming in the normal direction of multiple pings reveals that there are two irregular sublayers at depths of about 16 and $60 \mathrm{~m}$ beneath the seafloor. Their thicknesses are both about $15-20 \mathrm{~m}$. (3) Simulations based on a simple model compare favorably with the experimental data, and the results suggest that volumetric inhomogeneities can be identified by examining the ping-to-ping correlation of the vertically reflected field. (4) The backscattered field is determined by first subtracting the coherently reflected portion of the field and then beamforming the residual portion at the desired grazing angles; the behavior of the backscattered field correlates well with the aforementioned irregular sublayers. [Work supported by ONR.]

\section{2:15}

4pUW6. Backscattering data analysis using multiple constraint beamforming. D. Li, D. Tang, and G. V. Frisk (Dept. of Appl. Ocean Phys. and Eng., Woods Hole Oceanogr. Inst., Woods Hole, MA 02543)

Using a vertical receiving array to measure bottom backscattering is clearly advantageous over the single receiver configuration. In the single receiver case, one uses arrival time information and the assumption that the scatterers are on the seafloor to obtain backscattering strength as a function of grazing angle; a vertical array, on the other hand, can be steered in various desired directions without invoking the aforementioned assumption. However, sediment stratification causes reflections in the normal direction, which are generally much stronger than the scattered signals at oblique angles. If one uses conventional beamforming, the reflected signals in the normal direction may therefore contaminate the signals received at oblique angles through sidelobe leakage. Therefore a multiple constraint beamforming technique is applied which substantially reduces the sidelobe level in the normal direction and enables one to measure backscattering strength at oblique angles. This technique is employed in the analysis of a deep ocean backscattering data set, and it is found that sediment inhomogeneities rather than seafoor roughness are the primary cause of backscattering. [Work supported by ONR.]

\section{2:30}

4pUW7. Spatial coherence and rough bottom scattering in shallow water. R. Dwi Susanto and Henrik Schmidt (Dept. of Ocean Eng., MIT, Cambridge, MA 02139)

Shallow-water acoustic data collected off Mallorca in March 1993 are analyzed for spatial coherence, and their relation to rough bottom scattering is investigated. Bistatic reverberation data were collected by a horizontal receiver array with equispaced hydrophones suspended $1 \mathrm{~m}$ above the bottom. A flextentional transducer towed behind the ship was used as a source, generating a $209-\mathrm{dB}$ source level at center frequency of $400 \mathrm{~Hz}$, with 5 -ms signal length, and 10 -s interval. The ship made a hexagonal run with the horizontal receiver array inside the track. A theoretical source model has been designed to approximate the actual source pulse, and is used to match-filter the data. The modal arrivals are clearly identified in the resulting impulse responses, allowing for mode-by-mode analysis. The total field is decomposed into a mean field (coherent field) and scattered field (incoherent field) by a stacking procedure. The spatial coherence is then calculated using a correlation and a coherence function. The magnitude square of the complex coherence function (MSC) of the total field was 0.9 while the MSC of the scattered field was 0.1 , which suggested that the bottom roughness was very small, allowing for modeling by perturbation theory.

\section{2:45}

4pUW8. Scattering due to sound-speed inhomogeneities in ocean waveguides. Brian Tracey and Henrik Schmidt (Dept. of Ocean Eng., MIT, 77 Massachusetts Ave., Bldg. 5-007, Cambridge, MA 02139)

A self-consistent perturbation method for three-dimensional acoustic scattering due to sound-speed inhomogeneities has been developed. This method allows calculation of the mean-field attenuation due to scattering, as well as the second-order statistics of the reverberant field. Propagation in a shallow ocean overlying a sediment bottom containing sound-speed inhomogeneities is considered as an application. A modal expansion of the acoustic field is assumed and (mean-field) modal attenuation coefficients are calculated. The attenuation coefficient calculation is straightforward and is suitable for use with standard normal-mode codes. Numerical results show this work is consistent with an earlier approach to plane-wave scattering [D. Tang and G. Frisk, J. Acoust. Soc. Am. 90, 2751-2756 (1991)], and illustrate the importance of scattering into the continuous spectrum. The spatial correlation and intensity of the reverberant field in the waveguide are calculated. [Work supported by ONR High Latitude Program.]

\section{3:00-3:15 Break}

\section{3:15}

4pUW9. Bistatic reverberation from an ocean ridge. Nicholas $C$. Makris, Lilimar Avelino, and Richard Menis (Naval Res. Lab., Washington, DC 20375)

Bistatic reverberation from a highly lineated ridge in the mid-Atlantic was measured by two research vessels during the ARSRP Main Acoustics Experiment of 1993. Each vessel used a vertical source array and a horizontal receiving array to collect bistatic and monostatic data over a full range of incident and scattered angles with respect to the ridge axis. Highresolution bathymetry reveals that the ridge protrudes above conjugate depth for roughly $50 \mathrm{~km}$, and is characterized by a number of extended scarps running parallel to the ridge axis. Sufficient excess depth exists around the ridge to isolate it with waterborne propagation paths from $1 / 2$ convergence zone $(\mathrm{CZ})$ range $(\sim 33 \mathrm{~km})$ where low-frequency returns register precisely with steep scarps. Significant variations in received level from a given scarp occur as a function of incident and scattered angle. The most prominent returns register with scarps facing the receiving array. Registration between returns from high-resolution waveforms $(\sim 10 \mathrm{~m}$ in range) and fine-scale bathymetry is used to elucidate the fundamental scattering process. Comparisons with monostatic measurements of the same ridge from $1 / 2 \mathrm{CZ}$ and $21 / 2 \mathrm{CZ}$ indicate how fine-scale structure from a given scarp is smeared in long-range reverberation.

\section{3:30}

4pUW10. Acoustic reverberation from a laboratory model of a shelf break Saimu Li and C. S. Clay (Geophys. and Polar Res. Ctr., Univ. of Wisconsin-Madison, 1215 W. Dayton St., Madison, WI 53706)

The structure of continental boundaries such as the east coast of the United States has a shallow sloping bottom that changes to a steeper slope at the shelf break. Our laboratory acoustic model has exaggerated slopes. The "shelf" has a $10.8^{\circ}$ slope; the bottom changes to a $50^{\circ}$ slope at the 
shelf break. The surface and bottom of the acoustic model are drywall construction board. The source is a spark and the receiver is a small microphone $(2 \mathrm{~mm} \times 2 \mathrm{~mm})$. The Biot-Tolstoy exact time domain solution was used as a basis for numerical calculations [I. Tolstoy and C. S. Clay, Ocean Acoustics (American Institute of Physics, New York, 1987), Appendix 5]. Arrivals are reflected paths, diffractions from the shelf break, and reflected diffractions. The existence and amplitudes of arrivals depended on source and receiver locations. For example, a source location in the inner shelf gave reverberations that were mainly from the $10.8^{\circ}$ wedge. A source position beyond the shelf break gave reverberations that were mainly from the $50^{\circ}$ part of the model. Experimental signals matched the theory in amplitudes and arrival times.

\section{$3: 45$}

4pUW11. Underwater acoustic measurements with a digital ocean bottom seismometer. David M. F. Chapman, John C. Osler, W. Cary Risley (Defence Res. Establishment Atlantic, P.O. Box 1012, Dartmouth, NS B2Y 3Z7, Canada), and D. Jackson Dodds (GeoAcoust., Inc., Aurora, ON LAG 4J9, Canada)

A digital ocean bottom seismometer (OBS) has been constructed comprising a hydrophone, three $4.5-\mathrm{Hz}$ geophones in a symmetric orthogonal configuration, 24-bit sigma-delta analogue-to-digital conversion, and a radio telemetry link to a ship-board monitoring and recording laboratory. Careful attention to self-noise floors and OBS/seabed coupling has resulted in an instrument capable of accurately measuring ambient noise on both hydrophone and geophone sensors in the $1-50-\mathrm{Hz}$ range. A vibrator mounted in the OBS, controllable from the receiving platform, allows in situ calibration of the OBS response to seabed motion. The instrument's design, construction, deployment, and operation will be described briefly, followed by some examples of the unusual ambient noise data sets that have been collected. At one thinly sedimented site, a very-low-frequency banded structure was observed in the ambient noise on the horizontal geophone channels only; at many sites, a strong $6-\mathrm{Hz}$ signal associated with offshore drilling activity was observed intermittently in all the geophone signals but was not seen in the hydrophone signals.

\section{4:00}

4pUW12. Backscattering in shallow-water waveguides caused by obstacles on the seafloor. Finn B. Jensen (SACLANT Undersea Res. Ctr., I-19138 La Spezia, Italy)

As a result of a recent workshop on low-frequency reverberation and scattering modeling organized by S. A. Chin-Bing and D. B. King at NRL-SSC, it became evident that accurate wave-theoretic models are available for studying feature backscattering in ocean waveguides. A well tested, two-way coupled mode code [R. B. Evans, J. Acoust. Soc. Am. 74, 188-195 (1983)] is applied to compute backscattering from a single obstacle on the seafloor. Of particular interest is the effect of height and slope of the scattering facet on the reverberant field. It is shown that only steep slopes cause strong backscattering.

\section{4:15}

4pUW13. Average acoustic field in shallow water I: Active sonar performance prediction. Ji-Xun Zhou, Xue-Zhen Zhang, Peter H. Rogers, and Gary W. Caille (School of Mech. Eng., Georgia Inst. of Technol., Atlanta, GA 30332)
The seabed dominates the shallow-water acoustics problem, but bottom properties and bottom scattering mechanism are usually poorly known. Detailed interference patterns in shallow water are thus not always physically meaningful for engineering applications. A simple analytical expression averaged over frequency or space can sometimes give better insight into some physical problems. In this presentation, an average angular power spectrum method for calculating shallow-water sound propagation, reverberation, noise, and spatial coherence is briefly introduced. The method is based on normal-mode and ray-mode analogies and originally appeared in Chinese papers which are not available in English [Zhou, Acta Acoust. Sin. 5, 86-99 (1980); Acta Ocean. Sin. 1, 212-217 (1979)]. Taking active sonar performance prediction as an example, analytical expressions for range/depth dependences of the sound propagation, average reverburation intensity, and echo-reverberation ratio will be given for several typical cases, including Pekeris shallow water, wedged continental shelf, and negative gradient waveguide. [Work supported by ONR.]

\section{$4: 30$}

4pUW14. Average acoustic field in shallow water II: Reverberation and its inverse problem. Ji-Xun Zhou and Xue-Zhen Zhang (School of Mech. Eng., Georgia Inst. of Technol., Atlanta, GA 30332 and Inst. of Acoust., Academia Sinica, Beijing, People's Republic of China)

Average reverberation intensity and the vertical correlation coefficient for reverberation, obtained from Pekeris shallow water, are offered as possible simple test cases for numerical modeling of reverberation and bottom scattering mechanism analysis. Measurements were conducted at three different areas in the Yellow Sea and the East China Sea with water depths of 40,29 , and $52 \mathrm{~m}$. These three locations have very different seabed sediments, with mean grain diameters of $0.35,0.07$, and $0.02 \mathrm{~mm}$, and porosities of $35.5 \%, 46.2 \%$, and $56.1 \%$, respectively. Wideband explosive sources with $1000-\mathrm{g}$ TNT charges were used to get $1 / 3$-octave reverberation data with center frequencies ranging from 200 to $5000 \mathrm{~Hz}$. Using theoretical results obtained from the angular spectrum method, both small-angle bottom reflectivity and scattering strength are derived from high reverberation-noise ratio data, including reverberation intensity and vertical coherence in shallow water. [Work supported by ONR and LAAS.]

\section{4:45}

4pUW15. Scattering of seismic interface waves from pointlike obstacles in unconsolidated sediments. Eric Smith, Anthony Atchley, John A. Behrens, Preston S. Wilson, Benjamin T. Wolz, and Thomas G. Muir (Appl. Res. Labs., Univ. of Texas at Austin, P.O. Box 8029, Austin, TX 78713-8029)

The concept of an active, bistatic, seismoacoustic interface-wave sonar is investigated theoretically and experimentally. The goal of the theory is to relate the scattering of seismoacoustic interface waves from material inhomogeneities and objects buried beneath an interface to their target strengths. This is done in a multipole scattering expansion, which takes a simple and efficient form in the limit that the inclusions can be treated as pointlike perturbations of the properties of the medium. Experiments were performed in a sandy beach area of the Gulf of Mexico to demonstrate the concept. The experiments included methods for the selective excitation of interface waves, and examined the propagation of seismic interface waves in unconsolidated wet sand, both water-saturated and unsaturated. The boundary conditions and target strengths of a collection of buried objects were measured, using a variety of pulse-echo and continuous-wave sonar configurations. 


\title{
Meeting of Accredited Standards Committee S3 on Bioacoustics
}

to be held jointly with the

\section{U.S. Technical Advisory Group (TAG) Meetings for ISO/TC 43 Acoustics, IEC/TC 29 Electroacoustics, and ISO/TC 108/SC4 Human Exposure to Mechanical Vibration and Shock}

\author{
T. A. Frank, Chair S3
}

Penn State University Speech and Hearing Clinic, 110 Moore Building, University Park, Pennsylvania 16802

R. F. Burkard, Vice Chair S3

Boston University Dept. of Communication Disorders, 635 Commonwealth Avenue, Boston, Massachusetts 02215

P. D. Schomer, Chair, U.S. Technical Advisory Group (TAG) for ISO/TC 43, Acoustics

U.S. CERL, P.O. Box 4005, Champaign, Illinois 61820

H. E. von Gierke, Vice Chair, U.S. Technical Advisory Group (TAG) for ISO/TC 43, Acoustics and ISO/TC 108/SC4, Human Exposure to Mechanical Vibration and Shock

1325 Meadow Lane, Yellow Springs, Ohio 45387

V. Nedzelnitsky, U.S. Technical Advisor (TA) for IEC/TC 29, Electroacoustics

National Institute of Standards and Technology (NIST), Building 233, Room A149, Gaithersburg, Maryland 20899

Standards Committee S3 on Bioacoustics. The current status of standards under preparation will be discussed. In addition to those topics of interest, including hearing conservation, noise, dosimeters, hearing aids, etc., consideration will be given to new standards which might be needed over the next few years. Open discussion of committee reports is encouraged.

The international activities in ISO/TC 43 Acoustics, and IEC/TC 29 Electroacoustics, and ISO/TC 108/SC4 Human Exposure to Mechanical Vibration and Shock, will also be discussed. The Chairs of the U.S. Technical Advisory Groups for ISO/TC 43 (H. E. von Gierke), and IEC/TC 29 (V. Nedzelnitsky), will report on current activities of these Technical Committees and Subcommittees, including their most recent meetings. (ISO/TC 108/SC4 met in Prague, Czech Republic in September 1994 and ISO/TC 43 and IEC/TC 29 both met in London, U.K. during November 1994.)

Scope of S3: Standards, specifications, methods of measurement and test, and terminology in the fields of mechanical shock and physiological acoustics, including aspects of general acoustics, shock, and vibration which pertain to biological safety, tolerance, and comfort.

\section{Session 5aEA}

\section{Engineering Acoustics: General Topics}

Murray S. Korman, Cochair

Department of Physics, U. S. Naval Academy, Annapolis, Maryland 21402

Janet Ellzey, Cochair

Department of Mechanical Engineering, University of Texas, Austin, Texas 78712

\section{Contributed Papers}

$8: 30$

5aEAl. Numerical simulation of the aerosound from the viscous layer of an impinging jet. Hani $G$. Alexander and William $C$. Meecham (Mech. Aerosp. and Nucl. Eng. Depl., Univ. of California, Los Angeles, CA 90024)

Noise generated by a jet impinging on a surface is of interest in itself for such applications as vertical takeoff. Here we consider the image ar- gument [see A. Powell, J. Acoust. Soc. Am. 32, 982 (1960) and W. C. Meecham, ibid. 37, 516 (1965)]. It shows that there can be no dipole sound for a large flat surface, only the volume, quadrupole aerosound. The sound generated by the viscous layer at the surface is neglected, and is examined by us here and shown to be indeed negligible. The simulation was carried out in two separate steps. The first step was to generate the hydrodynamic field which was compared to well-documented experimental results of similar flows. Once an adequate hydrodynamic field was generated, the 
theoretical treatment of Curle and Lighthill relating surface generated sound and the nature of aeroacoustic sound, respectively, was employed to get order-of-magnitude values for the sound intensity and develop directivity patterns. The source region considered was the viscous region near the surface. The $k \epsilon$ turbulence model was employed and did a fair job of depicting the fluid field. Velocity profiles of the free-jet region were very successful, while the model broke down some in the "wall jet" region of the flow. Surface pressure and shear stress distribution was in good agreement with experimental values. The acoustic values were then obtained and the viscous layer was found to generate very low intensity levels, in the neighborhood of $25 \mathrm{~dB}$, much less than the volume sound.

\section{8:45}

5aEA2. Two-sound-pressures theorem for aerodynamic sound from two-dimensional flows. Brenda Henderson (Dept. of Mech. Eng., Univ. of Houston, Houston, TX 77204-4792)

The three-sound-pressures theorem [A. Powell, J. Acoust. Soc. Am. 34, 902-906 (1962)] applies to sound generated by inviscid, incompressible, free flows when the source region is acoustically compact and shows that the acoustic far field must be reducible to lateral quadrupole radiation only. In two dimensions, the source region is not compact in the third dimension so it is not obvious that the three-sound-pressures theorem directly applies in this case. The two-sound-pressures theorem is developed by integrating Lighthill's source term over the "third" dimension and is shown to be satisfied by two-dimensional lateral quadrupole radiation. In all known two-dimensional situations, the two-sound-pressures theorem is satisfied. A simple two-dimensional line vortex problem involving the collision of four rectilinear vortices is presented as an illustration.

\section{9:00}

5aEA3. Symmetrical mode of rectangular choked jet screech. Dan Lin and Alan Powell (Dept. of Mech. Eng., Univ. of Houston, TX 77204-4792)

The screech and edge tones of rectangular jets had always been considered to be due to asymmetric (sinuous) jet instabilities, with out-ofphase sound fields across the jet plane. Kozu's 1991 screech measurements showed a discontinuity at $2.12 \leqslant R_{d}<2.16, R$ =pressure ratio, the wavelengths $\lambda$ below this being about $25 \%$ less than the empirical $\lambda / h$ $=k \sqrt{\left(R-R_{c}\right)}, h=$ small dimension of rectangular nozzle, $k=$ constant $=5.2$ (Powell), $=5.0$ (Krothapali), $R_{c}=$ critical value. However, Lin's 1992 schlieren photographs showed a symmetric (varicose) mode for edge tones at a pressure ratio $R=2.36$ and small nozzle-to-edge distances for the same nozzle, aspect ratio 4.8 , with wavelength about half that for (asymmetrical) screech. Lin's 1992 investigation of screech for $R<R_{d}$ showed unambiguous symmetry of the sound field, implying symmetrical (varicose) jet instabilities. The evidence consisted of signals from microphones symmetrically placed in the nozzle plane that showed unambiguous symmetry below the discontinuity and asymmetry above; correlation measurements were consistent with this (though of low coherence). Powell's phase criterion $\lambda / h=\left(1+M_{\text {con }}\right) / M_{\text {con }}$ is satisfied within $10 \%$ for $2.5 \leqslant R<3.6$, with significant discrepancies elsewhere.

\section{9:15}

5aEA4. Vortex sound in 2D: "vortex-force" and "vorticity-alone" forms. Alan Powell (Dept. of Mech. Eng., Univ. of Houston, TX 77204-4792)

In the contiguous method [J. Acoust. Soc. Am. 36, 830-832 (1964)] an incompressible inviscid flow causes pressure or velocity perturbations, $p_{\text {inc }}$ or $\mathbf{u}$, on geometrically distant surface (cylinder) that drives the contiguous external acoustic field. Solid surfaces are replaced by some appropriate vortex image system. The far field sound pressures are found to be $p(\mathbf{x})$ $=[1 / \sqrt{(8 \pi)}]\left[\left(\rho \omega^{1 / 2}\right) /\left(x^{1 / 2} c^{1 / 2}\right)\right] I_{D}^{\star}$ for dipole sound, with $c^{-3 / 2} I_{Q}^{*}$ for quadrupoles, $\rho=$ density, $c=$ sound speed, $I=$ sinusoidal source term (or its Fourier component or transform) of frequency $\omega, f(t) \equiv f(t-x / c)$. In terms of vortex force, $-\rho(\xi \wedge \mathbf{u})$, where $\zeta=\nabla \wedge \mathbf{u}=$ vorticity, $\nabla^{2}\left(p_{\text {inc }}+\rho u^{2} / 2\right)=-\rho \nabla \cdot(\zeta \wedge \mathbf{u})$ leads to $I_{D}=-\int(\zeta \wedge \mathbf{u})_{x}^{\prime} d S(\mathbf{y})$, with $f(t)^{\prime} \equiv \partial f / \partial t$ and $I_{Q}=-\int y_{x}(\zeta \wedge \mathbf{u})_{x}^{\prime \prime} d S(\mathbf{y})$. For vorticity alone, use $\mathbf{u}=\boldsymbol{\nabla} \wedge \mathbf{B}$, where $\nabla^{2} \mathbf{B}=-\zeta$, to get $I_{D}=\int\left(\mathbf{y} \wedge \zeta^{\prime \prime}\right)_{x} d S(\mathbf{y})$ and $I_{Q}$ $=\int(1 / 2) y_{x}\left(y \wedge \zeta^{\prime \prime \prime}\right)_{x} d S(y)$. (In $3 \mathrm{D}$, constants in the integrals are 1,1 and $1 / 2,1 / 3$, respectively.) The implications of these all being integral results are discussed; while the vortex force $-\rho(\zeta \wedge \mathbf{u})$ is a local flow property, $(y \wedge \zeta)$ is not.

5aEA5. Vortex sound: Equivalence of "vortex-force" and "vorticity-alone" forms. Alan Powell (Dept. of Mech. Eng., Univ. of Houston, TX 77204-4792)

A direct general proof is offered of the equivalence of the source integrals of the "vortex-force" and "vorticity-alone" formulations of aerodynamic dipole and quadrupole sound in both two and three dimensions as given in the previous paper. For the dipole (with solid surfaces replaced by an image system), take the expansion of $\nabla(\mathbf{a} \cdot \mathbf{b}), \mathbf{a}=\mathbf{y}, \mathbf{b}=(\zeta \wedge \mathbf{u})$, apply Helmholtz' equation $\zeta^{\prime}+\nabla \wedge(\zeta \wedge \mathbf{u})=0$, then after some manipulation and reduction integrate over 2-D or 3-D space and use Kelvin's transformations; finally, differentiate w.r.t. $t$ and take the $x$ component. For the quadrupole, form $(\mathbf{y} \cdot \hat{\mathbf{x}}) \nabla_{y} \cdot[\hat{\mathbf{x}}(\mathbf{a} \cdot \mathbf{b})]$ and proceed similarly, introducing $\int \mathbf{y} \cdot(\zeta \wedge \mathbf{u}) d V(\mathbf{y})^{\prime}=(\text { kinetic energy })^{\prime}=0$ in 3D but $\int \mathbf{y} \cdot(\zeta \wedge \mathbf{u}) d S(\mathbf{y})^{\prime}$ $=(\text { constant or } 0)^{\prime}=0$ in $2 \mathrm{D}$. Only kinematic relationships have been used apart from the foregoing hydrodynamic integral relationships for the quadrupole. Most transparently, in $2 \mathrm{D}$ for the dipole, if no vorticity is generated, $\zeta^{\prime}+\nabla \wedge(\zeta \wedge \mathbf{u})=D \zeta / D t=0$; so for moving vorticity $\zeta=\zeta_{0} \not\left(\mathbf{y}-\mathbf{y}_{0}\right), \mathbf{y}_{0}=\mathbf{y}_{0}(t)$, and $\zeta_{0}=$ constant. Then the vorticityalone form reduces directly to the vortex force form: $\int \mathbf{y} \wedge\left(\zeta_{0} \delta\right)^{\prime \prime} d S(\mathbf{y})=\int\left(\mathbf{y}_{0} \wedge \zeta_{0}\right) d S\left(\mathbf{y}_{0}\right)^{\prime \prime}=-\int\left(\zeta_{0} \wedge \mathbf{u}_{0}^{\prime}\right) d S\left(\mathbf{y}_{0}\right)$.

\section{9:45}

5aEA6. Active noise control for attenuation of low-frequency broadband jet engine exhaust noise. Jerome $P$. Smith, Ricardo $A$. Burdisso, Chris R. Fuller (Vib. and Acoust. Labs., Dept. of Mech. Eng., Virginia Polytech. Inst. and State Univ., Blacksburg, VA 24061-0238), and Rob Gibson (WYLE Labs., Arlington, VA 22202)

Low-frequency broadband jet engine exhaust noise radiated into the surrounding area has been a problem for facilities that perform ground jet engine run up operations such as military hush-houses. This low-frequency acoustic radiation is capable of propagating over long distances, creating a noise and vibration problem in nearby communities. In this paper, active noise control (ANC) is experimentally implemented to achieve attenuation of low-frequency turbo-fan jet engine exhaust noise in a nearby area. The control method is the feedforward filtered-x LMS algorithm and is implemented for both single-input, single-output and multiple-input, multipleoutput systems. The control inputs are generated by filtering a reference signal through adaptive FIR filters before being input to the control loudspeakers, and microphones are used to generate the reference and error signals. Attenuations of up to $15 \mathrm{~dB}$ are achieved in the 1/3-octave bands at the error sensor locations. The results also demonstrate a large area of reduction surrounding the error microphones with overall attenuations of up to $7 \mathrm{~dB}$ and generally agree with analytical predictions. Results are demonstrated for both a running jet engine in a small scale hush-house facility and an unsuppressed running jet engine. [Work sponsored by the U. S. Air Force.]

\section{0:00}

5aEA7. Theoretical evaluation of flow-induced noise in bull-mounted sonar arrays. Bertrand Dubus (Inst. d'Electron. et de Microélectron. du Nord, UMR CNRS 9929, Dépt. ISEN, 41 boulevard Vauban, 59046 Lille Cedex, France) and Christian Giangreco (DCN-Ingénierie Sud, DLSM, Le Brusc, 83140 Six Fours les Plages, France)

Future hull-mounted sonar arrays are likely to be limited by the effects of flow-induced acoustic noise on the sensors. These arrays are usually constituted by extended sensors embedded in an elastomer layer acting as outer decoupler (OD) mounted on a structural support plate (SSP). Predictive models of flow-induced noise are obtained by assuming specific 
mechanisms: (i) The direct path results from the detection by the sensors of the fluctuating pressures from the turbulent boundary layer after propagation through the OD [Ko et al., J. Acoust. Soc. Am. 85, 1469 (1989)]; (ii) the flexural noise is associated with the flexure of the extended sensors via their lateral sensitivity [Montgomery et al., J. Acoust. Soc. Am. 94, 1688 (1993)]; (iii) The vibrating SSP radiates acoustic pressures in the near field which are sensed by the array. The OD and SSP are assumed of infinite extent for the direct path and of finite extent otherwise. This paper presents an analytical model for the later path. Results are displayed in terms of wave-number-frequency spectrum of the radiated pressure and of frequency spectral density. The relative contribution of the different paths and the effect of various parameters of the array are discussed.

\section{0:15}

5aEA8. Instrumentation to generate a two-phase turbulent (bubble) submerged water jet for flow noise measurements. Murray $S$. Korman (Dept. of Phys., U. S. Naval Academy, Annapolis, MD 21402)

It has been demonstrated that the near-field pressure spectrum (generated by a turbulent submerged water jet) is enhanced when the turbulent flow is modified to become a two-phase flow containing air bubbles ["Proceedings of the 14th ICA, Beijing, China," Acoustica 76, supplement to No. 4, May (1992), paper B6-1, p. 70]. An amplification factor $G$ $=\int p_{\text {rwo-phase }}^{2} d f / \int p_{\text {single-phase }}^{2} d f$ is measured as a function of the gross void fraction $\beta$ of the air bubbles. Results showed that $G \sim \beta^{2}$ and $G \sim 20$ at $\beta=0.0065$. The range of void fraction was limited due to the use of the bubble maker (located at the jet nozzle entrance) which consisted of a fritted ceramic disk that was housed in a glass Buchner funnel and fed compressed nitrogen gas. It is possible to improve this apparatus by using carbonated water at high pressure in a nozzle that consists of an array of small holes in a thin circular plate. This arrangement can be controlled to yield small bubbles with an average diameter of about $100 \mu \mathrm{m}$ in water. Larger void fractions are possible and the necessary constraint of having the resonant bubble frequencies well above the region of the lowfrequency flow noise spectrum $(<500 \mathrm{~Hz})$ is obtained. A brief videotape will be shown. [Work supported in part by ONR.]

\section{0:30}

5aEA9. The combustive sound source: Combustion and bubble dynamics theory and experiment. Janet L. Ellzey (Dept. of Mech Eng., Univ. of Texas at Austin, Austin, TX 78712), Preston S. Wilson, and Thomas G. Muir (Univ. of Texas at Austin, Austin, TX 78713-8029)

In combustion systems, characteristics such as adiabatic flame temperature and laminar flame speed are highly dependent on the equivalence ratio, which is defined as the actual fuel/oxidizer ratio divided by the stoichiometric fuel/oxidizer ratio. Experimental results indicate that the acoustic output of the combustive sound source (CSS) also shows equivalence ratio dependency. Peak acoustic pressure produced by CSS is low for lean mixtures, rises to a maximum for near stoichiometric mixtures, and falls for rich mixtures. Experiments were also conducted to compare the acoustic output of CSS with the predictions of Rayleigh-Willis bubble theory. The measured acoustic output of CSS follows the trend of Rayleigh-Willis bubble theory but always falls below the absolute values. Energy losses that are not accounted for in the theory are probably responsible for the high predictions. Other factors that affect the acoustic output of CSS are discussed, including the shape of the combustion chamber, the source of ignition, the type of oxidizer and fuel, the presence of highpressure bubble collapses, and the generation of high-frequency components. [Work supported by ONR.]

\section{0:45}

5uEA10. High level, low-frequency, plasma-induced bubble transducer. Chris M. Young, Jay W. Benze, Randy D. Curry, Gary R. Hess, Ellis L. Loree, William M. Moeny, Forest E. White, James D. Wieting, and Niels K. Winsor (Tetra Corp., Albuquerque, NM 87109-4512)
Conventional low-frequency transducers are rather bulky, of limited efficiency, and may suffer from cavitation at high source levels. Plasmainduced bubble transducers may offer improvement in some of these limiting factors. An implementation method for these devices that has demonstrated precise energy control into the plasma has been developed. This has resulted in the ability to produce high level, high spectral efficiency sound at low frequency in a very compact geometry. Results are presented for sustained oscillations of a large plasma-induced bubble which has produced transient sound levels as high as $215 \mathrm{~dB}$. This offers the opportunity to produce very high sound levels on a cw basis. Our experiments have demonstrated the ability to stabilize bubble motion due to buoyancy, reexcite bubble oscillations with high efficiency, and the use of closed-loop feedback control for timing of energy injection. Experimental results of the acoustic output and bubble motion are presented. [Work supported by the U. S. Navy.]

\section{1:00}

5aEA11. Perturbation method applied to vibration monitoring of slotted beams. Xiu Ting C. Man and Robert D. Finch (Mech. Eng. Dept., Univ. of Houston, 4800 Calhoun Rd., Houston, TX 77204-4792)

Vibration signals can be used in monitoring the structural integrity of steel beams. If the beams are cracked or slotted, the resonant frequencies will decrease and the mode shapes will change depending on the size and the location of the defects. The current study involves applying a perturbation method in prediction of resonant frequencies and mode shapes of a slotted beam. Modal analysis techniques are used in estimation of modal parameters including resonant frequencies, damping, and mode shapes. The effect of using the modal analysis method on the frequency resolution will be discussed. A large discrepancy was observed between experimental frequency shifts and those predicted by the perturbation method for relatively large slots. As required by the method itself, perturbation to the system (in this case is slot) should be small, which will lead to small changes in modal parameters. These results are compared with those obtained previously [Man et al., J. Acoust. Soc. Am. 95, 2029-2037 (1994)] by solving the exact vibration equation for a slotted beam. It is clear that the perturbation technique is not accurate, although it does give correct qualitative predictions which are not readily apparent with the other approach.

\section{1:15}

5aEA12. Flexural wave reduction using a compliant baffle. Sung $H$. Ko (Naval Undersea Warfare Ctr. Detachment, New London, CT 06320)

A theoretical model was developed to evaluate the reduction of flexural waves which is generated by a line force applied on an infinite plate using a compliant baffle. The compliant baffle layer placed between the plate excited by a line force and a signal conditioning plate is designed for reducing pressure fluctuations from the vibrating plate. The baffle layer considered here is the compliant-tube array, modeled by Junger [J. Acoust. Soc. Am. 78, 1010 (1985)] to represent a homogeneous (dispersive) fluid layer. Effects of various parameters such as the aspect ratio of the compliant tube, the distance between tubes, and the damping of the tube material on the flexural wave reduction are presented. Calculations made for the nondispersive fluid layer are compared with those made for the dispersive fluid layer.

\section{1:30}

5aEA13. Rethinking the acoustics curriculum. Daniel R. Raichel (Dept. of Mech. Eng., Cooper Union, 51 Astor Pl., New York, NY 10003)

R. Bruce Lindsay's classic "wheel diagram" [Acoustics: Historical and Philosophical Development (Deaden, Hutchison and Ross, Stroudsburg, PA, 1972), p. 2] remains remarkably relevant today. The manifold categories of acoustics have not increased in number but deepened, rather, under the impetus of current $R \& D$ spurred by easy availability of computers. This situation has resulted in more material to cover in undergraduate and graduate courses for students of engineering and natural sciences. The necessity for total mastery of fundamentals remains, but the question arises regarding how much and what should be treated within time constraints. 
After a basic overview-type course (which must include laboratory experience) at least two additional courses should be provided in order to ensure that the undergraduate student has a sufficiently strong background in acoustics. The second course might deal almost exclusively with the theory and applications of ultrasonics, and the third course could be an independent study, whereby the student can pursue topics of specific interest under professorial guidance. For architecture majors, a special course in building acoustics should be a requisite--not so much for the purpose of making them acoustical experts as to inculcate in them an awareness of the acoustical implications of their work.

\title{
Session 5aNS
}

\section{Noise and Psychological and Physiological Acoustics: The Annoyance of Low-Level Environmental Sounds}

\author{
Daniel L. Johnson, Cochair \\ EG\&G Special Projects, Inc., P.O. Box 9100, Albuquerque, New Mexico 87111-9100 \\ Dana S. Hougland, Cochair \\ David L. Adams Associates, Inc., 1701 Boulder Street, Denver, Colorado 80211
}

Chair's Introduction-8:15

\section{Invited Papers}

8:20

5aNS1. Low levels of noise and resolution of their nuisance. John J. Van Houten (J. J. Van Houten and Associates, Inc., Irvine, CA 92714)

Noise ordinances provide objective standards which are used in the resolution of complaints, disputes, and land use compatibility concerns. However, application of the prevailing ordinance standard may indicate that compliance exists without adequately addressing the nuisance which motivated the complaint. The frustration of relentless noise and its related nuisance can be the motivation for litigation. Legal action may be the only remedy for its ultimate abatement. This paper will consider low levels of noise, less than the prevailing public agency standard, and will explore justification for the resolution of the nuisance which they may cause. Sources of such noise include a neighbor's pool pump/motor or air conditioner, industrial equipment, transformers, combustion burners, pumping units, water flow in pipes, etc. Noise ordinance standards which prevail in California will be reviewed, and nuisance criteria will be identified. In addition, case histories will be discussed which will provide examples of the resolution of the nuisance caused by low level noise.

\section{8:45}

5aNS2. A perceived low-frequency sound in Taos, New Mexico. Joe H. Mullins (Dept. of Mech. Eng., Univ. of New Mexico, Albuquerque, NM 87121) and Horace Poteet (Sandia Natl. Labs., Albuquerque, NM 87185-5800)

Persistent complaints of an annoying low-frequeney sound in Northern New Mexico, particularly in the vicinity of Taos, led to a request by members of the Congressional delegation of NM for an investigation. During the summer of 1993, in Taos, extensive simultaneous measurements were carried out of acoustic, seismic, electric, magnetic, and electromagnetic signals by a team from Sandia and Los Alamos National Laboratories, the Air Force Phillips Laboratory, and the University of New Mexico. Since anecdotal evidence and signal matching tests by the hearers implicated the frequencies between 30 to $100 \mathrm{~Hz}$, special attention was given to that range. However, no signals were found matching the description, and in particular no airborne audio signals in this range were found other than background, even though the acoustical detector was capable of measuring signals less than $-50 \mathrm{~dB}$ SPL. Subsequent complaints of similar sounds from widely distributed areas in the U.S., and a long history of these in the U.K. [R. N. Vasudevan and C. G. Gordon, Appl. Acoust. 10, 57-69 (1977)] have focused attention on human hearing in the 20-100 Hz range. New instruments are being developed and controlled clinical tests are planned with hearers and nonhearers in the Taos area.

\section{9:10}

5aNS3. Low-frequency acoustic measurements at Los Alamos. Rodney W. Whitaker (Los Alamos Natl. Lab., EES-5 MS F665, LANL, Los Alamos, NM 87545)

Los Alamos National Laboratory has had an active program of atmospheric infrasonic measurement for the last 10 years, in the frequency region of $0.1-10 \mathrm{~Hz}$. During this time, substantial data have been gathered on acoustic signals from underground nuclear tests, earthquakes, and large conventional explosions, often at long range. Normal operational activity, issues of long-range propagation, and interesting data examples will be discussed. Recent work related to a low level environmental noise problem will also be presented. 
5aNS4. Low level, low-frequency sounds: Measurement and analysis challenges. Alfred J. Bedard, Jr. (Natl. Oceanic and Atmospheric Admin., Environmental Technol. Lab., 325 Broadway, Boulder, CO 80303)

Low-frequency sounds from both natural and civilization sources can travel for long distances (tens or hundreds of kilometers), producing local regions of enhanced as well as reduced intensity depending upon range and time. Thus the idenlification of sources can be difficult, since merely mapping sound level in the direction of increasing intensity may nol help to identify distant sources. A brief review of some examples of low-frequency background sounds provides the basis for a discussion of potential methods for identifying sound sources in a complex propagation environment. At low frequencies $(<100 \mathrm{~Hz})$ it is more difficult to determine direction with simple, portable systems. Some practical approaches for low-frequency source location are described.

\title{
10:00-10:15 PANEL DISCUSSION
}

\author{
10:15-10:30 Break
}

\section{Contributed Papers}

\section{0:30}

5aNSS. Response of national park visitors to the sounds of aireraft overflights. William E. Robert (Harris Miller Miller \& Hanson, Inc., 15 New England Executive Park, Burlington, MA 01803)

The responses of over 750 national park visitors to sounds of aircraft overflights were measured at six study areas in the Grand Canyon, Hawaii Volcanoes, and Haleakala National Parks. Park visitors were observed as they entered each study area, and were asked to participate in a brief survey as they exited the area. The survey included questions about the visitor's reasons for visiting the park, and asked visitors to rate their responses to aircraft sounds on five-point scales. Simultaneously, in one to three positions representative of the study area, $A$-weighted sound levels were recorded at 1-s intervals using extremely low noise instrumentation. In addition, human observers maintained time-synchronized logs of aircraft audibility, types of aircraft audible, types of background sounds, and other factors. Several mitigating variables, such as background sound level and visitor expectations, were tested for their ability to improve the doseresponse relationships. Logistic regression was used to generate four separate dose-response relationships, relating two visitor responses (annoyance due to aircraft sounds, and interference of aircraft sounds with natural quiet) to two acoustic doses (percentage of time aircraft are audible, and aircraft A-weighted equivalent sound level). [Work supported by National Park Service.]

\section{0:45}

5aNS6. Long-term, low-cost noise monitoring systems. Eric V. McKinney and Robert D. Bruce (Colloboration in Sci. and Technol., Inc., 15835 Park Ten Place, Ste. 105, Houston, TX 77084-5131)

Long-term noise monitoring has been possible in the pası with large, expensive systems which were designed to withsland harsh environmental conditions including rain and cold temperatures while recording several months worth of interval data. For the past 7 years, CSTI and others have experimented with the use of noise dosimeters for outdoor measurements, monitoring sound levels for a few days up to 6 months. In order to use these monitors in the field, however, consideration must be given to issues such as how to protect the monitor and download the data since dosimeters are not sold as part of an integrated outdoor monitoring system. A waterproof, tamper-proof enclosure which holds the monitor and external battery, and a mast to position and protect the microphone are essential elements to such a system. Data downloading may be performed in the field with a portable computer or over a phone line. This paper will summarize experiences at solving some of the problems.

\section{1:00}

5aNS7. Automated monitoring of low level ambient noise levels. Nelson D. Lewis and William A. Russell, Jr. (Environmental Noise Prog., U.S. Army Ctr. for Health Promotion and Preventive Medicine (Provisional), Aberdeen Proving Ground, MD 21010-5422)
Automated monitoring of low level ambient noise levels presents many challenges. The obvious challenge is to have a noise monitor with a low noise floor, less than $20 \mathrm{dBA}$, so that the ambient can be monitored. Other challenges include designing a lightweight system that can be backpacked to the monitoring site, and the site selection. Site selection criteria include protecting the monitor from direct sunlight and heal, and locating the microphone so that the influence on the measurements from rustling leaves and wind will be minimal. This paper presents the results of our learning curve from three studies of low level ambient noise.

\section{1:15}

5aNS8. Homeowner judgments of the annoyance of individual heavy weapons blasts. George A. Luz, Nelson D. Lewis, and William A. Russell, Jr. (Bio-Acoustics Div., U.S. Army Ctr. for Health Promotion and Preventive Medicine, Aberdeen Proving Ground, MD 21010-5422)

Past studies of the annoyance of heavy weapons noise have shown the relationship between the yearly average exposure and average annoyance as reported by residents. However, no one has reported the relationship between individual weapons blasts and the annoyance reported by homeowners who were experiencing these events inside their own home. As part of a noise and vibration measurement study undertaken in response to community complaints, four homeowners made judgments of the annoyance of heavy weapons noise. When these judgments were plotted against the outdoor measured linear peak SPL, "moderate annoyance" was found to begin just above $115 \mathrm{~dB}$. This finding is consistent with a report by $L$. L. Pater in 1976 that there was a low probability of complaints from residents living in the vicinity of the Naval Surface Weapons Center, Dahlgren, VA, if weapons blasts were below $115-\mathrm{dB}$ peak at the complainant's home.

\section{1:30}

SaNS9. Annoyance of individual vehicle pass-by noise for light and heavy vehicles. Niek J. Versfeld, Joos Vos, and Frank W. M. Geurtsen (TNO Human Factors Res. Inst., P.O. Box 23, 3769 ZG Soesterberg, The Netherlands)

A laboratory experiment has been conducted to assess the noise annoyance of individual vehicle pass-bys as a function of sound level. Vehicle type varied from passenger cars to heavy tanks. Results showed that for each individual vehicle type, the A-weighted sound exposure level (SEL) was the most important predictor of the annoyance. However, at a given annoyance, the difference in level between different vehicle types could be as high as $11 \mathrm{dBA}$ SEL. The difference in level between the high-frequency part and the low-frequency part of the spectrum seemed to play a role in the annoyance, in that sounds containing relatively much high-frequency energy (e.g., passenger cars) were judged as being more annoying than those having relatively much low-frequency energy (such as tanks). [Work supported by the Ministry of Defence.] 
Results showed that, at a fixed equivalent sound level, and with the number of vehicles kept constant, annoyance hardly depended on the duration and

5aNS10. Effect of silent periods having short or long durations on the annoyance of vehicle sounds. Niek $J$. Versfeld, Joos Vos, and Frank W. M. Geurtsen (TNO Human Factors Res. Inst., P.O. Box 23, 3769 ZG Soesterberg, The Netherlands)

Two laboratory experiments were performed to study the effect on annoyance of noise concentration in time. The first (rating-scale) experiment dealt with the influence on annoyance of short-time silent periods (varying from 0 to $160 \mathrm{~s}$ ) in pass-by vehicle noise of 240 -s total duration. position in time of the silent period. In the second experiment subjects had to compare the annoyance of road traffic sounds with that of sounds from heavy vehicles (such as tanks). In deciding which fragment was more annoying, the subjects had to imagine that they were exposed to the road traffic sounds throughout the year, whereas the sounds of heavy vehicles were only audible during a certain part of the day, week, or year. Results indicate that, at a given equivalent sound level, concentration of the sounds in time reduces annoyance. [Work supported by the Ministry of Defence.]

FRIDAY MORNING, 2 DECEMBER 1994

SABINE ROOM, 8:15 TO 11:45 A.M.

\title{
Session 5aPA
}

\author{
Physical Acoustics: Scattering and Elastic Wave Propagation \\ Paul E. Barbone, Chair \\ Department of Aerospace and Mechanical Engineering, Boston University, Boston, Massachusetts 02215
}

\section{Contributed Papers}

\section{8:15}

5aPA1. Eigenfunction and eigenvalue analysis of scattering operators. T. Douglas Mast and Robert C. Waag (Dept. of Elec. Eng., Univ. of Rochester, Rochester, NY 14627)

Acoustic scattering by a given inhomogeneity can be compactly described by a scattering operator. This operator acts on the transmitted acoustic field to yield the scattered acoustic field on a measurement surface. For scattering at fixed frequency, the operator is known to admit a basis of eigenfunctions. When a finite number of transmit angles and receiving points is considered, the scattering operator can be represented as a matrix with an associated basis of eigenvectors. The present paper reports an investigation of the relationship between these eigenfunctions, eigenvectors, and associated eigenvalues and the characteristics of scattering objects, including their location, size, shape, orientation, and strength. Scattering operators are derived analytically for axisymmetric scatterers such as cylinders; in this case, the eigenfunctions of the operators take on simple trigonometric forms. Connections are noted between the eigenvalues and eigenfunctions of the scattering operators and the basis functions that appear in orthogonal function representations of the scattered fields. Scattering matrices for arbitrary scatterers are calculated using a coupled finite-element/integral equation method due to Kirsch and Monk [IMA J. Num. Anal. (to appear)]. Examples of the relationship between scatterer properties and eigenvalues and eigenvectors of the scattering matrix are presented.

\section{8:30}

5aPA2. Evidence for the existence of strong bending modes for signals scattered at oblique incidence from spheroidal shells. $M$. F. Werby (Naval Res. Lab., Code 7181, Stennis Space Center, MS) and N. A. Sidorovskaia (Univ. of New Orleans, New Orleans, LA)

In an earlier work [Werby et al., J. Acoust. Soc. Am. 85, 2365 (1989)] it was established that bending (flexing) modes are excited for signals scattering at oblique incidence from solid spheroidal shells. This was accomplished by comparing the exact $T$-matrix resonance predictions with those predicted from Timoshenko beam theory which accurately predicts the bending modes of a bar. One must then ask, do such modes exist for shells? One would expect that for fairly thick shells such modes do, but do they exist for thin shells too? In this study scattering of acoustical signals from elastic shells of various materials, aspect ratios, and shell thicknesses are examined. The study does indeed demonstrate the presence of bending modes even for very thin shells. It is interesting that for thick shells the resonance's manifest themselves as maximum amplitude returns while for thin shells they manifest themselves as minimum amplitude returns. The effect with the transitional nature of a rigidlike background is associated to a softlike background for the two extremes so that the return signals vary in their coherence from adding constructively to adding destructively over the thickness variation. The sensitivity of resonance locations as a function of the elastic parameters is also presented. [Work sponsored by NRL and ONR.]

\section{8:45}

5aPA3. Do pseudo-Scholte resonances exist? M. F. Werby (Naval Res. Lab., Code 7181, Stennis Space Center, MS) and N. A. Sidorovskaia (Univ. of New Orleans, New Orleans, LA)

In a pioneering work Talmant et al. [J. Acoust. Soc. Am. 86, 278 (1989)] established the presence of pseudo-Stonely resonances excited when acoustical signals scatter from shells at or near coincidence frequency. This notion was supported by the argument that Stoneley waves exist at the fluid-elastic interface of a plate when water is on one side and the other side is evacuated. It is known that nondispersive waterborne waves are excited at the fluid-water interface for that case in the frequency region around coincidence frequency. This coincided with the bounded shell case in which very narrow strong resonances corresponded with the waterborne waves and a broad envelope function corresponded with the onset of the flexural resonances which become manifest at coincidence frequency when the flexural waves become supersonic and thus radiated into the water. The envelope effect corresponds to an abrupt phase change of pressure at coincidence frequency. An analogous argument predicts the existence of pseudo-Scholte resonances. The analog for that case is a plate in which fluids of like properties exist on both sides. In that case waterborne waves exist over the entire frequency range. The implication is that if one scatters from such an object there should be a proliferation of waterborne waves and thus for closed shells an abundance of resonances associated with waterborne waves circumnavigating the shell should by present. Can the abundance of sharp peaks excited on fluid filled shells be explained in terms of this mechanism? This issue is examined and the question is answered. [Work sponsored by NRL and ONR.]

\section{9:00}

5aPA4. The study of pulse signals from elastic spheroidal shells near reflecting interfaces. N. A. Sidorovskaia (Dept. of Phys., Univ. of New Orleans, New Orleans, LA), Cleon Dean (Georgia Southem Univ., 
A formulation is presented that allows one to describe backscattered echoes from elastic shells near pressure release and rigid interfaces. This formulation is always consistent even at low frequencies and large distances from the interfaces and allows for the rapid reproduction of backseattered echoes over frequency ranges of practical interest. Numerical results are examined for pulse signals scaltering from various targets as a function of target material, distance from the interface, and the effect that the two interface extremes have on the detected signals. [Work sponsored by NRL and ONR.]

\section{9:15}

5aPA5. Acoustic scattering frnm a rigid sphere coated with multiple layers of absorbing materials: Eigenfunction expansion method. B. S. Sridhara (Ind. Studies Depl., Middle Tenn. State Univ., Murfreesboro, TN 37132) and Sadasiva M. Rao (Auburn Univ., Auburn, AL 36849)

Far-field scattered properties for a rigid sphere coated with several layers of absorbing materials have been obtained using the eigenfunction expansion method. The sphere coated with $m$ number of acoustic layers and placed in air, in a lossless situation, was considered. Compatibility conditions of sound pressures and sound velocities were applied at layer interfaces. The resulting $(2 m+1)$ complex linear algebraic equations involving material properties, and the spherical Bessel, Newman, and Hankel functions, were written in the matrix form. Computer codes were developed and the equations were solved for the coefficients which, along with other variables and parameters, were used to compute the scattered field properties. The formulation was verified by computing the far-field scattered parameter and comparing it with the available standard results. Far-field scattered properties were computed for a rigid sphere coated with a total of 12 different layers. In each case, the magnitude of the far-field scatter parameter was computed and plotted as a function of the angle in the range from $0^{\circ}$ to $180^{\circ}$. It was observed that the scatter parameter was sensitive to the change in the angle and the variation was relatively large in the range from $100^{\circ}$ to $160^{\circ}$ in the case of certain layers.

\section{9:30}

5aPA6. Comparison of experimentally obtained and theoretically predicted signature features in returued echoes from submerged targets insonified by short, broadband pulses. Hans C. Strifors, Torbjön Ståhısten (Natl. Defense Res. Establishment, S-17290 Stockholm, Sweden), and Guillermo C. Gaunaurd (Naval Surface Warfare Ctr., Silver Spring, MD 20903-5640)

Backscattered echoes are studied from submerged elastic targets in the frequency and lime-frequency domains when the targets are insonified by short, broadband sound pulses. The combined time-frequency approach seems to give the most information since it can show the time evolution of the resonance fealures that identify each target. The targets are either an air-filled spherical shell or various solid brass or steel spheres. The incident waveform is generated by weighting a sinusoidal signal with a Blackman time window of a few cycles width. Experimentally obtained data are processed in the time-frequency domain using a pseudo-Wigner distribution (PWD). The associated time window is Gaussian, and its width is adjusted to suppress the interference of cross-terms in the PWD, yet retaining the desired property of time-frequency concentrating the extracted features. Each PWD is displayed in a 3D surface plot using progressively lighter color shades (pseudocolors) determined by the height of the surface (or the magnitude of the PWD), and that surface plot is also projected onto the time-frequency plane to produce an informative $2 \mathrm{D}$ intensity image plot. For each target, the time development of significant resonance features extracted from returned echoes is examined and favorably compared with the corresponding theoretically predicted PWD signatures.
10:00

5aPA7. A new generalized $k$-space ( $G k S)$ method for transient elastodynamic scattering problems. Qing-Huo Liu (Schlumberger-Doll Res., Old Quarry Rd., Ridgefield, CT 06877)

A conventional approach to simulating transient elastic wave propagation in inhomogeneous media has been the finite-difference (FD) method. However, the FD method requires a large number of grids in order to obtain accurale results. This is because in conventional FD schemes, second-order (sometimes higher-order) differences are used to approximate the spatial derivatives. In this work, a new generalized $k$-space (GkS) method is described for elastodynamic scattering problems. From its integral representation in spatial-frequency $(r-f)$ domain, a local equation is derived for the displacement field in spectral-frequency $(k-f)$ domain. This equation becomes a time-convolution equation in spectral-time $(k-t)$ domain. Using two temporal propagators, compressional and shear, this time-convolution equation can be converled into two time-stepping equations, which become much easier to solve. Hence, at each time step, the solution is first obtained in the $\boldsymbol{k}-\boldsymbol{t}$ domain, and then transformed to the $\boldsymbol{r}-\boldsymbol{t}$ domain by using spatial FFT. Since the GkS method uses the Fourier transform to represent the spatial derivatives, it is much more accurate than the FD method. Numerical examples show that the GkS method with only four grids per wavelength can achieve the same accuracy as the FD method with 16 grids per wavelength.

\section{0:15}

5aPA8. Large-scale 3D finite-difference simulation of elastic wave propagation in borehole environments. Qing-Huo Liu, Eric Schoen, François Daube, Curt Randall, Hsui-lin Liu, and Ping Lee (Schlumberger-Doll Res., Old Quarry Rd., Ridgefield, CT 06877)

Elastic wave propagation in realistic borehole environments is very complex because of the presence of borehole, dipping beds, and other irregular scatterers. In order to understand this complex wave propagation phenomenon for sonic logging applications, a three-dimensional finitedifference (FD) method was used to simulate elastic wave propagation on a parallel computing architecture. The FD scheme solves the first-order elaslic wave equations with central differencing in both space and time via staggered grids. Liao's absorbing boundary condition is used to reduce artificial reflections from the finite computational domain. In this work, uniform grids in Cartesian coordinates are used to discretize the inhomogeneous medium. Because of the staircase approximation of the circular borehole, it is observed that the discretization requirement is quite different for monopole and dipole sources. Several methods are suggested to remedy this problem. The results from the FD method were validated by other methods available for several special geometries. Numerical examples will be shown to demonstrate the interaction of elastic waves with borehole and the surrounding geologic structures. Applications to interpretation of sonic logging will be illustrated.

\section{0:30}

5aPA9. Three-dimensional dyadic Green's function for elastic waves in multilayer cylindrical structures. Cai-Cheng Lu (Dept. of Electrical and Comput. Eng., Univ. of Illinois, Urbana, IL 61801) and Qing-Huo Liu (Schlumberger-Doll Res., Old Quarry Rd., Ridgefield, CT 06877)

Multilayer cylindrical structures are encountered in many applications. Elastic waves generated by an arbitrary source in such a structure can be found by using the corresponding three-dimensional dyadic Green's function. In this work, a 3-D dyadic Green's function for the displacement field is derived for elastic wave propagation in coaxial cylindrical structures with an arbitrary number of layers. The primary and reflection parts of the dyadic Green's function are first written in terms of the Fourier transform of $z$ (axial coordinate) and Fourier series of $\theta$ (azimuthal coordinate). In this transform domain, the boundary conditions are then imposed so that the reflection coefficients can be calculated for each $k_{z}$ and $n$, which are, respectively, the transform variables of $z$ and $\theta$. The spatial dyadic Green's 
function is then obtained by inverse transforming this solution in $k_{z}-n$ domain. Once this dyadic Green's function is found, elastic waves due to any source in the cylindrical structure can be obtained by integrating the Green's function with the source. The numerical results are validated against previous results for special geometries. Several applications of this dyadic Green's function will be shown for layered structures commonly used in acoustic well logging.

\section{0:45}

5aPA10. Leaky Rayleigh wave scattering from elastic media with microstructure. Yuan Zhang and Richard L. Weaver (Dept. of Theor. and Appl. Mech., Univ. of Illinois, 216 Talbot Lab., 104 S. Wright St., Urbana, IL 61801)

Scattering of leaky Rayleigh wave from a flat fluid-solid interface is studied. The fluid half-space is taken to be ideal and homogeneous while the solid half-space has randomly inhomogeneous anisotropic elastic constants due to the microstructure of the material. For plane waves incident from the fluid onto the interface at the critical Rayleigh angle, the singly scattered incoherent field is obtained by utilizing a first Born approximation. When the solid is a crystal aggregate and when the correlation function is of exponential form, the mean-square scattered signal level is found to be inversely proportional to the dimensionless frequency in the highfrequency limit but proportional to the third power of frequency in the low-frequency limit. Numerical results are given for water-aluminum interface scattering. [Work supported by NSF.]

\section{1:00}

5aPA11. An asymptotic model for compressional and shear wave excitation in plates by ultrasonic transducers. Smaine Zeroug and Fred E. Stanke (Schlumberger-Doll Res., Old Quarry Rd., Ridgefield, CT 06877)

Pulsed beams excited and detected by ultrasonic transducers are routinely used to characterize elastic structures. An efficient model for transducer-structure interactions is presented and applied to transmission through a fluid-loaded plate with a transmitter-receiver pair. Each transducer is modeled as several complex-transducer points (CTPs) [Zeroug et al., Review of Progress in QNDE, edited by Thompson and Chimenti (Plenum, New York, 1994), Vol. 13], which behave as reciprocal, electroacoustic quasi-Gaussian transducers. The interaction of each CTP transmitter-receiver pair with the plate is solved by wave-number spectral decomposition and ray expansions, resulting in a sum of multiply reflected beams propagating within the plate. The resulting time-harmonic beam integrals are approximated asymptotically and transformed to the time domain to yield the received voltage as a finite sum of multiply reflected compressional $(P)$, shear $(S)$, and coupled $(P-S)$ arrivals. Comparison with experiments shows that (a) three CTPs accurately model the flat circular transducers, (b) the efficient asymptotic solution is accurate when the observed arrivals are distinct in time, and (c) at normal incidence, the $\boldsymbol{S}$ and $\boldsymbol{P}-\boldsymbol{S}$ arrivals which are excited by the transducers' finite spectrum necessitate higher-order asymptotic expansions. This approach can be gen- eralized to transducers' arbitrary orientation, multilayered and cylindrical configurations.

\section{1:15}

5aPA12. Transmitted beam distortion and rotation due to mode conversion between acoustic and shear waves at liquid-solid interfaces. B. J. Landsberger, M. F. Hamilton, and Yu. A. Il'insky (Dept. of Mech. Eng., Univ. of Texas at Austin, Austin, TX 78712-1063)

An experiment was performed with a pulsed sound beam incident on a 6-cm-thick aluminum block immersed in water. Tone bursts were radiated at $1 \mathrm{MHz}$ by a circular source of radius $1.2 \mathrm{~cm}$, which was positioned 10 $\mathrm{cm}$ away from one side of the block. Transmitted acoustic beam patterns were measured in the water beyond the opposite side. Attention was devoted to angles of incidence in the neighborhood of the critical angle for compression waves in the block $\left(\sim 13.5^{\circ}\right)$. The use of pulses, and the strong refraction of the compression wave in the block, enabled isolation of effects associated with the shear wave. Distortion in the form of multiple beam splitting, and up to $4^{\circ}$ increase in the effective propagation direction of the transmitted beam (relative to the angle of the incident sound beam), were observed. Computations based on standard Fouriertransform techniques, but excluding contributions due to the compression wave in the block, yield good quantitative agreement with experiment. For the conditions in this experiment, calculations indicate that the distortion and rotation of the transmitted sound beam are due mainly to the mode conversion at the second interface. [Work supported by AASERT and ONR.]

\section{1:30}

5aPA13. A model of a confocal ultrasonic inspection system for interfaces. E. Yogeswaren and John G. Harris (Theor. Appl. Mech. UIUC, 216 Talbot Lab., 104 S. Wright St., Urbana, IL 61801)

A mathematical model describing how a confocal arrangement of two focused ultrasonic transducers is used to interrogate a complex interface between two materials by scanning the focal point across the interface is outlined. A complex interface is one that has roughness and partial contact at several length scales, many of which are equal to or smaller than the wavelength in the material. When the focused ultrasound strikes such an interface, though the focal region be small, multiple scattering takes place among the scatterers within and adjacent to the focal region, making it unclear how the interface is being sampled. To clarify this issue a specific interface model, consisting of a planar array of multiple small cracks having arbitrary lengths and spacings, is used. This interface is interrogated by a focused, antiplane shear wave. The model shows that what is measured are the multiply scattered signals averaged over the aperture of the transducer, and that the dominant contribution comes from the scattered signals that phase match to the interrogating signal. Expressions relating the modeled reflected and transmitted signals to the convolution of the incident wave field with the crack-opening displacements at the focal region are given. Numerical examples are worked out for similar and contrasting materials on each side of the interface. [Work supported by the NSF.] 


\title{
Session 5aSA
}

\section{Structural Acoustics and Vibration: Numerical and Analytic Methods}

\author{
Courtney B. Burroughs, Chair \\ Applied Research Laboratory, Pennsylvania State University, P.O. Box 30, State College, Pennsylvania 16804
}

\section{Contributed Papers}

8:30

5aSAl. An acoustic boundary element method using analytical/numerical matching applied to a fluid loaded plate. Ronald J. Epstein and Donald B. Bliss (Mech. Eng. and Mater. Sci., Duke Univ., Box 90300, Durham, NC 27708-90300)

Analytical/numerical matching (ANM) is a hybrid scheme combining a low-resolution global numerical solution with a high-resolution local analytical solution to form a composite solution. ANM is applied to a harmonically vibrating flat plate in two dimensions to calculate the radiated acoustic field and the associated fluid loading. The problem utilizes overlapping smoothed doublets, and local corrections to calculate the doublet strength distribution along the plate. A smoothing length scale is introduced that is larger than the smallest physical scale, and smaller than the largest physical scalc. The global low-resolution solution is calculated numerically using smoothed doublets, and converges quickly. Local corrections are done with high-resolution local analytical solutions. The global numerical solution is asymptotically matched to the local analytical solutions via a matching solution. The matching solution cancels the global solution in the near field, and cancels the local solution in the far field. The method is very robust, offering an insensitivity to collocation point position. ANM provides high-resolution calculations from low-resolution numerics with analytical corrections, while avoiding the subtlety involving singular integral equations, and their numerical implementation. [Work supported by ONR.]

\section{$8: 45$}

5aSA2. A 3-D finite element model for sound transmission through a double-plate system with isotropic elastic porous materials. Raymond Panneton, Noureddine Atalla (G.A.U.S., Dépt. Génie Mëcanique, Univ. de Sherbrooke, Sherbrooke, PQ J1K 2R1, Canada), and J.-F. Allard (Univ. du Maine, Le Mans, France)

The prediction of sound transmission through multilayer structures is of utmost importance in aircrafts, buildings, and other engineering applications. In view of optimizing the transmission loss, the finite element method is an interesting mean to model such structures since it permits one to account for complex structure geometries and to model accurately the boundary conditions. In this paper, a 3-D-finite element model is developed to evaluate the normal incidence transmission loss through a doubleplate system with cavity absorption. The cavity is filled with an airsaturated isotropic elastic porous materials. The model uses a two-field finite element procedure for the porous medium based on the Biot theory. Since the Biot theory considers only the energy dissipation due to the viscous effects, the frequency-dependent bulk modulus of the air, worked out by Champoux and Allard [J. Appl. Phys. 70, 2182-2191 (1991)], is adopted to account for the energy dissipation due to thermal exchanges. Also, two sets of field variables are considered: the $u-U$ and the $u-P$ sets, where $u$ and $U$ are the solid and fluid displacements and $P$ is the pore-fluid pressure. Both approaches are developed and compared through numerical simulations. [Work supported by Bombardier, Inc., Canadair, and N.S.E.R.C.]

\section{9:00}

5aSA3. Radiation sensitivity of baffled plates to structural perturbation. Steven R. Hahn and Aldo A. Ferri (Mech. Eng. Dept., Georgia Inst. of Technol., MS 0405, Atlanta, GA 30332)

The analysis of acoustic radiation and scattering from submerged elastic structures is an important and challenging problem. Often, numetical solutions are hampered by the fact that the acoustic pressure field can be very sensitive to structural detail. In this presentation, a baffled plate submerged in a semi-infinite acoustic medium is used study this sensitive. A finite element description of the structure and a boundary element description of the fluid are used to model the plate-fluid-baffle arrangement. The sensitivity information is obtained by applying perturbation techniques to the matrix equations that arise, yielding exact derivatives of the chosen field variable. Several types of local structural perturbations (point masses, springs, dampers, and ribs) as well as global perturbations (Young's modulus, density, damping) are considered. The nature of the structural sensitivity is examined and some investigation is made into what types of structural features have the greatest impact on radiation and scattering. Finally, the use of the sensitivity information for engineering and design is discussed.

\section{9:15}

5aSA4. Minimization of the sound power radiated by a cubic structure using velocity constraints. Daniel $H$. Kruger, J. Adin Mann, III, and Ted Weigandt (2019 Black Eng. Bldg., Iowa State Univ., Ames, IA 50011)

Sound power radiated by a cubic structure as a function of normal surface velocity was written using the boundary element method $[\mathrm{K}$. $\mathrm{A}$. Cunefare and G. H. Koopman, J. Vib. Acoust. 113, 387-394 (1991)]. This function was minimized by a gradient method with linear and second-order velocity constraints. The constraints were formulated to reflect physical limitations in the ability of constrained layer damping material to modify structural velocity. Complex velocily phase was held constant while amplitude was allowed only to decrease. Initially, simulated velocity patterns were used to test the method and software created. Sound pawer reductions of $3 \mathrm{~dB}$ were achieved while modifying the normal velocity amplitude over only $0.46 \%$ of the surface area of the cube at a single frequency. Later, velocity of a real structure was measured using a laser vibrometer. Minimum sound power and corresponding velocity distributions were calculated for nondimensional $k a$ ranging from 0.5 to 3.0. [Work supported by NSF, Contract No. MSS-9103377.]

\section{9:30}

5aSA5. Structural intensity in an infinite cylindrical shell driven by normal forces. Donald J. McDowell, Jr. (Naval Undersea Warfare Crr., Div. Newport, Detachment New London, Code 423, New London, CT 06320). Courtney B. Burroughs (Penn Stale Univ., State College, PA 16801), and Carlos M. Godoy (Naval Undersea Warfare Cir., Newport, RI 02841)

The structural intensity formulation provided by Williams [J. Acoust. Soc. Am. 89, 1615-1622 (1991)] is used to consider the flow of power 
along an infinite length, thin cylindrical shell driven by a harmonic force applied in the radial direction. Finite element modeling is used to show that the active component of the time-averaged intensity oscillates with respect to the axial coordinate of the shell at a wave number given by the difference between the bending and longitudinal wave numbers. The reactive component of the intensity also oscillates at this rate and is in phase quadrature with the active component. This suggests that the total power at any point along the shell is constant and power is exchanged between wave types. The stored (reactive) power is shown to be associated with circumferential expansions and contractions of the shell.

\section{9:45}

5aSA6. Balance of energy between scattered and reflected waves resulting from an axial surface wave incident on a discontinuity of a fluid-loaded cylindrical shell. Steven L. Means (Graduate Prog. in Acoust., New ARL Bldg., Penn State Univ., University Park, PA 16801)

There is a branch of the dispersion curve of fluid-loaded cylindrical shells in which fluid mass and an "effeclive" spring stiffness balance at low frequencies. This leads to a surface wave with a wave number which becomes increasingly close to the acoustic wave number as the frequency tends to zero. When such a surface wave, propagating along the axis of the cylinder, is incident on a shell discontinuity, energy is scattered back along the cylinder's axis and into the surrounding fluid. The present paper examines the balance of the energy between the reflected and the scattered waves. Comparisons are made with analogous surface waves scattered by a discontinuity of an elastic plate on an elastic foundation.

\section{0:00-10:15 Break}

10:15

5aSA7. A numerical experiment on the coupling of structural-borne sound into an aircraft interior. Brian H. Houston, Martin H. Marcus, Earl G. Williams, Douglas M. Photiadis, and J. A. Bucaro (Naval Res. Lab., Washington, DC 20375-5350)

Numerical experiments have been carried out to study the broadband structural acoustics of an aircraftlike shell under point excitation. The dominant mechanisms that lead to the enhancement of interior acoustic modes of this finite shell are examined. To this end, the shell response is studied for both the fluid-loaded and in vacuo cases. A rigid shell calculation was also performed to determine the importance of the wall compliance on the interior acoustics. It was found that the low-frequency shell response is not dependent on the free-wave dispersions, and is driven by the interior volume acoustic modes. At higher frequencies, the shell and fluid are coupled at shell wave numbers determined by the free-wave dispersions. In general, the shell and fluid couple at primarily one circumferential mode.

\section{0:30}

5aSA8. Implementation of infinite elements in adaptive multilevel substructuring for structural acoustic calculations. Paul D. DSouza and Jeffrey K. Bennighof (Dept. of Aerospace Eng. and Eng. Mech., Univ. of Texas at Austin, Auslin, TX 78712-1085)

A procedure is presented for incorporating infinite element modeling of the external acoustic medium in structural acoustic calculations using adaptive multilevel substructuring. The coupled structure-fluid system is modeled using finite elements for the structure, and for the acoustic fluid external to the structure and within a circumscribing prolate spheroid, and infinite elements external to the prolate spheroid, following the formulation of Bumett (1994). The adaptive multilevel substructuring approach transforms the structure model from the original finite element one in terms of nodal displacements, to a much more efficient one in terms of responses in approximate substructure vibration modes, which are selectively included in the model to optimize accuracy per degree of freedom. This approach is also used on the finite element portion of the acoustic fluid model, and results in a high level of model reduction there. Transformation of the infinite element portion of the acoustic fluid model is not practical because of its frequency dependence. The transformation process results in a linear system that is generally reduced in order, having a sparse symmetric coefficient matrix amenable to the use of a profile solver. The procedure is demonstrated on numerical examples in three dimensions. [Work supported by ONR.]

\section{0:45}

5aSA9. Wavelet frame expansions and wavelet response functions. Taner Önsay (Dept. of Mech. Eng., Michigan State Univ., East Lansing, MI 48824)

Wavelet frame expansions are utilized to introduce "wavelet response functions" (WRF). The convolution integral is replaced by an efficient synthesis scheme by incorporating multiresolution and wavelet frame expansion concepts. Wavelet frame expansion is used to partition a wide spectral range into multiresolution frequency bands. The response from each resolution level is obtained by using a sampling interval that is matched with the bandwidth. The contributions coming from different resolution levels are combined to construct the overall system response. In the applications, the attention is focused on the synthesis of the transient time-domain vibration response of proportionally damped $\left(B_{n}=\eta, f_{n}\right.$, $n=$ modes) wideband linear systems. Naturally, such systems, having impulse response durations that are inversely proportional to the modal bandwidths $\left(T_{\max _{n}}=2.2 / B_{n}\right)$, require a multiresolution synthesis scheme during the construction of their transient response, which involves fastdecaying high-frequency components that are superimposed on top of slowly decaying low-frequency modal responses. The proposed construction technique averts the time-domain aliasing problem associated with the DFT schemes. The effectiveness of the wavelet frame synthesis scheme is demonstrated by considering the construction of the transient vibration response of a finite dispersive system.

\section{1:00}

SaSA10. Wave packet synthesis via the wavelet transform. David M. Drumheller, David H. Hughes, Brian O'Connor, and Charles F. Gaumond (Acoust. Div. Naval Res. Lab., Washington, DC 20375-5350)

A wavelet transform is used to synthesize distinct contributions from the monostatic form function for a randomly ribbed, complex end-capped, finite cylindrical shell. In order to achieve satisfactory wave packets, as measured by their cross-correlation with the original impulse response, two wavelet transforms are used, a temporal wavelet transform and a spectral wavelet transform. This is in contrast to synthesizing wave packets from the Wigner distribution where just one two-dimensional surface was necessary. However, interference terms between components in the Wigner distribution, which is quadratic in the underlying signal, are absent in the linear wavelet transform. This allows for a more facile synthesis of the acoustic excitations. The interference terms in the Wigner distribution can sometimes fall at points in the time-frequency plane where a signal component exists, making it difficult to synthesize that component without corruption caused by the presence of other compounds in the form function.

\section{1:15}

5aSA11. Apparent anomalies in the forced vibration of a damped beam. Mauro Pierucci and Wensen Liu (Group in Appl. Mech., College of Eng., San Diego State Univ., San Diego, CA 92182-1311)

An infinitely long Bernoulli beam with linear damping is acted upon by a localized force given by $\delta(x) u(t) e^{t \omega t}$. The results are then obtained in wave-number space and the inversion is carried out by using an FFT algorithm. Several interesting and not previously reported results will be presented. The solution in the transform domain is composed of four terms: two transient terms with wave numbers equal to $k \sqrt{\eta}$ and $k$ and two steady-state terms; one propagates energy into the far field while the other is a decaying localized disturbance. The disturbance created by this near field sloshes energy back and forth near the location of the forcing function. The apparent backward traveling wave which is present in the steadystate condition is not due to the localized continuous reflection of energy 
from the distributed damping but is due to the requirement that the beam vibration has to have continuity of displacement and slope. The force responsible for the continuity of the slope is the culprit for this apparent phenomena. If the forcing function is $\cos \omega t$, the steady-state solution can be obtained within 20 periods, while if the function $\sin \omega t$ is used, then the steady-state solution cannot be obtained until more than $10^{6}$ periods.

\section{1:30}

5aSA12. SEA with exterior fluid loading: An open channeling. Paul E. Barbone (Depl. of Aerospace and Mech. Eng., 110 Cummington St., Boston Univ., Boston, MA 02215) and D. G. Crighton (Univ. of Cambridge, Cambridge CB3 9EW, UK)
We describe the vibration of a submerged elastic solid within a statistical energy analysis (SEA) framework. We discuss the modal structure of a submerged elastic solid, and contrast it to that assumed in traditional SEA analyses. In parlicular, submerged solids have higher modal density than their in vacuo counterparts, much higher levels of (frequency dependent) damping, and possibly strong coupling between the fluid and solid. Further, modes of submerged structures do not form a complete set, and the energy of oscillation in a fluid/solid mode is often irretrievable, independent of the coupling in the system. These results pinpoint the shortcomings of SEA in addressing exterior fluid loading. We close by identifying research directions for the advancement of SEA toward incorporating exterior fluid loading. [Work supported by ONR.]

Special Note: Posters to be presented in Session 5pSP will be on display in Ballroom A from 8:00 a.m. to 5:00 p.m.

FRIDAY MORNING, 2 DECEMBER 1994

WEDGWOOD ROOM, 9:00 TO 11:35 A.M.

\author{
Session 5aSP
}

\title{
Speech Communication: Models of Speech Production
}

\author{
Ingo R. Titze, Chair \\ Speech Pathology and Audiology, University of Iowa, Iowa City, Iowa 52242-1012
}

Chair's Intraduction-9:00

\section{Contributed Papers}

9:05

5aSP1. Characterization of noise during glottal vibration: Measurements performed on a mechanical model. B. Y. Reis, ${ }^{\text {a) }} \mathrm{C}$. H. Coker, and R. A. Kubli (AT\&T Bell Labs., Murray Hill, NJ 07974)

Speech synthesis and coding schemes commonly incorporate a random noise component during voicing. It is hard to isolate this noise in recorded speech because of the inaccuracy of characterizing a modulated random process from a few nonidentical periods with a strong periodic component. Direct measurement on humans poses many clinical difficulties. Computational hydrodynamic modeling is difficult, and reliable modeling of turbulence is an unsolved problem. Here, direct steady-state measurements are made on a detailed, life-size mechanical model. A molded rubber model of the vocal cords is driven synchronously with the sampling clock so that precise repetitions of pitch periods are generated. The random component of the recording is isolated by subtracting away the periodic component (obtained by ensemble averaging across 1000 pitch periods). The noise power and its spectrum al each point in the pitch period are estimated by ensemble averaging the autocorrelations of the noise. The noise results are well explained in terms of the directly measured transglottal pressure and glottal area. In high-passed human speech, bursts of noise appear to overlap the closed-glollis interval. To explain this, it is necessary to include transit time for glottal air bursts to reach an obstruction $2-3 \mathrm{~cm}$ downstream. ${ }^{\text {a) }}$ Also a student at MIT.

\section{9:20}

5aSP2. The normal modes of incompressible vocal fold tissues. David A. Berry and Ingo R. Titze (Natl. Ctr. for Voice and Speech, Dept. of Speech Pathol. and Audiol., Univ. of lowa, Iowa City, 1A 52242)

Much of the theoretical groundwork for treating vocal fold vibrations as viscoelastic waves in a continuum can be found in earlier publications [Titze and Strong, J. Acoust. Soc. Am. 57, 736-744 (1975) and Titze, J.
Acoust. Soc. Am. 60, 1366-1380 (1976)]. Both of these papers are based on small-amplitude vibrations where linearization is assumed to be valid, allowing normal modes and natural frequencies to be calculated. Although vocal fold tissues are known to be nearly incompressible, the first analytic expression for the normal modes of vocal fold tissues were based on the assumption of complete compressibility. The present study shows how the analytic normal modes deform and how the natural frequencies of oscillation shifl as the vocal fold tissues are gradually changed from being strictly compressible to absolutely incompressible, leaving all other factors constant. [This research was supported by Grant No. P60 DC00976 from the National Institute on Deafness and Other Communication Disorders.]

\section{9:35}

5aSP3. A Navier-Stokes solution of laryngeal flow during vocal fold oscillations. Fariborz Alipour and Ingo Titze (Dept. of Speech Pathol. and Audiol. and the Natl. Ctr. for Voice and Speech, Univ. of lowa, lowa Cily, IA 52242-1012)

Dynamic modeling of vocal fold tissue movement and laryngeal airflow was combined in a computer simulations for the purpose of voice production. A finite-element model was used for the solution of tissue mechanics and a finite volume method was used in the solution of NavierStokes equations for the airflow. A so-called "shadow method" simulated the glottal constriction in the flow model to avoid the complexity of grid movement. The two-dimensional flow equations were solved in an iterative manner until the given transglottal pressure was approximated. The flow solution was then used in the estimation of the aerodynamic forces on the tissue, required in the finite element solution of tissue movement. The results indicate that glottal velocity profiles are parabolic, with maximum velocity in the exiting jet reaching $35 \mathrm{~m} / \mathrm{s}$. The time-varying Reynolds number in the glottis can reach up to 1500 at a lung pressure of $8 \mathrm{~cm}$ water. The jet velocity waveform is similar to that of an excised larynx and the pressure profiles are similar to those of steady flows in physical models. 
Also, the displacement of the inferior portion of glottis shows previously described phase differences with the superior portions. [Work supported by NIDCD Grant No. DC00831-03.]

\section{$9: 50$}

SaSP4. Stress-strain response of the human vocal ligament and its effect on $\boldsymbol{F}_{\mathbf{0}}$ control. Ingo R. Titze, Young B. Min, and Fariborz Alipour-Haghighi (Natl. Ctr. for Voice and Speech and Dept. of Speech Pathol. and Audiol., Univ. of Iowa, Iowa City, IA 52242)

The longitudinal elastic properties of the human vocal ligament were quantified by stress-strain measurements and by modeling the response mathematically. Human ligaments were obtained from surgery and autopsy cases. They were dissected, mounted, and stretched with a dual-servo ergometer to measure force versus elongation and to convert the results into stress and strain. To calculate a longitudinal Young's modulus, the stressstrain curves were fitted with polynomial and exponential functions and differentiated. Young's modulus was separately defined in the low and high strain regions. The mean Young's modulus for the low strain region was $33.1 \pm 10.4 \mathrm{kPa}$. In the high strain region, $A$ and $B$ parameters for an exponential fit in the high strain portion were $1.4 \pm 1.0$ and $9.6 \pm 1.2 \mathrm{kPa}$, respectively. The stress-strain and Young's modulus curves showed the typical hysteresis and nonlinearity seen previously in other vocal fold tissues (muscle and mucosa), but the nonlinearity was most profound for the vocal ligament. The effect of these results on $F_{0}$ control will be discussed. [Work supported by NIDCD Grant No. DC00976.]

\section{0:05}

5aSP5. A dynamical systems model representation of the $F 0$ fluctuation of voice. Yuki Kakita and Hitoshi Okamoto (Dept. of Electron., Kanazawa Inst. Tech. (KIT), 7-1 Ohgigaoka, Nonoichi-Machi, Kanazawa-Minami, 921 Japan)

This paper proposes a simple model of generating time series of $F 0$ (fundamental frequency) fluctuation of voice from the viewpoint of the dynamical systems generating the irregularity. The data examined were the $F 0$ fluctuation for two kinds of voice, the normal and the pathological (perceived as "rough"). The time series of $F 0$ were obtained from the speech waveform of sustained utterances. The voice samples analyzed were for 20 normal (for five male and three female subjects) and 39 pathological (rough) (for sixteen male and one female subjects). By examining the time series and the pseudo-phase-portraits, a simple twodimensional mathematical model generating the time series of fluctuation is proposed by combining the characteristics of the Hénon map ("folding" operation) and the Delayed logistic map ("twisting" operation). Using the model, basic aspects of the fluctuations as well as of the pseudo-phaseportraits are successfully described with a single control parameter. The model simulation also suggests that a resultant intermittent type of fluctuation manifests a chaotic behavior which is actually observed in a pathological rough voice. The transition of fluctuation from the normal voice to the pathological voice is simulated by this model.

\section{0:20-10:35 Break}

\section{0:35}

5aSP6. Multiphonic vocalizations: A multifactor analysis of Tibetan monk chanting. Claudio F. Milstein and Thomas Shipp (Dept. of Speech and Hear. Sci., Univ. of Arizona, Bldg. 71, Tucson, AZ 85721)

Multiphonic chanting refers to highly specialized vocal techniques wherein one person is able to sing two or more tones simultaneously. Tibetan chanting is one of these techniques. Five Tibetan monks were studied (two low chanters and three high chanters). Digital recordings, EGG, airflow glottograms, oral fiberoptic laryngoscopy, and perceptual data were analyzed. Spectra from both types of chanters presented a display of extremely well defined $F_{1}$ and $F_{2}$ tuned to specific harmonics (harmonics 5 and 9 for low chanters and harmonics 2 and 4 for high chanters). Low chanters showed unusual activity at the vocal fold level and were able to maintain the multiphonic quality and formant tuning despite significant modifications of the vocal tract. It is suggested that the larynx is responsible for the production of the multiple tones in low chanters. Low and high chanters appear to use different strategies to achieve the same multiphonic effect. Our findings corroborate earlier findings by Smith, Stevens, and Tomlinson [J. Acoust. Soc. Am. 41, 1262-1264 (1967)] for low chanters.

\section{0:50}

5aSP7. Three-dimensional tongue shapes of sibilant fricatives. Shrikanth Narayanan, Abeer Alwan (Dept. of Elec. Eng., 66-147E Eng. IV, UCLA, 405 Hilgard Ave., Los Angeles, CA 90024), and Kate Haker (Cedars-Sinai Med. Ctr., Los Angeles, CA 90048)

In this study, 3-D tongue shapes reconstructed from MR images obtained during sustained production of the fricatives $/ \mathrm{s}, \int, \mathrm{z}, 3 /$ by four speakers are analyzed. Images were collected in the coronal, axial, and sagittal planes using a GE SIGNA $1.5 \mathrm{~T}$ machine with image slice thickness of 3 $\mathrm{mm}$ and no interscan spacing. Results show that the tongue body for $/ \mathrm{s} /$ and $/ z /$ is significantly lowered behind the constriction with respect to the tip and back of the tongue; the tongue body has flat or slightly convex contours in the constriction region that become significantly concave behind the constriction. The degree of concavity is speaker dependent, and decreasing concavity is observed as the posterior pharyngeal wall is approached. For $/ \mathrm{S} /$ and $/ 3 /$, the tongue body rises slightly along its midline before it starts sloping towards its posterior end; the tongue shape behind the constriction is convex (two subjects) or flat (two subjects) and gradually turns concave towards the posterior region. The "grooving" effect in $/ \mathrm{s}, \mathrm{z} /$ contributes to a relatively abrupt increase in the area function behind the constriction when compared to $/ \int, 3 /$. Similarly, raising of the tongue back in $/ \mathrm{s}, 2 /$ results in smaller areas near the velum when compared to $/ \int, 3 /$. The acoustic significance of these articulatory shapes are discussed. [Work supported in part by NSF.]

\section{1:05}

5aSP8. Tongue structural model: Integrating MRI data and anatomical structure into a finite element model of the tongue. Chao-Min Wu (Biomed. Eng. Ctr., Ohio State Univ., Columbus, $\mathrm{OH}$ 43210 and ATR Human Inf. Process. Res. Labs., Kyoto, Japan) and Reiner Wilhelms-Tricarico (MIT, Cambridge, MA 02139)

The goal of this research is to obtain a three-dimensional (3-D) tongue structural model as a reference placement for dynamic simulations of the tongue during speech articulation. The tongue structural model adapted to each individual's anatomy will be developed by integrating tongue shape information from MRI scans and anatomical drawings to form an anatomical model. The data structure of this model will be a finite element mesh. Interactive programs written in MATLAB are developed to (i) manually extract tissue boundary information from MRI data of the tongue and other oral structure, (ii) combine this information with the anatomical drawings of cross sections of the human tongue to form an anatomical model, and (iii) fit a basic finite element model (topological model) to the anatomical model to form the tongue structural model for a sample vowel gesture of each subject. Both processes (ii) and (iii) will be achieved by a method of 3-D nonuniform geometrical mapping using a generalized thin-plate spline mapping. Two tongue structural models (one for a Caucasian native American male and another for an Asian native Japanese male) will be built, using MRI data obtained during a sustained vowel production. [Work supported in part by a NSF grant to Dr. O. Fujimura.]

\section{1:20}

5aSP9. Determining vocal tract shape with small section length given only a few formants. T. V. Ananthapadmanabha (Voice and Speech Syst., 53, "Girinivas," Temple St., Malleswaram, Bangalore 560 003, India)

Vocal tract shape made up of cylindrical sections of equal length can be determined given the formant data of vowel sounds; the number of sections 
equals twice the number of formants. For an adult male speaker, use of four formants, results in a large section length of $2.125 \mathrm{~cm}$. If section length of $0.5 \mathrm{~cm}$ is desired, 17 formants have to be specified which appears impractical. A method for obtaining vocal tract shape with small section lengths, given only a few lower formants (up to fourth or fifth) is presented. For vocal tract of $M$ sections, only the lower $M / 2$ formants are independent and upper formants are constrained by the lower formants.
This constraint gives unrealistic locations for the upper formants. One method for predicting the upper formants would be to use those of a uniform tube of same length as that of the vocal tract. For a desired section length (say $0.5 \mathrm{~cm}$ ), the required formants (say, 17) can be specified by using the given lower formants (say, four) and predicted upper formants (say, from 5 to 17). Validation of the method using $x$-ray data and simulated vocal tract shapes is presented.

\title{
Session 5aUW
}

\section{Underwater Acoustics: Propagation I}

\author{
Daniel Wurmser, Chair \\ Naval Research Laboratory, Code 7144, Washington, DC 20375-5350
}

\section{Contributed Papers}

\section{8:00}

5aUW1. A new parabolic equation for range-dependent sound speeds. Roger Dashen (Phys. Dept., Univ. of California, San Diego, La Jolla, CA 92037), Daniel Wurmser, and Gregory Orris (Naval Res. Lab., Washington, DC 20375-5350)

A new parabolic equation for range-dependent sound speeds has been developed by modifying a procedure borrowed from quantum mechanics. The forward and backward propagating solutions are decoupled order by order by repeated applications of the Foldy-Wouthuysen transformation. The result includes energy-conserving terms which are proportional to the second range derivative of the sound speed squared. Physically, the technique has identified the terms responsible for heretofore anomalous refractive effects which accumulate during propagation as a result of rapid oscillations between the forward and backward propagating solutions. Moreover, this phenomenon does not result in permanent energy transfer berween the two types of solutions. [Work supported by ONR.]

\section{8:15}

5aUW2. Range-stepping algorithm for the Helmholtz equation. Dalcio K. Dacol (Naval Res. Lab., Washingıon, DC 20375-5350)

The usual range-stepping algorithms used to obtain an approximate solution to the Helmholtz equation are based on the parabolic approximation and restricted to the forward propagating component of the solution. A complete solution of the Helmholtz equation in an inhomogeneous medium must also include backpropagating waves, that is, waves scattered towards the source by inhomogeneities. The inclusion of such effects in a numerically feasible full-wave approach to acoustic propagation is a problem of continual interest in ocean acoustics. This problem has been studied using the method of coupled amplitudes. Theoretical developments on the application of this technique to ocean acoustics will be discussed as well as numerical applications to the ASA range-dependent benchmark problems. Numerical application of this method involves a fourfold increase in the number of arithmetic operations as compared with that required for solving the same problem using a PE approach. However, one does get the backscattered field and one not does have to approximate square roots of differential operators as one must do in the PE approach. Since an elliptic equation is being solved one encoumters numerical instabilities; how to deal with them will be discussed.

\section{8:30}

5aUW3. A numerical method for random two-way propagation in the Helmholtz equation. Roger M. Oba (Naval Res. Lab., Stennis Space Center, MS 39529-5004)
Consider a strongly stratified ocean with randomness for which numerical computation of average complex pressure with two-way propagation is to be made. Let depth dependence be piecewise continuously differentiable and sediment be modeled by a fluid bottom. Within a fixed range interval, model uncertainty by an ensemble dependence upon some parameter in a continuously differentiable way, and the range dependence to be piecewise constant over independent intervals. For a single frequency source at a known depth, analysis for one element of the ensemble proceeds via the Helmholtz equation in radial symmetry and satisfies an outward radiation condition at large range. A previously developed modification for finite length range intervals in finite depth of the KohlerPapanicolaou coupled mode equations in the forward scattering approximation [R. M. Oba, Acoust. Lett. 16, 56-61 (1992)] computes the transmission loss and phase for the average solutions for one-way propagation. This paper presents a reformulation of this method to two-way propagation of average solutions. This analysis will also show the development and computation of averaged scattering type operators. [Work supported by Naval Research Laboratory and ONR.]

\section{8:45}

5aUW4. Nonparabolic marching algorithm for sound field calculation in the inhomogeneous ocean waveguide. A. Voronovich (NOAA/ERL/Environmental Technol. Lab., 325 Broadway, Boulder, CO 80303)

A new algorithm is proposed for calculating sound field in the inhomogeneous ocean waveguide. It does not involve parabolic approximation and at least for 2D inhomogeneities can be considered as exact. On the other hand, it is "marching" and can be as easily implemented as a parabolic equation. For inhomogeneity with final horizontal extent, the algorithm calculates, in particular, the scattering matrix for acoustic modes interacting with inhomogeneity. 11 is significant that the approach is able to calculate not only the acoustic field itself, but propagation times of different modes as well. The cases of uneven boundary and media with losses can be also dealt with. Thus the algorithm is able to provide an exact solution of the Helmholtz equation with 2D inhomogeneities both for shallow- and deep-water situations. The algorithm can be easily generalized for the case of the moving media and/or density variations. The corresponding code does not require great computer memory or speed and can be performed on a conventional PC. Numerical simulations of sound propagation through strong inhomogeneity related to subpolar front will be presented. Two types of mode interaction appearing for two-channel situations will be discussed. [Work supported by ONR.] 
5aUW5. Comparing the performance of split-step algorithms for coastal acoustics problems. David B. King, Stanley A. Chin-Bing (Naval Res. Lab., Stennis Space Center, MS 39529), and Michael D. Collins (Naval Res. Lab., Washington, DC 20375)

The split-step Fourier algorithm [R. H. Hardin and F. D. Tappert, SLAM Rev. 15, 423 (1973); S. M. Flatté and F. D. Tappert, J. Acoust. Soc. Am. 58, 1151-1159 (1975)] has been an important technique for solving range-dependent ocean acoustics problems for more than two decades. Since this algorithm is restricted to leading-order asymptotics, parabolic equation solutions based on finite-difference algorithms have been developed for handling effects that require higher-order asymptotics, such as wide propagation angles, extensive bottom interaction, and elastic and poroelastic waves. The split-step Fourier algorithm has remained in widespread use despite the improved accuracy and capability of finitedifference solutions. The split-step Padé algorithm [M. D. Collins, J. Acoust. Soc. Am. 93, 1736-1742 (1993); M. D. Collins, J. Acoust. Soc. Am. 96, 382-385 (1994)], which includes higher-order asymptotics, is orders of magnitude faster than standard finite-difference solutions. Results will be presented for representative test problems to illustrate that the split-step Padé algorithm is also more efficient than the split-step Fourier solution for the coastal acoustics problems that are currently of interest.

9:15

5aUW6. Direct solution of the acoustic wave equation in underwater environments with attenuation using the finite difference method. R. A. Zingarelli (Naval Res. Lab., Acoust. Simulation Sect., Stennis Space Center, MS 39529)

A finite difference solution to the acoustic wave equation for real pressure fields which incorporates sediment attenuation has been implemented. The differencing method is a centered space/centered time solution of the undamped wave equation, with absorbing boundaries simulated by an offset space/forward time solution of the radiation condition equation. This algorithm executes efficiently on SIMD and MIMD supercomputers, and gives an acceptable solution to the ASA ideal wedge benchmark problem. Stability, memory, and exccution time considerations will be presented. Two basic attenuation methods, an ad hoc absorption term and a Lax solution of the damped wave equation, along with several differencing schemes for each, were implemented. Results and runtimes for $\mathrm{CW}$ and time domain benchmarks and other problems incorporating bottom attenuation will be presented and discussed. [Work supported by ONR.]

\section{9:30}

5aUW7. A model for wave propagation in an inhomogeneous elastic medium. Raymond J. Nagem (Dept. of Aerospace and Mech. Eng., Boston Univ., 110 Cummington St., Boston, MA 02215), Ding Lee, and Tien-Chay Chen (Naval Undersea Warfare Ctr., New London, CT 06320)

A perturbation method is used to derive equations for wave propagation in an inhomogeneous elastic medium. The formulation is three dimensional, and is written explicitly in cylindrical coordinates so that it can include property variations in the depth, range, and azimuth directions. A factorization is used to convert the equations into an operator form which distinguishes outgoing waves from incoming waves. This factorization removes the requirement for a far-field boundary condition. For large-scale numerical problems, the factorization produces a tremendous reduction in computation time and in memory requirements. The elastic equations are written in a way that is directly analogous to previous work in ocean acoustics. With appropriate fluid-elastic interface conditions, the elastic and acoustic equations may be used to study the fluid-elastic interactions which are important in shallow-water environments, especially the propagation of shear waves in the ocean bottom. In this paper, the theoretical development of the elastic equations are presented and their merits and applications are discussed. [This work was jointly supported by the U.S. Office of Naval Research and the U.S. Naval Undersea Warfare Center.]
5aUW8. Convergence properties of wide-angle techniques. David Yevick (Dept. of Electrical Eng., Queen's Univ., Kingston, ON K7L 3N6, Canada)

The relative accuracy of various high-angle propagation methods were examined for the case of a rapidly expanding point source. It was established that the removal of evanescent modes in wide-angle calculations through, e.g., hybrid Fourier/Lanczos or Fourier/Padé algorithms [J. Acoust. Soc. Am. 96, 396 (1994)], greatly improves the numerical predictions of such techniques. Alternatively, procedures in which the imaginary part of the Thomson-Chapman propagation operator is applied after each propagation step may also be employed to increase the rate of convergence of standard unitary methods. Further, it was found that for moderate step lengths and highly divergent fields the errors implicit in rational approximations to the square-root operator are comparable to those associated with analogous approximations to the full propagator as well as with Chebyszev expansions of the exponent of the propagation operator with degree about twice that of the equivalent Pade method. Finally, a heuristic but useful relationship between the rate of convergence of a given Lanczos technique and the Taylor series expansion of the underlying propagation operator is outlined.

\section{0:00-10:15 Break}

\section{0:15}

5aUW9. High-frequency propagation in a turbulent flow. David $M$. Farmer, Daniela Di Iorio, and Ward Cartier (Inst. of Ocean Sci., 9860 W. Saanich Rd., Sidney, BC V8L 4B2, Canada)

As part of the new ONR-initiative in moderate-to-high-frequency acoustics, a pilot experiment will be carried out in a shallow tidally forced channel in September 1994 to compare acoustic variability with direct measurements of turbulent velocity and sound-speed fluctuations. The acoustic configuration will consist of a linear array of four sources on one side, and four receivers on the other $(f=67.5 \mathrm{kHz}, L \approx 700 \mathrm{~m})$, together with a single source and receiver for reciprocal transmission. Separation of reciprocal transmission from reception of the other four sources allows a repetition rate for all sources of $5 \mathrm{~Hz}$. The spatial filter will be applied to the acoustic data so as to focus the measurement on the central portion of the channel, at which location a moored turbulence sensor (R. Lueck) will be deployed at the same depth as the acoustic path. Supplementary measurements will be acquired with a multiple frequency backscatter sonar, moored current meters, and pressure gauges, and a neutrally buoyant float to measure the larger scale turbulent motions (E. D'Asaro, APL-UW and D. Veenstra, IOS). The experimental work will be discussed in the context of prior studies of forward scattering from turbulence and preliminary results will be presented.

\section{0:30}

5aUW10. Acoustic scintillation and random focusing effects in the ocean. John R. Potter (Marine Phys. Lab., Scripps Inst. of Oceanogr., La Jolla, CA 92093-0238)

Small-scale oceanographic variability becomes increasingly relevant to acoustic propagation as the frequency of interest exceeds a few $\mathrm{kHz}$. The intensity effects are generally unspectacular at very close or distant ranges; little scattering has yet occurred in the near field and stochastic limits apply in the far field. At intermediate ranges random focusing can greatly enhance the intensity in small volumetric regions. These predicted highintensity "ribbons" result from an accrual of multiple forward scattering of individual wavefronts and appear in addition to multipath effects. An experiment was performed in the eastern Mediterranean during 1986 using $3.6-\mathrm{kH} 2$ pulses transmitted over ranges of $11-23 \mathrm{~km}$ to investigate this effect. Observations were made over vertical and horizontal apertures of $200-250 \mathrm{~m}$ and $4-4.5 \mathrm{~km}$, respectively. The experiment is described and the random focusing is confirmed. The acoustic signals traveling along individual ray paths are observed to develop randomly focussed regions 6-18 dB above normal levels over regions some $1 \mathrm{~km}$ long and $20 \mathrm{~m}$ deep. 
The strength and size of these anomalies are consistent with predictions from multiple scattering theory. Understanding these features creates the possibility of inversion for a statistical description of the small-scale oceanography in addition to permitting constructive tactical use and improved quantitative estimates of system performance. [Work supported by ONR.]

\section{0:45}

5aUW11. ErTect of random volume and surface fiuctuations on propagation in shallow water. Terry $E$. Ewart and Daniel Rouseff (Appl. Phys. Lab., Univ. of Washington, Seattle, WA 98105)

The potential difficulties associated with modeling acoustic propagation in shallow-water environments are well documented. Larger scale deterministic features combine with random fluctuations in the water column, sediment, sea surface, and bottom to produce an extremely complicated propagation regime. The extent to which each of these features needs to be included in realistic propagation models remains to be quantified. Towards this end, results from a series of detailed simulations generated using the parabolic equation (PE) method are presented. Beginning with a deterministic downward refracting sound-speed profile and a known sloping bottom, realizations of the random features are sequentially added to the simulation. Pierson-Moskowitz surface waves, power-law bottom roughness, and representative bottom absorption are used to model the medium. The individual and cumulative effects of these scattering mechanisms on the acoustic wavefront are evaluated. Successive interactions with a random bottom are studied. The results are quantified by using a modal decomposition and also by examining the local arrival angle of energy as a function of depth. [Work supported by ONR.]

\section{1:00}

5aUW12. Pulse propagation in a range-dependent turbulent ocean. W. R. Chang, S. H. Dong, and J. H. Tarng (Dept. of Commun. Eng., Chiao-Tung Univ., Hsin-Chu, Taiwan)

In this research, an acoustic pulse propagating in a turbulent ocean with slightly range-dependent vertical sound-speed profile is investigated. A numerical approach called the split-step method, based on the parabolic equation, is used to simulate each frequency component of the pulse to propagate through the ocean. A random phase screen is generated to include the effects of random fluctuation of sound-speed profile in each step. The average acoustic pulse profile and root-mean-square pulse width are evaluated for different fluctuation strengths and scale lengths of turbulence. Some results are compared with those computed by theoretical approach. It is found that in each realization the pulse echoes, due to the multipath propagation, are changed in arrival times, pulse shapes, and numbers because of the random fluctuation of the sound-speed profile. The broadening of the pulse width is varied for different depths of the receiving position. An initial Gaussian-shaped pulse is considered. For the simulations, a Gaussian-beam source is used instead of a point source. Here, the sound-speed profile is assumed to be changed uniformly in the range direction.

\section{1:15}

5aUW13. Intensity statistics of acoustic waves scattered by internal waves. Roger Dashen and James Gerber (Univ. of California, San Diego, La Jolla, CA 92093-0319)

Intensity correlations for ocean acoustic propagation show markedly different behavior in the partially saturated regime than in the fully saturated regime. In contrast to the known exponential decay of temporal intensity correlations in full saturation, partial saturation correlations fall off very slowly. This phenomenon is a consequence of the peculiar time dependence of internal waves, and does not occur in other physical situations, such as atmospheric optics. Results of a numerical simulation which demonsirate the effect are presented.

\section{1:30}

5aUW14. Site selection for a long-term acoustic study of the temporal variability in a shallow-water environment. Ted Farrell, Rigo Torres, Cola Atkinson, and Greg Duckworth (BBN Syst. and Technol., 1300 N. 17th St., Ste. 1200, Arlington, VA 22209)

A site selection study was performed to determine the best location for an environmental-acoustic measurement system to be deployed for two years in shallow water. The study searched for continental United States (CONUS) locations where the environmental parameters are sufficiently variable such that, over time, the acoustic environment at these locations will be similar to the environments found at multiple littoral regions of interest outside of CONUS. Shipping densities, sound-speed profiles, wind speed, rainfall, bathymetry, and sediment characteristics were investigated using a MATLAB ${ }^{\mathscr{O}}$ based geographic information system. As part of the investigation, measures of variability were defined for each environmental variable. In addition, automatic classification of sound velocity profiles was implemented on more than $\mathbf{4 4 0 0 0}$ measured profiles from the areas of interest. The results of the study show that several CONUS areas have enough environmental variability to satisfy the requirements of a long-term measurement program. The systen developed for this effort significantly reduced the time required to analyze and visualize large amounts of environmental data. The system also provides a means to couple environmental data into various acoustic models. [Work sponsored by ONR.]

\section{1:45}

5aUW15. Coregistration of received signals with bathymetry using ARTIST. Vincent H. Lupien and J. Robert Fricke (Dept. of Ocean Eng., MIT, Rm. 5-435, 77 Massachusetts Ave., Cambridge, MA 02139)

As part of the ARSRP, a software package called Acoustical RayTracing Insonification Software (ARTIST) was developed which performs the coregistration of received signals to areas of the seafloor for direct paths. The transmitting and receiving array beampatterns are projected onto the actual seafloor by ray-tracing to yield two insonification patterns. The $(x, y)$ loci of ray contacts with the seafloor are then triangulated and shadow-zone processed so that the interior of each triangle is inside the illuminated region. Using a bivariate interpolator over each triangle of the insonification patterns, an intersection pattern is generated on a rectangular grid, yielding among other parameters: two-way travel times, TL, beampattern values, and grazing angles. Only the points which lie in the illuminated zones of both the receiver and transmitter are kept. By using another bivariate interpolator over each patch of the intersection pattern, pairs of time contours are generated and for each patch intersected by a contour; the fractional area lying inside the time window is calculated. The final step in the coregistration is to select only those patches which are most loudly insonified for each time window. The patches do not have to be adjacent and the amplitude criteria are user-specified. [Work supported by ONR.]

\section{2:00}

5aUW16. Diffraction of acoustical waves by spatially localized inhomogeneities in oceanic waveguides. Sergcy M. Gorsky, Vitaly A. Zverev, and Alexander I. Khil'ko (Inst. of Appl. Phys. of Russian Academy of Sci., Hydrophys. Dept., 46 Uljanova St., Nizhny Novgorod 603600, Russia)

Diffraction of the acoustic fields by spatially localized inhomogeneities in oceanic waveguides has been investigated. The formula for scattering matrix of diffracted waveguide modes has been obtained using a "shortwave" approximation. The spatial-frequency diffraction patterns for different locations of inhomogeneities, sources and receivers, and properties both of the spatial-localized inhomogeneities and the waveguides were investigated numerically. The possibilities of diffracted fields measurement in natural waveguides were investigated under the conditions of physical modeling in the ultrasonic frequency region. The significance of physical modeling methods for investigation of diffraction phenomena in ocean was demonstrated. It was shown that the efficiency of diffracted field observa- 
tions can be increased using results of calculation and measurements under model conditions. The spatial-temporal filtration by horizontal and vertical arrays and by matching processing of complex pulse illumination signals were discussed as methods for isolation of diffracted signals against the background of noise of randomly distributed oceanic inhomogeneities. Besides that, a systematic concept of the structure of perturbed signals in waveguides and an analysis of the possibilities of its observations in layered waveguides were developed.

FRIDAY AFTERNOON, 2 DECEMBER 1994

PECOS ROOM, 1:00 TO 3:30 P.M.

\title{
Session 5pEA
}

\section{Engineering Acoustics: Ultrasonic Transducers and Measurements}

\author{
Magne Vestrheim, Cochair \\ Department of Physics, University of Bergen, Allegt 55, N-5007, Bergen, Norway \\ John Behrens, Cochair \\ Applied Research Laboratories, The University of Texas, P.O. Box 8029, Austin, Texas 78713-8029
}

\section{Contributed Papers}

\section{1:00}

5pEA1. Modified lead titanate with Eu and Mn for high-frequency ultrasonic transducer. E. Suaste, J. Leybón, V. H. Salazar (Cinvestav I.P.N., Dept. of Electrical Eng., Bioelectron. Section, AV. IPN 2508 Mexico D. F.), and O. Perez-Martínez (Cuba)

The conventional materials, such as PZT ceramics, show a high electromechanical coupling factor; however, they must be acoustically matched to tissue in order to facilitate transmission and reception over a broad frequency band. Application of this ceramic in linear arrays entails additional complexity. Each element in an array must be subdivided into several subelements to suppress coupling unwanted lateral modes. Thus piezoelectric ceramics with large electromechanical anisotropies (the electromechanical coupling factor for thickness dilatational vibration $K_{t}$ is much large than that for planar extensional vibration $K_{p}$ ) have recently come to be developed for use as high-frequency ultrasonic transducer materials. $\mathrm{PbTiO}_{3}$ ceramics modified with alkaline metals or rare earth have been extensively investigated because of their promise as a piezoelectrical materials for high-frequency and high-temperature applications. In $\mathrm{Pb}\left[\left(\mathrm{Co}_{1 / 2} \mathrm{~W}_{1 / 2}\right)_{0.04} \mathrm{Ti}_{0.96}\right] \mathrm{O}_{3}$ ceramics Yamashita et al. reported that the electromechanical coupling factor for thickness dilatational vibration $K_{t}$ increasing of poling field but coupling factor for planar extensional vibration $K_{p}$ was negligibly small. In $\left(\mathrm{Pb}_{0.88} \mathbf{L n}_{0.08}\right)\left(\mathrm{Ti}_{0.98} \mathbf{M n}_{0.02}\right)$ ceramics ( $\mathbf{L n}$ $=\mathrm{La}, \mathrm{Nd}, \mathrm{Sm}$, and Gd) Takeuchi et al. found that the Sm ion was excellent in the substitution of the $\mathrm{Pb}$ ion to make large electromechanical anisotropy between longitudinal and transverse mode vibration. Electromechanical properties of $\mathrm{PbTiO}_{3}$ ceramics modified by the partial substitution of $\mathrm{Eu}$ for $\mathrm{Pb}$ and $\mathrm{Mn}$ for $\mathrm{Ti}$ are examined, and the results are compared with the partial substitution of $\mathrm{Sm}$ and other elements of the lanthanides group.

\section{1:15}

5pEA2. Transit time determination in a one-way ultrasonic measurement system. Magne Vestrheim, Steinar Vervik, and Murugendran Kanagasundram (Dept. of Phys., Univ. of Bergen, Allégt. 55, N-5007 Bergen, Norway)

Frequency and time domain analysis are used to study the transit time determination in a one-way ultrasonic measurement system for gases at around $200 \mathrm{kHz}$. Effects of transducers, diffraction, and interfacing to electronics on pulse forms and transit times are considered. Comparisons are made with experimental results using two transducers mounted in a stabilized acoustic chamber where fluctuations can be reduced so as not to affect amplitude and time measurement accuracies. By utilizing a fitted model for the transmitting responses of the transducers together with measured impedance data and the assumption of reciprocity, a useful modeling of the signal transmission through the system is achieved. The effects of receiving electronics and cabling are also measured and included. The results are used to discuss effects on the transit times for recent precision multibeam ultrasonic flowmeters used in the transport of natural gas, and at resolution and accuracies required in the zero calibration of such meters. [Work performed in cooperation with Christian Michelsen Research, Bergen.]

\section{$1: 30$}

5pEA3. A phase analysis of an ultrasonic pulse signal for measuring travel time. Eunbang Lee and Shigeo Ohtsuki (Precision and Intelligence Lab., Tokyo Inst. of Technol., Nagatsuta 4259, Midori-Ku, Yokohama 227, Japan)

A pulse signal has been utilized extensively for estimating travel time in an ultrasonic field. Travel time can be measured by calculating the time difference between transmitted signal and received signal. In a conventional method, time resolution is limited to its pulse width. Also, the received signal has an influence on the transducer characteristic and propagation characteristic and is distorted from the transmitted signal. It is not easy to compare between two different signals for measuring travel time. A technique to analyze the pulse signal by phase for measuring travel time is proposed. This technique can be applied to both a continuous-wave method and an impulse wave method for measuring travel time. The major feature is that the fluctuation of signals dependent on time is displayed in time domain, faithfully. Another feature is a method for identifying different amplitude signals, because this technique is independent of their amplitudes and $\mathrm{dc}$ bias. This technique is useful and acceptable for estimating time information such as travel time.

\section{$1: 45$}

5pEA4. Separation of overlapping reflections for ultrasonic NDE applications. Richard D. Patton (Raspet Flight Res. Lab., Dept. of Mech. Eng., Drawer ME, Miss. State Univ., Mississippi State, MS 39759), Patrick C. Marks, and James Bennett (Mississippi State Univ.)

Since individual layers in a composite material return their own reflections, it is necessary for ultrasonic NDE to separate a received signal into a series of reflections. This becomes more complicated when the layers are thinner than the wavelength of the ultrasonic signal used for testing, resulting in overlapping reflections. The Gauss linearization minimization method was used to separate overlapping reflections [J. V. Beck and $\mathrm{K}$. J. Arnold, Parameter Estimation in Engineering and Science (Wiley, New York, 1977)]. This algorithm fits a pulse obtained from the pulser receiver to the measured data. Amplitude and time delay estimates are made for each reflection. Multilayer Plexiglas samples with subwavelength thick- 
nesses at 1 but not at $5 \mathrm{MHz}$ were fabricated to verify the performance of the algorithm. Each sample was tested at 1 and at 5 MHz. The algorithm provided curve fits for the $1-\mathrm{MHz}$ data that were almost identical to the theoretical results. The maximum error was $8 \%$ and the average error was $2 \%$. Each of the reflections predicted by the curve fits were identified and verified in the 5-MHz data. [Work supported by the Mississippi NSF EPSCoR grant.]

\section{2:00}

5pEA5. Remote sensing of sheet metal texture in aluminum alloys. Shermann Min, Lawrence Peng, Wei-yang Lu, and Darcy Hughes (Sandia Natl. Labs., Livermore, CA 94551)

Metal forming operations such as deep drawing, stretching, bending, hemming, etc. are all significantly influenced by the degrec of crystallographic anisotropy (texture) present within the workpiece. An effort is underway at Sandia National Laboratories/CA to develop two noncontact ultrasonic systems for the measurement of texture in aluminum sheets. Although the effect of stress wave velocities on elastic anisotropy is well known, its ability to predict plastic behavior has yet to be firmly established. Ultrasonic measurements, which hold the potential for remote, inprocess screening, will be shown to exhibit a high degree of correlation with current techniques for measuring texture, which are off-line, destructive, and often inaccurate. Results from an electromagnetic acoustic transducer (EMAT) system are used as a baseline comparison for a more promising noncontact lechnique involving the optical generation and detection of ultrasound, commonly referred to as laser ultrasonics (LU). In addition to displaying the level of anisotropy, slowness curves obtained from these systems suggest the ability to distinguish between different types of texture, as predicted from theory.

\section{2:15}

5pEA6. Techniques and instruments for investigating the ultrasonic analogs of magnetooptic effects. Vladimir V. Gudkov (Inst. for Metal Phys. of Russian Academy of Sci., 18 Kovalevskaya St., GSP-170, Ekaterinburg 620219, Russia)

Magnetoacoustic phenomena involving alteration of the polarization of a transverse wave (namely, the ultrasonic analogs of the Faraday and Cotton-Mouton effects) have been found in yttrium iron garnet [H. Matthews and R. C. LeCraw, Phys. Rev. Lett. 8, 397 (1962); B. Luthi, Phys. Lett. 3, 285 (1963)]. As the ellipticity $E$ and the angle $F$ of rotation of the polarization plane are rather large in magneto-ordered materials, it was possible to make the first measurements using distortion of the exponential decay of the ultrasonic echo pulses in the oscilloscope screen. After discovering these effects in metals, a precise technique for $E$ and $F$ evaluation has been required. A technique for measuring $F$ has been suggested by Boyd and Gavenda [Phys. Rev. 152, 645 (1966)]. It is based on measurements of amplitude versus magnetic field for two cases: field parallel and antiparallel to the wave vector. In the present paper both some original generalizations of this technique as well as a device which enables simultaneous measurements of $F$ and $E$ are reported. In addition, an instrument for visual observation of the ellipsoid of polarization and the direction of the major ellipsoid axis is described.

\section{2:30}

5pEA7. Optimum frequency range for partial discharge detection in high-voltage transformers using ultrasonic methods. Joseph Kurusingal (Ctr. for Mater. Technol., Univ. of Technol., P.O. Box 123, Broadway, NSW 2007, Australia)

Partial discharge (PD) erosion of insulation is one of the deteriorative mechanisms in transformers and high-voltage plants leading to early failure. Ultrasonic detection techniques are used regularly for location of PDs when electrical method [IEC Pub. 76/IEC 270] used in routine tests or DGA results indicate the presence of PD. The technique uses either externally or internally mounted ultrasonic transducers (J. Unsworth, J. Kurusingal, and R. E. James, Proceedings of ICPADM 94, IEEE). Unfortunately ultrasonic technique requires expert supervision to distinguish PD signal patterns from some typical transformer noise patterns. Moreover, transducers are selected based on commercial availability or for their ability to reject radio interference. A study was conducted to determine the optimum frequency range for PD detection to give maximum range sensitivity and acoustic interference rejection. This paper presents transformer noise recorded in the field as well as in the laboratory with externally and internally mounted transducers. The data records are Fourier analyzed and transducer response corrected to obtain the acoustic noise spectra. It is found that there is an upper cutoff frequency for transformer acoustic noise which is around $75 \mathrm{kHz}$ and signals originating from $\mathrm{PD}$ have frequency components much beyond this range. Factors which influence range sensitivity are also discussed.

\section{2:45}

5pEA8. A dual-transducer acoustic time domain correlation technique for instantaneous blood flow velocity measurement. E. J. Kim and S. W. Yoon (Dept. of Phys., Sung Kyun Kwan Univ., Suwon 440-746, Republic of Korea)

A dual-transducer acoustic time domain correlation technique has been developed to measure instantaneous blood flow speed and direction. The experiment was performed with an artificial blood flow system in a water tank. The volumetric flow velocity as well as the instantaneous flow velocity at any sites in an artificial blood vessel can be measured with the dual-transducer technique. In the steady flow measurements, the volumetric flow rates estimated from the dual-transducer technique were within the error range of $8 \%$ from those of the hydrodynamic flow measurements. Even in the turbulent flow measurements, the instantaneous flow speed and direction can be determined at any sites in the vessel with the dualtransducer acoustic time domain correlation technique.

\section{3:00}

5pEA9. Temperature variation due to ultrasonic absorption in a protein solution. D. O. Shin, E. J. Kim, and S. W. Yoon (Dept. of Phys., Sung Kyun Kwan Univ., Suwon 440-746, Republic of Korea)

The temperature variation due to ultrasonic absorption in a protein solution has been experimentally investigated. Egg white and $10.6 \%$ ovalbumin solution were used as the samples of protein solutions. For ultrasonic absorption the continuous sound waves of 3.5, 5.0, 7.5, and 10.0 $\mathrm{MHz}$ from focused transducers were incident upon the samples of finite volume, respectively. The temperature variation was measured at the focal region of an ultrasonic beam in the sample. The measurements showed that the temperature variation in the egg white was much larger than that in the $10.6 \%$ ovalbumin solution. This discrepancy might be due to the differences in protein composition.

\section{3:15}

5pEA10. Investigation of Lamb waves for sensor applications in semiconductor processing. F. Levent Degertekin, Victor Honein, and Butrus T. Khuri-Yakub (E. L. Ginzton Lab., Stanford Univ., Stanford, CA 94305)

Considering the semiconductor wafers as anisotropic plates, Lamb wave propagation characteristics are related to various parameters dynamically changing during processing. Particularly, the effect of wafer temperature and thin-film growth on phase velocity of zeroth order Lamb waves are analyzed theoretically. These modes are chosen to enable single mode operation in silicon wafers for frequency-thickness product $\left(f^{*} d\right)$ less than $3 \mathrm{MHz} \mathrm{mm}$. The surface impedance approach is used in theoretical modeling of propagation in general multilayered anisotropic solid structures. Temperature, thin film, and their combined sensitivity figures are calculated for commonly used structures in semiconductor processing. The results are interpreted to obtain optimum ultrasonic sensor system parameters for certain applications. For example, in case of aluminum films on (100) silicon, antisymmetric mode in $\langle 100\rangle$ direction shows negligible sensitivity to thin films around $f^{*} d=0.8 \mathrm{MHz} \mathrm{mm}$. The same figure for silicon oxide film is $1.57 \mathrm{MHz} \mathrm{mm}$. In a combined measurement, a temperature sensor using these system parameters to measure phase velocity will be minimally affected by thin films, enabling an isolated temperature measurement. Practical implementations of the results in rapid thermal processing environment are also discussed in the paper. [Work supported by SRC.] 


\title{
Speech Communication: Voice Quality, Speech Training, Phonetics, and Prosody (Poster Session)
}

\author{
Randy L. Diehl, Chair \\ Department of Psychology, University of Texas at Austin, 300 Mezes, Austin, Texas 78712
}

\section{Contributed Papers}

To allow extended viewing time, all posters will be on display from 8:00 a.m. to 5:00 p.m. The session will be held from 1:00 to 5:00 p.m. To allow contributors an opportunity to see other posters, contributors of odd-numbered papers will be at their posters from 1:00 to 3:00 p.m., and contributors of even-numbered papers will be at their posters from 3:00 to 5:00 p.m.

5pSP1. The dimensional structure of the lexicon. Lee H. Wurm and Douglas A. Vakoch (Dept. of Psychol., State Univ. of New York at Stony Brook, Stony Brook, NY 11794-2500)

Past research indicates that the lexical access of emotion words is influenced by the dimensions of evaluation and activity. Increased reaction time to a lexical decision task using emotion words and nonwords was predicted by low loadings on evaluation and activity as determined by ratings on semantic differential seales [L. H. Wurm and D. A. Vakoch, J. Acoust. Soc. Am. 95, 2974 (1994)]. The current study examines the extent to which evaluation, activity, and potency influence lexical access of words more representative of the entire lexicon [C. E. Osgood, J. Pers. Soc. Psychol. 12, 194-199 (1969)]. Implications for a dimensional model of the structure of the lexicon are discussed.

5pSP2. Teaching phonetics to undergraduates at the University of Washington. Sharon Hargus (Dept. of Linguist., GN-40 Padelford Hall, Univ. of Washington, Seattle, WA 98195) and Katharine Davis (Univ. of Washington, Seattle, WA 98195)

In 1993, the Instrumentation and Laboratory Improvement Program of the National Science Foundation awarded $\$ 23,960$ to the University of Washington (who provided matching funds) for phonetics facilities for undergraduate education. In accordance with NSF guidelines, a summary of the progress to date is presented here. Funds were used to purchase a DOS machine and a Macintosh, which run software including programs for signal processing, speech synthesis, statistics, word processing, and graphics. Also purchased were analog and digital tape recorders, a mixer, microphones, and other speech analysis equipment including a nasometer and an airflow meter. A sound-treated booth in which to do recordings and perception experiments has been built. This laboratory has enabled us to offer a comprehensive course in Linguistic Phonetics at the undergraduate level, and has allowed us to supplement the offerings of other courses. For example, undergraduates in the Dept. of Asian Studies learned about pronunciation in Japanese, students in the English Phonology and Morphology course learned about individual differences in the implementation of phonology, and students in the Language Acquisition course learned about the phonetic characteristics of "motherese." There are plans for additional equipment which will enable us to teach students about speech perception as well.

5pSP3. Intelligibility training for foreign-accented speech: A preliminary study. Catherine L. Rogers (Commun. Disord. Technol., Inc., 205 S. Walnut St., Bloomington, IN 47404 and Indiana Univ.), Jonathan M. Dalby (Commun. Disord. Technol., Inc.), and Gladys DeVane (Commun. Disord. Technol., Inc. and Indiana Univ.)

A computer-based speech training system, the Indiana Speech Training Aid (ISTRA), has been shown to be clinically effective for improving the speech of hearing-impaired and phonologically disordered individuals [Kewley-Port et al., Clin. Ling. Phon. 5, 13-38 (1991)]. The potential value of speech recognition technology for the training of foreign-accented speech, using an overall measure of speech quality as feedback, was assessed. Phonological errors in English spoken by two native Mandarin speakers were analyzed and several training targets selected. One consonant and one vowel contrast each were selected for training using ISTRA, and by a speech-language pathologist (SLP). Pre- and posttraining tokens were rated for quality by a listener jury. Results showed significant improvement for both the ISTRA-trained consonant $/ /$, and the SLP-trained consonant $/ \theta /$. However, only one of the two vowel contrasts for each speaker improved significantly. ISTRA-trained sounds which showed significant improvement did so for both trained and untrained words. Two untrained phonemes, $/ \mathrm{r} /$ and $/ \mathrm{U} /$, also improved significantly, possibly indicating a generalization of improved sounds to other phonological distinctions in English. These results are promising as regards the usefulness of speech recognition technology for pronunciation training. [Work supported by NIHDCD SBIR Program.]

5pSP4. Training effects for identification of synthetic speech tokens for listeners with normal and impaired hearing. Amy R. Horwitz and Christopher W. Turner (Dept. of Commun. Sci. and Disord., $805 \mathrm{~S}$. Crouse Ave., Syracuse, NY 13244-2280)

Training effects for identification of speechlike stimuli having frequency, amplitude, and temporal characteristics based upon natural consonant-vowel (CV) speech tokens were measured. Two types of dynamic speech stimuli were synthesized. Specifically, in the first type formants were represented by pure tones and in the second by sweeping three "formant" filters across a voicing-source spectrum, to more closely approximate natural speech. Following a brief familiarization period, normalhearing and hearing-impaired subjects listened to and identified six CV syllables in a closed-set format. The order of presentation for the type of synthesized speech tokens was counterbalanced so that initially half of the subjects were tested with the pure-tone speech syllables while the other half were tested with the broader "voiced format" speech. Data were collected over the course of several months. Substantial training effects were observed for both types of speech stimuli, with all subjects ultimately achieving similar identification scores for both types of syllables. [Work supported by NIDCD].

5pSP5. Vocal correlates of interpersonal issues. Douglas A. Vakoch, Dean A. Pollina, and Lee H. Wurm (Dept. of Psychol., State Univ. of New York at Stony Brook, Stony Brook, NY 11794-2500)

Previous studies have related different interpersonal styles to the fundamental frequency $(F 0)$ of subjects' speech. For example, high ratings of pitch have been associated with sclf-attributions of dominance and affiliation [K. R. Scherer, J. Psychol. Res. 3, 281-298 (1974)], which are two dimensions along which interpersonal issues have been defined [L. E. Alden, J. S. Wiggins, and A. L. Pincus, J. Pers. Assessment 55, 521-536 (1990); L. S. Benjamin, Psychol. Rev. 81, 392-425 (1974)]. Similarly, subjects with greater pitch variability have been judged to be more benevolent [G. B. Ray, Commun. Monographs 53, 266-276 (1986)]. Previous studies, however, have failed to focus on the problematic aspects of interpersonal functioning. The current study reports on the acoustical correlates of six types of interpersonal problems: (1) excessive concern about the evaluations of others, (2) impact of past abuse, (3) dissatisfaction with therapist, (4) excessive responsibility for partner, (5) fear of abandonment, 
and (6) ineffective interpersonal responses. For females only, a higher mean $F 0$ was predictive of both a greater sense of excessive responsibility for partner $(p<0.05)$ and an increased fear of abandonment $(p<0.05)$.

5pSP6. Neuroticism and openness are predicted by fundamental frequency of speech. Douglas A. Vakoch, Dean A. Pollina, and Lee H. Wurm (Dept. of Psychol., State Univ. of New York at Stony Brook, Stony Brook, NY 11794-2500)

The fundamental frequency $(F 0)$ of speech has been used for examining both transient emotional states [D. A. Vakoch, J. Acoust. Soc. Am. 95, 2974 (1994)] and more stable personality traits. For example, low perceived pitch has been associated with attributions of high emotional stability [K. R. Scherer, Eur. J. Soc. Psychol. 8, 467-487 (1978)]. In the current study, characteristics derived from $F 0$ were related to five personality traits as measured by the NEO-PI: neuroticism, extroversion, openness, agreeableness, and conscientiousness. A high degree of openness was predicted by a high mean $F 0$ of an utterance $(p<0.025)$ and by a great variability in $F 0$ within an utterance $(p<0.004)$. The best predictor of neuroticism was peak $F 0$ within an utterance, with subjects scoring high on this scale having a higher maximum $F 0(p<0.021)$. The latter finding, in conjunction with previous research on attributions of emotional stability, suggests a convergence between objective acoustical measures and subjective perceptions of personality.

5pSP7. Allophonic variation in spoken and sung speech. Ray Daniloff, Kathy Wolf, George Larsen (Dept. of Speech and Hear. Sci., Univ. of North Texas, P.O. Box 5008, Denton, TX 76203-0008), and Lee Evans (Univ. of North Texas, Denton, TX 76203-0008)

Research suggests that sung and spoken speech differ systematically in terms of laryngeal, intonational, rhythmic, and articulatory performance. In the present study we examined control of pronunciation rules [Ladefoged, A Course in Phonetics (HBJ, 1993)] in singers. Three doctoral students of vocal performance sang and then spoke a set of sentences. Simple melodies were composed to fit the rhythmic figure of each sentence. The sentences contained five examples of each of 24 pronunciation rules of English. Recordings were transcribed, rhythm and stress patterns noted, and acoustic analysis was performed (GW Soundscope) to ascertain the degree to which each pronunciation rule was/was not implemented in sung versus spoken production. Results indicate that singing induces both systematic and nonsystematic changes in pronunciation across singers which can be related to the more stereotyped production strategies associated with singing.

5pSP8. Effects of speaking rate, focal stress, and distinctive vowel length on syllable-internal timing in Norwegian. Dawn M. Behne and Bente Moxness (Linguist. Inst., Univ. of Trondheim, N-7055 Dragvoll, Norway)

The relative timing of consonants and vowels of a syllable can be affected by factors such as speaking rate, focal stress, and distinctive vowel length. Speaking rate and focal stress tend to have a global affect on syllable-internal timing, whereas the effect of distinctive vowel length in Norwegian is generally believed to be localized to the syllable rhyme. When these factors converge on a syllable, the syllable-internal timing simultaneously reflects their concurrent effects. The goal of the present study is to examine and characterize the concurrent effects of speaking rate, focal stress, and distinctive vowel length on syllable-internal timing of C1VC2 components. Conversations were developed in which 12 Norwegian CVCs containing /i, o, a, i:, o:, a:/ were either focused or nonfocused by the discourse. The set of conversations was produced at three speaking rates by native speakers of Norwegian. Results indicate that speaking rate and focal stress have an overall affect on the timing of components in the syllable. In addition, the effects of distinctive vowel length and focal stress on components of the syllable rhyme were found to be interdependent across speaking rates. These findings are discussed in terms of syllable- and rhyme-internal timing.

5pSP9. Forward and backward presentation of Chinese and English speech in the verbal transformation efrect. Magdalene H. Chalikia, Rachel Waldstein, and William J. Stech (Dept. of Psychol., Moorhead State Univ., Moorhead, MN 56563)
When hearing a word which is continuously repeated, listeners report that the word seems to change into different forms and then vacillates among these forms. This auditory illusion has been called the verbal transformation effect [R. M. Warren, Br. J. Psychol. 17, 249-258 (1961)] and is considered to be caused by the lack of verbal context, produced by the repetition. This study further investigated the role of context in the interpretation of auditory stimulation. Twenty-six listeners heard the individual, continuous, repetition of six stimuli: an English sentence, an English word, and a Chinese word (each played forward and backward). It was assumed that listeners will report verbal transformations with all stimuli except the English sentence, since only this condition contained sufficient contextual cues. Results showed that both forms of the English sentence were treated in the same manner, and that significantly more verbal transformations were reported with the words.

5pSP10. Control experiments on the frequency scale of speech intonation. Dik J. Hermes and H. H. Rump (Inst. for Percept. Res. IPO, P.O. Box 513, $5600 \mathrm{MB}$ Eindhoven, The Netherlands)

According to Hermes and Van Gestel [J. Acoust. Soc. Am. 90, 97-102 (1991)], prominences of accent-lending pitch movements in different registers are equal when their excursion sizes are equal on the ERB-rate scale. A reanalysis of their experiments showed that an octave relationship between the high pitch levels in the different registers may have been crucial. In order to test whether this factor influenced the outcome of their experiments, adjustment experiments were performed with stimuli in different low and high registers. The results, supporting the ERB-rate scale hypothesis of Hermes and Van Gestel, showed that the octave relationship had no systematic influence on the results. In an additional experiment, stimuli were resynthesized from a male and a female voice in order to test the effect of the speaker's natural pitch range. The male and female stimuli gave about the same results. In sum, the effect of pitch excursion on prominence is very robust. An unexpected finding was that, on average, the excursions of the pitch movements in the high register were about $0.15 \mathrm{E}$ (0.5-1 semitone) larger than those of the pitch movements in the low register when lending equal prominence.

5pSP11. Some control experiments on a model for prominence perception. H. H. Rump and Dik J. Hermes (Inst. for Percept. Res. IPO, P.O. Box 513, 5600 MB Eindhoven, The Netherlands)

According to the model proposed by Hermes and Rump [J. Acoust. Soc. Am. (in press)] the prominence lent by accent-lending pitch movements is proportional to the pitch-level difference between the vowel nucleus of the accented syllable and the vowel nucleus of the preceding syllable. The pitch level on the lower declination line or baseline and the pitch level on the upper declination line or topline are thus regarded as equally important. By varying the height of the pitch levels on the baselines this assumption was tested against the alternative assumption that listeners equated pitch levels on the topline of each of the stimuli when adjusting prominences to be equal. Results showed that pitch levels on the toplines were adjusted to be more or less equal, but that the pitch-level difference between the low and the high pitch level influenced the adjusted height of the high pitch level, too. It is concluded that the proposed model makes claims about the relevance of the position of the low pitch level which is too strong. The role of pitch levels in relation to the perception of prominence will be discussed.

5pSP12. Prosodic characteristics of register variation in the read speech of adult native speakers of French. Andrea G. Levitt and Jeannine Geoffrion (Wellesley College, 106 Central St., Wellesley, MA 02181-8289)

Ten female native speakers of French were asked to read two paragraphs in each of three register styles: to a friend (native), to a nonnative speaker (foreigner talk), and to a 1-year-old child (child-directed speech). There were significant differences in the speech rate, number and length of pauses, and mean $F 0$ and $F 0$ range among the three speech styles. Speech rate was significantly different for each of the registers, with foreigner talk the slowest, native speech the fastest, and child-directed speech in between the other two. A similar pattern of significant differences emerged for pause length and number of pauses, again with foreigner talk showing the most pauses and the longest average pause length, native speech showing 
the fewest and shortest pauses, and child-directed speech in between the other two. Mean $F 0$ was significantly higher for child-directed speech than for the other two registers, which did not differ from one another. The three registers did differ significantly from one another in terms of $F 0$ range, with child-directed speech showing the greatest $F 0$ range, native speech the smallest, and foreigner talk in between the other two. [Work supported by NIH Grant No. 1 R15 HD28173-01.]

5pSP13. Prosodic cues of repetitions in Spanish spontaneous discourse. Pilar Munday (Inst. for Speech and Lang. Sci., NYU, 719 Broadway, 5th fl., New York, NY 10003)

When Spanish speakers repeat words or phrases in discourse, some repetitions are due to false starts or hesitations while others are used for emphasis or clarity. These will be referred to as "hesitations" and "emphasis" repetitions, respectively. Although the purpose of the repetition can often be determined from discourse context or part of speech, this study shows that there are also prosodic cues that serve to distinguish the two types. The speech data consist of all repetitions uttered by a male Colombian speaker over the course of a 1-h spontaneous conversations. The following acoustic information was obtained for each utterance and its repetition: duration, peak amplitude, average amplitude, and duration of any intervening pause. Pauses occur more often and tend to be longer in hesitation repetitions. Contrary to previously reported findings for English, however, a pause is frequently not present. The first element of the hesitation repetition tends to be longer than the second. Amplitude does not appear to differentiate the two types of repetitions. The results suggest that durations is the most important cue for distinguishing between types of repetitions.

5pSP14. Perceptual centers as an index of speech rhythm. Charles Andrew Harsín and Kerry P. Green (Natl. Ctr. for Neurogenic Commun. Disord., Univ. of Arizona, Tucson, AZ 85721)

One of the obstacles to investigating speech rhythm has been the difficulty in locating the syllabic beat. This study attempts to address that difficulty by using perceptual centers (p-centers) as an index of speech rhythm. P-centers of syllables extracted from natural utterances were determined both by listeners using a method of adjustment procedure and by an acoustic p-center model. The phonetic structure (syllabic onset) and stress patterns of the syllables in the utterances were varied, and the effects of these manipulations on the utterances' rhythms were investigated. As expected, the p-centers of the syllables varied systematically with their own phonetic structure. Preliminary findings indicate that the p-center of the syllables also changes along with the p-center of the previous or subsequent syllable so as to maintain a relatively constant interval between the two p-centers. The study also examines the effects on rhythm of altering stress patterns, by determining whether unstressed syllables might influence p-centers. The results will be discussed in terms of dynamic constraints which might affect speech production. [Work supported in part by National Multipurpose Research and Training Center Grant No. P60 DC01409 from the National Institute on Deafness and Other Communication Disorders.]

5pSP15. Perceptual centers are affected by stress location in English disyllables. Alan Bell and Debra Halperin Biasca (Dept. of Linguist., Box 295, Univ. of Colorado, Boulder, CO 80309)

The perceplual centers of English disyllables were investigated in two experiments. Six subjects participated in the first experiment, and five in the second. The first experiment compared initially stressed disyllables with five onsets whose duration varied from 4 (batter) to $180 \mathrm{~ms}$ (fatter). Its results demonstrated that inilially stressed disyllables with longer onsets have perceptual centers that are displaced relatively further from the acoustic beginning of the word by about the same amount as the difference in the onset durations, in agreement with earlier research on English monosyllables [e.g., A. Cooper, D. Whalen, and C. Fowler, Percept. Psychophys. 39, 187-196 (1986)]. The second experiment compared initially stressed disyllables with disyllables composed of a reduced first syllable and a stressed second syllable, e.g., com'mute vs 'comet, holding the total duration of all items constant. Again, longer onsets produced later perceptual centers for both stress locations, but the perceptual centers of finally stressed disyllables were later than those of initially stressed ones by about
$50 \mathrm{~ms}$, or roughly half the duration from the beginning of the unstressed vowel to the beginning of the stressed vowel.

5pSP16. Perceptual centers in Japanese disyllables. Alan Bell (Dept. of Linguist., Box 295, Univ. of Colorado, Boulder, CO 80309) and Yasunori Morishima (Dept. of Psychol., Box 345, Univ. of Colorado, Boulder, CO 80309)

Most research on perceptual centers has been based on monosyllables in languages with stress; indeed the term "stress beat" is sometimes used for the same phenomenon. Accent in Japanese is mainly realized by pitch; amplitude and duration are relatively unimportant. Perceptual centers in disyllabic words perceived by four Japanese were used to investigate the characteristics of perceptual centers in the context of pitch accent. Perceptual centers occur later in disyllables with longer consonant onsets, by a magnitude comparable to that found for stressed monosyllables [e.g., Cooper et al., Percept. Psychophys. 39, 187-196 (1986)] and for Japanese monosyllables [Hoequist, Lang. Speech 26, 367-376 (1983)]. The effect of lengthening the tail (the portion following the initial consonant) of disyllables also corresponded in magnitude to that found previously. Accent placement had little or no effect. The same effect of onset duration was found for initially accented and finally accented disyllables whose duration and amplitude contour were held constant. A small accent difference with respect to the effect of tail duration was found in words of the same duration but retaining differences in amplitude contour. The results are consonant with the greater prominence effects found for amplitude and duration than for pitch in rhythmic perception.

5pSP17. Spectral analysis of amplitude envelopes of bandpass filtered speech. King-Leung Kong (Dept. of Psychol., Univ. of Hong Kong, Hong Kong)

The amplitude envelopes of rectified bandpass filtered speech have been found to provide useful cues for speech perception [K. W. Grant, L. D. Braida, and R. J. Renn, J. Acoust. Soc. Am. 95, 1065-1073 (1994)]. An analog terminal was built to yield 25 such envelopes from filters with center (carrier) frequencies from 150 to $4850 \mathrm{~Hz}$. Each envelope was then subjected to another round of bandpass filtering and rectification to yield a modulation spectrum of up to nine channels with center (modulation) frequencies from half the carrier frequency to $700 \mathrm{~Hz}$. The spectra were examined for cues for the identification of voicing, fundamental frequency, and consonants. Voicing was generally characterized by the concentration of formant energy at a single carrier and modulation frequency, corresponding to the formant and fundamental frequencies, respectively. The second formant of the front vowel $/ \mathrm{i} /$ and nasal release sometimes exhibited bimodal modulation spectra, suggesting multiple sources of modulation. Stop consonants and fricatives were characterized by elements scattered at high carrier and modulation frequencies whose occurrences might not coincide. Some consonants could be identified with elements at specific modulation frequencies: e.g., /g/ and /j/ suggested a 700-Hz source modulating carriers whose frequencies depended on the following vowel.

5pSP18. Prediction of speech transmission quality of wideband and narrow-band telephone handsets using short-time sub-band analysis and psychoacoustic models. Winfried Krebber (Inst. f. El. Nachrichtentechnik, Aachen Univ. of Technol. (RWTH), D-52056 Aachen Germany)

A predictor is presented which estimates the mean opinion score (MOS) for a given speech sample from speech and noise transfer characteristics of a specific handset applied to an artificial ear. The critical band rate excitation pattern is computed in 50-ms blocks for original and distorted speech signals and additive room noise. For each block three psychoacoustic parameters are computed: An intelligibility index $(I)$ is evaluated using SNR analysis in each sub-band and considering simultaneous masking effects. Naturalness $(N)$ is estimated by spectral distance between original and distorted speech. A loudness index $(L)$ is derived from loudness (computed similar to ISO532) using a trapezoid function: $L$ decreases if the loudness which is exceeded in $10 \%$ of time is lower than 15 sone or higher than 45 sone. The MOS is predicted as a weighted sum of $I, N$, and $L$. The prediction results were verified by an opinion test including totally 442 speech samples of several talkers which were filtered simulating typical transfer characteristics of handsets and presented in a noisy environ- 
ment. Speech level and SNR were varied in a wide range. The comparison proves a good correlation ( $p=0.93$ for all samples and $p=0.96$ for speech levels below $76 \mathrm{~dB}$ SPL).

5pSP19. Cepstrogram and its application to speech analysis Chiwei Che and Qiguang Lin (CAIP Ctr., Rutgers Univ., Piscataway, NJ 08855-1390)

Cepstrum coefficients (including their different variants and/or derivatives) are nowadays commonly utilized in automatic speech recognition systems. Most adaptation algorithms for robust speech recognition also make use of cepstrum coefficients. It is therefore important to have a tool which can efficiently calculate and display cepstra. A computer program has been recently developed to display the cepstrogram of speech signals. The program has the following features: (1) It computes both linear prediction based and FFT based cepstra; (2) it displays cepstrum coefficients and their derivatives as a function of time and cepstrum index, as well as cepstrum coefficients at a fixed frame (section displays); (3) it generates synthesized spectra from cepstrum coefficients. In addition, the program can be used to segment and playback speech waveforms. The program has been utilized to study variations in cepstrum coefficients caused by speaker variability and channel differences. The tool is a useful supplement to conventional spectrograms for speech analysis. (The program is coded in $C$ and runs in the $\mathrm{X}$-window environment. It is available upon request.)

5pSP20. The PEACC method of characterization of dynamic aspects of speech. Jim Talley (Dept. of Linguist., 501 Calhoun Hall, Univ. of Texas at Austin, Austin, TX 78712)

In the phonetics/speech perception community, the assertion that $\mathrm{dy}$ namic aspects of the speech signal are employed in robust speech decoding is not particularly controversial. Though an increasing number of studies are addressing the various dynamic cues of speech, quantitative, analytical research is hampered somewhat by a lack of established methods. Methods for studying dynamic phenomena are much less well developed than those for more static properties. This paper proposes a new method for characterizing dynamic aspects of speech, the Piecewise Exponential Approximation with Continuity Constraints (PEACC) method, to help remedy this state of affairs. As its name suggests, PEACC performs piecewise fitting of exponential segments- $\gamma e^{\alpha x}+\beta,-\infty<x \leqslant 0-$ to the sampled signal. Dynamic programming is utilized in global sequence optimization where MSE is minimized within the solution space permitted by the constraints on continuity. This method has broad applicability and produces lowdistortion fits at a specifiable level of detail; however, its principle strength, from the perspective of speech science research, is that its resulting signal transition parameters have direct, intuitive interpretations. The paper concludes with a brief examination of the results of applying PEACC to a corpus of formant track data. [Work supported by NSF.]

5pSP21. Analysis of quality factors in synthetic speech produced by rules. Eri Miyazawa and Hiromi Nagabuchi (NTT Telecommun. Networks Labs., Midori-cho, Musashino-city, Tokyo, 180 Japan)

This paper investigates how various factors affect the quality of synthetic speech produced by rules. Using rules to synthesize speech will be an important techrique for providing various telecommunication services in future intelligent networks. The quality of synthetic speech is generally measured by subjectively evaluating the speech from the viewpoint of intelligibility, or by comparing it with the quality of other types of synthetic speech. However, the development of a practical speech synthesis method for use in telecommunication networks requires an overall quality evaluation, including intelligibility and naturalness. The quality should be compared with that of natural telephone speech. To establish an overall quality evaluation method, the effects of several factors on the overall quality (expressed by MOS) of speech synthesized by several Japanese text-to-speech systems are quantitatively compared with the effects of using additive speech-correlated white noise as a natural speech material. Experimental results show that such factors as subject, listening experience, average pitch frequency, and text affect synthetic speech more than natural speech. Quality evaluation characteristics due to these factors are discussed and an overall quality evaluation method for synthetic speech is proposed.
5pSP22. Possible usages of LPC error for the estimation of voice characteristics. Sungbok Lee (Central Inst. for the Deaf, $818 \mathrm{~S}$. Euclid, St. Louis, MO 63110)

Using the autocorrelation method, LPC error signals were computed as differences between the original waves and the predicted waves at each time instant. Analyses of synthesized and human vowel sounds show that the shapes and spectral contents of the instantaneous error signals are almost identical with those of the voice source for synthesized vowel sounds, and that the instants of glottal closure are well defined as maximally negative peaks in the error signals for both kinds of vowel sounds. Time integration of error signals was also performed. For male voices the shapes of integrated and instantaneous error signals are found to be very similar with those of EGG and differentiated EGG signals, respectively. This result supports the possibility that open and closed phases of the vocal folds within each glottal cycle can be determined from such LPC error signals. It was also found that automatic detection of valleys (or peaks) in the integrated error signal may be a robust pitch estimation method for voiced speech segments including synthetic diplophonic sounds. These and other results suggest that these simple LPC error analyses may provide significant information about the voice source.

5pSP23. Speech stress classification and feature analysis using a neural network classifier. Brian D. Womack and John H. L. Hansen (Robust Speech Processing Lab., Dept. of Electrical Eng., Duke Univ., Box 90291, Durham, NC 27708-0291)

Variability in speech production due to task induced stress contributes significantly to loss in speech processing performance. A new neural network algorithm is formulated which classifies speech under stress using the SUSAS stress database. Five spectral parameter representations (Mel, $\Delta$-Mel, $\Delta^{2}$-Mel, Auto-Corr-Mel, and Cross-Corr-Mel cepstral) are considered as potential stressed speech relayers. Eleven stress conditions are considered which include Angry, Clear, Fast, Lombard, Loud, Normal, Question, Slow, and Soft speech. Stressed speech classification using these features is evaluated with respect to (i) pairwise class separability, (ii) an objective measure of pairwise and global feature separability, and (iii) analysis of articulatory vocal tract area functions. Perturbations in speech production under stress are reflected to varying degrees in the five speech feature representations. A neural network classifier over phoneme partitions can achieve good speaker dependent classification performance (80.6\%) when stress conditions are combined into six groups of related stress domains. The implication of reliable stress classification will be discussed for applications such as monitoring speaker state, improving naturalness of speech coding, and increasing robustness of speech recognizers in adverse conditions.

5pSP24. Many-valued logic is reflected in formant location and spectral composition. Dean A. Pollina, Douglas A. Vakoch, and Lee H. Wurm (Dept. of Psychol., State Univ. of New York at Stony Brook, Stony Brook, NY 11794-2500)

Variation in the truth value of propositions has been shown to yield changes in cognitive event-related potentials (ERPs) [D. A. Pollina and N. K. Squires, Psychophys. 30, S52 (1993)]. Given the connection between the arousal of the central nervous system and peripheral systems, the same paradigm that was used to detect many-valued beliefs via ERPs was used to detect degree of belief by an acoustical analysis of the voice. Degree of belief was manipulated by having subjects solve a murder mystery, after which they learned facts associated with three people: (1) the person they most suspected of committing the murder, (2) the person they judged next most likely to be the murderer, and (3) the person they knew could not be the murderer. Articulatory precision of subjects' utterances was measured by formant location and spectral composition [F. J. Tolkmitt and K. R. Scherer, J. Exp. Psychol. Human Percept. Perform. 12, 302-313 (1986)]. These measures were related to the degree to which subjects believed each statement to be true.

5pSP25. Lie detection based on phonatory processes. Dean $A$ Pollina, Douglas A. Vakoch, and Lee H. Wurm (Dept. of Psychol., State Univ. of New York at Stony Brook, Stony Brook, NY 11794-2500)

Deception has been shown to be associated with changes in the fundamental frequency $(F 0)$ of vocalizations. For example, during attempted 
deception, $F 0$ typically increases [P. Ekman, W. V. Friesen, and K. R. Scherer, Semiotica 16, 23-27 (1976); L. A. Streeter, R. M. Kraus, V. Geller, C. Olson, and W. Apple, J. Pers. Soc. Psychol. 35, 345-350 (1977)]. The current study examines changes in $F 0$ for the identical utter- ances at two points in time: prior to establishing any belief about the statement, and after having a belief. By analyzing the same phrase at baseline and after the belief manipulation, more precise measurements corresponding to belief can be made than in previous studies.

FRIDAY AFTERNOON, 2 DECEMBER 1994

BALLROOM B, 1:15 TO 5:00 P.M.

\title{
Session 5pUW
}

\section{Underwater Acoustics: Propagation II}

\author{
Henrik Schmidt, Chair \\ Department of Ocean Engineering, Massachusetts Institute of Technology, Cambridge, Massachusetts 02139
}

Contributed Papers

1:15

5pUW1. Single-mode excitation in a laboratory waveguide using feedback control. John R. Buck (Dept. of Appl. Ocean Phys. and Eng., Woods Hole Oceanogr. Inst., Woods Hole, MA 02543 and Res. Lab. of Electron., MIT, 77 Massachusetts Ave., Cambridge, MA 02139-4307), Mark Johnson, James C. Preisig, and Josko Catipovic (Woods Hole Oceanogr. Inst., Woods Hole, MA 02543)

Previous work formulated an adaptive algorithm for exciting a single mode in a shallow-water waveguide using feedback control [Buck $e$ t al., J. Acoust. Soc. Am. 95, 2927(A) (1994)]. This paper presents results obtained using this algorithm in a laboratory waveguide experiment under a variety of conditions. Unlike previous laboratory single-mode excitation experiments [Clay and Huang, J. Acoust. Soc. Am. 67, 792-794 (1980); Gazanhes and Garnier, ibid. 69, 963-969 (1981)], our algorithm does not require a priori environmental knowledge, but only the sound-speed profile at the feedback array. The experiment results to be presented demonstrate the algorithm's convergence behavior and robustness to environmental mismatch. If time permits, present experimental results illustrating the ability of the algorithm to track time variations in the channel will also be presented. [Work supported by ONR and ARPA.]

\section{$1: 30$}

5pUW2. Advances in underwater acoustic normal-mode computations. Scott J. Levinson, Carol V. Sheppard, and Stephen K. Mitchell (Appl. Res. Labs., Univ. of Texas at Austin, Austin, TX 78713)

Recently, an efficient, numerically robust algorithm for calculating acoustic normal modes was shown to have more than an order of a magnitude improvement in speed while maintaining high accuracy over other algorithms (submitted to JASA by the authors, June '94). A limitation remains, however, in its dependence on presampled wave numbers in the search for the roots of the characteristic equation (eigenvalues). Since coarse wave-number presampling may result in some eigenvalues remaining undetected, the presampling part of this code is the most time consuming portion-particularly for multichannel environments. We introduce a new approach which overcomes this problem. Analytic derivatives (up to sixth order) are calculated to locally approximate the numerically robust characteristic equation with Pade' approximants when finding successive eigenvalues. The requirement of wave-number presampling is thereby avoided and eigenvalue root finding is achieved with a superlinear convergence property. Its speed and accuracy will be compared with previous methods for single and multichannel ocean acoustic environments. [Work supported by the Advanced Surveillance Acoustic Prediction System (ASAPS) Development for IUSS Program of the Space and Naval Warfare Systems Command (SPAWAR, PMW 181-1).]
$1: 45$

5pUW3. An efficient broadband normal-mode model for acoustoelastic ocean environments. Evan K. Westwood (Appl. Res. Labs., P.O. Box 8029, Univ. of Texas at Austin, Austin, TX 78713-8029)

A recently developed normal-mode model [E. K. Westwood et al., J. Acoust. Soc. Am. 95, 2908(A) (1994)] for acoustoelastic ocean environments has been extended to perform broadband calculations. The set of complex mode eigenvalues at the highest frequency of interest is first found. Then each eigenvalue $k_{n}$ is tracked as a function of frequency down to the lowest frequency of interest. Analytic derivatives with respect to frequency $d k_{n} / d \omega$ are used to extrapolate in frequency, and step sizes are adjusted adaptively. When the step size exceeds the FFT bin width, linear interpolation of eigenvalues may be used. Examples of broadband calculations are given. [Work supported by the ARL Internal Research and Development Program.]

\section{2:00}

5pUW4. A method for including lateral waves in normal-mode calculations. Evan K. Westwood (Appl. Res. Labs., P.O. Box 8029, Univ. of Texas at Austin, Austin, TX 78713-8029)

A method for including the lateral (or head) wave in a normal-mode propagation model [E. K. Westwood et al., J. Acoust. Soc. Am. 95, 2908(A) (1994)] for acoustoelastic ocean environments has been developed. The method consists of replacing the original lower half-space with a thick layer having a constant sound speed but a small gradient in atrenuation. Below the attenuating layer, the new lower half-space is given parameters such that there is no mismatch at the half-space interface. As a result, (1) the branch point corresponding to the critical angle in the halfspace is moved upward in the complex $k$ plane (where $k$ is horizontal wave number), (2) the Pekeris branch line integral is also moved upward such that it becomes negligible, and (3) modes appear in the complex $k$ plane between the old and new branch points. The cost of introducing the thick layer is not great because Airy function solutions are used in each layer. Removal of the Pekeris branch line integral, in conjunction with including the leaky modes, makes the mode solution represent the complete acoustic field. [Work supported by the ARL Internal Research and Development Program.]

\section{2:15}

5pUW5. A study of the influence of an offshore rise on low-frequency modal propagation. Thomas $N$. Lawrence, Wade Trappe, and Nancy R. Bedford (Appl. Res. Labs., Univ. of Texas at Austin, P.O. Box 8029, Austin, TX 78713)

Simple up slope propagation of underwater acoustic energy can usually be modeled by an adiabatic normal-mode approximation, provided the 
slope is not too severe. Bottom interacting paths in such an environment are usually sufficiently attenuated to make consideration of mode coupling unnecessary. An environment further complicated by an offshore rise can cause additional acoustic energy to be introduced into lower-order modes due to bottom interaction. These low-order modes will then propagate in deep water until encountering the continental shelf. Experimental results from the Arctic Ocean between 25 and $45 \mathrm{~Hz}$ suggest such propagation conditions. These results, and their interpretation in the context of a coupled mode study will be presented. Emphasis will be placed on the conditions under which mode coupling will occur, and when it must be considered in prediction and localization problems. The coupled mode model, COUPLE [R. B. Evans, J. Acoust. Soc. Am. 74, 188-195 (1983)], is used as the vehicle for this study. Additional approximation schemes of obtaining a coupled mode result will be explored with the objective of obtaining a faster numerical solution. Comparisons will be made with adiabatic normal mode, and parabolic equations models.

\section{2:30}

5pUW6. Trans-Arctic propagation effects of changes in ice morphology. Henrik Schmidt and Brian Tracey (Dept. of Ocean Eng., MIT, Cambridge, MA 02139)

The objective of the Trans-Arctic Propagation (TAP) experiment carried out in the spring of 1994 was to investigate the feasibility of using acoustic thermometry for inferring changes in the Arctic climate [P. Mikhalevsky, J. Acoust. Soc. Am. 94, 1760(A) (1993)]. An important issue is which acoustic property will provide the strongest climate change signature. The sensitivity of various properties of the acoustic field to changes in ice volume was investigated theoretically. A perturbation formulation for 3-D scattering from rough ice has been combined with the KRAKEN normalmode code to provide a model of the second-order statistics of the acoustic field. Such a hybrid model has been shown to provide excellent agreement with historical Arctic transmission loss data [LePage and Schmidt, J. Acoust. Soc. Am. 96, 1783-1795 (1994)]. We use this model to investigate the sensitivity of deterministic properties of the coherent field, such as modal loss, and modal phase and group velocity, to change in ice thickness and roughness statistics. In addition, the model has been use to investigate the sensitivity to changes in the ice cover of the higher-order statistics such as cross-modal correlation, and spatial correlation in general. Comparing these theoretical sensitivity measures, we discuss the potential performance of both deterministic and statistic acoustic thermometry in the Arctic.

\section{2:45}

5pUW7. An efficient algorithm for calculating depth dependent modal phase and for mode identification in normal-mode models based on the Airy equation. J. LeMond and Robert A. Koch (Appl. Res. Labs., Univ. of Texas at Austin, Austin, TX 78713)

The underwater acoustic normal modes of multichannel environments may exhibit closely spaced eigenvalues that require a fine horizontal wavenumber sample in models based on the Airy equation. Also, for broadband applications the maximum frequency difference that permits accurate interpolation is limited by the frequency difference of the modal depth functions. A straightforward and numerically efficient algorithm to construct a monotonic depth-dependent phase using the properties of the Airy functions is presented that significantly reduces the computational burdens imposed by these constraints. The total phase change of a mode across the depth of the waveguide gives the mode number, modulo $\pi$, which is essential in adiabatic normal-mode calculations for range variable environments because the acoustic field must be propagated from one environment to the next mode by mode. [Work supported by the Advanced Surveillance and Prediction System (ASAPS) Program of the Space and Naval Warfare Systems Command (SPAWAR, PMW 181-14).]

\section{3:00-3:15 Break}

\section{$3: 15$}

5pUW8. Shallow-water time series simulation using normal-mode theory in range-independent and range-dependent environments. Geoffrey S. Royal, David P. Knobles, and Robert A. Koch (Appl. Res. Labs., Univ. of Texas at Austin, Austin, TX 78713)

Broadband normal-mode model analyses of propagation in a shallowwater range-independent environment and in a wedge environment are presented. The model is first used to calculate received time series from a point source for a range invariant shallow-water acoustic environment with a constant water sound-speed medium and a penetrable bottom. Range invariant mode model time series are compared to actual experimental data [C. Tindle et al., J. Acoust. Soc. Am. 81, 288-293 (1987)] and to time series that were calculated using a ray model that includes beam displacement [E. Westwood and C. Tindle, J. Acoust. Soc. Am. 81, 1752-1761 (1987)]. The time series from both the mode and ray models agree with each other and with the measured data. In addition, time series from an adiabatic mode model for a shallow-water wedge environment are compared to the experimental results obtained by C. Tindle et al. [Work supported by IR\&D Fund at Applied Research Laboratories, The University of Texas at Austin.]

\section{3:30}

5pUW9. Characterizing nonadiabatic ocean acoustic environments. R. L. Field, T. H. Ruppel, and M. K. Broadhead (Code 7173, Naval Res. Lab., Stennis Space Center, MS 39529-5004)

In range-dependent environments, the adiabatic assumption, which neglects discrete mode coupling, is often made. Up continental shelves and in shallow-water regions, abrupt changes in the environment make this assumption suspect. An issue in these environments is the degree to which the adiabatic assumption is valid. Transmission loss calculations using both a parabolic equation and coupled mode models show, that for steep slopes, much more energy reaches the shelf from deep water than predicted by the adiabatic approximation. This increase in shelf energy is shown to be due to discrete mode coupling. In this study, changes in energy flux with range are used as a criterion for when the adiabatic assumption breaks down. The flux is computed from a parabolic equation model in upslope environments as a function of range; frequency, and slope angle. It is shown that rapid flux variations are good predictors of when the adiabatic approximation breaks down and mode coupling becomes significant. [Work supported by ONR, Program Element 602435N, with technical management provided by the Naval Research Laboratory.]

\section{3:45}

5pUW10. Compound-matrix methods for computation of wave fields in horizontally layered fluid-solid media. Sven Ivansson (Dept. of Hydroacoust. and Seismol., Natl. Defence Res. Establishment (FOA), S-172 90 Stockholm, Sweden)

The wave field is decomposed into its frequency-wave-number components, and a compound-matrix ODE theory is formulated for multiregion fluid-solid media using multipoint boundary conditions. Forward propagation of compound entities is done to a matching depth, from which stabilized backward propagation to the receivers is performed. Wavenumber integration as well as modal synthesis is covered. Source arrays and receivers may be arbitrarily located. An appropriate adjoint problem is defined and solved. The formula for modal excitation coefficients is generalized to cover leaky modes, which have an exponential increase with depth. Three methods for stable and automatic computation of modal depth functions, in solid as well as fluid regions, are proposed. In work by M. B. Porter and E. L. Reiss [J. Acoust. Soc. Am. 77, 1760-1767 (1985)] the mode shapes in the solid bottom were left aside, and the method by $F$. Schwab et al. [Bull. Seismol. Soc. Am. 74, 1555-1578 (1984)] involves experimentation with a cutoff depth for an artificial homogeneous halfspace. For a medium composed of homogeneous layers, it is shown how efficient computations are obtained by writing the propagator matrices 
involved as products of sparse matrices, which are applied in sequence. Numerical stability can be achieved without artificially splitting thick homogeneous layers.

\section{4:00}

5pUW11. A time-dependent solution of a wave traversing a wedge by a finite length ping ray trace technique. Elmer White (Naval Res. Lab., Stennis Space Center, MS and 130 Moonraker Dr., Slidell, LA 70458)

A technique has been developed which demonstrates that an acoustic wave can be simulated using modified ray trace theory for acoustic modeling in shallow water. The resulting time-dependent wave and the transmission loss data closely compares to that benchmarked in the PE Workshop II Proceedings of the Second Parabolic Equation Workshop published in May 1993, edited by S. A. Chin-Bing, D. B. King, J. A. Davis, and R. B. Evans. The solution approximating test case 2, a range-dependent environment demonstrates forward and backscatter characteristics which agree with both PE and normal-mode theory in its behavior patterns. A VCR composite of time images generated by the code TRNG.F will be shown which demonstrates the sound wave pattern as it traverses a wedge. Transmission loss data are compared to PE generated TL to verify its accuracy.

\section{4:15}

5pUW12. Finding eigenrays in environments prone to numerical instability and chaos by directly optimizing the travel time integral. Martin A. Mazur and Kenneth E. Gilbert (Appl. Res. Lab. and the Graduate Prog. in Acoust., Penn State Univ., P.O. Box 30, State College, PA 16804)

In classical ray tracing, eigenrays are determined by a "shooting" approach whereby the launch angles of rays are varied until the rays intersect the receiver. In nonseparable range-dependent environments, the ray paths computed by conventional methods are sometimes chaotic, thereby putting a fundamental limit on the accuracy of ray tracing. Previous researchers [M. D. Collins and W. A. Kuperman, "Overcoming ray chaos," J. Acoust. Soc. Am. 95, 3167-3170 (1994)] have suggested that ray chaos can be overcome by recasting the problem in terms of Fermat's principle of minimum propagation time. The problem then becomes amenable to so-called "direct methods" of optimization theory. For the specialized duct investigated by Collins and Kuperman we have easily found eigenrays using a simple Rayleigh-Ritz method for directly minimizing the travel time integral. The structure of the ray equations for the duct suggests, however, that the numerical instability may actually be due to "stiffness" rather than chaos. To investigate chaos independently of nu- merical issues such as stiffness, we consider several nonseparable, rangedependent ducts with known piecewise analytic ray solutions. Results obtained from a shooting method and from Fermat's principle for several such ducts are presented and discussed. [Work supported by ONR.]

\section{4:30}

5pUW13. The acoustic wave equation as a finite difference. Michael L. Oelze (Dept. of Phys., Harding Univ., 900 East Ctr., Searcy, AR 72149-0001)

The classical wave equation used to describe acoustic phenomena was converted to a finite difference equation. This was done by constraining a sound packet to be in a box of finite length. By using certain mathematical techniques, recursion relations were found which enabled the amplitude of sound to be measured at every point in the box. This form of the equation is useful in modeling certain acoustic phenomena. The finite difference equation is especially useful in calculating wave reflection/transmission phenomena. The classical way of calculating reflection/transmission was by calculating a separate wave for each reflected or transmitted wave. The finite difference equation method cancels the need for many different waves and encompasses all interference from these waves.

\section{$4: 45$}

5pUW14. Time-domain propagation of signals in a strongly refractive shallow-water waveguide and the extraction of ocean properties. N. A. Sidorovskaia (Dept. of Phys., Univ. of New Orleans, New Orleans, LA), A. I. Khil'Ko (Russian Academy of Science, Nizshy Novgorod 603600, Russia), and M. F. Werby (Naval Res. Lab., Stennis Space Center, MS)

The complex pressure from a point source is calculated over the frequency range between 1 and $5 \mathrm{kHz}$ in steps of $0.2 \mathrm{~Hz}$ as a function of depth and range for a strongly ducted shallow-water waveguide. The waveguide is composed of an $80-\mathrm{m}$ water column over a fluid half-space defined by a compressional velocity of $1650 \mathrm{~m} / \mathrm{s}$ and a density of 1.6 relative to water. Attenuation in the bottom is $0.25 \mathrm{~dB} / \lambda$. The velocity in the water column varies from 1540 at the surface to $1513 \mathrm{~m} / \mathrm{s}$ at the bottom where a pronounced gradient exists between 30 and $60 \mathrm{~m}$, with the remaining regions being weakly dependent on depth. A series of time-domain calculations are carried out from the stored frequency data for pulses in terms of (1) pulse widths, (2) central frequency, (3) depth of detection, and (4) range of detection. The central notion is that the pulse amplitude is strongly correlated with depth due to ducting effects. Further, pulse widths and the principle arrival time adds more information on ocean properties such as the velocity profile and some aspect of seafloor geoacoustic characteristics. Unambiguous systematics that suggest a tool useful for extraction of ocean data are reported. [Work sponsored by ONR.] 